Prepared in cooperation with the Indiana Department of Environmental Management

\title{
Low-Flow Characteristics for Selected Streams in Indiana
}

By Kathleen K. Fowler and John T. Wilson

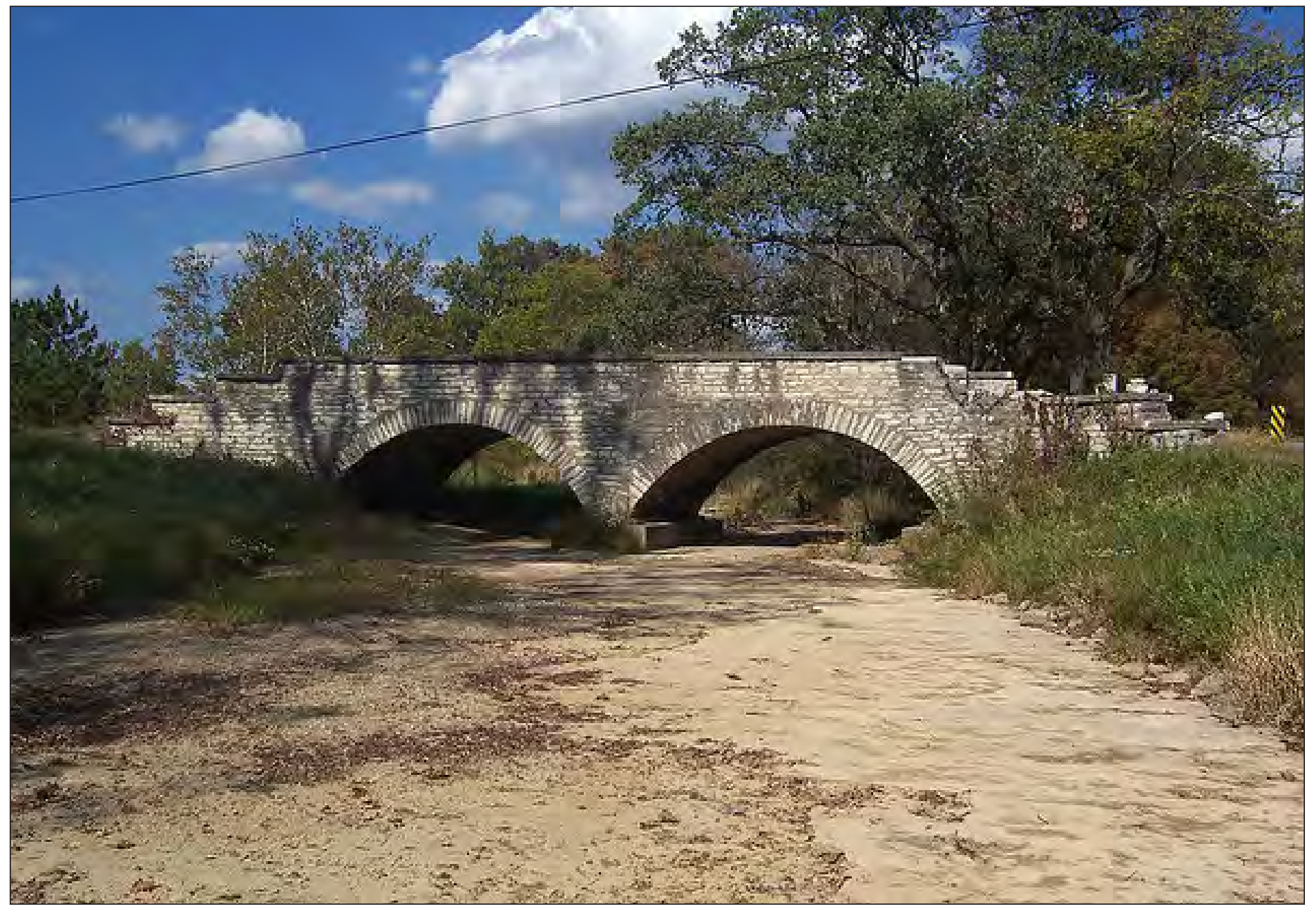

Scientific Investigations Report 2014-5242 
Cover: Shepard Bridge on Old Michigan Road over Big Creek in Ripley County, Indiana. Photo by Jim Grey, 2008. More information available at http://bridgehunter.com/in/ripley/6900032/. 


\section{Low-Flow Characteristics for Selected Streams in Indiana}

By Kathleen K. Fowler and John T. Wilson

Prepared in cooperation with the Indiana Department of Environmental Management

Scientific Investigations Report 2014-5242 


\title{
U.S. Department of the Interior SALLY JEWELL, Secretary
}

\section{U.S. Geological Survey Suzette M. Kimball, Acting Director}

\author{
U.S. Geological Survey, Reston, Virginia: 2015
}

For more information on the USGS - the Federal source for science about the Earth, its natural and living resources, natural hazards, and the environment-visit http://www.usgs.gov or call 1-888-ASK-USGS.

For an overview of USGS information products, including maps, imagery, and publications, visit http://www.usgs.gov/pubprod/.

To order this and other USGS information products, visit http://store.usgs.gov/.

Any use of trade, firm, or product names is for descriptive purposes only and does not imply endorsement by the U.S. Government.

Although this information product, for the most part, is in the public domain, it also may contain copyrighted materials as noted in the text. Permission to reproduce copyrighted items must be secured from the copyright owner.

Suggested citation:

Fowler, K.K., and Wilson, J.T., 2015, Low-flow characteristics for selected streams in Indiana: U.S. Geological Survey Scientific Investigations Report 2014-5242, 353 p., http://dx.doi.org/10.3133/sir20145242.

ISSN 2328-0328 (online) 


\section{Contents}

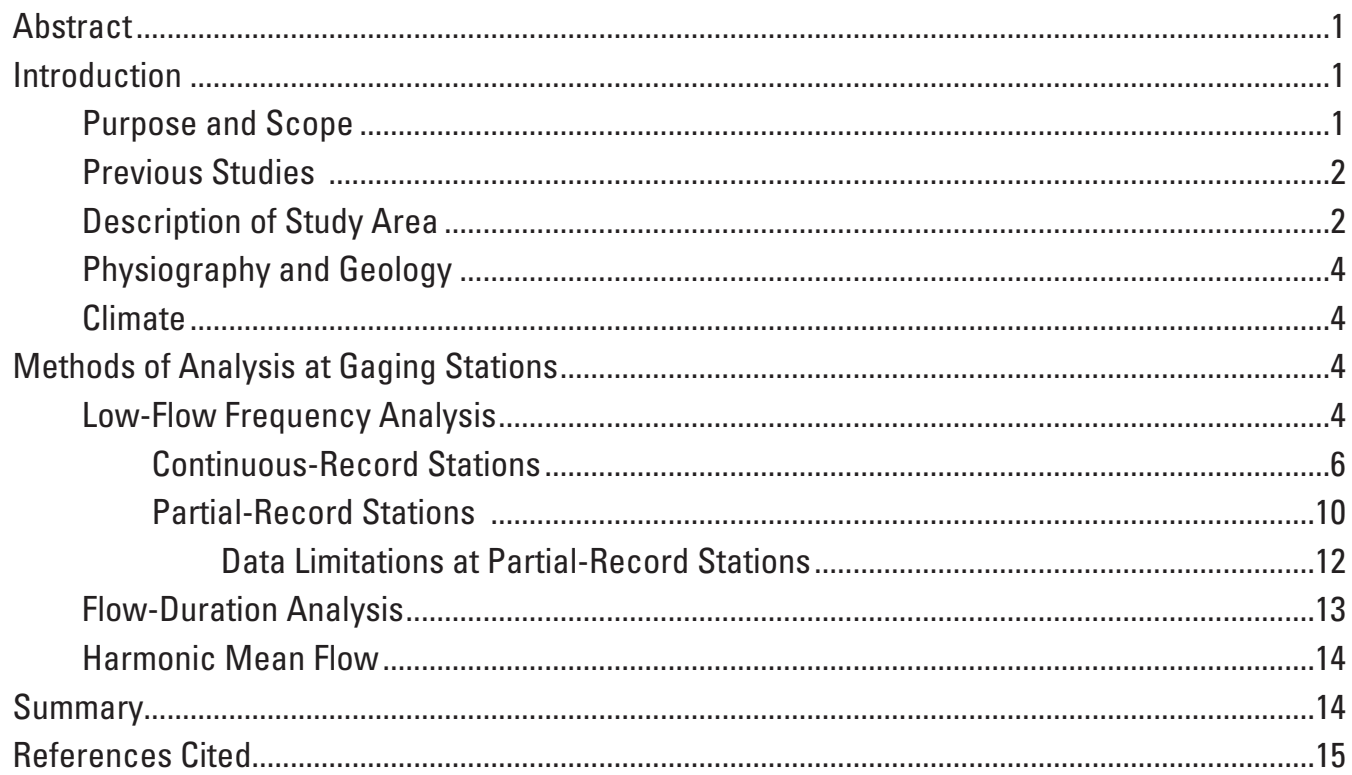

\section{Figures}

1. Map showing physiographic regions and major drainage basins in Indiana ....................3

2. Graph showing average annual precipitation and temperature in Indiana, by climatic division, for the period 1981-2010.

3. Graph showing part of a hypothetical annual hydrograph showing periods for which the daily discharges are averaged to give the 1-day, 7-day, and 30-day low flows for

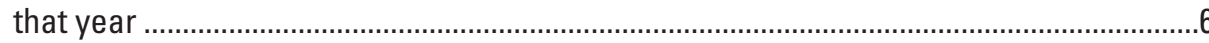

4. Map showing continuous-record streamgaging stations in Indiana ...............................7

5. Graph showing continuous-record streamgaging stations in Marion County, Indiana ...8

6. Graph showing low-flow frequency curve for Whitewater River near Hagerstown,

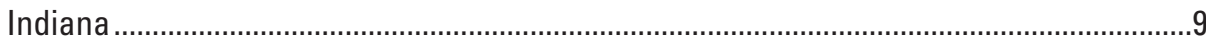

7. Map showing partial-record streamgaging stations in Indiana .....................................11

8. Graphical method of estimating low-flow characteristics at a partial-record station from known characteristics at a continuous-record station ...............................12

9. Graph showing flow-duration curves for a stream in southern Indiana (Big Creek) and a stream in northern Indiana (Pigeon Creek) ..........................................................13

\section{Tables}

1. Low-flow characteristics for continuous-record streamgaging stations in Indiana .......17

2. Low-flow characteristics for partial-record streamgaging stations in Indiana.............290

Tables 1 and 2 in Excel file format are available at http://dx.doi.org/10.3133/sir20145242. 


\section{Acknowledgments}

The authors wish to thank the many local, State, and Federal agencies that have cooperated in the funding for the operation and maintenance of the gages used for this study, especially the U.S. Army Corps of Engineers - Louisville District, the Indiana Department of Natural Resources, and the Indiana Department of Environmental Management. Special thanks are given to Indiana Department of Environmental Management for their cooperation in this study.

\section{Conversion Factors}

Inch-pound to International System of Units (SI)

\begin{tabular}{|c|c|c|}
\hline Multiply & By & To obtain \\
\hline \multicolumn{3}{|c|}{ Length } \\
\hline foot $(\mathrm{ft})$ & 0.3048 & meter $(\mathrm{m})$ \\
\hline mile (mi) & 1.609 & kilometer $(\mathrm{km})$ \\
\hline \multicolumn{3}{|c|}{ Area } \\
\hline square mile $\left(\mathrm{mi}^{2}\right)$ & 2.590 & square kilometer $\left(\mathrm{km}^{2}\right)$ \\
\hline \multicolumn{3}{|c|}{ Flow rate } \\
\hline cubic foot per second $\left(\mathrm{ft}^{3} / \mathrm{s}\right)$ & 0.02832 & cubic meter per second $\left(\mathrm{m}^{3} / \mathrm{s}\right)$ \\
\hline
\end{tabular}

Horizontal coordinate information is referenced to the North American Datum of 1927 (NAD 27) or 1983 (NAD 83) as noted. 


\title{
Low-Flow Characteristics for Selected Streams in Indiana
}

\author{
By Kathleen K. Fowler and John T. Wilson
}

\section{Abstract}

The management and availability of Indiana's water resources increase in importance every year. Specifically, information on low-flow characteristics of streams is essential to State water-management agencies. These agencies need lowflow information when working with issues related to irrigation, municipal and industrial water supplies, fish and wildlife protection, and the dilution of waste. Industrial, municipal, and other facilities must obtain National Pollutant Discharge Elimination System (NPDES) permits if their discharges go directly to surface waters. The Indiana Department of Environmental Management (IDEM) requires low-flow statistics in order to administer the NPDES permit program. Low-flowfrequency characteristics were computed for 272 continuousrecord stations. The information includes low-flow-frequency analysis, flow-duration analysis, and harmonic mean for the continuous-record stations. For those stations affected by some form of regulation, low-flow frequency curves are based on the longest period of homogeneous record under current conditions. Low-flow-frequency values and harmonic mean flow (if sufficient data were available) were estimated for the 166 partial-record stations. Partial-record stations are ungaged sites where streamflow measurements were made at base flow.

\section{Introduction}

The management and availability of Indiana's water resources increase in importance every year. Knowledge of the magnitude and frequency of low flows for streams is essential for water-supply planning and design, waste-load allocation, reservoir design, maintenance of aquatic life, and understanding the quantity and quality of water that is used for irrigation and recreation. Industrial, municipal, and other facilities must obtain National Pollutant Discharge Elimination System (NPDES) permits if their discharges go directly to surface waters (U.S Environmental Protection Agency, 2012a). As authorized by the Clean Water Act, the NPDES permit program limits water pollution by regulating point sources that discharge pollutants into waters of the United States. In Indiana, the NPDES permit program is administered by the
Indiana Department of Environmental Management (IDEM) on the basis of low-flow statistics.

The U.S. Geological Survey (USGS), in cooperation with State and Federal agencies, has collected daily streamflow data in Indiana since 1928. This continually growing data base is important in all aspects of water-resources investigations. Presented in this report are the low-flow characteristics for 272 continuous-record stations and 166 partial-record stations in Indiana. The information includes low-flow-frequency analysis (the annual 1-day, 10-year, 1Q10; the 7-day, 10- year, 7Q10; and the 30-day, 10-year, 30Q10 low flows), flow-duration analysis, and harmonic mean flow computations for the continuous-record sites. For the 166 partial-record stations, lowflow-frequency statistics (1Q10, 7Q10, and 30Q10 low flows) and the harmonic mean flow were estimated. Partial-record stations are ungaged sites where streamflow measurements were made at base flow. In this report "ungaged" means sites where there is no device that records the stage of the river or stream. The same low-flow characteristics estimated at partial-record stations were estimated for continuous-record stations where less than 10 years of record were available. Low-flow characteristics are based on available data through September 2011, except for selected short-term continuous-record stations, where data extending through September 2013 were used.

The analysis of low-flow characteristics of Indiana streams described and presented in this report was undertaken by the USGS in cooperation with IDEM.

\section{Purpose and Scope}

The purpose of this report is to present low-flow characteristics for 152 active continuous-record gaging stations, 120 inactive continuous-record gaging stations, and 166 partialrecord stations at Indiana streams. This information is needed for management decisions by State and local officials concerned with water supplies, pollution management, and fish and wildlife preservation. In particular, this report develops low-flow statistics needed by IDEM for purposes including the development of Total Maximum Daily Loads (TMDLs), 305b/303d programs (U.S. Environmental Protection Agency, $2012 b$ ), watershed planning, and the administration of the Indiana NPDES permit program. 
Low-flow characteristics were determined for 230 continuous-record stations with at least 10 years of record. An additional 42 continuous-record stations have less than 10 years of record; these sites were compared with an index site, and low-flow characteristics were estimated by correlation of concurrent flows at the short-term and long-term (index) stations. Continuous-record stations are streamgaging stations for which daily streamflow is computed and stored. Most of these stations currently are active; some have been discontinued. Natural flow conditions, based on the entire period of record, are represented for most sites. The analyses for stations at which flows are affected by significant regulation used only the period of record affected by regulation; the natural flow conditions prior to regulation were not included in the frequency, harmonic mean, and flow-duration analyses for regulated streams. For this report, streamflow is considered regulated if more than 10 percent of the contributing drainage area is subject to significant control of discharge (such as the control of outflow from reservoirs). When a stream is significantly regulated, the remarks section of table 1 specifies that only the current regulated period is used. Activities such as occasional water withdrawals, mining operations, or discharges into a stream also can affect flow but are not considered significant. Continuous-record stations with less than 10 years of record were treated as partial-record stations for estimating low-flow frequency and harmonic mean flows. The average daily discharge and percentiles for the flow-duration curve are provided for all of the continuous-record stations regardless of the length of the period of record, including records having less than 10 years. Frequency analysis for each of the continuous-record stations was done by fitting a logPearson type III curve to observed annual minimum data and by making a graphical adjustment to the fitted curve where the plotted data points and curve did not agree closely (Riggs, 1972). Calculations were based on the climatic year (April 1 through March 31).

Daily flow-duration information includes the percentage of days a particular discharge was equaled or exceeded. Duration tables for each continuous-record station were computed with the daily mean discharges for the entire period of record of complete water years. For those continuous-record stations having significant regulation, only the current, regulated period of record was used.

The terms "flow," "streamflow," and "discharge" are synonymous and are used interchangeably in this report. All three terms refer to the volume of water that passes a given point within a given period of time; all are expressed in units of cubic feet per second $\left(\mathrm{ft}^{3} / \mathrm{s}\right)$.

\section{Previous Studies}

Previous low-flow investigations of Indiana streams by the USGS include those by Hoggatt (1962), Rohne (1972), Stewart (1983), and Fowler and Wilson (1996). This report is similar to and is an update of these earlier reports. Arihood and Glatfelter (1986) presented a method for estimating 7-day low flows at the 10- and 2-year recurrence intervals (7Q10 and 7Q2) for ungaged streams in central and northern Indiana, using equations that require the contributing drainage area and a flow-duration ratio for the area of interest. The ArihoodGlatfelter equations were developed on the basis of the flow characteristics published in Stewart (1983). Arvin (1989) presented statistical summaries for streamflow data in Indiana that included flow-duration tables and annual low flows for selected consecutive days.

Statistical summaries like those presented in Arvin (1989) are available online for selected stations and are based on daily mean discharge through September 2006 (http://in.water.usgs. gov/dvstats/).

\section{Description of Study Area}

The State of Indiana spans 36,418 square miles $\left(\mathrm{mi}^{2}\right)$ in the east-central United States. The study area (fig. 1) consists of the entire State. The major drainage basins in Indiana (fig. 1) are the Great Lakes Drainage Basin, which includes the St. Joseph Basin, the Lake Michigan Basin, and the Maumee Basin; the Upper Mississippi River Basin, which includes the Kankakee Basin; the Wabash Drainage Basin, which includes the upper, middle, and lower Wabash Basins, the West Fork White Basin, the East Fork White Basin, and the Patoka Basin; and the Ohio River Tributary Drainage Basin, which includes the Whitewater Basin and minor tributaries to the Ohio River (Ohio Basin).

Nearly eighty percent of Indiana is drained by streams that discharge into the Ohio River. The largest river in Indiana, the Wabash, drains 32,910 $\mathrm{mi}^{2}$ (including parts of Ohio and Illinois). The White River, a tributary to the Wabash, has two subbasins of nearly equal size - the West Fork and the East Fork - with a total drainage area of $11,349 \mathrm{mi}^{2}$. The Whitewater River, which drains $1,369 \mathrm{mi}^{2}$, discharges into the Great Miami River in southern Ohio. The Kankakee and Iroquois Rivers are part of the Illinois River drainage; they drain about 7 percent of Indiana $\left(2,581 \mathrm{mi}^{2}\right)$ and flow westward into Illinois. Approximately 10 percent of Indiana is drained by three rivers that are part of the Great Lakes Basin. The Calumet and St. Joseph Rivers drain into Lake Michigan, and the Maumee River drains into Lake Erie. Both Lake Michigan and Lake Erie are part of the Saint Lawrence River drainage. Streams in the extreme south and southeast area of the State drain directly into the Ohio River. 


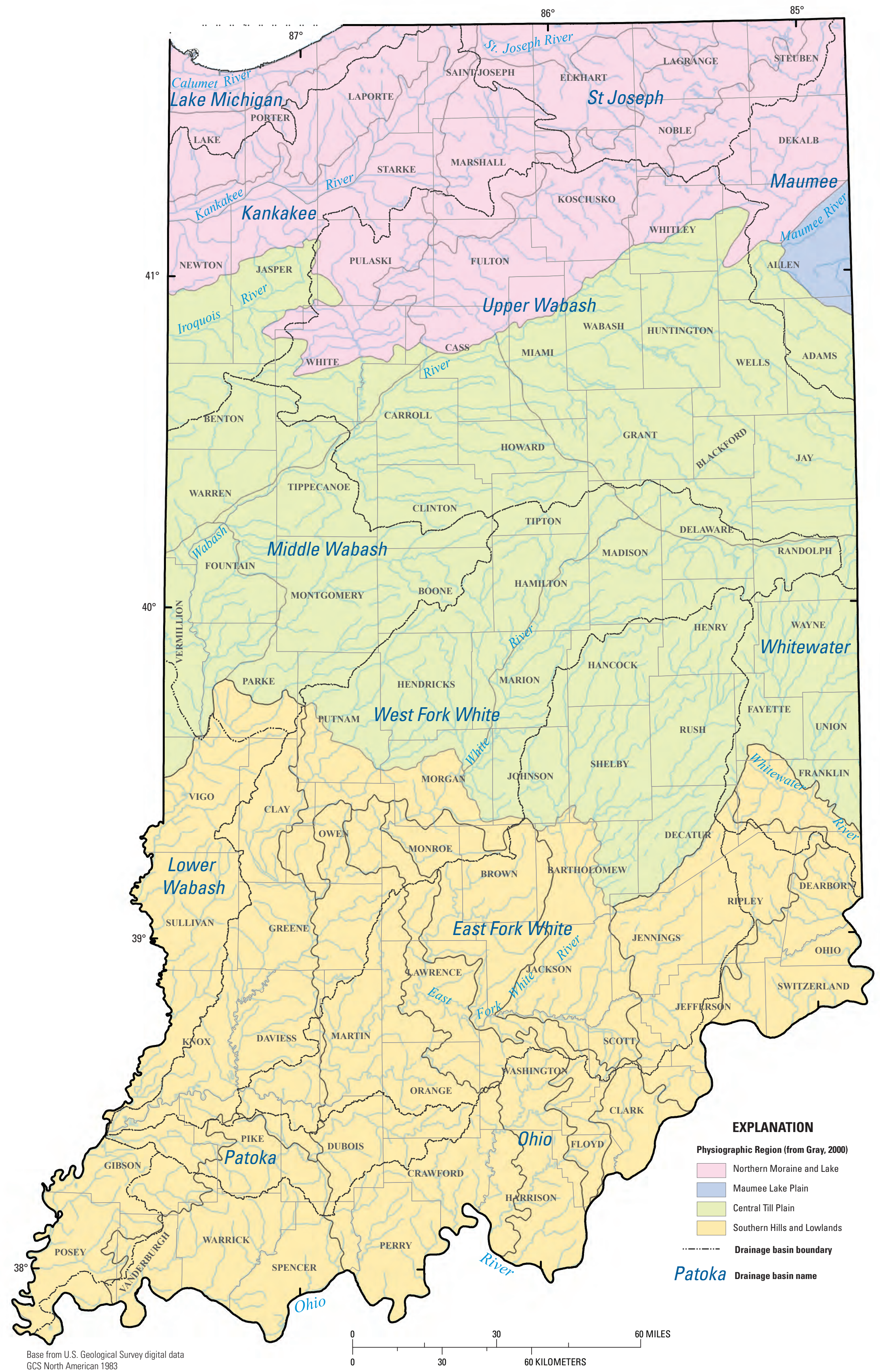




\section{Physiography and Geology}

Indiana can be divided into four broad physiographic zones that are based on similarities in topography and geology (Gray, 2000) (fig. 1). The Northern Moraine and Lake Region is mostly glacial in origin and generally has more relief than the central zone. The Central Till Plain Region is a depositional plain of low relief underlain by thick glacial till that has been modified by postglacial stream erosion. The small eastern zone, the Maumee Lake Plain Region, is part of a larger zone in Ohio that is fairly flat with a few low ridges of beach silt and sand. Landforms in the southern zone, the Southern Hills and Lowlands Region, are formed from degradational processes, such as weathering and stream erosion. A detailed description of the subdivisions of each region can be found in Indiana Geological Survey Special Report 61, "Physiographic Divisions of Indiana" (Gray, 2000).

Precipitation and geology directly influence the streams in Indiana. Streams in the northern, glaciated part of the State tend to have higher sustained base flows than those in the nonglaciated southern part.

\section{Climate}

Indiana climate is classified as continental and temperate; the summers tend to be hot and humid, whereas winters tend to be cold and damp. The transitional seasons of spring and fall are prone to frequent changes in weather. The growing season extends approximately from April through October. A well-defined, north-south climatic gradient across the State provides a cool, temperate climate in the north and a warm, temperate climate in the south. High humidity and frequent variations in temperature are characteristic.

Average annual precipitation ranges from 38.1 inches in northeast Indiana to 46.6 inches in the south-central area (fig. 2; National Oceanic and Atmospheric Administration, 2014). May is typically the wettest month of the year, with average rainfall between 4 and 5 inches across the State. Average rainfall decreases slightly as summer progresses. Autumn months are drier, with 3 inches of rainfall typical in each month. Indiana winters are the driest time of year, with less than 3 inches of precipitation commonly received each month. February is the driest month of the year statewide, then precipitation increases in March and April as the spring soilmoisture recharge season begins.

Annual precipitation is usually adequate for water needs, but an uneven distribution in the summer can limit crops. Mild to severe droughts occasionally occur in the summer when evaporation is highest and dependence on rainfall is greatest for crops. Groundwater storage is generally abundant in the north and central areas where there are glacial deposits. Underlying bedrock with shallow soils limits ground water storage in much of south-central Indiana (Scheeringa, 2002).
The average annual precipitation in figure 2 shows that precipitation increased from the period 1961-1990 (Fowler and Wilson, 1996) to the period 1981-2010. On average, annual precipitation increased about 1.7 inches in the northern divisions, 2.2 inches in the central divisions, and 1.3 inches in the southern divisions.

Average annual temperatures range from 49.9 degrees Fahrenheit $\left({ }^{\circ} \mathrm{F}\right)$ in northeast Indiana to $54.9^{\circ} \mathrm{F}$ in southwest Indiana. January is typically the coldest month of the year, with normal daily maximum temperatures ranging from 31 to $38^{\circ} \mathrm{F}$ north to south across Indiana. Normal January minimums range between 15 and $21^{\circ} \mathrm{F}$ north to south. July is the warmest month, with daily maximums averaging 80 to $83^{\circ} \mathrm{F}$ and minimums 63 to $65^{\circ} \mathrm{F}$ north to south (Scheeringa, 2002). The average annual temperatures in figure 2 show the temperatures have slightly increased from the period 1961-1990. In the northern divisions, temperature has increased about $0.6^{\circ} \mathrm{F}$; in the central division, $0.8^{\circ} \mathrm{F}$; and in the southern divisions, $0.1^{\circ} \mathrm{F}$.

\section{Methods of Analysis at Gaging Stations}

Streamflow is a continuous process that varies over time. Streamflow characteristics in river basins can change in response to climate changes and human alteration such as the installation of reservoirs and dams, withdrawals for water supply, and inputs from wastewater treatment facilities. These changes can affect low-flow statistics. For this study, low-flow characteristics were determined by frequency analysis, flowduration analysis, and harmonic mean computation.

\section{Low-Flow Frequency Analysis}

Frequency curves relate the magnitude of a variable to the frequency of occurrence (Riggs, 1968). In low-flow investigations, the frequency curves relate the minimum average discharge for a given number of consecutive days ( $N$-day) to the recurrence interval in years ( $T$-year). To compute return period low-flow values, such as $7 \mathrm{Q} 10$, an annual time series of average consecutive 7-day minimum discharges was calculated for all continuous-record streamgaging stations.

For example, the 7-day, 10-year low flow (7Q10) is the minimum average discharge for 7 consecutive days, which has a 0.1 probability of not being exceeded in a given year. The recurrence interval is the reciprocal of the probability of recurrence. The recurrence interval also may be defined in general terms as the average interval of time between occurrences of low-flow events (for example, the 10-year low flow). Figure 3 is an example of how the 1-day, 7-day, and 30-day periods of minimum average discharge may look on a hydrograph. 


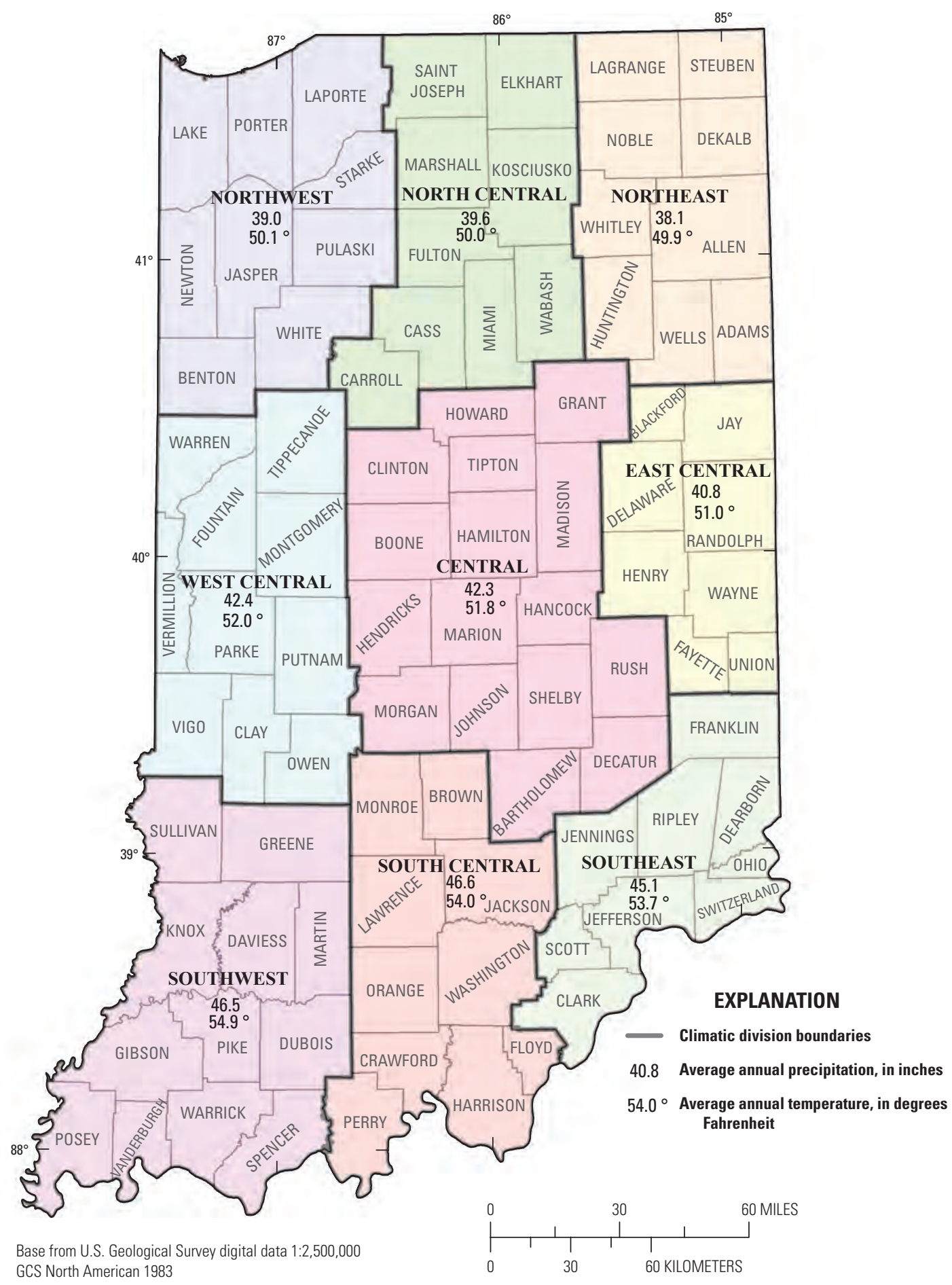

Figure 2. Average annual precipitation and temperature in Indiana, by climatic division, for the period 1981-2010. 


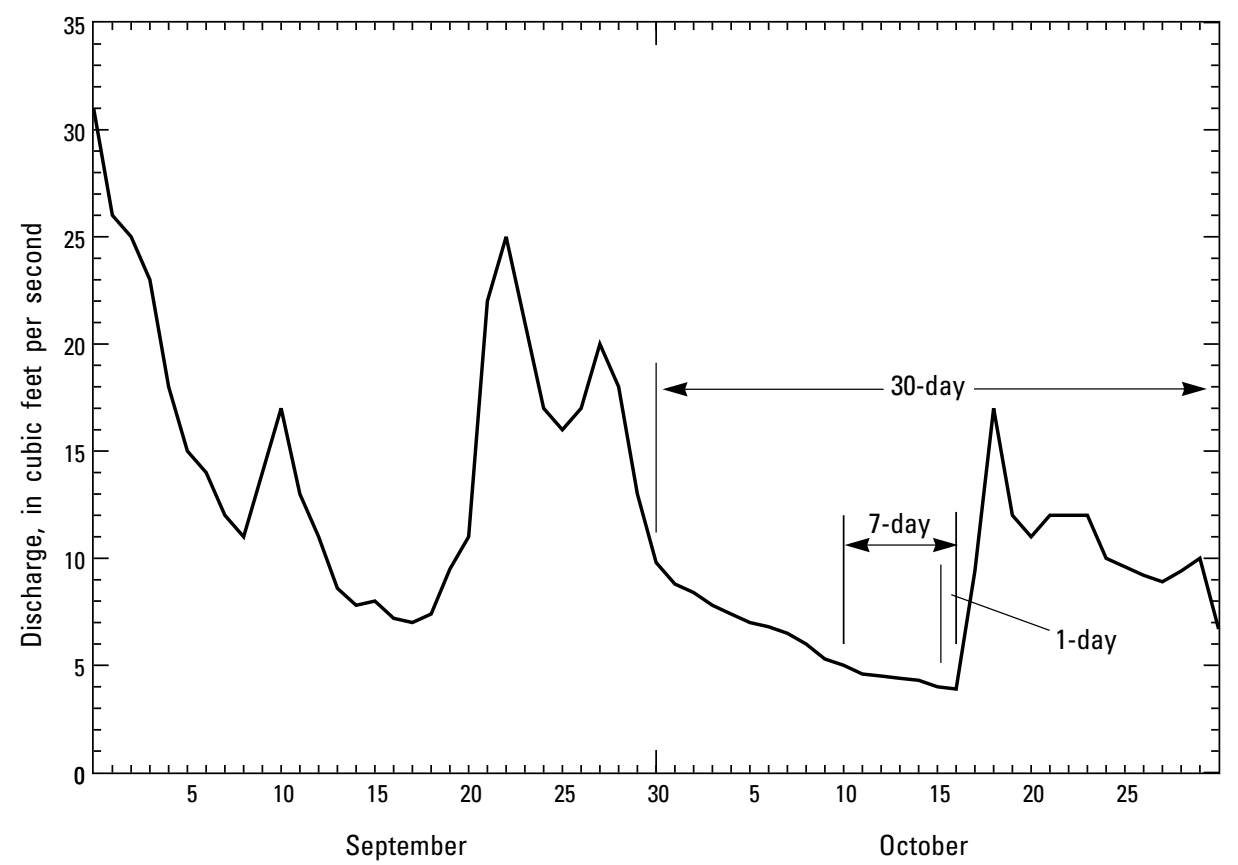

Figure 3. Part of a hypothetical annual hydrograph showing periods for which the daily discharges are averaged to give the 1-day, 7-day, and 30-day low flows for that year.

\section{Continuous-Record Stations}

Frequency analyses were computed for 230 continuousrecord stations in Indiana with at least 10 years of record (figs. 4 and 5). Frequency curves were defined by using a mathematical procedure for fitting the data to a log-Pearson type III distribution (Riggs, 1972). Figure 6 is an example of a frequency curve of 7-day low flows. Selected $N$-day, $T$-year low-flow characteristics can be computed from the following equation

$$
X_{T}=\bar{X}+K S
$$

where

$$
\begin{array}{cl}
X_{T} & \text { is selected } N \text {-day, } T \text {-year low flow, } \\
\bar{X} & \text { is mean of the logarithms of the } N \text {-day values, } \\
K & \text { is Pearson type III frequency factor for the } \\
& \text { value of skew coefficient computed for } \\
\text { the data and a recurrence interval of } T \\
\text { (nonexceedance probability of } p=1 / T \text { ), } \\
\text { and } \\
\text { is standard deviation of the logarithms of the } \\
N \text {-day values. }
\end{array}
$$

The USGS Surface-Water Statistics (SWSTAT) software was used to select annual minimum flows; compute the mean, standard deviation, and coefficient of skew; plot the fitted logPearson type III probability distribution; and plot the selected minimum flows versus recurrence intervals (Hutchison, 1975;
Lumb and others, 1990; Flynn and others, 1995). Plotting positions were determined from the equation

$$
R I=(n+1) / m
$$

where

$$
\begin{aligned}
R I & \text { is recurrence interval in years, } \\
n & \text { is number of years of record, and } \\
m & \text { is numerical rank, with the lowest flow being } 1 .
\end{aligned}
$$

This procedure produced acceptable fits to most sets of low-flow data; however, certain low-flow-frequency curves could not be fitted adequately for a complete dataset. Where the data points deviated significantly from the log-Pearson type III curve, a curve was drawn manually through the data points to provide better estimates of the flow characteristics. This method of graphical interpretation is illustrated in Riggs $(1972$, p. 5). The log-Pearson type III fitting procedure works best when the absolute value of skew of the logs is close to zero. (Skew is a measure of asymmetry of a frequency distribution.) In a few cases, calculations were not made because the $N$-day values varied over a large enough range that the absolute value of skew exceeded allowable limits. This situation typically occurred when extreme regulation dropped discharge for a short period of time to a fraction of the normal discharge. About 12 percent of the continuous-record stations had at least one of the three curves adjusted. Values determined from frequency analysis that were less than or equal to $0.05 \mathrm{ft}^{3} / \mathrm{s}$ were rounded to zero; those that were between 0.05 and $0.1 \mathrm{ft}^{3} / \mathrm{s}$ were rounded to $0.1 \mathrm{ft}^{3} / \mathrm{s}$. 


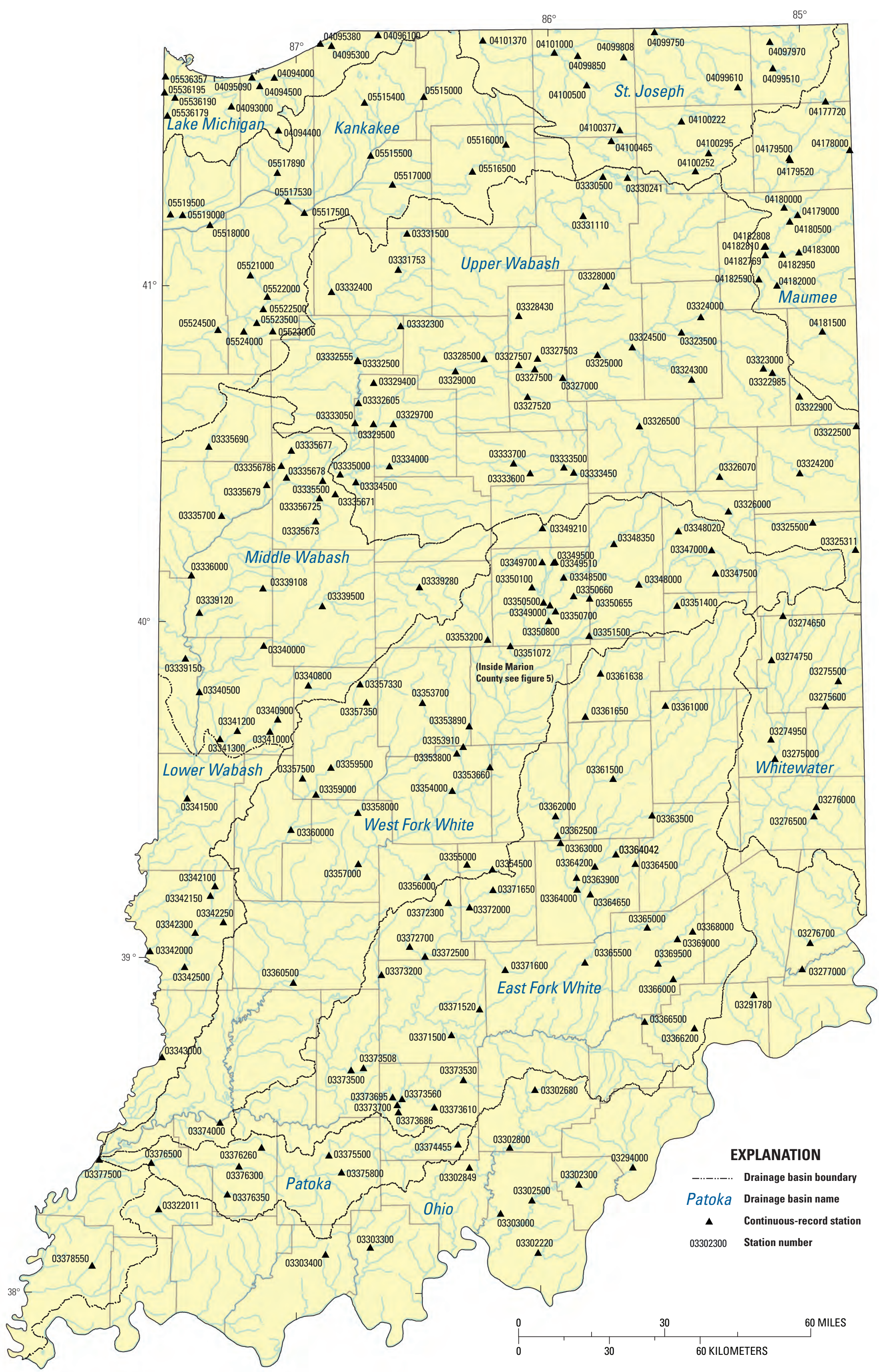

Base from U.S. Geological Survey digital dato

Base from U.S. Geological S
GCS North American 1983 


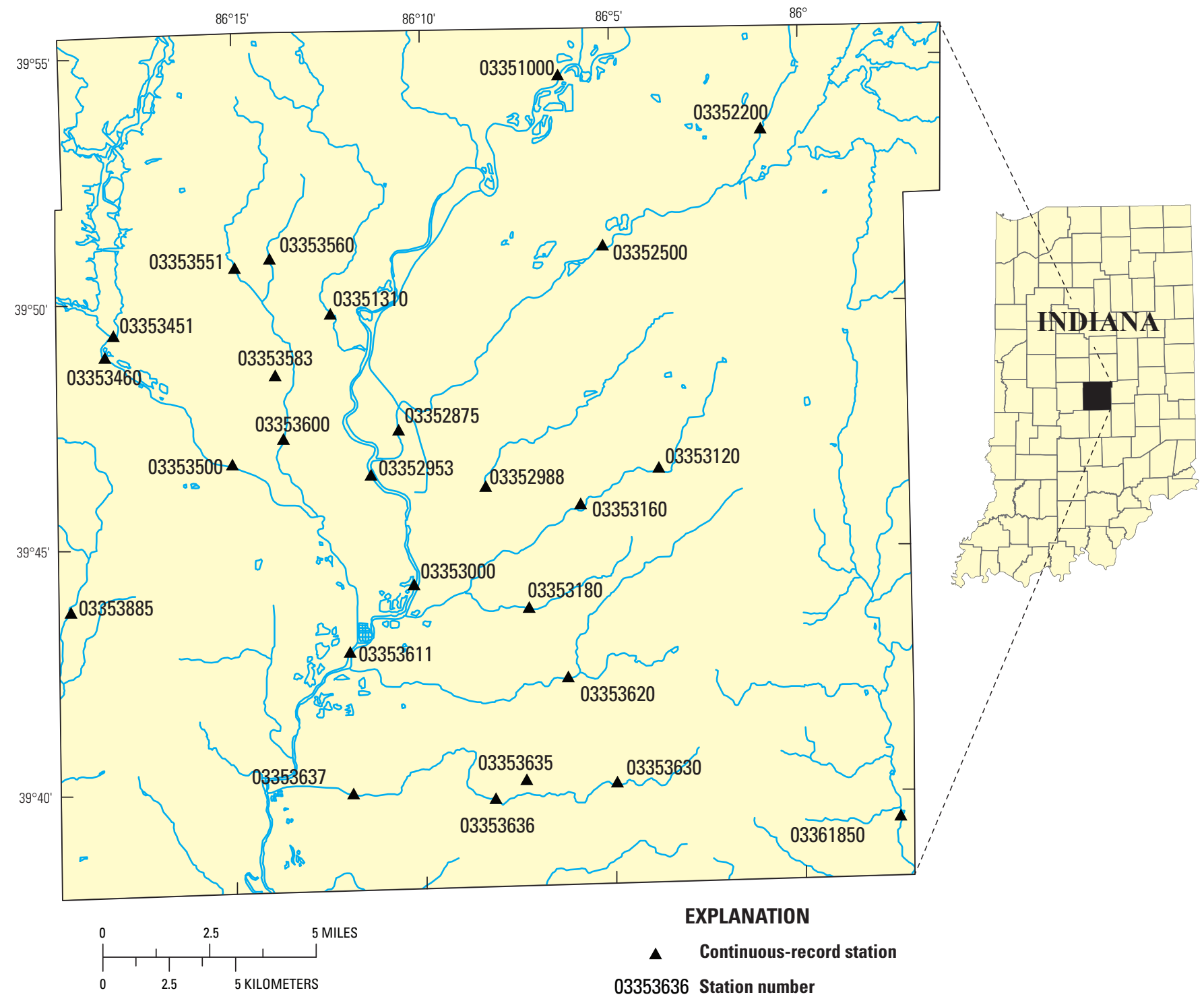

Figure 5. Continuous-record streamgaging stations in Marion County, Indiana. 


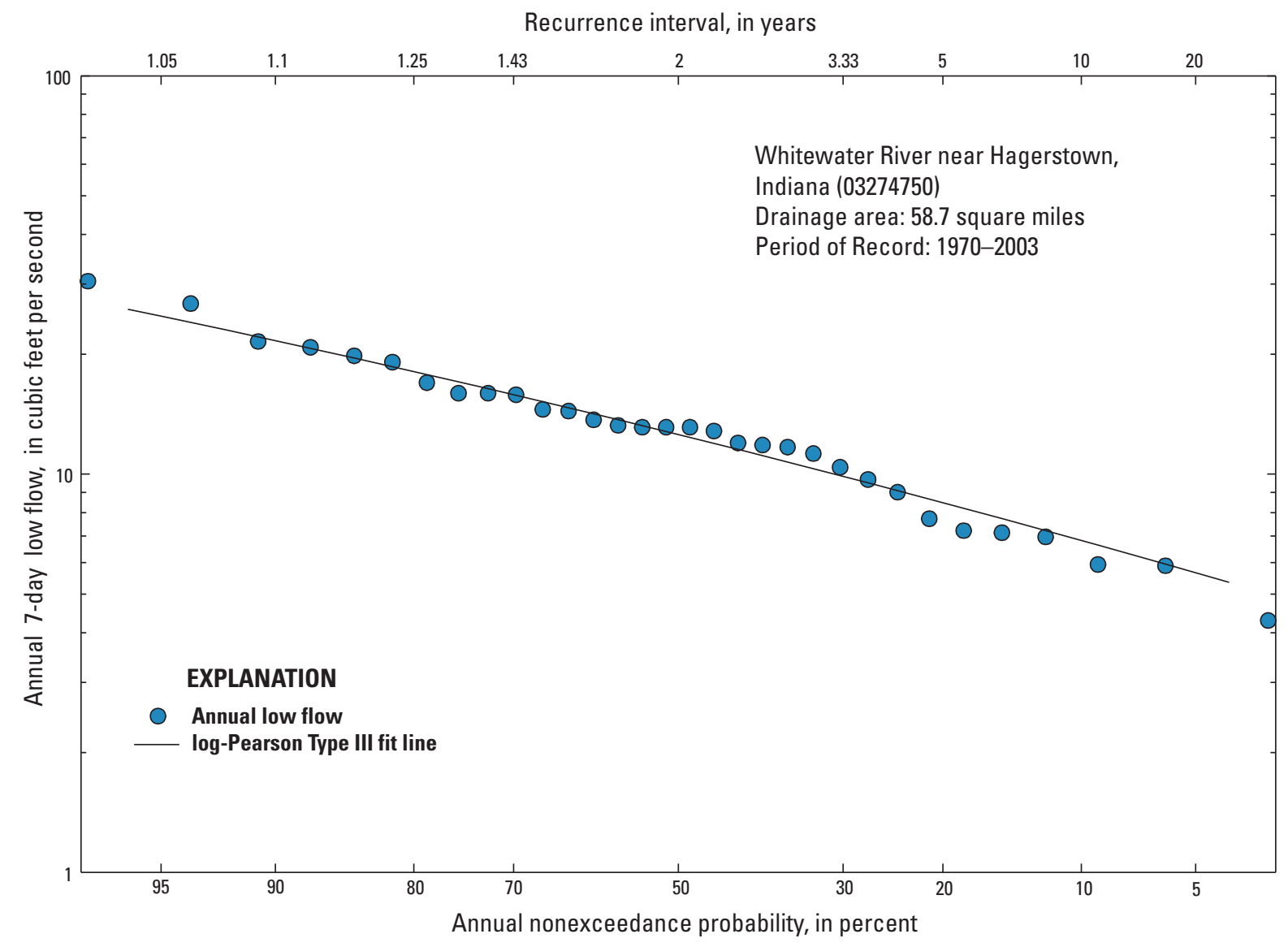

Figure 6. Low-flow frequency curve for Whitewater River near Hagerstown, Indiana. 
Riggs (1972) indicated that the reliability of a low-flow frequency curve is closely related to the length of record. Ideally, the record would be representative of the long-term-flow characteristics. In general, the longer the period of record, the more reliable the low-flow estimates. The period of record is listed in table 1 for all the continuous-record stations, several of which have been discontinued. Low-flow characteristics for stations with less than 10 years of record were estimated with the methods described in the section "Partial-Record Stations." The estimated low-flow characteristics at a given station will change as the period of record changes, especially if drought years or unusually high flow years are added to the record. A comparison was made between 126 continuous-record stations (with comparable periods of record) to look at the difference between the calculated 7Q10 values of the 1996 study and this study. The 7Q10 was selected because it is the most commonly used hydrologically based frequency value. Sixty-four of the stations (52 percent) showed a positive change in the flow value, with a mean relative percent difference (RPD) of 8.8 percent. Thirty-six stations (28 percent) showed a negative change in the flow value, with a mean RPD of -15 percent. Twenty-six stations (20 percent) showed no change.

Several continuous-record stations are affected by some form of regulation or diversion, which is noted in the "Remarks" section of the affected stations in table 1. For those stations affected by some form of regulation, low-flow frequency curves are based on the longest period of homogeneous record under current conditions.

\section{Partial-Record Stations}

Partial-record stations and continuous-record stations with less than 10 years of streamflow record have insufficient data for fitting the log-Pearson distribution. Partial-record stations are ungaged sites at which measurements of base flow are made. The base flows are related to the concurrent daily mean flows of a nearby continuous-record station (index station) for which a low-flow frequency curve has been defined. Riggs (1972) indicated that, generally, 8 to 10 measurements made on different streamflow recessions and in more than 1 year should define the relation to concurrent flows at the index station. Riggs (1972) also indicated that fewer measurements may suffice if some were made during a significant drought. Ideally, the watershed for the index station should be of similar terrain, drainage area, and geologic characteristics as that for the partial-record station. With this in mind, regional physiography (fig. 1), the flow-duration ratio map of Arihood and Glatfelter (1986), and proximity were considered during the selection of index stations. The location of the partialrecord stations is shown in figure 7.

Low-flow characteristics at partial-record stations were estimated by use of the mathematical technique described in Stedinger and Thomas (1985) or by the graphical correlation methods described in Riggs (1972). In both techniques, a relation between base-flow measurements at the partial-record stations and concurrent daily flows at index stations (using logarithms of flows) is developed. The Stedinger-Thomas technique defines the relation by means of least-squares regression analysis. The regression analysis and low-flow statistics at the index station are used to estimate the desired flow characteristics at the partial-record station. One of the key assumptions of the Stedinger-Thomas technique is that the frequency factor for the index station and the frequency factor for the partial-record station are the same. It is assumed that the frequency factors will be approximately equal if the sites are in similar hydrologic settings and have similar drainage areas. This is an important assumption of the method that must be considered when choosing index stations.

The Stedinger-Thomas technique was used as the primary method for estimating low-flow characteristics at the partialrecord stations because it is automated and, therefore, easily reproduced. However, hydrologic judgment is needed when selecting which measurements and concurrent daily flows to use because outliers can affect the slope of the linear regression. Ideally, it is recommended that there be at least 10 baseflow measurements at the partial-record station and that there be a linear relation between the base flows and the concurrent daily mean flows at the index station. Stedinger and Thomas (1985) recommend that the correlation coefficient exceed 0.70 .

Also, the Stedinger-Thomas technique uses logarithms of flows; zero flows cannot be used.

The Stedinger-Thomas technique was originally designed to estimate only the 7 Q10 but has been shown to adequately estimate other flow frequencies (1Q10 and 30Q10) (Thompson and Hoffman, 2006). The Stedinger-Thomas technique has been automated by the USGS and has the capability of using multiple index stations. The Stedinger-Thomas technique for partial-record stations is included in the regional hydrologic regression and NETwork analysis using Generalized Least Squares (GLSNet) software (Tasker and Stedinger, 1989; Flynn and others, 1995).

The graphical correlation method (Riggs, 1972) was used to estimate low-flow characteristics for the partial-record stations with zero flows or where the relation between base flows and concurrent daily mean flows was not well defined. Base-flow measurements at the partial-record station are plotted on log-log scale against concurrent daily mean flows at the index station, and a best-fit line relating the two is drawn. Points from the frequency curve defined for the index station are transferred through the best-fit line to obtain estimates of analogous frequency points at the partial-record station. An example of an application of the graphical method is shown in figure 8 .

The procedures outlined above also were used to estimate low-flow characteristics at continuous-record stations with less than 10 years of record (table 1). At some stations with a short period of record, daily values during base flow were used to augment the discharge measurements. 


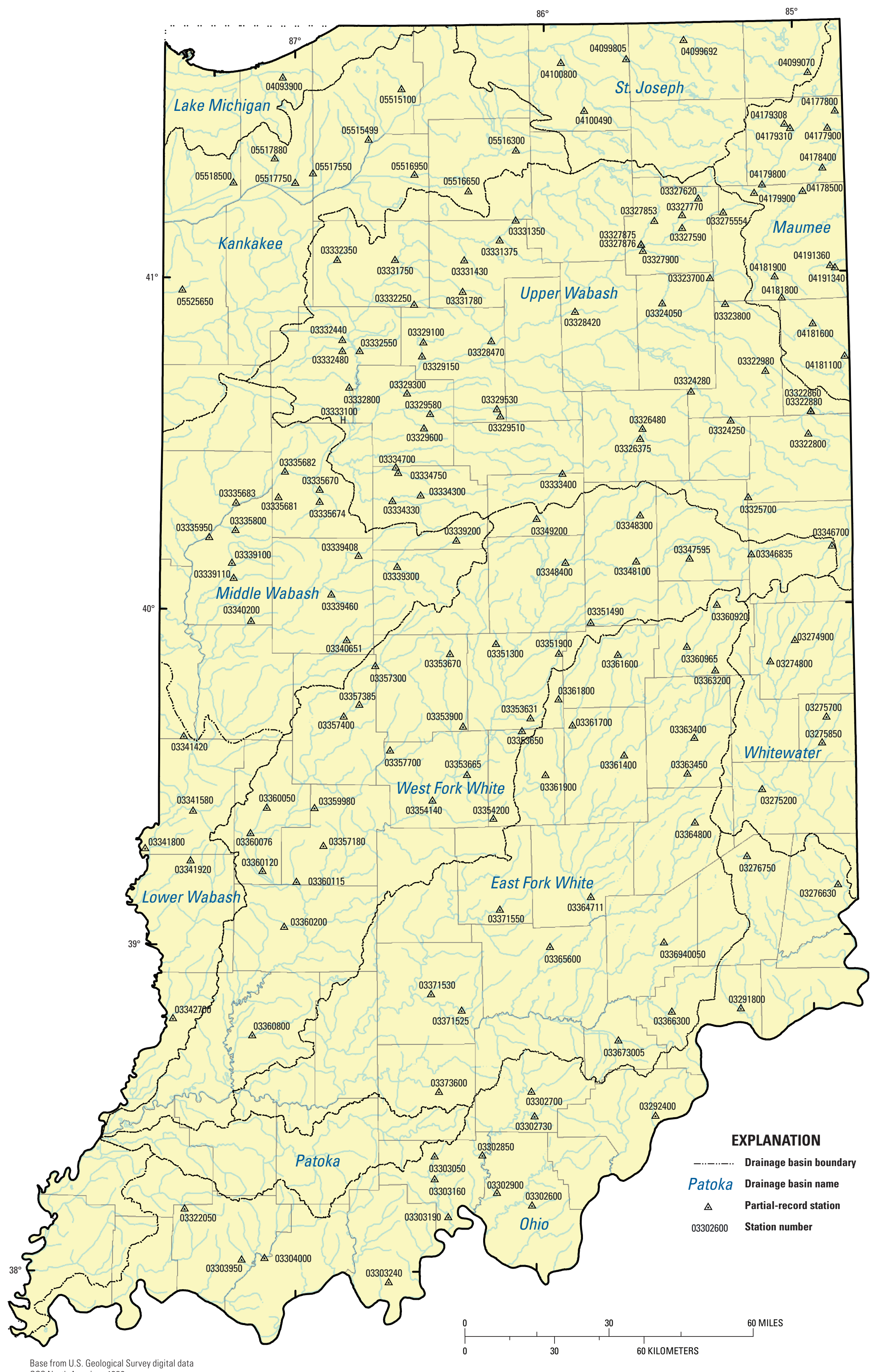

Base from U.S. Geological Survey digital data

Figure 7. Partial-record streamgaging stations in Indiana. 


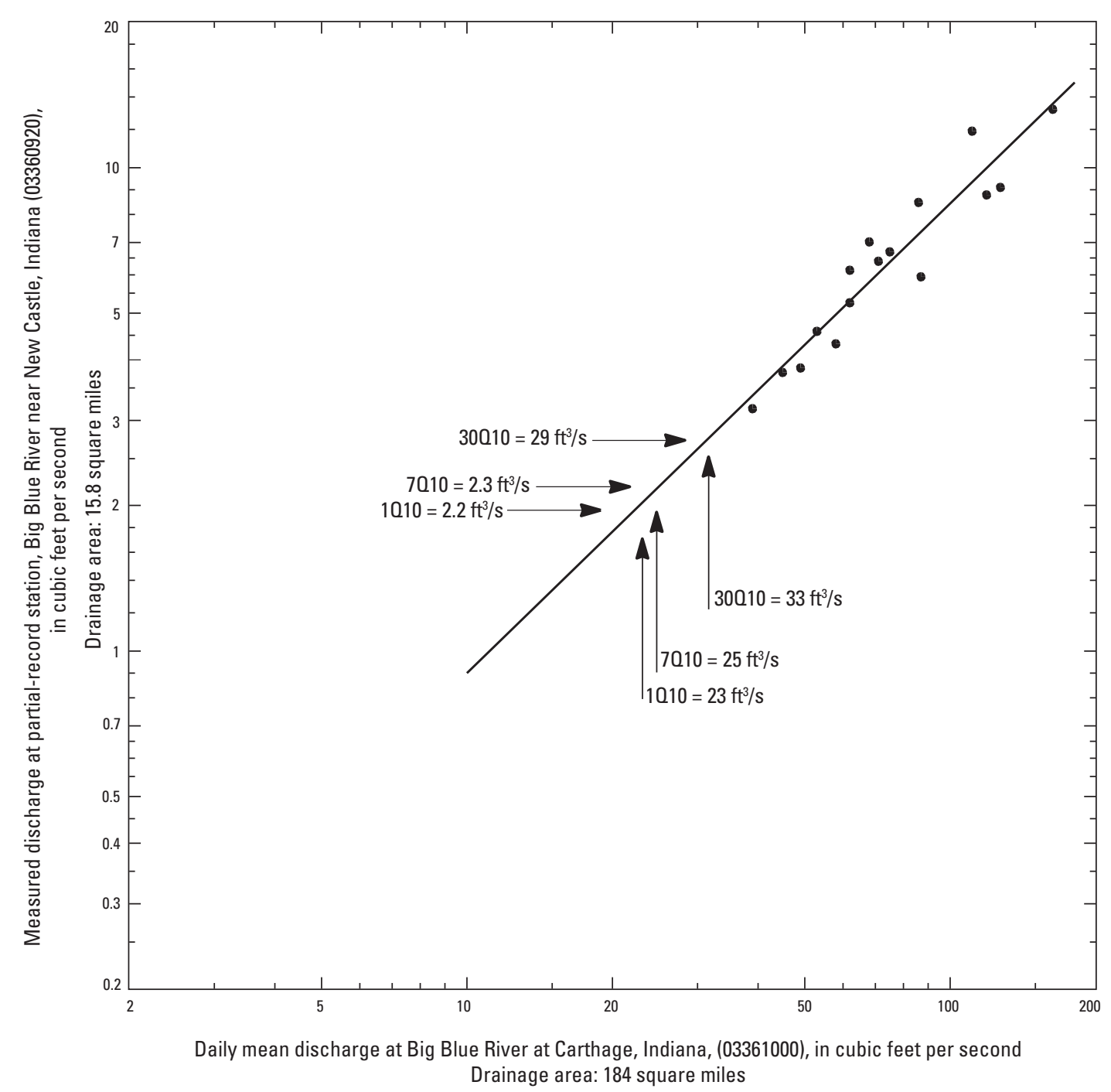

Figure 8. Graphical method of estimating low-flow characteristics at a partial-record station from known characteristics at a continuous-record station.

Low-flow characteristics were estimated at 166 partialrecord stations in Indiana (table 2). The estimated flow characteristics are limited to the 1Q10, the 7Q10, and 30Q10. Estimates of 1Q10, 7Q10 and 30Q10 that were less than or equal to $0.05 \mathrm{ft}^{3} / \mathrm{s}$ were rounded to zero; those that were between 0.05 and $0.1 \mathrm{ft}^{3} / \mathrm{s}$ were rounded to $0.1 \mathrm{ft}^{3} / \mathrm{s}$.

\section{Data Limitations at Partial-Record Stations}

Most of the base-flow measurements at partial-record stations were made in the 1960s and 1970s, presumably when cooperative programs were in place that emphasized gathering low-flow data at ungaged sites. There was also a short period of renewed interest in gathering low-flow data in 1988 and 1989 as a result of drought conditions in Indiana, the effects of which were documented by Fowler (1992). Therefore, it needs to be recognized that correlations between partialrecord stations and index stations are based on data that are decades old. There is no guarantee that a linear relation (slope) between a partial-record station and an index station is the same today as it was 30,40 , or 50 years ago. Because few new base-flow measurements have been collected, estimates of low-flow statistics at partial-record stations can be updated only by using index stations that are active or were active until recently. With this in mind, a priority was placed on selecting active gages as index stations. However, at some partial-record stations, the only suitable correlation was with a gage that was discontinued sometime after base-flow measurements were made at the partial-record station. 


\section{Flow-Duration Analysis}

A flow-duration curve is a cumulative frequency curve that shows the percentage of time that a specified discharge at a site was equaled or exceeded during a given period of record (Searcy, 1959). Flow-duration curves are useful for showing the flow characteristics of a stream throughout its range of discharge. The chronological sequence of flows is not a factor in the curve. This report contains flow-duration analyses for all of the continuous-record stations in Indiana (table 1). A flowduration analysis applies only to the period of record used to develop the curve, and the flow-duration curve represents the average curve for the period of record.

Only complete years of record are used for flow-duration analysis. The years used do not need to be consecutive, but conditions in the basin (artificial storage, diversions, or other manmade influences) need to be the same. By arranging flows according to frequency of occurrence and by plotting the flowduration curve, the combined effects of the factors influencing runoff are integrated into the curve. The shape of the curve is determined by the hydrologic and geologic characteristics of the drainage basin. The slope at the lower end of the curve is indicative of the perennial storage in the drainage basin; a gentle slope indicates a large amount of storage, and a steep slope indicates little storage. A gentle slope indicates sustained streamflow during dry periods. A curve with a steep slope indicates highly variable streamflow that is influenced by direct runoff. A flow-duration curve with a gentle slope indicates the presence of surface-water or groundwater storage; slow, constant release of the stored water tends to equalize the flow at the station (Searcy, 1959). Arihood and Glatfelter (1986) used the slope of the flow-duration curve between the 20-percent and 90-percent flow durations (F20/90) as an explanatory variable for estimating low-flow statistics at unregulated, ungaged streams, and referred to this as the flow-duration ratio.

Figure 9. Flow-duration curves for a stream in southern Indiana (Big Creek) and a stream in northern Indiana (Pigeon Creek).
In general, flow-duration curves with steep slopes are typical of streams in the hilly areas of southern Indiana, and curves with gentle slopes are typical of streams in the glaciated areas of northern Indiana. Figure 9 shows two flowduration curves for streams with drainage areas of similar size, one from southern Indiana and one from northern Indiana. Despite the similar drainage areas, flow characteristics differ. Big Creek (Posey County, fig. 4) is in an area of exposed or shallow bedrock with some unconsolidated alluvial or loess deposits (Gray, 2000), and its flow is highly variable and periodically goes to zero. The steep slope of the curve for Big Creek is reflected in the flow-duration ratio of 300 (F20/90). Pigeon Creek (Steuben County, fig. 4) has a smaller range of flow that was sustained 99.5 percent of the time above $5 \mathrm{ft}^{3} / \mathrm{s}$. The gentler slope of the curve for Pigeon Creek is reflected in the flow-duration ratio of 7.2 (F20/90). This sustained flow can be attributed to the unconsolidated deposits in the area and contributing groundwater (Gray, 2000). Sustained flows in the Central Till Plain are generally lower than those in the north, except in localized areas of higher channel permeability. Flowduration curves were generated with the SWSTAT software.

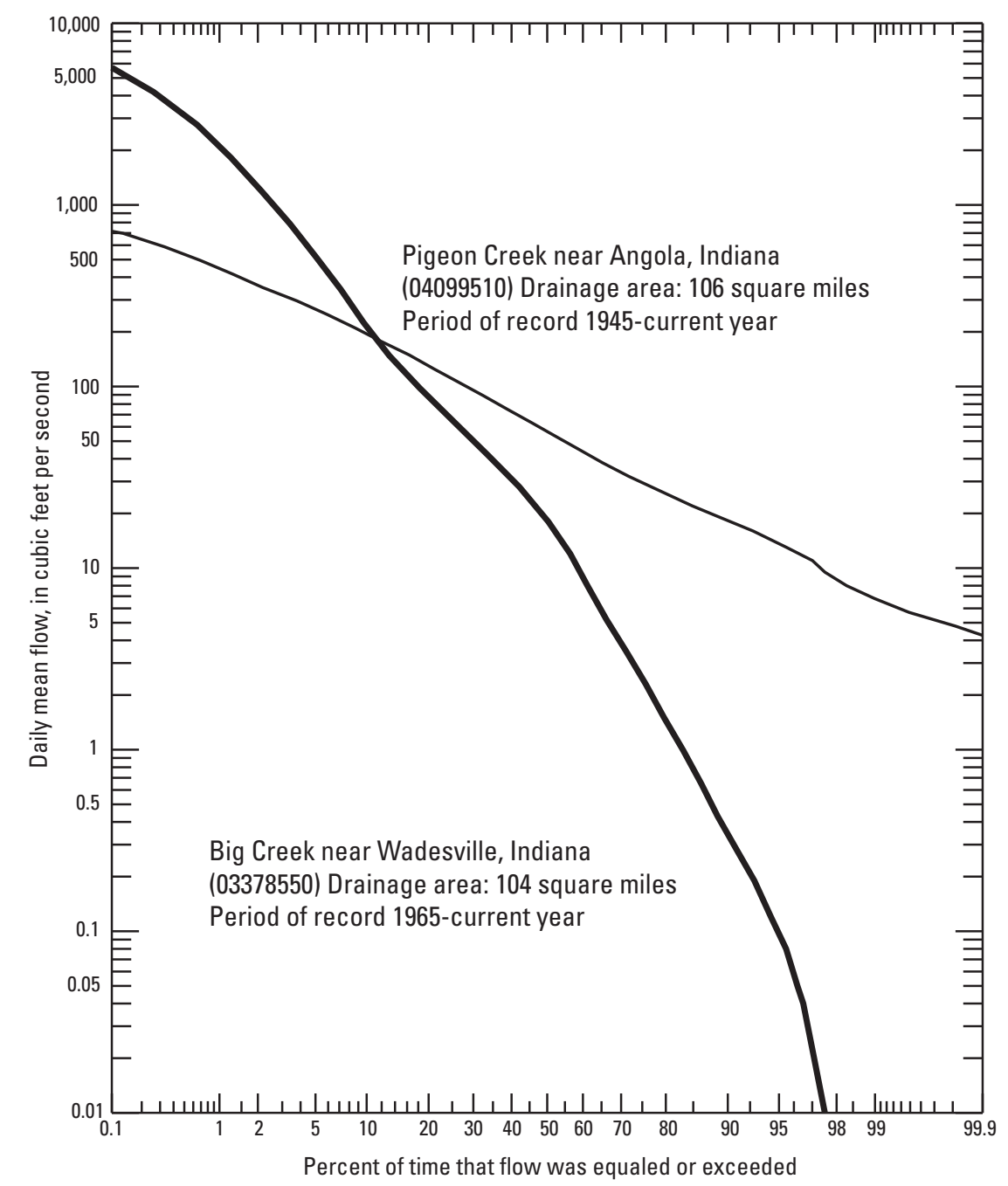




\section{Harmonic Mean Flow}

The harmonic mean flow statistic $\left(Q_{h}\right)$ can serve as a design flow for human health criteria that are based on lifetime exposures because it can be used to calculate the average exposure concentration of a contaminant for an average contaminant loading rate (Rossman 1990a; Straub, 2001; Koltun and Whitehead, 2002). Design flows are used in water-pollution control programs to provide adequate protection against pollutant exposure periods of a given duration (Rossman, 1990b). The harmonic mean flow value was calculated for each of the 230 streamgages with at least 10 years of record from the daily mean discharge record by using a computer program based on DFLOW, a computer program developed by the U.S. Environmental Protection Agency (Rossman, 1990b). The period of record used was the same as was used for the frequency analysis (the entire record or current conditions after regulation). The exposure concentration will be greater and more harmful on days with low flow than on days with high flows. The $Q_{h}$ statistic computed from a streamflow record generally is smaller than the corresponding arithmetic mean discharge, is adjusted for the days with zero flow, and gives greater weight to low daily mean discharges than high daily mean discharges. The $Q_{h}$ streamflow statistic is calculated as:

$$
Q_{h}=\left(\frac{N_{n z}}{N_{t}}\right)\left(N_{n z} / \sum_{i=1}^{N_{n z}} \frac{1}{Q_{i}}\right)
$$

where $Q_{i}$ is the mean streamflow for a given day, $N_{n z}$ is the number of non-zero daily mean streamflows and $N_{t}$ is the total number of daily mean streamflows. If no zero-flow days are in the record, the harmonic mean flow is equal to the reciprocal of the mean of the reciprocals of the daily mean streamflow data.

Harmonic mean flows at partial-record stations and continuous-record stations with less than 10 years of record were estimated by use of linear relations between base-flow measurements at the partial-record stations and concurrent daily mean flows at the index stations. If the Stedinger-Thomas correlation technique was used, the logarithm of the harmonic mean at the index station was used in the linear equation to solve for the harmonic mean at the partial-record station. If the graphical correlation method was used, the harmonic mean at the index station was transferred to the partial-record station as shown in figure 8 .

\section{Summary}

Low-flow characteristics for 272 continuous-record streamgaging stations and 166 partial-record stations in Indiana are presented in this report. A frequency analysis, flow-duration information, and the harmonic mean flow are computed for all continuous-record stations. For partial-record stations, the listed estimates are the 1-day, 7-day, and 30-day low flow at the 10-year recurrence interval and the harmonic mean flow (if sufficient data are available). Discussions of the statistical methods used are presented. The streams in Indiana are directly influenced by precipitation and geology. The average annual precipitation and temperature in Indiana increased from the period 1961-1990 to 1981-2010. Generally, streams in the glaciated northern zone of the State have higher sustained base flows than in the nonglaciated southern zone. Stream channels in northern Indiana tend to be highly permeable, and flows are more sustained because of the influence of groundwater. Sustained flows in the Central Till Plain are generally lower than those in the north, except in localized areas of higher channel permeability. The southern zone of the State has the most diverse physiography of the four zones. Throughout much of southern Indiana, however, there is little sustained base flow. In some areas of alluvial deposits, sustained flows are increased but are generally lowest in the south. 


\section{References Cited}

Arihood, L.D., and Glatfelter, D.R., 1986, Method for estimating low-flow characteristics of ungagged streams in Indiana: U.S. Geological Survey Open-File Report 86-323, 32 p.

Arvin, D.V., 1989, Statistical summary of streamflow data for Indiana: U.S. Geological Survey Open-File Report 89-62, $964 \mathrm{p}$.

Flynn, K.M., Hummel, P.R., Lumb, A.M., and Kittle, J.L., Jr., 1995, User's manual for ANNIE, Version 2, a computer program for interactive hydrologic data management: U.S. Geological Survey Water-Resources Investigations Report 95-4085, 211 p., http://water.usgs.gov/software/ANNIE/ code/doc/annie.pdf.

Fowler, K.K., 1992, Description and effects of 1988 drought on ground-water levels, streamflow, and reservoir levels in Indiana: U.S. Geological Survey Water-Resources Investigations Report 91-4100, $91 \mathrm{p}$.

Fowler, K.K., and Wilson, J.T., 1996, Low-flow characteristics of Indiana streams: U.S. Geological Survey WaterResources Investigations Report 96-4128, 313 p.

Gray, H.H., 2000, Physiographic divisions of Indiana: Indiana Geological Survey Special Report 61, Indiana University, $15 \mathrm{p} ., 1 \mathrm{pl}$.

Hoggatt, R.E., 1962, Low-flow characteristics of Indiana streams: Indiana Stream Pollution Control Board, $171 \mathrm{p}$.

Hutchison, N.E., comp., 1975 (revised 1984), WATSTORE user's guide, Volume 1, National Water Data Storage and Retrieval System: U.S. Geological Survey Open-File Report 75-426 [variously paged].

Koltun, G.F., and Whitehead, M.T., 2002, Techniques for estimating selected streamflow characteristics of rural, unregulated streams in Ohio: U.S. Geological Survey Water-Resources Investigations Report 02-4068, $50 \mathrm{p}$. (Also available at http://oh.water.usgs.gov/reports/wrir/ wrir02-4068.pdf.)

Lumb, A.M., Kittle, J.L., Jr., and Flynn, K.M., 1990, User's manual for ANNIE, a computer program for interactive hydrologic analyses and data management: U.S. Geological Survey Water-Resources Investigations Report 89-4080, $236 \mathrm{p}$.

National Oceanic and Atmospheric Administration, 2014, Climatological data, annual summary, Indiana, 2010: Asheville, N.C., National Climatic Data Center.

Riggs, H.C., 1968, Frequency curves: U.S. Geological Survey Techniques of Water-Resources Investigations, book 4, chap. A2, 15 p.
Riggs, H.C., 1972, Low-flow investigations: U.S. Geological Survey Techniques of Water-Resources Investigations, book 4, chap. B1, 18 p.

Rohne, P.B., Jr., 1972, Low-flow characteristics of Indiana streams: U.S. Geological Survey Open-File Report [unnumbered], $322 \mathrm{p}$.

Rossman, L.A., 1990a, Design stream flows based on harmonic means: Journal of Hydraulic Engineering, v. 116, no. 7, p. 946-950.

Rossman, L.A., 1990b, DFLOW user's manual: Cincinnati, Ohio, U.S. Environmental Protection Agency, Risk Reduction Engineering Laboratory, 26 p.

Scheeringa, K., 2002, About Indiana climate: Indiana State Climate Office Web page, accessed October 28, 2014, at http://iclimate.org/narrative.asp.

Searcy, J.K., 1959, Flow-duration curves: U.S. Geological Survey Water-Supply Paper 1542-A, 33 p.

Stedinger, J.R., and Thomas, W.O., Jr., 1985, Low-flow frequency estimation using base-flow measurements: U.S. Geological Survey Open-File Report 85-95, 22 p.

Stewart, J.A., 1983, Low-flow characteristics of Indiana streams: U.S. Geological Survey Open-File Report 82-1007, $277 \mathrm{p}$.

Straub, D.E., 2001, Low-flow characteristics of streams in Ohio through water year 1997: U.S. Geological Survey Water-Resources Investigations Report 01-4140, 415 p. (Also available at http://oh.water.usgs.gov/reports/wrir/ wrir01-4140.pdf.)

Tasker, G.D., and Stedinger, J.R., 1989, An operational GLS model for hydrologic regression: Journal of Hydrology, v. 111, p. 361-375.

Thompson, R.E., and Hoffman, S.A., 2006, Selected streamflow statistics and regression equations for predicting statistics at stream locations in Monroe County, Pennsylvania: U.S. Geological Survey Scientific Investigations Report 2006-5244, 45 p.

U.S. Environmental Protection Agency, 2012a, National Pollutant Discharge Elimination System Compliance Monitoring: U.S. Environmental Protection Agency Web page, accessed October 28, 2014, at http://www.epa.gov/compliance/monitoring/programs/cwa/npdes.html.

U.S. Environmental Protection Agency, 2012b, Information Concerning 2012 Clean Water Act Sections 303(d), 305(b), and 314 Integrated Reporting and Listing Decisions: U.S. Environmental Protection Agency Web page, accessed October 28, 2014, at http://water.epa.gov/lawsregs/ lawsguidance/cwa/tmdl/ir_memo_2012.cfm. 



\section{Table 1}

1. Low-flow characteristics for continuous-record streamgaging stations in Indiana.

Tables 1 and 2 in Excel file format are available at http://dx.doi.org/10.3133/sir20145242.

Table 1. Low-flow characteristics for continuous-record streamgaging stations in Indiana.

The following data sheets list station descriptions and low-flow characteristics for 230 continuous-record stations in Indiana with at least 10 years of record and an additional 42 stations with less than 10 years of record. Low-flow characteristics are based on available data through September 2011, except for selected short-term continuous-record stations, where data extending through September 2013 were used. The information includes low-flow-frequency analysis, flow-duration analysis, and harmonic mean flows (human health design flow) for the continuous-record stations. Design flows are used in water-pollution control programs to provide adequate protection against pollutant exposure periods of a given duration (Rossman, 1990b). Each continuous-record station is linked to the USGS NWIS Web interface. For those stations affected by some form of regulation, low-flow frequency curves are based on the longest period of homogeneous record under current conditions.

Locational coordinates are given in degree-minute-second format. In addition to standard abbreviations for compass directions, the following abbreviations are used throughout the data sheets:

$\begin{array}{ll}\mathrm{ft} & \text { feet } \\ \mathrm{ft}^{3} / \mathrm{s} & \text { cubic feet per second } \\ \text { Lat } & \text { latitude } \\ \text { long } & \text { longitude } \\ \mathrm{mi} & \text { mile } \\ \mathrm{mi}^{2} & \text { square mile } \\ \mathrm{R} . & \text { range } \\ \text { sec. } & \text { section } \\ \text { T. } & \text { township }\end{array}$


Great Miami River Basin

\section{WHITEWATER RIVER NEAR ECONOMY, IN}

Location. - Lat $40^{\circ} 00^{\prime} 15^{\prime \prime}$, long $85^{\circ} 06^{\prime} 56^{\prime \prime}$ referenced to North American Datum of 1927, in NW 1/4 NE 1/4 sec.19, T.18 N., R.13 E., Wayne County, IN, Hydrologic Unit 05080003, on right bank $15 \mathrm{ft}$ downstream from bridge on Wayne County Line Road, 1.7 mi upstream from Little Creek, 2.4 mi northwest of Economy, and at mile 91.9.

Drainage area.- $10.4 \mathrm{mi}^{2}$.

Period of record.- October 1970 to current year.

Average discharge. $-11.9 \mathrm{ft}^{3} / \mathrm{s}$

Minimum daily discharge. $-0.0 \mathrm{ft}^{3} / \mathrm{s}$

Human health (harmonic mean) design flow.- $1.6 \mathrm{ft}^{3} / \mathrm{s}$

Remarks. - None.

\begin{tabular}{ccc}
\hline \multicolumn{3}{c}{ Magnitude and frequency of annual low streamflow } \\
\hline $\begin{array}{c}\text { Lowest average streamflow, in } \mathrm{ft}^{3} / \mathrm{s} \text {, for indicated period of } \\
\text { consecutive days at an annual nonexceedance probability of } 0.1\end{array}$ \\
\hline $\mathbf{7}$ & $\mathbf{3 0}$ \\
\hline 0.2 & 0.3 & 0.3 \\
\hline
\end{tabular}

\begin{tabular}{cc|cc}
\hline Percentage of time streamflow was equaled or exceeded for \\
the period of record
\end{tabular}




\section{WHITEWATER RIVER NEAR HAGERSTOWN, IN}

Location. - Lat 39 $52^{\prime} 25^{\prime \prime}$, long $85^{\circ} 09^{\prime} 47^{\prime \prime}$ referenced to North American Datum of 1927, in NE 1/4 NE 1/4 sec.3, T.16 N., R.12 E., Wayne County, IN, Hydrologic Unit 05080003, on left bank at downstream side of bridge on Jerry Meyers Road, 1.0 mi upstream from Pronghorn Run, 1.5 mi north of Interstate 70, 2.0 mi downstream from Nettle Creek, 2.6 mi south of Hagerstown, and at mile 84.9.

Drainage area. $-58.7 \mathrm{mi}^{2}$.

Period of record.- October 1970 to 2003.

Average discharge. $-69.4 \mathrm{ft}^{3} / \mathrm{s}$

Minimum daily discharge. $-3.9 \mathrm{ft}^{3} / \mathrm{s}$

Human health (harmonic mean) design flow. $-28 \mathrm{ft}^{3} / \mathrm{s}$

Remarks. - None.

\begin{tabular}{ccc}
\hline \multicolumn{3}{c}{ Magnitude and frequency of annual low streamflow } \\
\hline $\begin{array}{c}\text { Lowest average streamflow, in } \mathrm{ft}^{3} / \mathrm{s} \text {, for indicated period of } \\
\text { consecutive days at an annual nonexceedance probability of } 0.1\end{array}$ \\
\hline 1 & $\mathbf{7}$ & $\mathbf{3 0}$ \\
\hline 6.2 & 6.8 & 8.4 \\
\hline
\end{tabular}

\begin{tabular}{cc|cc}
\hline \multicolumn{4}{c}{ Percentage of time streamflow was equaled or exceeded for } \\
the period of record
\end{tabular}


Great Miami River Basin

\section{LITTLE WILLIAMS CREEK AT CONNERSVILLE, IN}

Location. - Lat $39^{\circ} 18^{\prime} 16^{\prime \prime}$, long $85^{\circ} 10^{\prime} 20^{\prime \prime}$ referenced to North American Datum of 1927, in SW 1/4 NE 1/4 sec.27, T.14 N., R.12 E., Fayette County, IN, Hydrologic Unit 05080003, on downstream left bank wingwall of bridge on State Highway 44, $1.0 \mathrm{mi}$ west of Connersville, and $2.6 \mathrm{mi}$ upstream from mouth.

Drainage area. $-9.16 \mathrm{mi}^{2}$.

Period of record.- September 1968 to 1991.

Average discharge. $-9.90 \mathrm{ft}^{3} / \mathrm{s}$

Minimum daily discharge. $-0.0 \mathrm{ft}^{3} / \mathrm{s}$

Human health (harmonic mean) design flow. $-2.0 \mathrm{ft}^{3} / \mathrm{s}$

Remarks. - None.

\begin{tabular}{ccc}
\hline \multicolumn{3}{c}{ Magnitude and frequency of annual low streamflow } \\
\hline $\begin{array}{c}\text { Lowest average streamflow, in } \mathrm{ft}^{3} / \mathrm{s} \text {, for indicated period of } \\
\text { consecutive days at an annual nonexceedance probability of } 0.1\end{array}$ \\
\hline 1 & 7 & 30 \\
\hline 0.2 & 0.3 & 0.3 \\
\hline
\end{tabular}

Percentage of time streamflow was equaled or exceeded for the period of record

\begin{tabular}{cc|cc}
\hline $\begin{array}{c}\text { Percentage } \\
\text { of time }\end{array}$ & $\begin{array}{c}\text { Daily mean } \\
\text { streamflow } \\
\left(\mathbf{f t}^{3} / \mathbf{s}\right)\end{array}$ & $\begin{array}{c}\text { Percentage } \\
\text { of time }\end{array}$ & $\begin{array}{c}\text { Daily mean } \\
\text { streamflow } \\
\left(\mathbf{f t}^{3} / \mathbf{s}\right)\end{array}$ \\
\hline 99 & 0.2 & 40 & 6.6 \\
\hline 98 & 0.4 & 30 & 9.0 \\
\hline 95 & 0.7 & 20 & 13 \\
\hline 90 & 1.0 & 10 & 19 \\
\hline 80 & 1.6 & 5 & 31 \\
\hline 70 & 2.5 & 2 & 64 \\
\hline 60 & 3.7 & 1 & 107 \\
\hline 50 & 4.9 & & \\
\hline
\end{tabular}




\section{WHITEWATER RIVER NEAR ALPINE, IN}

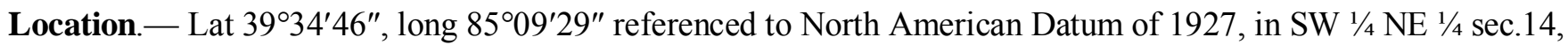
T.13 N., R.12 E., Fayette County, IN, Hydrologic Unit 05080003, on right bank $1117 \mathrm{ft}$ upstream from Wilson Creek, 5.1 mi upstream from Bear Creek, 0.4 mi upstream from bridge on County Road 480 South, 2.0 mi northeast of Alpine, and at mile 54.8.

Drainage area. $-522 \mathrm{mi}^{2}$.

Period of record.- October 1928 to current year. Prior to October 1936, published as West Fork Whitewater River near Alpine.

Average discharge. $-589 \mathrm{ft}^{3} / \mathrm{s}$.

Minimum daily discharge. $-30 \mathrm{ft}^{3} / \mathrm{s}$

Human health (harmonic mean) design flow. $-203 \mathrm{ft}^{3} / \mathrm{s}$

Remarks.- None.

\begin{tabular}{ccc}
\hline \multicolumn{3}{c}{ Magnitude and frequency of annual low flow } \\
\hline $\begin{array}{c}\text { Lowest average streamflow, in } \mathrm{ft}^{3} / \mathrm{s} \text {, for indicated period of } \\
\text { consecutive days at an annual nonexceedance probability of } 0.1\end{array}$ \\
\hline 7 & 7 & 30 \\
\hline 48 & 54 & 62 \\
\hline
\end{tabular}

\begin{tabular}{cc|cc}
\hline \multicolumn{3}{c}{ Percentage of time streamflow was equaled or exceeded for } \\
the period of record
\end{tabular}


Great Miami River Basin

\section{EAST FORK WHITEWATER RIVER AT RICHMOND, IN}

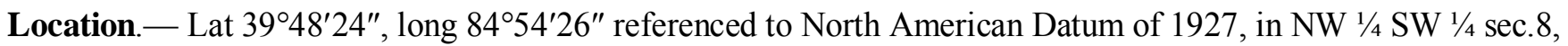
T.13 N., R.1 W., Wayne County, IN, Hydrologic Unit 05080003, on left bank $50 \mathrm{ft}$ downstream from highway bridge, 0.8 mi south of Richmond, 1.5 mi upstream from Short Creek, and at mi 33.4.

Drainage area.- $121 \mathrm{mi}^{2}$.

Period of record.- April 1949 to September 1978.

Average discharge. $-115 \mathrm{ft}^{3} / \mathrm{s}$.

Minimum daily discharge. $-1.2 \mathrm{ft}^{3} / \mathrm{s}$

Human health (harmonic mean) design flow. $-28 \mathrm{ft}^{3} / \mathrm{s}$

Remarks. - None.

\begin{tabular}{ccc}
\hline \multicolumn{3}{c}{ Magnitude and frequency of annual low flow } \\
\hline $\begin{array}{c}\text { Lowest average streamflow, in } \mathrm{ft}^{3} / \mathrm{s} \text {, for indicated period of } \\
\text { consecutive days at an annual nonexceedance probability of } 0.1\end{array}$ \\
\hline 1 & 7 & 30 \\
\hline 2.7 & 3.7 & 6.7 \\
\hline
\end{tabular}

\begin{tabular}{cc|cc}
\hline \multicolumn{3}{c}{ Percentage of time streamflow was equaled or exceeded for } \\
the period of record
\end{tabular}




\section{EAST FORK WHITEWATER RIVER AT ABINGTON, IN}

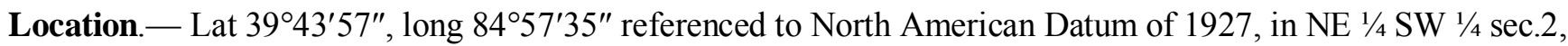
T.12 N., R.2 W., Wayne County, IN, Hydrologic Unit 05080003, $15 \mathrm{ft}$ downstream of bridge on county road at Abington, 3 mi downstream from Elkhorn Creek, 8 mi southwest of Richmond, and at mile 26.7.

Drainage area. $-200 \mathrm{mi}^{2}$.

Period of record. - October 1965 to current year.

Average discharge. $-240 \mathrm{ft}^{3} / \mathrm{s}$.

Minimum daily discharge. $-10 \mathrm{ft}^{3} / \mathrm{s}$

Human health (harmonic mean) design flow. $-76 \mathrm{ft}^{3} / \mathrm{s}$

Remarks.- None.

\begin{tabular}{ccc}
\hline \multicolumn{3}{c}{ Magnitude and frequency of annual low flow } \\
\hline $\begin{array}{c}\text { Lowest average streamflow, in } \mathrm{ft}^{3} / \mathrm{s} \text {, for indicated period of } \\
\text { consecutive days at an annual nonexceedance probability of } 0.1\end{array}$ \\
\hline 1 & $\mathbf{3}$ & $\mathbf{3 0}$ \\
\hline 14 & 16 & 20 \\
\hline
\end{tabular}

\begin{tabular}{cc|cc}
\hline \multicolumn{4}{c}{ Percentage of time streamflow was equaled or exceeded for } \\
the period of record
\end{tabular}


Great Miami River Basin

\section{EAST FORK WHITEWATER RIVER AT BROOKVILLE, IN}

Location. - Lat $39^{\circ} 26^{\prime} 02^{\prime \prime}$, long $85^{\circ} 00^{\prime} 12^{\prime \prime}$ referenced to North American Datum of 1927, in NE 1/4 NE $1 / 4 \mathrm{sec} .20$, T.9 N., R.2 W., Franklin County, IN, Hydrologic Unit 05080003, on right bank $100 \mathrm{ft}$ upstream from bridge on State Highway 101, at Brookville, 0.4 mi downstream from Brookville Lake, and 1.8 mi upstream from mouth.

Drainage area. $-380 \mathrm{mi}^{2}$.

Period of record.- March 1954 to September 1981 (discharge). October 1981 to September 2001 (discharge provided by U.S. Army Corps of Engineers).

Average discharge. $-413 \mathrm{ft}^{3} / \mathrm{s}$.

Minimum daily discharge. $-0.0 \mathrm{ft}^{3} / \mathrm{s}$.

Human health (harmonic mean) design flow. - $102 \mathrm{ft}^{3} / \mathrm{s}$.

Remarks. - Flow regulated by the U.S. Army Corps of Engineers from Brookville Lake since January 1974. Lowflow statistics are calculated for the regulated period, 1974 to 2001.

\begin{tabular}{ccc}
\hline \multicolumn{3}{c}{ Magnitude and frequency of annual low flow } \\
\hline $\begin{array}{c}\text { Lowest average streamflow, in } \mathrm{ft}^{3} / \mathrm{s} \text {, for indicated period of } \\
\text { consecutive days at an annual nonexceedance probability of } 0.1\end{array}$ \\
\hline $\mathbf{1}$ & $\mathbf{3}$ & $\mathbf{3 0}$ \\
\hline 0 & 19 & 27 \\
\hline
\end{tabular}

Percentage of time streamflow was equaled or exceeded for the period of record

\begin{tabular}{cc|cc}
\hline $\begin{array}{c}\text { Percentage } \\
\text { of time }\end{array}$ & $\begin{array}{c}\text { Daily mean } \\
\text { streamflow } \\
\left(\mathrm{ft}^{3} / \mathbf{s}\right)\end{array}$ & $\begin{array}{c}\text { Percentage } \\
\text { of time }\end{array}$ & $\begin{array}{c}\text { Daily mean } \\
\text { streamflow } \\
\left(\mathrm{ft}^{3} / \mathbf{s}\right)\end{array}$ \\
\hline 99 & 23 & 40 & 353 \\
\hline 98 & 28 & 30 & 443 \\
\hline 95 & 34 & 20 & 660 \\
\hline 90 & 42 & 10 & 1060 \\
\hline 80 & 55 & 5 & 1750 \\
\hline 70 & 80 & 2 & 2700 \\
\hline 60 & 142 & 1 & 3470 \\
\hline 50 & 230 & & \\
\hline
\end{tabular}




\section{WHITEWATER RIVER AT BROOKVILLE, IN}

Location. - Lat $39^{\circ} 24^{\prime} 24^{\prime \prime}$, long $85^{\circ} 00^{\prime} 46^{\prime \prime}$ referenced to North American Datum of 1927, in NE 1/4 NW 1/4 sec.32, T.9 N., R.2 W., Franklin County, IN, Hydrologic Unit 05080003, on right bank at downstream side of highway bridge, 0.3 mi downstream from East Fork Whitewater River, $1.1 \mathrm{mi}$ south of Brookville, and at mile 29.3.

Drainage area.- $1,224 \mathrm{mi}^{2}$.

Period of record. - June 1915 to September 1917, October 1917 to May 1920 (gage heights only), and July 1923 to current year.

Average discharge. - 1,370 $\mathrm{ft}^{3} / \mathrm{s}$.

Minimum daily discharge. $-60 \mathrm{ft}^{3} / \mathrm{s}$.

Human health (harmonic mean) design flow. - $528 \mathrm{ft}^{3} / \mathrm{s}$.

Remarks. - Flow regulated by the U.S. Army Corps of Engineers from Brookville Lake since January 1974. Lowflow statistics are calculated for the regulated period, 1974 to 2011.

\begin{tabular}{ccc}
\hline \multicolumn{3}{c}{ Magnitude and frequency of annual low flow } \\
\hline $\begin{array}{c}\text { Lowest average streamflow, in } \mathrm{ft}^{3} / \mathrm{s} \text {, for indicated period of } \\
\text { consecutive days at an annual nonexceedance probability of } \mathbf{0 . 1}\end{array}$ \\
\hline $\mathbf{1}$ & $\mathbf{7}$ & $\mathbf{3 0}$ \\
\hline 100 & 108 & 119 \\
\hline
\end{tabular}

\begin{tabular}{cc|cc}
\hline \multicolumn{5}{c}{ Percentage of time streamflow was equaled or exceeded for } \\
the period of record
\end{tabular}


Ohio River Basin

\section{SOUTH HOGAN CREEK NEAR DILLSBORO, IN}

Location.- Lat $39^{\circ} 01^{\prime} 47^{\prime \prime}$, long $85^{\circ} 02^{\prime} 17^{\prime \prime}$, in SW1/4NW1/4 sec.7, T.4 N., R.2 W., Dearborn County, Hydrologic Unit 05090203, on left downstream abutment of bridge on county road at Dillsboro Station, 1.2 mi northeast of Dillsboro, and 1.5 mi downstream from Whitaker Creek.

Drainage area.- $38.1 \mathrm{mi}^{2}$.

Period of record.- July 1961 to September 1993.

Average discharge. $-43.3 \mathrm{ft}^{3} / \mathrm{s}$.

Minimum daily discharge. $-0.0 \mathrm{ft}^{3} / \mathrm{s}$.

Human health (harmonic mean) design flow. $-0.6 \mathrm{ft}^{3} / \mathrm{s}$.

Remarks. - None.

\begin{tabular}{ccc}
\hline \multicolumn{3}{c}{ Magnitude and frequency of annual low flow } \\
\hline $\begin{array}{c}\text { Lowest average streamflow, in } \mathrm{ft}^{3} / \mathrm{s} \text {, for indicated period of } \\
\text { consecutive days at an annual nonexceedance probability of } 0.1\end{array}$ \\
\hline $\mathbf{1}$ & $\mathbf{3 0}$ \\
\hline 0.0 & 0.0 & 0.0 \\
\hline
\end{tabular}

Percentage of time streamflow was equaled or exceeded for the period of record

\begin{tabular}{cc|cc}
\hline $\begin{array}{c}\text { Percentage } \\
\text { of time }\end{array}$ & $\begin{array}{c}\text { Daily mean } \\
\text { streamflow } \\
\left(\mathrm{ft}^{3} / \mathbf{s}\right)\end{array}$ & $\begin{array}{c}\text { Percentage } \\
\text { of time }\end{array}$ & $\begin{array}{c}\text { Daily mean } \\
\text { streamflow } \\
\left(\mathrm{ft}^{3} / \mathbf{s}\right)\end{array}$ \\
\hline 99 & 0.0 & 40 & 14 \\
\hline 98 & 0.0 & 30 & 24 \\
\hline 95 & 0.0 & 20 & 40 \\
\hline 90 & 0.1 & 10 & 86 \\
\hline 80 & 0.7 & 5 & 192 \\
\hline 70 & 2.2 & 2 & 441 \\
\hline 60 & 4.8 & 1 & 664 \\
\hline 50 & 8.6 & & \\
\hline
\end{tabular}


Ohio River Basin

\section{LAUGHERY CREEK NEAR FARMERS RETREAT, IN}

Location.- Lat $38^{\circ} 57^{\prime} 08^{\prime \prime}$, long $85^{\circ} 04^{\prime} 15^{\prime \prime}$, in NW 1/4 SE 1/4 sec.2, T.4 N., R.3 W., Ohio County, IN, on right bank 2.4 mi southeast of Farmers Retreat, and 3.8 mi downstream from Bear Creek.

Drainage area. $-248 \mathrm{mi}^{2}$.

Period of record. - October 1940 to September 1973.

Average discharge. $-272 \mathrm{ft}^{3} / \mathrm{s}$.

Minimum daily discharge. - No flow at times most years.

Human health (harmonic mean) design flow. $-2.7 \mathrm{ft}^{3} / \mathrm{s}$

Remarks. - Some regulation by mill upstream from station.

\begin{tabular}{ccc}
\hline \multicolumn{3}{c}{ Magnitude and frequency of annual low flow } \\
\hline $\begin{array}{c}\text { Lowest average streamflow, in } \mathrm{ft}^{3} / \mathrm{s} \text {, for indicated period of } \\
\text { consecutive days at an annual nonexceedance probability of } 0.1\end{array}$ \\
\hline $\mathbf{1}$ & $\mathbf{7}$ & $\mathbf{3 0}$ \\
\hline 0.0 & 0.0 & 0.0 \\
\hline
\end{tabular}

\begin{tabular}{cc|cc}
\hline Percentage of time streamflow was equaled or exceeded for \\
the period of record
\end{tabular}


Ohio River Basin

\section{INDIAN-KENTUCK CREEK NEAR CANAAN, IN}

Location.- Lat $38^{\circ} 52^{\prime} 41^{\prime \prime}$, long $85^{\circ} 15^{\prime} 26^{\prime \prime}$ referenced to North American Datum of 1927, in SW 1/4 NW 1/4 Sec. 13 , T.5 N., R.11 E., Jefferson County, IN, Hydrologic Unit 05140101, on downstream end of left pier of bridge on State Highway 62, 1,500 ft upstream from Wilson Fork, 2.0 mi northeast of Canaan, and at mile 16.7.

Drainage area. $-27.5 \mathrm{mi}^{2}$.

Period of record. - October 1969 to current year.

Average discharge. $-39.2 \mathrm{ft}^{3} / \mathrm{s}$.

Minimum daily discharge. $-0.0 \mathrm{ft}^{3} / \mathrm{s}$.

Human health (harmonic mean) design flow. $-0.5 \mathrm{ft}^{3} / \mathrm{s}$.

Remarks. - Some regulation by mill upstream from station.

\begin{tabular}{ccc}
\hline \multicolumn{3}{c}{ Magnitude and frequency of annual low flow } \\
\hline $\begin{array}{c}\text { Lowest average streamflow, in } \mathrm{ft}^{3} / \mathrm{s} \text {, for indicated period of } \\
\text { consecutive days at an annual nonexceedance probability of } 0.1\end{array}$ \\
\hline $\mathbf{1}$ & $\mathbf{3 0}$ \\
\hline 0.0 & 0.0 & 0.0 \\
\hline
\end{tabular}

Percentage of time streamflow was equaled or exceeded for the period of record

\begin{tabular}{cc|cc}
\hline $\begin{array}{c}\text { Percentage } \\
\text { of time }\end{array}$ & $\begin{array}{c}\text { Daily mean } \\
\text { streamflow } \\
\left(\mathrm{ft}^{3} / \mathbf{s}\right)\end{array}$ & $\begin{array}{c}\text { Percentage } \\
\text { of time }\end{array}$ & $\begin{array}{c}\text { Daily mean } \\
\text { streamflow } \\
\left(\mathrm{ft}^{3} / \mathbf{s}\right)\end{array}$ \\
\hline 99 & 0.0 & 40 & 16 \\
\hline 98 & 0.0 & 30 & 24 \\
\hline 95 & 0.0 & 20 & 39 \\
\hline 90 & 0.1 & 10 & 84 \\
\hline 80 & 0.9 & 5 & 173 \\
\hline 70 & 2.8 & 2 & 377 \\
\hline 60 & 5.6 & 1 & 571 \\
\hline 50 & 10 & & \\
\hline
\end{tabular}


Ohio River Basin

\section{SILVER CREEK NEAR SELLERSBURG, IN}

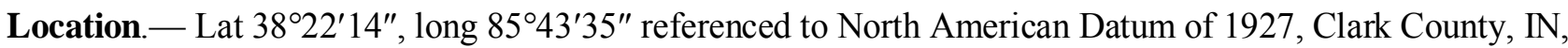
Hydrologic Unit 05140101, in lot 68, Clark Military Grant, on downstream side of Straws Mill bridge on Watson Road, $0.3 \mathrm{mi}$ downstream from Pleasant Run, $2.4 \mathrm{mi}$ southeast of Sellersburg, and $12.2 \mathrm{mi}$ upstream from mouth.

Drainage area. $-189 \mathrm{mi}^{2}$.

Period of record. - October 1954 to current year.

Average discharge. $-234 \mathrm{ft}^{3} / \mathrm{s}$.

Minimum daily discharge. $-0.0 \mathrm{ft}^{3} / \mathrm{s}$.

Human health (harmonic mean) design flow. $-5.1 \mathrm{ft}^{3} / \mathrm{s}$.

Remarks. - Some regulation by Deam Lake.

\begin{tabular}{ccc}
\hline \multicolumn{3}{c}{ Magnitude and frequency of annual low flow } \\
\hline $\begin{array}{c}\text { Lowest average streamflow, in } \mathrm{ft}^{3} / \mathrm{s} \text {, for indicated period of } \\
\text { consecutive days at an annual nonexceedance probability of } 0.1\end{array}$ \\
\hline $\mathbf{1}$ & $\mathbf{7}$ & $\mathbf{3 0}$ \\
\hline 0.0 & 0.1 & 0.4 \\
\hline
\end{tabular}

Percentage of time streamflow was equaled or exceeded for the period of record

\begin{tabular}{cc|cc}
\hline $\begin{array}{c}\text { Percentage } \\
\text { of time }\end{array}$ & $\begin{array}{c}\text { Daily mean } \\
\text { streamflow } \\
\left(\mathrm{ft}^{3} / \mathbf{s}\right)\end{array}$ & $\begin{array}{c}\text { Percentage } \\
\text { of time }\end{array}$ & $\begin{array}{c}\text { Daily mean } \\
\text { streamflow } \\
\left(\mathrm{ft}^{3} / \mathbf{s}\right)\end{array}$ \\
\hline 99 & 0.3 & 40 & 100 \\
\hline 98 & 0.5 & 30 & 158 \\
\hline 95 & 1.3 & 20 & 255 \\
\hline 90 & 3.1 & 10 & 515 \\
\hline 80 & 8.1 & 5 & 982 \\
\hline 70 & 17 & 2 & 2170 \\
\hline 60 & 33 & 1 & 3340 \\
\hline 50 & 59 & & \\
\hline
\end{tabular}


Ohio River Basin

\section{BUCK CREEK NEAR NEW MIDDLETOWN, IN}

Location. - Lat $38^{\circ} 07^{\prime} 13^{\prime \prime}$, long $86^{\circ} 05^{\prime} 17^{\prime \prime}$ referenced to North American Datum of 1927, in SE 1/4 NE $1 / 4$ sec.32, T.4 S., R.4 E., Harrison County, IN, Hydrologic Unit 05140104, on right bank at downstream side of bridge on State Highway 337, 0.6 mi downstream from South Fork Buck Creek, 3.6 mi southwest of New Middletown, and 14.6 mi upstream from mouth.

Drainage area.- $65.2 \mathrm{mi}^{2}$, of which $28.1 \mathrm{mi}^{2}$ does not contribute directly to surface runoff.

Period of record. - October 1969 to current year.

Average discharge. $-79.9 \mathrm{ft}^{3} / \mathrm{s}$

Minimum daily discharge. $-0.52 \mathrm{ft}^{3} / \mathrm{s}$.

Human health (harmonic mean) design flow. $-8.6 \mathrm{ft}^{3} / \mathrm{s}$.

Remarks.- None.

\begin{tabular}{ccc}
\hline \multicolumn{3}{c}{ Magnitude and frequency of annual low flow } \\
\hline $\begin{array}{c}\text { Lowest average streamflow, in } \mathrm{ft}^{3} / \mathrm{s} \text {, for indicated period of } \\
\text { consecutive days at an annual nonexceedance probability of } 0.1\end{array}$ \\
\hline $\mathbf{1}$ & $\mathbf{7}$ & $\mathbf{3 0}$ \\
\hline 0.7 & 0.8 & 1.3 \\
\hline
\end{tabular}

\begin{tabular}{cc|cc}
\hline Percentage of time streamflow was equaled or exceeded for \\
the period of record
\end{tabular}


Ohio River Basin

\section{LITTLE INDIAN CREEK NEAR GALENA, IN}

Location.- Lat $38^{\circ} 19^{\prime} 19^{\prime \prime}$, long $85^{\circ} 55^{\prime} 53^{\prime \prime}$, in NE $1 / 4 \mathrm{SW} 1 \frac{1}{4} \mathrm{sec} .23$, T.2 S., R.5 E., Floyd County, IN, Hydrologic Unit 05140104 , on right bank at downstream side of county road bridge, 2 mi south of Galena, 3.6 mi upstream from mouth, and 7.0 mi northwest of New Albany.

Drainage area. $-16.1 \mathrm{mi}^{2}$.

Period of record.- October 1968 to October 2003.

Average discharge. $-22.8 \mathrm{ft}^{3} / \mathrm{s}$.

Minimum daily discharge. $-0.0 \mathrm{ft}^{3} / \mathrm{s}$.

Human health (harmonic mean) design flow. $-0.6 \mathrm{ft}^{3} / \mathrm{s}$.

Remarks. - None.

Magnitude and frequency of annual low flow

Lowest average streamflow, in $\mathrm{ft}^{3} / \mathrm{s}$, for indicated period of consecutive days at an annual nonexceedance probability of 0.1

\begin{tabular}{ccc}
\hline $\mathbf{1}$ & $\mathbf{7}$ & $\mathbf{3 0}$ \\
\hline 0.0 & 0.0 & 0.0 \\
\hline
\end{tabular}

Percentage of time streamflow was equaled or exceeded for the period of record

\begin{tabular}{cc|cc}
\hline $\begin{array}{c}\text { Percentage } \\
\text { of time }\end{array}$ & $\begin{array}{c}\text { Daily mean } \\
\text { streamflow } \\
\left(\mathrm{ft}^{3} / \mathbf{s}\right)\end{array}$ & $\begin{array}{c}\text { Percentage } \\
\text { of time }\end{array}$ & $\begin{array}{c}\text { Daily mean } \\
\text { streamflow } \\
\left(\mathrm{ft}^{3} / \mathbf{s}\right)\end{array}$ \\
\hline 99 & 0.0 & 40 & 10.3 \\
\hline 98 & 0.0 & 30 & 15.9 \\
\hline 95 & 0.1 & 20 & 25 \\
\hline 90 & 0.2 & 10 & 46 \\
\hline 80 & 0.7 & 5 & 87 \\
\hline 70 & 1.7 & 2 & 187 \\
\hline 60 & 3.3 & 1 & 307 \\
\hline 50 & 6.1 & & \\
\hline
\end{tabular}


Ohio River Basin

\section{INDIAN CREEK NEAR CORYDON, IN}

Location. - Lat $38^{\circ} 16^{\prime} 35^{\prime \prime}$, long $86^{\circ} 06^{\prime} 35^{\prime \prime}$, in SW 1/4 SE 1/4 sec.6, T.3 S., R.4 E., Harrison County, IN, Hydrologic Unit 05140104, on upstream side of bridge on State Highway 335, 0.6 mi upstream from Raccoon Branch, $4.5 \mathrm{mi}$ north of Corydon, and at mile 33.7.

Drainage area.- $129 \mathrm{mi}^{2}$, of which $10.6 \mathrm{mi}^{2}$ does not contribute directly to surface runoff.

Period of record.— October 1943 to September 1993.

Average discharge. $-166 \mathrm{ft}^{3} / \mathrm{s}$.

Minimum daily discharge. $-0.0 \mathrm{ft}^{3} / \mathrm{s}$.

Human health (harmonic mean) design flow. $-3.4 \mathrm{ft}^{3} / \mathrm{s}$.

Remarks. - None.

\begin{tabular}{ccc}
\hline \multicolumn{3}{c}{ Magnitude and frequency of annual low flow } \\
\hline $\begin{array}{c}\text { Lowest average streamflow, in } \mathrm{ft}^{3} / \mathrm{s} \text {, for indicated period of } \\
\text { consecutive days at an annual nonexceedance probability of } 0.1\end{array}$ \\
\hline $\mathbf{7}$ & $\mathbf{7}$ & $\mathbf{3 0}$ \\
\hline 0.0 & 0.0 & 0.2 \\
\hline
\end{tabular}

\begin{tabular}{cc|cc}
\hline Percentage of time streamflow was equaled or exceeded for \\
the period of record
\end{tabular}


Ohio River Basin

\section{WEST FORK BLUE RIVER AT SALEM, IN}

Location. - Lat $38^{\circ} 36^{\prime} 19^{\prime \prime}$, long $86^{\circ} 05^{\prime} 40^{\prime \prime}$ referenced to North American Datum of 1927, in SW 1/4 SE $1 / 4 \mathrm{sec} .17$, T.2 N., R.4 E., Washington County, IN, Hydrologic Unit 05140104, on left bank at downstream side of bridge on East Market Street, 0.35 mi east of County Court House in Salem, 6.0 mi upstream from Hoggatt Branch, and $6.9 \mathrm{mi}$ upstream from mouth.

Drainage area. $-19.0 \mathrm{mi}^{2}$.

Period of record.- July 1970 to current year.

Average discharge. $-26.2 \mathrm{ft}^{3} / \mathrm{s}$.

Minimum daily discharge. $-0.0 \mathrm{ft}^{3} / \mathrm{s}$.

Human health (harmonic mean) design flow. $-0.8 \mathrm{ft}^{3} / \mathrm{s}$.

Remarks. - None.

\begin{tabular}{ccc}
\hline \multicolumn{3}{c}{ Magnitude and frequency of annual low flow } \\
\hline $\begin{array}{c}\text { Lowest average streamflow, in } \mathrm{ft}^{3} / \mathrm{s} \text {, for indicated period of } \\
\text { consecutive days at an annual nonexceedance probability of } 0.1\end{array}$ \\
\hline $\mathbf{1}$ & $\mathbf{7}$ & $\mathbf{3 0}$ \\
\hline 0.0 & 0.0 & 0.1 \\
\hline
\end{tabular}

Percentage of time streamflow was equaled or exceeded for the period of record

\begin{tabular}{cc|cc}
\hline $\begin{array}{c}\text { Percentage } \\
\text { of time }\end{array}$ & $\begin{array}{c}\text { Daily mean } \\
\text { streamflow } \\
\left(\mathrm{ft}^{3} / \mathbf{s}\right)\end{array}$ & $\begin{array}{c}\text { Percentage } \\
\text { of time }\end{array}$ & $\begin{array}{c}\text { Daily mean } \\
\text { streamflow } \\
\left(\mathrm{ft}^{3} / \mathbf{s}\right)\end{array}$ \\
\hline 99 & 0.0 & 40 & 13 \\
\hline 98 & 0.1 & 30 & 19 \\
\hline 95 & 0.2 & 20 & 30 \\
\hline 90 & 0.4 & 10 & 61 \\
\hline 80 & 1.3 & 5 & 104 \\
\hline 70 & 2.8 & 2 & 205 \\
\hline 60 & 4.9 & 1 & 316 \\
\hline 50 & 7.9 & & \\
\hline
\end{tabular}


Ohio River Basin

\section{BLUE RIVER AT FREDERICKSBURG, IN}

Location. - Lat $38^{\circ} 26^{\prime} 02^{\prime \prime}$, long $86^{\circ} 11^{\prime} 30^{\prime \prime}$ referenced to North American Datum of 1927, in NE 1/4 NW 1/4 sec.16, T.1 S., R.3 E., Washington County, IN, Hydrologic Unit 05140104, on downstream side of bridge on U.S. Highway 150 at Fredericksburg, 0.5 mi downstream from South Fork Blue River, and at mile 57.1.

Drainage area. $-283 \mathrm{mi}^{2}$, of which $76.9 \mathrm{mi}^{2}$ does not contribute directly to runoff.

Period of record. - July 1968 to current year.

Average discharge. $-347 \mathrm{ft}^{3} / \mathrm{s}$.

Minimum daily discharge. $-1.8 \mathrm{ft}^{3} / \mathrm{s}$.

Human health (harmonic mean) design flow. $-38 \mathrm{ft}^{3} / \mathrm{s}$.

Remarks. - None.

\begin{tabular}{ccc}
\hline \multicolumn{3}{c}{ Magnitude and frequency of annual low flow } \\
\hline $\begin{array}{c}\text { Lowest average streamflow, in } \mathrm{ft}^{3} / \mathrm{s} \text {, for indicated period of } \\
\text { consecutive days at an annual nonexceedance probability of } \mathbf{0 . 1}\end{array}$ \\
\hline $\mathbf{1}$ & $\mathbf{7}$ & $\mathbf{3 0}$ \\
\hline 2.8 & 3.4 & 4.9 \\
\hline
\end{tabular}

Percentage of time streamflow was equaled or exceeded for the period of record

\begin{tabular}{cc|cc}
\hline $\begin{array}{c}\text { Percentage } \\
\text { of time }\end{array}$ & $\begin{array}{c}\text { Daily mean } \\
\text { streamflow } \\
\left(\mathrm{ft}^{3} / \mathbf{s}\right)\end{array}$ & $\begin{array}{c}\text { Percentage } \\
\text { of time }\end{array}$ & $\begin{array}{c}\text { Daily mean } \\
\text { streamflow } \\
\left(\mathrm{ft}^{3} / \mathbf{s}\right)\end{array}$ \\
\hline 99 & 4.4 & 40 & 189 \\
\hline 98 & 5.9 & 30 & 279 \\
\hline 95 & 9.0 & 20 & 434 \\
\hline 90 & 14 & 10 & 794 \\
\hline 80 & 28 & 5 & 1270 \\
\hline 70 & 48 & 2 & 2470 \\
\hline 60 & 78 & 1 & 3990 \\
\hline 50 & 124 & & \\
\hline
\end{tabular}




\section{WHISKEY RUN AT MARENGO, IN}

Location. - Lat $38^{\circ} 22^{\prime} 32^{\prime \prime}$, long $86^{\circ} 20^{\prime} 41^{\prime \prime}$ referenced to North American Datum of 1927, in SW 1/4 NW 1/4 Sec.6, T.2 S., R.2 E., Crawford County, IN, Hydrologic Unit 05140104, on left (north) bank approximately $100 \mathrm{ft}$ upstream from bridge and the intersection of North Main Street and North Water Street in Marengo, known as Old Town, 0.1 mi northwest of the intersection of State Highway 64 and North Main Street in Marengo, and 0.6 mi west of the intersection of State Highway 64 and State Highway 66.

Drainage area. $-7.02 \mathrm{mi}^{2}$.

Period of record.- October 1968 to September1993.

Average discharge. $-5.27 \mathrm{ft}^{3} / \mathrm{s}$.

Minimum daily discharge. $-0.0 \mathrm{ft}^{3} / \mathrm{s}$.

Human health (harmonic mean) design flow. $-0.1 \mathrm{ft}^{3} / \mathrm{s}$.

Remarks. - None.

\begin{tabular}{ccc}
\hline \multicolumn{3}{c}{ Magnitude and frequency of annual low flow } \\
\hline $\begin{array}{c}\text { Lowest average streamflow, in } \mathrm{ft}^{3} / \mathrm{s} \text {, for indicated period of } \\
\text { consecutive days at an annual nonexceedance probability of } 0.1\end{array}$ \\
\hline $\mathbf{1}$ & $\mathbf{3 0}$ \\
\hline 0.0 & 0.0 & 0.0 \\
\hline
\end{tabular}

Percentage of time streamflow was equaled or exceeded for the period of record

\begin{tabular}{cc|cc}
\hline $\begin{array}{c}\text { Percentage } \\
\text { of time }\end{array}$ & $\begin{array}{c}\text { Daily mean } \\
\text { streamflow } \\
\left(\mathrm{ft}^{3} / \mathbf{s}\right)\end{array}$ & $\begin{array}{c}\text { Percentage } \\
\text { of time }\end{array}$ & $\begin{array}{c}\text { Daily mean } \\
\text { streamflow } \\
\left(\mathrm{ft}^{3} / \mathbf{s}\right)\end{array}$ \\
\hline 99 & 0.0 & 40 & 1.0 \\
\hline 98 & 0.0 & 30 & 2.2 \\
\hline 95 & 0.0 & 20 & 4.0 \\
\hline 90 & 0.0 & 10 & 9.6 \\
\hline 80 & 0.0 & 5 & 22 \\
\hline 70 & 0.1 & 2 & 53 \\
\hline 60 & 0.2 & 1 & 80 \\
\hline 50 & 0.3 & & \\
\hline
\end{tabular}


Ohio River Basin

\section{BLUE RIVER NEAR WHITE CLOUD, IN}

Location. - Lat $38^{\circ} 14^{\prime} 15^{\prime \prime}$, long $86^{\circ} 13^{\prime} 42^{\prime \prime}$ referenced to North American Datum of 1927, in NW 1/4 SE 1/4 sec.19, T.3 S., R.3 E., Harrison County, IN, Hydrologic Unit 05140104, on left bank 400 ft downstream from Spring Creek, $600 \mathrm{ft}$ upstream from bridge on Interstate 64, $0.2 \mathrm{mi}$ upstream from bridge on State Highway 62, $0.8 \mathrm{mi}$ north of White Cloud, and at mile 14.7.

Drainage area.- $476 \mathrm{mi}^{2}$, of which $192 \mathrm{mi}^{2}$ does not contribute directly to surface runoff.

Period of record.- April 1931 to current year.

Average discharge. $-677 \mathrm{ft}^{3} / \mathrm{s}$.

Minimum daily discharge. $-9.4 \mathrm{ft}^{3} / \mathrm{s}$.

Human health (harmonic mean) design flow. $-109 \mathrm{ft}^{3} / \mathrm{s}$.

Remarks.- None.

\begin{tabular}{ccc}
\hline \multicolumn{3}{c}{ Magnitude and frequency of annual low flow } \\
\hline $\begin{array}{c}\text { Lowest average streamflow, in } \mathrm{ft}^{3} / \mathrm{s} \text {, for indicated period of } \\
\text { consecutive days at an annual nonexceedance probability of } 0.1\end{array}$ \\
\hline $\mathbf{1}$ & $\mathbf{7}$ & $\mathbf{3 0}$ \\
\hline 13 & 14 & 19 \\
\hline
\end{tabular}

Percentage of time streamflow was equaled or exceeded for the period of record

\begin{tabular}{cc|cc}
\hline $\begin{array}{c}\text { Percentage } \\
\text { of time }\end{array}$ & $\begin{array}{c}\text { Daily mean } \\
\text { streamflow } \\
\left(\mathrm{ft}^{3} / \mathbf{s}\right)\end{array}$ & $\begin{array}{c}\text { Percentage } \\
\text { of time }\end{array}$ & $\begin{array}{c}\text { Daily mean } \\
\text { streamflow } \\
\left(\mathrm{ft}^{3} / \mathbf{s}\right)\end{array}$ \\
\hline 99 & 17 & 40 & 407 \\
\hline 98 & 20 & 30 & 578 \\
\hline 95 & 27 & 20 & 861 \\
\hline 90 & 39 & 10 & 1510 \\
\hline 80 & 69 & 5 & 2480 \\
\hline 70 & 113 & 2 & 4710 \\
\hline 60 & 179 & 1 & 7140 \\
\hline 50 & 277 & & \\
\hline
\end{tabular}




\section{MIDDLE FORK ANDERSON RIVER AT BRISTOW, IN}

Location. - Lat $38^{\circ} 08^{\prime} 20^{\prime \prime}$, long $86^{\circ} 43^{\prime} 16^{\prime \prime}$ referenced to North American Datum of 1927, in NW 1/4 SE 1/4 sec.27, T.4 S., R.3 W., Perry County, IN, Hydrologic Unit 05140201, on left bank at downstream side of bridge on State Highway 145 at Bristow, 2.0 mi downstream from Coon Branch, 6.0 mi upstream from Sulphur Fork Creek, and at mile 14.1.

Drainage area. $-39.8 \mathrm{mi}^{2}$.

Period of record. - August 1961 to current year.

Average discharge. $-58 \mathrm{ft}^{3} / \mathrm{s}$.

Minimum daily discharge $-0.0 \mathrm{ft}^{3} / \mathrm{s}$.

Human health (harmonic mean) design flow. $-0.9 \mathrm{ft}^{3} / \mathrm{s}$.

Remarks.- None.

\begin{tabular}{|c|c|c|c|}
\hline \multicolumn{4}{|c|}{ Magnitude and frequency of annual low flow } \\
\hline \multicolumn{4}{|c|}{$\begin{array}{l}\text { Lowest average streamflow, in } \mathrm{ft}^{3} / \mathrm{s} \text {, for indicated period of } \\
\text { consecutive days at an annual nonexceedance probability of } 0.1\end{array}$} \\
\hline 1 & \multicolumn{2}{|c|}{7} & 30 \\
\hline 0.0 & \multicolumn{2}{|c|}{0.0} & 0.0 \\
\hline \multicolumn{4}{|c|}{$\begin{array}{c}\text { Percentage of time streamflow was equaled or exceeded for } \\
\text { the period of record }\end{array}$} \\
\hline $\begin{array}{l}\text { Percentage } \\
\text { of time }\end{array}$ & $\begin{array}{l}\text { Daily mean } \\
\text { streamflow } \\
\left(\mathrm{ft}^{3} / \mathrm{s}\right)\end{array}$ & $\begin{array}{l}\text { Percentage } \\
\text { of time }\end{array}$ & $\begin{array}{l}\text { Daily mean } \\
\text { streamflow } \\
\left(\mathrm{ft}^{3} / \mathrm{s}\right)\end{array}$ \\
\hline 99 & 0.0 & 40 & 28 \\
\hline 98 & 0.0 & 30 & 44 \\
\hline 95 & 0.0 & 20 & 73 \\
\hline 90 & 0.3 & 10 & 156 \\
\hline 80 & 1.5 & 5 & 303 \\
\hline 70 & 3.9 & 2 & 472 \\
\hline 60 & 8.4 & 1 & 589 \\
\hline 50 & 17 & & \\
\hline
\end{tabular}


Ohio River Basin

\section{CROOKED CREEK NEAR SANTA CLAUS, IN}

Location. - Lat $38^{\circ} 07^{\prime} 05^{\prime \prime}$, long 86 53'24", in SW 1/4 SE 1/4 sec.31, T.4 S., R.4 W., Spencer County, IN, Hydrologic Unit 05140201, on right bank at upstream side of bridge on county road, 1.3 mi east of Santa Claus Post Office, and 1.8 mi upstream from unnamed right-bank tributary.

Drainage area. $-7.86 \mathrm{mi}^{2}$.

Period of record.- October 1969 to October 2003.

Average discharge. $-11.3 \mathrm{ft}^{3} / \mathrm{s}$.

Minimum daily discharge. $-0.0 \mathrm{ft}^{3} / \mathrm{s}$.

Human health (harmonic mean) design flow. $-0.2 \mathrm{ft}^{3} / \mathrm{s}$.

Remarks. - None.

\section{Magnitude and frequency of annual low flow}

Lowest average streamflow, in $\mathrm{ft}^{3} / \mathrm{s}$, for indicated period of consecutive days at an annual nonexceedance probability of 0.1

\begin{tabular}{ccc}
\hline $\mathbf{1}$ & $\mathbf{7}$ & $\mathbf{3 0}$ \\
\hline 0.0 & 0.0 & 0.0 \\
\hline
\end{tabular}

\begin{tabular}{cc|cc}
\hline Percentage of time streamflow was equaled or exceeded for \\
the period of record
\end{tabular}


Ohio River Basin

\section{PIGEON CREEK NEAR FORT BRANCH, IN}

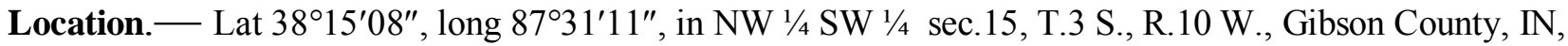
Hydrologic Unit 05140202, on right bank $20 \mathrm{ft}$ downstream from bridge on State Highway 168, 1.1 mi upstream from West Fork Pigeon Creek and 2.6 mi east of intersection of U.S. Highway 41 at Fort Branch.

Drainage area. $-35.4 \mathrm{mi}^{2}$.

Period of record.- October 1986 to October 2001.

Average discharge. $-38.0 \mathrm{ft}^{3} / \mathrm{s}$.

Minimum daily discharge. $-0.28 \mathrm{ft}^{3} / \mathrm{s}$.

Human health (harmonic mean) design flow. - $3.5 \mathrm{ft}^{3} / \mathrm{s}$.

Remarks. - None.

\section{Magnitude and frequency of annual low flow}

Lowest average streamflow, in $\mathrm{ft}^{3} / \mathrm{s}$, for indicated period of consecutive days at an annual nonexceedance probability of 0.1

\begin{tabular}{ccc}
\hline $\mathbf{1}$ & $\mathbf{7}$ & $\mathbf{3 0}$ \\
\hline 0.4 & 0.5 & 0.7 \\
\hline
\end{tabular}

\begin{tabular}{cc|cc}
\hline Percentage of time streamflow was equaled or exceeded for \\
the period of record
\end{tabular}


Wabash River Basin

\section{WABASH RIVER NEAR NEW CORYDON, IN}

Location. - Lat 40³3'50", long 8448'10", in NE 1/4 SE 1/4 sec. 3, T.24 N., R.15 E., Jay County, IN,Hydrologic Unit 05120101 , on left bank $10 \mathrm{ft}$ downstream from county bridge on Indiana-Ohio State line road, $2 \mathrm{mi}$ east of New Corydon, 2.8 mi downstream from Beaver Creek, and at mile 466.0.

Drainage area. $-262 \mathrm{mi}^{2}$.

Period of record.- April 1951 to September 1988.

Average discharge. $-200 \mathrm{ft}^{3} / \mathrm{s}$.

Minimum daily discharge. $-0.80 \mathrm{ft}^{3} / \mathrm{s}$.

Human health (harmonic mean) design flow. $-19 \mathrm{ft}^{3} / \mathrm{s}$.

Remarks.- Occasional regulation by Grand Lake, diversion from or into St. Marys River Basin, and into Miami and Erie Canals.

\begin{tabular}{ccc}
\hline \multicolumn{3}{c}{ Magnitude and frequency of annual low flow } \\
\hline $\begin{array}{c}\text { Lowest average streamflow, in } \mathrm{ft}^{3} / \mathrm{s}, \text { for indicated period of } \\
\text { consecutive days at an annual nonexceedance probability of } \mathbf{0 . 1}\end{array}$ \\
\hline $\mathbf{1}$ & $\mathbf{7}$ & $\mathbf{3 0}$ \\
\hline 1.9 & 2.3 & 4.1 \\
\hline
\end{tabular}

\begin{tabular}{cc|cc}
\hline Percentage of time streamflow was equaled or exceeded for \\
the period of record
\end{tabular}




\section{WABASH RIVER AT LINN GROVE, IN}

Location. - Lat $40^{\circ} 39^{\prime} 22^{\prime \prime}$, long $85^{\circ} 01^{\prime} 58^{\prime \prime}$ referenced to North American Datum of 1927, in SE 1/4 SE 1/4 Sec.34, T.26 N., R.13 E., Adams County, IN, Hydrologic Unit 05120101, (from USGS topographic quadrangle LINN GROVE, IN), on right bank $10 \mathrm{ft}$ downstream from bridge on State Highway 218, $800 \mathrm{ft}$ downstream from Shoemaker Ditch, 0.8 mi north of Linn Grove, and 2.2 mi upstream from Rice Ditch, 0.8 mi east of junction of State Highways 116 and 218, and 0.8 mi north of Linn Grove.

Drainage area. $-453 \mathrm{mi}^{2}$.

Period of record. - September 1964 to current year.

Average discharge. $-433 \mathrm{ft}^{3} / \mathrm{s}$.

Minimum daily discharge. $-4.0 \mathrm{ft}^{3} / \mathrm{s}$.

Human health (harmonic mean) design flow. $-40 \mathrm{ft}^{3} / \mathrm{s}$.

Remarks. - Occasional regulation by Grand Lake, diversion from or into St. Marys River Basin, and into Miami and Erie Canals.

\begin{tabular}{|c|c|c|c|}
\hline \multicolumn{4}{|c|}{ Magnitude and frequency of annual low flow } \\
\hline \multicolumn{4}{|c|}{$\begin{array}{l}\text { Lowest average streamflow, in } \mathrm{ft}^{3} / \mathrm{s} \text {, for indicated period of } \\
\text { consecutive days at an annual nonexceedance probability of } 0 .\end{array}$} \\
\hline 1 & \multicolumn{2}{|c|}{7} & 30 \\
\hline 4.9 & \multicolumn{2}{|c|}{5.7} & 7.3 \\
\hline \multicolumn{4}{|c|}{$\begin{array}{c}\text { Percentage of time streamflow was equaled or exceeded for } \\
\text { the period of record }\end{array}$} \\
\hline $\begin{array}{l}\text { Percentage } \\
\text { of time }\end{array}$ & $\begin{array}{l}\text { Daily mean } \\
\text { streamflow } \\
\left(\mathrm{ft}^{3} / \mathrm{s}\right)\end{array}$ & $\begin{array}{l}\text { Percentage } \\
\text { of time }\end{array}$ & $\begin{array}{l}\text { Daily mean } \\
\text { streamflow } \\
\left(\mathrm{ft}^{3} / \mathrm{s}\right)\end{array}$ \\
\hline 99 & 6.5 & 40 & 196 \\
\hline 98 & 7.3 & 30 & 303 \\
\hline 95 & 9.7 & 20 & 544 \\
\hline 90 & 13 & 10 & 1280 \\
\hline 80 & 23 & 5 & 2080 \\
\hline 70 & 40 & 2 & 3270 \\
\hline 60 & 71 & 1 & 4230 \\
\hline 50 & 124 & & \\
\hline
\end{tabular}


Wabash River Basin

\section{WABASH RIVER NEAR BLUFFTON, IN}

Location.- Lat $40^{\circ} 43^{\prime} 41^{\prime \prime}$, long $85^{\circ} 08^{\prime} 12^{\prime \prime}$ referenced to North American Datum of 1927, in NE 1/4 NW 1/4 Sec.11, T.26 N., R.12 E., Wells County, IN, Hydrologic Unit 05120101, on left bank $300 \mathrm{ft}$ downstream of bridge on County Road 450 East (State Highway 201), 0.95 mi south of State Highway 124, 2.5 mi southeast of Bluffton, and at mile 436.6 .

Drainage area. $-508 \mathrm{mi}^{2}$.

Period of record. - September 2001 to current year.

Average discharge. $-631 \mathrm{ft}^{3} / \mathrm{s}$.

Minimum daily discharge. $-5.9 \mathrm{ft}^{3} / \mathrm{s}$.

Human health (harmonic mean) design flow. $-61 \mathrm{ft}^{3} / \mathrm{s}$.

Remarks.- Occasional regulation by Grand Lake, diversion from or into St. Marys River Basin, and into Miami and Erie Canals.

\begin{tabular}{|c|c|c|c|}
\hline \multicolumn{4}{|c|}{ Magnitude and frequency of annual low flow } \\
\hline \multicolumn{4}{|c|}{$\begin{array}{l}\text { Lowest average streamflow, in } \mathrm{ft}^{3} / \mathrm{s} \text {, for indicated period of } \\
\text { consecutive days at an annual nonexceedance probability of } 0.1\end{array}$} \\
\hline 1 & \multicolumn{2}{|c|}{7} & 30 \\
\hline 6.0 & \multicolumn{2}{|c|}{6.8} & 9.4 \\
\hline \multicolumn{4}{|c|}{$\begin{array}{l}\text { Percentage of time streamflow was equaled or exceeded for } \\
\text { the period of record }\end{array}$} \\
\hline $\begin{array}{l}\text { Percentage } \\
\text { of time }\end{array}$ & $\begin{array}{c}\text { Daily mean } \\
\text { streamflow } \\
\left(\mathrm{ft}^{3} / \mathrm{s}\right)\end{array}$ & $\begin{array}{l}\text { Percentage } \\
\text { of time }\end{array}$ & $\begin{array}{c}\text { Daily mean } \\
\text { streamflow } \\
\left(\mathrm{ft}^{3} / \mathrm{s}\right)\end{array}$ \\
\hline 99 & 9.7 & 40 & 290 \\
\hline 98 & 12 & 30 & 488 \\
\hline 95 & 14 & 20 & 973 \\
\hline 90 & 20 & 10 & 1950 \\
\hline 80 & 35 & 5 & 2920 \\
\hline 70 & 62 & 2 & 4110 \\
\hline 60 & 124 & 1 & 5200 \\
\hline 50 & 196 & & \\
\hline
\end{tabular}




\section{WABASH RIVER AT BLUFFTON, IN}

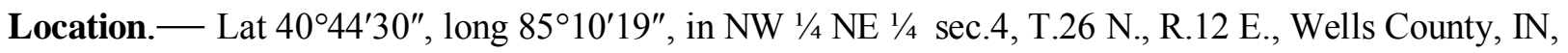
Hydrologic Unit 05120101, on downstream side of left abutment of Main Street (State Highway 1) bridge in Bluffton, 2 mi downstream from Sixmile Creek, and at mile 434.5.

Drainage area. $-532 \mathrm{mi}^{2}$.

Period of record. - October 1930 to September 1971.

Average discharge. $-387 \mathrm{ft}^{3} / \mathrm{s}$.

Minimum daily discharge. $-4.0 \mathrm{ft}^{3} / \mathrm{s}$.

Human health (harmonic mean) design flow. $-32 \mathrm{ft}^{3} / \mathrm{s}$.

Remarks. - None.

\section{Magnitude and frequency of annual low flow}

Lowest average streamflow, in $\mathrm{ft}^{3} / \mathrm{s}$, for indicated period of consecutive days at an annual nonexceedance probability of 0.1

\begin{tabular}{ccc}
\hline $\mathbf{1}$ & $\mathbf{7}$ & $\mathbf{3 0}$ \\
\hline 4.6 & 5.1 & 6.5 \\
\hline
\end{tabular}

\begin{tabular}{cc|cc}
\hline Percentage of time streamflow was equaled or exceeded for \\
the period of record
\end{tabular}




\section{WABASH RIVER AT HUNTINGTON, IN}

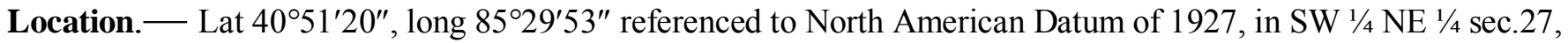
T.28 N., R.9 E., Huntington County, IN, Hydrologic Unit 05120101, on right bank at the Huntington Water and Light Plant, 2 mi south of Huntington, 2.4 mi downstream from Huntington Lake, 3.2 mi upstream from Little River, and at mile 409.0.

Drainage area. $-721 \mathrm{mi}^{2}$.

Period of record.- January 1951 to September 1976 (discharge). October 1976 to September 2001 (discharge provided by U.S. Army Corps of Engineers).

Average discharge. $-604 \mathrm{ft}^{3} / \mathrm{s}$.

Minimum daily discharge. $-0.0 \mathrm{ft}^{3} / \mathrm{s}$.

Human health (harmonic mean) design flow. - $101 \mathrm{ft}^{3} / \mathrm{s}$.

Remarks. - Flow regulated by Huntington Lake. Low-flow statistics are calculated for the regulated period, 1969 to 2001. Daily discharge computed from relation among discharge, head, and gate openings for Huntington Lake beginning Oct. 1, 1974.

\begin{tabular}{|c|c|c|c|}
\hline \multicolumn{4}{|c|}{ Magnitude and frequency of annual low flow } \\
\hline \multicolumn{4}{|c|}{$\begin{array}{l}\text { Lowest average streamflow, in } \mathrm{ft}^{3} / \mathrm{s} \text {, for indicated period of } \\
\text { consecutive days at an annual nonexceedance probability of } 0 .\end{array}$} \\
\hline 1 & \multicolumn{2}{|c|}{7} & 30 \\
\hline 8.2 & \multicolumn{2}{|c|}{16} & 23 \\
\hline \multicolumn{4}{|c|}{$\begin{array}{c}\text { Percentage of time streamflow was equaled or exceeded for } \\
\text { the period of record }\end{array}$} \\
\hline $\begin{array}{l}\text { Percentage } \\
\text { of time }\end{array}$ & $\begin{array}{l}\text { Daily mean } \\
\text { streamflow } \\
\left(\mathrm{ft}^{3} / \mathrm{s}\right)\end{array}$ & $\begin{array}{l}\text { Percentage } \\
\text { of time }\end{array}$ & $\begin{array}{l}\text { Daily mean } \\
\text { streamflow } \\
\left(\mathrm{ft}^{3} / \mathrm{s}\right)\end{array}$ \\
\hline 99 & 19 & 40 & 280 \\
\hline 98 & 22 & 30 & 457 \\
\hline 95 & 27 & 20 & 912 \\
\hline 90 & 42 & 10 & 2040 \\
\hline 80 & 68 & 5 & 3050 \\
\hline 70 & 93 & 2 & 3930 \\
\hline 60 & 128 & 1 & 4590 \\
\hline 50 & 195 & & \\
\hline
\end{tabular}




\section{LITTLE RIVER NEAR HUNTINGTON, IN}

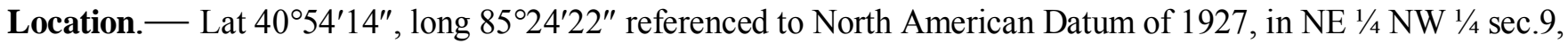
T.28 N., R.10 E., Huntington County, IN, Hydrologic Unit 05120101, on right bank on upstream side of former highway bridge, $0.5 \mathrm{mi}$ upstream of County Road 200 East bridge, 5 mi east of Huntington, and at mile 7.5.

Drainage area. $-263 \mathrm{mi}^{2}$.

Period of record.- October 1943 to current year. Prior to January 1944, monthly discharge only.

Average discharge. $-247 \mathrm{ft}^{3} / \mathrm{s}$.

Minimum daily discharge. $-1.1 \mathrm{ft}^{3} / \mathrm{s}$.

Human health (harmonic mean) design flow. $-37 \mathrm{ft}^{3} / \mathrm{s}$.

Remarks.- None.

\begin{tabular}{|c|c|c|c|}
\hline \multicolumn{4}{|c|}{ Magnitude and frequency of annual low flow } \\
\hline \multicolumn{4}{|c|}{$\begin{array}{l}\text { Lowest average streamflow, in } \mathrm{ft}^{3} / \mathrm{s} \text {, for indicated period of } \\
\text { consecutive days at an annual nonexceedance probability of } 0.1\end{array}$} \\
\hline 1 & \multicolumn{2}{|c|}{7} & 30 \\
\hline 4.4 & \multicolumn{2}{|c|}{5.4} & 7.5 \\
\hline \multicolumn{4}{|c|}{$\begin{array}{l}\text { Percentage of time streamflow was equaled or exceeded for } \\
\text { the period of record }\end{array}$} \\
\hline $\begin{array}{l}\text { Percentage } \\
\text { of time }\end{array}$ & $\begin{array}{l}\text { Daily mean } \\
\text { streamflow } \\
\left(\mathrm{ft}^{3} / \mathrm{s}\right)\end{array}$ & $\begin{array}{l}\text { Percentage } \\
\text { of time }\end{array}$ & $\begin{array}{l}\text { Daily mean } \\
\text { streamflow } \\
\left(\mathrm{ft}^{3} / \mathrm{s}\right)\end{array}$ \\
\hline 99 & 6.2 & 40 & 103 \\
\hline 98 & 7.5 & 30 & 153 \\
\hline 95 & 11 & 20 & 271 \\
\hline 90 & 16 & 10 & 628 \\
\hline 80 & 25 & 5 & 1240 \\
\hline 70 & 35 & 2 & 2160 \\
\hline 60 & 51 & 1 & 2750 \\
\hline 50 & 73 & & \\
\hline
\end{tabular}


Wabash River Basin

\section{SALAMONIE RIVER AT PORTLAND, IN}

Location.- Lat $40^{\circ} 25^{\prime} 40^{\prime \prime}$, long $85^{\circ} 02^{\prime} 20^{\prime \prime}$, in NE $1 / 4$ SE 1/4 sec.23, T.23 N., R.13 E., Jay County, IN, Hydrologic Unit 05120102, on right bank at downstream side of county road bridge, $2.3 \mathrm{mi}$ downstream from Butternut Creek, 3.2 mi west of Portland, 3.7 mi downstream from Little Salamonie River, and at mile 70.5.

Drainage area. $-85.6 \mathrm{mi}^{2}$.

Period of record.-September 1959 to 1993.

Average discharge. $-75.3 \mathrm{ft}^{3} / \mathrm{s}$.

Minimum daily discharge. $-0.40 \mathrm{ft}^{3} / \mathrm{s}$.

Human health (harmonic mean) design flow. $-6.1 \mathrm{ft}^{3} / \mathrm{s}$.

Remarks. - Natural flow partially affected by effluent.

\begin{tabular}{|c|c|c|c|}
\hline \multicolumn{4}{|c|}{ Magnitude and frequency of annual low flow } \\
\hline \multicolumn{4}{|c|}{$\begin{array}{l}\text { Lowest average streamflow, in } \mathrm{ft}^{3} / \mathrm{s} \text {, for indicated period of } \\
\text { consecutive days at an annual nonexceedance probability of } 0.1\end{array}$} \\
\hline 1 & \multicolumn{2}{|c|}{7} & 30 \\
\hline 0.6 & \multicolumn{2}{|c|}{1.0} & 1.4 \\
\hline \multicolumn{4}{|c|}{$\begin{array}{l}\text { Percentage of time streamflow was equaled or exceeded for } \\
\text { the period of record }\end{array}$} \\
\hline $\begin{array}{l}\text { Percentage } \\
\text { of time }\end{array}$ & $\begin{array}{l}\text { Daily mean } \\
\text { streamflow } \\
\left(\mathrm{ft}^{3} / \mathrm{s}\right)\end{array}$ & $\begin{array}{l}\text { Percentage } \\
\text { of time }\end{array}$ & $\begin{array}{c}\text { Daily mean } \\
\text { streamflow } \\
\left(\mathrm{ft}^{3} / \mathrm{s}\right)\end{array}$ \\
\hline 99 & 1.2 & 40 & 20 \\
\hline 98 & 1.4 & 30 & 32 \\
\hline 95 & 1.8 & 20 & 61 \\
\hline 90 & 2.3 & 10 & 156 \\
\hline 80 & 3.5 & 5 & 356 \\
\hline 70 & 5.3 & 2 & 817 \\
\hline 60 & 8.1 & 1 & 1220 \\
\hline 50 & 13 & & \\
\hline
\end{tabular}




\section{SALAMONIE RIVER NEAR WARREN, IN}

Location.- Lat $40^{\circ} 42^{\prime} 45^{\prime \prime}$, long $85^{\circ} 27^{\prime} 13^{\prime \prime}$ referenced to North American Datum of 1927 , in SE $1 / 4$ SE $1 / 4$ sec.12, T.26 N., R.9 E., Huntington County, IN, Hydrologic Unit 05120102, on right bank at downstream side of bridge on County Road 800 South, 0.4 mi downstream from Detamore Ditch, 0.4 mi downstream from Interstate 69, $0.8 \mathrm{mi}$ upstream from concrete and stone dam, 2.4 mi northwest of Warren, and at mile 30.0.

Drainage area. $-425 \mathrm{mi}^{2}$.

Period of record. - March 1957 to current year.

Average discharge. $-418 \mathrm{ft}^{3} / \mathrm{s}$.

Minimum daily discharge. $-0.09 \mathrm{ft}^{3} / \mathrm{s}$.

Human health (harmonic mean) design flow. $-50 \mathrm{ft}^{3} / \mathrm{s}$.

Remarks.- None.

\begin{tabular}{|c|c|c|c|}
\hline \multicolumn{4}{|c|}{ Magnitude and frequency of annual low flow } \\
\hline \multicolumn{4}{|c|}{$\begin{array}{l}\text { Lowest average streamflow, in } \mathrm{ft}^{3} / \mathrm{s} \text {, for indicated period of } \\
\text { consecutive days at an annual nonexceedance probability of } 0.1\end{array}$} \\
\hline 1 & \multicolumn{2}{|c|}{7} & 30 \\
\hline 6.8 & \multicolumn{2}{|c|}{8.1} & 10 \\
\hline \multicolumn{4}{|c|}{$\begin{array}{l}\text { Percentage of time streamflow was equaled or exceeded for } \\
\text { the period of record }\end{array}$} \\
\hline $\begin{array}{l}\text { Percentage } \\
\text { of time }\end{array}$ & $\begin{array}{c}\text { Daily mean } \\
\text { streamflow } \\
\left(\mathrm{ft}^{3} / \mathrm{s}\right)\end{array}$ & $\begin{array}{l}\text { Percentage } \\
\text { of time }\end{array}$ & $\begin{array}{c}\text { Daily mean } \\
\text { streamflow } \\
\left(\mathrm{ft}^{3} / \mathrm{s}\right)\end{array}$ \\
\hline 99 & 9.4 & 40 & 138 \\
\hline 98 & 11 & 30 & 219 \\
\hline 95 & 14 & 20 & 408 \\
\hline 90 & 18 & 10 & 1070 \\
\hline 80 & 31 & 5 & 2250 \\
\hline 70 & 48 & 2 & 3870 \\
\hline 60 & 69 & 1 & 4980 \\
\hline 50 & 96 & & \\
\hline
\end{tabular}


Wabash River Basin

\section{SALAMONIE RIVER AT DORA, IN}

Location.- Lat $40^{\circ} 48^{\prime} 42^{\prime \prime}$, long $85^{\circ} 41^{\prime} 02^{\prime \prime}$ referenced to North American Datum of 1927, in NE 1/4 NE 1/4 Sec.12, T.27 N., R.7 E., Wabash County, IN, Hydrologic Unit 05120102, on right bank, 0.4 mi downstream from Salamonie Lake, $1.5 \mathrm{mi}$ northwest of Dora, and $3.0 \mathrm{mi}$ upstream from mouth.

Drainage area. $-557 \mathrm{mi}^{2}$.

Period of record.- November 1923 to September 1976 (discharge). October 1976 to September 2001 (discharge provided by U.S. Army Corps of Engineers).

Average discharge. $-515 \mathrm{ft}^{3} / \mathrm{s}$.

Minimum daily discharge. $-0.70 \mathrm{ft}^{3} / \mathrm{s}$.

Human health (harmonic mean) design flow. $-82 \mathrm{ft}^{3} / \mathrm{s}$.

Remarks. - Flow regulated by Salamonie Lake since April 1967. Low-flow statistics are calculated for the regulated period 1967 to 2001 and include periods of extreme regulation.

\begin{tabular}{|c|c|c|c|}
\hline \multicolumn{4}{|c|}{ Magnitude and frequency of annual low flow } \\
\hline \multicolumn{4}{|c|}{$\begin{array}{l}\text { Lowest average streamflow, in } \mathrm{ft}^{3} / \mathrm{s} \text {, for indicated period of } \\
\text { consecutive days at an annual nonexceedance probability of } 0.1\end{array}$} \\
\hline 1 & \multicolumn{2}{|c|}{7} & 30 \\
\hline 11 & \multicolumn{2}{|c|}{16} & 18 \\
\hline \multicolumn{4}{|c|}{$\begin{array}{l}\text { Percentage of time streamflow was equaled or exceeded for } \\
\text { the period of record }\end{array}$} \\
\hline $\begin{array}{l}\text { Percentage } \\
\text { of time }\end{array}$ & $\begin{array}{l}\text { Daily mean } \\
\text { streamflow } \\
\left(\mathrm{ft}^{3} / \mathrm{s}\right)\end{array}$ & $\begin{array}{l}\text { Percentage } \\
\text { of time }\end{array}$ & $\begin{array}{l}\text { Daily mean } \\
\text { streamflow } \\
\left(\mathrm{ft}^{3} / \mathrm{s}\right)\end{array}$ \\
\hline 99 & 17 & 40 & 340 \\
\hline 98 & 20 & 30 & 470 \\
\hline 95 & 22 & 20 & 758 \\
\hline 90 & 25 & 10 & 1590 \\
\hline 80 & 47 & 5 & 2470 \\
\hline 70 & 88 & 2 & 3570 \\
\hline 60 & 136 & 1 & 4310 \\
\hline 50 & 229 & & \\
\hline
\end{tabular}




\section{WABASH RIVER AT WABASH, IN}

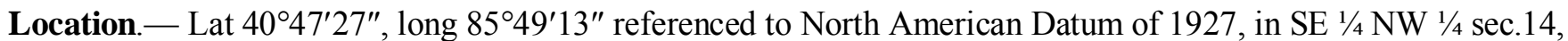
T.27 N., R.6 E., Wabash County, IN, Hydrologic Unit 05120101, on right bank $200 \mathrm{ft}$ downstream of Wabash Street bridge in Wabash, $500 \mathrm{ft}$ north of intersection of State Highway 15 and Columbus Street, 0.5 mi downstream from railroad bridge, $7 \mathrm{mi}$ downstream from Salamonie River, $12 \mathrm{mi}$ upstream from Mississinewa River, and at mi 387.2.

Drainage area. $-1,768 \mathrm{mi}^{2}$.

Period of record.- August 1923 to current year.

Average discharge. - $1,610 \mathrm{ft}^{3} / \mathrm{s}$.

Minimum daily discharge. $-17 \mathrm{ft}^{3} / \mathrm{s}$.

Human health (harmonic mean) design flow. $-425 \mathrm{ft}^{3} / \mathrm{s}$.

Remarks. - Flow regulated by Salamonie Lake beginning April 1967, Huntington Lake beginning October 1976, and Grand Lake Reservoir. Low-flow statistics are calculated for the regulated period, 1969 to 2011.

\begin{tabular}{cccc}
\hline \multicolumn{5}{c}{ Magnitude and frequency of annual low flow } \\
\hline \multicolumn{4}{c}{ Lowest average streamflow, in $\mathrm{ft}^{3} / \mathrm{s}$, for indicated period of } \\
consecutive days at an annual nonexceedance probability of 0.1
\end{tabular}


Wabash River Basin

\section{LITTLE MISSISSINEWA RIVER AT UNION CITY, IN}

Location.- Lat $40^{\circ} 11^{\prime} 46^{\prime \prime}$, long $84^{\circ} 49^{\prime} 45^{\prime \prime}$ referenced to North American Datum of 1927, in SE 1/4 SE 1/4 Sec.26, T.18 N., R.1 W., Randolph County, IN, Hydrologic Unit 05120103, on right bank $85 \mathrm{ft}$ downstream from Westinghouse Road, $0.5 \mathrm{mi}$ downstream from Little Ditch, $0.8 \mathrm{mi}$ upstream from City Drain, and $1.2 \mathrm{mi}$ west of the Post Office in Union City.

Drainage area. $-9.67 \mathrm{mi}^{2}$.

Period of record.-October 1982 to 1997.

Average discharge. $-10.1 \mathrm{ft}^{3} / \mathrm{s}$.

Minimum daily discharge. $-0.0 \mathrm{ft}^{3} / \mathrm{s}$.

Human health (harmonic mean) design flow. $-0.3 \mathrm{ft} 3 / \mathrm{s}$.

Remarks. - None.

\begin{tabular}{|c|c|c|c|}
\hline \multicolumn{4}{|c|}{ Magnitude and frequency of annual low flow } \\
\hline \multicolumn{4}{|c|}{$\begin{array}{l}\text { Lowest average streamflow, in } \mathrm{ft}^{3} / \mathrm{s} \text {, for indicated period of } \\
\text { consecutive days at an annual nonexceedance probability of } 0.1\end{array}$} \\
\hline 1 & \multicolumn{2}{|c|}{7} & 30 \\
\hline 0.0 & \multicolumn{2}{|c|}{0.0} & 0.0 \\
\hline \multicolumn{4}{|c|}{$\begin{array}{l}\text { Percentage of time streamflow was equaled or exceeded for } \\
\text { the period of record }\end{array}$} \\
\hline $\begin{array}{l}\text { Percentage } \\
\text { of time }\end{array}$ & $\begin{array}{l}\text { Daily mean } \\
\text { streamflow } \\
\left(\mathrm{ft}^{3} / \mathrm{s}\right)\end{array}$ & $\begin{array}{l}\text { Percentage } \\
\text { of time }\end{array}$ & $\begin{array}{l}\text { Daily mean } \\
\text { streamflow } \\
\left(\mathrm{ft}^{3} / \mathrm{s}\right)\end{array}$ \\
\hline 99 & 0.0 & 40 & 4.3 \\
\hline 98 & 0.0 & 30 & 6.5 \\
\hline 95 & 0.0 & 20 & 12 \\
\hline 90 & 0.1 & 10 & 28 \\
\hline 80 & 0.4 & 5 & 50 \\
\hline 70 & 1.1 & 2 & 79 \\
\hline 60 & 1.9 & 1 & 106 \\
\hline 50 & 2.9 & & \\
\hline
\end{tabular}




\section{MISSISSINEWA RIVER NEAR RIDGEVILLE, IN}

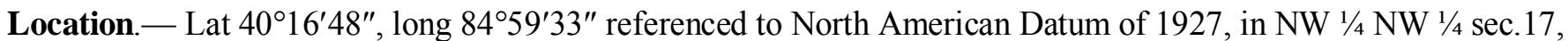
T.21 N., R.14 E., Randolph County, IN, Hydrologic Unit 05120103, on left bank $800 \mathrm{ft}$ upstream from county road bridge, 0.6 mi downstream from Mud Creek, 2 mi east of Ridgeville, and at mile 99.7.

Drainage area.- $133 \mathrm{mi}^{2}$.

Period of record. - August 1946 to current year.

Average discharge. $-137 \mathrm{ft}^{3} / \mathrm{s}$.

Minimum daily discharge. $-0.10 \mathrm{ft}^{3} / \mathrm{s}$.

Human health (harmonic mean) design flow. $-10 \mathrm{ft}^{3} / \mathrm{s}$.

Remarks. - None.

\begin{tabular}{cccc}
\hline \multicolumn{4}{c}{ Magnitude and frequency of annual low flow } \\
\hline \multicolumn{4}{c}{ Lowest average streamflow, in $\mathrm{ft}^{3}$ /s, for indicated period of } \\
consecutive days at an annual nonexceedance probability of 0.1
\end{tabular}


Wabash River Basin

\section{MISSISSINEWA RIVER NEAR EATON, IN}

Location. - Lat $40^{\circ} 19^{\prime} 08^{\prime \prime}$, long $85^{\circ} 19^{\prime} 10^{\prime \prime}$ referenced to North American Datum of 1927, in NW 1/4 NE 1/4 sec.31, T.22 N., R.11 E., Delaware County, IN, on right bank at downstream side of bridge, $1.5 \mathrm{mi}$ upstream from Estey Creek, and $2.5 \mathrm{mi}$ southeast of Eaton.

Drainage area. $-310 \mathrm{mi}^{2}$.

Period of record.-March 1952 to September 1971.

Average discharge. $-266 \mathrm{ft}^{3} / \mathrm{s}$.

Minimum daily discharge. $-2.0 \mathrm{ft}^{3} / \mathrm{s}$.

Human health (harmonic mean) design flow. $-25 \mathrm{ft}^{3} / \mathrm{s}$.

Remarks.- None.

\begin{tabular}{|c|c|c|c|}
\hline \multicolumn{4}{|c|}{ Magnitude and frequency of annual low flow } \\
\hline \multicolumn{4}{|c|}{$\begin{array}{l}\text { Lowest average streamflow, in } \mathrm{ft}^{3} / \mathrm{s} \text {, for indicated period of } \\
\text { consecutive days at an annual nonexceedance probability of } 0.1\end{array}$} \\
\hline 1 & \multicolumn{2}{|c|}{7} & 30 \\
\hline 2.4 & \multicolumn{2}{|c|}{2.9} & 3.7 \\
\hline \multicolumn{4}{|c|}{$\begin{array}{l}\text { Percentage of time streamflow was equaled or exceeded for } \\
\text { the period of record }\end{array}$} \\
\hline $\begin{array}{l}\text { Percentage } \\
\text { of time }\end{array}$ & $\begin{array}{l}\text { Daily mean } \\
\text { streamflow } \\
\left(\mathrm{ft}^{3} / \mathrm{s}\right)\end{array}$ & $\begin{array}{l}\text { Percentage } \\
\text { of time }\end{array}$ & $\begin{array}{l}\text { Daily mean } \\
\text { streamflow } \\
\left(\mathrm{ft}^{3} / \mathrm{s}\right)\end{array}$ \\
\hline 99 & 3.6 & 40 & 86 \\
\hline 98 & 4.8 & 30 & 134 \\
\hline 95 & 6.5 & 20 & 254 \\
\hline 90 & 9.1 & 10 & 590 \\
\hline 80 & 16 & 5 & 1330 \\
\hline 70 & 25 & 2 & 2490 \\
\hline 60 & 39 & 1 & 3580 \\
\hline 50 & 59 & & \\
\hline
\end{tabular}




\section{BIG LICK CREEK NEAR HARTFORD CITY, IN}

Location.- Lat $40^{\circ} 25^{\prime} 20^{\prime \prime}$, long $85^{\circ} 21^{\prime} 04^{\prime \prime}$ sec.23, T.23 N., R.10 E., Blackford County, IN, Hydrologic Unit 05120103, on right bank $6 \mathrm{ft}$ downstream from bridge on County Road 100 East, and 2.0 mi southeast of Hartford City.

Drainage area. $-29.2 \mathrm{mi}^{2}$.

Period of record.- July 1971 to October 2003.

Average discharge. $-28.8 \mathrm{ft}^{3} / \mathrm{s}$.

Minimum daily discharge. $-0.19 \mathrm{ft}^{3} / \mathrm{s}$.

Human health (harmonic mean) design flow. $-3 \mathrm{ft}^{3} / \mathrm{s}$.

Remarks.- None.

\begin{tabular}{ccc}
\hline \multicolumn{3}{c}{ Magnitude and frequency of annual low flow } \\
\hline $\begin{array}{c}\text { Lowest average streamflow, in } \mathrm{ft}^{3} / \mathrm{s} \text {, for indicated period of } \\
\text { consecutive days at an annual nonexceedance probability of } \mathbf{0 . 1}\end{array}$ \\
\hline $\mathbf{1}$ & $\mathbf{7}$ & $\mathbf{3 0}$ \\
\hline 0.3 & 0.5 & 0.7 \\
\hline
\end{tabular}

Percentage of time streamflow was equaled or exceeded for the period of record

\begin{tabular}{cc|cc}
\hline $\begin{array}{c}\text { Percentage } \\
\text { of time }\end{array}$ & $\begin{array}{c}\text { Daily mean } \\
\text { streamflow } \\
\left(\mathrm{ft}^{3} / \mathbf{s}\right)\end{array}$ & $\begin{array}{c}\text { Percentage } \\
\text { of time }\end{array}$ & $\begin{array}{c}\text { Daily mean } \\
\text { streamflow } \\
\left(\mathrm{ft}^{3} / \mathbf{s}\right)\end{array}$ \\
\hline 99 & 0.5 & 40 & 8.6 \\
\hline 98 & 0.6 & 30 & 14 \\
\hline 95 & 0.8 & 20 & 25 \\
\hline 90 & 1.2 & 10 & 67 \\
\hline 80 & 2.0 & 5 & 140 \\
\hline 70 & 2.9 & 2 & 280 \\
\hline 60 & 4.1 & 1 & 411 \\
\hline 50 & 5.9 & & \\
\hline
\end{tabular}


Wabash River Basin

\section{MISSISSINEWA RIVER AT MARION, IN}

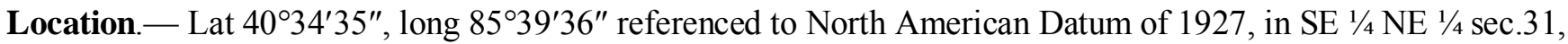
T.25 N., R.8 E., Grant County, IN, Hydrologic Unit 05120103, on left bank 12 ft downstream from Highland Avenue bridge in Marion, $0.1 \mathrm{mi}$ downstream from old mill dam, 1.0 mi upstream from Hummel Creek, $4.6 \mathrm{mi}$ downstream from Lugar Creek, and at mile 35.8.

Drainage area.-682 $\mathrm{mi}^{2}$.

Period of record. - September 1923 to current year.

Average discharge. $-654 \mathrm{ft}^{3} / \mathrm{s}$.

Minimum daily discharge. $-3.4 \mathrm{ft}^{3} / \mathrm{s}$.

Human health (harmonic mean) design flow. $-120 \mathrm{ft}^{3} / \mathrm{s}$.

Remarks. - Flow periodically regulated by dam 0.1 mile above station..

\begin{tabular}{|c|c|c|c|}
\hline \multicolumn{4}{|c|}{ Magnitude and frequency of annual low flow } \\
\hline \multicolumn{4}{|c|}{$\begin{array}{l}\text { Lowest average streamflow, in } \mathrm{ft}^{3} / \mathrm{s} \text {, for indicated period of } \\
\text { consecutive days at an annual nonexceedance probability of } 0.1\end{array}$} \\
\hline 1 & \multicolumn{2}{|c|}{7} & 30 \\
\hline 7.2 & \multicolumn{2}{|c|}{20} & 30 \\
\hline \multicolumn{4}{|c|}{$\begin{array}{l}\text { Percentage of time streamflow was equaled or exceeded for } \\
\text { the period of record }\end{array}$} \\
\hline $\begin{array}{l}\text { Percentage } \\
\text { of time }\end{array}$ & $\begin{array}{l}\text { Daily mean } \\
\text { streamflow } \\
\left(\mathrm{ft}^{3} / \mathrm{s}\right)\end{array}$ & $\begin{array}{l}\text { Percentage } \\
\text { of time }\end{array}$ & $\begin{array}{l}\text { Daily mean } \\
\text { streamflow } \\
\left(\mathrm{ft}^{3} / \mathrm{s}\right)\end{array}$ \\
\hline 99 & 25 & 40 & 296 \\
\hline 98 & 31 & 30 & 436 \\
\hline 95 & 40 & 20 & 708 \\
\hline 90 & 50 & 10 & 1540 \\
\hline 80 & 70 & 5 & 2930 \\
\hline 70 & 102 & 2 & 5260 \\
\hline 60 & 146 & 1 & 7390 \\
\hline 50 & 210 & & \\
\hline
\end{tabular}




\section{MISSISSINEWA RIVER AT PEORIA, IN}

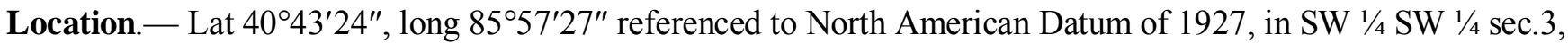
T.26 N., R.5 E., Miami County, IN, Hydrologic Unit 05120103, on right bank at Peoria, 0.6 mi downstream from Mississinewa Lake, $6.5 \mathrm{mi}$ southeast of Peru, and 6.7 mi upstream from mouth.

Drainage area. $-808 \mathrm{mi}^{2}$.

Period of record.- October 1952 to September 1976 (discharge). October 1976 to September 2001 (discharge provided by U.S. Army Corps of Engineers).

Average discharge. $-734 \mathrm{ft}^{3} / \mathrm{s}$.

Minimum daily discharge. $-0.0 \mathrm{ft}^{3} / \mathrm{s}$.

Human health (harmonic mean) design flow. $-169 \mathrm{ft}^{3} / \mathrm{s}$.

Remarks. - Flow regulated by Mississinewa Lake since April 1968. Low-flow statistics are calculated for the regulated period, 1968 to 2001. Records of daily discharge provided by U.S. Army Corps of Engineers beginning Oct. 1, 1976.

\begin{tabular}{ccc}
\hline \multicolumn{3}{c}{ Magnitude and frequency of annual low flow } \\
\hline $\begin{array}{c}\text { Lowest average streamflow, in } \mathrm{ft}^{3} / \mathrm{s}, \text { for indicated period of } \\
\text { consecutive days at an annual nonexceedance probability of } \mathbf{0 . 1}\end{array}$ \\
\hline $\mathbf{1}$ & $\mathbf{7}$ & $\mathbf{3 0}$ \\
\hline 7.0 & 16 & 21 \\
\hline
\end{tabular}

Percentage of time streamflow was equaled or exceeded for the period of record

\begin{tabular}{cc|cc}
\hline $\begin{array}{c}\text { Percentage } \\
\text { of time }\end{array}$ & $\begin{array}{c}\text { Daily mean } \\
\text { streamflow } \\
\left(\mathrm{ft}^{3} / \mathbf{s}\right)\end{array}$ & $\begin{array}{c}\text { Percentage } \\
\text { of time }\end{array}$ & $\begin{array}{c}\text { Daily mean } \\
\text { streamflow } \\
\left(\mathrm{ft}^{3} / \mathbf{s}\right)\end{array}$ \\
\hline 99 & 26 & 40 & 474 \\
\hline 98 & 33 & 30 & 659 \\
\hline 95 & 47 & 20 & 1080 \\
\hline 90 & 65 & 10 & 2190 \\
\hline 80 & 115 & 5 & 3430 \\
\hline 70 & 174 & 2 & 4670 \\
\hline 60 & 248 & 1 & 5400 \\
\hline 50 & 350 & & \\
\hline
\end{tabular}


Wabash River Basin

\section{WABASH RIVER AT PERU, IN}

Location. - Lat $40^{\circ} 45^{\prime} 00^{\prime \prime}$, long $86^{\circ} 04^{\prime} 00^{\prime \prime}$ referenced to North American Datum of 1983, in SE $1 / 4$ NE $1 / 4$ Sec.32, T.27 N., R.4 E., Miami County, IN, Hydrologic Unit 05120101, on right bank at upstream side of bridge on U.S. Highway 31, 0.5 mi southwest of Peru, 4.4 mi downstream from Mississinewa River, and at mile 370.5.

Drainage area. $-2,686 \mathrm{mi}^{2}$.

Period of record.- August 1943 to current year.

Average discharge. $-2,559 \mathrm{ft}^{3} / \mathrm{s}$.

Minimum daily discharge. $-72 \mathrm{ft}^{3} / \mathrm{s}$.

Human health (harmonic mean) design flow. $-809 \mathrm{ft}^{3} / \mathrm{s}$.

Remarks. - Flow regulated by Mississinewa Lake since April 1968. Low-flow statistics are calculated for the regulated period, 1968 to 2001. Records of daily discharge provided by U.S. Army Corps of Engineers beginning Oct. 1, 1976.

\begin{tabular}{|c|c|c|c|}
\hline \multicolumn{4}{|c|}{ Magnitude and frequency of annual low flow } \\
\hline \multicolumn{4}{|c|}{$\begin{array}{l}\text { Lowest average streamflow, in } \mathrm{ft}^{3} / \mathrm{s} \text {, for indicated period of } \\
\text { consecutive days at an annual nonexceedance probability of } 0.1\end{array}$} \\
\hline 1 & \multicolumn{2}{|c|}{7} & 30 \\
\hline 124 & \multicolumn{2}{|c|}{144} & 189 \\
\hline \multicolumn{4}{|c|}{$\begin{array}{c}\text { Percentage of time streamflow was equaled or exceeded for } \\
\text { the period of record }\end{array}$} \\
\hline $\begin{array}{l}\text { Percentage } \\
\text { of time }\end{array}$ & $\begin{array}{c}\text { Daily mean } \\
\text { streamflow } \\
\left(\mathrm{ft}^{3} / \mathrm{s}\right)\end{array}$ & $\begin{array}{l}\text { Percentage } \\
\text { of time }\end{array}$ & $\begin{array}{c}\text { Daily mean } \\
\text { streamflow } \\
\left(\mathrm{ft}^{3} / \mathrm{s}\right)\end{array}$ \\
\hline 99 & 166 & 40 & 1770 \\
\hline 98 & 188 & 30 & 2920 \\
\hline 95 & 239 & 20 & 5070 \\
\hline 90 & 323 & 10 & 7750 \\
\hline 80 & 509 & 5 & 9570 \\
\hline 70 & 705 & 2 & 11600 \\
\hline 60 & 946 & 1 & 13100 \\
\hline 50 & 1280 & & \\
\hline
\end{tabular}




\section{PRAIRIE DITCH AT PERU, IN}

Location. - Lat $40^{\circ} 46^{\prime} 54^{\prime \prime}$, long $86^{\circ} 03^{\prime} 19^{\prime \prime}$ referenced to North American Datum of 1927 , in SE $1 / 4$ SE $1 / 4 \sec .15$, T.27 N., R.4 E., Miami County, IN, Hydrologic Unit 05120101, on downstream left edge near culvert on Lovers Lane, $200 \mathrm{ft}$ south Highway 24, 0.24 mi northeast from intersection of Highways 19 and 24, and at mile 6.06.

Drainage area. $-2.0 \mathrm{mi}^{2}$.

Period of record. - September 2007 to current year.

Average discharge. $-0.94 \mathrm{ft}^{3} / \mathrm{s}$.

Minimum daily discharge. $-0.0 \mathrm{ft}^{3} / \mathrm{s}$.

Human health (harmonic mean) design flow. $-0.0 \mathrm{ft}^{3} / \mathrm{s}$.

Remarks. - This site has less than 10 years of record.

\begin{tabular}{|c|c|c|c|}
\hline \multicolumn{4}{|c|}{ Magnitude and frequency of annual low flow } \\
\hline \multicolumn{4}{|c|}{$\begin{array}{l}\text { Lowest average streamflow, in } \mathrm{ft}^{3} / \mathrm{s} \text {, for indicated period of } \\
\text { consecutive days at an annual nonexceedance probability of } 0.1\end{array}$} \\
\hline 1 & \multicolumn{2}{|c|}{7} & 30 \\
\hline 0.0 & \multicolumn{2}{|c|}{0.0} & 0.0 \\
\hline \multicolumn{4}{|c|}{$\begin{array}{l}\text { Percentage of time streamflow was equaled or exceeded for } \\
\text { the period of record }\end{array}$} \\
\hline $\begin{array}{l}\text { Percentage } \\
\text { of time }\end{array}$ & $\begin{array}{l}\text { Daily mean } \\
\text { streamflow } \\
\left(\mathrm{ft}^{3} / \mathrm{s}\right)\end{array}$ & $\begin{array}{l}\text { Percentage } \\
\text { of time }\end{array}$ & $\begin{array}{l}\text { Daily mean } \\
\text { streamflow } \\
\left(\mathrm{ft}^{3} / \mathrm{s}\right)\end{array}$ \\
\hline 99 & 0.0 & 40 & 0.1 \\
\hline 98 & .0 & 30 & .2 \\
\hline 95 & .0 & 20 & .4 \\
\hline 90 & .0 & 10 & 1.0 \\
\hline 80 & .0 & 5 & 2.1 \\
\hline 70 & .0 & 2 & 7.5 \\
\hline 60 & .0 & 1 & 22 \\
\hline 50 & .1 & & \\
\hline
\end{tabular}


Wabash River Basin

\section{PRAIRIE DITCH NEAR PERU, IN}

Location. - Lat 40 $45^{\prime} 49^{\prime \prime}$, long $86^{\circ} 07^{\prime} 43^{\prime \prime}$ referenced to North American Datum of 1927, in NE 1/4 NW 1/4 NW 1/4 sec.30, T.27 N., R.4 E., Miami County, IN, Hydrologic Unit 05120101, on the downstream right edge abutment of bridge \#B-076 on Division Road, 0.06 mi west of Hwy 31, 20.2 mi north of Kokomo and at mile 1.41.

Drainage area. $-11.1 \mathrm{mi}^{2}$.

Period of record. - September 2007 to current year.

Average discharge. - $13.0 \mathrm{ft}^{3} / \mathrm{s}$.

Minimum daily discharge. $-0.23 \mathrm{ft}^{3} / \mathrm{s}$.

Human health (harmonic mean) design flow. $-4.4 \mathrm{ft}^{3} / \mathrm{s}$.

Remarks. - This site has less than 10 years of record.

\begin{tabular}{ccc}
\hline \multicolumn{3}{c}{ Magnitude and frequency of annual low flow } \\
\hline $\begin{array}{c}\text { Lowest average streamflow, in } \mathrm{ft}^{3} / \mathrm{s} \text {, for indicated period of } \\
\text { consecutive days at an annual nonexceedance probability of } \mathbf{0 . 1}\end{array}$ \\
\hline $\mathbf{1}$ & $\mathbf{7}$ & $\mathbf{3 0}$ \\
\hline 0.2 & 0.3 & 1.6 \\
\hline
\end{tabular}

Percentage of time streamflow was equaled or exceeded for the period of record

\begin{tabular}{cc|cc}
\hline $\begin{array}{c}\text { Percentage } \\
\text { of time }\end{array}$ & $\begin{array}{c}\text { Daily mean } \\
\text { streamflow } \\
\left(\mathrm{ft}^{3} / \mathbf{s}\right)\end{array}$ & $\begin{array}{c}\text { Percentage } \\
\text { of time }\end{array}$ & $\begin{array}{c}\text { Daily mean } \\
\text { streamflow } \\
\left(\mathrm{ft}^{3} / \mathbf{s}\right)\end{array}$ \\
\hline 99 & 0.6 & 40 & 9.8 \\
\hline 98 & 1.0 & 30 & 14 \\
\hline 95 & 1.8 & 20 & 20 \\
\hline 90 & 2.4 & 10 & 30 \\
\hline 80 & 3.2 & 5 & 42 \\
\hline 70 & 4.0 & 2 & 59 \\
\hline 60 & 5.1 & 1 & 90 \\
\hline 50 & 6.7 & & \\
\hline
\end{tabular}




\section{PIPE CREEK NEAR BUNKER HILL, IN}

Location.- Lat $40^{\circ} 40^{\prime} 06^{\prime \prime}$, long $86^{\circ} 05^{\prime} 44^{\prime \prime}$ referenced to North American Datum of 1983 in NE 1/4 SE $1 / 4$ sec.29, T.26 N., R.4 E., Miami County, IN, Hydrologic Unit 05120101, on right bank $150 \mathrm{ft}$ downstream from bridge on County Road 125 West, 0.5 mi northeast of Bunker Hill, and at mile 11.4.

Drainage area. - $159 \mathrm{mi}^{2}$.

Period of record. - May 1968 to October 2003.

Average discharge. $-152 \mathrm{ft}^{3} / \mathrm{s}$.

Minimum daily discharge. $-2.9 \mathrm{ft}^{3} / \mathrm{s}$.

Human health (harmonic mean) design flow. $-28 \mathrm{ft}^{3} / \mathrm{s}$.

Remarks.- None.

\begin{tabular}{|c|c|c|c|}
\hline \multicolumn{4}{|c|}{ Magnitude and frequency of annual low flow } \\
\hline \multicolumn{4}{|c|}{$\begin{array}{l}\text { Lowest average streamflow, in } \mathrm{ft}^{3} / \mathrm{s} \text {, for indicated period of } \\
\text { consecutive days at an annual nonexceedance probability of } 0.1\end{array}$} \\
\hline 1 & \multicolumn{2}{|c|}{7} & 30 \\
\hline 4.1 & \multicolumn{2}{|c|}{4.7} & 5.6 \\
\hline \multicolumn{4}{|c|}{$\begin{array}{l}\text { Percentage of time streamflow was equaled or exceeded for } \\
\text { the period of record }\end{array}$} \\
\hline $\begin{array}{l}\text { Percentage } \\
\text { of time }\end{array}$ & $\begin{array}{l}\text { Daily mean } \\
\text { streamflow } \\
\left(\mathrm{ft}^{3} / \mathrm{s}\right)\end{array}$ & $\begin{array}{l}\text { Percentage } \\
\text { of time }\end{array}$ & $\begin{array}{l}\text { Daily mean } \\
\text { streamflow } \\
\left(\mathrm{ft}^{3} / \mathrm{s}\right)\end{array}$ \\
\hline 99 & 4.9 & 40 & 76 \\
\hline 98 & 6.0 & 30 & 111 \\
\hline 95 & 7.8 & 20 & 180 \\
\hline 90 & 11 & 10 & 369 \\
\hline 80 & 17 & 5 & 654 \\
\hline 70 & 28 & 2 & 1160 \\
\hline 60 & 41 & 1 & 1550 \\
\hline 50 & 56 & & \\
\hline
\end{tabular}


Wabash River Basin

\section{EEL RIVER AT NORTH MANCHESTER, IN}

Location.- Lat 4059'38", long 8546'53" referenced to North American Datum of 1927, in NE 1/4 SE $1 / 4$ sec.6, T.29 N., R.7 E., Wabash County, IN, Hydrologic Unit 05120104, on right bank $300 \mathrm{ft}$ upstream from New Wabash Road bridge in North Manchester, $400 \mathrm{ft}$ downstream of New York Central railroad bridge, 0.4 mi downstream from Pony Creek, and at mile 51.0.

Drainage area. $-417 \mathrm{mi}^{2}$.

Period of record. - October 1929 to current year.

Average discharge. $-394 \mathrm{ft}^{3} / \mathrm{s}$.

Minimum daily discharge. $-16 \mathrm{ft}^{3} / \mathrm{s}$.

Human health (harmonic mean) design flow. $-142 \mathrm{ft}^{3} / \mathrm{s}$.

Remarks.- None.

\begin{tabular}{|c|c|c|c|}
\hline \multicolumn{4}{|c|}{ Magnitude and frequency of annual low flow } \\
\hline \multicolumn{4}{|c|}{$\begin{array}{l}\text { Lowest average streamflow, in } \mathrm{ft}^{3} / \mathrm{s} \text {, for indicated period of } \\
\text { consecutive days at an annual nonexceedance probability of } 0.1\end{array}$} \\
\hline 1 & \multicolumn{2}{|c|}{7} & 30 \\
\hline 32 & \multicolumn{2}{|c|}{39} & 47 \\
\hline \multicolumn{4}{|c|}{$\begin{array}{c}\text { Percentage of time streamflow was equaled or exceeded for } \\
\text { the period of record }\end{array}$} \\
\hline $\begin{array}{l}\text { Percentage } \\
\text { of time }\end{array}$ & $\begin{array}{l}\text { Daily mean } \\
\text { streamflow } \\
\left(\mathrm{ft}^{3} / \mathrm{s}\right)\end{array}$ & $\begin{array}{l}\text { Percentage } \\
\text { of time }\end{array}$ & $\begin{array}{l}\text { Daily mean } \\
\text { streamflow } \\
\left(\mathrm{ft}^{3} / \mathrm{s}\right)\end{array}$ \\
\hline 99 & 42 & 40 & 241 \\
\hline 98 & 48 & 30 & 331 \\
\hline 95 & 58 & 20 & 502 \\
\hline 90 & 68 & 10 & 938 \\
\hline 80 & 86 & 5 & 1570 \\
\hline 70 & 109 & 2 & 2430 \\
\hline 60 & 140 & 1 & 3060 \\
\hline 50 & 182 & & \\
\hline
\end{tabular}




\section{WEESAU CREEK NEAR DEEDSVILLE, IN}

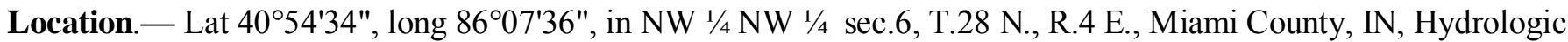
Unit 05120104, on left bank $100 \mathrm{ft}$ downstream from bridge on County Road 1000 North, and 1.5 mi west of Deedsville.

Drainage area. $-8.87 \mathrm{mi}^{2}$.

Period of record.- October 1970 to 2001.

Average discharge. $-10.5 \mathrm{ft}^{3} / \mathrm{s}$.

Minimum daily discharge. $-0.2 \mathrm{ft}^{3} / \mathrm{s}$.

Human health (harmonic mean) design flow. $-2.5 \mathrm{ft}^{3} / \mathrm{s}$.

Remarks. - None.

\begin{tabular}{|c|c|c|c|}
\hline \multicolumn{4}{|c|}{ Magnitude and frequency of annual low flow } \\
\hline \multicolumn{4}{|c|}{$\begin{array}{l}\text { Lowest average streamflow, in } \mathrm{ft}^{3} / \mathrm{s} \text {, for indicated period of } \\
\text { consecutive days at an annual nonexceedance probability of } 0.1\end{array}$} \\
\hline 1 & \multicolumn{2}{|c|}{7} & 30 \\
\hline 0.3 & \multicolumn{2}{|c|}{0.4} & 0.5 \\
\hline \multicolumn{4}{|c|}{$\begin{array}{l}\text { Percentage of time streamflow was equaled or exceeded for } \\
\text { the period of record }\end{array}$} \\
\hline $\begin{array}{l}\text { Percentage } \\
\text { of time }\end{array}$ & $\begin{array}{l}\text { Daily mean } \\
\text { streamflow } \\
\left(\mathrm{ft}^{3} / \mathrm{s}\right)\end{array}$ & $\begin{array}{l}\text { Percentage } \\
\text { of time }\end{array}$ & $\begin{array}{l}\text { Daily mean } \\
\text { streamflow } \\
\left(\mathrm{ft}^{3} / \mathrm{s}\right)\end{array}$ \\
\hline 99 & 0.5 & 40 & 5.8 \\
\hline 98 & 0.6 & 30 & 8.3 \\
\hline 95 & 0.8 & 20 & 13 \\
\hline 90 & 1.0 & 10 & 25 \\
\hline 80 & 1.5 & 5 & 44 \\
\hline 70 & 2.2 & 2 & 73 \\
\hline 60 & 3.1 & 1 & 99 \\
\hline 50 & 4.3 & & \\
\hline
\end{tabular}




\section{EEL RIVER NEAR LOGANSPORT, IN}

Location. - Lat $40^{\circ} 46^{\prime} 58^{\prime \prime}$, long $86^{\circ} 15^{\prime} 53^{\prime \prime}$ referenced to North American Datum of 1983, in NE 1/4 SE 1/4 Sec.14, T.27 N., R.2 E., Cass County, IN, Hydrologic Unit 05120104, on right bank at upstream side of bridge on County Road 150 North, 5.5 mi northeast of Logansport, and 7.4 mi upstream from mouth.

Drainage area. $-789 \mathrm{mi}^{2}$.

Period of record. - July 1943 to current year.

Average discharge. $-789 \mathrm{ft}^{3} / \mathrm{s}$.

Minimum daily discharge. $-70 \mathrm{ft}^{3} / \mathrm{s}$.

Human health (harmonic mean) design flow. $-330 \mathrm{ft}^{3} / \mathrm{s}$.

Remarks.- None.

\begin{tabular}{|c|c|c|c|}
\hline \multicolumn{4}{|c|}{ Magnitude and frequency of annual low flow } \\
\hline \multicolumn{4}{|c|}{$\begin{array}{l}\text { Lowest average streamflow, in } \mathrm{ft}^{3} / \mathrm{s} \text {, for indicated period of } \\
\text { consecutive days at an annual nonexceedance probability of } 0.1\end{array}$} \\
\hline 1 & \multicolumn{2}{|c|}{7} & 30 \\
\hline 98 & \multicolumn{2}{|c|}{105} & 117 \\
\hline \multicolumn{4}{|c|}{$\begin{array}{l}\text { Percentage of time streamflow was equaled or exceeded for } \\
\text { the period of record }\end{array}$} \\
\hline $\begin{array}{l}\text { Percentage } \\
\text { of time }\end{array}$ & $\begin{array}{l}\text { Daily mean } \\
\text { streamflow } \\
\left(\mathrm{ft}^{3} / \mathrm{s}\right)\end{array}$ & $\begin{array}{l}\text { Percentage } \\
\text { of time }\end{array}$ & $\begin{array}{l}\text { Daily mean } \\
\text { streamflow } \\
\left(\mathrm{ft}^{3} / \mathrm{s}\right)\end{array}$ \\
\hline 99 & 104 & 40 & 533 \\
\hline 98 & 115 & 30 & 706 \\
\hline 95 & 135 & 20 & 1040 \\
\hline 90 & 158 & 10 & 1800 \\
\hline 80 & 200 & 5 & 2880 \\
\hline 70 & 252 & 2 & 4430 \\
\hline 60 & 322 & 1 & 5730 \\
\hline 50 & 412 & & \\
\hline
\end{tabular}




\section{WABASH RIVER AT LOGANSPORT, IN}

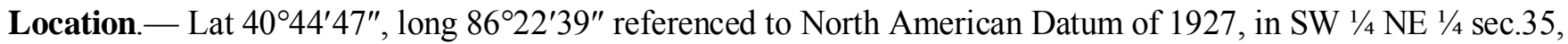
T.27 N., R.1 E., Cass County, IN, Hydrologic Unit 05120105, on left bank, $150 \mathrm{ft}$ downstream from Cicott Street bridge in Logansport, 1,000 ft downstream from Eel River, $0.85 \mathrm{mi}$ upstream of U.S. Highway 35, and at mile 353.7 .

Drainage area. $-3,779 \mathrm{mi}^{2}$.

Period of record. - May 1923 to current year.

Average discharge. $-3,543 \mathrm{ft}^{3} / \mathrm{s}$.

Minimum daily discharge. $-135 \mathrm{ft}^{3} / \mathrm{s}$.

Human health (harmonic mean) design flow. - 1,420 $\mathrm{ft}^{3} / \mathrm{s}$.

Remarks. - Flow partially regulated by Huntington Lake, Salamonie Lake, and Mississinewa Lake. Low-flow statistics are calculated for the regulated period, 1969 to 2011.

\begin{tabular}{|c|c|c|c|}
\hline \multicolumn{4}{|c|}{ Magnitude and frequency of annual low flow } \\
\hline \multicolumn{4}{|c|}{$\begin{array}{l}\text { Lowest average streamflow, in } \mathrm{ft}^{3} / \mathrm{s} \text {, for indicated period of } \\
\text { consecutive days at an annual nonexceedance probability of } 0.1\end{array}$} \\
\hline 1 & \multicolumn{2}{|c|}{7} & 30 \\
\hline 278 & \multicolumn{2}{|c|}{304} & 379 \\
\hline \multicolumn{4}{|c|}{$\begin{array}{l}\text { Percentage of time streamflow was equaled or exceeded for } \\
\text { the period of record }\end{array}$} \\
\hline $\begin{array}{l}\text { Percentage } \\
\text { of time }\end{array}$ & $\begin{array}{l}\text { Daily mean } \\
\text { streamflow } \\
\left(\mathrm{ft}^{3} / \mathrm{s}\right)\end{array}$ & $\begin{array}{l}\text { Percentage } \\
\text { of time }\end{array}$ & $\begin{array}{l}\text { Daily mean } \\
\text { streamflow } \\
\left(\mathrm{ft}^{3} / \mathrm{s}\right)\end{array}$ \\
\hline 99 & 340 & 40 & 2570 \\
\hline 98 & 384 & 30 & 4140 \\
\hline 95 & 484 & 20 & 6970 \\
\hline 90 & 637 & 10 & 10600 \\
\hline 80 & 886 & 5 & 13500 \\
\hline 70 & 1140 & 2 & 16300 \\
\hline 60 & 1430 & 1 & 18700 \\
\hline 50 & 1860 & & \\
\hline
\end{tabular}


Wabash River Basin

\section{RATTLESNAKE CREEK NEAR PATTON, IN}

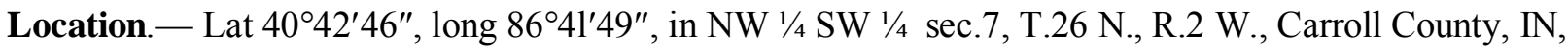
Hydrologic Unit 05120105 , on left bank $5 \mathrm{ft}$ downstream from bridge on County Road $900 \mathrm{West}$, and $2.5 \mathrm{mi}$ northeast of Patton.

Drainage area. $-6.83 \mathrm{mi}^{2}$.

Period of record.- October 1968 to September 1993.

Average discharge. $-7.0 \mathrm{ft}^{3} / \mathrm{s}$.

Minimum daily discharge. $-0.06 \mathrm{ft}^{3} / \mathrm{s}$.

Human health (harmonic mean) design flow. $-1.2 \mathrm{ft}^{3} / \mathrm{s}$.

Remarks.- None.

\begin{tabular}{|c|c|c|c|}
\hline \multicolumn{4}{|c|}{ Magnitude and frequency of annual low flow } \\
\hline \multicolumn{4}{|c|}{$\begin{array}{l}\text { Lowest average streamflow, in } \mathrm{ft}^{3} / \mathrm{s} \text {, for indicated period of } \\
\text { consecutive days at an annual nonexceedance probability of } 0.1\end{array}$} \\
\hline 1 & \multicolumn{2}{|c|}{7} & 30 \\
\hline 0.1 & \multicolumn{2}{|c|}{0.1} & 0.1 \\
\hline \multicolumn{4}{|c|}{$\begin{array}{l}\text { Percentage of time streamflow was equaled or exceeded for } \\
\text { the period of record }\end{array}$} \\
\hline $\begin{array}{l}\text { Percentage } \\
\text { of time }\end{array}$ & $\begin{array}{l}\text { Daily mean } \\
\text { streamflow } \\
\left(\mathrm{ft}^{3} / \mathrm{s}\right)\end{array}$ & $\begin{array}{l}\text { Percentage } \\
\text { of time }\end{array}$ & $\begin{array}{l}\text { Daily mean } \\
\text { streamflow } \\
\left(\mathrm{ft}^{3} / \mathrm{s}\right)\end{array}$ \\
\hline 99 & 0.1 & 40 & 4.2 \\
\hline 98 & 0.2 & 30 & 5.9 \\
\hline 95 & 0.3 & 20 & 8.6 \\
\hline 90 & 0.5 & 10 & 17 \\
\hline 80 & 1.0 & 5 & 28 \\
\hline 70 & 1.6 & 2 & 47 \\
\hline 60 & 2.2 & 1 & 64 \\
\hline 50 & 3.1 & & \\
\hline
\end{tabular}




\section{WABASH RIVER AT DELPHI, IN}

Location.- Lat 40 35'26", long 86 $41^{\prime} 54^{\prime \prime}$, in SE 1/4 SE 1/4 sec.24, T.25 N., R.3 W., Carroll County, IN, on downstream side of second pier from left abutment of highway bridge, $1 \mathrm{mi}$ west of Delphi, $1.6 \mathrm{mi}$ upstream from Deer Creek, 8.6 mi upstream from Tippecanoe River, and at mile 330.8.

Drainage area. $-4,072 \mathrm{mi}^{2}$.

Period of record. - October 1939 to September 1971.

Average discharge. $-3,324 \mathrm{ft}^{3} / \mathrm{s}$.

Minimum daily discharge. $-158 \mathrm{ft}^{3} / \mathrm{s}$.

Human health (harmonic mean) design flow. $-934 \mathrm{ft}^{3} / \mathrm{s}$.

Remarks. - Flow partially regulated by Huntington Reservoir, Salamonie Reservoir, and Mississinewa Reservoir.

\begin{tabular}{|c|c|c|c|}
\hline \multicolumn{4}{|c|}{ Magnitude and frequency of annual low flow } \\
\hline \multicolumn{4}{|c|}{$\begin{array}{l}\text { Lowest average streamflow, in } \mathrm{ft}^{3} / \mathrm{s} \text {, for indicated period of } \\
\text { consecutive days at an annual nonexceedance probability of } 0.1\end{array}$} \\
\hline 1 & \multicolumn{2}{|c|}{7} & 30 \\
\hline 206 & \multicolumn{2}{|c|}{218} & 244 \\
\hline \multicolumn{4}{|c|}{$\begin{array}{l}\text { Percentage of time streamflow was equaled or exceeded for } \\
\text { the period of record }\end{array}$} \\
\hline $\begin{array}{l}\text { Percentage } \\
\text { of time }\end{array}$ & $\begin{array}{c}\text { Daily mean } \\
\text { streamflow } \\
\left(\mathrm{ft}^{3} / \mathrm{s}\right)\end{array}$ & $\begin{array}{l}\text { Percentage } \\
\text { of time }\end{array}$ & $\begin{array}{l}\text { Daily mean } \\
\text { streamflow } \\
\left(\mathrm{ft}^{3} / \mathrm{s}\right)\end{array}$ \\
\hline 99 & 227 & 40 & 1910 \\
\hline 98 & 270 & 30 & 2720 \\
\hline 95 & 331 & 20 & 4350 \\
\hline 90 & 408 & 10 & 8640 \\
\hline 80 & 551 & 5 & 13800 \\
\hline 70 & 753 & 2 & 22000 \\
\hline 60 & 1020 & 1 & 29300 \\
\hline 50 & 1400 & & \\
\hline
\end{tabular}


Wabash River Basin

\section{DEER CREEK NEAR DELPHI, IN}

Location. - Lat $40^{\circ} 35^{\prime} 25^{\prime \prime}$, long 86 $37^{\prime} 17^{\prime \prime}$ referenced to North American Datum of 1927, in NE 1/4 NE 1/4 Sec.27, T.25 N., R.2 W., Carroll County, IN, Hydrologic Unit 05120105, on downstream side of left wingwall of county road bridge, $0.85 \mathrm{mi}$ south of Sharp Point Cemetery, 3.0 mi northeast of Delphi Post Office, and 4.5 mi upstream from mouth.

Drainage area. $-274 \mathrm{mi}^{2}$.

Period of record.- October 1943 to current year.

Average discharge. $-257 \mathrm{ft}^{3} / \mathrm{s}$.

Minimum daily discharge. $-6.2 \mathrm{ft}^{3} / \mathrm{s}$.

Human health (harmonic mean) design flow. $-66 \mathrm{ft}^{3} / \mathrm{s}$.

Remarks. - None.

\begin{tabular}{ccc}
\hline \multicolumn{3}{c}{ Magnitude and frequency of annual low flow } \\
\hline $\begin{array}{c}\text { Lowest average streamflow, in } \mathrm{ft}^{3} / \mathrm{s} \text {, for indicated period of } \\
\text { consecutive days at an annual nonexceedance probability of } 0.1\end{array}$ \\
\hline $\mathbf{1}$ & $\mathbf{7}$ & $\mathbf{3 0}$ \\
\hline 11 & 12 & 15 \\
\hline
\end{tabular}

Percentage of time streamflow was equaled or exceeded for the period of record

\begin{tabular}{cc|cc}
\hline $\begin{array}{c}\text { Percentage } \\
\text { of time }\end{array}$ & $\begin{array}{c}\text { Daily mean } \\
\text { streamflow } \\
\left(\mathrm{ft}^{3} / \mathbf{s}\right)\end{array}$ & $\begin{array}{c}\text { Percentage } \\
\text { of time }\end{array}$ & $\begin{array}{c}\text { Daily mean } \\
\text { streamflow } \\
\left(\mathrm{ft}^{3} / \mathbf{s}\right)\end{array}$ \\
\hline 99 & 14 & 40 & 151 \\
\hline 98 & 17 & 30 & 212 \\
\hline 95 & 22 & 20 & 315 \\
\hline 90 & 28 & 10 & 583 \\
\hline 80 & 41 & 5 & 987 \\
\hline 70 & 57 & 2 & 1730 \\
\hline 60 & 81 & 1 & 2410 \\
\hline 50 & 110 & & \\
\hline
\end{tabular}




\section{TIPPECANOE RIVER AT NORTH WEBSTER, IN}

Location. - Lat $41^{\circ} 18^{\prime} 58^{\prime \prime}$, long $85^{\circ} 41^{\prime} 32^{\prime \prime}$ referenced to North American Datum of 1927, in SE 1/4 NE 1/4 Sec.15, T.33 N., R.7 E., Kosciusko County, IN, Hydrologic Unit 05120106, on right upstream corner of State Road 13 bridge, at the intersection of State Road 13 and County Road 550 North, 0.4 mi southeast of North Webster, and 0.5 mi north of intersection of State Road 13 and 500 North.

Drainage area. $-49.3 \mathrm{mi}^{2}$.

Period of record. - May 1986 to current year.

Average discharge. $-47.6 \mathrm{ft}^{3} / \mathrm{s}$.

Minimum daily discharge. $-0.06 \mathrm{ft}^{3} / \mathrm{s}$.

Human health (harmonic mean) design flow. $-12 \mathrm{ft}^{3} / \mathrm{s}$.

Remarks. - Flow regulated at times by dams at Webster Lake, 0.25 mi upstream.

\begin{tabular}{|c|c|c|c|}
\hline \multicolumn{4}{|c|}{ Magnitude and frequency of annual low flow } \\
\hline \multicolumn{4}{|c|}{$\begin{array}{l}\text { Lowest average streamflow, in } \mathrm{ft}^{3} / \mathrm{s} \text {, for indicated period of } \\
\text { consecutive days at an annual nonexceedance probability of } 0.1\end{array}$} \\
\hline 1 & \multicolumn{2}{|c|}{7} & 30 \\
\hline 0.4 & \multicolumn{2}{|c|}{0.9} & 1.9 \\
\hline \multicolumn{4}{|c|}{$\begin{array}{l}\text { Percentage of time streamflow was equaled or exceeded for } \\
\text { the period of record }\end{array}$} \\
\hline $\begin{array}{l}\text { Percentage } \\
\text { of time }\end{array}$ & $\begin{array}{c}\text { Daily mean } \\
\text { streamflow } \\
\left(\mathrm{ft}^{3} / \mathrm{s}\right)\end{array}$ & $\begin{array}{l}\text { Percentage } \\
\text { of time }\end{array}$ & $\begin{array}{c}\text { Daily mean } \\
\text { streamflow } \\
\left(\mathrm{ft}^{3} / \mathrm{s}\right)\end{array}$ \\
\hline 99 & 1.6 & 40 & 40 \\
\hline 98 & 2.2 & 30 & 55 \\
\hline 95 & 3.4 & 20 & 77 \\
\hline 90 & 5.4 & 10 & 119 \\
\hline 80 & 9.6 & 5 & 163 \\
\hline 70 & 15 & 2 & 228 \\
\hline 60 & 20 & 1 & 275 \\
\hline 50 & 29 & & \\
\hline
\end{tabular}


Wabash River Basin

\section{TIPPECANOE RIVER AT OSWEGO, IN}

Location. - Lat $41^{\circ} 19^{\prime} 14^{\prime \prime}$, long $85^{\circ} 47^{\prime} 21^{\prime \prime}$ referenced to North American Datum of 1927, in NE 1/4 NE 1/4 Sec.14, T.33 N., R.6 E., Kosciusko County, IN, Hydrologic Unit 05120106, on left bank $50 \mathrm{ft}$ downstream from dam at Tippecanoe Lake Outlet in Oswego, 3 mi east of Leesburg, and at mile 158.9.

Drainage area. $-113 \mathrm{mi}^{2}$.

Period of record.- October 1949 to current year.

Average discharge. $-108 \mathrm{ft}^{3} / \mathrm{s}$.

Minimum daily discharge. $-0.08 \mathrm{ft}^{3} / \mathrm{s}$.

Human health (harmonic mean) design flow. $-27 \mathrm{ft}^{3} / \mathrm{s}$.

Remarks. - Periodic regulation by gates at Tippecanoe Lake outlet.

\begin{tabular}{ccc}
\hline \multicolumn{3}{c}{ Magnitude and frequency of annual low flow } \\
\hline $\begin{array}{c}\text { Lowest average streamflow, in } \mathrm{ft}^{3} / \mathrm{s} \text {, for indicated period of } \\
\text { consecutive days at an annual nonexceedance probability of } \mathbf{0 . 1}\end{array}$ \\
\hline $\mathbf{7}$ & $\mathbf{3 0}$ \\
\hline 1.7 & 2.7 & 4.8 \\
\hline
\end{tabular}

Percentage of time streamflow was equaled or exceeded for the period of record

\begin{tabular}{cc|cc}
\hline $\begin{array}{c}\text { Percentage } \\
\text { of time }\end{array}$ & $\begin{array}{c}\text { Daily mean } \\
\text { streamflow } \\
\left(\mathrm{ft}^{3} / \mathbf{s}\right)\end{array}$ & $\begin{array}{c}\text { Percentage } \\
\text { of time }\end{array}$ & $\begin{array}{c}\text { Daily mean } \\
\text { streamflow } \\
\left(\mathrm{ft}^{3} / \mathbf{s}\right)\end{array}$ \\
\hline 99 & 3.9 & 40 & 102 \\
\hline 98 & 5.9 & 30 & 134 \\
\hline 95 & 9.9 & 20 & 177 \\
\hline 90 & 15 & 10 & 250 \\
\hline 80 & 26 & 5 & 316 \\
\hline 70 & 40 & 2 & 398 \\
\hline 60 & 56 & 1 & 491 \\
\hline 50 & 76 & & \\
\hline
\end{tabular}




\section{WALNUT CREEK NEAR WARSAW, IN}

Location.- Lat $41^{\circ} 12^{\prime} 17^{\prime \prime}$, long 85 52'11" referenced to North American Datum of 1927, in NW 1/4 NE 1/4 sec.30, T.32 N., R.6 E., Kosciusko County, IN, Hydrologic Unit 05120106, on left bank $10 \mathrm{ft}$ upstream from bridge on County Road 200 South, 0.3 mi downstream from small right-bank tributary, and 2.5 mi south of Court House in Warsaw.

Drainage area. - $19.6 \mathrm{mi}^{2}$.

Period of record.- October 1969 to October 2003.

Average discharge. $-17.0 \mathrm{ft}^{3} / \mathrm{s}$.

Minimum daily discharge. $-0.4 \mathrm{ft}^{3} / \mathrm{s}$.

Human health (harmonic mean) design flow. $-4.8 \mathrm{ft}^{3} / \mathrm{s}$.

Remarks. - Flow occasionally regulated by lakes upstream.

\begin{tabular}{ccc}
\hline \multicolumn{3}{c}{ Magnitude and frequency of annual low flow } \\
\hline $\begin{array}{c}\text { Lowest average streamflow, in } \mathrm{ft}^{3} / \mathrm{s} \text {, for indicated period of } \\
\text { consecutive days at an annual nonexceedance probability of } 0.1\end{array}$ \\
\hline $\mathbf{1}$ & $\mathbf{3 0}$ \\
\hline 0.5 & 0.6 & 0.9 \\
\hline
\end{tabular}

Percentage of time streamflow was equaled or exceeded for the period of record

\begin{tabular}{cc|cc}
\hline $\begin{array}{c}\text { Percentage } \\
\text { of time }\end{array}$ & $\begin{array}{c}\text { Daily mean } \\
\text { streamflow } \\
\left(\mathrm{ft}^{3} / \mathbf{s}\right)\end{array}$ & $\begin{array}{c}\text { Percentage } \\
\text { of time }\end{array}$ & $\begin{array}{c}\text { Daily mean } \\
\text { streamflow } \\
\left(\mathrm{ft}^{3} / \mathbf{s}\right)\end{array}$ \\
\hline 99 & 0.8 & 40 & 15 \\
\hline 98 & 0.9 & 30 & 20 \\
\hline 95 & 1.3 & 20 & 28 \\
\hline 90 & 1.9 & 10 & 43 \\
\hline 80 & 3.2 & 5 & 61 \\
\hline 70 & 4.9 & 2 & 86 \\
\hline 60 & 7.4 & 1 & 11 \\
\hline 50 & 11 & & \\
\hline
\end{tabular}


Wabash River Basin

\section{TIPPECANOE RIVER NEAR ORA, IN}

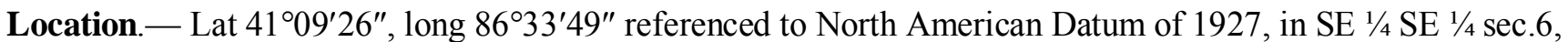
T.31 N., R.1 W., Pulaski County, IN, Hydrologic Unit 05120106, on right bank at downstream side of bridge on County Road 700 East, 1.0 mi upstream from Bartee Ditch, 2.5 mi downstream from House Ditch. 1.3 mi southwest of Ora, and at river mile 78.5.

Drainage area. $-856 \mathrm{mi}^{2}$.

Period of record. - September 1943 to current year.

Average discharge. $-888 \mathrm{ft}^{3} / \mathrm{s}$.

Minimum daily discharge. $-87 \mathrm{ft}^{3} / \mathrm{s}$.

Human health (harmonic mean) design flow. $-471 \mathrm{ft}^{3} / \mathrm{s}$.

Remarks. - None.

\begin{tabular}{|c|c|c|c|}
\hline \multicolumn{4}{|c|}{ Magnitude and frequency of annual low flow } \\
\hline \multicolumn{4}{|c|}{$\begin{array}{l}\text { Lowest average streamflow, in } \mathrm{ft}^{3} / \mathrm{s} \text {, for indicated period of } \\
\text { consecutive days at an annual nonexceedance probability of } 0.1\end{array}$} \\
\hline 1 & \multicolumn{2}{|c|}{7} & 30 \\
\hline 133 & \multicolumn{2}{|c|}{138} & 153 \\
\hline \multicolumn{4}{|c|}{$\begin{array}{l}\text { Percentage of time streamflow was equaled or exceeded for } \\
\text { the period of record }\end{array}$} \\
\hline $\begin{array}{l}\text { Percentage } \\
\text { of time }\end{array}$ & $\begin{array}{l}\text { Daily mean } \\
\text { streamflow } \\
\left(\mathrm{ft}^{3} / \mathrm{s}\right)\end{array}$ & $\begin{array}{l}\text { Percentage } \\
\text { of time }\end{array}$ & $\begin{array}{l}\text { Daily mean } \\
\text { streamflow } \\
\left(\mathrm{ft}^{3} / \mathrm{s}\right)\end{array}$ \\
\hline 99 & 142 & 40 & 781 \\
\hline 98 & 156 & 30 & 1010 \\
\hline 95 & 185 & 20 & 1350 \\
\hline 90 & 223 & 10 & 1890 \\
\hline 80 & 297 & 5 & 2470 \\
\hline 70 & 378 & 2 & 3320 \\
\hline 60 & 480 & 1 & 4180 \\
\hline 50 & 616 & & \\
\hline
\end{tabular}




\section{TIPPECANOE RIVER AT WINAMAC, IN}

Location. - Lat $41^{\circ} 02^{\prime} 59^{\prime \prime}$, long $86^{\circ} 35^{\prime} 57^{\prime \prime}$ referenced to North American Datum of 1927, in SW 1/4 NW 1/4 sec.13, T.30 N., R.2 W., Pulaski County, IN, Hydrologic Unit 05120106, on the northeast corner of the Washington Street bridge in Winamac, 0.3 mi downstream of the city park, 2 mi north of U.S. Highway 35 bridge, and at mile 70.3.

Drainage area. $-942 \mathrm{mi}^{2}$.

Period of record.- August 2001 to current year.

Average discharge. $-1,031 \mathrm{ft}^{3} / \mathrm{s}$.

Minimum daily discharge. $-4.8 \mathrm{ft}^{3} / \mathrm{s}$.

Human health (harmonic mean) design flow. $-451 \mathrm{ft}^{3} / \mathrm{s}$.

Remarks. - None.

\begin{tabular}{ccc}
\hline \multicolumn{3}{c}{ Magnitude and frequency of annual low flow } \\
\hline $\begin{array}{c}\text { Lowest average streamflow, in } \mathrm{ft}^{3} / \mathrm{s} \text {, for indicated period of } \\
\text { consecutive days at an annual nonexceedance probability of } \mathbf{0 . 1}\end{array}$ \\
\hline $\mathbf{7}$ & $\mathbf{3 0}$ \\
\hline 183 & 185 & 198 \\
\hline
\end{tabular}

Percentage of time streamflow was equaled or exceeded for the period of record

\begin{tabular}{cc|cc}
\hline $\begin{array}{c}\text { Percentage } \\
\text { of time }\end{array}$ & $\begin{array}{c}\text { Daily mean } \\
\text { streamflow } \\
\left(\mathbf{f t}^{3} / \mathbf{s}\right)\end{array}$ & $\begin{array}{c}\text { Percentage } \\
\text { of time }\end{array}$ & $\begin{array}{c}\text { Daily mean } \\
\text { streamflow } \\
\left(\mathrm{ft}^{3} / \mathbf{s}\right)\end{array}$ \\
\hline 99 & 191 & 40 & 980 \\
\hline 98 & 205 & 30 & 1250 \\
\hline 95 & 242 & 20 & 1620 \\
\hline 90 & 290 & 10 & 2130 \\
\hline 80 & 357 & 5 & 2650 \\
\hline 70 & 446 & 2 & 3580 \\
\hline 60 & 576 & 1 & 4430 \\
\hline 50 & 758 & & \\
\hline
\end{tabular}


Wabash River Basin

\section{LITTLE INDIAN CREEK NEAR ROYAL CENTER, IN}

Location.- Lat 40 $52^{\prime} 53^{\prime \prime}$, long 86 35'26" referenced to North American Datum of 1927, in NE 1/4 NW 1/4 sec.13, T.28 N., R.2 W., White County, IN, Hydrologic Unit 05120106, on right bank at downstream side of county road bridge, 2.9 mi upstream from mouth, 3.2 mi downstream from Fredericks Ditch, and 4.8 mi northwest of Royal Center Post Office.

Drainage area. $-35.0 \mathrm{mi}^{2}$.

Period of record.- July 1959 to September 1973.

Average discharge. $-28.7 \mathrm{ft}^{3} / \mathrm{s}$.

Minimum daily discharge. $-0.50 \mathrm{ft}^{3} / \mathrm{s}$.

Human health (harmonic mean) design flow. $-7.1 \mathrm{ft}^{3} / \mathrm{s}$.

Remarks.- None.

\begin{tabular}{|c|c|c|c|}
\hline \multicolumn{4}{|c|}{ Magnitude and frequency of annual low flow } \\
\hline \multicolumn{4}{|c|}{$\begin{array}{l}\text { Lowest average streamflow, in } \mathrm{ft}^{3} / \mathrm{s} \text {, for indicated period of } \\
\text { consecutive days at an annual nonexceedance probability of } 0.1\end{array}$} \\
\hline 1 & \multicolumn{2}{|c|}{7} & 30 \\
\hline 0.6 & \multicolumn{2}{|c|}{0.7} & 1.2 \\
\hline \multicolumn{4}{|c|}{$\begin{array}{l}\text { Percentage of time streamflow was equaled or exceeded for } \\
\text { the period of record }\end{array}$} \\
\hline $\begin{array}{l}\text { Percentage } \\
\text { of time }\end{array}$ & $\begin{array}{c}\text { Daily mean } \\
\text { streamflow } \\
\left(\mathrm{ft}^{3} / \mathrm{s}\right)\end{array}$ & $\begin{array}{l}\text { Percentage } \\
\text { of time }\end{array}$ & $\begin{array}{c}\text { Daily mean } \\
\text { streamflow } \\
\left(\mathrm{ft}^{3} / \mathrm{s}\right)\end{array}$ \\
\hline 99 & 0.8 & 40 & 20 \\
\hline 98 & 1.3 & 30 & 27 \\
\hline 95 & 2.1 & 20 & 39 \\
\hline 90 & 3.3 & 10 & 66 \\
\hline 80 & 5.0 & 5 & 108 \\
\hline 70 & 7.5 & 2 & 183 \\
\hline 60 & 11 & 1 & 239 \\
\hline 50 & 15 & & \\
\hline
\end{tabular}




\section{BIG MONON CREEK NEAR FRANCESVILLE, IN}

Location. - Lat 4059'03", long 86 $51^{\prime} 43^{\prime \prime}$ referenced to North American Datum of 1927, in NW 1/4 NE 1/4 sec.10, T.29 N., R.4 W., Pulaski County, IN, Hydrologic Unit 05120106, on right bank at downstream side of county road bridge, 1.1 mi east of Francesville, 1.6 mi downstream from right bank tributary, and $10.2 \mathrm{mi}$ upstream from mouth.

Drainage area.- $152 \mathrm{mi}^{2}$.

Period of record. - August 1959 to September 1973.

Average discharge. $-144 \mathrm{ft}^{3} / \mathrm{s}$.

Minimum daily discharge. $-8.50 \mathrm{ft}^{3} / \mathrm{s}$.

Human health (harmonic mean) design flow. $-55 \mathrm{ft}^{3} / \mathrm{s}$.

Remarks.- None.

\begin{tabular}{|c|c|c|c|}
\hline \multicolumn{4}{|c|}{ Magnitude and frequency of annual low flow } \\
\hline \multicolumn{4}{|c|}{$\begin{array}{l}\text { Lowest average streamflow, in } \mathrm{ft}^{3} / \mathrm{s} \text {, for indicated period of } \\
\text { consecutive days at an annual nonexceedance probability of } 0.1\end{array}$} \\
\hline 1 & \multicolumn{2}{|c|}{7} & 30 \\
\hline 9.1 & \multicolumn{2}{|c|}{9.9} & 13 \\
\hline \multicolumn{4}{|c|}{$\begin{array}{l}\text { Percentage of time streamflow was equaled or exceeded for } \\
\text { the period of record }\end{array}$} \\
\hline $\begin{array}{l}\text { Percentage } \\
\text { of time }\end{array}$ & $\begin{array}{l}\text { Daily mean } \\
\text { streamflow } \\
\left(\mathrm{ft}^{3} / \mathrm{s}\right)\end{array}$ & $\begin{array}{l}\text { Percentage } \\
\text { of time }\end{array}$ & $\begin{array}{l}\text { Daily mean } \\
\text { streamflow } \\
\left(\mathrm{ft}^{3} / \mathrm{s}\right)\end{array}$ \\
\hline 99 & 13 & 40 & 108 \\
\hline 98 & 15 & 30 & 144 \\
\hline 95 & 18 & 20 & 199 \\
\hline 90 & 23 & 10 & 308 \\
\hline 80 & 36 & 5 & 464 \\
\hline 70 & 51 & 2 & 748 \\
\hline 60 & 69 & 1 & 1050 \\
\hline 50 & 88 & & \\
\hline
\end{tabular}


Wabash River Basin

\section{TIPPECANOE RIVER NEAR MONTICELLO, IN}

Location. - Lat 40 46 $48^{\prime \prime}$, long $86^{\circ} 45^{\prime} 36^{\prime \prime}$ referenced to North American Datum of 1927 in NW 1/4 NE $1 / 4$ sec.21, T.27 N., R.3 W., White County, IN, Hydrologic Unit 05120106, at Norway plant of Northern Indiana Public Service Co., 2 mi north of Monticello, and at mile 32.0.

Drainage area. $-1,732 \mathrm{mi}^{2}$.

Period of record.- October 1931 to 1981.

Average discharge. $-1,508 \mathrm{ft}^{3} / \mathrm{s}$.

Minimum daily discharge. $-103 \mathrm{ft}^{3} / \mathrm{s}$.

Human health (harmonic mean) design flow. $-709 \mathrm{ft}^{3} / \mathrm{s}$.

Remarks. - Discharge computed on basis of records of operation of power plant and flow over dam.

\begin{tabular}{|c|c|c|c|}
\hline \multicolumn{4}{|c|}{ Magnitude and frequency of annual low flow } \\
\hline \multicolumn{4}{|c|}{$\begin{array}{l}\text { Lowest average streamflow, in } \mathrm{ft}^{3} / \mathrm{s} \text {, for indicated period of } \\
\text { consecutive days at an annual nonexceedance probability of } 0.1\end{array}$} \\
\hline 1 & \multicolumn{2}{|c|}{7} & 30 \\
\hline 132 & \multicolumn{2}{|c|}{183} & 213 \\
\hline \multicolumn{4}{|c|}{$\begin{array}{l}\text { Percentage of time streamflow was equaled or exceeded for } \\
\text { the period of record }\end{array}$} \\
\hline $\begin{array}{l}\text { Percentage } \\
\text { of time }\end{array}$ & $\begin{array}{l}\text { Daily mean } \\
\text { streamflow } \\
\left(\mathrm{ft}^{3} / \mathrm{s}\right)\end{array}$ & $\begin{array}{l}\text { Percentage } \\
\text { of time }\end{array}$ & $\begin{array}{c}\text { Daily mean } \\
\text { streamflow } \\
\left(\mathrm{ft}^{3} / \mathrm{s}\right)\end{array}$ \\
\hline 99 & 192 & 40 & 1220 \\
\hline 98 & 212 & 30 & 1590 \\
\hline 95 & 269 & 20 & 2190 \\
\hline 90 & 331 & 10 & 3400 \\
\hline 80 & 464 & 5 & 4730 \\
\hline 70 & 587 & 2 & 6840 \\
\hline 60 & 737 & 1 & 8580 \\
\hline 50 & 952 & & \\
\hline
\end{tabular}




\section{TIPPECANOE RIVER AT NORWAY, IN}

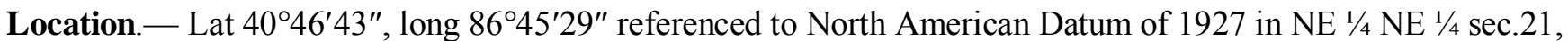
T.27 N., R.3 W., White County, IN, Hydrologic Unit 05120106, on upstream side of bridge on Francis St., $2.6 \mathrm{mi}$ northeast of Monticello, $16.2 \mathrm{mi}$ west of Logansport, and at river mile 31.9.

Drainage area. $-1,760 \mathrm{mi}^{2}$.

Period of record.- December 2008 to current year.

Average discharge. $-1,935 \mathrm{ft}^{3} / \mathrm{s}$.

Minimum daily discharge. $-205 \mathrm{ft}^{3} / \mathrm{s}$.

Human health (harmonic mean) design flow. - $920 \mathrm{ft}^{3} / \mathrm{s}$.

Remarks. - This site has less than 10 years of record. The extreme regulation by upstream dam causes unnatural flow conditions.

\begin{tabular}{|c|c|c|c|}
\hline \multicolumn{4}{|c|}{ Magnitude and frequency of annual low flow } \\
\hline \multicolumn{4}{|c|}{$\begin{array}{l}\text { Lowest average streamflow, in } \mathrm{ft}^{3} / \mathrm{s} \text {, for indicated period of } \\
\text { consecutive days at an annual nonexceedance probability of } 0.1\end{array}$} \\
\hline 1 & \multicolumn{2}{|c|}{7} & 30 \\
\hline 204 & \multicolumn{2}{|c|}{246} & 304 \\
\hline \multicolumn{4}{|c|}{$\begin{array}{l}\text { Percentage of time streamflow was equaled or exceeded for } \\
\text { the period of record }\end{array}$} \\
\hline $\begin{array}{l}\text { Percentage } \\
\text { of time }\end{array}$ & $\begin{array}{l}\text { Daily mean } \\
\text { streamflow } \\
\left(\mathrm{ft}^{3} / \mathrm{s}\right)\end{array}$ & $\begin{array}{l}\text { Percentage } \\
\text { of time }\end{array}$ & $\begin{array}{l}\text { Daily mean } \\
\text { streamflow } \\
\left(\mathrm{ft}^{3} / \mathrm{s}\right)\end{array}$ \\
\hline 99 & 256 & 40 & 1650 \\
\hline 98 & 278 & 30 & 2120 \\
\hline 95 & 329 & 20 & 2710 \\
\hline 90 & 415 & 10 & 3800 \\
\hline 80 & 522 & 5 & 5060 \\
\hline 70 & 674 & 2 & 7230 \\
\hline 60 & 968 & 1 & 9000 \\
\hline 50 & 1240 & & \\
\hline
\end{tabular}


Wabash River Basin

\section{TIPPECANOE RIVER BELOW OAKDALE DAM, IN}

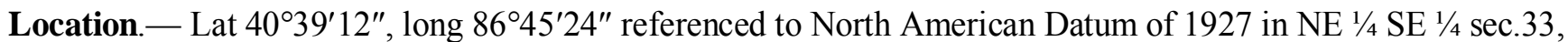
T.26 N., R.3 W., Carroll County, IN, Hydrologic Unit 05120106, on the downstream side of the bridge on CR 725 N, 0.6 mi west of Co. Rd. 1150 W, 0.9 mi east of Springboro Road, and 8.4 mi northwest of Delphi.

Drainage area. $-1,790 \mathrm{mi}^{2}$.

Period of record.- December 2008 to current year.

Average discharge.- $1,875 \mathrm{ft}^{3} / \mathrm{s}$.

Minimum daily discharge. $-179 \mathrm{ft}^{3} / \mathrm{s}$.

Human health (harmonic mean) design flow. $-963 \mathrm{ft}^{3} / \mathrm{s}$.

Remarks. - This site has less than 10 years of record. The extreme regulation by upstream dam causes unnatural flow conditions.

\begin{tabular}{ccc}
\hline \multicolumn{3}{c}{ Magnitude and frequency of annual low flow } \\
\hline $\begin{array}{c}\text { Lowest average streamflow, in } \mathrm{ft}^{3} / \mathrm{s} \text {, for indicated period of } \\
\text { consecutive days at an annual nonexceedance probability of } \mathbf{0 . 1}\end{array}$ \\
\hline $\mathbf{1}$ & $\mathbf{7}$ & $\mathbf{3 0}$ \\
\hline 188 & 231 & 291 \\
\hline
\end{tabular}

Percentage of time streamflow was equaled or exceeded for the period of record

\begin{tabular}{cc|cc}
\hline $\begin{array}{c}\text { Percentage } \\
\text { of time }\end{array}$ & $\begin{array}{c}\text { Daily mean } \\
\text { streamflow } \\
\left(\mathrm{ft}^{3} / \mathbf{s}\right)\end{array}$ & $\begin{array}{c}\text { Percentage } \\
\text { of time }\end{array}$ & $\begin{array}{c}\text { Daily mean } \\
\text { streamflow } \\
\left(\mathbf{f t}^{3} / \mathbf{s}\right)\end{array}$ \\
\hline 99 & 265 & 40 & 1610 \\
\hline 98 & 282 & 30 & 2150 \\
\hline 95 & 312 & 20 & 2880 \\
\hline 90 & 432 & 10 & 4080 \\
\hline 80 & 543 & 5 & 5570 \\
\hline 70 & 695 & 2 & 7800 \\
\hline 60 & 950 & 1 & 9390 \\
\hline 50 & 1230 & & \\
\hline
\end{tabular}




\section{TIPPECANOE RIVER NEAR DELPHI, IN}

Location. - Lat $40^{\circ} 35^{\prime} 38^{\prime \prime}$, long $86^{\circ} 46^{\prime} 12^{\prime \prime}$ referenced to North American Datum of 1927, in SW 1/4 SW 1/4 sec.21, T. 25 N., R.3 W., Carroll County, IN, Hydrologic Unit 05120106, on left bank $20 \mathrm{ft}$ upstream from bridge on State Highway 18, 1,400 ft east of Springboro, 5 mi west of Delphi, 8.1 mi downstream from Big Creek, and at mile 8.7.

Drainage area. $-1,869 \mathrm{mi}^{2}$.

Period of record. - October 1987 to current year.

Average discharge. $-2,075 \mathrm{ft}^{3} / \mathrm{s}$.

Minimum daily discharge. $-131 \mathrm{ft}^{3} / \mathrm{s}$.

Human health (harmonic mean) design flow. - 1,080 $\mathrm{ft}^{3} / \mathrm{s}$.

Remarks. - Flow regulated by upstream reservoirs.

\begin{tabular}{ccc}
\hline \multicolumn{3}{c}{ Magnitude and frequency of annual low flow } \\
\hline $\begin{array}{c}\text { Lowest average streamflow, in } \mathrm{ft}^{3} / \mathrm{s} \text {, for indicated period of } \\
\text { consecutive days at an annual nonexceedance probability of } 0.1\end{array}$ \\
\hline $\mathbf{1}$ & $\mathbf{3 0}$ \\
\hline 164 & 208 & 271 \\
\hline
\end{tabular}

Percentage of time streamflow was equaled or exceeded for the period of record

\begin{tabular}{cc|cc}
\hline $\begin{array}{c}\text { Percentage } \\
\text { of time }\end{array}$ & $\begin{array}{c}\text { Daily mean } \\
\text { streamflow } \\
\left(\mathrm{ft}^{3} / \mathbf{s}\right)\end{array}$ & $\begin{array}{c}\text { Percentage } \\
\text { of time }\end{array}$ & $\begin{array}{c}\text { Daily mean } \\
\text { streamflow } \\
\left(\mathrm{ft}^{3} / \mathbf{s}\right)\end{array}$ \\
\hline 99 & 286 & 40 & 1870 \\
\hline 98 & 329 & 30 & 2380 \\
\hline 95 & 406 & 20 & 3070 \\
\hline 90 & 495 & 10 & 4350 \\
\hline 80 & 699 & 5 & 5750 \\
\hline 70 & 913 & 2 & 7770 \\
\hline 60 & 1170 & 1 & 9380 \\
\hline 50 & 1480 & & \\
\hline
\end{tabular}


Wabash River Basin

\section{WILDCAT CREEK NEAR JEROME, IN}

Location.- Lat $40^{\circ} 26^{\prime} 29^{\prime \prime}$, long $85^{\circ} 55^{\prime} 08^{\prime \prime}$ referenced to North American Datum of 1927, in NE 1/4 SE 1/4 sec.14, T.23 N., R.5 E., Howard County, IN, Hydrologic Unit 05120107, on right bank at downstream side of bridge on County Road 1100 East, 0.5 mi downstream from Mud Creek, 1.5 mi southeast of Jerome, and at mile 79.9.

Drainage area. $-146 \mathrm{mi}^{2}$.

Period of record.- July 1961 to current year.

Average discharge. $-151 \mathrm{ft}^{3} / \mathrm{s}$.

Minimum daily discharge. $-0.47 \mathrm{ft}^{3} / \mathrm{s}$.

Human health (harmonic mean) design flow. $-13 \mathrm{ft}^{3} / \mathrm{s}$.

Remarks. - None.

\begin{tabular}{|c|c|c|c|}
\hline \multicolumn{4}{|c|}{ Magnitude and frequency of annual low flow } \\
\hline \multicolumn{4}{|c|}{$\begin{array}{l}\text { Lowest average streamflow, in } \mathrm{ft}^{3} / \mathrm{s} \text {, for indicated period of } \\
\text { consecutive days at an annual nonexceedance probability of } 0.1\end{array}$} \\
\hline 1 & \multicolumn{2}{|c|}{7} & 30 \\
\hline 1.2 & \multicolumn{2}{|c|}{1.4} & 1.8 \\
\hline \multicolumn{4}{|c|}{$\begin{array}{l}\text { Percentage of time streamflow was equaled or exceeded for } \\
\text { the period of record }\end{array}$} \\
\hline $\begin{array}{l}\text { Percentage } \\
\text { of time }\end{array}$ & $\begin{array}{l}\text { Daily mean } \\
\text { streamflow } \\
\left(\mathrm{ft}^{3} / \mathrm{s}\right)\end{array}$ & $\begin{array}{l}\text { Percentage } \\
\text { of time }\end{array}$ & $\begin{array}{l}\text { Daily mean } \\
\text { streamflow } \\
\left(\mathrm{ft}^{3} / \mathrm{s}\right)\end{array}$ \\
\hline 99 & 1.6 & 40 & 71 \\
\hline 98 & 2.0 & 30 & 107 \\
\hline 95 & 3.0 & 20 & 180 \\
\hline 90 & 4.7 & 10 & 387 \\
\hline 80 & 8.8 & 5 & 660 \\
\hline 70 & 18 & 2 & 1170 \\
\hline 60 & 30 & 1 & 1640 \\
\hline 50 & 47 & & \\
\hline
\end{tabular}




\section{WILDCAT CREEK AT GREENTOWN, IN}

Location.- Lat $40^{\circ} 27^{\prime}$, long $85^{\circ} 57^{\prime}$, referenced to North American Datum of 1927, on line between secs. 9 and 10 , T.23 N., R.5 E., Howard County, IN, Hydrologic Unit 05120107, on left bank at downstream side of bridge on State Highway 213, $1.5 \mathrm{mi}$ south of Greentown.

Drainage area. $-168 \mathrm{mi}^{2}$.

Period of record.- October 1944 to June 1961.

Average discharge. $-152 \mathrm{ft}^{3} / \mathrm{s}$.

Minimum daily discharge. $-1.0 \mathrm{ft}^{3} / \mathrm{s}$.

Human health (harmonic mean) design flow. $-16 \mathrm{ft}^{3} / \mathrm{s}$.

Remarks. - None.

\begin{tabular}{|c|c|c|c|}
\hline \multicolumn{4}{|c|}{ Magnitude and frequency of annual low flow } \\
\hline \multicolumn{4}{|c|}{$\begin{array}{l}\text { Lowest average streamflow, in } \mathrm{ft}^{3} / \mathrm{s} \text {, for indicated period of } \\
\text { consecutive days at an annual nonexceedance probability of } 0.1\end{array}$} \\
\hline 1 & \multicolumn{2}{|c|}{7} & 30 \\
\hline 1.1 & \multicolumn{2}{|c|}{1.4} & 2.2 \\
\hline \multicolumn{4}{|c|}{$\begin{array}{l}\text { Percentage of time streamflow was equaled or exceeded for } \\
\text { the period of record }\end{array}$} \\
\hline $\begin{array}{l}\text { Percentage } \\
\text { of time }\end{array}$ & $\begin{array}{l}\text { Daily mean } \\
\text { streamflow } \\
\left(\mathrm{ft}^{3} / \mathrm{s}\right)\end{array}$ & $\begin{array}{l}\text { Percentage } \\
\text { of time }\end{array}$ & $\begin{array}{l}\text { Daily mean } \\
\text { streamflow } \\
\left(\mathrm{ft}^{3} / \mathrm{s}\right)\end{array}$ \\
\hline 99 & 1.9 & 40 & 76 \\
\hline 98 & 2.5 & 30 & 117 \\
\hline 95 & 4.0 & 20 & 194 \\
\hline 90 & 5.9 & 10 & 377 \\
\hline 80 & 12 & 5 & 675 \\
\hline 70 & 21 & 2 & 1190 \\
\hline 60 & 35 & 1 & 1630 \\
\hline 50 & 51 & & \\
\hline
\end{tabular}


Wabash River Basin

\section{KOKOMO CREEK NEAR KOKOMO, IN}

Location. - Lat $40^{\circ} 26^{\prime} 28^{\prime \prime}$, long $86^{\circ} 05^{\prime} 20^{\prime \prime}$ referenced to North American Datum of 1927, in NW 1/4 SW 1/4 sec.16, T.23 N., R.4 E., Howard County, IN, Hydrologic Unit 05120107, on left bank at upstream side of bridge on County Road 200 East, $0.5 \mathrm{mi}$ south of County Road 200 South, $2.6 \mathrm{mi}$ southeast of intersection of U.S. Highways 31 and 35 in Kokomo, and 4.2 mi upstream from mouth.

Drainage area. $-24.7 \mathrm{mi}^{2}$.

Period of record. - July 1959 to Jan. 2012.

Average discharge. $-24.4 \mathrm{ft}^{3} / \mathrm{s}$.

Minimum daily discharge. $-0.01 \mathrm{ft}^{3} / \mathrm{s}$.

Human health (harmonic mean) design flow. $-1.96 \mathrm{ft}^{3} / \mathrm{s}$.

Remarks. - None.

Magnitude and frequency of annual low flow

Lowest average streamflow, in $\mathrm{ft}^{3} / \mathrm{s}$, for indicated period of consecutive days at an annual nonexceedance probability of 0.1

\begin{tabular}{ccc}
\hline $\mathbf{1}$ & $\mathbf{7}$ & $\mathbf{3 0}$ \\
\hline 0.1 & 0.2 & 0.3 \\
\hline
\end{tabular}

Percentage of time streamflow was equaled or exceeded for the period of record

\begin{tabular}{cc|cc}
\hline $\begin{array}{c}\text { Percentage } \\
\text { of time }\end{array}$ & $\begin{array}{c}\text { Daily mean } \\
\text { streamflow } \\
\left(\mathrm{ft}^{3} / \mathbf{s}\right)\end{array}$ & $\begin{array}{c}\text { Percentage } \\
\text { of time }\end{array}$ & $\begin{array}{c}\text { Daily mean } \\
\text { streamflow } \\
\left(\mathrm{ft}^{3} / \mathbf{s}\right)\end{array}$ \\
\hline 99 & 0.2 & 40 & 12 \\
\hline 98 & 0.3 & 30 & 18 \\
\hline 95 & 0.5 & 20 & 30 \\
\hline 90 & 0.8 & 10 & 60 \\
\hline 80 & 1.7 & 5 & 106 \\
\hline 70 & 2.9 & 2 & 193 \\
\hline 60 & 5.1 & 1 & 274 \\
\hline 50 & 7.8 & & \\
\hline
\end{tabular}




\section{WILDCAT CREEK AT KOKOMO, IN}

Location.- Lat $40^{\circ} 28^{\prime} 15.3^{\prime \prime}$, long $86^{\circ} 09^{\prime} 10.6^{\prime \prime}$ referenced to North American Datum of 1983, in SW 1/4 NE 1/4 sec.2, T.23 N., R.3 E., Howard County, IN, Hydrologic Unit 05120107, on right bank on property of Kokomo Sewage Treatment Plant in Kokomo, $250 \mathrm{ft}$ downstream from Kokomo Creek, $1.0 \mathrm{mi}$ upstream from Dixon Road bridge, and at mile 62.9 .

Drainage area. $-242 \mathrm{mi}^{2}$.

Period of record. - October 1955 to current year.

Average discharge. $-249 \mathrm{ft}^{3} / \mathrm{s}$.

Minimum daily discharge. $-7.2 \mathrm{ft}^{3} / \mathrm{s}$.

Human health (harmonic mean) design flow. $-59 \mathrm{ft}^{3} / \mathrm{s}$.

Remarks. - Some regulation by Kokomo Reservoirs Nos. 1 and 2 (combined capacity 4,170 acre-ft, used for municipal water supply) and by Kokomo Sewage Treatment Plant. Prior to May 9, 1986, recording gage at site 0.4 mi downstream at present datum.

\begin{tabular}{ccc}
\hline \multicolumn{3}{c}{ Magnitude and frequency of annual low flow } \\
\hline $\begin{array}{c}\text { Lowest average streamflow, in } \mathrm{ft}^{3} / \mathrm{s} \text {, for indicated period of } \\
\text { consecutive days at an annual nonexceedance probability of } \mathbf{0 . 1}\end{array}$ \\
\hline $\mathbf{7}$ & $\mathbf{7}$ & $\mathbf{3 0}$ \\
\hline 12 & 14 & 16 \\
\hline
\end{tabular}

Percentage of time streamflow was equaled or exceeded for the period of record

\begin{tabular}{cc|cc}
\hline $\begin{array}{c}\text { Percentage } \\
\text { of time }\end{array}$ & $\begin{array}{c}\text { Daily mean } \\
\text { streamflow } \\
\left(\mathrm{ft}^{3} / \mathbf{s}\right)\end{array}$ & $\begin{array}{c}\text { Percentage } \\
\text { of time }\end{array}$ & $\begin{array}{c}\text { Daily mean } \\
\text { streamflow } \\
\left(\mathrm{ft}^{3} / \mathbf{s}\right)\end{array}$ \\
\hline 99 & 14 & 40 & 130 \\
\hline 98 & 17 & 30 & 191 \\
\hline 95 & 21 & 20 & 306 \\
\hline 90 & 25 & 10 & 600 \\
\hline 80 & 34 & 5 & 1020 \\
\hline 70 & 47 & 2 & 1770 \\
\hline 60 & 66 & 1 & 2480 \\
\hline 50 & 92 & & \\
\hline
\end{tabular}


Wabash River Basin

\section{WILDCAT CREEK AT OWASCO, IN}

Location. - Lat $40^{\circ} 27^{\prime} 50^{\prime \prime}$, long $86^{\circ} 38^{\prime} 15^{\prime \prime}$ referenced to North American Datum of 1927, in SE 1/4 SE 1/4 Sec.4, T.23 N., R.2 W., Carroll County, IN, Hydrologic Unit 05120107, on left bank $200 \mathrm{ft}$ downstream from bridge on State Highway 39, $0.5 \mathrm{mi}$ northwest of Owasco, $8.7 \mathrm{mi}$ south of Delphi, and $15 \mathrm{mi}$ upstream from South Fork Wildcat Creek.

Drainage area. $-396 \mathrm{mi}^{2}$.

Period of record. - October 1943 to September 1973, and October 1988 to current year.

Average discharge. $-397 \mathrm{ft}^{3} / \mathrm{s}$.

Minimum daily discharge. $-12 \mathrm{ft}^{3} / \mathrm{s}$.

Human health (harmonic mean) design flow. $-104 \mathrm{ft}^{3} / \mathrm{s}$.

Remarks. - Some regulation at low stages for municipal water supply by Kokomo Water Company since 1955.

\begin{tabular}{ccc}
\hline \multicolumn{3}{c}{ Magnitude and frequency of annual low flow } \\
\hline $\begin{array}{c}\text { Lowest average streamflow, in } \mathrm{ft}^{3} / \mathrm{s} \text {, for indicated period of } \\
\text { consecutive days at an annual nonexceedance probability of } 0.1\end{array}$ \\
\hline $\mathbf{7}$ & $\mathbf{3 0}$ \\
\hline 18 & 21 & 25 \\
\hline
\end{tabular}

Percentage of time streamflow was equaled or exceeded for the period of record

\begin{tabular}{cc|cc}
\hline $\begin{array}{c}\text { Percentage } \\
\text { of time }\end{array}$ & $\begin{array}{c}\text { Daily mean } \\
\text { streamflow } \\
\left(\mathrm{ft}^{3} / \mathbf{s}\right)\end{array}$ & $\begin{array}{c}\text { Percentage } \\
\text { of time }\end{array}$ & $\begin{array}{c}\text { Daily mean } \\
\text { streamflow } \\
\left(\mathrm{ft}^{3} / \mathbf{s}\right)\end{array}$ \\
\hline 99 & 24 & 40 & 240 \\
\hline 98 & 27 & 30 & 336 \\
\hline 95 & 35 & 20 & 507 \\
\hline 90 & 44 & 10 & 936 \\
\hline 80 & 63 & 5 & 1550 \\
\hline 70 & 90 & 2 & 2560 \\
\hline 60 & 128 & 1 & 3390 \\
\hline 50 & 175 & & \\
\hline
\end{tabular}




\section{SOUTH FORK WILDCAT CREEK NEAR LAFAYETTE, IN}

Location. - Lat $40^{\circ} 25^{\prime} 04^{\prime \prime}$, long $86^{\circ} 46^{\prime} 05^{\prime \prime}$ referenced to North American Datum of 1927, in SW 1/4 SW 1/4 sec.21, T.23 N., R.3 W., Tippecanoe County, IN, Hydrologic Unit 05120107, on right bank $40 \mathrm{ft}$ upstream from bridge on State Highway 26, 0.5 mi upstream from Middle Fork, 4.4 mi upstream from mouth, and 5 mi east of Lafayette.

Drainage area. $-243 \mathrm{mi}^{2}$.

Period of record. - October 1943 to current year.

Average discharge. $-245 \mathrm{ft}^{3} / \mathrm{s}$.

Minimum daily discharge. $-15 \mathrm{ft}^{3} / \mathrm{s}$.

Human health (harmonic mean) design flow. $-79 \mathrm{ft}^{3} / \mathrm{s}$.

Remarks. - None.

Magnitude and frequency of annual low flow

Lowest average streamflow, in $\mathrm{ft}^{3} / \mathrm{s}$, for indicated period of consecutive days at an annual nonexceedance probability of 0.1

\begin{tabular}{ccc}
\hline $\mathbf{1}$ & $\mathbf{7}$ & $\mathbf{3 0}$ \\
\hline 19 & 20 & 23 \\
\hline
\end{tabular}

Percentage of time streamflow was equaled or exceeded for the period of record

\begin{tabular}{cc|cc}
\hline $\begin{array}{c}\text { Percentage } \\
\text { of time }\end{array}$ & $\begin{array}{c}\text { Daily mean } \\
\text { streamflow } \\
\left(\mathrm{ft}^{3} / \mathbf{s}\right)\end{array}$ & $\begin{array}{c}\text { Percentage } \\
\text { of time }\end{array}$ & $\begin{array}{c}\text { Daily mean } \\
\text { streamflow } \\
\left(\mathrm{ft}^{3} / \mathbf{s}\right)\end{array}$ \\
\hline 99 & 22 & 40 & 150 \\
\hline 98 & 24 & 30 & 202 \\
\hline 95 & 29 & 20 & 296 \\
\hline 90 & 36 & 10 & 536 \\
\hline 80 & 48 & 5 & 902 \\
\hline 70 & 64 & 2 & 1580 \\
\hline 60 & 86 & 1 & 2250 \\
\hline 50 & 114 & & \\
\hline
\end{tabular}


Wabash River Basin

\section{WILDCAT CREEK NEAR LAFAYETTE, IN}

Location.- 40 $26^{\prime} 26^{\prime \prime}$, long 86 $49^{\prime} 45^{\prime \prime}$ referenced to North American Datum of 1927, in SW 1/4 NW 1/4 sec.13, T.23 N., R.4 W., Tippecanoe County, IN, Hydrologic Unit 05120107, on right bank about $200 \mathrm{ft}$ downstream of bridge on County Road 2A East, 2.8 mi downstream from South Fork Wildcat Creek, 3.7 mi northeast of courthouse in Lafayette, and 4.8 mi upstream from mouth.

Drainage area. $-794 \mathrm{mi}^{2}$.

Period of record.- May 1954 to current year.

Average discharge. $-804 \mathrm{ft}^{3} / \mathrm{s}$.

Minimum daily discharge. $-47 \mathrm{ft}^{3} / \mathrm{s}$.

Human health (harmonic mean) design flow. $-260 \mathrm{ft}^{3} / \mathrm{s}$.

Remarks. - None.

Magnitude and frequency of annual low flow

Lowest average streamflow, in $\mathrm{ft}^{3} / \mathrm{s}$, for indicated period of consecutive days at an annual nonexceedance probability of 0.1

\begin{tabular}{ccc}
\hline $\mathbf{1}$ & $\mathbf{7}$ & $\mathbf{3 0}$ \\
\hline 59 & 63 & 74 \\
\hline
\end{tabular}

Percentage of time streamflow was equaled or exceeded for the period of record

\begin{tabular}{cc|cc}
\hline $\begin{array}{c}\text { Percentage } \\
\text { of time }\end{array}$ & $\begin{array}{c}\text { Daily mean } \\
\text { streamflow } \\
\left(\mathrm{ft}^{3} / \mathbf{s}\right)\end{array}$ & $\begin{array}{c}\text { Percentage } \\
\text { of time }\end{array}$ & $\begin{array}{c}\text { Daily mean } \\
\text { streamflow } \\
\left(\mathrm{ft}^{3} / \mathbf{s}\right)\end{array}$ \\
\hline 99 & 67 & 40 & 497 \\
\hline 98 & 76 & 30 & 686 \\
\hline 95 & 94 & 20 & 1030 \\
\hline 90 & 115 & 10 & 1840 \\
\hline 80 & 156 & 5 & 3070 \\
\hline 70 & 211 & 2 & 5090 \\
\hline 60 & 283 & 1 & 6580 \\
\hline 50 & 376 & & \\
\hline
\end{tabular}




\section{WABASH RIVER AT LAFAYETTE, IN}

Location. - Lat 40²5'19", long 86 53'49" referenced to North American Datum of 1927, in NE 1/4 SW 1/4 Sec.20, T.23 N., R.4 W., Tippecanoe County, IN, Hydrologic Unit 05120108, on right bank $20 \mathrm{ft}$ downstream from Brown St. in Lafayette, 0.2 mi upstream from Main St. bridge, $0.3 \mathrm{mi}$ downstream from Harrison Memorial Bridge, $5.1 \mathrm{mi}$ downstream from Wildcat Creek, and at river mile 311.9.

Drainage area. $-7,267 \mathrm{mi}^{2}$.

Period of record.- October 1953 to current year.

Average discharge. $-6,871 \mathrm{ft}^{3} / \mathrm{s}$.

Minimum daily discharge. $-399 \mathrm{ft}^{3} / \mathrm{s}$.

Human health (harmonic mean) design flow. $-3,520 \mathrm{ft}^{3} / \mathrm{s}$.

Remarks. - Flow partially regulated by upstream reservoirs and power development. Low-flow statistics are calculated for the regulated period, 1969 to 1993.

\begin{tabular}{|c|c|c|c|}
\hline \multicolumn{4}{|c|}{ Magnitude and frequency of annual low flow } \\
\hline \multicolumn{4}{|c|}{$\begin{array}{l}\text { Lowest average streamflow, in } \mathrm{ft}^{3} / \mathrm{s} \text {, for indicated period of } \\
\text { consecutive days at an annual nonexceedance probability of } 0.1\end{array}$} \\
\hline 1 & \multicolumn{2}{|c|}{7} & 30 \\
\hline 824 & \multicolumn{2}{|c|}{897} & 1080 \\
\hline \multicolumn{4}{|c|}{$\begin{array}{l}\text { Percentage of time streamflow was equaled or exceeded for } \\
\text { the period of record }\end{array}$} \\
\hline $\begin{array}{l}\text { Percentage } \\
\text { of time }\end{array}$ & $\begin{array}{l}\text { Daily mean } \\
\text { streamflow } \\
\left(\mathrm{ft}^{3} / \mathrm{s}\right)\end{array}$ & $\begin{array}{l}\text { Percentage } \\
\text { of time }\end{array}$ & $\begin{array}{l}\text { Daily mean } \\
\text { streamflow } \\
\left(\mathrm{ft}^{3} / \mathrm{s}\right)\end{array}$ \\
\hline 99 & 933 & 40 & 5720 \\
\hline 98 & 1100 & 30 & 8050 \\
\hline 95 & 1360 & 20 & 12000 \\
\hline 90 & 1660 & 10 & 18100 \\
\hline 80 & 2230 & 5 & 24100 \\
\hline 70 & 2830 & 2 & 31400 \\
\hline 60 & 3540 & 1 & 37600 \\
\hline 50 & 4440 & & \\
\hline
\end{tabular}


Wabash River Basin

\section{ELLIOTT DITCH NEAR LAFAYETTE, IN}

Location. - Lat $40^{\circ} 22^{\prime} 58^{\prime \prime}$, long $87^{\circ} 50^{\prime} 52^{\prime \prime}$, referenced to North American Datum of 1927, in SW 1/4 NW 1/4 sec.2, T.22 N., R.4 W., Tippecanoe County, IN, Hydrologic Unit 05120108, on left bank in the northwest corner of Tippecanoe County Highway Department property, $3.5 \mathrm{mi}$ southeast of Lafayette courthouse, $3.6 \mathrm{mi}$ southwest of I65 , and at river mile 4.58 .

Drainage area. $-11.8 \mathrm{mi}^{2}$.

Period of record.- April 2008 to current year.

Average discharge. $-6.9 \mathrm{ft}^{3} / \mathrm{s}$.

Minimum daily discharge. $-0.00 \mathrm{ft}^{3} / \mathrm{s}$.

Human health (harmonic mean) design flow. $-0.6 \mathrm{ft}^{3} / \mathrm{s}$.

Remarks. - This site has less than 10 years of record.

\begin{tabular}{|c|c|c|c|}
\hline \multicolumn{4}{|c|}{ Magnitude and frequency of annual low flow } \\
\hline \multicolumn{4}{|c|}{$\begin{array}{l}\text { Lowest average streamflow, in } \mathrm{ft}^{3} / \mathrm{s} \text {, for indicated period of } \\
\text { consecutive days at an annual nonexceedance probability of } 0.1\end{array}$} \\
\hline 1 & \multicolumn{2}{|c|}{7} & 30 \\
\hline 0.0 & \multicolumn{2}{|c|}{0.0} & 0.2 \\
\hline \multicolumn{4}{|c|}{$\begin{array}{c}\text { Percentage of time streamflow was equaled or exceeded for } \\
\text { the period of record }\end{array}$} \\
\hline $\begin{array}{l}\text { Percentage } \\
\text { of time }\end{array}$ & $\begin{array}{l}\text { Daily mean } \\
\text { streamflow } \\
\left(\mathrm{ft}^{3} / \mathrm{s}\right)\end{array}$ & $\begin{array}{l}\text { Percentage } \\
\text { of time }\end{array}$ & $\begin{array}{l}\text { Daily mean } \\
\text { streamflow } \\
\left(\mathrm{ft}^{3} / \mathrm{s}\right)\end{array}$ \\
\hline 99 & 0 & 40 & 3.6 \\
\hline 98 & 0 & 30 & 5.1 \\
\hline 95 & 0.2 & 20 & 8.2 \\
\hline 90 & 0.3 & 10 & 16 \\
\hline 80 & 0.7 & 5 & 28 \\
\hline 70 & 1.2 & 2 & 50 \\
\hline 60 & 1.8 & 1 & 70 \\
\hline 50 & 2.6 & & \\
\hline
\end{tabular}




\section{ELLIOTT DITCH NEAR ELSTON, IN}

Location. - Lat $40^{\circ} 22^{\prime} 15^{\prime \prime}$, long 86 $6^{\circ} 4^{\prime} 34^{\prime \prime}$ referenced to North American Datum of 1927, in NW 1/4 NE 1/4 sec.7, T.22 N., R.4 W., Tippecanoe County, IN, Hydrologic Unit 05120108, on downstream side of bridge, $1.3 \mathrm{mi}$ southwest of Old 231, 7.5 mi southwest of I-65.

Drainage area. - $18.0 \mathrm{mi}^{2}$.

Period of record. - March 2009 to current year.

Average discharge. $-14.6 \mathrm{ft}^{3} / \mathrm{s}$.

Minimum daily discharge. $-0.31 \mathrm{ft}^{3} / \mathrm{s}$.

Human health (harmonic mean) design flow. $-3.4 \mathrm{ft}^{3} / \mathrm{s}$.

Remarks. - This site has less than 10 years of record.

\begin{tabular}{|c|c|c|c|}
\hline \multicolumn{4}{|c|}{ Magnitude and frequency of annual low flow } \\
\hline \multicolumn{4}{|c|}{$\begin{array}{l}\text { Lowest average streamflow, in } \mathrm{ft}^{3} / \mathrm{s} \text {, for indicated period of } \\
\text { consecutive days at an annual nonexceedance probability of } 0.1\end{array}$} \\
\hline 1 & \multicolumn{2}{|c|}{7} & 30 \\
\hline 0.3 & \multicolumn{2}{|c|}{0.3} & 0.6 \\
\hline \multicolumn{4}{|c|}{$\begin{array}{l}\text { Percentage of time streamflow was equaled or exceeded for } \\
\text { the period of record }\end{array}$} \\
\hline $\begin{array}{l}\text { Percentage } \\
\text { of time }\end{array}$ & $\begin{array}{l}\text { Daily mean } \\
\text { streamflow } \\
\left(\mathrm{ft}^{3} / \mathrm{s}\right)\end{array}$ & $\begin{array}{l}\text { Percentage } \\
\text { of time }\end{array}$ & $\begin{array}{l}\text { Daily mean } \\
\text { streamflow } \\
\left(\mathrm{ft}^{3} / \mathrm{s}\right)\end{array}$ \\
\hline 99 & 0.4 & 40 & 8.8 \\
\hline 98 & 0.5 & 30 & 12 \\
\hline 95 & 0.9 & 20 & 17 \\
\hline 90 & 1.3 & 10 & 28 \\
\hline 80 & 2.4 & 5 & 47 \\
\hline 70 & 3.5 & 2 & 91 \\
\hline 60 & 5.1 & 1 & 140 \\
\hline 50 & 6.8 & & \\
\hline
\end{tabular}




\section{LITTLE WEA CREEK AT SOUTH RAUB, IN}

Location.- Lat $40^{\circ} 18^{\prime} 05^{\prime \prime}$, long $86^{\circ} 55^{\prime} 25^{\prime \prime}$, referenced to North American Datum of 1927, in NE 1/4 NE 1/4 sec.1, T.21 N., R.5 W., Tippecanoe County, IN, Hydrologic Unit 05120108, on upstream side of bridge on CR 800 South, $1.0 \mathrm{mi}$ west of Hwy 231, $0.52 \mathrm{mi}$ west of South Raub, $8.3 \mathrm{mi}$ south-southwest of courthouse in Lafayette, and $7.0 \mathrm{mi}$ upstream from mouth.

Drainage area. $-17.3 \mathrm{mi}^{2}$.

Period of record. - March 2009 to current year.

Average discharge. $-22.1 \mathrm{ft}^{3} / \mathrm{s}$

Minimum daily discharge. $-1.5 \mathrm{ft}^{3} / \mathrm{s}$.

Human health (harmonic mean) design flow. $-8.3 \mathrm{ft}^{3} / \mathrm{s}$.

Remarks. - This site has less than 10 years of record. -- indicates that there was poor correlation with the index station: no values determined.

\begin{tabular}{|c|c|c|c|}
\hline \multicolumn{4}{|c|}{ Magnitude and frequency of annual low flow } \\
\hline \multicolumn{4}{|c|}{$\begin{array}{l}\text { Lowest average streamflow, in } \mathrm{ft}^{3} / \mathrm{s} \text {, for indicated period of } \\
\text { consecutive days at an annual nonexceedance probability of } 0.1\end{array}$} \\
\hline 1 & \multicolumn{2}{|c|}{7} & 30 \\
\hline -- & \multicolumn{2}{|c|}{--} & -- \\
\hline \multicolumn{4}{|c|}{$\begin{array}{l}\text { Percentage of time streamflow was equaled or exceeded for } \\
\text { the period of record }\end{array}$} \\
\hline $\begin{array}{l}\text { Percentage } \\
\text { of time }\end{array}$ & $\begin{array}{l}\text { Daily mean } \\
\text { streamflow } \\
\left(\mathrm{ft}^{3} / \mathrm{s}\right)\end{array}$ & $\begin{array}{l}\text { Percentage } \\
\text { of time }\end{array}$ & $\begin{array}{l}\text { Daily mean } \\
\text { streamflow } \\
\left(\mathrm{ft}^{3} / \mathrm{s}\right)\end{array}$ \\
\hline 99 & 2.1 & 40 & 16 \\
\hline 98 & 2.4 & 30 & 19 \\
\hline 95 & 3.3 & 20 & 26 \\
\hline 90 & 4.0 & 10 & 43 \\
\hline 80 & 5.0 & 5 & 73 \\
\hline 70 & 6.2 & 2 & 138 \\
\hline 60 & 8.5 & 1 & 196 \\
\hline 50 & 12 & & \\
\hline
\end{tabular}




\section{MARSHALL DITCH NEAR MONTMORENCI, IN}

Location.- Lat $40^{\circ} 30^{\prime} 42^{\prime \prime}$, long $87^{\circ} 01^{\prime} 10^{\prime \prime}$, referenced to North American Datum of 1927, in NW 1/4 SW 1/4 sec.20, T.24 N., R.5 W., Tippecanoe County, IN, Hydrologic Unit 05120108, on right bank at mile 1.7, and 2.9 mi northeast of Montmorenci.

Drainage area. $-1.58 \mathrm{mi}^{2}$.

Period of record.- October 1990 to 1995.

Average discharge. $-1.49 \mathrm{ft}^{3} / \mathrm{s}$.

Minimum daily discharge. $-0.00 \mathrm{ft}^{3} / \mathrm{s}$.

Human health (harmonic mean) design flow. $-0.2 \mathrm{ft}^{3} / \mathrm{s}$.

Remarks. - This site has less than 10 years of record.

\begin{tabular}{|c|c|c|c|}
\hline \multicolumn{4}{|c|}{ Magnitude and frequency of annual low flow } \\
\hline \multicolumn{4}{|c|}{$\begin{array}{l}\text { Lowest average streamflow, in } \mathrm{ft}^{3} / \mathrm{s} \text {, for indicated period of } \\
\text { consecutive days at an annual nonexceedance probability of } 0.1\end{array}$} \\
\hline 1 & \multicolumn{2}{|c|}{7} & 30 \\
\hline 0.0 & \multicolumn{2}{|c|}{0.0} & 0.0 \\
\hline \multicolumn{4}{|c|}{$\begin{array}{l}\text { Percentage of time streamflow was equaled or exceeded for } \\
\text { the period of record }\end{array}$} \\
\hline $\begin{array}{l}\text { Percentage } \\
\text { of time }\end{array}$ & $\begin{array}{l}\text { Daily mean } \\
\text { streamflow } \\
\left(\mathrm{ft}^{3} / \mathrm{s}\right)\end{array}$ & $\begin{array}{l}\text { Percentage } \\
\text { of time }\end{array}$ & $\begin{array}{l}\text { Daily mean } \\
\text { streamflow } \\
\left(\mathrm{ft}^{3} / \mathrm{s}\right)\end{array}$ \\
\hline 99 & 0.0 & 40 & 0.7 \\
\hline 98 & 0.0 & 30 & 0.9 \\
\hline 95 & 0.0 & 20 & 1.3 \\
\hline 90 & 0.0 & 10 & 2.5 \\
\hline 80 & 0.1 & 5 & 5.0 \\
\hline 70 & 0.2 & 2 & 12 \\
\hline 60 & 0.4 & 1 & 20 \\
\hline 50 & 0.5 & & \\
\hline
\end{tabular}




\section{INDIAN CREEK NEAR MONTMORENCI, IN}

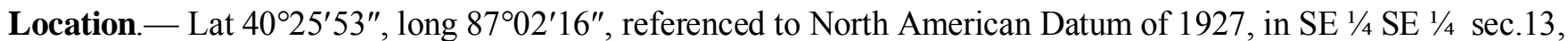
T.23 N., R.6 W., Tippecanoe County, IN, HydrologicUnit 05120108, on right bank 1.8 mi upstream from mouth, 1.4 mi downstream from Goose Creek and 3.0 mi southwest of Montmorenci.

Drainage area. $-27.8 \mathrm{mi}^{2}$.

Period of record.- October 1990 to September 1994.

Average discharge. $-27.9 \mathrm{ft}^{3} / \mathrm{s}$.

Minimum daily discharge. $-0.08 \mathrm{ft}^{3} / \mathrm{s}$.

Human health (harmonic mean) design flow. $-2.6 \mathrm{ft}^{3} / \mathrm{s}$.

Remarks. - This site has less than 10 years of record.

\begin{tabular}{|c|c|c|c|}
\hline \multicolumn{4}{|c|}{ Magnitude and frequency of annual low flow } \\
\hline \multicolumn{4}{|c|}{$\begin{array}{l}\text { Lowest average streamflow, in } \mathrm{ft}^{3} / \mathrm{s} \text {, for indicated period of } \\
\text { consecutive days at an annual nonexceedance probability of } 0.1\end{array}$} \\
\hline 1 & \multicolumn{2}{|c|}{7} & 30 \\
\hline 0.1 & \multicolumn{2}{|c|}{0.2} & 0.3 \\
\hline \multicolumn{4}{|c|}{$\begin{array}{l}\text { Percentage of time streamflow was equaled or exceeded for } \\
\text { the period of record }\end{array}$} \\
\hline $\begin{array}{l}\text { Percentage } \\
\text { of time }\end{array}$ & $\begin{array}{c}\text { Daily mean } \\
\text { streamflow } \\
\left(\mathrm{ft}^{3} / \mathrm{s}\right)\end{array}$ & $\begin{array}{l}\text { Percentage } \\
\text { of time }\end{array}$ & $\begin{array}{l}\text { Daily mean } \\
\text { streamflow } \\
\left(\mathrm{ft}^{3} / \mathrm{s}\right)\end{array}$ \\
\hline 99 & 0.2 & 40 & 15 \\
\hline 98 & 0.3 & 30 & 20 \\
\hline 95 & 0.7 & 20 & 29 \\
\hline 90 & 1.4 & 10 & 55 \\
\hline 80 & 3.0 & 5 & 108 \\
\hline 70 & 5.5 & 2 & 192 \\
\hline 60 & 8.2 & 1 & 312 \\
\hline 50 & 12 & & \\
\hline
\end{tabular}




\section{LITTLE PINE CREEK NEAR MONTMORENCI, IN}

Location.- Lat $40^{\circ} 28^{\prime} 02^{\prime \prime}$, long $87^{\circ} 03^{\prime} 29^{\prime \prime}$, referenced to North American Datum of 1927 , in NE $1 / 4$ SE $1 / 4 \mathrm{sec} .2$,

T.23 N., R.6 W., Tippecanoe County, IN, Hydrologic Unit 05120108, on downstream side of bridge, 1.5 mi west of CR 350 N, 2 mi southwest of Montmorenci, and 15.5 mi west of I-65.

Drainage area. $-21.7 \mathrm{mi}^{2}$.

Period of record.- March 2009 to current year.

Average discharge. $-23.0 \mathrm{ft}^{3} / \mathrm{s}$.

Minimum daily discharge. $-0.0 \mathrm{ft}^{3} / \mathrm{s}$.

Human health (harmonic mean) design flow. $-0.4 \mathrm{ft}^{3} / \mathrm{s}$.

Remarks. - This site has less than 10 years of record.

\begin{tabular}{|c|c|c|c|}
\hline \multicolumn{4}{|c|}{ Magnitude and frequency of annual low flow } \\
\hline \multicolumn{4}{|c|}{$\begin{array}{l}\text { Lowest average streamflow, in } \mathrm{ft}^{3} / \mathrm{s} \text {, for indicated period of } \\
\text { consecutive days at an annual nonexceedance probability of } 0.1\end{array}$} \\
\hline 1 & \multicolumn{2}{|c|}{7} & 30 \\
\hline 0.0 & \multicolumn{2}{|c|}{0.0} & 0.0 \\
\hline \multicolumn{4}{|c|}{$\begin{array}{l}\text { Percentage of time streamflow was equaled or exceeded for } \\
\text { the period of record }\end{array}$} \\
\hline $\begin{array}{l}\text { Percentage } \\
\text { of time }\end{array}$ & $\begin{array}{l}\text { Daily mean } \\
\text { streamflow } \\
\text { (ft } 3 / s)\end{array}$ & $\begin{array}{l}\text { Percentage } \\
\text { of time }\end{array}$ & $\begin{array}{l}\text { Daily mean } \\
\text { streamflow } \\
\left(\mathrm{ft}^{3} / \mathrm{s}\right)\end{array}$ \\
\hline 99 & 0 & 40 & 13 \\
\hline 98 & 0 & 30 & 18 \\
\hline 95 & 0.1 & 20 & 29 \\
\hline 90 & 0.4 & 10 & 57 \\
\hline 80 & 1 & 5 & 93 \\
\hline 70 & 2.6 & 2 & 156 \\
\hline 60 & 5.2 & 1 & 249 \\
\hline 50 & 8.8 & & \\
\hline
\end{tabular}




\section{LITTLE PINE CREEK AT GREEN HILL, IN}

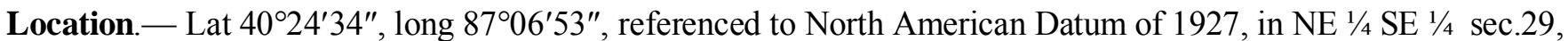
T.23 N., R.6 W., Warren County, IN, Hydrologic Unit 05120108, on right bank at southwest edge of Green Hill, 1.2 mile downstream from Armstrong Creek and at river mile 6.1.

Drainage area. $-42.3 \mathrm{mi}^{2}$.

Period of record. - October 1990 to September 1995.

Average discharge. $-49.0 \mathrm{ft}^{3} / \mathrm{s}$.

Minimum daily discharge. $-0.55 \mathrm{ft}^{3} / \mathrm{s}$.

Human health (harmonic mean) design flow. $-7.8 \mathrm{ft}^{3} / \mathrm{s}$.

Remarks. - This site has less than 10 years of record.

\begin{tabular}{|c|c|c|c|}
\hline \multicolumn{4}{|c|}{ Magnitude and frequency of annual low flow } \\
\hline \multicolumn{4}{|c|}{$\begin{array}{l}\text { Lowest average streamflow, in } \mathrm{ft}^{3} / \mathrm{s} \text {, for indicated period of } \\
\text { consecutive days at an annual nonexceedance probability of } 0 \text {. }\end{array}$} \\
\hline 1 & \multicolumn{2}{|c|}{7} & 30 \\
\hline 0.4 & \multicolumn{2}{|c|}{0.7} & 1.0 \\
\hline \multicolumn{4}{|c|}{$\begin{array}{l}\text { Percentage of time streamflow was equaled or exceeded for } \\
\text { the period of record }\end{array}$} \\
\hline $\begin{array}{l}\text { Percentage } \\
\text { of time }\end{array}$ & $\begin{array}{l}\text { Daily mean } \\
\text { streamflow } \\
\left(\mathrm{ft}^{3} / \mathrm{s}\right)\end{array}$ & $\begin{array}{l}\text { Percentage } \\
\text { of time }\end{array}$ & $\begin{array}{l}\text { Daily mean } \\
\text { streamflow } \\
\left(\mathrm{ft}^{3} / \mathrm{s}\right)\end{array}$ \\
\hline 99 & 0.9 & 40 & 32 \\
\hline 98 & 1.1 & 30 & 44 \\
\hline 95 & 1.6 & 20 & 64 \\
\hline 90 & 2.7 & 10 & 114 \\
\hline 80 & 6.8 & 5 & 197 \\
\hline 70 & 13 & 2 & 293 \\
\hline 60 & 18 & 1 & 370 \\
\hline 50 & 25 & & \\
\hline
\end{tabular}




\section{MUD PINE CREEK NEAR OXFORD, IN}

Location. - Lat $40^{\circ} 31^{\prime} 24^{\prime \prime}$, long $87^{\circ} 20^{\prime} 30^{\prime \prime}$ referenced to North American Datum of 1927, in NE 1/4 SE 1/4 sec.17, T.24 N., R. 8 W., Benton County, IN, Hydrologic Unit 05120108, on right bank $5 \mathrm{ft}$ downstream from county road bridge, $0.3 \mathrm{mi}$ north of Chase, $2 \mathrm{mi}$ east of Boswell, and $5 \mathrm{mi}$ west of Oxford.

Drainage area. $-39.4 \mathrm{mi}^{2}$.

Period of record. - June 1971 to October 2003.

Average discharge. $-42.8 \mathrm{ft}^{3} / \mathrm{s}$.

Minimum daily discharge. $-0.01 \mathrm{ft}^{3} / \mathrm{s}$.

Human health (harmonic mean) design flow. $-2.7 \mathrm{ft}^{3} / \mathrm{s}$.

Remarks.- None.

\begin{tabular}{|c|c|c|c|}
\hline \multicolumn{4}{|c|}{ Magnitude and frequency of annual low flow } \\
\hline \multicolumn{4}{|c|}{$\begin{array}{l}\text { Lowest average streamflow, in } \mathrm{ft}^{3} / \mathrm{s} \text {, for indicated period of } \\
\text { consecutive days at an annual nonexceedance probability of } 0.1\end{array}$} \\
\hline 1 & \multicolumn{2}{|c|}{7} & 30 \\
\hline 0.1 & \multicolumn{2}{|c|}{0.2} & 0.4 \\
\hline \multicolumn{4}{|c|}{$\begin{array}{l}\text { Percentage of time streamflow was equaled or exceeded for } \\
\text { the period of record }\end{array}$} \\
\hline $\begin{array}{l}\text { Percentage } \\
\text { of time }\end{array}$ & $\begin{array}{l}\text { Daily mean } \\
\text { streamflow } \\
\left(\mathrm{ft}^{3} / \mathrm{s}\right)\end{array}$ & $\begin{array}{l}\text { Percentage } \\
\text { of time }\end{array}$ & $\begin{array}{l}\text { Daily mean } \\
\text { streamflow } \\
\left(\mathrm{ft}^{3} / \mathrm{s}\right)\end{array}$ \\
\hline 99 & 0.3 & 40 & 24 \\
\hline 98 & 0.4 & 30 & 35 \\
\hline 95 & 0.6 & 20 & 54 \\
\hline 90 & 0.9 & 10 & 101 \\
\hline 80 & 2.3 & 5 & 161 \\
\hline 70 & 5.4 & 2 & 299 \\
\hline 60 & 10 & 1 & 462 \\
\hline 50 & 16 & & \\
\hline
\end{tabular}




\section{BIG PINE CREEK NEAR WILLIAMSPORT, IN}

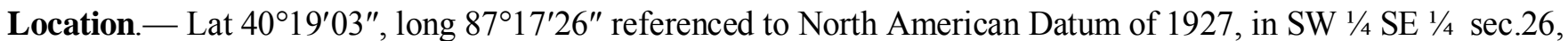
T.22 N., R.8 W., Warren County, IN, Hydrologic Unit 05120108, on downstream side of county road bridge, $1.6 \mathrm{mi}$ north of Williamsport city limits, and 3.7 mi upstream from mouth.

Drainage area. $-323 \mathrm{mi}^{2}$.

Period of record.-October 1955 to September 1987.

Average discharge. $-270 \mathrm{ft}^{3} / \mathrm{s}$.

Minimum daily discharge. $-6.5 \mathrm{ft}^{3} / \mathrm{s}$.

Human health (harmonic mean) design flow. $-52 \mathrm{ft}^{3} / \mathrm{s}$.

Remarks.-None.

\begin{tabular}{|c|c|c|c|}
\hline \multicolumn{4}{|c|}{ Magnitude and frequency of annual low flow } \\
\hline \multicolumn{4}{|c|}{$\begin{array}{l}\text { Lowest average streamflow, in } \mathrm{ft}^{3} / \mathrm{s} \text {, for indicated period of } \\
\text { consecutive days at an annual nonexceedance probability of } 0.1\end{array}$} \\
\hline 1 & \multicolumn{2}{|c|}{7} & 30 \\
\hline 7.6 & \multicolumn{2}{|c|}{8.1} & 10 \\
\hline \multicolumn{4}{|c|}{$\begin{array}{l}\text { Percentage of time streamflow was equaled or exceeded for } \\
\text { the period of record }\end{array}$} \\
\hline $\begin{array}{l}\text { Percentage } \\
\text { of time }\end{array}$ & $\begin{array}{l}\text { Daily mean } \\
\text { streamflow } \\
\left(\mathrm{ft}^{3} / \mathrm{s}\right)\end{array}$ & $\begin{array}{l}\text { Percentage } \\
\text { of time }\end{array}$ & $\begin{array}{l}\text { Daily mean } \\
\text { streamflow } \\
\left(\mathrm{ft}^{3} / \mathrm{s}\right)\end{array}$ \\
\hline 99 & 8.9 & 40 & 168 \\
\hline 98 & 11 & 30 & 236 \\
\hline 95 & 15 & 20 & 353 \\
\hline 90 & 19 & 10 & 635 \\
\hline 80 & 31 & 5 & 1080 \\
\hline 70 & 52 & 2 & 1790 \\
\hline 60 & 83 & 1 & 2330 \\
\hline 50 & 122 & & \\
\hline
\end{tabular}




\section{WABASH RIVER AT COVINGTON, IN}

Location. - Lat $40^{\circ} 08^{\prime} 24^{\prime \prime}$, long $87^{\circ} 24^{\prime} 24^{\prime \prime}$ referenced to North American Datum of 1927, in NE 1/4 NW 1/4 sec.35, T.20 N., R.9 W., Warren County, IN, Hydrologic Unit 05120108, on right approach to old U.S. Highway 136 bridge at Covington, $2.9 \mathrm{mi}$ downstream from Oppossum Run, $3.6 \mathrm{mi}$ upstream from Spring Creek, and at river mile 271.1.

Drainage area. $-8,218 \mathrm{mi}^{2}$.

Period of record. - October 1939 to current year.

Average discharge. $-7,902 \mathrm{ft}^{3} / \mathrm{s}$.

Minimum daily discharge. $-487 \mathrm{ft}^{3} / \mathrm{s}$.

Human health (harmonic mean) design flow. $-4,120 \mathrm{ft}^{3} / \mathrm{s}$.

Remarks. - Flow partially regulated by upstream reservoirs. Low-flow statistics are calculated for the regulated period, 1969 to 2011.

\begin{tabular}{|c|c|c|c|}
\hline \multicolumn{4}{|c|}{ Magnitude and frequency of annual low flow } \\
\hline \multicolumn{4}{|c|}{$\begin{array}{l}\text { Lowest average streamflow, in } \mathrm{ft}^{3} / \mathrm{s} \text {, for indicated period of } \\
\text { consecutive days at an annual nonexceedance probability of } 0 \text {. }\end{array}$} \\
\hline 1 & \multicolumn{2}{|c|}{7} & 30 \\
\hline 1020 & \multicolumn{2}{|c|}{1080} & 1290 \\
\hline \multicolumn{4}{|c|}{$\begin{array}{l}\text { Percentage of time streamflow was equaled or exceeded for } \\
\text { the period of record }\end{array}$} \\
\hline $\begin{array}{l}\text { Percentage } \\
\text { of time }\end{array}$ & $\begin{array}{c}\text { Daily mean } \\
\text { streamflow } \\
\left(\mathrm{ft}^{3} / \mathrm{s}\right)\end{array}$ & $\begin{array}{l}\text { Percentage } \\
\text { of time }\end{array}$ & $\begin{array}{c}\text { Daily mean } \\
\text { streamflow } \\
\left(\mathrm{ft}^{3} / \mathrm{s}\right)\end{array}$ \\
\hline 99 & 1190 & 40 & 6600 \\
\hline 98 & 1320 & 30 & 9180 \\
\hline 95 & 1620 & 20 & 13600 \\
\hline 90 & 1960 & 10 & 20700 \\
\hline 80 & 2600 & 5 & 26800 \\
\hline 70 & 3250 & 2 & 33200 \\
\hline 60 & 4100 & 1 & 39500 \\
\hline 50 & 5170 & & \\
\hline
\end{tabular}




\section{EAST FORK COAL CREEK NEAR HILLSBORO, IN}

Location. - Lat $40^{\circ} 06^{\prime} 06^{\prime \prime}$, long $87^{\circ} 07^{\prime} 54^{\prime \prime}$ referenced to North American Datum of 1927, in NW 1/4 SW 1/4 sec.8, T.19 N., R.6 W., Fountain County, IN, Hydrologic Unit 05120108, at center pier on downstream side of bridge on County Road 700 East, 1.5 mi east of Hillsboro, 3.7 mi northwest of Waynetown, and 9.6 mi upstream from mouth.

Drainage area. $-33.4 \mathrm{mi}^{2}$.

Period of record.- September 1968 to September 1991.

Average discharge. $-37.3 \mathrm{ft}^{3} / \mathrm{s}$.

Minimum daily discharge. $-2.1 \mathrm{ft}^{3} / \mathrm{s}$.

Human health (harmonic mean) design flow. $-14 \mathrm{ft}^{3} / \mathrm{s}$.

Remarks.- None.

\begin{tabular}{|c|c|c|c|}
\hline \multicolumn{4}{|c|}{ Magnitude and frequency of annual low flow } \\
\hline \multicolumn{4}{|c|}{$\begin{array}{l}\text { Lowest average streamflow, in } \mathrm{ft}^{3} / \mathrm{s} \text {, for indicated period of } \\
\text { consecutive days at an annual nonexceedance probability of } 0.1\end{array}$} \\
\hline 1 & \multicolumn{2}{|c|}{7} & 30 \\
\hline 3.1 & \multicolumn{2}{|c|}{3.4} & 4.3 \\
\hline \multicolumn{4}{|c|}{$\begin{array}{l}\text { Percentage of time streamflow was equaled or exceeded for } \\
\text { the period of record }\end{array}$} \\
\hline $\begin{array}{l}\text { Percentage } \\
\text { of time }\end{array}$ & $\begin{array}{l}\text { Daily mean } \\
\text { streamflow } \\
\left(\mathrm{ft}^{3} / \mathrm{s}\right)\end{array}$ & $\begin{array}{l}\text { Percentage } \\
\text { of time }\end{array}$ & $\begin{array}{l}\text { Daily mean } \\
\text { streamflow } \\
\text { (fts/s) }\end{array}$ \\
\hline 99 & 4.0 & 40 & 24 \\
\hline 98 & 4.5 & 30 & 30 \\
\hline 95 & 5.5 & 20 & 42 \\
\hline 90 & 6.9 & 10 & 72 \\
\hline 80 & 9.2 & 5 & 120 \\
\hline 70 & 12 & 2 & 235 \\
\hline 60 & 15 & 1 & 351 \\
\hline 50 & 19 & & \\
\hline
\end{tabular}




\section{COAL CREEK AT COAL CREEK, IN}

Location. - Lat $40^{\circ} 01^{\prime} 42^{\prime \prime}$, long $87^{\circ} 22^{\prime} 30^{\prime \prime}$ referenced to North American Datum of 1927, in SW 1/4 SW 1/4 sec. 6 , T.18N., R.8.W., Fountain County, IN, on downstream side of county road bridge, 0.7 mile southeast of Coal Creek.

Drainage area. $-214 \mathrm{mi}^{2}$.

Period of record. - October 1964 to September 1972.

Average discharge. $-178 \mathrm{ft}^{3} / \mathrm{s}$.

Minimum daily discharge. $-6.0 \mathrm{ft}^{3} / \mathrm{s}$.

Human health (harmonic mean) design flow. $-47 \mathrm{ft}^{3} / \mathrm{s}$.

Remarks. - This site has less than10 years of record.

\begin{tabular}{ccc}
\hline \multicolumn{3}{c}{ Magnitude and frequency of annual low flow } \\
\hline $\begin{array}{c}\text { Lowest average streamflow, in } \mathrm{ft}^{3} / \mathrm{s} \text {, for indicated period of } \\
\text { consecutive days at an annual nonexceedance probability of } 0.1\end{array}$ \\
\hline $\mathbf{1}$ & $\mathbf{7}$ & $\mathbf{3 0}$ \\
\hline 7.6 & 8.3 & 10 \\
\hline
\end{tabular}

\begin{tabular}{cc|cc}
\hline \multicolumn{3}{c}{ Percentage of time streamflow was equaled or exceeded for } \\
the period of record \\
\hline $\begin{array}{c}\text { Percentage } \\
\text { of time }\end{array}$ & $\begin{array}{c}\text { Daily mean } \\
\text { streamflow } \\
\left(\mathrm{ft}^{3} / \mathbf{s}\right)\end{array}$ & $\begin{array}{c}\text { Percentage } \\
\text { of time }\end{array}$ & $\begin{array}{c}\text { Daily mean } \\
\text { streamflow } \\
\left(\mathrm{ft}^{3} / \mathrm{s}\right)\end{array}$ \\
\hline 99 & 8.3 & 40 & 104 \\
\hline 98 & 9.7 & 30 & 140 \\
\hline 95 & 14 & 20 & 199 \\
\hline 90 & 22 & 10 & 347 \\
\hline 80 & 31 & 5 & 579 \\
\hline 70 & 43 & 2 & 1180 \\
\hline 60 & 60 & 1 & 1850 \\
\hline 50 & 80 & & \\
\hline
\end{tabular}




\section{LITTLE VERMILLION CREEK NEAR NEWPORT, IN}

Location.- Lat 39 $53^{\prime} 32^{\prime \prime}$, long $87^{\circ} 25^{\prime} 42^{\prime \prime}$ referenced to North American Datum of 1927, in SW 1/4 NW 1/4 sec. 27 , T.17N., R.9W., Vermillion County, IN, on downstream side of bridge on State Highway 63, 1.2 mi northwest of Newport, and 6 mi upstream from mouth.

Drainage area. $-237 \mathrm{mi}^{2}$.

Period of record. - October 1964 to September 1972.

Average discharge. $-186 \mathrm{ft}^{3} / \mathrm{s}$.

Minimum daily discharge. $-5.2 \mathrm{ft}^{3} / \mathrm{s}$.

Human health (harmonic mean) design flow. $-5.5 \mathrm{ft}^{3} / \mathrm{s}$.

Remarks. - This site has less than10 years of record.

\begin{tabular}{|c|c|c|c|}
\hline \multicolumn{4}{|c|}{ Magnitude and frequency of annual low flow } \\
\hline \multicolumn{4}{|c|}{$\begin{array}{l}\text { Lowest average streamflow, in } \mathrm{ft}^{3} / \mathrm{s} \text {, for indicated period of } \\
\text { consecutive days at an annual nonexceedance probability of } 0.1\end{array}$} \\
\hline 1 & \multicolumn{2}{|c|}{7} & 30 \\
\hline 0.2 & \multicolumn{2}{|c|}{0.2} & 0.4 \\
\hline \multicolumn{4}{|c|}{$\begin{array}{l}\text { Percentage of time streamflow was equaled or exceeded for } \\
\text { the period of record }\end{array}$} \\
\hline $\begin{array}{l}\text { Percentage } \\
\text { of time }\end{array}$ & $\begin{array}{l}\text { Daily mean } \\
\text { streamflow } \\
\left(\mathrm{ft}^{3} / \mathrm{s}\right)\end{array}$ & $\begin{array}{l}\text { Percentage } \\
\text { of time }\end{array}$ & $\begin{array}{l}\text { Daily mean } \\
\text { streamflow } \\
\left(\mathrm{ft}^{3} / \mathrm{s}\right)\end{array}$ \\
\hline 99 & 0.2 & 40 & 112 \\
\hline 98 & 0.4 & 30 & 162 \\
\hline 95 & 2.3 & 20 & 234 \\
\hline 90 & 5.4 & 10 & 431 \\
\hline 80 & 12 & 5 & 693 \\
\hline 70 & 27 & 2 & 1310 \\
\hline 60 & 48 & 1 & 1950 \\
\hline 50 & 77 & & \\
\hline
\end{tabular}




\section{PRAIRIE CREEK NEAR LEBANON, IN}

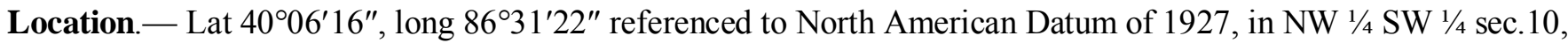
T.19 N., R.1 W., Boone County, IN, Hydrologic Unit 05120110, on right bank $50 \mathrm{ft}$ upstream from bridge on County Road 450 North, 4.0 mi upstream from Deer Creek, 4.9 mi northwest of Lebanon, and 7.7 mi upstream from mouth.

Drainage area. $-33.2 \mathrm{mi}^{2}$.

Period of record. - October 1987 to current year.

Average discharge. $-41.0 \mathrm{ft}^{3} / \mathrm{s}$.

Minimum daily discharge. $-0.03 \mathrm{ft}^{3} / \mathrm{s}$.

Human health (harmonic mean) design flow. $-6.7 \mathrm{ft}^{3} / \mathrm{s}$.

Remarks.- None.

\begin{tabular}{|c|c|c|c|}
\hline \multicolumn{4}{|c|}{ Magnitude and frequency of annual low flow } \\
\hline \multicolumn{4}{|c|}{$\begin{array}{l}\text { Lowest average streamflow, in } \mathrm{ft}^{3} / \mathrm{s} \text {, for indicated period of } \\
\text { consecutive days at an annual nonexceedance probability of } 0.1\end{array}$} \\
\hline 1 & \multicolumn{2}{|c|}{7} & 30 \\
\hline 0.3 & \multicolumn{2}{|c|}{0.5} & 1.2 \\
\hline \multicolumn{4}{|c|}{$\begin{array}{l}\text { Percentage of time streamflow was equaled or exceeded for } \\
\text { the period of record }\end{array}$} \\
\hline $\begin{array}{l}\text { Percentage } \\
\text { of time }\end{array}$ & $\begin{array}{c}\text { Daily mean } \\
\text { streamflow } \\
\left(\mathrm{ft}^{3} / \mathrm{s}\right)\end{array}$ & $\begin{array}{l}\text { Percentage } \\
\text { of time }\end{array}$ & $\begin{array}{c}\text { Daily mean } \\
\text { streamflow } \\
\left(\mathrm{ft}^{3} / \mathrm{s}\right)\end{array}$ \\
\hline 99 & 0.8 & 40 & 21 \\
\hline 98 & 1.3 & 30 & 29 \\
\hline 95 & 2.1 & 20 & 47 \\
\hline 90 & 3.0 & 10 & 87 \\
\hline 80 & 4.9 & 5 & 150 \\
\hline 70 & 7.4 & 2 & 305 \\
\hline 60 & 11 & 1 & 494 \\
\hline 50 & 15 & & \\
\hline
\end{tabular}




\section{SUGAR CREEK AT CRAWFORDSVILLE, IN}

Location. - Lat 40² $2^{\prime} 56^{\prime \prime}$, long 86 $53^{\prime} 58^{\prime \prime}$ referenced to North American Datum of 1927, in SW 1/4 NW 1/4 sec.32, T.19 N., R.4 W., Montgomery County, IN, Hydrologic Unit 05120110, on left bank $327 \mathrm{ft}$ upstream from Crawfordsville Electric Light and Power Co. dam at Crawfordsville, $700 \mathrm{ft}$ upstream from bridge on U.S. Highway 231, 1.0 mi downstream from Walnut Fork Sugar Creek, and at mile 40.4.

Drainage area. $-509 \mathrm{mi}^{2}$.

Period of record.- June 1938 to current year.

Average discharge. $-507 \mathrm{ft}^{3} / \mathrm{s}$.

Minimum daily discharge. $-2.4 \mathrm{ft}^{3} / \mathrm{s}$.

Human health (harmonic mean) design flow. $-76 \mathrm{ft}^{3} / \mathrm{s}$.

Remarks.- None.

\begin{tabular}{ccc}
\hline \multicolumn{3}{c}{ Magnitude and frequency of annual low flow } \\
\hline $\begin{array}{c}\text { Lowest average streamflow, in } \mathrm{ft}^{3} / \mathrm{s} \text {, for indicated period of } \\
\text { consecutive days at an annual nonexceedance probability of } 0.1\end{array}$ \\
\hline $\mathbf{1}$ & $\mathbf{3 0}$ \\
\hline 8.0 & 8.9 & 12 \\
\hline
\end{tabular}

Percentage of time streamflow was equaled or exceeded for the period of record

\begin{tabular}{cc|cc}
\hline $\begin{array}{c}\text { Percentage } \\
\text { of time }\end{array}$ & $\begin{array}{c}\text { Daily mean } \\
\text { streamflow } \\
\left(\mathrm{ft}^{3} / \mathbf{s}\right)\end{array}$ & $\begin{array}{c}\text { Percentage } \\
\text { of time }\end{array}$ & $\begin{array}{c}\text { Daily mean } \\
\text { streamflow } \\
\left(\mathrm{ft}^{3} / \mathbf{s}\right)\end{array}$ \\
\hline 99 & 12 & 40 & 258 \\
\hline 98 & 15 & 30 & 379 \\
\hline 95 & 21 & 20 & 605 \\
\hline 90 & 30 & 10 & 1210 \\
\hline 80 & 50 & 5 & 2110 \\
\hline 70 & 83 & 2 & 3820 \\
\hline 60 & 128 & 1 & 5470 \\
\hline 50 & 184 & & \\
\hline
\end{tabular}




\section{SUGAR CREEK NEAR BYRON, IN}

Location. - Lat $39^{\circ} 55^{\prime} 52^{\prime \prime}$, long $87^{\circ} 07^{\prime} 33^{\prime \prime}$ referenced to North American Datum of 1927, in NW 1/4 SW 1/4 sec.8, T.17 N., R.6 W., Parke County, IN, Hydrologic Unit 05120110, on right bank $30 \mathrm{ft}$ upstream from county highway bridge, 2.5 mi northwest of Byron, 5 mi downstream from Indian Creek.

Drainage area. $-670 \mathrm{mi}^{2}$.

Period of record.- October 1940 to September 1971.

Average discharge. $-628 \mathrm{ft}^{3} / \mathrm{s}$.

Minimum daily discharge. $-12 \mathrm{ft}^{3} / \mathrm{s}$.

Human health (harmonic mean) design flow. - $130 \mathrm{ft}^{3} / \mathrm{s}$.

Remarks.- None.

\begin{tabular}{|c|c|c|c|}
\hline \multicolumn{4}{|c|}{ Magnitude and frequency of annual low flow } \\
\hline \multicolumn{4}{|c|}{$\begin{array}{l}\text { Lowest average streamflow, in } \mathrm{ft}^{3} / \mathrm{s} \text {, for indicated period of } \\
\text { consecutive days at an annual nonexceedance probability of } 0.1\end{array}$} \\
\hline 1 & \multicolumn{2}{|c|}{7} & 30 \\
\hline 21 & \multicolumn{2}{|c|}{22} & 28 \\
\hline \multicolumn{4}{|c|}{$\begin{array}{l}\text { Percentage of time streamflow was equaled or exceeded for } \\
\text { the period of record }\end{array}$} \\
\hline $\begin{array}{l}\text { Percentage } \\
\text { of time }\end{array}$ & $\begin{array}{l}\text { Daily mean } \\
\text { streamflow } \\
\left(\mathrm{ft}^{3} / \mathrm{s}\right)\end{array}$ & $\begin{array}{l}\text { Percentage } \\
\text { of time }\end{array}$ & $\begin{array}{l}\text { Daily mean } \\
\text { streamflow } \\
\left(\mathrm{ft}^{3} / \mathrm{s}\right)\end{array}$ \\
\hline 99 & 25 & 40 & 328 \\
\hline 98 & 31 & 30 & 466 \\
\hline 95 & 41 & 20 & 718 \\
\hline 90 & 54 & 10 & 1410 \\
\hline 80 & 78 & 5 & 2430 \\
\hline 70 & 113 & 2 & 4710 \\
\hline 60 & 163 & 1 & 6910 \\
\hline 50 & 233 & & \\
\hline
\end{tabular}




\section{WABASH RIVER AT MONTEZUMA, IN}

Location. - Lat $39^{\circ} 47^{\prime} 33^{\prime \prime}$, long $87^{\circ} 22^{\prime} 26^{\prime \prime}$ referenced to North American Datum of 1927, in SE 1/4 NE 1/4 Sec.35, T.16 N., R.9 W., Parke County, IN, Hydrologic Unit 05120108, on left bank $20 \mathrm{ft}$ upstream from bridge on U.S. Highway 36 at Montezuma, 2.0 mi upstream from Big Raccoon Creek, 4.9 mi downstream from Sugar Creek, and at mile 240.0.

Drainage area. $-11,118 \mathrm{mi}^{2}$.

Period of record. - October 1927 to current year.

Average discharge. $-10,440 \mathrm{ft}^{3} / \mathrm{s}$.

Minimum daily discharge. $-571 \mathrm{ft}^{3} / \mathrm{s}$.

Human health (harmonic mean) design flow. $-5,190 \mathrm{ft}^{3} / \mathrm{s}$.

Remarks. - Flow partially regulated by upstream reservoirs. Low-flow statistics are calculated for the regulated period, 1969 to 2011.

\begin{tabular}{|c|c|c|c|}
\hline \multicolumn{4}{|c|}{ Magnitude and frequency of annual low flow } \\
\hline \multicolumn{4}{|c|}{$\begin{array}{l}\text { Lowest average streamflow, in } \mathrm{ft}^{3} / \mathrm{s} \text {, for indicated period of } \\
\text { consecutive days at an annual nonexceedance probability of } 0 \text {. }\end{array}$} \\
\hline 1 & \multicolumn{2}{|c|}{7} & 30 \\
\hline 981 & \multicolumn{2}{|c|}{1050} & 1580 \\
\hline \multicolumn{4}{|c|}{$\begin{array}{c}\text { Percentage of time streamflow was equaled or exceeded for } \\
\text { the period of record }\end{array}$} \\
\hline $\begin{array}{l}\text { Percentage } \\
\text { of time }\end{array}$ & $\begin{array}{c}\text { Daily mean } \\
\text { streamflow } \\
\left(\mathrm{ft}^{3} / \mathrm{s}\right)\end{array}$ & $\begin{array}{l}\text { Percentage } \\
\text { of time }\end{array}$ & $\begin{array}{c}\text { Daily mean } \\
\text { streamflow } \\
\left(\mathrm{ft}^{3} / \mathrm{s}\right)\end{array}$ \\
\hline 99 & 1310 & 40 & 9250 \\
\hline 98 & 1520 & 30 & 12700 \\
\hline 95 & 1900 & 20 & 18500 \\
\hline 90 & 2340 & 10 & 27700 \\
\hline 80 & 3240 & 5 & 35700 \\
\hline 70 & 4200 & 2 & 48400 \\
\hline 60 & 5490 & 1 & 59400 \\
\hline 50 & 7100 & & \\
\hline
\end{tabular}




\section{BIG RACCOON CREEK NEAR FINCASTLE, IN}

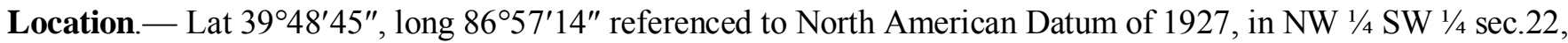
T.16 N., R.5 W., Putnam County, IN, Hydrologic Unit 05120108, on downstream side of County Road 1000N bridge, 1.6 mi upstream from Ramp Creek, $3.7 \mathrm{mi}$ west-northwest of Fincastle, 19.7 mi southwest of Crawfordsville.

Drainage area. $-139 \mathrm{mi}^{2}$.

Period of record. - August 1957 to current year.

Average discharge. $-149 \mathrm{ft}^{3} / \mathrm{s}$.

Minimum daily discharge. $-0.85 \mathrm{ft}^{3} / \mathrm{s}$.

Human health (harmonic mean) design flow. $-17 \mathrm{ft}^{3} / \mathrm{s}$.

Remarks. - Prior to October 1963, published as Raccoon Creek near Fincastle.

\begin{tabular}{|c|c|c|c|}
\hline \multicolumn{4}{|c|}{ Magnitude and frequency of annual low flow } \\
\hline \multicolumn{4}{|c|}{$\begin{array}{l}\text { Lowest average streamflow, in } \mathrm{ft}^{3} / \mathrm{s} \text {, for indicated period of } \\
\text { consecutive days at an annual nonexceedance probability of } 0.1\end{array}$} \\
\hline 1 & \multicolumn{2}{|c|}{7} & 30 \\
\hline 1.6 & \multicolumn{2}{|c|}{1.9} & 2.5 \\
\hline \multicolumn{4}{|c|}{$\begin{array}{l}\text { Percentage of time streamflow was equaled or exceeded for } \\
\text { the period of record }\end{array}$} \\
\hline $\begin{array}{l}\text { Percentage } \\
\text { of time }\end{array}$ & $\begin{array}{l}\text { Daily mean } \\
\text { streamflow } \\
\left(\mathrm{ft}^{3} / \mathrm{s}\right)\end{array}$ & $\begin{array}{l}\text { Percentage } \\
\text { of time }\end{array}$ & $\begin{array}{l}\text { Daily mean } \\
\text { streamflow } \\
\left(\mathrm{ft}^{3} / \mathrm{s}\right)\end{array}$ \\
\hline 99 & 2.0 & 40 & 78 \\
\hline 98 & 2.6 & 30 & 111 \\
\hline 95 & 3.8 & 20 & 171 \\
\hline 90 & 6.2 & 10 & 319 \\
\hline 80 & 12 & 5 & 544 \\
\hline 70 & 23 & 2 & 1090 \\
\hline 60 & 38 & 1 & 1800 \\
\hline 50 & 55 & & \\
\hline
\end{tabular}




\section{BIG RACCOON CREEK AT FERNDALE, IN}

Location. - Lat $39^{\circ} 42^{\prime} 41^{\prime \prime}$, long $87^{\circ} 04^{\prime} 17^{\prime \prime}$ referenced to North American Datum of 1927, in SE $1 / 4$ SE $1 / 4$ sec. 28 , T.15 N., R.6 W., Parke County, IN, Hydrologic Unit 05120108, on right bank at upstream side of bridge on New Discovery Road, 0.5 mi downstream from Cecil M. Harden Lake, 3.7 mi upstream from Rocky Fork Creek, and at mile 33.3.

Drainage area. $-222 \mathrm{mi}^{2}$.

Period of record.- October 1956 to September 2001.

Average discharge. $-225 \mathrm{ft}^{3} / \mathrm{s}$.

Minimum daily discharge. $-2.7 \mathrm{ft}^{3} / \mathrm{s}$. No flow at times due to regulation.

Human health (harmonic mean) design flow. $-50 \mathrm{ft}^{3} / \mathrm{s}$.

Remarks. - Flow regulated by Cecil M. Harden Lake since December 1960. Records of daily discharge provided by U.S. Army Corps of Engineers beginning Oct. 1, 1976. Low-flow statistics are calculated for the regulated period, 1961 to 2001.

\begin{tabular}{|c|c|c|c|}
\hline \multicolumn{4}{|c|}{ Magnitude and frequency of annual low flow } \\
\hline \multicolumn{4}{|c|}{$\begin{array}{l}\text { Lowest average streamflow, in } \mathrm{ft}^{3} / \mathrm{s} \text {, for indicated period of } \\
\text { consecutive days at an annual nonexceedance probability of } 0.1\end{array}$} \\
\hline 1 & \multicolumn{2}{|c|}{7} & 30 \\
\hline 9.4 & \multicolumn{2}{|c|}{13} & 15 \\
\hline \multicolumn{4}{|c|}{$\begin{array}{c}\text { Percentage of time streamflow was equaled or exceeded for } \\
\text { the period of record }\end{array}$} \\
\hline $\begin{array}{l}\text { Percentage } \\
\text { of time }\end{array}$ & $\begin{array}{l}\text { Daily mean } \\
\text { streamflow } \\
\left(\mathrm{ft}^{3} / \mathrm{s}\right)\end{array}$ & $\begin{array}{l}\text { Percentage } \\
\text { of time }\end{array}$ & $\begin{array}{l}\text { Daily mean } \\
\text { streamflow } \\
\left(\mathrm{ft}^{3} / \mathrm{s}\right)\end{array}$ \\
\hline 99 & 15 & 40 & 132 \\
\hline 98 & 16 & 30 & 210 \\
\hline 95 & 18 & 20 & 364 \\
\hline 90 & 20 & 10 & 677 \\
\hline 80 & 28 & 5 & 962 \\
\hline 70 & 39 & 2 & 1280 \\
\hline 60 & 55 & 1 & 1530 \\
\hline 50 & 91 & & \\
\hline
\end{tabular}




\section{BIG RACCOON CREEK AT MANSFIELD, IN}

Location.- Lat $39^{\circ} 41^{\prime} 00^{\prime \prime}$, long $87^{\circ} 07^{\prime} 00^{\prime \prime}$ referenced to North American Datum of 1927, in sec.8, T.14 N., R.6 W., Parke County, Hydrologic Unit 05120108, at bridge on State Highway 59 at Mansfield, 1 mi downstream from Rocky Fork.

Drainage area. $-240 \mathrm{mi}^{2}$.

Period of record. - 1939 to 1958.

Average discharge. $-230 \mathrm{ft}^{3} / \mathrm{s}$.

Minimum daily discharge. $-1.4 \mathrm{ft}^{3} / \mathrm{s}$ due to regulation.

Human health (harmonic mean) design flow. $-28 \mathrm{ft}^{3} / \mathrm{s}$.

Remarks. - Some regulation at low flow by mill upstream of the gage.

\begin{tabular}{|c|c|c|c|}
\hline \multicolumn{4}{|c|}{ Magnitude and frequency of annual low flow } \\
\hline \multicolumn{4}{|c|}{$\begin{array}{l}\text { Lowest average streamflow, in } \mathrm{ft}^{3} / \mathrm{s} \text {, for indicated period of } \\
\text { consecutive days at an annual nonexceedance probability of } 0.1\end{array}$} \\
\hline 1 & \multicolumn{2}{|c|}{7} & 30 \\
\hline 2.4 & \multicolumn{2}{|c|}{3.1} & 3.6 \\
\hline \multicolumn{4}{|c|}{$\begin{array}{l}\text { Percentage of time streamflow was equaled or exceeded for } \\
\text { the period of record }\end{array}$} \\
\hline $\begin{array}{l}\text { Percentage } \\
\text { of time }\end{array}$ & $\begin{array}{l}\text { Daily mean } \\
\text { streamflow } \\
\left(\mathrm{ft}^{3} / \mathrm{s}\right)\end{array}$ & $\begin{array}{l}\text { Percentage } \\
\text { of time }\end{array}$ & $\begin{array}{l}\text { Daily mean } \\
\text { streamflow } \\
\left(\mathrm{ft}^{3} / \mathrm{s}\right)\end{array}$ \\
\hline 99 & 2.7 & 40 & 124 \\
\hline 98 & 3.8 & 30 & 175 \\
\hline 95 & 7.2 & 20 & 256 \\
\hline 90 & 13 & 10 & 457 \\
\hline 80 & 20 & 5 & 790 \\
\hline 70 & 34 & 2 & 1680 \\
\hline 60 & 55 & 1 & 2740 \\
\hline 50 & 85 & & \\
\hline
\end{tabular}




\section{LITTLE RACCOON CREEK NEAR CATLIN, IN}

Location.- Lat $39^{\circ} 40^{\prime} 38^{\prime \prime}$, long $87^{\circ} 13^{\prime} 38^{\prime \prime}$ referenced to North American Datum of 1927, in NE 1/4 NW 1/4 sec.7, T.14 N., R.7 W., Parke County, IN, Hydrologic Unit 05120108, on left bank at downstream side of county road bridge, $300 \mathrm{ft}$ downstream from small left-bank tributary, $0.4 \mathrm{mi}$ upstream from Sunderland Branch, 1.2 mi northeast of Catlin, 2.4 mi upstream from Weisner Branch, and 3.8 mi upstream from mouth.

Drainage area.- $134 \mathrm{mi}^{2}$.

Period of record. - October 1957 to September 1971.

Average discharge. $-117 \mathrm{ft}^{3} / \mathrm{s}$.

Minimum daily discharge. $-4.1 \mathrm{ft}^{3} / \mathrm{s}$.

Human health (harmonic mean) design flow. $-20 \mathrm{ft}^{3} / \mathrm{s}$.

Remarks. - None.

\begin{tabular}{|c|c|c|c|}
\hline \multicolumn{4}{|c|}{ Magnitude and frequency of annual low flow } \\
\hline \multicolumn{4}{|c|}{$\begin{array}{l}\text { Lowest average streamflow, in } \mathrm{ft}^{3} / \mathrm{s} \text {, for indicated period of } \\
\text { consecutive days at an annual nonexceedance probability of } 0.1\end{array}$} \\
\hline 1 & \multicolumn{2}{|c|}{7} & 30 \\
\hline 4.2 & \multicolumn{2}{|c|}{4.5} & 5.3 \\
\hline \multicolumn{4}{|c|}{$\begin{array}{l}\text { Percentage of time streamflow was equaled or exceeded for } \\
\text { the period of record }\end{array}$} \\
\hline $\begin{array}{l}\text { Percentage } \\
\text { of time }\end{array}$ & $\begin{array}{l}\text { Daily mean } \\
\text { streamflow } \\
\left(\mathrm{ft}^{3} / \mathrm{s}\right)\end{array}$ & $\begin{array}{l}\text { Percentage } \\
\text { of time }\end{array}$ & $\begin{array}{l}\text { Daily mean } \\
\text { streamflow } \\
\left(\mathrm{ft}^{3} / \mathrm{s}\right)\end{array}$ \\
\hline 99 & 4.9 & 40 & 64 \\
\hline 98 & 5.4 & 30 & 94 \\
\hline 95 & 6.8 & 20 & 136 \\
\hline 90 & 9.0 & 10 & 238 \\
\hline 80 & 14 & 5 & 385 \\
\hline 70 & 20 & 2 & 723 \\
\hline 60 & 30 & 1 & 1320 \\
\hline 50 & 45 & & \\
\hline
\end{tabular}


Wabash River Basin

\section{BIG RACCOON CREEK AT COXVILLE, IN}

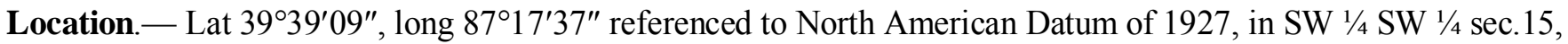
T.14 N., R. 8 W., Parke County, IN, Hydrologic Unit 05120108, on right bank at downstream side of covered bridge on county road at Coxville, $0.8 \mathrm{mi}$ upstream from Rock Run, $1.5 \mathrm{mi}$ downstream from Little Raccoon Creek, $2.1 \mathrm{mi}$ northwest of Rosedale, and at mile 13.1 .

Drainage area. $-448 \mathrm{mi}^{2}$.

Period of record.- October 1956 to September 1988, October 1992 to current year.

Average discharge. $-513 \mathrm{ft}^{3} / \mathrm{s}$.

Minimum daily discharge. $-6.5 \mathrm{ft}^{3} / \mathrm{s}$.

Human health (harmonic mean) design flow. $-170 \mathrm{ft}^{3} / \mathrm{s}$.

Remarks. - Flow regulated by Cecil M. Harden Lake. Low-flow statistics are calculated for the regulated period, 1962 to 1988 and 1992 to 2011. Prior to October 1963, published as Raccoon Creek at Coxville.

\begin{tabular}{cccc}
\hline \multicolumn{5}{c}{ Magnitude and frequency of annual low flow } \\
\hline \multicolumn{4}{c}{ Lowest average streamflow, in $\mathrm{ft}^{3} / \mathbf{s}$, for indicated period of } \\
consecutive days at an annual nonexceedance probability of 0.1
\end{tabular}




\section{WABASH RIVER AT TERRE HAUTE, IN}

Location. - Lat $39^{\circ} 28^{\prime} 33^{\prime \prime}$, long $87^{\circ} 25^{\prime} 08^{\prime \prime}$ referenced to North American Datum of 1927, in NE 1/4 NW 1/4 sec.21, T.12 N., R.9 W., Vigo County, IN, Hydrologic Unit 05120111, on left bank at Indiana America Water Company, Inc., 1st and Elm Streets in Terre Haute, 3.0 mi upstream from Sugar Creek, and 3.6 mi downstream from Lost Creek, and at mile 215.

Drainage area. $-12,263 \mathrm{mi}^{2}$.

Period of record. - February 1905 to July 1906, October 1927 to current year.

Average discharge. $-11,460 \mathrm{ft}^{3} / \mathrm{s}$.

Minimum daily discharge. $-701 \mathrm{ft}^{3} / \mathrm{s}$.

Human health (harmonic mean) design flow. - 5,890 $\mathrm{ft}^{3} / \mathrm{s}$.

Remarks. - Flow partially regulated by upstream reservoirs. Low-flow statistics are calculated for the regulated period, 1969 to 2011.

\begin{tabular}{cccc}
\hline \multicolumn{4}{c}{ Magnitude and frequency of annual low flow } \\
\hline \multicolumn{4}{c}{1300} \\
Lowest average streamflow, in $\mathrm{ft}^{3} / \mathbf{s}$, for indicated period of \\
consecutive days at an annual nonexceedance probability of 0.1
\end{tabular}




\section{WABASH RIVER AT RIVERTON, IN}

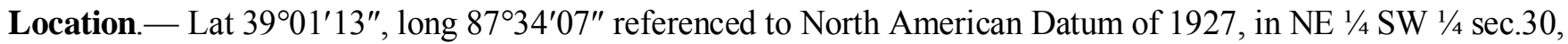
T.7 N., R.10 W., Sullivan County, IN, Hydrologic Unit 05120111, on left bank at downstream side of Illinois Central Railroad bridge at Riverton, 0.5 mi downstream from Turtle Creek, 2 mi south of Merom, and at mile 162.0.

Drainage area. - 13,161 $\mathrm{mi}^{2}$.

Period of record. - October 1938 to current year.

Average discharge. $-12,730 \mathrm{ft}^{3} / \mathrm{s}$.

Minimum daily discharge. $-858 \mathrm{ft}^{3} / \mathrm{s}$.

Human health (harmonic mean) design flow. $-6,520 \mathrm{ft}^{3} / \mathrm{s}$.

Remarks. - Flow partially regulated by upstream reservoirs. Low-flow statistics are calculated for the regulated period, 1969 to 2011.

\begin{tabular}{|c|c|c|c|}
\hline \multicolumn{4}{|c|}{ Magnitude and frequency of annual low flow } \\
\hline \multicolumn{4}{|c|}{$\begin{array}{l}\text { Lowest average streamflow, in } \mathrm{ft}^{3} / \mathrm{s} \text {, for indicated period of } \\
\text { consecutive days at an annual nonexceedance probability of } 0 \text {. }\end{array}$} \\
\hline 1 & \multicolumn{2}{|c|}{7} & 30 \\
\hline 1550 & \multicolumn{2}{|c|}{1660} & 1940 \\
\hline \multicolumn{4}{|c|}{$\begin{array}{l}\text { Percentage of time streamflow was equaled or exceeded for } \\
\text { the period of record }\end{array}$} \\
\hline $\begin{array}{l}\text { Percentage } \\
\text { of time }\end{array}$ & $\begin{array}{c}\text { Daily mean } \\
\text { streamflow } \\
\left(\mathrm{ft}^{3} / \mathrm{s}\right)\end{array}$ & $\begin{array}{l}\text { Percentage } \\
\text { of time }\end{array}$ & $\begin{array}{c}\text { Daily mean } \\
\text { streamflow } \\
\left(\mathrm{ft}^{3} / \mathrm{s}\right)\end{array}$ \\
\hline 99 & 1720 & 40 & 11,500 \\
\hline 98 & 1990 & 30 & 15,500 \\
\hline 95 & 2430 & 20 & 22,600 \\
\hline 90 & 2980 & 10 & 34,000 \\
\hline 80 & 4070 & 5 & 42,700 \\
\hline 70 & 5240 & 2 & 54,400 \\
\hline 60 & 6750 & 1 & 64,100 \\
\hline 50 & 8710 & & \\
\hline
\end{tabular}




\section{BUSSERON CREEK NEAR HYMERA, IN}

Location.- Lat $39^{\circ} 12^{\prime} 54^{\prime \prime}$, long $87^{\circ} 18^{\prime} 41^{\prime \prime}$ referenced to North American Datum of 1927, in NW 1/4 NW 1/4 sec.21, T.9 N., R.8 W., Sullivan County, IN, Hydrologic Unit 05120111, on right bank at downstream side of bridge on County Road 900 North, 1.3 mi upstream from East Fork Busseron Creek, 1.9 mi northwest of Hymera, $4.1 \mathrm{mi}$ upstream from West Fork Busseron Creek, and at mile 30.3.

Drainage area. $-16.7 \mathrm{mi}^{2}$.

Period of record. - June 1966 to October 2003.

Average discharge. $-18.5 \mathrm{ft}^{3} / \mathrm{s}$.

Minimum daily discharge. $-0.0 \mathrm{ft}^{3} / \mathrm{s}$.

Human health (harmonic mean) design flow. $-0.3 \mathrm{ft}^{3} / \mathrm{s}$.

Remarks.- None.

\begin{tabular}{|c|c|c|c|}
\hline \multicolumn{4}{|c|}{ Magnitude and frequency of annual low flow } \\
\hline \multicolumn{4}{|c|}{$\begin{array}{l}\text { Lowest average streamflow, in } \mathrm{ft}^{3} / \mathrm{s} \text {, for indicated period of } \\
\text { consecutive days at an annual nonexceedance probability of } 0.1\end{array}$} \\
\hline 1 & \multicolumn{2}{|c|}{7} & 30 \\
\hline 0.0 & \multicolumn{2}{|c|}{0.0} & 0.0 \\
\hline \multicolumn{4}{|c|}{$\begin{array}{l}\text { Percentage of time streamflow was equaled or exceeded for } \\
\text { the period of record }\end{array}$} \\
\hline $\begin{array}{l}\text { Percentage } \\
\text { of time }\end{array}$ & $\begin{array}{l}\text { Daily mean } \\
\text { streamflow } \\
\left(\mathrm{ft}^{3} / \mathrm{s}\right)\end{array}$ & $\begin{array}{l}\text { Percentage } \\
\text { of time }\end{array}$ & $\begin{array}{l}\text { Daily mean } \\
\text { streamflow } \\
\left(\mathrm{ft}^{3} / \mathrm{s}\right)\end{array}$ \\
\hline 99 & 0.0 & 40 & 6.5 \\
\hline 98 & 0.0 & 30 & 12 \\
\hline 95 & 0.0 & 20 & 21 \\
\hline 90 & 0.1 & 10 & 45 \\
\hline 80 & 0.4 & 5 & 79 \\
\hline 70 & 1.0 & 2 & 153 \\
\hline 60 & 2.0 & 1 & 252 \\
\hline 50 & 3.7 & & \\
\hline
\end{tabular}




\section{WEST FORK BUSSERON CREEK NEAR HYMERA, IN}

Location.- Lat $39^{\circ} 11^{\prime} 10^{\prime \prime}$, long $87^{\circ} 19^{\prime} 44^{\prime \prime}$ referenced to North American Datum of 1927, in NW 1/4 NW 1/4 sec. 32 , T.9 N., R.8 W., Sullivan County, IN, Hydrologic Unit 05120111, on right bank at downstream side of bridge on State Highway 48; 1.4 mi upstream from mouth, 1.5 mi west of Hymera, and 3.7 mi east of U.S. Highway 41.

Drainage area. $-14.4 \mathrm{mi}^{2}$.

Period of record.- October 1966 to September 1986.

Average discharge. $-13.8 \mathrm{ft}^{3} / \mathrm{s}$.

Minimum daily discharge. - No flow at times most years.

Human health (harmonic mean) design flow. $-0.4 \mathrm{ft}^{3} / \mathrm{s}$.

Remarks.- None.

\begin{tabular}{|c|c|c|c|}
\hline \multicolumn{4}{|c|}{ Magnitude and frequency of annual low flow } \\
\hline \multicolumn{4}{|c|}{$\begin{array}{l}\text { Lowest average streamflow, in } \mathrm{ft}^{3} / \mathrm{s} \text {, for indicated period of } \\
\text { consecutive days at an annual nonexceedance probability of } 0.1\end{array}$} \\
\hline 1 & \multicolumn{2}{|c|}{7} & 30 \\
\hline 0.0 & \multicolumn{2}{|c|}{0.0} & 0.0 \\
\hline \multicolumn{4}{|c|}{$\begin{array}{l}\text { Percentage of time streamflow was equaled or exceeded for } \\
\text { the period of record }\end{array}$} \\
\hline $\begin{array}{l}\text { Percentage } \\
\text { of time }\end{array}$ & $\begin{array}{l}\text { Daily mean } \\
\text { streamflow } \\
\left(\mathrm{ft}^{3} / \mathrm{s}\right)\end{array}$ & $\begin{array}{l}\text { Percentage } \\
\text { of time }\end{array}$ & $\begin{array}{l}\text { Daily mean } \\
\text { streamflow } \\
\left(\mathrm{ft}^{3} / \mathrm{s}\right)\end{array}$ \\
\hline 99 & 0.0 & 40 & 4.1 \\
\hline 98 & 0.0 & 30 & 6.8 \\
\hline 95 & 0.1 & 20 & 12 \\
\hline 90 & 0.1 & 10 & 29 \\
\hline 80 & 0.3 & 5 & 60 \\
\hline 70 & 0.8 & 2 & 137 \\
\hline 60 & 1.4 & 1 & 230 \\
\hline 50 & 2.5 & & \\
\hline
\end{tabular}




\section{MUD CREEK NEAR DUGGER, IN}

Location. - Lat $39^{\circ} 06^{\prime} 28^{\prime \prime}$, long $87^{\circ} 16^{\prime} 42^{\prime \prime}$ referenced to North American Datum of 1927, in SE 1/4 NE $1 / 4$ sec.27, T.8 N., R.8 W., Sullivan County, Hydrologic Unit 05120111, on right bank at downstream side of bridge on County Road 700 East, 0.6 mi north of County Road 100 North, 1.7 mi upstream from mouth, and 2.5 mi northwest of Dugger.

Drainage area. $-11.9 \mathrm{mi}^{2}$.

Period of record.- June 1966 to September 1981.

Average discharge. $-14.1 \mathrm{ft}^{3} / \mathrm{s}$.

Minimum daily discharge. $-0.40 \mathrm{ft}^{3} / \mathrm{s}$.

Human health (harmonic mean) design flow. $-3.7 \mathrm{ft}^{3} / \mathrm{s}$.

Remarks. - Flow affected by surface-mined areas.

\begin{tabular}{|c|c|c|c|}
\hline \multicolumn{4}{|c|}{ Magnitude and frequency of annual low flow } \\
\hline \multicolumn{4}{|c|}{$\begin{array}{l}\text { Lowest average streamflow, in } \mathrm{ft}^{3} / \mathrm{s} \text {, for indicated period of } \\
\text { consecutive days at an annual nonexceedance probability of } 0 \text {. }\end{array}$} \\
\hline 1 & \multicolumn{2}{|c|}{7} & 30 \\
\hline 0.4 & \multicolumn{2}{|c|}{0.7} & 0.9 \\
\hline \multicolumn{4}{|c|}{$\begin{array}{l}\text { Percentage of time streamflow was equaled or exceeded for } \\
\text { the period of record }\end{array}$} \\
\hline $\begin{array}{l}\text { Percentage } \\
\text { of time }\end{array}$ & $\begin{array}{l}\text { Daily mean } \\
\text { streamflow } \\
\left(\mathrm{ft}^{3} / \mathrm{s}\right)\end{array}$ & $\begin{array}{l}\text { Percentage } \\
\text { of time }\end{array}$ & $\begin{array}{l}\text { Daily mean } \\
\text { streamflow } \\
\left(\mathrm{ft}^{3} / \mathrm{s}\right)\end{array}$ \\
\hline 99 & 0.7 & 40 & 8.7 \\
\hline 98 & 1.0 & 30 & 12 \\
\hline 95 & 1.3 & 20 & 17 \\
\hline 90 & 1.7 & 10 & 29 \\
\hline 80 & 2.5 & 5 & 50 \\
\hline 70 & 3.5 & 2 & 99 \\
\hline 60 & 4.9 & 1 & 149 \\
\hline 50 & 6.6 & & \\
\hline
\end{tabular}




\section{BUSSERON CREEK NEAR SULLIVAN, IN}

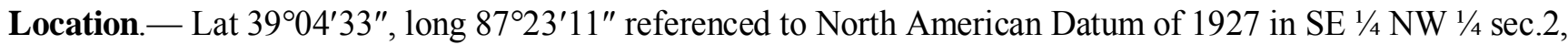
T.7 N., R.9 W., Sullivan County, IN, Hydrologic Unit 05120111, on left bank at upstream side of bridge on State Highway 54, $1.5 \mathrm{mi}$ southeast of Sullivan, $1.6 \mathrm{mi}$ east of intersection of U.S. Highway 41 and State Highway 54, 1.7 mi upstream from Buttermilk Creek, and at mile 16.7.

Drainage area. $-138 \mathrm{mi}^{2}$.

Period of record.- June 1966 to September 1986.

Average discharge. $-154 \mathrm{ft}^{3} / \mathrm{s}$.

Minimum daily discharge. $-0.90 \mathrm{ft}^{3} / \mathrm{s}$.

Human health (harmonic mean) design flow. - $15 \mathrm{ft}^{3} / \mathrm{s}$.

Remarks. - Flow affected by surface-mined areas.

\begin{tabular}{|c|c|c|c|}
\hline \multicolumn{4}{|c|}{ Magnitude and frequency of annual low flow } \\
\hline \multicolumn{4}{|c|}{$\begin{array}{l}\text { Lowest average streamflow, in } \mathrm{ft}^{3} / \mathrm{s} \text {, for indicated period of } \\
\text { consecutive days at an annual nonexceedance probability of } 0.1\end{array}$} \\
\hline 1 & \multicolumn{2}{|c|}{7} & 30 \\
\hline 1.7 & \multicolumn{2}{|c|}{2.2} & 3.1 \\
\hline \multicolumn{4}{|c|}{$\begin{array}{c}\text { Percentage of time streamflow was equaled or exceeded for } \\
\text { the period of record }\end{array}$} \\
\hline $\begin{array}{l}\text { Percentage } \\
\text { of time }\end{array}$ & $\begin{array}{l}\text { Daily mean } \\
\text { streamflow } \\
\left(\mathrm{ft}^{3} / \mathrm{s}\right)\end{array}$ & $\begin{array}{l}\text { Percentage } \\
\text { of time }\end{array}$ & $\begin{array}{l}\text { Daily mean } \\
\text { streamflow } \\
\left(\mathrm{ft}^{3} / \mathrm{s}\right)\end{array}$ \\
\hline 99 & 2.6 & 40 & 67 \\
\hline 98 & 2.9 & 30 & 107 \\
\hline 95 & 4.0 & 20 & 181 \\
\hline 90 & 5.9 & 10 & 389 \\
\hline 80 & 11 & 5 & 717 \\
\hline 70 & 18 & 2 & 1290 \\
\hline 60 & 28 & 1 & 1690 \\
\hline 50 & 43 & & \\
\hline
\end{tabular}




\section{BUSSERON CREEK NEAR CARLISLE, IN}

Location. - Lat $38^{\circ} 58^{\prime} 27^{\prime \prime}$, long $87^{\circ} 25^{\prime} 33^{\prime \prime}$ referenced to North American Datum, in NW 1/4 survey 17, Vincennes Tract, Sullivan County, IN, Hydrologic Unit 05120111, on left downstream side of bridge on State Highway 58, $1.5 \mathrm{mi}$ northwest of Carlisle, and $6.7 \mathrm{mi}$ upstream from mouth.

Drainage area. $-228 \mathrm{mi}^{2}$.

Period of record.- October 1943 to October 2003, February 2007 to current year.

Average discharge. - $239 \mathrm{ft}^{3} / \mathrm{s}$.

Minimum daily discharge. $-0.00 \mathrm{ft}^{3} / \mathrm{s}$.

Human health (harmonic mean) design flow. - $10 \mathrm{ft}^{3} / \mathrm{s}$.

Remarks. - Flow affected by surface-mined areas.

\begin{tabular}{|c|c|c|c|}
\hline \multicolumn{4}{|c|}{ Magnitude and frequency of annual low flow } \\
\hline \multicolumn{4}{|c|}{$\begin{array}{l}\text { Lowest average streamflow, in } \mathrm{ft}^{3} / \mathrm{s} \text {, for indicated period of } \\
\text { consecutive days at an annual nonexceedance probability of } 0.1\end{array}$} \\
\hline 1 & \multicolumn{2}{|c|}{7} & 30 \\
\hline 0.6 & \multicolumn{2}{|c|}{0.9} & 1.4 \\
\hline \multicolumn{4}{|c|}{$\begin{array}{l}\text { Percentage of time streamflow was equaled or exceeded for } \\
\text { the period of record }\end{array}$} \\
\hline $\begin{array}{l}\text { Percentage } \\
\text { of time }\end{array}$ & $\begin{array}{l}\text { Daily mean } \\
\text { streamflow } \\
\left(\mathrm{ft}^{3} / \mathrm{s}\right)\end{array}$ & $\begin{array}{l}\text { Percentage } \\
\text { of time }\end{array}$ & $\begin{array}{c}\text { Daily mean } \\
\text { streamflow } \\
\left(\mathrm{ft}^{3} / \mathrm{s}\right)\end{array}$ \\
\hline 99 & 0.7 & 40 & 93 \\
\hline 98 & 1.4 & 30 & 154 \\
\hline 95 & 3.3 & 20 & 270 \\
\hline 90 & 6.1 & 10 & 668 \\
\hline 80 & 13 & 5 & 1240 \\
\hline 70 & 23 & 2 & 1990 \\
\hline 60 & 37 & 1 & 2680 \\
\hline 50 & 60 & & \\
\hline
\end{tabular}




\section{WABASH RIVER AT VINCENNES, IN}

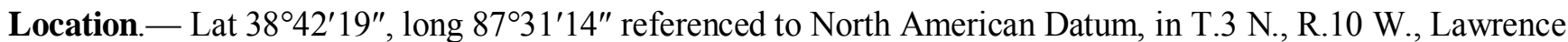
County, IL, Hydrologic Unit 05120111, on right bank $30 \mathrm{ft}$ east of Illinois State Highway 33, $300 \mathrm{ft}$ upstream from Kelso Creek, $570 \mathrm{ft}$ downstream from U.S. Highway 50 bridge, $5.1 \mathrm{mi}$ downstream from Maria Creek, $7.5 \mathrm{mi}$ upstream from Embarras River, and at mile 129.6.

Drainage area. $-13,706 \mathrm{mi}^{2}$.

Period of record. - October 1929 to September 1994.

Average discharge. $-12,370 \mathrm{ft}^{3} / \mathrm{s}$.

Minimum daily discharge. $-770 \mathrm{ft}^{3} / \mathrm{s}$.

Human health (harmonic mean) design flow. $-6,980 \mathrm{ft}^{3} / \mathrm{s}$.

Remarks. - Flow partially regulated by upstream reservoirs. Low-flow statistics are calculated for the regulated period, 1969 to 1994.

\begin{tabular}{cccc}
\hline \multicolumn{4}{c}{ Magnitude and frequency of annual low flow } \\
\hline \multicolumn{4}{c}{1830} \\
Lowest average streamflow, in $\mathrm{ft}^{3} / \mathbf{s}$, for indicated period of \\
consecutive days at an annual nonexceedance probability of 0.1
\end{tabular}




\section{WHITE RIVER AT MUNCIE, IN}

Location.- Lat $40^{\circ} 12^{\prime} 15^{\prime \prime}$, long $85^{\circ} 23^{\prime} 14^{\prime \prime}$ referenced to North American Datum of 1927, in NE 1/4 NE 1/4 Sec.9, T.20 N., R.10 E., Delaware County, IN, Hydrologic Unit 05120201, on right bank $200 \mathrm{ft}$ downstream from Walnut Street bridge in Muncie, 6 mi upstream from Bell Creek, and at mile 315.8.

Drainage area. $-241 \mathrm{mi}^{2}$.

Period of record. - November 1930 to current year. Prior to October 1948, published as West Fork White River at Muncie.

Average discharge. $-232 \mathrm{ft}^{3} / \mathrm{s}$.

Minimum daily discharge. $-1.1 \mathrm{ft}^{3} / \mathrm{s}$.

Human health (harmonic mean) design flow. $-31 \mathrm{ft}^{3} / \mathrm{s}$.

Remarks. - Natural flow affected by regulation of Prairie Creek Reservoir and by diversion of municipal water supply by Muncie Water Works Co. above gage.

\begin{tabular}{cc|cc}
\hline \multicolumn{5}{c}{ Magnitude and frequency of annual low flow } \\
\hline \multicolumn{4}{c}{2.9} \\
Lowest average streamflow, in $\mathrm{ft}^{3} / \mathbf{s}$, for indicated period of \\
consecutive days at an annual nonexceedance probability of 0.1
\end{tabular}




\section{BUCK CREEK NEAR MUNCIE, IN}

Location.- Lat $40^{\circ} 08^{\prime} 05^{\prime \prime}$, long $85^{\circ} 22^{\prime} 25^{\prime \prime}$ referenced to North American Datum of 1927, in SW 1/4 SE $1 / 4$ sec.34, T.20 N., R.10 E., Delaware County, IN, Hydrologic Unit 05120201, on left bank at downstream side of bridge on County Road 400 South, 1.0 mi upstream from Muncie Water Works Co. pumping station, 4.2 mi southeast of Court House in Muncie, and at mile 10.6.

Drainage area. $-35.5 \mathrm{mi}^{2}$.

Period of record.- October 1954 to October 2003.

Average discharge. $-38.5 \mathrm{ft}^{3} / \mathrm{s}$.

Minimum daily discharge. $-4.7 \mathrm{ft}^{3} / \mathrm{s}$.

Human health (harmonic mean) design flow. $-22 \mathrm{ft}^{3} / \mathrm{s}$.

Remarks. - None.

\begin{tabular}{|c|c|c|c|}
\hline \multicolumn{4}{|c|}{ Magnitude and frequency of annual low flow } \\
\hline \multicolumn{4}{|c|}{$\begin{array}{l}\text { Lowest average streamflow, in } \mathrm{ft}^{3} / \mathrm{s} \text {, for indicated period of } \\
\text { consecutive days at an annual nonexceedance probability of } 0 \text {. }\end{array}$} \\
\hline 1 & \multicolumn{2}{|c|}{7} & 30 \\
\hline 6.7 & \multicolumn{2}{|c|}{7.5} & 8.5 \\
\hline \multicolumn{4}{|c|}{$\begin{array}{c}\text { Percentage of time streamflow was equaled or exceeded for } \\
\text { the period of record }\end{array}$} \\
\hline $\begin{array}{l}\text { Percentage } \\
\text { of time }\end{array}$ & $\begin{array}{c}\text { Daily mean } \\
\text { streamflow } \\
\left(\mathrm{ft}^{3} / \mathrm{s}\right)\end{array}$ & $\begin{array}{l}\text { Percentage } \\
\text { of time }\end{array}$ & $\begin{array}{c}\text { Daily mean } \\
\text { streamflow } \\
\left(\mathrm{ft}^{3} / \mathrm{s}\right)\end{array}$ \\
\hline 99 & 8.0 & 40 & 30 \\
\hline 98 & 8.6 & 30 & 36 \\
\hline 95 & 10 & 20 & 45 \\
\hline 90 & 12 & 10 & 68 \\
\hline 80 & 16 & 5 & 108 \\
\hline 70 & 19 & 2 & 193 \\
\hline 60 & 22 & 1 & 272 \\
\hline 50 & 25 & & \\
\hline
\end{tabular}




\section{WHITE RIVER AT ANDERSON, IN}

Location.- Lat $40^{\circ} 06^{\prime} 20^{\prime \prime}$, long $85^{\circ} 40^{\prime} 16^{\prime \prime}$ referenced to North American Datum of 1927, in NW 1/4 NW 1/4 sec.18, T.19 N., R.8 E., Madison County, IN, Hydrologic Unit 05120201, on downstream side of abandoned Twelfth Street bridge abutment, $250 \mathrm{ft}$ upstream from municipal water-supply plant in Anderson, 1 mi upstream from Killbuck Creek, and at mile 293.3.

Drainage area. $-406 \mathrm{mi}^{2}$.

Period of record. - October 1931 to September 1993.

Average discharge. $-394 \mathrm{ft}^{3} / \mathrm{s}$.

Minimum daily discharge. $-9.1 \mathrm{ft}^{3} / \mathrm{s}$.

Human health (harmonic mean) design flow. - $135 \mathrm{ft}^{3} / \mathrm{s}$.

Remarks.- Prior to Sept. 15, 1973, the City of Anderson diverted water for its municipal supply above the gage then in use.

\begin{tabular}{|c|c|c|c|}
\hline \multicolumn{4}{|c|}{ Magnitude and frequency of annual low flow } \\
\hline \multicolumn{4}{|c|}{$\begin{array}{l}\text { Lowest average streamflow, in } \mathrm{ft}^{3} / \mathrm{s} \text {, for indicated period of } \\
\text { consecutive days at an annual nonexceedance probability of } 0.1\end{array}$} \\
\hline 1 & \multicolumn{2}{|c|}{7} & 30 \\
\hline 25 & \multicolumn{2}{|c|}{29} & 36 \\
\hline \multicolumn{4}{|c|}{$\begin{array}{c}\text { Percentage of time streamflow was equaled or exceeded for } \\
\text { the period of record }\end{array}$} \\
\hline $\begin{array}{l}\text { Percentage } \\
\text { of time }\end{array}$ & $\begin{array}{l}\text { Daily mean } \\
\text { streamflow } \\
\left(\mathrm{ft}^{3} / \mathrm{s}\right)\end{array}$ & $\begin{array}{l}\text { Percentage } \\
\text { of time }\end{array}$ & $\begin{array}{l}\text { Daily mean } \\
\text { streamflow } \\
\left(\mathrm{ft}^{3} / \mathrm{s}\right)\end{array}$ \\
\hline 99 & 30 & 40 & 246 \\
\hline 98 & 38 & 30 & 332 \\
\hline 95 & 50 & 20 & 492 \\
\hline 90 & 64 & 10 & 917 \\
\hline 80 & 87 & 5 & 1520 \\
\hline 70 & 112 & 2 & 2720 \\
\hline 60 & 145 & 1 & 3840 \\
\hline 50 & 187 & & \\
\hline
\end{tabular}




\section{KILLBUCK CREEK NEAR GASTON, IN}

Location.- Lat $40^{\circ} 15^{\prime} 45^{\prime \prime}$, long $85^{\circ} 30^{\prime} 53^{\prime \prime}$ referenced to North American Datum of 1927, in SE 1/4 SW 1/4 Sec.16, T.21 N., R.9 E., Delaware County, IN, Hydrologic Unit 05120201, on right bank $30 \mathrm{ft}$ upstream from bridge on County Road 500 North, 3.6 mi southwest of Gaston, and at mile 15.6.

Drainage area. $-25.5 \mathrm{mi}^{2}$.

Period of record.- June 1968 to September 1991.

Average discharge. $-26.4 \mathrm{ft}^{3} / \mathrm{s}$.

Minimum daily discharge. $-0.76 \mathrm{ft}^{3} / \mathrm{s}$.

Human health (harmonic mean) design flow. $-6.9 \mathrm{ft}^{3} / \mathrm{s}$.

Remarks.- None.

\begin{tabular}{|c|c|c|c|}
\hline \multicolumn{4}{|c|}{ Magnitude and frequency of annual low flow } \\
\hline \multicolumn{4}{|c|}{$\begin{array}{l}\text { Lowest average streamflow, in } \mathrm{ft}^{3} / \mathrm{s} \text {, for indicated period of } \\
\text { consecutive days at an annual nonexceedance probability of } 0.1\end{array}$} \\
\hline 1 & \multicolumn{2}{|c|}{7} & 30 \\
\hline 1.0 & \multicolumn{2}{|c|}{1.1} & 1.3 \\
\hline \multicolumn{4}{|c|}{$\begin{array}{l}\text { Percentage of time streamflow was equaled or exceeded for } \\
\text { the period of record }\end{array}$} \\
\hline $\begin{array}{l}\text { Percentage } \\
\text { of time }\end{array}$ & $\begin{array}{l}\text { Daily mean } \\
\text { streamflow } \\
\left(\mathrm{ft}^{3} / \mathrm{s}\right)\end{array}$ & $\begin{array}{l}\text { Percentage } \\
\text { of time }\end{array}$ & $\begin{array}{l}\text { Daily mean } \\
\text { streamflow } \\
\left(\mathrm{ft}^{3} / \mathrm{s}\right)\end{array}$ \\
\hline 99 & 1.2 & 40 & 16 \\
\hline 98 & 1.5 & 30 & 22 \\
\hline 95 & 2.0 & 20 & 33 \\
\hline 90 & 3.0 & 10 & 59 \\
\hline 80 & 4.7 & 5 & 100 \\
\hline 70 & 6.4 & 2 & 179 \\
\hline 60 & 8.7 & 1 & 253 \\
\hline 50 & 12 & & \\
\hline
\end{tabular}




\section{WHITE RIVER AT RAIBLE AVENUE AT ANDERSON, IN}

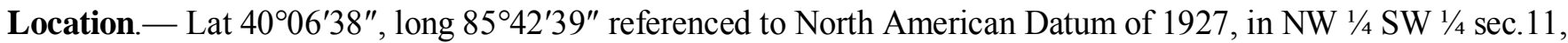
T.19 N., R.7 E., Madison County, IN, Hydrologic Unit 05120201, on the upstream side of bridge in southeast quadrant of Raible Avenue and White River, $0.3 \mathrm{mi}$ upstream of waste-water treatment plant, 2 mi downstream of Killbuck Creek, and 3.0 mi downstream of the municipal power plant in Anderson.

Drainage area. $-519 \mathrm{mi}^{2}$.

Period of record. - September 1999 to current year.

Average discharge. $-660 \mathrm{ft}^{3} / \mathrm{s}$.

Minimum daily discharge. $-62 \mathrm{ft}^{3} / \mathrm{s}$.

Human health (harmonic mean) design flow. $-253 \mathrm{ft}^{3} / \mathrm{s}$.

Remarks. - Flow may be affected at times by upstream regulation.

\begin{tabular}{|c|c|c|c|}
\hline \multicolumn{4}{|c|}{ Magnitude and frequency of annual low flow } \\
\hline \multicolumn{4}{|c|}{$\begin{array}{l}\text { Lowest average streamflow, in } \mathrm{ft}^{3} / \mathrm{s} \text {, for indicated period of } \\
\text { consecutive days at an annual nonexceedance probability of } 0 \text {. }\end{array}$} \\
\hline 1 & \multicolumn{2}{|c|}{7} & 30 \\
\hline 69 & \multicolumn{2}{|c|}{72} & 80 \\
\hline \multicolumn{4}{|c|}{$\begin{array}{l}\text { Percentage of time streamflow was equaled or exceeded for } \\
\text { the period of record }\end{array}$} \\
\hline $\begin{array}{l}\text { Percentage } \\
\text { of time }\end{array}$ & $\begin{array}{l}\text { Daily mean } \\
\text { streamflow } \\
\left(\mathrm{ft}^{3} / \mathrm{s}\right)\end{array}$ & $\begin{array}{l}\text { Percentage } \\
\text { of time }\end{array}$ & $\begin{array}{l}\text { Daily mean } \\
\text { streamflow } \\
\left(\mathrm{ft}^{3} / \mathrm{s}\right)\end{array}$ \\
\hline 99 & 78 & 40 & 433 \\
\hline 98 & 85 & 30 & 558 \\
\hline 95 & 96 & 20 & 781 \\
\hline 90 & 114 & 10 & 1330 \\
\hline 80 & 154 & 5 & 2240 \\
\hline 70 & 202 & 2 & 4160 \\
\hline 60 & 262 & 1 & 6360 \\
\hline 50 & 338 & & \\
\hline
\end{tabular}




\section{PIPE CREEK AT FRANKTON, IN}

Location. - Lat 40 $13^{\prime} 38^{\prime \prime}$, long $85^{\circ} 45^{\prime} 58^{\prime \prime}$ referenced to North American Datum of 1927, in SE 1/4 NE 1/4 sec.31, T.21 N., R.7 E., Madison County, IN, Hydrologic Unit 05120201, on right bank $20 \mathrm{ft}$ downstream from bridge on County Road 500 West, at northeast edge of Frankton, 1.88 mi downstream of Plummer Brook mouth, and at mile 10.35 .

Drainage area.- $113 \mathrm{mi}^{2}$.

Period of record.- May 1968 to October 2003.

Average discharge. $-109 \mathrm{ft}^{3} / \mathrm{s}$.

Minimum daily discharge. $-2.0 \mathrm{ft}^{3} / \mathrm{s}$.

Human health (harmonic mean) design flow. $-23 \mathrm{ft}^{3} / \mathrm{s}$.

Remarks.- None.

\begin{tabular}{|c|c|c|c|}
\hline \multicolumn{4}{|c|}{ Magnitude and frequency of annual low flow } \\
\hline \multicolumn{4}{|c|}{$\begin{array}{l}\text { Lowest average streamflow, in } \mathrm{ft}^{3} / \mathrm{s} \text {, for indicated period of } \\
\text { consecutive days at an annual nonexceedance probability of } 0.1\end{array}$} \\
\hline 1 & \multicolumn{2}{|c|}{7} & 30 \\
\hline 3.6 & \multicolumn{2}{|c|}{4.0} & 4.9 \\
\hline \multicolumn{4}{|c|}{$\begin{array}{l}\text { Percentage of time streamflow was equaled or exceeded for } \\
\text { the period of record }\end{array}$} \\
\hline $\begin{array}{l}\text { Percentage } \\
\text { of time }\end{array}$ & $\begin{array}{l}\text { Daily mean } \\
\text { streamflow } \\
\left(\mathrm{ft}^{3} / \mathrm{s}\right)\end{array}$ & $\begin{array}{l}\text { Percentage } \\
\text { of time }\end{array}$ & $\begin{array}{c}\text { Daily mean } \\
\text { streamflow } \\
\left(\mathrm{ft}^{3} / \mathrm{s}\right)\end{array}$ \\
\hline 99 & 4.3 & 40 & 56 \\
\hline 98 & 5.5 & 30 & 79 \\
\hline 95 & 7.2 & 20 & 124 \\
\hline 90 & 9.2 & 10 & 251 \\
\hline 80 & 15 & 5 & 463 \\
\hline 70 & 21 & 2 & 847 \\
\hline 60 & 30 & 1 & 1170 \\
\hline 50 & 41 & & \\
\hline
\end{tabular}




\section{WHITE RIVER NEAR NOBLESVILLE, IN}

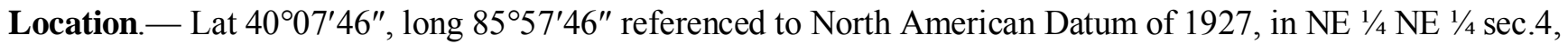
T.19 N., R.5 E., Hamilton County, IN, Hydrologic Unit 05120201, near center of span on downstream side of highway bridge, $1 \mathrm{mi}$ west of Strawtown, $7 \mathrm{mi}$ northeast of Noblesville, $9.5 \mathrm{mi}$ upstream from Cicero Creek, and at mile 277.4.

Drainage area. $-828 \mathrm{mi}^{2}$.

Period of record.- May 1915 to September 1926, October 1928 to September 1974.

Average discharge. $-794 \mathrm{ft}^{3} / \mathrm{s}$.

Minimum daily discharge. $-39 \mathrm{ft}^{3} / \mathrm{s}$.

Human health (harmonic mean) design flow. $-252 \mathrm{ft}^{3} / \mathrm{s}$.

Remarks. - None.

\begin{tabular}{|c|c|c|c|}
\hline \multicolumn{4}{|c|}{ Magnitude and frequency of annual low flow } \\
\hline \multicolumn{4}{|c|}{$\begin{array}{l}\text { Lowest average streamflow, in } \mathrm{ft}^{3} / \mathrm{s} \text {, for indicated period of } \\
\text { consecutive days at an annual nonexceedance probability of } 0.1\end{array}$} \\
\hline 1 & \multicolumn{2}{|c|}{7} & 30 \\
\hline 60 & \multicolumn{2}{|c|}{66} & 76 \\
\hline \multicolumn{4}{|c|}{$\begin{array}{l}\text { Percentage of time streamflow was equaled or exceeded for } \\
\text { the period of record }\end{array}$} \\
\hline $\begin{array}{l}\text { Percentage } \\
\text { of time }\end{array}$ & $\begin{array}{c}\text { Daily mean } \\
\text { streamflow } \\
\left(\mathrm{ft}^{3} / \mathrm{s}\right)\end{array}$ & $\begin{array}{l}\text { Percentage } \\
\text { of time }\end{array}$ & $\begin{array}{c}\text { Daily mean } \\
\text { streamflow } \\
\left(\mathrm{ft}^{3} / \mathrm{s}\right)\end{array}$ \\
\hline 99 & 67 & 40 & 470 \\
\hline 98 & 77 & 30 & 659 \\
\hline 95 & 94 & 20 & 993 \\
\hline 90 & 114 & 10 & 1820 \\
\hline 80 & 147 & 5 & 2980 \\
\hline 70 & 192 & 2 & 5260 \\
\hline 60 & 255 & 1 & 7550 \\
\hline 50 & 350 & & \\
\hline
\end{tabular}




\section{WHITE RIVER AT NOBLESVILLE, IN}

Location.- Lat $40^{\circ} 02^{\prime} 49^{\prime \prime}$, long $86^{\circ} 01^{\prime} 02^{\prime \prime}$ referenced to North American Datum of 1927 , in SE $1 / 4$ SE $1 / 4 \sec .36$, T.19 N., R.4 E., Hamilton County, IN, Hydrologic Unit 05120201, on right bank at downstream side of Logan Street bridge in Noblesville, $1.5 \mathrm{mi}$ upstream from Cicero Creek, $5.1 \mathrm{mi}$ downstream from dam at Clare, and at mile 263.5.

Drainage area. $-858 \mathrm{mi}^{2}$.

Period of record. - October 1946 to current year.

Average discharge. $-906 \mathrm{ft}^{3} / \mathrm{s}$.

Minimum daily discharge. $-44 \mathrm{ft}^{3} / \mathrm{s}$.

Human health (harmonic mean) design flow. $-316 \mathrm{ft}^{3} / \mathrm{s}$.

Remarks. - Flow partially regulated by the power plant above the station.

\begin{tabular}{ccc}
\hline \multicolumn{3}{c}{ Magnitude and frequency of annual low flow } \\
\hline $\begin{array}{c}\text { Lowest average streamflow, in } \mathrm{ft}^{3} / \mathrm{s} \text {, for indicated period of } \\
\text { consecutive days at an annual nonexceedance probability of } \mathbf{0 . 1}\end{array}$ \\
\hline $\mathbf{1}$ & $\mathbf{3 0}$ \\
\hline 70 & 82 & 96 \\
\hline
\end{tabular}

Percentage of time streamflow was equaled or exceeded for the period of record

\begin{tabular}{cc|cc}
\hline $\begin{array}{c}\text { Percentage } \\
\text { of time }\end{array}$ & $\begin{array}{c}\text { Daily mean } \\
\text { streamflow } \\
\left(\mathrm{ft}^{3} / \mathbf{s}\right)\end{array}$ & $\begin{array}{c}\text { Percentage } \\
\text { of time }\end{array}$ & $\begin{array}{c}\text { Daily mean } \\
\text { streamflow } \\
\left(\mathrm{ft}^{3} / \mathbf{s}\right)\end{array}$ \\
\hline 99 & 90 & 40 & 571 \\
\hline 98 & 100 & 30 & 780 \\
\hline 95 & 118 & 20 & 1140 \\
\hline 90 & 142 & 10 & 2050 \\
\hline 80 & 187 & 5 & 3410 \\
\hline 70 & 248 & 2 & 5590 \\
\hline 60 & 327 & 1 & 7710 \\
\hline 50 & 430 & & \\
\hline
\end{tabular}


White River Basin

\section{CICERO CREEK AT TIPTON, IN}

Location. - Lat $40^{\circ} 16^{\prime} 37^{\prime \prime}$, long $86^{\circ} 02^{\prime} 33^{\prime \prime}$, referenced to North American Datum of 1927, in SW 1/4 SE 1/4 sec.11, T.21 N., R.4 E., Tipton County, IN, Hydrologic Unit 05120201, on left downstream northeast corner of brick building, $400 \mathrm{ft}$ west of S.R. 19 bridge, $0.3 \mathrm{mi}$ south southwest of courthouse, and at mile 27.9.

Drainage area. $-81.3 \mathrm{mi}^{2}$.

Period of record. - October 2007 to current year.

Average discharge. $-111 \mathrm{ft}^{3} / \mathrm{s}$.

Minimum daily discharge. $-0.06 \mathrm{ft}^{3} / \mathrm{s}$.

Human health (harmonic mean) design flow. $-3.6 \mathrm{ft}^{3} / \mathrm{s}$.

Remarks. - This site has less than 10 years of record.

\begin{tabular}{|c|c|c|c|}
\hline \multicolumn{4}{|c|}{ Magnitude and frequency of annual low flow } \\
\hline \multicolumn{4}{|c|}{$\begin{array}{l}\text { Lowest average streamflow, in } \mathrm{ft}^{3} / \mathrm{s} \text {, for indicated period of } \\
\text { consecutive days at an annual nonexceedance probability of } 0.1\end{array}$} \\
\hline 1 & \multicolumn{2}{|c|}{7} & 30 \\
\hline 0.4 & \multicolumn{2}{|c|}{0.5} & 0.8 \\
\hline \multicolumn{4}{|c|}{$\begin{array}{l}\text { Percentage of time streamflow was equaled or exceeded for } \\
\text { the period of record }\end{array}$} \\
\hline $\begin{array}{l}\text { Percentage } \\
\text { of time }\end{array}$ & $\begin{array}{l}\text { Daily mean } \\
\text { streamflow } \\
\left(\mathrm{ft}^{3} / \mathrm{s}\right)\end{array}$ & $\begin{array}{l}\text { Percentage } \\
\text { of time }\end{array}$ & $\begin{array}{l}\text { Daily mean } \\
\text { streamflow } \\
\left(\mathrm{ft}^{3} / \mathrm{s}\right)\end{array}$ \\
\hline 99 & 0.5 & 40 & 50 \\
\hline 98 & 0.6 & 30 & 74 \\
\hline 95 & 0.9 & 20 & 136 \\
\hline 90 & 1.4 & 10 & 304 \\
\hline 80 & 3.0 & 5 & 508 \\
\hline 70 & 7.6 & 2 & 888 \\
\hline 60 & 20 & 1 & 1210 \\
\hline 50 & 34 & & \\
\hline
\end{tabular}




\section{CICERO CREEK NEAR ARCADIA, IN}

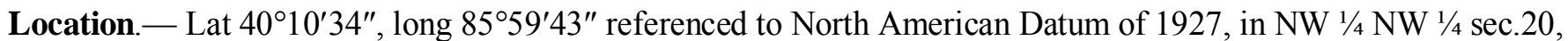
T.20 N., R.5 E., Hamilton County, IN, Hydrologic Unit 05120201, on left bank at downstream side of bridge, $1.5 \mathrm{mi}$ east of Arcadia, $12.5 \mathrm{mi}$ upstream from Morse Dam, and at mile 17.2.

Drainage area. $-131 \mathrm{mi}^{2}$.

Period of record.- October 1954 to September 1976.

Average discharge. $-122 \mathrm{ft}^{3} / \mathrm{s}$.

Minimum daily discharge. $-0.50 \mathrm{ft}^{3} / \mathrm{s}$.

Human health (harmonic mean) design flow. - $10 \mathrm{ft}^{3} / \mathrm{s}$.

Remarks. - None.

\begin{tabular}{ccc}
\hline \multicolumn{3}{c}{ Magnitude and frequency of annual low flow } \\
\hline $\begin{array}{c}\text { Lowest average streamflow, in } \mathrm{ft}^{3} / \mathrm{s} \text {, for indicated period of } \\
\text { consecutive days at an annual nonexceedance probability of } 0.1\end{array}$ \\
\hline $\mathbf{7}$ & $\mathbf{3 0}$ \\
\hline 0.8 & 1.1 & 1.5 \\
\hline
\end{tabular}

Percentage of time streamflow was equaled or exceeded for the period of record

\begin{tabular}{cc|cc}
\hline $\begin{array}{c}\text { Percentage } \\
\text { of time }\end{array}$ & $\begin{array}{c}\text { Daily mean } \\
\text { streamflow } \\
\left(\mathrm{ft}^{3} / \mathbf{s}\right)\end{array}$ & $\begin{array}{c}\text { Percentage } \\
\text { of time }\end{array}$ & $\begin{array}{c}\text { Daily mean } \\
\text { streamflow } \\
\left(\mathrm{ft}^{3} / \mathbf{s}\right)\end{array}$ \\
\hline 99 & 1.2 & 40 & 61 \\
\hline 98 & 1.6 & 30 & 94 \\
\hline 95 & 2.1 & 20 & 153 \\
\hline 90 & 3.4 & 10 & 309 \\
\hline 80 & 7.7 & 5 & 548 \\
\hline 70 & 15 & 2 & 904 \\
\hline 60 & 25 & 1 & 1270 \\
\hline 50 & 40 & & \\
\hline
\end{tabular}




\section{CICERO CREEK AT ARCADIA, IN}

Location. - Lat $40^{\circ} 10^{\prime} 28^{\prime \prime}$, long $86^{\circ} 00^{\prime} 02^{\prime \prime}$ referenced to North American Datum of 1927, in NW 1/4 NE 1/4 sec.19, T.20 N., R.5 E., Hamilton County, IN, Hydrologic Unit 05120201, affixed to the southeast abutment of the Mt.

Pleasant Road bridge over Cicero Creek, $600 \mathrm{ft}$ south of 266th Street, $3500 \mathrm{ft}$ east of State Road 19, 0.9 mi north of 256th Street, and 1.2 mile west of Fall Road.

Drainage area. $-131 \mathrm{mi}^{2}$.

Period of record. - July 12, 2004, to current year.

Average discharge. $-167 \mathrm{ft}^{3} / \mathrm{s}$.

Minimum daily discharge. $-0.73 \mathrm{ft}^{3} / \mathrm{s}$.

Human health (harmonic mean) design flow. $-12 \mathrm{ft}^{3} / \mathrm{s}$.

Remarks. - This site has less than10 years of record.

\begin{tabular}{c}
\hline \multicolumn{4}{c}{ Magnitude and frequency of annual low flow } \\
\hline \multicolumn{4}{c}{ Lowest average streamflow, in $\mathrm{ft}^{3}$ /s, for indicated period of } \\
consecutive days at an annual nonexceedance probability of 0.1
\end{tabular}




\section{LITTLE CICERO CREEK NEAR ARCADIA, IN}

Location. - Lat $40^{\circ} 10^{\prime} 32^{\prime \prime}$, long $86^{\circ} 02^{\prime} 45^{\prime \prime}$ referenced to North American Datum of 1927, in NE 1/4 NW 1/4 sec.23, T.20 N., R.4 E., Hamilton County, IN, Hydrologic Unit 05120201, on left bank at downstream side of county road bridge, $0.5 \mathrm{mi}$ downstream from Taylor Creek, $1.3 \mathrm{mi}$ west of Arcadia, $3.9 \mathrm{mi}$ upstream from mouth, and $9.3 \mathrm{mi}$ northwest of Noblesville.

Drainage area. $-40.4 \mathrm{mi}^{2}$.

Period of record. - October 1955 to September 1976.

Average discharge. $-39.7 \mathrm{ft}^{3} / \mathrm{s}$.

Minimum daily discharge. - No flow at times during many years.

Human health (harmonic mean) design flow. $-2 \mathrm{ft}^{3} / \mathrm{s}$.

Remarks. - None.

\begin{tabular}{|c|c|c|c|}
\hline \multicolumn{4}{|c|}{ Magnitude and frequency of annual low flow } \\
\hline \multicolumn{4}{|c|}{$\begin{array}{l}\text { Lowest average streamflow, in } \mathrm{ft}^{3} / \mathrm{s} \text {, for indicated period of } \\
\text { consecutive days at an annual nonexceedance probability of } 0.1\end{array}$} \\
\hline 1 & \multicolumn{2}{|c|}{7} & 30 \\
\hline 0.0 & \multicolumn{2}{|c|}{0.0} & 0.0 \\
\hline \multicolumn{4}{|c|}{$\begin{array}{l}\text { Percentage of time streamflow was equaled or exceeded for } \\
\text { the period of record }\end{array}$} \\
\hline $\begin{array}{l}\text { Percentage } \\
\text { of time }\end{array}$ & $\begin{array}{l}\text { Daily mean } \\
\text { streamflow } \\
\left(\mathrm{ft}^{3} / \mathrm{s}\right)\end{array}$ & $\begin{array}{l}\text { Percentage } \\
\text { of time }\end{array}$ & $\begin{array}{l}\text { Daily mean } \\
\text { streamflow } \\
\left(\mathrm{ft}^{3} / \mathrm{s}\right)\end{array}$ \\
\hline 99 & 0.0 & 40 & 18 \\
\hline 98 & 0.0 & 30 & 28 \\
\hline 95 & 0.3 & 20 & 50 \\
\hline 90 & 0.8 & 10 & 97 \\
\hline 80 & 1.8 & 5 & 172 \\
\hline 70 & 3.5 & 2 & 320 \\
\hline 60 & 6.8 & 1 & 466 \\
\hline 50 & 11 & & \\
\hline
\end{tabular}




\section{HINKLE CREEK NEAR CICERO, IN}

Location. - Lat $40^{\circ} 06^{\prime} 05^{\prime \prime}$, long $86^{\circ} 05^{\prime} 10^{\prime \prime}$ referenced to North American Datum of 1927, in NW 1/4 NW 1/4 sec.16, T.19 N., R.4 E., Hamilton County, IN, Hydrologic Unit 05120201, on left bank at downstream side of county road bridge, $3.7 \mathrm{mi}$ above mouth, $4.0 \mathrm{mi}$ upstream from Morse Reservoir Dam, $4.2 \mathrm{mi}$ southwest of Cicero, and $5.7 \mathrm{mi}$ northwest of Noblesville.

Drainage area.- $18.5 \mathrm{mi}^{2}$.

Period of record. - October 1955 to September 1976.

Average discharge. $-19.6 \mathrm{ft}^{3} / \mathrm{s}$.

Minimum daily discharge. $-0.07 \mathrm{ft}^{3} / \mathrm{s}$.

Human health (harmonic mean) design flow. $-2.2 \mathrm{ft}^{3} / \mathrm{s}$.

Remarks.- None.

\begin{tabular}{|c|c|c|c|}
\hline \multicolumn{4}{|c|}{ Magnitude and frequency of annual low flow } \\
\hline \multicolumn{4}{|c|}{$\begin{array}{l}\text { Lowest average streamflow, in } \mathrm{ft}^{3} / \mathrm{s} \text {, for indicated period of } \\
\text { consecutive days at an annual nonexceedance probability of } 0.1\end{array}$} \\
\hline 1 & \multicolumn{2}{|c|}{7} & 30 \\
\hline 0.1 & \multicolumn{2}{|c|}{0.2} & 0.3 \\
\hline \multicolumn{4}{|c|}{$\begin{array}{c}\text { Percentage of time streamflow was equaled or exceeded for } \\
\text { the period of record }\end{array}$} \\
\hline $\begin{array}{l}\text { Percentage } \\
\text { of time }\end{array}$ & $\begin{array}{l}\text { Daily mean } \\
\text { streamflow } \\
\left(\mathrm{ft}^{3} / \mathrm{s}\right)\end{array}$ & $\begin{array}{l}\text { Percentage } \\
\text { of time }\end{array}$ & $\begin{array}{c}\text { Daily mean } \\
\text { streamflow } \\
\left(\mathrm{ft}^{3} / \mathrm{s}\right)\end{array}$ \\
\hline 99 & 0.2 & 40 & 9.5 \\
\hline 98 & 0.4 & 30 & 14 \\
\hline 95 & 0.6 & 20 & 21 \\
\hline 90 & 0.9 & 10 & 43 \\
\hline 80 & 1.5 & 5 & 74 \\
\hline 70 & 2.5 & 2 & 146 \\
\hline 60 & 4.1 & 1 & 250 \\
\hline 50 & 6.4 & & \\
\hline
\end{tabular}




\section{CICERO CREEK AT NOBLESVILLE, IN}

Location. - Lat $40^{\circ} 03^{\prime} 20^{\prime \prime}$, long $86^{\circ} 02^{\prime} 30^{\prime \prime}$ referenced to North American Datum of 1927, in NW 1/4 NE $1 / 4$ sec.35, T.19 N., R.4 E., Hamilton County, IN, Hydrologic Unit 05120201, on right bank $150 \mathrm{ft}$ downstream from bridge on Stage Highway 38, $1.0 \mathrm{mi}$ northwest of Noblesville, $1.6 \mathrm{mi}$ downstream from Morse Reservoir, 1.9 mi downstream from Hinkle Creek, and 3.2 mi upstream from mouth.

Drainage area. $-216 \mathrm{mi}^{2}$.

Period of record.- July 1950 to September 1980 and October 1985 to September 1992.

Average discharge. $-197 \mathrm{ft}^{3} / \mathrm{s}$.

Minimum daily discharge. $-0.25 \mathrm{ft}^{3} / \mathrm{s}$.

Human health (harmonic mean) design flow. $-9.5 \mathrm{ft}^{3} / \mathrm{s}$.

Remarks. - Flow regulated by Morse Reservoir. Low-flow statistics are calculated for the regulated period 1958 to 1980, and 1985 to 1992.

\begin{tabular}{|c|c|c|c|}
\hline \multicolumn{4}{|c|}{ Magnitude and frequency of annual low flow } \\
\hline \multicolumn{4}{|c|}{$\begin{array}{l}\text { Lowest average streamflow, in } \mathrm{ft}^{3} / \mathrm{s} \text {, for indicated period of } \\
\text { consecutive days at an annual nonexceedance probability of } 0.1\end{array}$} \\
\hline 1 & \multicolumn{2}{|c|}{7} & 30 \\
\hline 0.6 & \multicolumn{2}{|c|}{0.8} & 1.1 \\
\hline \multicolumn{4}{|c|}{$\begin{array}{l}\text { Percentage of time streamflow was equaled or exceeded for } \\
\text { the period of record }\end{array}$} \\
\hline $\begin{array}{l}\text { Percentage } \\
\text { of time }\end{array}$ & $\begin{array}{c}\text { Daily mean } \\
\text { streamflow } \\
\left(\mathrm{ft}^{3} / \mathrm{s}\right)\end{array}$ & $\begin{array}{l}\text { Percentage } \\
\text { of time }\end{array}$ & $\begin{array}{c}\text { Daily mean } \\
\text { streamflow } \\
\left(\mathrm{ft}^{3} / \mathrm{s}\right)\end{array}$ \\
\hline 99 & 0.9 & 40 & 105 \\
\hline 98 & 1.1 & 30 & 153 \\
\hline 95 & 1.7 & 20 & 241 \\
\hline 90 & 2.6 & 10 & 479 \\
\hline 80 & 11 & 5 & 848 \\
\hline 70 & 26 & 2 & 1490 \\
\hline 60 & 46 & 1 & 2090 \\
\hline 50 & 72 & & \\
\hline
\end{tabular}


White River Basin

\section{STONY CREEK AT FISHERSBURG, IN}

Location. - Lat $40^{\circ} 03^{\prime} 58^{\prime \prime}$, long 85 $51^{\prime} 47^{\prime \prime}$ referenced to North American Datum of 1927, in NE 1/4 SE 1/4 Sec.29, T.19 N., R.6 E., Hamilton County, IN, Hydrologic Unit 05120201, on middle of bridge on CR 1000W, 1 mi from Lapel, 5.8 mi northwest of I-69, and at river mile 11.71

Drainage area. $-17.5 \mathrm{mi}^{2}$.

Period of record. - October 2008 to current year.

Average discharge. $-21.7 \mathrm{ft}^{3} / \mathrm{s}$.

Minimum daily discharge. $-0.27 \mathrm{ft}^{3} / \mathrm{s}$.

Human health (harmonic mean) design flow. $-3.0 \mathrm{ft}^{3} / \mathrm{s}$.

Remarks. - This site has less than10 years of record.

\begin{tabular}{ccc}
\hline \multicolumn{3}{c}{ Magnitude and frequency of annual low flow } \\
\hline $\begin{array}{c}\text { Lowest average streamflow, in } \mathrm{ft}^{3} / \mathrm{s} \text {, for indicated period of } \\
\text { consecutive days at an annual nonexceedance probability of } \mathbf{0 . 1}\end{array}$ \\
\hline $\mathbf{1}$ & $\mathbf{7}$ & $\mathbf{3 0}$ \\
\hline 0.1 & 0.1 & 0.2 \\
\hline
\end{tabular}

Percentage of time streamflow was equaled or exceeded for the period of record

\begin{tabular}{cc|cc}
\hline $\begin{array}{c}\text { Percentage } \\
\text { of time }\end{array}$ & $\begin{array}{c}\text { Daily mean } \\
\text { streamflow } \\
\left(\mathrm{ft}^{3} / \mathbf{s}\right)\end{array}$ & $\begin{array}{c}\text { Percentage } \\
\text { of time }\end{array}$ & $\begin{array}{c}\text { Daily mean } \\
\text { streamflow } \\
\left(\mathrm{ft}^{3} / \mathbf{s}\right)\end{array}$ \\
\hline 99 & 0.3 & 40 & 12 \\
\hline 98 & 0.6 & 30 & 16 \\
\hline 95 & 0.8 & 20 & 26 \\
\hline 90 & 1.0 & 10 & 50 \\
\hline 80 & 2.4 & 5 & 86 \\
\hline 70 & 3.6 & 2 & 151 \\
\hline 60 & 5.6 & 1 & 219 \\
\hline 50 & 8.5 & & \\
\hline
\end{tabular}




\section{WILLIAM LOCK DITCH NEAR DURBIN, IN}

Location. - Lat $40^{\circ} 04^{\prime} 25^{\prime \prime}$, long $85^{\circ} 55^{\prime} 30^{\prime \prime}$ referenced to North American Datum of 1927, in SW 1/4 SE 1/4 SE 1/4 sec.23, T.19 N., R.5 E., Hamilton County, IN, Hydrologic Unit 05120201, on the downstream side middle of two concrete culverts at on Mystic Road, $2.4 \mathrm{mi}$ from 196th St., $8.8 \mathrm{mi}$ from Highway 37, and at mile 0.35.

Drainage area. $-11.0 \mathrm{mi}^{2}$.

Period of record. - September 2008 to current year.

Average discharge. - $16.4 \mathrm{ft}^{3} / \mathrm{s}$.

Minimum daily discharge. $-0.27 \mathrm{ft}^{3} / \mathrm{s}$.

Human health (harmonic mean) design flow. $-5.1 \mathrm{ft}^{3} / \mathrm{s}$.

Remarks. - This site has less than10 years of record.

\begin{tabular}{|c|c|c|c|}
\hline \multicolumn{4}{|c|}{ Magnitude and frequency of annual low flow } \\
\hline \multicolumn{4}{|c|}{$\begin{array}{l}\text { Lowest average streamflow, in } \mathrm{ft}^{3} / \mathrm{s} \text {, for indicated period of } \\
\text { consecutive days at an annual nonexceedance probability of } 0.1\end{array}$} \\
\hline 1 & \multicolumn{2}{|c|}{7} & 30 \\
\hline 0.3 & \multicolumn{2}{|c|}{0.4} & 0.8 \\
\hline \multicolumn{4}{|c|}{$\begin{array}{l}\text { Percentage of time streamflow was equaled or exceeded for } \\
\text { the period of record }\end{array}$} \\
\hline $\begin{array}{l}\text { Percentage } \\
\text { of time }\end{array}$ & $\begin{array}{l}\text { Daily mean } \\
\text { streamflow } \\
\text { (ft } 3 / \mathrm{s})\end{array}$ & $\begin{array}{l}\text { Percentage } \\
\text { of time }\end{array}$ & $\begin{array}{l}\text { Daily mean } \\
\text { streamflow } \\
\left(\mathrm{ft}^{3} / \mathrm{s}\right)\end{array}$ \\
\hline 99 & 1.1 & 40 & 12 \\
\hline 98 & 1.4 & 30 & 16 \\
\hline 95 & 1.8 & 20 & 24 \\
\hline 90 & 2.4 & 10 & 42 \\
\hline 80 & 3.5 & 5 & 59 \\
\hline 70 & 4.6 & 2 & 94 \\
\hline 60 & 6.1 & 1 & 129 \\
\hline 50 & 8.7 & & \\
\hline
\end{tabular}




\section{STONEY CREEK NEAR NOBLESVILLE, IN}

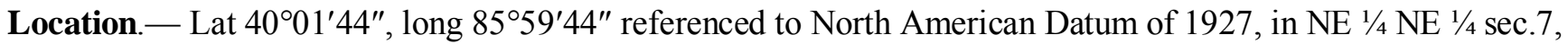
T.18 N., R.5 E., Hamilton County, IN, Hydrologic Unit 05120201, on right bank, between dual bridges on State Road 37, 1.2 mi south of intersection of State Road 38 and State Road 37, 1.4 mi upstream from mouth, and $1.4 \mathrm{mi}$ southeast of Noblesville.

Drainage area. $-50.8 \mathrm{mi}^{2}$.

Period of record. - July 1967 to current year.

Average discharge. $-54.2 \mathrm{ft}^{3} / \mathrm{s}$.

Minimum daily discharge. $-0.88 \mathrm{ft}^{3} / \mathrm{s}$.

Human health (harmonic mean) design flow. $-15 \mathrm{ft}^{3} / \mathrm{s}$.

Remarks.- None.

\begin{tabular}{ccc}
\hline \multicolumn{3}{c}{ Magnitude and frequency of annual low flow } \\
\hline $\begin{array}{c}\text { Lowest average streamflow, in } \mathrm{ft}^{3} / \mathrm{s} \text {, for indicated period of } \\
\text { consecutive days at an annual nonexceedance probability of } 0.1\end{array}$ \\
\hline $\mathbf{7}$ & $\mathbf{3 0}$ \\
\hline 2.4 & 2.6 & 3.2 \\
\hline
\end{tabular}

Percentage of time streamflow was equaled or exceeded for the period of record

\begin{tabular}{cc|cc}
\hline $\begin{array}{c}\text { Percentage } \\
\text { of time }\end{array}$ & $\begin{array}{c}\text { Daily mean } \\
\text { streamflow } \\
\left(\mathrm{ft}^{3} / \mathbf{s}\right)\end{array}$ & $\begin{array}{c}\text { Percentage } \\
\text { of time }\end{array}$ & $\begin{array}{c}\text { Daily mean } \\
\text { streamflow } \\
\left(\mathrm{ft}^{3} / \mathbf{s}\right)\end{array}$ \\
\hline 99 & 3.0 & 40 & 34 \\
\hline 98 & 3.5 & 30 & 47 \\
\hline 95 & 4.6 & 20 & 69 \\
\hline 90 & 6.2 & 10 & 122 \\
\hline 80 & 9.2 & 5 & 194 \\
\hline 70 & 13 & 2 & 337 \\
\hline 60 & 18 & 1 & 508 \\
\hline 50 & 25 & & \\
\hline
\end{tabular}




\section{WHITE RIVER AT 146TH ST NEAR NOBLESVILLE, IN}

Location.- Lat $40^{\circ} 00^{\prime} 01^{\prime \prime}$, long $86^{\circ} 01^{\prime} 21^{\prime \prime}$ referenced to North American Datum of 1927, in SW 1/4 SE 1/4 Sec.13, T.17 N., R.4 E., Hamilton County, IN, Hydrologic Unit 05120201, on the southeast corner of the 146th Street bridge, 25 feet south of centerline of eastbound lanes, $1035 \mathrm{ft}$ west of Allisonville Road, and at mile 258.96.

Drainage area. $-1,142 \mathrm{mi}^{2}$.

Period of record. - July 2006 to current year.

Average discharge. $-1,518 \mathrm{ft}^{3} / \mathrm{s}$.

Minimum daily discharge. $-150 \mathrm{ft}^{3} / \mathrm{s}$.

Human health (harmonic mean) design flow. $-403 \mathrm{ft}^{3} / \mathrm{s}$.

Remarks. - This site has less than 10 years of record.

\begin{tabular}{ccc}
\hline \multicolumn{3}{c}{ Magnitude and frequency of annual low flow } \\
\hline $\begin{array}{c}\text { Lowest average streamflow, in } \mathrm{ft}^{3} / \mathrm{s} \text {, for indicated period of } \\
\text { consecutive days at an annual nonexceedance probability of } \mathbf{0 . 1}\end{array}$ \\
\hline $\mathbf{1}$ & $\mathbf{7}$ & $\mathbf{3 0}$ \\
\hline 84 & 99 & 116 \\
\hline
\end{tabular}

Percentage of time streamflow was equaled or exceeded for the period of record

\begin{tabular}{cc|cc}
\hline $\begin{array}{c}\text { Percentage } \\
\text { of time }\end{array}$ & $\begin{array}{c}\text { Daily mean } \\
\text { streamflow } \\
(\mathbf{f t} 3 / \mathbf{s})\end{array}$ & $\begin{array}{c}\text { Percentage } \\
\text { of time }\end{array}$ & $\begin{array}{c}\text { Daily mean } \\
\text { streamflow } \\
\left(\mathrm{ft}^{3} / \mathbf{s}\right)\end{array}$ \\
\hline 99 & 158 & 40 & 990 \\
\hline 98 & 166 & 30 & 1380 \\
\hline 95 & 183 & 20 & 2080 \\
\hline 90 & 206 & 10 & 3480 \\
\hline 80 & 258 & 5 & 5970 \\
\hline 70 & 332 & 2 & 9400 \\
\hline 60 & 469 & 1 & 12600 \\
\hline 50 & 711 & & \\
\hline
\end{tabular}




\section{WHITE RIVER NEAR NORA, IN}

Location.- Lat 39 $54^{\prime} 38^{\prime \prime}$, long $86^{\circ} 06^{\prime} 20^{\prime \prime}$ referenced to North American Datum of 1927, in NW 1/4 NW 1/4 sec.20,

T.17 N., R.4 E., Marion County, IN, Hydrologic Unit 05120201, on right upstream corner of bridge on 82nd Street, 2 mi east of Nora, 3.75 mi upstream of the Indianapolis Water Company dam at Broad Ripple, $14 \mathrm{mi}$ upstream from Fall Creek, and at mile 247.9.

Drainage area. $-1,219 \mathrm{mi}^{2}$.

Period of record. - October 1929 to current year.

Average discharge. $-1,201 \mathrm{ft}^{3} / \mathrm{s}$.

Minimum daily discharge. $-49 \mathrm{ft}^{3} / \mathrm{s}$.

Human health (harmonic mean) design flow. - $442 \mathrm{ft}^{3} / \mathrm{s}$.

Remarks. - Prior to October 1948, published as West Fork White River near Nora. Flow partially regulated by Morse Reservoir. Low-flow statistics calculated for regulated period, 1957 to 2011.

\begin{tabular}{ccc}
\hline \multicolumn{3}{c}{ Magnitude and frequency of annual low flow } \\
\hline $\begin{array}{c}\text { Lowest average streamflow, in } \mathrm{ft}^{3} / \mathrm{s} \text {, for indicated period of } \\
\text { consecutive days at an annual nonexceedance probability of } \mathbf{0 . 1}\end{array}$ \\
\hline $\mathbf{1}$ & $\mathbf{3 0}$ \\
\hline 114 & 123 & 139 \\
\hline
\end{tabular}

Percentage of time streamflow was equaled or exceeded for the period of record

\begin{tabular}{cc|cc}
\hline $\begin{array}{c}\text { Percentage } \\
\text { of time }\end{array}$ & $\begin{array}{c}\text { Daily mean } \\
\text { streamflow } \\
\left(\mathrm{ft}^{3} / \mathbf{s}\right)\end{array}$ & $\begin{array}{c}\text { Percentage } \\
\text { of time }\end{array}$ & $\begin{array}{c}\text { Daily mean } \\
\text { streamflow } \\
\left(\mathbf{f t}^{3} / \mathbf{s}\right)\end{array}$ \\
\hline 99 & 132 & 40 & 822 \\
\hline 98 & 141 & 30 & 1140 \\
\hline 95 & 163 & 20 & 1650 \\
\hline 90 & 191 & 10 & 2900 \\
\hline 80 & 257 & 5 & 4690 \\
\hline 70 & 348 & 2 & 7680 \\
\hline 60 & 464 & 1 & 10300 \\
\hline 50 & 614 & & \\
\hline
\end{tabular}




\section{WILLIAMS CREEK AT 96 ${ }^{\text {TH }}$ STREET, INDIANAPOLIS, IN}

Location.- Lat $39^{\circ} 55^{\prime} 37^{\prime \prime}$, long $86^{\circ} 10^{\prime} 20^{\prime \prime}$ referenced to North American Datum of 1927, in SE 1/4 SW 1/4 SE $1 / 4$ sec.10, T.17 N., R.3 E., Hamilton County, IN, Hydrologic Unit 05120201, on upstream left edge of bridge, $0.63 \mathrm{mi}$ east of 96th Street and Ditch Road, 0.75 mi west of 96th Street and Highway 31, and at mile 5.13.

Drainage area. $-16.3 \mathrm{mi}^{2}$.

Period of record.- August 2007 to current year.

Average discharge. $-26.4 \mathrm{ft}^{3} / \mathrm{s}$.

Minimum daily discharge. $-0.08 \mathrm{ft}^{3} / \mathrm{s}$.

Human health (harmonic mean) design flow. $-2.9 \mathrm{ft}^{3} / \mathrm{s}$.

Remarks. - This site has less than 10 years of record.

\begin{tabular}{cccc}
\hline \multicolumn{5}{c}{ Magnitude and frequency of annual low flow } \\
\hline \multicolumn{4}{c}{ Lowest average streamflow, in $\mathrm{ft}^{3} / \mathbf{s}$, for indicated period of } \\
consecutive days at an annual nonexceedance probability of 0.1
\end{tabular}




\section{CROOKED CREEK AT INDIANAPOLIS, IN}

Location. - Lat $39^{\circ} 49^{\prime} 47^{\prime \prime}$, long $86^{\circ} 12^{\prime} 22^{\prime \prime}$ referenced to North American Datum of 1927, in NW 1/4 SE 1/4 Sec.16, T.16 N., R.3 E., Marion County, IN, Hydrologic Unit 05120201, on left bank $150 \mathrm{ft}$ downstream from 42nd Street bridge in Indianapolis, at river mile 1.6, 2.3 mi west-northwest of burial plot of John Dillinger in Crown Hill Cemetery, and $2.35 \mathrm{mi}$ northeast of Indianapolis Motor Speedway.

Drainage area. $-17.9 \mathrm{mi}^{2}$.

Period of record. - June 1969 current year.

Average discharge. $-20.3 \mathrm{ft}^{3} / \mathrm{s}$.

Minimum daily discharge. $-0.0 \mathrm{ft}^{3} / \mathrm{s}$.

Human health (harmonic mean) design flow. $-3.8 \mathrm{ft}^{3} / \mathrm{s}$.

Remarks.- None.

\begin{tabular}{ccc}
\hline \multicolumn{3}{c}{ Magnitude and frequency of annual low flow } \\
\hline $\begin{array}{c}\text { Lowest average streamflow, in } \mathrm{ft}^{3} / \mathrm{s} \text {, for indicated period of } \\
\text { consecutive days at an annual nonexceedance probability of } \mathbf{0 . 1}\end{array}$ \\
\hline $\mathbf{1}$ & $\mathbf{3 0}$ \\
\hline 0.2 & 0.4 & 0.9 \\
\hline
\end{tabular}

Percentage of time streamflow was equaled or exceeded for the period of record

\begin{tabular}{cc|cc}
\hline $\begin{array}{c}\text { Percentage } \\
\text { of time }\end{array}$ & $\begin{array}{c}\text { Daily mean } \\
\text { streamflow } \\
\left(\mathrm{ft}^{3} / \mathbf{s}\right)\end{array}$ & $\begin{array}{c}\text { Percentage } \\
\text { of time }\end{array}$ & $\begin{array}{c}\text { Daily mean } \\
\text { streamflow } \\
\left(\mathrm{ft}^{3} / \mathbf{s}\right)\end{array}$ \\
\hline 99 & 0.5 & 40 & 11 \\
\hline 98 & 0.7 & 30 & 16 \\
\hline 95 & 1.1 & 20 & 23 \\
\hline 90 & 1.7 & 10 & 42 \\
\hline 80 & 2.8 & 5 & 71 \\
\hline 70 & 4.2 & 2 & 135 \\
\hline 60 & 5.9 & 1 & 225 \\
\hline 50 & 8.0 & & \\
\hline
\end{tabular}




\section{SUGAR CREEK NEAR MIDDLETOWN, IN}

Location.- Lat $40^{\circ} 02^{\prime} 27^{\prime \prime}$, long $85^{\circ} 31^{\prime} 30^{\prime \prime}$ referenced to North American Datum of 1927, in NW 1/4 SE 1/4 Sec.5, T.18 N., R.9 E., Henry County, IN, Hydrologic Unit 05120201, on right bank $90 \mathrm{ft}$ upstream from bridge on County Road 750 North, 1 mi southeast of Middletown.

Drainage area. $-5.8 \mathrm{mi}^{2}$.

Period of record.- October 1968 to September 1989.

Average discharge. $-5.76 \mathrm{ft}^{3} / \mathrm{s}$.

Minimum daily discharge. $-0.02 \mathrm{ft}^{3} / \mathrm{s}$.

Human health (harmonic mean) design flow. $-0.4 \mathrm{ft}^{3} / \mathrm{s}$.

Remarks.- None.

\begin{tabular}{|c|c|c|c|}
\hline \multicolumn{4}{|c|}{ Magnitude and frequency of annual low flow } \\
\hline \multicolumn{4}{|c|}{$\begin{array}{l}\text { Lowest average streamflow, in } \mathrm{ft}^{3} / \mathrm{s} \text {, for indicated period of } \\
\text { consecutive days at an annual nonexceedance probability of } 0.1\end{array}$} \\
\hline 1 & \multicolumn{2}{|c|}{7} & 30 \\
\hline 0.0 & \multicolumn{2}{|c|}{0.0} & 0.1 \\
\hline \multicolumn{4}{|c|}{$\begin{array}{l}\text { Percentage of time streamflow was equaled or exceeded for } \\
\text { the period of record }\end{array}$} \\
\hline $\begin{array}{l}\text { Percentage } \\
\text { of time }\end{array}$ & $\begin{array}{l}\text { Daily mean } \\
\text { streamflow } \\
\left(\mathrm{ft}^{3} / \mathrm{s}\right)\end{array}$ & $\begin{array}{l}\text { Percentage } \\
\text { of time }\end{array}$ & $\begin{array}{c}\text { Daily mean } \\
\text { streamflow } \\
\left(\mathrm{ft}^{3} / \mathrm{s}\right)\end{array}$ \\
\hline 99 & 0.1 & 40 & 2.4 \\
\hline 98 & 0.1 & 30 & 3.6 \\
\hline 95 & 0.1 & 20 & 5.9 \\
\hline 90 & 0.2 & 10 & 13 \\
\hline 80 & 0.3 & 5 & 25 \\
\hline 70 & 0.5 & 2 & 49 \\
\hline 60 & 0.9 & 1 & 73 \\
\hline 50 & 1.5 & & \\
\hline
\end{tabular}




\section{FALL CREEK NEAR FORTVILLE, IN}

Location.- Lat $39^{\circ} 57^{\prime} 17^{\prime \prime}$, long 85 $52^{\prime} 03^{\prime \prime}$ referenced to North American Datum of 1927, in NW 1/4 NE 1/4 sec.5, T.17 N., R.6 E., Hamilton County, IN, Hydrologic Unit 05120201, on right bank $100 \mathrm{ft}$ downstream from bridge on State Highway 238, 0.2 mi downstream from Lick Creek, 2 mi northwest of Fortville, and at mile 26.1.

Drainage area. $-169 \mathrm{mi}^{2}$.

Period of record. - July 1941 to current year.

Average discharge.- $184 \mathrm{ft}^{3} / \mathrm{s}$.

Minimum daily discharge. $-7.0 \mathrm{ft}^{3} / \mathrm{s}$.

Human health (harmonic mean) design flow. $-68 \mathrm{ft}^{3} / \mathrm{s}$.

Remarks. - None.

\begin{tabular}{|c|c|c|c|}
\hline \multicolumn{4}{|c|}{ Magnitude and frequency of annual low flow } \\
\hline \multicolumn{4}{|c|}{$\begin{array}{l}\text { Lowest average streamflow, in } \mathrm{ft}^{3} / \mathrm{s} \text {, for indicated period of } \\
\text { consecutive days at an annual nonexceedance probability of } 0.1\end{array}$} \\
\hline 1 & \multicolumn{2}{|c|}{7} & 30 \\
\hline 15 & \multicolumn{2}{|c|}{16} & 19 \\
\hline \multicolumn{4}{|c|}{$\begin{array}{l}\text { Percentage of time streamflow was equaled or exceeded for } \\
\text { the period of record }\end{array}$} \\
\hline $\begin{array}{l}\text { Percentage } \\
\text { of time }\end{array}$ & $\begin{array}{l}\text { Daily mean } \\
\text { streamflow } \\
\left(\mathrm{ft}^{3} / \mathrm{s}\right)\end{array}$ & $\begin{array}{l}\text { Percentage } \\
\text { of time }\end{array}$ & $\begin{array}{l}\text { Daily mean } \\
\text { streamflow } \\
\left(\mathrm{ft}^{3} / \mathrm{s}\right)\end{array}$ \\
\hline 99 & 18 & 40 & 125 \\
\hline 98 & 21 & 30 & 164 \\
\hline 95 & 26 & 20 & 231 \\
\hline 90 & 33 & 10 & 380 \\
\hline 80 & 43 & 5 & 607 \\
\hline 70 & 57 & 2 & 1070 \\
\hline 60 & 75 & 1 & 1590 \\
\hline 50 & 96 & & \\
\hline
\end{tabular}




\section{MUD CREEK AT INDIANAPOLIS, IN}

Location.- Lat $39^{\circ} 53^{\prime} 30^{\prime \prime}$, long $86^{\circ} 00^{\prime} 57^{\prime \prime}$ referenced to North American Datum of 1927, in SE 1/4 NE 1/4 Sec.25, T.17 N., R.4 E., Marion County, IN, Hydrologic Unit 05120201, on left bank at downstream side of Lantern Road bridge, 0.2 mi northeast of 75th Street and Sargent Road intersection, 1.5 mi upstream from mouth, and $2.0 \mathrm{mi}$ southeast of Castleton.

Drainage area. $-42.4 \mathrm{mi}^{2}$.

Period of record.- May 1958 to September 1976.

Average discharge. $-37.2 \mathrm{ft}^{3} / \mathrm{s}$.

Minimum daily discharge. $-0.2 \mathrm{ft}^{3} / \mathrm{s}$.

Human health (harmonic mean) design flow. $-5.5 \mathrm{ft}^{3} / \mathrm{s}$.

Remarks.- None.

\begin{tabular}{|c|c|c|c|}
\hline \multicolumn{4}{|c|}{ Magnitude and frequency of annual low flow } \\
\hline \multicolumn{4}{|c|}{$\begin{array}{l}\text { Lowest average streamflow, in } \mathrm{ft}^{3} / \mathrm{s} \text {, for indicated period of } \\
\text { consecutive days at an annual nonexceedance probability of } 0 \text {. }\end{array}$} \\
\hline 1 & \multicolumn{2}{|c|}{7} & 30 \\
\hline 0.4 & \multicolumn{2}{|c|}{0.5} & 0.6 \\
\hline \multicolumn{4}{|c|}{$\begin{array}{c}\text { Percentage of time streamflow was equaled or exceeded for } \\
\text { the period of record }\end{array}$} \\
\hline $\begin{array}{l}\text { Percentage } \\
\text { of time }\end{array}$ & $\begin{array}{c}\text { Daily mean } \\
\text { streamflow } \\
\left(\mathrm{ft}^{3} / \mathrm{s}\right)\end{array}$ & $\begin{array}{l}\text { Percentage } \\
\text { of time }\end{array}$ & $\begin{array}{c}\text { Daily mean } \\
\text { streamflow } \\
\left(\mathrm{ft}^{3} / \mathrm{s}\right)\end{array}$ \\
\hline 99 & 0.6 & 40 & 20 \\
\hline 98 & 0.9 & 30 & 28 \\
\hline 95 & 1.6 & 20 & 45 \\
\hline 90 & 2.4 & 10 & 89 \\
\hline 80 & 4.0 & 5 & 153 \\
\hline 70 & 5.9 & 2 & 274 \\
\hline 60 & 8.8 & 1 & 422 \\
\hline 50 & 14 & & \\
\hline
\end{tabular}




\section{FALL CREEK AT MILLERSVILLE, IN}

Location.- Lat $39^{\circ} 51^{\prime} 07^{\prime \prime}$, long $86^{\circ} 05^{\prime} 15^{\prime \prime}$ referenced to North American Datum of 1927, in NE 1/4 NE 1/4 Sec.9, T.16 N., R.4 E., Marion County, IN, Hydrologic Unit 05120201, on right bank at downstream side of Emerson Way bridge at Millersville, $2.4 \mathrm{mi}$ upstream of Keystone Avenue, $2.9 \mathrm{mi}$ downstream of Interstate 465, and $9.2 \mathrm{mi}$ upstream from mouth.

Drainage area. $-298 \mathrm{mi}^{2}$.

Period of record. - October 1929 to current year.

Average discharge. $-307 \mathrm{ft}^{3} / \mathrm{s}$.

Minimum daily discharge. $-7.8 \mathrm{ft}^{3} / \mathrm{s}$.

Human health (harmonic mean) design flow. $-114 \mathrm{ft}^{3} / \mathrm{s}$.

Remarks. - Flow regulated by Geist Reservoir. Low-flow statistics calculated for the regulated period, 1943 to 1993.

\begin{tabular}{cc|cc}
\hline \multicolumn{5}{c}{ Magnitude and frequency of annual low flow } \\
\hline \multicolumn{4}{c}{ Lowest average streamflow, in $\mathrm{ft}^{3} / \mathrm{s}$, for indicated period of } \\
consecutive days at an annual nonexceedance probability of $\mathbf{0 . 1}$
\end{tabular}




\section{FALL CREEK AT 16 ${ }^{\text {TH }}$ STREET AT INDIANAPOLIS, IN}

Location.- Lat $39^{\circ} 47^{\prime} 20^{\prime \prime}$, long $86^{\circ} 10^{\prime} 40^{\prime \prime}$ referenced to North American Datum of 1927, in SW 1/4 NW 1/4 sec.35, T.16 N., R.3 E., Marion County, IN, Hydrologic Unit 05120201, on left bank $120 \mathrm{ft}$ upstream from 16th Street on Aqueduct Street, 1.3 mi upstream from mouth.

Drainage area. $-317 \mathrm{mi}^{2}$.

Period of record.- October 1985 to September 1991.

Average discharge. $-337 \mathrm{ft}^{3} / \mathrm{s}$.

Minimum daily discharge. $-18 \mathrm{ft}^{3} / \mathrm{s}$.

Human health (harmonic mean) design flow. $-110 \mathrm{ft}^{3} / \mathrm{s}$.

Remarks. - This site has less than 10 years of record. Natural flow affected by regulation of Geist Reservoir and by diversion of municipal water supply of Indianapolis.

\begin{tabular}{ccc}
\hline \multicolumn{3}{c}{ Magnitude and frequency of annual low flow } \\
\hline $\begin{array}{c}\text { Lowest average streamflow, in } \mathrm{ft}^{3} / \mathrm{s}, \text { for indicated period of } \\
\text { consecutive days at an annual nonexceedance probability of } \mathbf{0 . 1}\end{array}$ \\
\hline $\mathbf{1}$ & $\mathbf{7}$ & $\mathbf{3 0}$ \\
\hline 18 & 23 & 28 \\
\hline
\end{tabular}

Percentage of time streamflow was equaled or exceeded for the period of record

\begin{tabular}{rr|rc}
\hline $\begin{array}{c}\text { Percentage } \\
\text { of time }\end{array}$ & $\begin{array}{c}\text { Daily mean } \\
\text { streamflow } \\
\left(\mathrm{ft}^{3} / \mathbf{s}\right)\end{array}$ & $\begin{array}{c}\text { Percentage } \\
\text { of time }\end{array}$ & $\begin{array}{c}\text { Daily mean } \\
\text { streamflow } \\
\left(\mathrm{ft}^{3} / \mathbf{s}\right)\end{array}$ \\
\hline 99 & 26 & 40 & 249 \\
\hline 98 & 29 & 30 & 322 \\
\hline 95 & 36 & 20 & 437 \\
\hline 90 & 46 & 10 & 739 \\
\hline 80 & 63 & 5 & 1250 \\
\hline 70 & 93 & 2 & 2000 \\
\hline 60 & 136 & 1 & 2640 \\
\hline 50 & 188 & & \\
\hline
\end{tabular}


White River Basin

\section{WHITE RIVER AT MICHIGAN STREET AT INDIANAPOLIS, IN}

Location.- Lat $39^{\circ} 46^{\prime} 29^{\prime \prime}$, long $86^{\circ} 11^{\prime} 23^{\prime \prime}$ referenced to North American Datum of 1927, in SE 1/4 NW 1/4 Sec.3, T.15 N., R.3 E., Marion County, IN, Hydrologic Unit 05120201, on the southwest corner of the Michigan Street bridge, $0.34 \mathrm{mi}$ downstream of 10th Street bridge, $0.38 \mathrm{mi}$ downstream of the mouth of Fall Creek, 1.7 mi northwest of Monument Circle, and at mile 233.3.

Drainage area. $-1,622 \mathrm{mi}^{2}$.

Period of record. - September 2004 to current year.

Average discharge. $-2,083 \mathrm{ft}^{3} / \mathrm{s}$.

Minimum daily discharge. $-52 \mathrm{ft}^{3} / \mathrm{s}$.

Human health (harmonic mean) design flow. $-383 \mathrm{ft}^{3} / \mathrm{s}$.

Remarks. - This site has less than 10 years of record.

\begin{tabular}{ccc}
\hline \multicolumn{3}{c}{ Magnitude and frequency of annual low flow } \\
\hline $\begin{array}{c}\text { Lowest average streamflow, in } \mathrm{ft}^{3} / \mathrm{s} \text {, for indicated period of } \\
\text { consecutive days at an annual nonexceedance probability of } 0.1\end{array}$ \\
\hline $\mathbf{1}$ & $\mathbf{3 0}$ \\
\hline 57 & 68 & 88 \\
\hline
\end{tabular}

Percentage of time streamflow was equaled or exceeded for the period of record

\begin{tabular}{cc|cc}
\hline $\begin{array}{c}\text { Percentage } \\
\text { of time }\end{array}$ & $\begin{array}{c}\text { Daily mean } \\
\text { streamflow } \\
\left(\mathrm{ft}^{3} / \mathbf{s}\right)\end{array}$ & $\begin{array}{c}\text { Percentage } \\
\text { of time }\end{array}$ & $\begin{array}{c}\text { Daily mean } \\
\text { streamflow } \\
\left(\mathrm{ft}^{3} / \mathbf{s}\right)\end{array}$ \\
\hline 99 & 119 & 40 & 1400 \\
\hline 98 & 132 & 30 & 1880 \\
\hline 95 & 156 & 20 & 2780 \\
\hline 90 & 199 & 10 & 4790 \\
\hline 80 & 317 & 5 & 7970 \\
\hline 70 & 479 & 2 & 12700 \\
\hline 60 & 722 & 1 & 17400 \\
\hline 50 & 1050 & & \\
\hline
\end{tabular}




\section{POGUES RUN AT VERMONT STREET AT INDIANAPOLIS, IN}

Location. - Lat $39^{\circ} 46^{\prime} 20^{\prime \prime}$, long $86^{\circ} 08^{\prime} 22^{\prime \prime}$ referenced to North American Datum of 1927, in SW 1/4 NW 1/4 SW 1/4 sec.6, T.15 N., R.4 E., Marion County, IN, Hydrologic Unit 05120201, on the downstream left edge bank at bridge, $0.2 \mathrm{mi}$ east of I-65/I-70, $0.4 \mathrm{mi}$ north of Washington Street, and at river mile 2.43.

Drainage area. $-9.74 \mathrm{mi}^{2}$.

Period of record. - January 2007 to current year.

Average discharge. $-7.33 \mathrm{ft}^{3} / \mathrm{s}$.

Minimum daily discharge. $-0.0 \mathrm{ft}^{3} / \mathrm{s}$.

Human health (harmonic mean) design flow. $-0.6 \mathrm{ft}^{3} / \mathrm{s}$.

Remarks. - This site has less than 10 years of record.

\begin{tabular}{cccc}
\hline \multicolumn{5}{c}{ Magnitude and frequency of annual low flow } \\
\hline \multicolumn{4}{c}{ Lowest average streamflow, in ft 3 /s, for indicated period of } \\
consecutive days at an annual nonexceedance probability of 0.1
\end{tabular}




\section{WHITE RIVER AT INDIANAPOLIS, IN}

Location.- Lat 39 $44^{\prime} 14^{\prime \prime}$, long $86^{\circ} 10^{\prime} 08^{\prime \prime}$ referenced to North American Datum of 1927, in SE 1/4 SW 1/4 Sec.14, T.15 N., R.3 E., Marion County, IN, Hydrologic Unit 05120201, on left bank under Raymond Street bridge in Indianapolis, $2.3 \mathrm{mi}$ upstream from Eagle Creek, 2.9 mi upstream from Indianapolis Power and Light Company dam, 3.7 mi downstream from Fall Creek, and at mile 229.2.

Drainage area. $-1,635 \mathrm{mi}^{2}$.

Period of record.- March 1904 to July 1906 and April 1930 to current year.

Average discharge.- $-1,532 \mathrm{ft}^{3} / \mathrm{s}$.

Minimum daily discharge. $-8.0 \mathrm{ft}^{3} / \mathrm{s}$.

Human health (harmonic mean) design flow. $-414 \mathrm{ft}^{3} / \mathrm{s}$.

Remarks. - Stage-discharge relation affected at times by large releases from Eagle Creek and by variable leakage at Indianapolis Power and Light Company dam. Natural flow affected by regulation of Morse Reservoir and Geist Reservoir and by diversion of municipal water supply by the Indianapolis Water Company. Low-flow statistics are calculated for the regulated period, 1943 to 2011.

\begin{tabular}{cc|cc}
\hline \multicolumn{5}{c}{ Magnitude and frequency of annual low flow } \\
\hline \multicolumn{4}{c}{7} \\
Lowest average streamflow, in $\mathrm{ft}^{3} / \mathbf{s}$, for indicated period of \\
consecutive days at an annual nonexceedance probability of 0.1
\end{tabular}




\section{PLEASANT RUN AT ARLINGTON AVENUE AT INDIANAPOLIS, IN}

Location.- Lat $39^{\circ} 46^{\prime} 33^{\prime \prime}$, long $86^{\circ} 03^{\prime} 50^{\prime \prime}$ referenced to North American Datum of 1927, in SW 1/4 NW 1/4 sec.2, T.15 N., R.4 E., Marion County, IN, Hydrologic Unit 05120201, on right bank $46 \mathrm{ft}$ upstream from Arlington Avenue bridge in Indianapolis, 0.5 mi downstream from small left-bank tributary, and at mile 7.9.

Drainage area. $-7.58 \mathrm{mi}^{2}$.

Period of record.- December 1959 to current year.

Average discharge. $-8.76 \mathrm{ft}^{3} / \mathrm{s}$.

Minimum daily discharge. $-0.0 \mathrm{ft}^{3} / \mathrm{s}$.

Human health (harmonic mean) design flow. $-1.1 \mathrm{ft}^{3} / \mathrm{s}$.

Remarks.- None.

\begin{tabular}{|c|c|c|c|}
\hline \multicolumn{4}{|c|}{ Magnitude and frequency of annual low flow } \\
\hline \multicolumn{4}{|c|}{$\begin{array}{l}\text { Lowest average streamflow, in } \mathrm{ft}^{3} / \mathrm{s} \text {, for indicated period of } \\
\text { consecutive days at an annual nonexceedance probability of } 0.1\end{array}$} \\
\hline 1 & \multicolumn{2}{|c|}{7} & 30 \\
\hline 0.0 & \multicolumn{2}{|c|}{0.1} & 0.3 \\
\hline \multicolumn{4}{|c|}{$\begin{array}{l}\text { Percentage of time streamflow was equaled or exceeded for } \\
\text { the period of record }\end{array}$} \\
\hline $\begin{array}{l}\text { Percentage } \\
\text { of time }\end{array}$ & $\begin{array}{c}\text { Daily mean } \\
\text { streamflow } \\
\left(\mathrm{ft}^{3} / \mathrm{s}\right)\end{array}$ & $\begin{array}{l}\text { Percentage } \\
\text { of time }\end{array}$ & $\begin{array}{c}\text { Daily mean } \\
\text { streamflow } \\
\left(\mathrm{ft}^{3} / \mathrm{s}\right)\end{array}$ \\
\hline 99 & 0.1 & 40 & 2.9 \\
\hline 98 & 0.2 & 30 & 4.5 \\
\hline 95 & 0.3 & 20 & 7.6 \\
\hline 90 & 0.5 & 10 & 19 \\
\hline 80 & 0.8 & 5 & 39 \\
\hline 70 & 1.1 & 2 & 80 \\
\hline 60 & 1.5 & 1 & 126 \\
\hline 50 & 2.0 & & \\
\hline
\end{tabular}




\section{PLEASANT RUN AT BROOKVILLE ROAD AT INDIANAPOLIS, IN}

Location. - Lat $39^{\circ} 45^{\prime} 52^{\prime \prime}$, long $86^{\circ} 05^{\prime} 43^{\prime \prime}$ referenced to North American Datum of 1927, in NE 1/4 NW 1/4 sec.9, T.15 N., R.4 E., Marion County, IN, Hydrologic Unit 05120201, on right bank at downstream side of Brookville Road bridge in Indianapolis, $2.2 \mathrm{mi}$ downstream from Arlington Avenue, and at mile 5.7.

Drainage area.- $-10.1 \mathrm{mi}^{2}$.

Period of record.- November 1959 to May 1981.

Average discharge. $-10.1 \mathrm{ft}^{3} / \mathrm{s}$.

Minimum daily discharge. $-0.0 \mathrm{ft}^{3} / \mathrm{s}$.

Human health (harmonic mean) design flow. $-1.1 \mathrm{ft}^{3} / \mathrm{s}$.

Remarks. - None.

\begin{tabular}{ccc}
\hline \multicolumn{3}{c}{ Magnitude and frequency of annual low flow } \\
\hline $\begin{array}{c}\text { Lowest average streamflow, in } \mathrm{ft}^{3} / \mathrm{s} \text {, for indicated period of } \\
\text { consecutive days at an annual nonexceedance probability of } 0.1\end{array}$ \\
\hline $\mathbf{7}$ & $\mathbf{3 0}$ \\
\hline 0.0 & 0.0 & 0.1 \\
\hline
\end{tabular}

Percentage of time streamflow was equaled or exceeded for the period of record

\begin{tabular}{cc|cc}
\hline $\begin{array}{c}\text { Percentage } \\
\text { of time }\end{array}$ & $\begin{array}{c}\text { Daily mean } \\
\text { streamflow } \\
\left(\mathrm{ft}^{3} / \mathbf{s}\right)\end{array}$ & $\begin{array}{c}\text { Percentage } \\
\text { of time }\end{array}$ & $\begin{array}{c}\text { Daily mean } \\
\text { streamflow } \\
\left(\mathrm{ft}^{3} / \mathbf{s}\right)\end{array}$ \\
\hline 99 & 0.0 & 40 & 3.5 \\
\hline 98 & 0.0 & 30 & 5.2 \\
\hline 95 & 0.0 & 20 & 8.7 \\
\hline 90 & 0.4 & 10 & 21 \\
\hline 80 & 0.8 & 5 & 42 \\
\hline 70 & 1.3 & 2 & 93 \\
\hline 60 & 1.8 & 1 & 152 \\
\hline 50 & 2.5 & & \\
\hline
\end{tabular}




\section{BEAN CREEK AT INDIANAPOLIS, IN}

Location. - Lat $39^{\circ} 43^{\prime} 45^{\prime \prime}$, long $86^{\circ} 07^{\prime} 14^{\prime \prime}$ referenced to North American Datum of 1927 in NW 1/4 SW 1/4 sec.20, T.15 N., R.4 E., Marion County, Hydrologic Unit 05120201, on left bank 80 ft upstream from Keystone Avenue bridge and west edge of Sarah Shank Golf Course in Indianapolis, and at mile 1.8.

Drainage area. $-4.4 \mathrm{mi}^{2}$.

Period of record. - October 1970 to September 1993.

Average discharge. $-5.37 \mathrm{ft}^{3} / \mathrm{s}$.

Minimum daily discharge. $-0.30 \mathrm{ft}^{3} / \mathrm{s}$.

Human health (harmonic mean) design flow. $-2.4 \mathrm{ft}^{3} / \mathrm{s}$.

Remarks. - None.

\begin{tabular}{ccc}
\hline \multicolumn{3}{c}{ Magnitude and frequency of annual low flow } \\
\hline $\begin{array}{c}\text { Lowest average streamflow, in } \mathrm{ft}^{3} / \mathrm{s} \text {, for indicated period of } \\
\text { consecutive days at an annual nonexceedance probability of } 0.1\end{array}$ \\
\hline $\mathbf{1}$ & $\mathbf{7}$ & $\mathbf{3 0}$ \\
\hline 0.5 & 0.6 & 0.9 \\
\hline
\end{tabular}

Percentage of time streamflow was equaled or exceeded for the period of record

\begin{tabular}{cc|cc}
\hline $\begin{array}{c}\text { Percentage } \\
\text { of time }\end{array}$ & $\begin{array}{c}\text { Daily mean } \\
\text { streamflow } \\
\left(\mathrm{ft}^{3} / \mathbf{s}\right)\end{array}$ & $\begin{array}{c}\text { Percentage } \\
\text { of time }\end{array}$ & $\begin{array}{c}\text { Daily mean } \\
\text { streamflow } \\
\left(\mathrm{ft}^{3} / \mathbf{s}\right)\end{array}$ \\
\hline 99 & 0.7 & 40 & 3.3 \\
\hline 98 & 0.8 & 30 & 4.2 \\
\hline 95 & 1.0 & 20 & 6.1 \\
\hline 90 & 1.3 & 10 & 11 \\
\hline 80 & 1.7 & 5 & 19 \\
\hline 70 & 2.0 & 2 & 33 \\
\hline 60 & 2.3 & 1 & 48 \\
\hline 50 & 2.7 & & \\
\hline
\end{tabular}




\section{EAGLE CREEK AT ZIONSVILLE, IN}

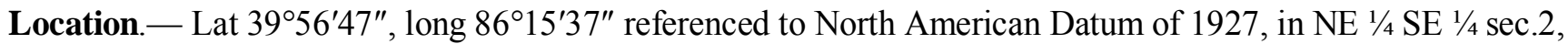
T.17 N., R.2 E., Boone County, IN, Hydrologic Unit 05120201, on right upstream end of Zionsville Road bridge over Eagle Creek, 0.15 mi south of Highway 334, 0.34 mi downstream from Long Branch Ditch, 1.0 mi downstream from Little Eagle Creek, and at mile 24.4.

Drainage area. $-106 \mathrm{mi}^{2}$.

Period of record.- October 1957 to current year.

Average discharge. $-106 \mathrm{ft}^{3} / \mathrm{s}$.

Minimum daily discharge. $-0.0 \mathrm{ft}^{3} / \mathrm{s}$.

Human health (harmonic mean) design flow. $-2.7 \mathrm{ft}^{3} / \mathrm{s}$.

Remarks.- None.

\begin{tabular}{c}
\hline \multicolumn{5}{c}{ Magnitude and frequency of annual low flow } \\
\hline \multicolumn{4}{c}{ Lowest average streamflow, in $\mathrm{ft}^{3} / \mathrm{s}$, for indicated period of } \\
consecutive days at an annual nonexceedance probability of 0.1
\end{tabular}




\section{EAGLE CREEK AT CLERMONT, IN}

Location. - Lat $39^{\circ} 48^{\prime} 52^{\prime \prime}$, long $86^{\circ} 18^{\prime} 19^{\prime \prime}$ referenced to North American Datum of 1927, in NW 1/4 SW 1/4 sec.22, T.16 N., R.2 E., Marion County, IN, Hydrologic Unit 05120201, on right bank at bridge abutment at the end of Salt Lake Road, $400 \mathrm{ft}$ north of intersection of Salt Lake Road and Dandy Trail, 0.9 mi northeast of Clermont, and at mile 11.4.

Drainage area. $-164 \mathrm{mi}^{2}$.

Period of record. - June 2006 to current year.

Average discharge. $-213 \mathrm{ft}^{3} / \mathrm{s}$.

Minimum daily discharge. $-6.5 \mathrm{ft}^{3} / \mathrm{s}$.

Human health (harmonic mean) design flow. $-26 \mathrm{ft}^{3} / \mathrm{s}$.

Remarks. - This site has less than 10 years of record.

\begin{tabular}{ccc}
\hline \multicolumn{3}{c}{ Magnitude and frequency of annual low flow } \\
\hline $\begin{array}{c}\text { Lowest average streamflow, in } \mathrm{ft}^{3} / \mathrm{s} \text {, for indicated period of } \\
\text { consecutive days at an annual nonexceedance probability of } 0.1\end{array}$ \\
\hline $\mathbf{1}$ & $\mathbf{7}$ & $\mathbf{3 0}$ \\
\hline 6.5 & 8.6 & 9.2 \\
\hline
\end{tabular}

Percentage of time streamflow was equaled or exceeded for the period of record

\begin{tabular}{cc|cc}
\hline $\begin{array}{c}\text { Percentage } \\
\text { of time }\end{array}$ & $\begin{array}{c}\text { Daily mean } \\
\text { streamflow } \\
\left(\mathrm{ft}^{3} / \mathbf{s}\right)\end{array}$ & $\begin{array}{c}\text { Percentage } \\
\text { of time }\end{array}$ & $\begin{array}{c}\text { Daily mean } \\
\text { streamflow } \\
\left(\mathrm{ft}^{3} / \mathbf{s}\right)\end{array}$ \\
\hline 99 & 8.3 & 40 & 93 \\
\hline 98 & 8.8 & 30 & 171 \\
\hline 95 & 10 & 20 & 271 \\
\hline 90 & 12 & 10 & 511 \\
\hline 80 & 14 & 5 & 925 \\
\hline 70 & 16 & 2 & 1580 \\
\hline 60 & 18 & 1 & 2370 \\
\hline 50 & 38 & & \\
\hline
\end{tabular}




\section{EAGLE CREEK AT INDIANAPOLIS, IN}

Location. - Lat $39^{\circ} 46^{\prime} 40^{\prime \prime}$, long $86^{\circ} 15^{\prime} 01^{\prime \prime}$ referenced to North American Datum of 1927, in NW 1/4 NW 1/4 sec.6, T.15 N., R.3 E., Marion County, IN, Hydrologic Unit 05120201, on the upstream side of bridge on Lynhurst Drive, approximately $600 \mathrm{ft}$ south of intersection of West 10th Street and Lynhurst Drive, $0.5 \mathrm{mi}$ downstream from West 10th Street bridge, $1.0 \mathrm{mi}$ upstream from Vermont Street bridge, 3.0 mi upstream from Little Eagle Creek, and 7.1 mi upstream from mouth.

Drainage area. $-174 \mathrm{mi}^{2}$.

Period of record. - November 1938 to current year.

Average discharge. $-163 \mathrm{ft}^{3} / \mathrm{s}$.

Minimum daily discharge. $-0.0 \mathrm{ft}^{3} / \mathrm{s}$.

Human health (harmonic mean) design flow. $-22 \mathrm{ft}^{3} / \mathrm{s}$.

Remarks. - Flow regulated since November 1969 by Eagle Creek Reservoir, 4.7 mi upstream (see station 03353450). Low-flow statistics are calculated for the regulated period, 1970 to 2011.

\begin{tabular}{|c|c|c|c|}
\hline \multicolumn{4}{|c|}{ Magnitude and frequency of annual low flow } \\
\hline \multicolumn{4}{|c|}{$\begin{array}{l}\text { Lowest average streamflow, in } \mathrm{ft}^{3} / \mathrm{s} \text {, for indicated period of } \\
\text { consecutive days at an annual nonexceedance probability of } 0.1\end{array}$} \\
\hline 1 & \multicolumn{2}{|c|}{7} & 30 \\
\hline 3.2 & \multicolumn{2}{|c|}{3.9} & 5.1 \\
\hline \multicolumn{4}{|c|}{$\begin{array}{l}\text { Percentage of time streamflow was equaled or exceeded for } \\
\text { the period of record }\end{array}$} \\
\hline $\begin{array}{l}\text { Percentage } \\
\text { of time }\end{array}$ & $\begin{array}{l}\text { Daily mean } \\
\text { streamflow } \\
\left(\mathrm{ft}^{3} / \mathrm{s}\right)\end{array}$ & $\begin{array}{l}\text { Percentage } \\
\text { of time }\end{array}$ & $\begin{array}{l}\text { Daily mean } \\
\text { streamflow } \\
\left(\mathrm{ft}^{3} / \mathrm{s}\right)\end{array}$ \\
\hline 99 & 3.6 & 40 & 76 \\
\hline 98 & 4.8 & 30 & 136 \\
\hline 95 & 7.1 & 20 & 213 \\
\hline 90 & 9.7 & 10 & 422 \\
\hline 80 & 14 & 5 & 736 \\
\hline 70 & 17 & 2 & 1390 \\
\hline 60 & 24 & 1 & 2040 \\
\hline 50 & 35 & & \\
\hline
\end{tabular}




\section{LITTLE EAGLE CREEK AT 52ND STREET AT INDIANAPOLIS, IN}

Location.- Lat $39^{\circ} 50^{\prime} 45^{\prime \prime}$, long $86^{\circ} 14^{\prime} 55^{\prime \prime}$ referenced to North American Datum of 1927 in NE 1/4 SW 1/4 sec.7, T.16 N., R.2 E., Marion County, IN, Hydrologic Unit 05120201, on right bank at downstream side of West 52nd Street, 0.4 mi east of Lafayette Road, 1.1 mi upstream from Guion Creek, and at mile 7.2

Drainage area. $-6.28 \mathrm{mi}^{2}$.

Period of record.- October 1989 to September 2000.

Average discharge. $-9.78 \mathrm{ft}^{3} / \mathrm{s}$.

Minimum daily discharge. $-0.10 \mathrm{ft}^{3} / \mathrm{s}$.

Human health (harmonic mean) design flow. $-1.7 \mathrm{ft}^{3} / \mathrm{s}$.

Remarks. - None.

\begin{tabular}{|c|c|c|c|}
\hline \multicolumn{4}{|c|}{ Magnitude and frequency of annual low flow } \\
\hline \multicolumn{4}{|c|}{$\begin{array}{l}\text { Lowest average streamflow, in } \mathrm{ft}^{3} / \mathrm{s} \text {, for indicated period of } \\
\text { consecutive days at an annual nonexceedance probability of } 0.1\end{array}$} \\
\hline 1 & \multicolumn{2}{|c|}{7} & 30 \\
\hline 0.1 & \multicolumn{2}{|c|}{0.2} & 0.4 \\
\hline \multicolumn{4}{|c|}{$\begin{array}{l}\text { Percentage of time streamflow was equaled or exceeded for } \\
\text { the period of record }\end{array}$} \\
\hline $\begin{array}{l}\text { Percentage } \\
\text { of time }\end{array}$ & $\begin{array}{l}\text { Daily mean } \\
\text { streamflow } \\
\left(\mathrm{ft}^{3} / \mathrm{s}\right)\end{array}$ & $\begin{array}{l}\text { Percentage } \\
\text { of time }\end{array}$ & $\begin{array}{l}\text { Daily mean } \\
\text { streamflow } \\
\left(\mathrm{ft}^{3} / \mathrm{s}\right)\end{array}$ \\
\hline 99 & 0.2 & 40 & 4.2 \\
\hline 98 & 0.3 & 30 & 6.0 \\
\hline 95 & 0.5 & 20 & 9.5 \\
\hline 90 & 0.7 & 10 & 20 \\
\hline 80 & 1.1 & 5 & 38 \\
\hline 70 & 1.7 & 2 & 80 \\
\hline 60 & 2.4 & 1 & 136 \\
\hline 50 & 3.2 & & \\
\hline
\end{tabular}




\section{GUION CREEK ABOVE 52ND STREET AT INDIANAPOLIS, IN}

Location.- Lat 39 $50^{\prime} 45^{\prime \prime}$, long $86^{\circ} 13^{\prime} 57^{\prime \prime}$ referenced to North American Datum of 1927, in NW 1/4 SW 1/4 sec.08., T.16 N., R.3 E., Marion County, Hydrologic Unit 05120201, on right bank $25 \mathrm{ft}$ upstream from private bridge, 0.2 mi north of West 52nd Street along Guion Road, and $1.25 \mathrm{mi}$ upstream of the confluence with Little Eagle Creek.

Drainage area. $-4.10 \mathrm{mi}^{2}$.

Period of record.- October 1989 to September 2001.

Average discharge. $-4.72 \mathrm{ft}^{3} / \mathrm{s}$.

Minimum daily discharge. $-0.00 \mathrm{ft}^{3} / \mathrm{s}$.

Human health (harmonic mean) design flow. $-0.3 \mathrm{ft}^{3} / \mathrm{s}$.

Remarks.- None.

\begin{tabular}{|c|c|c|c|}
\hline \multicolumn{4}{|c|}{ Magnitude and frequency of annual low flow } \\
\hline \multicolumn{4}{|c|}{$\begin{array}{l}\text { Lowest average streamflow, in } \mathrm{ft}^{3} / \mathrm{s} \text {, for indicated period of } \\
\text { consecutive days at an annual nonexceedance probability of } 0.1\end{array}$} \\
\hline 1 & \multicolumn{2}{|c|}{7} & 30 \\
\hline 0.0 & \multicolumn{2}{|c|}{0.0} & 0.0 \\
\hline \multicolumn{4}{|c|}{$\begin{array}{l}\text { Percentage of time streamflow was equaled or exceeded for } \\
\text { the period of record }\end{array}$} \\
\hline $\begin{array}{l}\text { Percentage } \\
\text { of time }\end{array}$ & $\begin{array}{l}\text { Daily mean } \\
\text { streamflow } \\
\left(\mathrm{ft}^{3} / \mathrm{s}\right)\end{array}$ & $\begin{array}{l}\text { Percentage } \\
\text { of time }\end{array}$ & $\begin{array}{l}\text { Daily mean } \\
\text { streamflow } \\
\left(\mathrm{ft}^{3} / \mathrm{s}\right)\end{array}$ \\
\hline 99 & 0.0 & 40 & 2.1 \\
\hline 98 & 0.0 & 30 & 3.1 \\
\hline 95 & 0.1 & 20 & 4.9 \\
\hline 90 & 0.2 & 10 & 9.9 \\
\hline 80 & 0.4 & 5 & 20 \\
\hline 70 & 0.7 & 2 & 40 \\
\hline 60 & 1.0 & 1 & 60 \\
\hline 50 & 1.5 & & \\
\hline
\end{tabular}




\section{FALCON CREEK AT 30TH STREET AT INDIANAPOLIS, IN}

Location.- Lat 39 $48^{\prime} 33^{\prime \prime}$, long 86 $6^{\circ} 3^{\prime} 56^{\prime \prime}$ referenced to North American Datum of 1927, in NW 1/4 NW 1/4 sec.29, T.16 N., R.03 E., Marion County, IN, Hydrologic Unit 05120201, on left bank, $150 \mathrm{ft}$ downstream from bridge on West 30th Street, $0.6 \mathrm{mi}$ west of Lafayette Road, and $0.6 \mathrm{mi}$ upstream of confluence with Little Eagle Creek.

Drainage area. $-4.15 \mathrm{mi}^{2}$.

Period of record.- October 1989 to September 2001.

Average discharge. $-4.30 \mathrm{ft}^{3} / \mathrm{s}$.

Minimum daily discharge. $-0.00 \mathrm{ft}^{3} / \mathrm{s}$.

Human health (harmonic mean) design flow. $-0.6 \mathrm{ft}^{3} / \mathrm{s}$.

Remarks.- None.

\begin{tabular}{ccc}
\hline \multicolumn{3}{c}{ Magnitude and frequency of annual low flow } \\
\hline $\begin{array}{c}\text { Lowest average streamflow, in } \mathrm{ft}^{3} / \mathrm{s} \text {, for indicated period of } \\
\text { consecutive days at an annual nonexceedance probability of } \mathbf{0 . 1}\end{array}$ \\
\hline $\mathbf{1}$ & $\mathbf{7}$ & $\mathbf{3 0}$ \\
\hline 0.1 & 0.1 & 0.2 \\
\hline
\end{tabular}

Percentage of time streamflow was equaled or exceeded for the period of record

\begin{tabular}{cc|cc}
\hline $\begin{array}{c}\text { Percentage } \\
\text { of time }\end{array}$ & $\begin{array}{c}\text { Daily mean } \\
\text { streamflow } \\
\left(\mathrm{ft}^{3} / \mathbf{s}\right)\end{array}$ & $\begin{array}{c}\text { Percentage } \\
\text { of time }\end{array}$ & $\begin{array}{c}\text { Daily mean } \\
\text { streamflow } \\
\left(\mathrm{ft}^{3} / \mathbf{s}\right)\end{array}$ \\
\hline 99 & 0.1 & 40 & 1.6 \\
\hline 98 & 0.1 & 30 & 2.5 \\
\hline 95 & 0.2 & 20 & 4.2 \\
\hline 90 & 0.3 & 10 & 9.5 \\
\hline 80 & 0.4 & 5 & 19 \\
\hline 70 & 0.6 & 2 & 37 \\
\hline 60 & 0.8 & 1 & 57 \\
\hline 50 & 1.2 & & \\
\hline
\end{tabular}




\section{LITTLE EAGLE CREEK AT SPEEDWAY, IN}

Location. - Lat $39^{\circ} 47^{\prime} 15^{\prime \prime}$, long $86^{\circ} 13^{\prime} 43^{\prime \prime}$ referenced to North American Datum of 1927, in NE 1/4 SW 1/4 sec.32, T.16 N., R.3 E., Marion County, IN, Hydrologic Unit 05120201, on right bank at downstream side of 16th Street bridge in Speedway, 0.6 mi upstream from Dry Run, and 2.3 mi upstream from mouth.

Drainage area. $-24.3 \mathrm{mi}^{2}$, including $5.57 \mathrm{mi}^{2}$ from Dry Run basin. Since June 1964, part of the flow from the $5.57 \mathrm{mi}^{2}$ of Dry Run basin has been diverted into Little Eagle Creek above gage.

Period of record. - October 1959 to current year.

Average discharge. $-27.2 \mathrm{ft}^{3} / \mathrm{s}$ (for water years 1965 to current year).

Minimum daily discharge. $-0.00 \mathrm{ft}^{3} / \mathrm{s}$.

Human health (harmonic mean) design flow. $-2.9 \mathrm{ft}^{3} / \mathrm{s}$.

Remarks. - None.

\begin{tabular}{ccc}
\hline \multicolumn{3}{c}{ Magnitude and frequency of annual low flow } \\
\hline $\begin{array}{c}\text { Lowest average streamflow, in } \mathrm{ft}^{3} / \mathrm{s} \text {, for indicated period of } \\
\text { consecutive days at an annual nonexceedance probability of } \mathbf{0 . 1}\end{array}$ \\
\hline $\mathbf{1}$ & $\mathbf{7}$ & $\mathbf{3 0}$ \\
\hline 0.1 & 0.2 & 0.6 \\
\hline
\end{tabular}

Percentage of time streamflow was equaled or exceeded for the period of record

\begin{tabular}{cc|cc}
\hline $\begin{array}{c}\text { Percentage } \\
\text { of time }\end{array}$ & $\begin{array}{c}\text { Daily mean } \\
\text { streamflow } \\
\left(\mathrm{ft}^{3} / \mathbf{s}\right)\end{array}$ & $\begin{array}{c}\text { Percentage } \\
\text { of time }\end{array}$ & $\begin{array}{c}\text { Daily mean } \\
\text { streamflow } \\
\left(\mathrm{ft}^{3} / \mathbf{s}\right)\end{array}$ \\
\hline 99 & 0.2 & 40 & 13 \\
\hline 98 & 0.4 & 30 & 18 \\
\hline 95 & 0.8 & 20 & 29 \\
\hline 90 & 1.4 & 10 & 57 \\
\hline 80 & 2.6 & 5 & 104 \\
\hline 70 & 4.2 & 2 & 198 \\
\hline 60 & 6.1 & 1 & 305 \\
\hline 50 & 8.7 & & \\
\hline
\end{tabular}




\section{WHITE RIVER AT STOUT GEN. STN. AT INDIANAPOLIS, IN}

Location.- Lat $39^{\circ} 42^{\prime} 52^{\prime \prime}$, long $86^{\circ} 12^{\prime} 02^{\prime \prime}$ referenced to North American Datum of 1927, in SE $1 / 4$ NE $1 / 4$ sec.28, T.15 N., R.3 E., Marion County, IN, Hydrologic Unit 05120201, on right bank 0.34 mi above confluence with Lick Creek, $0.63 \mathrm{mi}$ west of South Harding Street, $1.42 \mathrm{mi}$ east of Lockburn Street and $1.46 \mathrm{mi}$ south of Raymond Street, and at mile 226.3.

Drainage area. $-1,898 \mathrm{mi}^{2}$.

Period of record. - October 1992 to current year.

Average discharge. $-2,162 \mathrm{ft}^{3} / \mathrm{s}$.

Minimum daily discharge. $-150 \mathrm{ft}^{3} / \mathrm{s}$.

Human health (harmonic mean) design flow. $-752 \mathrm{ft}^{3} / \mathrm{s}$.

Remarks. - Natural flow affected by regulation of Morse Reservoir, Geist Reservoir, and Eagle Creek Reservoir and by diversion of municipal water supply by the Indianapolis Water Company.

\begin{tabular}{|c|c|c|c|}
\hline \multicolumn{4}{|c|}{ Magnitude and frequency of annual low flow } \\
\hline \multicolumn{4}{|c|}{$\begin{array}{l}\text { Lowest average streamflow, in } \mathrm{ft}^{3} / \mathrm{s} \text {, for indicated period of } \\
\text { consecutive days at an annual nonexceedance probability of } 0.1\end{array}$} \\
\hline 1 & \multicolumn{2}{|c|}{7} & 30 \\
\hline 152 & \multicolumn{2}{|c|}{171} & 222 \\
\hline \multicolumn{4}{|c|}{$\begin{array}{c}\text { Percentage of time streamflow was equaled or exceeded for } \\
\text { the period of record }\end{array}$} \\
\hline $\begin{array}{l}\text { Percentage } \\
\text { of time }\end{array}$ & $\begin{array}{c}\text { Daily mean } \\
\text { streamflow } \\
\left(\mathrm{ft}^{3} / \mathrm{s}\right)\end{array}$ & $\begin{array}{l}\text { Percentage } \\
\text { of time }\end{array}$ & $\begin{array}{l}\text { Daily mean } \\
\text { streamflow } \\
\left(\mathrm{ft}^{3} / \mathrm{s}\right)\end{array}$ \\
\hline 99 & 189 & 40 & 1520 \\
\hline 98 & 212 & 30 & 2030 \\
\hline 95 & 263 & 20 & 2890 \\
\hline 90 & 318 & 10 & 4880 \\
\hline 80 & 439 & 5 & 7870 \\
\hline 70 & 615 & 2 & 12600 \\
\hline 60 & 838 & 1 & 16900 \\
\hline 50 & 1130 & & \\
\hline
\end{tabular}




\section{LICK CREEK AT INDIANAPOLIS, IN}

Location.- Lat $39^{\circ} 42^{\prime} 21^{\prime \prime}$, long $86^{\circ} 06^{\prime} 13^{\prime \prime}$ referenced to North American Datum of 1927, in NE 1/4 NE 1/4 sec.32, T.15 N., R.4 E., Marion County, IN, Hydrologic Unit 05120201, on left bank, at upstream side of Sherman Drive bridge in Indianapolis, $0.35 \mathrm{mi}$ downstream of Beach Creek mouth, $5.1 \mathrm{mi}$ west of Wanamaker, IN, and at mile 6.2.

Drainage area. $-15.6 \mathrm{mi}^{2}$.

Period of record. - October 1970 to current year.

Average discharge. $-21.1 \mathrm{ft}^{3} / \mathrm{s}$.

Minimum daily discharge. $-0.05 \mathrm{ft}^{3} / \mathrm{s}$.

Human health (harmonic mean) design flow. $-2.6 \mathrm{ft}^{3} / \mathrm{s}$.

Remarks. - None.

\begin{tabular}{|c|c|c|c|}
\hline \multicolumn{4}{|c|}{ Magnitude and frequency of annual low flow } \\
\hline \multicolumn{4}{|c|}{$\begin{array}{l}\text { Lowest average streamflow, in } \mathrm{ft}^{3} / \mathrm{s} \text {, for indicated period of } \\
\text { consecutive days at an annual nonexceedance probability of } 0.1\end{array}$} \\
\hline 1 & \multicolumn{2}{|c|}{7} & 30 \\
\hline 0.1 & \multicolumn{2}{|c|}{0.2} & 0.5 \\
\hline \multicolumn{4}{|c|}{$\begin{array}{l}\text { Percentage of time streamflow was equaled or exceeded for } \\
\text { the period of record }\end{array}$} \\
\hline $\begin{array}{l}\text { Percentage } \\
\text { of time }\end{array}$ & $\begin{array}{c}\text { Daily mean } \\
\text { streamflow } \\
\left(\mathrm{ft}^{3} / \mathrm{s}\right)\end{array}$ & $\begin{array}{l}\text { Percentage } \\
\text { of time }\end{array}$ & $\begin{array}{l}\text { Daily mean } \\
\text { streamflow } \\
\left(\mathrm{ft}^{3} / \mathrm{s}\right)\end{array}$ \\
\hline 99 & 0.2 & 40 & 10 \\
\hline 98 & 0.4 & 30 & 15 \\
\hline 95 & 0.7 & 20 & 23 \\
\hline 90 & 1.2 & 10 & 47 \\
\hline 80 & 2.4 & 5 & 84 \\
\hline 70 & 3.7 & 2 & 167 \\
\hline 60 & 5.2 & 1 & 243 \\
\hline 50 & 7.2 & & \\
\hline
\end{tabular}




\section{LITTLE BUCK CREEK NEAR SOUTHPORT, IN}

Location. - Lat $39^{\circ} 40^{\prime} 11^{\prime \prime}$, long $86^{\circ} 04^{\prime} 57^{\prime \prime}$ referenced to North American Datum of 1927, in SW 1/4 SW 1/4 sec.10, T.14 N., T.4 E., Marion County, Hydrologic Unit 05120201, on right bank $5 \mathrm{ft}$ upstream from Emerson Avenue bridge in Indianapolis, $1.1 \mathrm{mi}$ downstream from Bunker Creek, and $2.5 \mathrm{mi}$ upstream from Derbyshire Creek.

Drainage area. $-5.75 \mathrm{mi}^{2}$.

Period of record.- October 1989 to September 2000.

Average discharge. $-8.54 \mathrm{ft}^{3} / \mathrm{s}$.

Minimum daily discharge. $-0.00 \mathrm{ft}^{3} / \mathrm{s}$.

Human health (harmonic mean) design flow. $-0.2 \mathrm{ft}^{3} / \mathrm{s}$.

Remarks. - None.

\begin{tabular}{|c|c|c|c|}
\hline \multicolumn{4}{|c|}{ Magnitude and frequency of annual low flow } \\
\hline \multicolumn{4}{|c|}{$\begin{array}{l}\text { Lowest average streamflow, in } \mathrm{ft}^{3} / \mathrm{s} \text {, for indicated period of } \\
\text { consecutive days at an annual nonexceedance probability of } 0.1\end{array}$} \\
\hline 1 & \multicolumn{2}{|c|}{7} & 30 \\
\hline 0.0 & \multicolumn{2}{|c|}{0.0} & 0.0 \\
\hline \multicolumn{4}{|c|}{$\begin{array}{l}\text { Percentage of time streamflow was equaled or exceeded for } \\
\text { the period of record }\end{array}$} \\
\hline $\begin{array}{l}\text { Percentage } \\
\text { of time }\end{array}$ & $\begin{array}{l}\text { Daily mean } \\
\text { streamflow } \\
\left(\mathrm{ft}^{3} / \mathrm{s}\right)\end{array}$ & $\begin{array}{l}\text { Percentage } \\
\text { of time }\end{array}$ & $\begin{array}{l}\text { Daily mean } \\
\text { streamflow } \\
\left(\mathrm{ft}^{3} / \mathrm{s}\right)\end{array}$ \\
\hline 99 & 0.0 & 40 & 3.5 \\
\hline 98 & 0.0 & 30 & 5.2 \\
\hline 95 & 0.0 & 20 & 8.3 \\
\hline 90 & 0.1 & 10 & 17 \\
\hline 80 & 0.4 & 5 & 36 \\
\hline 70 & 0.8 & 2 & 78 \\
\hline 60 & 1.5 & 1 & 119 \\
\hline 50 & 2.4 & & \\
\hline
\end{tabular}


Wabash River Basin

\section{DERBYSHIRE CREEK AT SOUTHPORT, IN}

Location. - Lat $39^{\circ} 40^{\prime} 15^{\prime \prime}$, long $86^{\circ} 07^{\prime} 21^{\prime \prime}$ referenced to North American Datum of 1927, in NE 1/4 SE 1/4 Sec.07, T.14 N., R.04 E., Marion County, IN, Hydrologic Unit 05120201, on left bank, $10 \mathrm{ft}$ downstream from bridge on Derbyshire Road, 0.25 mi south of Fairhope Drive, and 0.3 mi upstream from mouth.

Drainage area. $-1.76 \mathrm{mi}^{2}$.

Period of record.- September 1989 to December 2001.

Average discharge. $-2.68 \mathrm{ft}^{3} / \mathrm{s}$.

Minimum daily discharge. $-0.03 \mathrm{ft}^{3} / \mathrm{s}$.

Human health (harmonic mean) design flow. $-0.4 \mathrm{ft}^{3} / \mathrm{s}$.

Remarks. - None.

\begin{tabular}{ccc}
\hline \multicolumn{3}{c}{ Magnitude and frequency of annual low flow } \\
\hline $\begin{array}{c}\text { Lowest average streamflow, in } \mathrm{ft}^{3} / \mathrm{s} \text {, for indicated period of } \\
\text { consecutive days at an annual nonexceedance probability of } 0.1\end{array}$ \\
\hline $\mathbf{7}$ & $\mathbf{7 0}$ \\
\hline 0.0 & 0.0 & 0.1 \\
\hline
\end{tabular}

\begin{tabular}{cc|cc}
\hline \multicolumn{3}{c}{ Percentage of time streamflow was equaled or exceeded for } \\
the period of record
\end{tabular}




\section{LITTLE BUCK CREEK AT SOUTHPORT, IN}

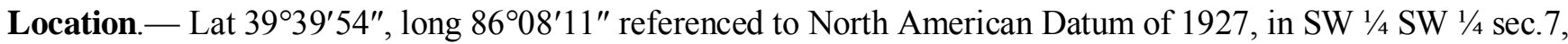
T.14 N., R.4 E., Marion County, IN, Hydrologic Unit 05120201, on left bank $50 \mathrm{ft}$ downstream from Southport Road bridge in Indianapolis, 0.6 mi west of U.S. Highway 31, and at mile 9.52.

Drainage area. $-10.8 \mathrm{mi}^{2}$.

Period of record.- October 1989 to December 2001.

Average discharge. $-16.3 \mathrm{ft}^{3} / \mathrm{s}$.

Minimum daily discharge. $-0.09 \mathrm{ft}^{3} / \mathrm{s}$.

Human health (harmonic mean) design flow. $-1.7 \mathrm{ft}^{3} / \mathrm{s}$.

Remarks.- None.

Magnitude and frequency of annual low flow

Lowest average streamflow, in $\mathrm{ft}^{3} / \mathrm{s}$, for indicated period of consecutive days at an annual nonexceedance probability of 0.1

\begin{tabular}{ccc}
\hline $\mathbf{1}$ & $\mathbf{7}$ & $\mathbf{3 0}$ \\
\hline 0.1 & 0.1 & 0.2 \\
\hline
\end{tabular}

Percentage of time streamflow was equaled or exceeded for the period of record

\begin{tabular}{cc|cc}
\hline $\begin{array}{c}\text { Percentage } \\
\text { of time }\end{array}$ & $\begin{array}{c}\text { Daily mean } \\
\text { streamflow } \\
\left(\mathrm{ft}^{3} / \mathbf{s}\right)\end{array}$ & $\begin{array}{c}\text { Percentage } \\
\text { of time }\end{array}$ & $\begin{array}{c}\text { Daily mean } \\
\text { streamflow } \\
\left(\mathrm{ft}^{3} / \mathbf{s}\right)\end{array}$ \\
\hline 99 & 0.2 & 40 & 8.0 \\
\hline 98 & 0.2 & 30 & 12 \\
\hline 95 & 0.4 & 20 & 18 \\
\hline 90 & 0.7 & 10 & 33 \\
\hline 80 & 1.6 & 5 & 61 \\
\hline 70 & 2.7 & 2 & 134 \\
\hline 60 & 4.1 & 1 & 181 \\
\hline 50 & 5.8 & & \\
\hline
\end{tabular}




\section{LITTLE BUCK CREEK NEAR INDIANAPOLIS, IN}

Location. - Lat $39^{\circ} 40^{\prime} 00^{\prime \prime}$, long $86^{\circ} 11^{\prime} 47^{\prime \prime}$ referenced to North American Datum of 1927, in SW 1/4 SW 1/4 sec.10, T.14 N., R.3 E., Marion County, IN, Hydrologic Unit 05120201, on right bank, $10 \mathrm{ft}$ upstream from bridge on South Belmont Street, $0.75 \mathrm{mi}$ west of State Road 37, $1.5 \mathrm{mi}$ south of Interstate 465, and $2.2 \mathrm{mi}$ above mouth.

Drainage area. $-17.0 \mathrm{mi}^{2}$.

Period of record.- October 1989 to current year.

Average discharge. $-23.5 \mathrm{ft}^{3} / \mathrm{s}$.

Minimum daily discharge. $-0.00 \mathrm{ft}^{3} / \mathrm{s}$.

Human health (harmonic mean) design flow. - $1.5 \mathrm{ft}^{3} / \mathrm{s}$.

Remarks.- None.

\begin{tabular}{|c|c|c|c|}
\hline \multicolumn{4}{|c|}{ Magnitude and frequency of annual low flow } \\
\hline \multicolumn{4}{|c|}{$\begin{array}{l}\text { Lowest average streamflow, in } \mathrm{ft}^{3} / \mathrm{s} \text {, for indicated period of } \\
\text { consecutive days at an annual nonexceedance probability of } 0.1\end{array}$} \\
\hline 1 & \multicolumn{2}{|c|}{7} & 30 \\
\hline 0.0 & \multicolumn{2}{|c|}{0.0} & 0.0 \\
\hline \multicolumn{4}{|c|}{$\begin{array}{l}\text { Percentage of time streamflow was equaled or exceeded for } \\
\text { the period of record }\end{array}$} \\
\hline $\begin{array}{l}\text { Percentage } \\
\text { of time }\end{array}$ & $\begin{array}{l}\text { Daily mean } \\
\text { streamflow } \\
\left(\mathrm{ft}^{3} / \mathrm{s}\right)\end{array}$ & $\begin{array}{l}\text { Percentage } \\
\text { of time }\end{array}$ & $\begin{array}{l}\text { Daily mean } \\
\text { streamflow } \\
\left(\mathrm{ft}^{3} / \mathrm{s}\right)\end{array}$ \\
\hline 99 & 0.0 & 40 & 13 \\
\hline 98 & 0.0 & 30 & 18 \\
\hline 95 & 0.0 & 20 & 26 \\
\hline 90 & 0.0 & 10 & 49 \\
\hline 80 & 1.7 & 5 & 87 \\
\hline 70 & 4.0 & 2 & 179 \\
\hline 60 & 6.1 & 1 & 280 \\
\hline 50 & 8.8 & & \\
\hline
\end{tabular}




\section{WHITE RIVER AT WAVERLY, IN}

Location. - Lat 39³3'35", long 86 $16^{\prime} 29^{\prime \prime}$ referenced to North American Datum of 1927, in NW 1/4 NE 1/4 sec.23., T.13., R.2E., Morgan County, IN, Hydrologic Unit 05120201, on left bank $82 \mathrm{ft}$ upstream from bridge on State Highway 144, 0.6 mi downstream from North Bluff Creek, and at mile 211.0.

Drainage area. $-2,026 \mathrm{mi}^{2}$.

Period of record.- July 1986 to September 1988.

Average discharge. $-1,253 \mathrm{ft}^{3} / \mathrm{s}$.

Minimum daily discharge. $-247 \mathrm{ft}^{3} / \mathrm{s}$.

Human health (harmonic mean) design flow. $-743 \mathrm{ft}^{3} / \mathrm{s}$.

Remarks. - This site has less than 10 years of record.

\begin{tabular}{ccc}
\hline \multicolumn{3}{c}{ Magnitude and frequency of annual low flow } \\
\hline $\begin{array}{c}\text { Lowest average streamflow, in } \mathrm{ft}^{3} / \mathrm{s} \text {, for indicated period of } \\
\text { consecutive days at an annual nonexceedance probability of } \mathbf{0 . 1}\end{array}$ \\
\hline $\mathbf{1}$ & $\mathbf{3 0}$ \\
\hline 268 & 279 & 296 \\
\hline
\end{tabular}

\begin{tabular}{cc|cc}
\hline \multicolumn{5}{c}{ Percentage of time streamflow was equaled or exceeded for } \\
the period of record
\end{tabular}




\section{WEST FORK WHITE LICK CREEK AT DANVILLE, IN}

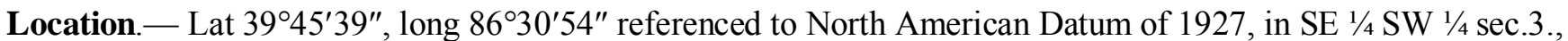
T.15 N., R.1 W., Hendricks County, IN, Hydrologic Unit 05120201, at Danville Filtration Plant, $600 \mathrm{ft}$ upstream of U.S. Highway 36 bridge, 0.6 mi upstream from small left-bank tributary, and 7 mi west of Avon.

Drainage area. $-28.8 \mathrm{mi}^{2}$.

Period of record. - May 1958 to October 2003.

Average discharge. $-31.4 \mathrm{ft}^{3} / \mathrm{s}$.

Minimum daily discharge. $-0.0 \mathrm{ft}^{3} / \mathrm{s}$.

Human health (harmonic mean) design flow. $-0.5 \mathrm{ft}^{3} / \mathrm{s}$.

Remarks. - Low flow affected by releases from Danville Filtration Plant.

\begin{tabular}{ccc}
\hline \multicolumn{3}{c}{ Magnitude and frequency of annual low flow } \\
\hline $\begin{array}{c}\text { Lowest average streamflow, in } \mathrm{ft}^{3} / \mathrm{s} \text {, for indicated period of } \\
\text { consecutive days at an annual nonexceedance probability of } 0.1\end{array}$ \\
\hline $\mathbf{1}$ & $\mathbf{3 0}$ \\
\hline 0.0 & 0.0 & 0.0 \\
\hline
\end{tabular}

Percentage of time streamflow was equaled or exceeded for the period of record

\begin{tabular}{cc|cc}
\hline $\begin{array}{c}\text { Percentage } \\
\text { of time }\end{array}$ & $\begin{array}{c}\text { Daily mean } \\
\text { streamflow } \\
\left(\mathrm{ft}^{3} / \mathbf{s}\right)\end{array}$ & $\begin{array}{c}\text { Percentage } \\
\text { of time }\end{array}$ & $\begin{array}{c}\text { Daily mean } \\
\text { streamflow } \\
\left(\mathrm{ft}^{3} / \mathbf{s}\right)\end{array}$ \\
\hline 99 & 0.0 & 40 & 14 \\
\hline 98 & 0.0 & 30 & 21 \\
\hline 95 & 0.1 & 20 & 35 \\
\hline 90 & 0.2 & 10 & 74 \\
\hline 80 & 0.8 & 5 & 136 \\
\hline 70 & 2.2 & 2 & 250 \\
\hline 60 & 4.8 & 1 & 384 \\
\hline 50 & 8.4 & & \\
\hline
\end{tabular}




\section{WHITE LICK CREEK AT MOORSEVILLE, IN}

Location.- Lat $39^{\circ} 36^{\prime} 28^{\prime \prime}$, long $86^{\circ} 22^{\prime} 56^{\prime \prime}$ referenced to North American Datum of 1927, in NE 1/4 SE 1/4 Sec.35, T.14 N., R.1 E., Morgan County, IN, Hydrologic Unit 05120201, on bridge rail center of upstream side of bridge on State Highway 42 at Mooresville, 0.9 mi downstream from McCracken Creek, 2.0 mi upstream from East Fork White Lick Creek, and at mile 11.4.

Drainage area. $-212 \mathrm{mi}^{2}$.

Period of record. - August 1957 to current year.

Average discharge. $-234 \mathrm{ft}^{3} / \mathrm{s}$.

Minimum daily discharge. $-0.68 \mathrm{ft}^{3} / \mathrm{s}$.

Human health (harmonic mean) design flow. $-36 \mathrm{ft}^{3} / \mathrm{s}$.

Remarks. - Pumpage from a well field upstream of gage may affect low flows.

\begin{tabular}{|c|c|c|c|}
\hline \multicolumn{4}{|c|}{ Magnitude and frequency of annual low flow } \\
\hline \multicolumn{4}{|c|}{$\begin{array}{l}\text { Lowest average streamflow, in } \mathrm{ft}^{3} / \mathrm{s} \text {, for indicated period of } \\
\text { consecutive days at an annual nonexceedance probability of } 0.1\end{array}$} \\
\hline 1 & \multicolumn{2}{|c|}{7} & 30 \\
\hline 3.0 & \multicolumn{2}{|c|}{3.9} & 5.6 \\
\hline \multicolumn{4}{|c|}{$\begin{array}{l}\text { Percentage of time streamflow was equaled or exceeded for } \\
\text { the period of record }\end{array}$} \\
\hline $\begin{array}{l}\text { Percentage } \\
\text { of time }\end{array}$ & $\begin{array}{l}\text { Daily mean } \\
\text { streamflow } \\
\left(\mathrm{ft}^{3} / \mathrm{s}\right)\end{array}$ & $\begin{array}{l}\text { Percentage } \\
\text { of time }\end{array}$ & $\begin{array}{l}\text { Daily mean } \\
\text { streamflow } \\
\left(\mathrm{ft}^{3} / \mathrm{s}\right)\end{array}$ \\
\hline 99 & 4.6 & 40 & 133 \\
\hline 98 & 6.1 & 30 & 185 \\
\hline 95 & 9.4 & 20 & 280 \\
\hline 90 & 15 & 10 & 505 \\
\hline 80 & 27 & 5 & 834 \\
\hline 70 & 42 & 2 & 1580 \\
\hline 60 & 66 & 1 & 2570 \\
\hline 50 & 96 & & \\
\hline
\end{tabular}




\section{EAST FORK WHITE LICK CREEK AT BRIDGEPORT, IN}

Location. - Lat $39^{\circ} 43^{\prime} 45^{\prime \prime}$, long $86^{\circ} 19^{\prime} 22^{\prime \prime}$ referenced to North American Datum of 1927, in sec.21, T.15 N., R.2 E., Marion County, IN, Hydrologic Unit 05120201, on the left upstream side of abandoned bridge, on the west side of Bridgeport, 3.2 mi west of I-465 and at river mile 11.48.

Drainage area. $-23.6 \mathrm{mi}^{2}$.

Period of record.- October 2006 to current year.

Average discharge. $-38.5 \mathrm{ft}^{3} / \mathrm{s}$.

Minimum daily discharge. $-1.0 \mathrm{ft}^{3} / \mathrm{s}$.

Human health (harmonic mean) design flow. $-5.3 \mathrm{ft}^{3} / \mathrm{s}$.

Remarks. - This site has less than 10 years of record.

\begin{tabular}{|c|c|c|c|}
\hline \multicolumn{4}{|c|}{ Magnitude and frequency of annual low flow } \\
\hline \multicolumn{4}{|c|}{$\begin{array}{l}\text { Lowest average streamflow, in } \mathrm{ft}^{3} / \mathrm{s} \text {, for indicated period of } \\
\text { consecutive days at an annual nonexceedance probability of } 0.1\end{array}$} \\
\hline 1 & \multicolumn{2}{|c|}{7} & 30 \\
\hline 0.7 & \multicolumn{2}{|c|}{0.8} & 1.1 \\
\hline \multicolumn{4}{|c|}{$\begin{array}{c}\text { Percentage of time streamflow was equaled or exceeded for } \\
\text { the period of record }\end{array}$} \\
\hline $\begin{array}{l}\text { Percentage } \\
\text { of time }\end{array}$ & $\begin{array}{c}\text { Daily mean } \\
\text { streamflow } \\
\left(\mathrm{ft}^{3} / \mathrm{s}\right)\end{array}$ & $\begin{array}{l}\text { Percentage } \\
\text { of time }\end{array}$ & $\begin{array}{c}\text { Daily mean } \\
\text { streamflow } \\
\left(\mathrm{ft}^{3} / \mathrm{s}\right)\end{array}$ \\
\hline 99 & 1.5 & 40 & 24 \\
\hline 98 & 1.8 & 30 & 32 \\
\hline 95 & 2.2 & 20 & 45 \\
\hline 90 & 2.8 & 10 & 76 \\
\hline 80 & 4.7 & 5 & 124 \\
\hline 70 & 8.3 & 2 & 232 \\
\hline 60 & 13 & 1 & 395 \\
\hline 50 & 18 & & \\
\hline
\end{tabular}




\section{EAST FORK WHITE LICK CREEK NEAR CAMBY, IN}

Location.- Lat $39^{\circ} 41^{\prime} 22^{\prime \prime}$, long $86^{\circ} 20^{\prime} 00^{\prime \prime}$ referenced to North American Datum of 1927, in SE 1/4 SW 1/4 NE 1/4 sec.5, T.14 N., R.2 E., Hendricks County, IN, Hydrologic Unit 05120201, on right upstream wingwall of bridge, 0.15 mi north of intersection of I-70 and 6 Points Road (Ronald Regan Expressway), 1 mi north of U.S Hwy 40, 3 mi west of Indianapolis and at river mile 8.24.

Drainage area. $-33.0 \mathrm{mi}^{2}$.

Period of record. - November 2006 to current year.

Average discharge. $-57.7 \mathrm{ft}^{3} / \mathrm{s}$

Minimum daily discharge. $-1.1 \mathrm{ft}^{3} / \mathrm{s}$.

Human health (harmonic mean) design flow. $-7.7 \mathrm{ft}^{3} / \mathrm{s}$.

Remarks. - This site has less than 10 years of record.

\begin{tabular}{|c|c|c|c|}
\hline \multicolumn{4}{|c|}{ Magnitude and frequency of annual low flow } \\
\hline \multicolumn{4}{|c|}{$\begin{array}{l}\text { Lowest average streamflow, in } \mathrm{ft}^{3} / \mathrm{s} \text {, for indicated period of } \\
\text { consecutive days at an annual nonexceedance probability of } 0 .\end{array}$} \\
\hline 1 & \multicolumn{2}{|c|}{7} & 30 \\
\hline 1.6 & \multicolumn{2}{|c|}{1.9} & 2.3 \\
\hline \multicolumn{4}{|c|}{$\begin{array}{l}\text { Percentage of time streamflow was equaled or exceeded for } \\
\text { the period of record }\end{array}$} \\
\hline $\begin{array}{l}\text { Percentage } \\
\text { of time }\end{array}$ & $\begin{array}{l}\text { Daily mean } \\
\text { streamflow } \\
\left(\mathrm{ft}^{3} / \mathrm{s}\right)\end{array}$ & $\begin{array}{l}\text { Percentage } \\
\text { of time }\end{array}$ & $\begin{array}{l}\text { Daily mean } \\
\text { streamflow } \\
\left(\mathrm{ft}^{3} / \mathrm{s}\right)\end{array}$ \\
\hline 99 & 1.7 & 40 & 33 \\
\hline 98 & 2 & 30 & 47 \\
\hline 95 & 3 & 20 & 70 \\
\hline 90 & 4.1 & 10 & 116 \\
\hline 80 & 6.6 & 5 & 194 \\
\hline 70 & 11 & 2 & 371 \\
\hline 60 & 17 & 1 & 643 \\
\hline 50 & 24 & & \\
\hline
\end{tabular}


White River Basin

\section{EAST FORK WHITE LICK CREEK NEAR MOORSEVILLE, IN}

Location. - Lat 39 $37^{\prime} 42^{\prime \prime}$, long $86^{\circ} 21^{\prime} 26^{\prime \prime}$ referenced to North American Datum of 1927, in SE 1/4 NE 1/4 NW 1/4 sec.30, T.14 N., R.2 E., Morgan County, IN, Hydrologic Unit 05120201, on right bank $150 \mathrm{ft}$ upstream of covered footbridge in Pioneer Park, $25 \mathrm{ft}$ downstream of Silon Creek, $8.7 \mathrm{mi}$ southwest of Old 67 and at 2.86 river mile.

Drainage area. - $48.0 \mathrm{mi}^{2}$.

Period of record.- March 2007 to current year.

Average discharge. $-76.6 \mathrm{ft}^{3} / \mathrm{s}$.

Minimum daily discharge. $-3.3 \mathrm{ft}^{3} / \mathrm{s}$.

Human health (harmonic mean) design flow. $-11 \mathrm{ft}^{3} / \mathrm{s}$.

Remarks. - This site has less than 10 years of record.

\begin{tabular}{|c|c|c|c|}
\hline \multicolumn{4}{|c|}{ Magnitude and frequency of annual low flow } \\
\hline \multicolumn{4}{|c|}{$\begin{array}{l}\text { Lowest average streamflow, in } \mathrm{ft}^{3} / \mathrm{s} \text {, for indicated period of } \\
\text { consecutive days at an annual nonexceedance probability of } 0.1\end{array}$} \\
\hline 1 & \multicolumn{2}{|c|}{7} & 30 \\
\hline 1.4 & \multicolumn{2}{|c|}{1.8} & 2.4 \\
\hline \multicolumn{4}{|c|}{$\begin{array}{l}\text { Percentage of time streamflow was equaled or exceeded for } \\
\text { the period of record }\end{array}$} \\
\hline $\begin{array}{l}\text { Percentage } \\
\text { of time }\end{array}$ & $\begin{array}{l}\text { Daily mean } \\
\text { streamflow } \\
\left(\mathrm{ft}^{3} / \mathrm{s}\right)\end{array}$ & $\begin{array}{l}\text { Percentage } \\
\text { of time }\end{array}$ & $\begin{array}{l}\text { Daily mean } \\
\text { streamflow } \\
\left(\mathrm{ft}^{3} / \mathrm{s}\right)\end{array}$ \\
\hline 99 & 4.4 & 40 & 48 \\
\hline 98 & 4.9 & 30 & 61 \\
\hline 95 & 5.6 & 20 & 82 \\
\hline 90 & 6.8 & 10 & 142 \\
\hline 80 & 11 & 5 & 255 \\
\hline 70 & 19 & 2 & 486 \\
\hline 60 & 28 & 1 & 846 \\
\hline 50 & 38 & & \\
\hline
\end{tabular}




\section{WHITE RIVER NEAR CENTERTON, IN}

Location. - Lat $39^{\circ} 29^{\prime} 51^{\prime \prime}$, long $86^{\circ} 24^{\prime} 02^{\prime \prime}$ referenced to North American Datum of 1927, in NE 1/4 NE 1/4 Sec.10, T.12 N., R.1 E., Morgan County, IN, Hydrologic Unit 05120201, on right bank at upstream side of bridge on Blue Bluff Road, 0.8 mi downstream from White Lick Creek, 1 mi south of Centerton, and at mile 199.3.

Drainage Area . $-2,444 \mathrm{mi}^{2}$.

Period of record.- October 1930 to March 1932, October 1946 to current year.

Average discharge. $-2,670 \mathrm{ft}^{3} / \mathrm{s}$.

Minimum daily discharge. $-138 \mathrm{ft}^{3} / \mathrm{s}$

Human health (harmonic mean) design flow. - $1001 \mathrm{ft}^{3} / \mathrm{s}$.

Remarks. - Flow may be affected by upstream reservoirs. Low-flow statistics are calculated for the regulated period, 1948 to 2011.

\begin{tabular}{|c|c|c|c|}
\hline \multicolumn{4}{|c|}{ Magnitude and frequency of annual low flow } \\
\hline \multicolumn{4}{|c|}{$\begin{array}{l}\text { Lowest average streamflow, in } \mathrm{ft}^{3} / \mathrm{s} \text {, for indicated period of } \\
\text { consecutive days at an annual nonexceedance probability of } 0.1\end{array}$} \\
\hline 1 & \multicolumn{2}{|c|}{7} & 30 \\
\hline 260 & \multicolumn{2}{|c|}{274} & 297 \\
\hline \multicolumn{4}{|c|}{$\begin{array}{l}\text { Percentage of time streamflow was equaled or exceeded for } \\
\text { the period of record }\end{array}$} \\
\hline $\begin{array}{l}\text { Percentage } \\
\text { of time }\end{array}$ & $\begin{array}{c}\text { Daily mean } \\
\text { streamflow } \\
\left(\mathrm{ft}^{3} / \mathrm{s}\right)\end{array}$ & $\begin{array}{l}\text { Percentage } \\
\text { of time }\end{array}$ & $\begin{array}{c}\text { Daily mean } \\
\text { streamflow } \\
\left(\mathrm{ft}^{3} / \mathrm{s}\right)\end{array}$ \\
\hline 99 & 283 & 40 & 1870 \\
\hline 98 & 302 & 30 & 2500 \\
\hline 95 & 353 & 20 & 3570 \\
\hline 90 & 427 & 10 & 6030 \\
\hline 80 & 598 & 5 & 9530 \\
\hline 70 & 813 & 2 & 15500 \\
\hline 60 & 1090 & 1 & 20000 \\
\hline 50 & 1420 & & \\
\hline
\end{tabular}


Wabash River Basin

\section{BEANBLOSSOM CREEK AT BEANBLOSSOM, IN}

Location. - Lat $39^{\circ} 15^{\prime} 45^{\prime \prime}$, long $86^{\circ} 14^{\prime} 55^{\prime \prime}$ referenced to North American Datum of 1927, in SW 1/4 NW 1/4 sec.31, T.10 N., R.3 E., Brown County, IN, Hydrologic Unit 05120202, on right bank $25 \mathrm{ft}$ upstream from bridge on State Highway 135, $0.3 \mathrm{mi}$ south of Beanblossom, $2.7 \mathrm{mi}$ upstream from North Fork Beanblossom Creek, and at mile 42.1 .

Drainage area. $-14.6 \mathrm{mi}^{2}$.

Period of record.- October 1951 to September 1993.

Average discharge. $-16.0 \mathrm{ft}^{3} / \mathrm{s}$.

Minimum daily discharge. $-0.0 \mathrm{ft}^{3} / \mathrm{s}$

Human health (harmonic mean) design flow. $-0.5 \mathrm{ft}^{3} / \mathrm{s}$.

Remarks. - None.

\begin{tabular}{|c|c|c|c|}
\hline \multicolumn{4}{|c|}{ Magnitude and frequency of annual low flow } \\
\hline \multicolumn{4}{|c|}{$\begin{array}{l}\text { Lowest average streamflow, in } \mathrm{ft}^{3} / \mathrm{s} \text {, for indicated period of } \\
\text { consecutive days at an annual nonexceedance probability of } 0.1\end{array}$} \\
\hline 1 & \multicolumn{2}{|c|}{7} & 30 \\
\hline 0.0 & \multicolumn{2}{|c|}{0.0} & 0.0 \\
\hline \multicolumn{4}{|c|}{$\begin{array}{c}\text { Percentage of time streamflow was equaled or exceeded for } \\
\text { the period of record }\end{array}$} \\
\hline $\begin{array}{l}\text { Percentage } \\
\text { of time }\end{array}$ & $\begin{array}{l}\text { Daily mean } \\
\text { streamflow } \\
\left(\mathrm{ft}^{3} / \mathrm{s}\right)\end{array}$ & $\begin{array}{l}\text { Percentage } \\
\text { of time }\end{array}$ & $\begin{array}{l}\text { Daily mean } \\
\text { streamflow } \\
\left(\mathrm{ft}^{3} / \mathrm{s}\right)\end{array}$ \\
\hline 99 & 0.0 & 40 & 6.4 \\
\hline 98 & 0.0 & 30 & 11 \\
\hline 95 & 0.0 & 20 & 18 \\
\hline 90 & 0.0 & 10 & 35 \\
\hline 80 & 0.3 & 5 & 64 \\
\hline 70 & 0.8 & 2 & 135 \\
\hline 60 & 1.9 & 1 & 228 \\
\hline 50 & 3.6 & & \\
\hline
\end{tabular}




\section{BEAR CREEK NEAR TREVLAC, IN}

Location. - Lat $39^{\circ} 16^{\prime} 40^{\prime \prime}$, long $86^{\circ} 20^{\prime} 45^{\prime \prime}$ referenced to North American Datum of 1927, in NE 1/4 NE 1/4 sec.30, T.10 N., R.2 E., Brown County, IN, Hydrologic Unit 05120202, on left bank $15 \mathrm{ft}$ west of Bear Creek Road, $100 \mathrm{ft}$ upstream from Slippery Elm Shoot Road ford, $1.1 \mathrm{mi}$ northwest of Trevlac, and $1.3 \mathrm{mi}$ upstream from mouth.

Drainage area. $-6.94 \mathrm{mi}^{2}$.

Period of record. - May 1952 to September 1973.

Average discharge. $-6.69 \mathrm{ft}^{3} / \mathrm{s}$

Minimum daily discharge. $-0.0 \mathrm{ft}^{3} / \mathrm{s}$

Human health (harmonic mean) design flow. $-0.3 \mathrm{ft}^{3} / \mathrm{s}$.

Remarks.- None.

Magnitude and frequency of annual low flow

Lowest average streamflow, in $\mathrm{ft}^{3} / \mathrm{s}$, for indicated period of consecutive days at an annual nonexceedance probability of 0.1

\begin{tabular}{ccc}
\hline $\mathbf{1}$ & $\mathbf{7}$ & $\mathbf{3 0}$ \\
\hline 0.0 & 0.0 & 0.0 \\
\hline
\end{tabular}

Percentage of time streamflow was equaled or exceeded for the period of record

\begin{tabular}{|c|c|c|c|}
\hline $\begin{array}{c}\text { Percentage } \\
\text { of time }\end{array}$ & $\begin{array}{c}\text { Daily mean } \\
\text { streamflow } \\
\left(\mathrm{ft}^{3} / \mathrm{s}\right)\end{array}$ & $\begin{array}{l}\text { Percentage } \\
\text { of time }\end{array}$ & $\begin{array}{c}\text { Daily mean } \\
\text { streamflow } \\
\left(\mathrm{ft}^{3} / \mathrm{s}\right)\end{array}$ \\
\hline 99 & 0.0 & 40 & 2.2 \\
\hline 98 & 0.0 & 30 & 4.0 \\
\hline 95 & 0.0 & 20 & 7.2 \\
\hline 90 & 0.0 & 10 & 16 \\
\hline 80 & 0.1 & 5 & 30 \\
\hline 70 & 0.2 & 2 & 62 \\
\hline 60 & 0.5 & 1 & 103 \\
\hline 50 & 1.2 & & \\
\hline
\end{tabular}




\section{BEANBLOSSOM CREEK AT DOLAN, IN}

Location. - Lat $39^{\circ} 14^{\prime} 30^{\prime \prime}$, long $86^{\circ} 29^{\prime} 57^{\prime \prime}$ referenced to North American Datum of 1927, in NW 1/4 SW 1/4 sec.2, T.9 N., R.1 W., Monroe County, IN, Hydrologic Unit 05120202, on downstream side of pier of highway bridge at Dolan, $5.8 \mathrm{mi}$ northeast of Bloomington, $8.2 \mathrm{mi}$ downstream from Lake Lemon, and $21.5 \mathrm{mi}$ upstream from mouth.

Drainage area. $-100 \mathrm{mi}^{2}$.

Period of record.- April 1946 to September 1978.

Average discharge. $-111 \mathrm{ft}^{3} / \mathrm{s}$.

Minimum daily discharge. $-0.0 \mathrm{ft}^{3} / \mathrm{s}$

Human health (harmonic mean) design flow. $-18 \mathrm{ft}^{3} / \mathrm{s}$.

Remarks. - Flow regulated by Lake Lemon 8.2 mi upstream. Low-flow statistics calculated for the regulated period, 1953 to 1978.

\begin{tabular}{ccc}
\hline \multicolumn{3}{c}{ Magnitude and frequency of annual low flow } \\
\hline $\begin{array}{c}\text { Lowest average streamflow, in } \mathrm{ft}^{3} / \mathrm{s} \text {, for indicated period of } \\
\text { consecutive days at an annual nonexceedance probability of } 0.1\end{array}$ \\
\hline $\mathbf{1}$ & $\mathbf{7 0}$ & $\mathbf{3 0}$ \\
\hline 1.6 & 2.9 & 8.0 \\
\hline
\end{tabular}

Percentage of time streamflow was equaled or exceeded for the period of record

\begin{tabular}{cc|cc}
\hline $\begin{array}{c}\text { Percentage } \\
\text { of time }\end{array}$ & $\begin{array}{c}\text { Daily mean } \\
\text { streamflow } \\
\left(\mathrm{ft}^{3} / \mathbf{s}\right)\end{array}$ & $\begin{array}{c}\text { Percentage } \\
\text { of time }\end{array}$ & $\begin{array}{c}\text { Daily mean } \\
\text { streamflow } \\
\left(\mathrm{ft}^{3} / \mathbf{s}\right)\end{array}$ \\
\hline 99 & 4.4 & 40 & 37 \\
\hline 98 & 6.5 & 30 & 63 \\
\hline 95 & 11 & 20 & 110 \\
\hline 90 & 13 & 10 & 232 \\
\hline 80 & 16 & 5 & 419 \\
\hline 70 & 19 & 2 & 820 \\
\hline 60 & 22 & 1 & 1220 \\
\hline 50 & 26 & & \\
\hline
\end{tabular}




\section{WHITE RIVER AT SPENCER, IN}

Location. - Lat $39^{\circ} 16^{\prime} 52^{\prime \prime}$, long $86^{\circ} 45^{\prime} 44^{\prime \prime}$ referenced to North American Datum of 1927, in NE 1/4 NE 1/4 Sec.29, T.10 N., R.3 W., Owen County, IN, Hydrologic Unit 05120202, on right bank at upstream side of county road bridge at the south edge of Spencer, 3.3 mi upstream from McBrides Creek, 14 mi northwest of Bloomington, and at mile 165.9 .

Drainage area. $-2,988 \mathrm{mi}^{2}$.

Period of record. - July 1925 to September 1971.

Average discharge. $-2,961 \mathrm{ft}^{3} / \mathrm{s}$.

Minimum daily discharge. $-135 \mathrm{ft}^{3} / \mathrm{s}$

Human health (harmonic mean) design flow. $-936 \mathrm{ft}^{3} / \mathrm{s}$.

Remarks. - Natural flow of stream affected by storage reservoirs.

\begin{tabular}{ccc}
\hline \multicolumn{3}{c}{ Magnitude and frequency of annual low flow } \\
\hline $\begin{array}{c}\text { Lowest average streamflow, in } \mathrm{ft}^{3} / \mathrm{s} \text {, for indicated period of } \\
\text { consecutive days at an annual nonexceedance probability of } \mathbf{0 . 1}\end{array}$ \\
\hline $\mathbf{7}$ & $\mathbf{3 0}$ \\
\hline 215 & 226 & 259 \\
\hline
\end{tabular}

Percentage of time streamflow was equaled or exceeded for the period of record

\begin{tabular}{cc|cc}
\hline $\begin{array}{c}\text { Percentage } \\
\text { of time }\end{array}$ & $\begin{array}{c}\text { Daily mean } \\
\text { streamflow } \\
\left(\mathrm{ft}^{3} / \mathbf{s}\right)\end{array}$ & $\begin{array}{c}\text { Percentage } \\
\text { of time }\end{array}$ & $\begin{array}{c}\text { Daily mean } \\
\text { streamflow } \\
\left(\mathrm{ft}^{3} / \mathbf{s}\right)\end{array}$ \\
\hline 99 & 237 & 40 & 1930 \\
\hline 98 & 277 & 30 & 2690 \\
\hline 95 & 329 & 20 & 3900 \\
\hline 90 & 402 & 10 & 6900 \\
\hline 80 & 542 & 5 & 11500 \\
\hline 70 & 730 & 2 & 18300 \\
\hline 60 & 1020 & 1 & 24100 \\
\hline 50 & 1410 & & \\
\hline
\end{tabular}




\section{BIG WALNUT CREEK NEAR ROACHDALE, IN}

Location.- Lat $39^{\circ} 48^{\prime} 58^{\prime \prime}$, long $86^{\circ} 45^{\prime} 12^{\prime \prime}$ referenced to North American Datum of 1927, in SE 1/4 NW 1/4 sec.21, T.16 N., R.3 W., Putnam County, IN, Hydrologic Unit 05120203, on right upstream bank at County Road 1100S bridge, 3.4 mi southeast of Roachdale, $9.06 \mathrm{mi}$ upstream from confluence with Plum Creek, and at mile 39.16.

Drainage area. $-131 \mathrm{mi}^{2}$.

Period of record.- October 2001 to current year.

Average discharge. $-171 \mathrm{ft}^{3} / \mathrm{s}$.

Minimum daily discharge. $-0.0 \mathrm{ft}^{3} / \mathrm{s}$.

Human health (harmonic mean) design flow. $-20 \mathrm{ft}^{3} / \mathrm{s}$.

Remarks.- None.

\begin{tabular}{|c|c|c|c|}
\hline \multicolumn{4}{|c|}{ Magnitude and frequency of annual low flow } \\
\hline \multicolumn{4}{|c|}{$\begin{array}{l}\text { Lowest average streamflow, in } \mathrm{ft}^{3} / \mathrm{s} \text {, for indicated period of } \\
\text { consecutive days at an annual nonexceedance probability of } 0.1\end{array}$} \\
\hline 1 & \multicolumn{2}{|c|}{7} & 30 \\
\hline 1.3 & \multicolumn{2}{|c|}{1.4} & 1.9 \\
\hline \multicolumn{4}{|c|}{$\begin{array}{l}\text { Percentage of time streamflow was equaled or exceeded for } \\
\text { the period of record }\end{array}$} \\
\hline $\begin{array}{l}\text { Percentage } \\
\text { of time }\end{array}$ & $\begin{array}{l}\text { Daily mean } \\
\text { streamflow } \\
\left(\mathrm{ft}^{3} / \mathrm{s}\right)\end{array}$ & $\begin{array}{l}\text { Percentage } \\
\text { of time }\end{array}$ & $\begin{array}{l}\text { Daily mean } \\
\text { streamflow } \\
\left(\mathrm{ft}^{3} / \mathrm{s}\right)\end{array}$ \\
\hline 99 & 1.5 & 40 & 100 \\
\hline 98 & 2.2 & 30 & 140 \\
\hline 95 & 5.0 & 20 & 216 \\
\hline 90 & 7.7 & 10 & 378 \\
\hline 80 & 16 & 5 & 639 \\
\hline 70 & 31 & 2 & 1210 \\
\hline 60 & 52 & 1 & 1960 \\
\hline 50 & 74 & & \\
\hline
\end{tabular}




\section{PLUM CREEK NEAR BAINBRIDGE, IN}

Location. - Lat $39^{\circ} 45^{\prime} 42^{\prime \prime}$, long $86^{\circ} 43^{\prime} 46^{\prime \prime}$ referenced to North American Datum of 1927, in SW 1/4 SE $1 / 4 \mathrm{sec} .3$, T.15 N., R.3 W., Putnam County, IN, Hydrologic Unit 05120203, on right upstream wingwall of bridge on U.S. Highway 36, $0.5 \mathrm{mi}$ west of Groveland, and $4.5 \mathrm{mi}$ east of Bainbridge.

Drainage area. $-3.0 \mathrm{mi}^{2}$.

Period of record.- July 1969 to current year.

Average discharge. $-3.71 \mathrm{ft}^{3} / \mathrm{s}$.

Minimum daily discharge. $-0.0 \mathrm{ft}^{3} / \mathrm{s}$.

Human health (harmonic mean) design flow. - $0.1 \mathrm{ft}^{3} / \mathrm{s}$.

Remarks.- None.

\begin{tabular}{|c|c|c|c|}
\hline \multicolumn{4}{|c|}{ Magnitude and frequency of annual low flow } \\
\hline \multicolumn{4}{|c|}{$\begin{array}{l}\text { Lowest average streamflow, in } \mathrm{ft}^{3} / \mathrm{s} \text {, for indicated period of } \\
\text { consecutive days at an annual nonexceedance probability of } 0.1\end{array}$} \\
\hline 1 & \multicolumn{2}{|c|}{7} & 30 \\
\hline 0.0 & \multicolumn{2}{|c|}{0.0} & 0.0 \\
\hline \multicolumn{4}{|c|}{$\begin{array}{c}\text { Percentage of time streamflow was equaled or exceeded for } \\
\text { the period of record }\end{array}$} \\
\hline $\begin{array}{l}\text { Percentage } \\
\text { of time }\end{array}$ & $\begin{array}{c}\text { Daily mean } \\
\text { streamflow } \\
\left(\mathrm{ft}^{3} / \mathrm{s}\right)\end{array}$ & $\begin{array}{l}\text { Percentage } \\
\text { of time }\end{array}$ & $\begin{array}{c}\text { Daily mean } \\
\text { streamflow } \\
\left(\mathrm{ft}^{3} / \mathrm{s}\right)\end{array}$ \\
\hline 99 & 0.0 & 40 & 1.6 \\
\hline 98 & 0.0 & 30 & 2.6 \\
\hline 95 & 0.0 & 20 & 4.2 \\
\hline 90 & 0.0 & 10 & 7.8 \\
\hline 80 & 0.1 & 5 & 14 \\
\hline 70 & 0.3 & 2 & 31 \\
\hline 60 & 0.6 & 1 & 52 \\
\hline 50 & 1.1 & & \\
\hline
\end{tabular}




\section{BIG WALNUT CREEK NEAR REELSVILLE, IN}

Location. - Lat 39 $32^{\prime} 11^{\prime \prime}$, long $86^{\circ} 58^{\prime} 35^{\prime \prime}$ referenced to North American Datum of 1927, in NW 1/4 SW 1/4 sec.28, T.13 N., R.5 W., Putnam County, IN, Hydrologic Unit 05120203, on left bank at downstream side of county highway bridge, $1.5 \mathrm{mi}$ southwest of Reelsville, $3.8 \mathrm{mi}$ southwest of Manhattan, and 4.1 mi upstream from Mill Creek.

Drainage area.- $326 \mathrm{mi}^{2}$.

Period of record. - July 1949 to September 2002.

Average discharge. $-353.8 \mathrm{ft}^{3} / \mathrm{s}$.

Minimum daily discharge. $-1.4 \mathrm{ft}^{3} / \mathrm{s}$.

Human health (harmonic mean) design flow. $-54 \mathrm{ft}^{3} / \mathrm{s}$.

Remarks. - Flow partly regulated by U.S. Soil Conservation Service control structures on tributaries to Little Walnut Creek beginning in 1971. Published as Eel River near Reelsville, October 1952 to September 1956.

\begin{tabular}{|c|c|c|c|}
\hline \multicolumn{4}{|c|}{ Magnitude and frequency of annual low flow } \\
\hline \multicolumn{4}{|c|}{$\begin{array}{l}\text { Lowest average streamflow, in } \mathrm{ft}^{3} / \mathrm{s} \text {, for indicated period of } \\
\text { consecutive days at an annual nonexceedance probability of } 0.1\end{array}$} \\
\hline 1 & \multicolumn{2}{|c|}{7} & 30 \\
\hline 3.8 & \multicolumn{2}{|c|}{4.6} & 5.6 \\
\hline \multicolumn{4}{|c|}{$\begin{array}{l}\text { Percentage of time streamflow was equaled or exceeded for } \\
\text { the period of record }\end{array}$} \\
\hline $\begin{array}{l}\text { Percentage } \\
\text { of time }\end{array}$ & $\begin{array}{l}\text { Daily mean } \\
\text { streamflow } \\
\left(\mathrm{ft}^{3} / \mathrm{s}\right)\end{array}$ & $\begin{array}{l}\text { Percentage } \\
\text { of time }\end{array}$ & $\begin{array}{l}\text { Daily mean } \\
\text { streamflow } \\
\left(\mathrm{ft}^{3} / \mathrm{s}\right)\end{array}$ \\
\hline 99 & 5.6 & 40 & 209 \\
\hline 98 & 8.1 & 30 & 291 \\
\hline 95 & 14 & 20 & 436 \\
\hline 90 & 22 & 10 & 781 \\
\hline 80 & 43 & 5 & 1280 \\
\hline 70 & 70 & 2 & 2440 \\
\hline 60 & 106 & 1 & 3810 \\
\hline 50 & 151 & & \\
\hline
\end{tabular}




\section{MILL CREEK NEAR CATARACT, IN}

Location. - Lat $39^{\circ} 26^{\prime} 00^{\prime \prime}$, long $86^{\circ} 45^{\prime} 48^{\prime \prime}$ referenced to North American Datum of 1927, in NE 1/4 SE 1/4 Sec.32, T.12 N., R.3 W., Owen County, IN, Hydrologic Unit 05120203, on right bank at downstream side of bridge on U.S. Highway 231, 3 mi east of Cataract, $5.7 \mathrm{mi}$ south of Cloverdale, and at mile 17.5.

Drainage area. $-245 \mathrm{mi}^{2}$.

Period of record.- July 1949 to current year.

Average discharge. $-281 \mathrm{ft}^{3} / \mathrm{s}$.

Minimum daily discharge. $-0.10 \mathrm{ft}^{3} / \mathrm{s}$.

Human health (harmonic mean) design flow. $-21 \mathrm{ft}^{3} / \mathrm{s}$.

Remarks.- Nov. 8, 1949 to Sept. 22, 1968, water-stage recorder at site $100 \mathrm{ft}$ upstream at same datum.

\begin{tabular}{ccc}
\hline \multicolumn{3}{c}{ Magnitude and frequency of annual low flow } \\
\hline $\begin{array}{c}\text { Lowest average streamflow, in } \mathrm{ft}^{3} / \mathrm{s}, \text { for indicated period of } \\
\text { consecutive days at an annual nonexceedance probability of } \mathbf{0 . 1}\end{array}$ \\
\hline $\mathbf{1}$ & $\mathbf{7}$ & $\mathbf{3 0}$ \\
\hline 1.4 & 1.8 & 2.9 \\
\hline
\end{tabular}

\begin{tabular}{cc|cc}
\hline Percentage of time streamflow was equaled or exceeded for \\
the period of record
\end{tabular}




\section{MILL CREEK NEAR MANHATTAN, IN}

Location.- Lat $39^{\circ} 29^{\prime} 15^{\prime \prime}$, long $86^{\circ} 55^{\prime} 29^{\prime \prime}$ referenced to North American Datum of 1927, in SE 1/4 SE 1/4 sec.11, T.12 N., R.5 W., Putnam County, IN, Hydrologic Unit 05120203, on left bank 0.3 mi upstream from Cagles Mill Dam, 0.4 mi downstream from Cagles Mill Lake, 1.3 mi upstream from Deer Creek, 5.0 mi south of Manhattan, and at mile 2.3.

Drainage area.- $294 \mathrm{mi}^{2}$.

Period of record.- October 1938 to September 2001.

Average discharge. $-321 \mathrm{ft}^{3} / \mathrm{s}$.

Minimum daily discharge. $-0.0 \mathrm{ft}^{3} / \mathrm{s}$.

Human health (harmonic mean) design flow. $-21 \mathrm{ft}^{3} / \mathrm{s}$.

Remarks. - Flow regulated by U.S. Army Corps of Engineers from Cagles Mill Lake since July 1953. Records of daily discharge provided by U.S. Army Corps of Engineers beginning Oct. 1, 1976. Low-flow statistics calculated for the regulated period, 1953 to 2001.

\begin{tabular}{|c|c|c|c|}
\hline \multicolumn{4}{|c|}{ Magnitude and frequency of annual low flow } \\
\hline \multicolumn{4}{|c|}{$\begin{array}{l}\text { Lowest average streamflow, in } \mathrm{ft}^{3} / \mathrm{s} \text {, for indicated period of } \\
\text { consecutive days at an annual nonexceedance probability of } 0 .\end{array}$} \\
\hline 1 & \multicolumn{2}{|c|}{7} & 30 \\
\hline 0.9 & \multicolumn{2}{|c|}{1.4} & 2.4 \\
\hline \multicolumn{4}{|c|}{$\begin{array}{l}\text { Percentage of time streamflow was equaled or exceeded for } \\
\text { the period of record }\end{array}$} \\
\hline $\begin{array}{l}\text { Percentage } \\
\text { of time }\end{array}$ & $\begin{array}{l}\text { Daily mean } \\
\text { streamflow } \\
\left(\mathrm{ft}^{3} / \mathrm{s}\right)\end{array}$ & $\begin{array}{l}\text { Percentage } \\
\text { of time }\end{array}$ & $\begin{array}{l}\text { Daily mean } \\
\text { streamflow } \\
\left(\mathrm{ft}^{3} / \mathrm{s}\right)\end{array}$ \\
\hline 99 & 1.8 & 40 & 132 \\
\hline 98 & 2.4 & 30 & 227 \\
\hline 95 & 4.3 & 20 & 487 \\
\hline 90 & 13 & 10 & 1210 \\
\hline 80 & 22 & 5 & 1690 \\
\hline 70 & 44 & 2 & 2110 \\
\hline 60 & 77 & 1 & 2340 \\
\hline 50 & 102 & & \\
\hline
\end{tabular}




\section{DEER CREEK NEAR PUTNAMVILLE, IN}

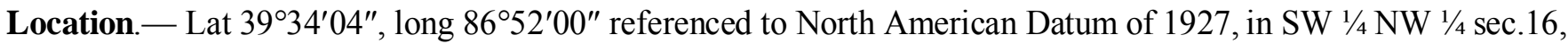
T.13 N., R.4 W., Putnam County, IN, Hydrologic Unit 05120203, on right bank at downstream side of bridge on State Highway 243, $0.4 \mathrm{mi}$ southwest of Putnamville, 0.4 mi downstream from small left-bank tributary, and $0.8 \mathrm{mi}$ downstream from Limestone Creek.

Drainage area. $-59.0 \mathrm{mi}^{2}$.

Period of record.— October 1954 to September 1965, October 1967 to September 1972.

Average discharge. $-59.9 \mathrm{ft}^{3} / \mathrm{s}$.

Minimum daily discharge. $-0.00 \mathrm{ft}^{3} / \mathrm{s}$.

Human health (harmonic mean) design flow. $-2.5 \mathrm{ft}^{3} / \mathrm{s}$.

Remarks. - None.

\begin{tabular}{|c|c|c|c|}
\hline \multicolumn{4}{|c|}{ Magnitude and frequency of annual low flow } \\
\hline \multicolumn{4}{|c|}{$\begin{array}{l}\text { Lowest average streamflow, in } \mathrm{ft}^{3} / \mathrm{s} \text {, for indicated period of } \\
\text { consecutive days at an annual nonexceedance probability of } 0.1\end{array}$} \\
\hline 1 & \multicolumn{2}{|c|}{7} & 30 \\
\hline 0.1 & \multicolumn{2}{|c|}{0.1} & 0.2 \\
\hline \multicolumn{4}{|c|}{$\begin{array}{l}\text { Percentage of time streamflow was equaled or exceeded for } \\
\text { the period of record }\end{array}$} \\
\hline $\begin{array}{l}\text { Percentage } \\
\text { of time }\end{array}$ & $\begin{array}{l}\text { Daily mean } \\
\text { streamflow } \\
\left(\mathrm{ft}^{3} / \mathrm{s}\right)\end{array}$ & $\begin{array}{l}\text { Percentage } \\
\text { of time }\end{array}$ & $\begin{array}{l}\text { Daily mean } \\
\text { streamflow } \\
\left(\mathrm{ft}^{3} / \mathrm{s}\right)\end{array}$ \\
\hline 99 & 0.1 & 40 & 27 \\
\hline 98 & 0.2 & 30 & 39 \\
\hline 95 & 0.6 & 20 & 59 \\
\hline 90 & 1.2 & 10 & 108 \\
\hline 80 & 3.1 & 5 & 204 \\
\hline 70 & 6.1 & 2 & 477 \\
\hline 60 & 11 & 1 & 879 \\
\hline 50 & 18 & & \\
\hline
\end{tabular}


Wabash River Basin

\section{EEL RIVER AT BOWLING GREEN, IN}

Location. - Lat $39^{\circ} 22^{\prime} 58^{\prime \prime}$, long $87^{\circ} 01^{\prime} 14^{\prime \prime}$ referenced to North American Datum of 1927, in NE 1/4 NE 1/4 Sec.24, T.11 N., R.6 W., Clay County, IN, Hydrologic Unit 05120203, on left bank $500 \mathrm{ft}$ downstream from bridge on State Highway 46 at Bowling Green, 0.2 mi downstream from Jordan Creek, 15 mi northwest of Spencer, and at mile 38.4.

Drainage area. $-830 \mathrm{mi}^{2}$.

Period of record. - January 1931 to current year.

Average discharge. - $930 \mathrm{ft}^{3} / \mathrm{s}$.

Minimum daily discharge. $-11 \mathrm{ft}^{3} / \mathrm{s}$.

Human health (harmonic mean) design flow. $-175 \mathrm{ft}^{3} / \mathrm{s}$.

Remarks. - Flow regulated by Cagles Mill Lake. Low-flow statistics calculated for the regulated period, 1953 to 2011.

\begin{tabular}{|c|c|c|c|}
\hline \multicolumn{4}{|c|}{ Magnitude and frequency of annual low flow } \\
\hline \multicolumn{4}{|c|}{$\begin{array}{l}\text { Lowest average streamflow, in } \mathrm{ft}^{3} / \mathrm{s} \text {, for indicated period of } \\
\text { consecutive days at an annual nonexceedance probability of } 0.1\end{array}$} \\
\hline 1 & \multicolumn{2}{|c|}{7} & 30 \\
\hline 22 & \multicolumn{2}{|c|}{23} & 27 \\
\hline \multicolumn{4}{|c|}{$\begin{array}{c}\text { Percentage of time streamflow was equaled or exceeded for } \\
\text { the period of record }\end{array}$} \\
\hline $\begin{array}{l}\text { Percentage } \\
\text { of time }\end{array}$ & $\begin{array}{l}\text { Daily mean } \\
\text { streamflow } \\
\left(\mathrm{ft}^{3} / \mathrm{s}\right)\end{array}$ & $\begin{array}{l}\text { Percentage } \\
\text { of time }\end{array}$ & $\begin{array}{l}\text { Daily mean } \\
\text { streamflow } \\
\left(\mathrm{ft}^{3} / \mathrm{s}\right)\end{array}$ \\
\hline 99 & 24 & 40 & 729 \\
\hline 98 & 30 & 30 & 1210 \\
\hline 95 & 43 & 20 & 1690 \\
\hline 90 & 65 & 10 & 2260 \\
\hline 80 & 118 & 5 & 2880 \\
\hline 70 & 189 & 2 & 4530 \\
\hline 60 & 298 & 1 & 6620 \\
\hline 50 & 449 & & \\
\hline
\end{tabular}




\section{WHITE RIVER AT NEWBERRY, IN}

Location. - Lat $38^{\circ} 55^{\prime} 39^{\prime \prime}$, long $87^{\circ} 00^{\prime} 41^{\prime \prime}$ referenced to North American Datum of 1927, in NE 1/4 NW 1/4 sec.30, T.6 N., R.5 W., Greene County, IN, Hydrologic Unit 05120202, on left bank, 0.4 mi upstream from bridge on State Highway 57 at Newberry, $2.0 \mathrm{mi}$ downstream from Doans Creek, and at mile 112.4.

Drainage area.- $-4,688 \mathrm{mi}^{2}$.

Period of record. - September 1928 to current year.

Average discharge. $-5,084 \mathrm{ft}^{3} / \mathrm{s}$.

Minimum daily discharge. $-200 \mathrm{ft}^{3} / \mathrm{s}$.

Human health (harmonic mean) design flow. - 1,779 $\mathrm{ft}^{3} / \mathrm{s}$.

Remarks. - Flow regulated upstream reservoirs. Low-flow statistics are calculated for the regulated period, 1943 to 2011.

\begin{tabular}{ccc}
\hline \multicolumn{3}{c}{ Magnitude and frequency of annual low flow } \\
\hline $\begin{array}{c}\text { Lowest average streamflow, in } \mathrm{ft}^{3} / \mathrm{s} \text {, for indicated period of } \\
\text { consecutive days at an annual nonexceedance probability of } 0.1\end{array}$ \\
\hline $\mathbf{1}$ & $\mathbf{7}$ & $\mathbf{3 0}$ \\
\hline 377 & 393 & 433 \\
\hline
\end{tabular}

Percentage of time streamflow was equaled or exceeded for the period of record

\begin{tabular}{cc|cc}
\hline $\begin{array}{c}\text { Percentage } \\
\text { of time }\end{array}$ & $\begin{array}{c}\text { Daily mean } \\
\text { streamflow } \\
\left(\mathrm{ft}^{3} / \mathbf{s}\right)\end{array}$ & $\begin{array}{c}\text { Percentage } \\
\text { of time }\end{array}$ & $\begin{array}{c}\text { Daily mean } \\
\text { streamflow } \\
\left(\mathrm{ft}^{3} / \mathbf{s}\right)\end{array}$ \\
\hline 99 & 402 & 40 & 4060 \\
\hline 98 & 441 & 30 & 5570 \\
\hline 95 & 549 & 20 & 7690 \\
\hline 90 & 713 & 10 & 12400 \\
\hline 80 & 1080 & 5 & 18600 \\
\hline 70 & 1530 & 2 & 26700 \\
\hline 60 & 2170 & 1 & 34000 \\
\hline 50 & 2950 & & \\
\hline
\end{tabular}




\section{BIG BLUE RIVER AT CARTHAGE, IN}

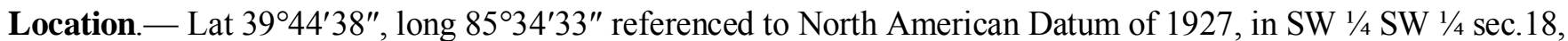
T.15 N., R.9 E., Rush County, IN, Hydrologic Unit 05120204, on right bank $300 \mathrm{ft}$ upstream from highway bridge, $0.5 \mathrm{mi}$ northwest of Carthage, $2.2 \mathrm{mi}$ downstream from Three Mile Creek, and at mile 50.7.

Drainage area.- $184 \mathrm{mi}^{2}$.

Period of record.- October 1950 to March 2004.

Average discharge. $-205 \mathrm{ft}^{3} / \mathrm{s}$.

Minimum daily discharge. $-17 \mathrm{ft}^{3} / \mathrm{s}$.

Human health (harmonic mean) design flow. $-98 \mathrm{ft}^{3} / \mathrm{s}$.

Remarks. - Flow partly regulated by Big Blue River Conservancy District control structures on tributaries to Big Blue River beginning in 1969. Prior to October 1961, published as Blue River at Carthage.

\begin{tabular}{|c|c|c|c|}
\hline \multicolumn{4}{|c|}{ Magnitude and frequency of annual low flow } \\
\hline \multicolumn{4}{|c|}{$\begin{array}{l}\text { Lowest average streamflow, in } \mathrm{ft}^{3} / \mathrm{s} \text {, for indicated period of } \\
\text { consecutive days at an annual nonexceedance probability of } 0 \text {. }\end{array}$} \\
\hline 1 & \multicolumn{2}{|c|}{7} & 30 \\
\hline 23 & \multicolumn{2}{|c|}{25} & 33 \\
\hline \multicolumn{4}{|c|}{$\begin{array}{l}\text { Percentage of time streamflow was equaled or exceeded for } \\
\text { the period of record }\end{array}$} \\
\hline $\begin{array}{l}\text { Percentage } \\
\text { of time }\end{array}$ & $\begin{array}{l}\text { Daily mean } \\
\text { streamflow } \\
\left(\mathrm{ft}^{3} / \mathrm{s}\right)\end{array}$ & $\begin{array}{l}\text { Percentage } \\
\text { of time }\end{array}$ & $\begin{array}{l}\text { Daily mean } \\
\text { streamflow } \\
\left(\mathrm{ft}^{3} / \mathrm{s}\right)\end{array}$ \\
\hline 99 & 30 & 40 & 142 \\
\hline 98 & 34 & 30 & 182 \\
\hline 95 & 42 & 20 & 248 \\
\hline 90 & 51 & 10 & 407 \\
\hline 80 & 65 & 5 & 641 \\
\hline 70 & 78 & 2 & 1110 \\
\hline 60 & 95 & 1 & 1610 \\
\hline 50 & 117 & & \\
\hline
\end{tabular}




\section{BIG BLUE RIVER AT SHELBYVILLE, IN}

Location.- Lat 39 $31^{\prime} 43^{\prime \prime}$, long $85^{\circ} 46^{\prime} 56^{\prime \prime}$ referenced to North American Datum of 1927, in SE 1/4 SE $1 / 4 \mathrm{sec} .31$, T.13 N., R.7 E., Shelby County, IN, Hydrologic Unit 05120204, on left bank 0.2 mi downstream from bridge on State Highway 9 in Shelbyville, 0.6 mi downstream from Little Blue River, and at mile 23.9.

Drainage area. $-421 \mathrm{mi}^{2}$.

Period of record. - September 1943 to current year.

Average discharge. $-492 \mathrm{ft}^{3} / \mathrm{s}$

Minimum daily discharge. $-27 \mathrm{ft}^{3} / \mathrm{s}$.

Human health (harmonic mean) design flow. $-174 \mathrm{ft}^{3} / \mathrm{s}$.

Remarks. - Prior to October 1961, published as Blue River at Shelbyville.

\begin{tabular}{|c|c|c|c|}
\hline \multicolumn{4}{|c|}{ Magnitude and frequency of annual low flow } \\
\hline \multicolumn{4}{|c|}{$\begin{array}{l}\text { Lowest average streamflow, in } \mathrm{ft}^{3} / \mathrm{s} \text {, for indicated period of } \\
\text { consecutive days at an annual nonexceedance probability of } 0.1\end{array}$} \\
\hline 1 & \multicolumn{2}{|c|}{7} & 30 \\
\hline 40 & \multicolumn{2}{|c|}{43} & 48 \\
\hline \multicolumn{4}{|c|}{$\begin{array}{l}\text { Percentage of time streamflow was equaled or exceeded for } \\
\text { the period of record }\end{array}$} \\
\hline $\begin{array}{l}\text { Percentage } \\
\text { of time }\end{array}$ & $\begin{array}{c}\text { Daily mean } \\
\text { streamflow } \\
\left(\mathrm{ft}^{3} / \mathrm{s}\right)\end{array}$ & $\begin{array}{l}\text { Percentage } \\
\text { of time }\end{array}$ & $\begin{array}{l}\text { Daily mean } \\
\text { streamflow } \\
\left(\mathrm{ft}^{3} / \mathrm{s}\right)\end{array}$ \\
\hline 99 & 43 & 40 & 328 \\
\hline 98 & 48 & 30 & 437 \\
\hline 95 & 61 & 20 & 623 \\
\hline 90 & 77 & 10 & 1060 \\
\hline 80 & 104 & 5 & 1720 \\
\hline 70 & 141 & 2 & 3040 \\
\hline 60 & 192 & 1 & 4310 \\
\hline 50 & 252 & & \\
\hline
\end{tabular}




\section{LEARY-WEBER DITCH AT MOHAWK, IN}

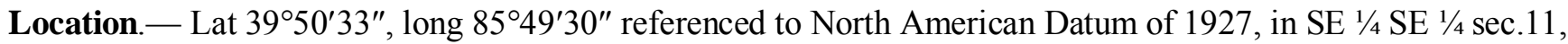
T.16 N., R.6 E., Hancock County, IN, Hydrologic Unit 05120204, $60 \mathrm{ft}$ upstream of bridge on County Road 400N, $0.33 \mathrm{mi}$ upstream of Sugar Creek, $0.70 \mathrm{mi}$ east of Mohawk, and $3.06 \mathrm{mi}$ southwest of Maxwell.

Drainage area. $-2.79 \mathrm{mi}^{2}$.

Period of record.- July 2002 to October 2007, November 6, 2009, to current year.

Average discharge. $-3.24 \mathrm{ft}^{3} / \mathrm{s}$.

Minimum daily discharge. $-0.00 \mathrm{ft}^{3} / \mathrm{s}$.

Human health (harmonic mean) design flow. $-0.2 \mathrm{ft}^{3} / \mathrm{s}$.

Remarks. - This site has less than 10 years of record.

\begin{tabular}{c}
\hline \multicolumn{4}{c}{ Magnitude and frequency of annual low flow } \\
\hline \multicolumn{4}{c}{ Lowest average streamflow, in $\mathrm{ft}^{3}$ /s, for indicated period of } \\
consecutive days at an annual nonexceedance probability of 0.1
\end{tabular}




\section{SUGAR CREEK AT NEW PALESTINE, IN}

Location.- Lat $39^{\circ} 42^{\prime} 51^{\prime \prime}$, long 85 $53^{\prime} 08^{\prime \prime}$ referenced to North American Datum of 1927, in SE 1/4 SW 1/4 sec.29, T.15 N., R.6 E., Hancock County, IN, Hydrologic Unit 05120204, on left bank $10 \mathrm{ft}$ downstream from bridge on County Road 450 West, 0.5 mi south of New Palestine, 3.1 mi upstream from Little Sugar Creek, and at mile 37.3.

Drainage area. $-93.9 \mathrm{mi}^{2}$.

Period of record.- October 1967 to current year.

Average discharge. $-108 \mathrm{ft}^{3} / \mathrm{s}$

Minimum daily discharge. $-0.11 \mathrm{ft}^{3} / \mathrm{s}$.

Human health (harmonic mean) design flow. $-20 \mathrm{ft}^{3} / \mathrm{s}$.

Remarks. - Prior to October 1961, published as Blue River at Shelbyville.

\begin{tabular}{|c|c|c|c|}
\hline \multicolumn{4}{|c|}{ Magnitude and frequency of annual low flow } \\
\hline \multicolumn{4}{|c|}{$\begin{array}{l}\text { Lowest average streamflow, in } \mathrm{ft}^{3} / \mathrm{s} \text {, for indicated period of } \\
\text { consecutive days at an annual nonexceedance probability of } 0.1\end{array}$} \\
\hline 1 & \multicolumn{2}{|c|}{7} & 30 \\
\hline 1.7 & \multicolumn{2}{|c|}{2.3} & 3.1 \\
\hline \multicolumn{4}{|c|}{$\begin{array}{l}\text { Percentage of time streamflow was equaled or exceeded for } \\
\text { the period of record }\end{array}$} \\
\hline $\begin{array}{l}\text { Percentage } \\
\text { of time }\end{array}$ & $\begin{array}{c}\text { Daily mean } \\
\text { streamflow } \\
\left(\mathrm{ft}^{3} / \mathrm{s}\right)\end{array}$ & $\begin{array}{l}\text { Percentage } \\
\text { of time }\end{array}$ & $\begin{array}{l}\text { Daily mean } \\
\text { streamflow } \\
\left(\mathrm{ft}^{3} / \mathrm{s}\right)\end{array}$ \\
\hline 99 & 3.2 & 40 & 65 \\
\hline 98 & 3.9 & 30 & 93 \\
\hline 95 & 5.9 & 20 & 142 \\
\hline 90 & 8.4 & 10 & 265 \\
\hline 80 & 15 & 5 & 453 \\
\hline 70 & 23 & 2 & 768 \\
\hline 60 & 35 & 1 & 1010 \\
\hline 50 & 48 & & \\
\hline
\end{tabular}




\section{BUCK CREEK AT ACTON, IN}

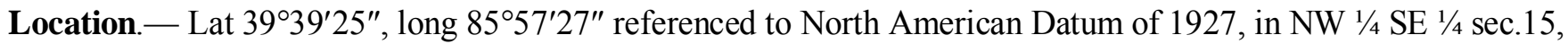
T.14 N., R.5 E., Marion County, IN, Hydrologic Unit 05120204, on left bank, 30 ft downstream from McGregor Road bridge, $0.5 \mathrm{mi}$ east of Acton, and $4.1 \mathrm{mi}$ upstream from mouth.

Drainage area. $-78.8 \mathrm{mi}^{2}$.

Period of record.- October 1967 to current year.

Average discharge. $-97.3 \mathrm{ft}^{3} / \mathrm{s}$.

Minimum daily discharge. $-0.20 \mathrm{ft}^{3} / \mathrm{s}$.

Human health (harmonic mean) design flow.- $14 \mathrm{ft}^{3} / \mathrm{s}$.

Remarks. - Low flow is affected by regulation.

\begin{tabular}{cc|cc}
\hline \multicolumn{5}{c}{ Magnitude and frequency of annual low flow } \\
\hline \multicolumn{4}{c}{ Lowest average streamflow, in $\mathrm{ft}^{3} / \mathbf{s}$, for indicated period of } \\
consecutive days at an annual nonexceedance probability of 0.1 \\
\hline $\mathbf{1}$ \\
\hline \multicolumn{4}{c}{1.4} \\
\hline 0.8
\end{tabular}




\section{YOUNGS CREEK NEAR EDINBURGH, IN}

Location.- Lat $39^{\circ} 25^{\prime} 08^{\prime \prime}$, long $86^{\circ} 00^{\prime} 18^{\prime \prime}$ referenced to North American Datum of 1927, in SE 1/4 SW 1/4 Sec.5, T.11 N., R.5 E., Johnson County, IN, Hydrologic Unit 05120204, on right bank at downstream side of County Road 400S bridge, $0.5 \mathrm{mi}$ southwest of Amity, $2.0 \mathrm{mi}$ upstream from mouth, and $5.0 \mathrm{mi}$ northwest of Edinburgh.

Drainage area. $-107 \mathrm{mi}^{2}$.

Period of record.- October 1942 to current year.

Average discharge. $-119 \mathrm{ft}^{3} / \mathrm{s}$

Minimum daily discharge. $-0.50 \mathrm{ft}^{3} / \mathrm{s}$.

Human health (harmonic mean) design flow. - $14 \mathrm{ft}^{3} / \mathrm{s}$.

Remarks. - Prior to June 30, 1955, non-recording gage at same site and datum.

\begin{tabular}{ccc}
\hline \multicolumn{3}{c}{ Magnitude and frequency of annual low flow } \\
\hline $\begin{array}{c}\text { Lowest average streamflow, in } \mathrm{ft}^{3} / \mathrm{s} \text {, for indicated period of } \\
\text { consecutive days at an annual nonexceedance probability of } 0.1\end{array}$ \\
\hline $\mathbf{1}$ & $\mathbf{3 0}$ \\
\hline 1.3 & 1.7 & 2.3 \\
\hline
\end{tabular}

Percentage of time streamflow was equaled or exceeded for the period of record

\begin{tabular}{cc|cc}
\hline $\begin{array}{c}\text { Percentage } \\
\text { of time }\end{array}$ & $\begin{array}{c}\text { Daily mean } \\
\text { streamflow } \\
\left(\mathrm{ft}^{3} / \mathbf{s}\right)\end{array}$ & $\begin{array}{c}\text { Percentage } \\
\text { of time }\end{array}$ & $\begin{array}{c}\text { Daily mean } \\
\text { streamflow } \\
\left(\mathrm{ft}^{3} / \mathbf{s}\right)\end{array}$ \\
\hline 99 & 2.1 & 40 & 60 \\
\hline 98 & 2.6 & 30 & 87 \\
\hline 95 & 3.6 & 20 & 139 \\
\hline 90 & 5.1 & 10 & 268 \\
\hline 80 & 9.0 & 5 & 464 \\
\hline 70 & 17 & 2 & 879 \\
\hline 60 & 27 & 1 & 1360 \\
\hline 50 & 42 & & \\
\hline
\end{tabular}




\section{SUGAR CREEK NEAR EDINBURGH, IN}

Location. - Lat 39²1'39.3", long 8559'53.1" referenced to North American Datum of 1983, in SW 1/4 SE 1/4 sec.29, T.11 N., R.5 E., Johnson County, IN, Hydrologic Unit 05120204, on upstream side of bridge of E800S going into Camp Atterbury, $1.3 \mathrm{mi}$ upstream from confluence with Blue River, $1.5 \mathrm{mi}$ northwest of Edinburgh, and at mile 1.3 .

Drainage area.- $474 \mathrm{mi}^{2}$.

Period of record. - October 1942 to current year.

Average discharge. $-531 \mathrm{ft}^{3} / \mathrm{s}$.

Minimum daily discharge. $-9.2 \mathrm{ft}^{3} / \mathrm{s}$.

Human health (harmonic mean) design flow. $-121 \mathrm{ft}^{3} / \mathrm{s}$.

Remarks.- None.

\begin{tabular}{ccc}
\hline \multicolumn{3}{c}{ Magnitude and frequency of annual low flow } \\
\hline $\begin{array}{c}\text { Lowest average streamflow, in } \mathrm{ft}^{3} / \mathrm{s} \text {, for indicated period of } \\
\text { consecutive days at an annual nonexceedance probability of } \mathbf{0 . 1}\end{array}$ \\
\hline $\mathbf{7}$ & $\mathbf{7 0}$ \\
\hline 18 & 20 & 24 \\
\hline
\end{tabular}

Percentage of time streamflow was equaled or exceeded for the period of record

\begin{tabular}{cc|cc}
\hline $\begin{array}{c}\text { Percentage } \\
\text { of time }\end{array}$ & $\begin{array}{c}\text { Daily mean } \\
\text { streamflow } \\
\left(\mathrm{ft}^{3} / \mathbf{s}\right)\end{array}$ & $\begin{array}{c}\text { Percentage } \\
\text { of time }\end{array}$ & $\begin{array}{c}\text { Daily mean } \\
\text { streamflow } \\
\left(\mathrm{ft}^{3} / \mathbf{s}\right)\end{array}$ \\
\hline 99 & 23 & 40 & 312 \\
\hline 98 & 26 & 30 & 436 \\
\hline 95 & 35 & 20 & 661 \\
\hline 90 & 48 & 10 & 1200 \\
\hline 80 & 73 & 5 & 2070 \\
\hline 70 & 110 & 2 & 3690 \\
\hline 60 & 163 & 1 & 5170 \\
\hline 50 & 228 & & \\
\hline
\end{tabular}




\section{DRIFTWOOD RIVER NEAR EDINBURGH, IN}

Location. - Lat $39^{\circ} 20^{\prime} 21^{\prime \prime}$, long $85^{\circ} 59^{\prime} 11^{\prime \prime}$ referenced to North American Datum of 1927, in NW 1/4 NW 1/4 sec.4, T.10 N., R.5 E., Bartholomew County, IN, Hydrologic Unit 05120204, on downstream side of Hendricks Ford Road bridge, 0.8 mi downstream from confluence of Big Blue River and Sugar Creek, 1.5 mi southwest of Edinburgh, and at mile 14.1 .

Drainage area.- $1,060 \mathrm{mi}^{2}$.

Period of record. - October 1941 to September 1990, February 2011 to current year.

Average discharge. - 1,186 $\mathrm{ft}^{3} / \mathrm{s}$.

Minimum daily discharge. $-50 \mathrm{ft}^{3} / \mathrm{s}$.

Human health (harmonic mean) design flow. $-378 \mathrm{ft}^{3} / \mathrm{s}$.

Remarks.- None.

\begin{tabular}{ccc}
\hline \multicolumn{3}{c}{ Magnitude and frequency of annual low flow } \\
\hline $\begin{array}{c}\text { Lowest average streamflow, in } \mathrm{ft}^{3} / \mathrm{s} \text {, for indicated period of } \\
\text { consecutive days at an annual nonexceedance probability of } 0.1\end{array}$ \\
\hline $\mathbf{1}$ & $\mathbf{7}$ & $\mathbf{3 0}$ \\
\hline 88 & 93 & 103 \\
\hline
\end{tabular}

Percentage of time streamflow was equaled or exceeded for the period of record

\begin{tabular}{cc|cc}
\hline $\begin{array}{c}\text { Percentage } \\
\text { of time }\end{array}$ & $\begin{array}{c}\text { Daily mean } \\
\text { streamflow } \\
\left(\mathrm{ft}^{3} / \mathbf{s}\right)\end{array}$ & $\begin{array}{c}\text { Percentage } \\
\text { of time }\end{array}$ & $\begin{array}{c}\text { Daily mean } \\
\text { streamflow } \\
\left(\mathrm{ft}^{3} / \mathbf{s}\right)\end{array}$ \\
\hline 99 & 97 & 40 & 781 \\
\hline 98 & 110 & 30 & 1090 \\
\hline 95 & 134 & 20 & 1580 \\
\hline 90 & 164 & 10 & 2720 \\
\hline 80 & 227 & 5 & 4370 \\
\hline 70 & 310 & 2 & 7030 \\
\hline 60 & 430 & 1 & 9570 \\
\hline 50 & 583 & & \\
\hline
\end{tabular}




\section{FLATROCK RIVER AT ST. PAUL, IN}

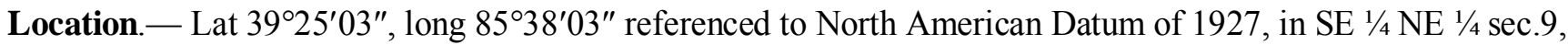
T.11 N., R.8 E., Shelby County, IN, Hydrologic Unit 05120205 , on right bank $500 \mathrm{ft}$ downstream from county road bridge, $0.8 \mathrm{mi}$ southwest of St. Paul, $1.5 \mathrm{mi}$ downstream from Mill Creek, and at mile 34.4.

Drainage area. $-303 \mathrm{mi}^{2}$.

Period of record.- October 1930 to current year.

Average discharge. $-339 \mathrm{ft}^{3} / \mathrm{s}$.

Minimum daily discharge. $-0.60 \mathrm{ft}^{3} / \mathrm{s}$.

Human health (harmonic mean) design flow. - $36 \mathrm{ft}^{3} / \mathrm{s}$.

Remarks. - Prior to October 1958, published as Flatrock Creek at St. Paul.

\begin{tabular}{ccc}
\hline \multicolumn{3}{c}{ Magnitude and frequency of annual low flow } \\
\hline $\begin{array}{c}\text { Lowest average streamflow, in } \mathrm{ft}^{3} / \mathrm{s} \text {, for indicated period of } \\
\text { consecutive days at an annual nonexceedance probability of } \mathbf{0 . 1}\end{array}$ \\
\hline $\mathbf{7}$ & $\mathbf{3 0}$ \\
\hline 2.0 & 2.5 & 4.2 \\
\hline
\end{tabular}

Percentage of time streamflow was equaled or exceeded for the period of record

\begin{tabular}{cc|cc}
\hline $\begin{array}{c}\text { Percentage } \\
\text { of time }\end{array}$ & $\begin{array}{c}\text { Daily mean } \\
\text { streamflow } \\
\left(\mathrm{ft}^{3} / \mathbf{s}\right)\end{array}$ & $\begin{array}{c}\text { Percentage } \\
\text { of time }\end{array}$ & $\begin{array}{c}\text { Daily mean } \\
\text { streamflow } \\
\left(\mathrm{ft}^{3} / \mathbf{s}\right)\end{array}$ \\
\hline 99 & 3.1 & 40 & 203 \\
\hline 98 & 5.4 & 30 & 290 \\
\hline 95 & 9.9 & 20 & 443 \\
\hline 90 & 16 & 10 & 798 \\
\hline 80 & 30 & 5 & 1320 \\
\hline 70 & 54 & 2 & 2270 \\
\hline 60 & 94 & 1 & 3310 \\
\hline 50 & 142 & & \\
\hline
\end{tabular}




\section{FLATROCK RIVER AT COLUMBUS, IN}

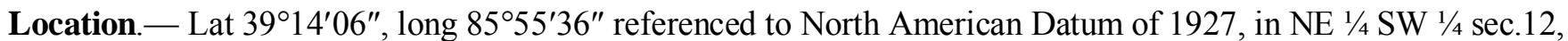
T.9 N., R.5 E., Bartholomew County, IN, Hydrologic Unit 05120205, on left bank at downstream side of bridge on U.S. Highway 31, 0.2 mi northwest of Columbus city limits, and 2.6 mi upstream from mouth.

Drainage area. $-534 \mathrm{mi}^{2}$.

Period of record.- October 1967 to current year.

Average discharge. $-636 \mathrm{ft}^{3} / \mathrm{s}$

Minimum daily discharge. $-13 \mathrm{ft}^{3} / \mathrm{s}$.

Human health (harmonic mean) design flow. $-163 \mathrm{ft}^{3} / \mathrm{s}$.

Remarks. - None.

\begin{tabular}{ccc}
\hline \multicolumn{3}{c}{ Magnitude and frequency of annual low flow } \\
\hline $\begin{array}{c}\text { Lowest average streamflow, in } \mathrm{ft}^{3} / \mathrm{s} \text {, for indicated period of } \\
\text { consecutive days at an annual nonexceedance probability of } \mathbf{0 . 1}\end{array}$ \\
\hline $\mathbf{7}$ & $\mathbf{3 0}$ \\
\hline 28 & 29 & 32 \\
\hline
\end{tabular}

Percentage of time streamflow was equaled or exceeded for the period of record

\begin{tabular}{cc|cc}
\hline $\begin{array}{c}\text { Percentage } \\
\text { of time }\end{array}$ & $\begin{array}{c}\text { Daily mean } \\
\text { streamflow } \\
\left(\mathbf{f t}^{3} / \mathbf{s}\right)\end{array}$ & $\begin{array}{c}\text { Percentage } \\
\text { of time }\end{array}$ & $\begin{array}{c}\text { Daily mean } \\
\text { streamflow } \\
\left(\mathrm{ft}^{3} / \mathbf{s}\right)\end{array}$ \\
\hline 99 & 29 & 40 & 440 \\
\hline 98 & 35 & 30 & 588 \\
\hline 95 & 45 & 20 & 827 \\
\hline 90 & 60 & 10 & 1390 \\
\hline 80 & 101 & 5 & 2200 \\
\hline 70 & 164 & 2 & 3880 \\
\hline 60 & 246 & 1 & 5590 \\
\hline 50 & 331 & & \\
\hline
\end{tabular}




\section{EAST FORK WHITE RIVER AT COLUMBUS, IN}

Location. - Lat $39^{\circ} 12^{\prime} 00^{\prime \prime}$, long $85^{\circ} 55^{\prime} 32^{\prime \prime}$ referenced to North American Datum of 1927, in NE 1/4 NW 1/4 sec.25, T.9 N., R.5 E., Bartholomew County, IN, Hydrologic Unit 05120205, on left bank at abutment of abandoned bridge at west end of Second Street in Columbus, 0.6 mi downstream from confluence of Driftwood River and Flatrock River, 1.3 mi upstream from Haw Creek, and at mile 238.7.

Drainage area.- $1,707 \mathrm{mi}^{2}$.

Period of record. - October 1947 to current year.

Average discharge. $-1,981 \mathrm{ft}^{3} / \mathrm{s}$.

Minimum daily discharge. $-85 \mathrm{ft}^{3} / \mathrm{s}$.

Human health (harmonic mean) design flow. $-626 \mathrm{ft}^{3} / \mathrm{s}$.

Remarks.- None.

\begin{tabular}{ccc}
\hline \multicolumn{3}{c}{ Magnitude and frequency of annual low flow } \\
\hline $\begin{array}{c}\text { Lowest average streamflow, in } \mathrm{ft}^{3} / \mathrm{s} \text {, for indicated period of } \\
\text { consecutive days at an annual nonexceedance probability of } \mathbf{0 . 1}\end{array}$ \\
\hline $\mathbf{1}$ & $\mathbf{7}$ & $\mathbf{3 0}$ \\
\hline 129 & 134 & 148 \\
\hline
\end{tabular}

Percentage of time streamflow was equaled or exceeded for the period of record

\begin{tabular}{cc|cc}
\hline $\begin{array}{c}\text { Percentage } \\
\text { of time }\end{array}$ & $\begin{array}{c}\text { Daily mean } \\
\text { streamflow } \\
\left(\mathrm{ft}^{3} / \mathbf{s}\right)\end{array}$ & $\begin{array}{c}\text { Percentage } \\
\text { of time }\end{array}$ & $\begin{array}{c}\text { Daily mean } \\
\text { streamflow } \\
\left(\mathrm{ft}^{3} / \mathbf{s}\right)\end{array}$ \\
\hline 99 & 135 & 40 & 1360 \\
\hline 98 & 158 & 30 & 1840 \\
\hline 95 & 204 & 20 & 2630 \\
\hline 90 & 261 & 10 & 4440 \\
\hline 80 & 372 & 5 & 6970 \\
\hline 70 & 535 & 2 & 11800 \\
\hline 60 & 763 & 1 & 16200 \\
\hline 50 & 1040 & & \\
\hline
\end{tabular}




\section{East Fork White River Basin}

\section{HAW CREEK AT HOPE, IN}

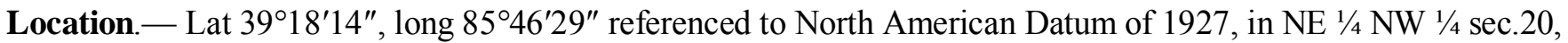
T.10 N., R.7 E., Bartholomew County, IN, Hydrologic Unit 05120205, on the upstream side of the bridge over Haw Creek, $600 \mathrm{ft}$ west of S.R. 9 on Jackson St., $14.5 \mathrm{mi}$ southeast from I-65, $18 \mathrm{mi}$ south of Shelbyville, and at river mile 13.5.

Drainage area. - $17.9 \mathrm{mi}^{2}$.

Period of record. - June 2010 to current year.

Average discharge. $-21.4 \mathrm{ft}^{3} / \mathrm{s}$.

Minimum daily discharge. $-0.0 \mathrm{ft}^{3} / \mathrm{s}$.

Human health (harmonic mean) design flow. $-0.2 \mathrm{ft}^{3} / \mathrm{s}$.

Remarks. - This site has less than 10 years of record.

\begin{tabular}{|c|c|c|c|}
\hline \multicolumn{4}{|c|}{ Magnitude and frequency of annual low flow } \\
\hline \multicolumn{4}{|c|}{$\begin{array}{l}\text { Lowest average streamflow, in } \mathrm{ft}^{3} / \mathrm{s} \text {, for indicated period of } \\
\text { consecutive days at an annual nonexceedance probability of } 0.1\end{array}$} \\
\hline 1 & \multicolumn{2}{|c|}{7} & 30 \\
\hline 0 & \multicolumn{2}{|c|}{0} & 0 \\
\hline \multicolumn{4}{|c|}{$\begin{array}{c}\text { Percentage of time streamflow was equaled or exceeded for } \\
\text { the period of record }\end{array}$} \\
\hline $\begin{array}{l}\text { Percentage } \\
\text { of time }\end{array}$ & $\begin{array}{l}\text { Daily mean } \\
\text { streamflow } \\
\left(\mathrm{ft}^{3} / \mathrm{s}\right)\end{array}$ & $\begin{array}{l}\text { Percentage } \\
\text { of time }\end{array}$ & $\begin{array}{l}\text { Daily mean } \\
\text { streamflow } \\
\left(\mathrm{ft}^{3} / \mathrm{s}\right)\end{array}$ \\
\hline 99 & 0 & 40 & 8.3 \\
\hline 98 & 0 & 30 & 15 \\
\hline 95 & 0 & 20 & 24 \\
\hline 90 & 0 & 10 & 49 \\
\hline 80 & 0 & 5 & 89 \\
\hline 70 & 0.2 & 2 & 201 \\
\hline 60 & 2.1 & 1 & 351 \\
\hline 50 & 4.8 & & \\
\hline
\end{tabular}




\section{HAW CREEK NEAR CLIFFORD, IN}

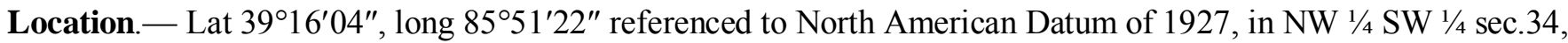
T.10 N., R.6 E., Bartholomew County, IN, Hydrologic Unit 05120205, on downstream side of bridge on County Road 450 North, 1.2 mi southeast of Clifford, 5.8 mi northeast of Columbus, and $7.6 \mathrm{mi}$ above mouth.

Drainage area. $-47.5 \mathrm{mi}^{2}$.

Period of record.- July 1967 to June 1992, June 22, 2010, to current year.

Average discharge. $-50.7 \mathrm{ft}^{3} / \mathrm{s}$.

Minimum daily discharge. $-0.0 \mathrm{ft}^{3} / \mathrm{s}$.

Human health (harmonic mean) design flow. $-5.8 \mathrm{ft}^{3} / \mathrm{s}$.

Remarks.- None.

\begin{tabular}{|c|c|c|c|}
\hline \multicolumn{4}{|c|}{ Magnitude and frequency of annual low flow } \\
\hline \multicolumn{4}{|c|}{$\begin{array}{l}\text { Lowest average streamflow, in } \mathrm{ft}^{3} / \mathrm{s} \text {, for indicated period of } \\
\text { consecutive days at an annual nonexceedance probability of } 0.1\end{array}$} \\
\hline 1 & \multicolumn{2}{|c|}{7} & 30 \\
\hline 0.5 & \multicolumn{2}{|c|}{0.6} & 0.8 \\
\hline \multicolumn{4}{|c|}{$\begin{array}{l}\text { Percentage of time streamflow was equaled or exceeded for } \\
\text { the period of record }\end{array}$} \\
\hline $\begin{array}{l}\text { Percentage } \\
\text { of time }\end{array}$ & $\begin{array}{l}\text { Daily mean } \\
\text { streamflow } \\
\left(\mathrm{ft}^{3} / \mathrm{s}\right)\end{array}$ & $\begin{array}{l}\text { Percentage } \\
\text { of time }\end{array}$ & $\begin{array}{c}\text { Daily mean } \\
\text { streamflow } \\
\left(\mathrm{ft}^{3} / \mathrm{s}\right)\end{array}$ \\
\hline 99 & 0.7 & 40 & 28 \\
\hline 98 & 0.9 & 30 & 40 \\
\hline 95 & 1.5 & 20 & 60 \\
\hline 90 & 2.3 & 10 & 108 \\
\hline 80 & 4.2 & 5 & 185 \\
\hline 70 & 7.9 & 2 & 362 \\
\hline 60 & 14 & 1 & 610 \\
\hline 50 & 20 & & \\
\hline
\end{tabular}




\section{CLIFTY CREEK AT HARTSVILLE, IN}

Location.- Lat $39^{\circ} 16^{\prime} 29^{\prime \prime}$, long $85^{\circ} 42^{\prime} 06^{\prime \prime}$ referenced to North American Datum of 1927, in NW 1/4 NW 1/4 sec.36, T.10 N., R.7 E., Bartholomew County, IN, Hydrologic Unit 05120206, at downstream side of left abutment of County Road 1150 E bridge, $0.2 \mathrm{mi}$ north of Hartsville, $5.9 \mathrm{mi}$ upstream from Duck Creek, and at mile 22.0.

Drainage area.- $91.4 \mathrm{mi}^{2}$.

Period of record.- February 1948 to current year.

Average discharge. $-105 \mathrm{ft}^{3} / \mathrm{s}$

Minimum daily discharge. $-0.0 \mathrm{ft}^{3} / \mathrm{s}$.

Human health (harmonic mean) design flow. $-1.6 \mathrm{ft}^{3} / \mathrm{s}$.

Remarks.- None.

Magnitude and frequency of annual low flow

Lowest average streamflow, in $\mathrm{ft}^{3} / \mathrm{s}$, for indicated period of consecutive days at an annual nonexceedance probability of 0.1

\begin{tabular}{ccc}
\hline $\mathbf{1}$ & $\mathbf{7}$ & $\mathbf{3 0}$ \\
\hline 0.0 & 0.0 & 0.0 \\
\hline
\end{tabular}

Percentage of time streamflow was equaled or exceeded for the period of record

\begin{tabular}{cc|cc}
\hline $\begin{array}{c}\text { Percentage } \\
\text { of time }\end{array}$ & $\begin{array}{c}\text { Daily mean } \\
\text { streamflow } \\
\left(\mathrm{ft}^{3} / \mathbf{s}\right)\end{array}$ & $\begin{array}{c}\text { Percentage } \\
\text { of time }\end{array}$ & $\begin{array}{c}\text { Daily mean } \\
\text { streamflow } \\
\left(\mathrm{ft}^{3} / \mathbf{s}\right)\end{array}$ \\
\hline 99 & 0.0 & 40 & 51 \\
\hline 98 & 0.0 & 30 & 77 \\
\hline 95 & 0.0 & 20 & 124 \\
\hline 90 & 0.8 & 10 & 234 \\
\hline 80 & 4.0 & 5 & 410 \\
\hline 70 & 11 & 2 & 807 \\
\hline 60 & 21 & 1 & 1280 \\
\hline 50 & 35 & & \\
\hline
\end{tabular}


Wabash River Basin

\section{CLIFTY CREEK NEAR COLUMBUS, IN}

Location.- Lat $39^{\circ} 11^{\prime} 07^{\prime \prime}$, long 85 52'30" referenced to North American Datum of 1927, in NW 1/4 NW 1/4 sec.33, T.9 N., R.6 E., Bartholomew County, IN, Hydrologic Unit 05120206, on left bank, at downstream side of left abutment of county highway bridge, 0.2 mi north of Hartsville, and 5.9 mi upstream from Duck Creek, and at mile 3.62 .

Drainage area. $-202 \mathrm{mi}^{2}$.

Period of record. - June 2006 to current year.

Average discharge. - $286 \mathrm{ft}^{3} / \mathrm{s}$.

Minimum daily discharge. $-1.8 \mathrm{ft}^{3} / \mathrm{s}$.

Human health (harmonic mean) design flow. - $16 \mathrm{ft}^{3} / \mathrm{s}$.

Remarks. - This site has less than 10 years of record.

\begin{tabular}{ccc}
\hline \multicolumn{3}{c}{ Magnitude and frequency of annual low flow } \\
\hline $\begin{array}{c}\text { Lowest average streamflow, in } \mathrm{ft}^{3} / \mathrm{s} \text {, for indicated period of } \\
\text { consecutive days at an annual nonexceedance probability of } 0.1\end{array}$ \\
\hline $\mathbf{1}$ & $\mathbf{7}$ & $\mathbf{3 0}$ \\
\hline 1.8 & 2.0 & 2.3 \\
\hline
\end{tabular}

Percentage of time streamflow was equaled or exceeded for the period of record

\begin{tabular}{cc|cc}
\hline $\begin{array}{c}\text { Percentage } \\
\text { of time }\end{array}$ & $\begin{array}{c}\text { Daily mean } \\
\text { streamflow } \\
\left(\mathrm{ft}^{3} / \mathbf{s}\right)\end{array}$ & $\begin{array}{c}\text { Percentage } \\
\text { of time }\end{array}$ & $\begin{array}{c}\text { Daily mean } \\
\text { streamflow } \\
\left(\mathrm{ft}^{3} / \mathbf{s}\right)\end{array}$ \\
\hline 99 & 2.2 & 40 & 140 \\
\hline 98 & 2.5 & 30 & 193 \\
\hline 95 & 3.1 & 20 & 304 \\
\hline 90 & 4.3 & 10 & 618 \\
\hline 80 & 8.6 & 5 & 1260 \\
\hline 70 & 22 & 2 & 2480 \\
\hline 60 & 62 & 1 & 3570 \\
\hline 50 & 101 & & \\
\hline
\end{tabular}




\section{SAND CREEK NEAR BREWERSVILLE, IN}

Location.- Lat $39^{\circ} 05^{\prime} 03^{\prime \prime}$, long 85'39'32", in NW 1/4 NE 1/4 sec.5, T.7 N., R.8 E., Jennings County, IN, Hydrologic Unit 05120206 , on left bank at downstream side of county highway bridge, $2.5 \mathrm{mi}$ west of Brewersville, $5.7 \mathrm{mi}$ upstream from Wyaloosing Creek, and 16.0 mi upstream from mouth.

Drainage area. $-155 \mathrm{mi}^{2}$.

Period of record. - February 1948 to September 1986.

Average discharge. $-173 \mathrm{ft}^{3} / \mathrm{s}$.

Minimum daily discharge. $-0.0 \mathrm{ft}^{3} / \mathrm{s}$.

Human health (harmonic mean) design flow. $-6.3 \mathrm{ft}^{3} / \mathrm{s}$.

Remarks.- None.

\begin{tabular}{|c|c|c|c|}
\hline \multicolumn{4}{|c|}{ Magnitude and frequency of annual low flow } \\
\hline \multicolumn{4}{|c|}{$\begin{array}{l}\text { Lowest average streamflow, in } \mathrm{ft}^{3} / \mathrm{s} \text {, for indicated period of } \\
\text { consecutive days at an annual nonexceedance probability of } 0.1\end{array}$} \\
\hline 1 & \multicolumn{2}{|c|}{7} & 30 \\
\hline 0.0 & \multicolumn{2}{|c|}{0.0} & 0.0 \\
\hline \multicolumn{4}{|c|}{$\begin{array}{c}\text { Percentage of time streamflow was equaled or exceeded for } \\
\text { the period of record }\end{array}$} \\
\hline $\begin{array}{l}\text { Percentage } \\
\text { of time }\end{array}$ & $\begin{array}{l}\text { Daily mean } \\
\text { streamflow } \\
\left(\mathrm{ft}^{3} / \mathrm{s}\right)\end{array}$ & $\begin{array}{l}\text { Percentage } \\
\text { of time }\end{array}$ & $\begin{array}{l}\text { Daily mean } \\
\text { streamflow } \\
\left(\mathrm{ft}^{3} / \mathrm{s}\right)\end{array}$ \\
\hline 99 & 0.0 & 40 & 77 \\
\hline 98 & 0.1 & 30 & 114 \\
\hline 95 & 1.6 & 20 & 186 \\
\hline 90 & 3.8 & 10 & 380 \\
\hline 80 & 9.4 & 5 & 738 \\
\hline 70 & 18 & 2 & 1430 \\
\hline 60 & 30 & 1 & 2110 \\
\hline 50 & 49 & & \\
\hline
\end{tabular}


Wabash River Basin

\section{EAST FORK WHITE RIVER AT SEYMOUR, IN}

Location.- Lat $38^{\circ} 58^{\prime} 57^{\prime \prime}$, long 85 $53^{\prime} 57^{\prime \prime}$ referenced to North American Datum of 1927, in NW 1/4 NE 1/4 sec.7, T.6 N., R.6 E., Jackson County, IN, Hydrologic Unit 05120206, on left bank 1,700 ft downstream from county road highway bridge, $1 \mathrm{mi}$ north of Seymour, $9.5 \mathrm{mi}$ downstream from Sand Creek, and at mile 214.6.

Drainage area.- $-2,341 \mathrm{mi}^{2}$.

Period of record. - October 1927 to current year.

Average discharge. $-2,688 \mathrm{ft}^{3} / \mathrm{s}$.

Minimum daily discharge. $-86 \mathrm{ft}^{3} / \mathrm{s}$.

Human health (harmonic mean) design flow. $-775 \mathrm{ft}^{3} / \mathrm{s}$.

Remarks. - None.

\begin{tabular}{|c|c|c|c|}
\hline \multicolumn{4}{|c|}{ Magnitude and frequency of annual low flow } \\
\hline \multicolumn{4}{|c|}{$\begin{array}{l}\text { Lowest average streamflow, in } \mathrm{ft}^{3} / \mathrm{s} \text {, for indicated period of } \\
\text { consecutive days at an annual nonexceedance probability of } 0.1\end{array}$} \\
\hline 1 & \multicolumn{2}{|c|}{7} & 30 \\
\hline 168 & \multicolumn{2}{|c|}{179} & 195 \\
\hline \multicolumn{4}{|c|}{$\begin{array}{l}\text { Percentage of time streamflow was equaled or exceeded for } \\
\text { the period of record }\end{array}$} \\
\hline $\begin{array}{l}\text { Percentage } \\
\text { of time }\end{array}$ & $\begin{array}{l}\text { Daily mean } \\
\text { streamflow } \\
\left(\mathrm{ft}^{3} / \mathrm{s}\right)\end{array}$ & $\begin{array}{l}\text { Percentage } \\
\text { of time }\end{array}$ & $\begin{array}{l}\text { Daily mean } \\
\text { streamflow } \\
\left(\mathrm{ft}^{3} / \mathrm{s}\right)\end{array}$ \\
\hline 99 & 180 & 40 & 1760 \\
\hline 98 & 202 & 30 & 2440 \\
\hline 95 & 247 & 20 & 3530 \\
\hline 90 & 310 & 10 & 6110 \\
\hline 80 & 442 & 5 & 9770 \\
\hline 70 & 656 & 2 & 16900 \\
\hline 60 & 957 & 1 & 24100 \\
\hline 50 & 1310 & & \\
\hline
\end{tabular}




\section{GRAHAM CREEK NEAR VERNON, IN}

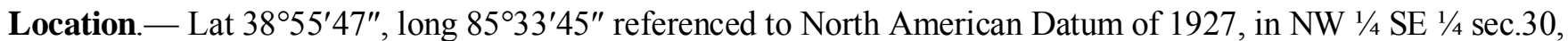
T.6 N., R.9 E., Jennings County, IN, Hydrologic Unit 05120207, on right bank $10 \mathrm{ft}$ upstream from State Highway 7, 4.7 mi southeast of Vernon, and 8.0 mi downstream from Little Graham Creek.

Drainage area. $-77.2 \mathrm{mi}^{2}$.

Period of record.- June 1955 to September 1973.

Average discharge. $-93.2 \mathrm{ft}^{3} / \mathrm{s}$.

Minimum daily discharge. $-0.0 \mathrm{ft}^{3} / \mathrm{s}$.

Human health (harmonic mean) design flow. $-1 \mathrm{ft}^{3} / \mathrm{s}$.

Remarks. - None.

Magnitude and frequency of annual low flow

Lowest average streamflow, in $\mathrm{ft}^{3} / \mathrm{s}$, for indicated period of consecutive days at an annual nonexceedance probability of 0.1

\begin{tabular}{ccc}
\hline $\mathbf{1}$ & $\mathbf{7}$ & $\mathbf{3 0}$ \\
\hline 0.0 & 0.0 & 0.0 \\
\hline
\end{tabular}

Percentage of time streamflow was equaled or exceeded for the period of record

\begin{tabular}{cc|cc}
\hline $\begin{array}{c}\text { Percentage } \\
\text { of time }\end{array}$ & $\begin{array}{c}\text { Daily mean } \\
\text { streamflow } \\
\left(\mathrm{ft}^{3} / \mathbf{s}\right)\end{array}$ & $\begin{array}{c}\text { Percentage } \\
\text { of time }\end{array}$ & $\begin{array}{c}\text { Daily mean } \\
\text { streamflow } \\
\left(\mathrm{ft}^{3} / \mathbf{s}\right)\end{array}$ \\
\hline 99 & 0.0 & 40 & 26 \\
\hline 98 & 0.0 & 30 & 43 \\
\hline 95 & 0.0 & 20 & 78 \\
\hline 90 & 0.3 & 10 & 188 \\
\hline 80 & 1.5 & 5 & 416 \\
\hline 70 & 4.2 & 2 & 964 \\
\hline 60 & 8.8 & 1 & 1510 \\
\hline 50 & 15 & & \\
\hline
\end{tabular}




\section{HARBERTS CREEK NEAR MADISON, IN}

Location.- Lat $38^{\circ} 46^{\prime} 55^{\prime \prime}$, long $85^{\circ} 29^{\prime} 08^{\prime \prime}$ referenced to North American Datum of 1927, in SW 1/4 SE 1/4 Sec.14, T.4 N., R.9 E., Jefferson County, IN, Hydrologic Unit 05120207, attached to left downstream wingwall of bridge on County Road 533 West, $0.2 \mathrm{mi}$ west of Smyrna, 3.7 mi upstream from Big Creek, and 4 mi northwest of Madison.

Drainage area.- $-9.31 \mathrm{mi}^{2}$.

Period of record.- August 1968 to October 2003.

Average discharge. $-13.6 \mathrm{ft}^{3} / \mathrm{s}$.

Minimum daily discharge. $-0.0 \mathrm{ft}^{3} / \mathrm{s}$.

Human health (harmonic mean) design flow. $-0.3 \mathrm{ft}^{3} / \mathrm{s}$.

Remarks. - None.

Magnitude and frequency of annual low flow

Lowest average streamflow, in $\mathrm{ft}^{3} / \mathrm{s}$, for indicated period of consecutive days at an annual nonexceedance probability of 0.1

\begin{tabular}{ccc}
\hline $\mathbf{1}$ & $\mathbf{7}$ & $\mathbf{3 0}$ \\
\hline 0.0 & 0.0 & 0.0 \\
\hline
\end{tabular}

Percentage of time streamflow was equaled or exceeded for the period of record

\begin{tabular}{cc|cc}
\hline $\begin{array}{c}\text { Percentage } \\
\text { of time }\end{array}$ & $\begin{array}{c}\text { Daily mean } \\
\text { streamflow } \\
\left(\mathrm{ft}^{3} / \mathbf{s}\right)\end{array}$ & $\begin{array}{c}\text { Percentage } \\
\text { of time }\end{array}$ & $\begin{array}{c}\text { Daily mean } \\
\text { streamflow } \\
\left(\mathrm{ft}^{3} / \mathbf{s}\right)\end{array}$ \\
\hline 99 & 0.0 & 40 & 4.1 \\
\hline 98 & 0.0 & 30 & 6.6 \\
\hline 95 & 0.0 & 20 & 12 \\
\hline 90 & 0.1 & 10 & 27 \\
\hline 80 & 0.4 & 5 & 62 \\
\hline 70 & 0.8 & 2 & 140 \\
\hline 60 & 1.5 & 1 & 225 \\
\hline 50 & 2.5 & & \\
\hline
\end{tabular}




\section{MUSCATATUCK RIVER NEAR DEPUTY, IN}

Location.- Lat $38^{\circ} 48^{\prime} 15^{\prime \prime}$, long $85^{\circ} 40^{\prime} 26^{\prime \prime}$ referenced to North American Datum of 1927, in SW 1/4 NE 1/4 Sec.7, T.4 N., R.8 E., Jefferson County, IN, Hydrologic Unit 05120207, on left bank approximately $100 \mathrm{ft}$ downstream of highway bridge, 1.4 mi northwest of Deputy, 1.9 mi upstream from Coffee Creek, 2.4 mi downstream from confluence of Graham Creek and Big Creek, and at mile 50.0.

Drainage area. $-293 \mathrm{mi}^{2}$.

Period of record. - November 1947 to current year.

Average discharge. $-373 \mathrm{ft}^{3} / \mathrm{s}$.

Minimum daily discharge. $-0.0 \mathrm{ft}^{3} / \mathrm{s}$.

Human health (harmonic mean) design flow. $-5.4 \mathrm{ft}^{3} / \mathrm{s}$.

Remarks. - None.

\begin{tabular}{ccc}
\hline \multicolumn{3}{c}{ Magnitude and frequency of annual low flow } \\
\hline $\begin{array}{c}\text { Lowest average streamflow, in } \mathrm{ft}^{3} / \mathrm{s} \text {, for indicated period of } \\
\text { consecutive days at an annual nonexceedance probability of } 0.1\end{array}$ \\
\hline $\mathbf{1}$ & $\mathbf{7}$ & $\mathbf{3 0}$ \\
\hline 0.0 & 0.0 & 0.2 \\
\hline
\end{tabular}

Percentage of time streamflow was equaled or exceeded for the period of record

\begin{tabular}{cc|cc}
\hline $\begin{array}{c}\text { Percentage } \\
\text { of time }\end{array}$ & $\begin{array}{c}\text { Daily mean } \\
\text { streamflow } \\
\left(\mathrm{ft}^{3} / \mathbf{s}\right)\end{array}$ & $\begin{array}{c}\text { Percentage } \\
\text { of time }\end{array}$ & $\begin{array}{c}\text { Daily mean } \\
\text { streamflow } \\
\left(\mathrm{ft}^{3} / \mathbf{s}\right)\end{array}$ \\
\hline 99 & 0.1 & 40 & 139 \\
\hline 98 & 0.2 & 30 & 222 \\
\hline 95 & 1.1 & 20 & 372 \\
\hline 90 & 3.8 & 10 & 805 \\
\hline 80 & 13 & 5 & 1630 \\
\hline 70 & 27 & 2 & 3480 \\
\hline 60 & 51 & 1 & 5550 \\
\hline 50 & 86 & & \\
\hline
\end{tabular}




\section{BRUSH CREEK NEAR NEBRASKA, IN}

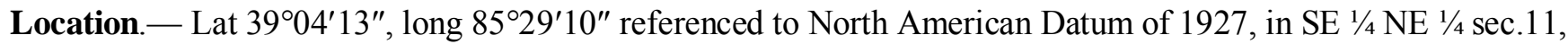
T.7 N., R.9 E., Jennings County, IN, Hydrologic Unit 05120207, at upstream side of bridge on right bank on County Road 675 E, 1.5 mi northwest of Nebraska, 2.9 mi northeast of Butlerville, and 3.6 mi upstream from Brush Creek Dam.

Drainage area. $-11.4 \mathrm{mi}^{2}$.

Period of record.- May 1955 to current year.

Average discharge. $-14.7 \mathrm{ft}^{3} / \mathrm{s}$.

Minimum daily discharge. $-0.0 \mathrm{ft}^{3} / \mathrm{s}$.

Human health (harmonic mean) design flow. $-0.3 \mathrm{ft}^{3} / \mathrm{s}$.

Remarks. - None.

\begin{tabular}{|c|c|c|c|}
\hline \multicolumn{4}{|c|}{ Magnitude and frequency of annual low flow } \\
\hline \multicolumn{4}{|c|}{$\begin{array}{l}\text { Lowest average streamflow, in } \mathrm{ft}^{3} / \mathrm{s} \text {, for indicated period of } \\
\text { consecutive days at an annual nonexceedance probability of } 0.1\end{array}$} \\
\hline 1 & \multicolumn{2}{|c|}{7} & 30 \\
\hline 0.0 & \multicolumn{2}{|c|}{0.0} & 0.0 \\
\hline \multicolumn{4}{|c|}{$\begin{array}{l}\text { Percentage of time streamflow was equaled or exceeded for } \\
\text { the period of record }\end{array}$} \\
\hline $\begin{array}{l}\text { Percentage } \\
\text { of time }\end{array}$ & $\begin{array}{l}\text { Daily mean } \\
\text { streamflow } \\
\left(\mathrm{ft}^{3} / \mathrm{s}\right)\end{array}$ & $\begin{array}{l}\text { Percentage } \\
\text { of time }\end{array}$ & $\begin{array}{c}\text { Daily mean } \\
\text { streamflow } \\
\left(\mathrm{ft}^{3} / \mathrm{s}\right)\end{array}$ \\
\hline 99 & 0.0 & 40 & 3.9 \\
\hline 98 & 0.0 & 30 & 6.2 \\
\hline 95 & 0.0 & 20 & 11 \\
\hline 90 & 0.0 & 10 & 26 \\
\hline 80 & 0.2 & 5 & 64 \\
\hline 70 & 0.6 & 2 & 158 \\
\hline 60 & 1.4 & 1 & 265 \\
\hline 50 & 2.4 & & \\
\hline
\end{tabular}


Wabash River Basin

\section{VERNON FORK MUSCATATUCK RIVER NEAR BUTLERVILLE, IN}

Location.- Lat $39^{\circ} 02^{\prime} 55^{\prime \prime}$, long 85³2'40" referenced to North American Datum of 1927, in NW 1/4 SE 1/4 sec.17, T.7 N., R.9 E., Jennings County, IN, Hydrologic Unit 05120207, on left bank 0.3 mi downstream from Muscatatuck State School dam, 1.1 mi downstream from Brush Creek, 2 mi northwest of Butlerville, and at mile 50.6.

Drainage area. $-85.9 \mathrm{mi}^{2}$.

Period of record. - February 1942 to December 2001.

Average discharge. $-95.5 \mathrm{ft}^{3} / \mathrm{s}$.

Minimum daily discharge. $-0.0 \mathrm{ft}^{3} / \mathrm{s}$.

Human health (harmonic mean) design flow. $-3.5 \mathrm{ft}^{3} / \mathrm{s}$.

Remarks. - Water supply for the Muscatatuck State School is diverted and the sewage effluent returned above station. Flow regulated by Brush Creek Reservoir. Low-flow statistics are calculated for the regulated period, 1943 to 2002. Prior to October 1960, published as North Fork of Vernon Fork near Butlerville..

\begin{tabular}{|c|c|c|c|}
\hline \multicolumn{4}{|c|}{ Magnitude and frequency of annual low flow } \\
\hline \multicolumn{4}{|c|}{$\begin{array}{l}\text { Lowest average streamflow, in } \mathrm{ft}^{3} / \mathrm{s} \text {, for indicated period of } \\
\text { consecutive days at an annual nonexceedance probability of } 0.1\end{array}$} \\
\hline 1 & \multicolumn{2}{|c|}{7} & 30 \\
\hline 0.1 & \multicolumn{2}{|c|}{0.2} & 0.5 \\
\hline \multicolumn{4}{|c|}{$\begin{array}{l}\text { Percentage of time streamflow was equaled or exceeded for } \\
\text { the period of record }\end{array}$} \\
\hline $\begin{array}{l}\text { Percentage } \\
\text { of time }\end{array}$ & $\begin{array}{l}\text { Daily mean } \\
\text { streamflow } \\
\left(\mathrm{ft}^{3} / \mathrm{s}\right)\end{array}$ & $\begin{array}{l}\text { Percentage } \\
\text { of time }\end{array}$ & $\begin{array}{l}\text { Daily mean } \\
\text { streamflow } \\
\left(\mathrm{ft}^{3} / \mathrm{s}\right)\end{array}$ \\
\hline 99 & 0.3 & 40 & 34 \\
\hline 98 & 0.4 & 30 & 53 \\
\hline 95 & 0.8 & 20 & 87 \\
\hline 90 & 1.5 & 10 & 186 \\
\hline 80 & 3.3 & 5 & 385 \\
\hline 70 & 6.6 & 2 & 875 \\
\hline 60 & 12 & 1 & 1480 \\
\hline 50 & 21 & & \\
\hline
\end{tabular}




\section{VERNON FORK MUSCATATUCK RIVER AT VERNON, IN}

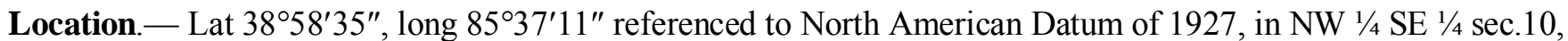
T.6 N., R.8 E., Jennings County, IN, Hydrologic Unit 05120207, at left upstream side of bridge, 1 mi southwest of Vernon, $3.1 \mathrm{mi}$ downstream from Otter Creek, and at mile 36.4.

Drainage area.- $-198 \mathrm{mi}^{2}$.

Period of record. - October 1939 to current year.

Average discharge. $-234 \mathrm{ft}^{3} / \mathrm{s}$.

Minimum daily discharge. $-0.0 \mathrm{ft}^{3} / \mathrm{s}$.

Human health (harmonic mean) design flow. $-6.2 \mathrm{ft}^{3} / \mathrm{s}$.

Remarks. - Diversion above station for municipal water supply of North Vernon and Vernon. Part of this diversion returned above gage as sewage effluent by North Vernon Sewage Treatment Plant. Some regulation at times at low flow by Old Timbers Lake on Jefferson Proving Grounds and Brush Creek Reservoir.

\begin{tabular}{|c|c|c|c|}
\hline \multicolumn{4}{|c|}{ Magnitude and frequency of annual low flow } \\
\hline \multicolumn{4}{|c|}{$\begin{array}{l}\text { Lowest average streamflow, in } \mathrm{ft}^{3} / \mathrm{s} \text {, for indicated period of } \\
\text { consecutive days at an annual nonexceedance probability of } 0.1\end{array}$} \\
\hline 1 & \multicolumn{2}{|c|}{7} & 30 \\
\hline 0.2 & \multicolumn{2}{|c|}{0.3} & 0.7 \\
\hline \multicolumn{4}{|c|}{$\begin{array}{l}\text { Percentage of time streamflow was equaled or exceeded for } \\
\text { the period of record }\end{array}$} \\
\hline $\begin{array}{l}\text { Percentage } \\
\text { of time }\end{array}$ & $\begin{array}{l}\text { Daily mean } \\
\text { streamflow } \\
\quad\left(\mathrm{ft}^{3} / \mathrm{s}\right)\end{array}$ & $\begin{array}{l}\text { Percentage } \\
\text { of time }\end{array}$ & $\begin{array}{l}\text { Daily mean } \\
\text { streamflow } \\
\left(\mathrm{ft}^{3} / \mathrm{s}\right)\end{array}$ \\
\hline 99 & 0.3 & 40 & 85 \\
\hline 98 & 0.6 & 30 & 134 \\
\hline 95 & 1.4 & 20 & 225 \\
\hline 90 & 2.9 & 10 & 486 \\
\hline 80 & 7.6 & 5 & 1000 \\
\hline 70 & 17 & 2 & 2200 \\
\hline 60 & 32 & 1 & 3400 \\
\hline 50 & 54 & & \\
\hline
\end{tabular}




\section{EAST FORK WHITE RIVER NEAR BEDFORD, IN}

Location.- Lat $38^{\circ} 46^{\prime} 13^{\prime \prime}$, long $86^{\circ} 24^{\prime} 35^{\prime \prime}$ referenced to North American Datum of 1927, in SW 1/4 NE 1/4 sec.21, T.4 N., R.1 E., Lawrence County, IN, Hydrologic Unit 05120208, on right downstream side of county road bridge, $0.4 \mathrm{mi}$ upstream from Mill Creek, $2.9 \mathrm{mi}$ downstream from Sugar Creek, 3.9 mi northeast of Mitchell, $7.8 \mathrm{mi}$ southeast of Bedford, and at mile 153.3.

Drainage area. $-3,861 \mathrm{mi}^{2}$.

Period of record.- May 1939 to current year.

Average discharge. $-4,517 \mathrm{ft}^{3} / \mathrm{s}$.

Minimum daily discharge. $-138 \mathrm{ft}^{3} / \mathrm{s}$.

Human health (harmonic mean) design flow.- 1,290 $\mathrm{ft}^{3} / \mathrm{s}$.

Remarks. - None.

\begin{tabular}{|c|c|c|c|}
\hline \multicolumn{4}{|c|}{ Magnitude and frequency of annual low flow } \\
\hline \multicolumn{4}{|c|}{$\begin{array}{l}\text { Lowest average streamflow, in } \mathrm{ft}^{3} / \mathrm{s} \text {, for indicated period of } \\
\text { consecutive days at an annual nonexceedance probability of } 0.1\end{array}$} \\
\hline 1 & \multicolumn{2}{|c|}{7} & 30 \\
\hline 253 & \multicolumn{2}{|c|}{272} & 290 \\
\hline \multicolumn{4}{|c|}{$\begin{array}{l}\text { Percentage of time streamflow was equaled or exceeded for } \\
\text { the period of record }\end{array}$} \\
\hline $\begin{array}{l}\text { Percentage } \\
\text { of time }\end{array}$ & $\begin{array}{c}\text { Daily mean } \\
\text { streamflow } \\
\left(\mathrm{ft}^{3} / \mathrm{s}\right)\end{array}$ & $\begin{array}{l}\text { Percentage } \\
\text { of time }\end{array}$ & $\begin{array}{c}\text { Daily mean } \\
\text { streamflow } \\
\left(\mathrm{ft}^{3} / \mathrm{s}\right)\end{array}$ \\
\hline 99 & 267 & 40 & 3250 \\
\hline 98 & 300 & 30 & 4540 \\
\hline 95 & 387 & 20 & 6720 \\
\hline 90 & 492 & 10 & 11100 \\
\hline 80 & 749 & 5 & 15600 \\
\hline 70 & 1170 & 2 & 23600 \\
\hline 60 & 1690 & 1 & 32100 \\
\hline 50 & 2370 & & \\
\hline
\end{tabular}




\section{BACK CREEK AT LEESVILLE, IN}

Location.- Lat $38^{\circ} 50^{\prime} 48^{\prime \prime}$, long $86^{\circ} 18^{\prime} 06^{\prime \prime}$ referenced to North American Datum of 1927, in SW 1/4 SE $1 / 4$ sec.21, T.5 N., R.2 E., Lawrence County, IN, Hydrologic Unit 05120208, on left bank at downstream side of county road bridge, 0.9 mi west of Leesville, 2.5 mi upstream from Jones Defeat Hollow, and 7 mi upstream of mouth.

Drainage area. $-24.1 \mathrm{mi}^{2}$.

Period of record. - October 1970 to October 2003.

Average discharge. $-36.1 \mathrm{ft}^{3} / \mathrm{s}$.

Minimum daily discharge. $-0.0 \mathrm{ft}^{3} / \mathrm{s}$.

Human health (harmonic mean) design flow. $-0.6 \mathrm{ft}^{3} / \mathrm{s}$.

Remarks.- None.

\begin{tabular}{ccc}
\hline \multicolumn{3}{c}{ Magnitude and frequency of annual low flow } \\
\hline $\begin{array}{c}\text { Lowest average streamflow, in } \mathrm{ft}^{3} / \mathrm{s} \text {, for indicated period of } \\
\text { consecutive days at an annual nonexceedance probability of } 0.1\end{array}$ \\
\hline $\mathbf{7}$ & $\mathbf{3 0}$ \\
\hline 0.0 & 0.0 & 0.0 \\
\hline
\end{tabular}

Percentage of time streamflow was equaled or exceeded for the period of record

\begin{tabular}{cc|cc}
\hline $\begin{array}{c}\text { Percentage } \\
\text { of time }\end{array}$ & $\begin{array}{c}\text { Daily mean } \\
\text { streamflow } \\
\left(\mathrm{ft}^{3} / \mathbf{s}\right)\end{array}$ & $\begin{array}{c}\text { Percentage } \\
\text { of time }\end{array}$ & $\begin{array}{c}\text { Daily mean } \\
\text { streamflow } \\
\left(\mathrm{ft}^{3} / \mathbf{s}\right)\end{array}$ \\
\hline 99 & 0.0 & 40 & 15 \\
\hline 98 & 0.0 & 30 & 24 \\
\hline 95 & 0.0 & 20 & 40 \\
\hline 90 & 0.3 & 10 & 79 \\
\hline 80 & 1.1 & 5 & 151 \\
\hline 70 & 2.7 & 2 & 311 \\
\hline 60 & 5.1 & 1 & 464 \\
\hline 50 & 9.2 & & \\
\hline
\end{tabular}




\section{SOUTH FORK SALT CREEK AT KURTZ, IN}

Location.- Lat $38^{\circ} 57^{\prime} 46^{\prime \prime}$, long $86^{\circ} 12^{\prime} 12^{\prime \prime}$ referenced to North American Datum of 1927, in SW 1/4 SW 1/4 sec.9, T.6 N., R.3 E., Jackson County, IN, Hydrologic Unit 05120208, on right bank at downstream side of county road bridge, at north edge of Kurtz, 0.8 mi upstream from right-bank tributary, and $6.1 \mathrm{mi}$ upstream from Little Salt Creek.

Drainage area. $-38.2 \mathrm{mi}^{2}$.

Period of record.- October 1960 to September 1971.

Average discharge. $-39.9 \mathrm{ft}^{3} / \mathrm{s}$.

Minimum daily discharge. $-0.0 \mathrm{ft}^{3} / \mathrm{s}$.

Human health (harmonic mean) design flow. $-0.4 \mathrm{ft}^{3} / \mathrm{s}$.

Remarks. - None.

\begin{tabular}{ccc}
\hline \multicolumn{3}{c}{ Magnitude and frequency of annual low flow } \\
\hline $\begin{array}{c}\text { Lowest average streamflow, in } \mathrm{ft}^{3} / \mathrm{s} \text {, for indicated period of } \\
\text { consecutive days at an annual nonexceedance probability of } 0.1\end{array}$ \\
\hline $\mathbf{1}$ & $\mathbf{3 0}$ \\
\hline 0.0 & 0.0 & 0.0 \\
\hline
\end{tabular}

Percentage of time streamflow was equaled or exceeded for the period of record

\begin{tabular}{cc|cc}
\hline $\begin{array}{c}\text { Percentage } \\
\text { of time }\end{array}$ & $\begin{array}{c}\text { Daily mean } \\
\text { streamflow } \\
\left(\mathrm{ft}^{3} / \mathbf{s}\right)\end{array}$ & $\begin{array}{c}\text { Percentage } \\
\text { of time }\end{array}$ & $\begin{array}{c}\text { Daily mean } \\
\text { streamflow } \\
\left(\mathrm{ft}^{3} / \mathbf{s}\right)\end{array}$ \\
\hline 99 & 0.0 & 40 & 8.4 \\
\hline 98 & 0.0 & 30 & 17 \\
\hline 95 & 0.0 & 20 & 35 \\
\hline 90 & 0.0 & 10 & 79 \\
\hline 80 & 0.0 & 5 & 161 \\
\hline 70 & 0.5 & 2 & 386 \\
\hline 60 & 1.7 & 1 & 688 \\
\hline 50 & 4.2 & & \\
\hline
\end{tabular}




\section{NORTH FORK SALT CREEK AT NASHVILLE, IN}

Location.- Lat $39^{\circ} 12^{\prime} 06^{\prime \prime}$, long $86^{\circ} 14^{\prime} 51^{\prime \prime}$ referenced to North American Datum of 1927, in NW 1/4 SW 1/4 Sec.19, T.9 N., R.3 E., Brown County, IN, Hydrologic Unit 05120208, on right bank $90 \mathrm{ft}$ downstream from bridge on State Highway 46, $800 \mathrm{ft}$ downstream from Greasy Creek, $0.4 \mathrm{mi}$ south of center of Nashville, and at mile 32.5.

Drainage area.- $76.1 \mathrm{mi}^{2}$.

Period of record. - July 1962 to September 1976.

Average discharge. $-78.9 \mathrm{ft}^{3} / \mathrm{s}$.

Minimum daily discharge. $-0.0 \mathrm{ft}^{3} / \mathrm{s}$.

Human health (harmonic mean) design flow. $-1.6 \mathrm{ft}^{3} / \mathrm{s}$.

Remarks. - None.

\begin{tabular}{ccc}
\hline \multicolumn{3}{c}{ Magnitude and frequency of annual low flow } \\
\hline $\begin{array}{c}\text { Lowest average streamflow, in } \mathrm{ft}^{3} / \mathrm{s} \text {, for indicated period of } \\
\text { consecutive days at an annual nonexceedance probability of } 0.1\end{array}$ \\
\hline $\mathbf{7}$ & $\mathbf{3 0}$ \\
\hline 0.0 & 0.0 & 0.0 \\
\hline
\end{tabular}

Percentage of time streamflow was equaled or exceeded for the period of record

\begin{tabular}{cc|cc}
\hline $\begin{array}{c}\text { Percentage } \\
\text { of time }\end{array}$ & $\begin{array}{c}\text { Daily mean } \\
\text { streamflow } \\
\left(\mathrm{ft}^{3} / \mathbf{s}\right)\end{array}$ & $\begin{array}{c}\text { Percentage } \\
\text { of time }\end{array}$ & $\begin{array}{c}\text { Daily mean } \\
\text { streamflow } \\
\left(\mathrm{ft}^{3} / \mathbf{s}\right)\end{array}$ \\
\hline 99 & 0.0 & 40 & 29 \\
\hline 98 & 0.0 & 30 & 53 \\
\hline 95 & 0.1 & 20 & 92 \\
\hline 90 & 0.5 & 10 & 185 \\
\hline 80 & 1.7 & 5 & 329 \\
\hline 70 & 3.9 & 2 & 610 \\
\hline 60 & 7.9 & 1 & 1110 \\
\hline 50 & 16 & & \\
\hline
\end{tabular}




\section{NORTH FORK SALT CREEK NEAR BELMONT, IN}

Location.- Lat $39^{\circ} 09^{\prime} 00^{\prime \prime}$, long $86^{\circ} 20^{\prime} 14^{\prime \prime}$ referenced to North American Datum of 1927, in SW 1/4 NW 1/4 sec.5, T.8 N., R.2 E., Brown County, IN, Hydrologic Unit 05120208, on right bank $15 \mathrm{ft}$ downstream from bridge on State Highway 46, $100 \mathrm{ft}$ upstream Schooner Creek, 0.7 mi northeast of Belmont, 6.5 mi upstream from Brummett Creek, and 20 mi upstream from mouth.

Drainage area. $-120 \mathrm{mi}^{2}$.

Period of record.- April 1946 to September 1971.

Average discharge. $-130 \mathrm{ft}^{3} / \mathrm{s}$.

Minimum daily discharge. $-0.0 \mathrm{ft}^{3} / \mathrm{s}$.

Human health (harmonic mean) design flow. $-1.8 \mathrm{ft}^{3} / \mathrm{s}$.

Remarks. - None.

\begin{tabular}{ccc}
\hline \multicolumn{3}{c}{ Magnitude and frequency of annual low flow } \\
\hline $\begin{array}{c}\text { Lowest average streamflow, in } \mathrm{ft}^{3} / \mathrm{s} \text {, for indicated period of } \\
\text { consecutive days at an annual nonexceedance probability of } 0.1\end{array}$ \\
\hline $\mathbf{1}$ & $\mathbf{3 0}$ \\
\hline 0.0 & 0.0 & 0.0 \\
\hline
\end{tabular}

Percentage of time streamflow was equaled or exceeded for the period of record

\begin{tabular}{cc|cc}
\hline $\begin{array}{c}\text { Percentage } \\
\text { of time }\end{array}$ & $\begin{array}{c}\text { Daily mean } \\
\text { streamflow } \\
\left(\mathrm{ft}^{3} / \mathbf{s}\right)\end{array}$ & $\begin{array}{c}\text { Percentage } \\
\text { of time }\end{array}$ & $\begin{array}{c}\text { Daily mean } \\
\text { streamflow } \\
\left(\mathrm{ft}^{3} / \mathbf{s}\right)\end{array}$ \\
\hline 99 & 0.0 & 40 & 42 \\
\hline 98 & 0.1 & 30 & 78 \\
\hline 95 & 0.1 & 20 & 135 \\
\hline 90 & 0.5 & 10 & 275 \\
\hline 80 & 2.6 & 5 & 542 \\
\hline 70 & 5.7 & 2 & 1270 \\
\hline 60 & 12 & 1 & 1850 \\
\hline 50 & 23 & & \\
\hline
\end{tabular}


Wabash River Basin

\section{STEPHENS CREEK NEAR BLOOMINGTON, IN}

Location. - Lat $39^{\circ} 10^{\prime} 11^{\prime \prime}$, long $86^{\circ} 25^{\prime} 07^{\prime \prime}$ referenced to North American Datum of 1927, in NE 1/4 NW 1/4 sec.4, T.8 N., R.1 E., Monroe County, IN, Hydrologic Unit 05120208, on downstream side of right pier of bridge on State Highway 46, $0.2 \mathrm{mi}$ downstream from Kerr Creek, $4.0 \mathrm{mi}$ west of Belmont, and $6.1 \mathrm{mi}$ east of Bloomington.

Drainage area.- $10.9 \mathrm{mi}^{2}$.

Period of record.- October 1970 to September 1991.

Average discharge. - $14.0 \mathrm{ft}^{3} / \mathrm{s}$.

Minimum daily discharge. $-0.0 \mathrm{ft}^{3} / \mathrm{s}$.

Human health (harmonic mean) design flow. $-0.4 \mathrm{ft}^{3} / \mathrm{s}$.

Remarks. - None.

\begin{tabular}{ccc}
\hline \multicolumn{3}{c}{ Magnitude and frequency of annual low flow } \\
\hline $\begin{array}{c}\text { Lowest average streamflow, in } \mathrm{ft}^{3} / \mathrm{s} \text {, for indicated period of } \\
\text { consecutive days at an annual nonexceedance probability of } 0.1\end{array}$ \\
\hline $\mathbf{7}$ & $\mathbf{3 0}$ \\
\hline 0.0 & 0.0 & 0.0 \\
\hline
\end{tabular}

Percentage of time streamflow was equaled or exceeded for the period of record

\begin{tabular}{cc|cc}
\hline $\begin{array}{c}\text { Percentage } \\
\text { of time }\end{array}$ & $\begin{array}{c}\text { Daily mean } \\
\text { streamflow } \\
\left(\mathrm{ft}^{3} / \mathbf{s}\right)\end{array}$ & $\begin{array}{c}\text { Percentage } \\
\text { of time }\end{array}$ & $\begin{array}{c}\text { Daily mean } \\
\text { streamflow } \\
\left(\mathrm{ft}^{3} / \mathbf{s}\right)\end{array}$ \\
\hline 99 & 0.0 & 40 & 6.5 \\
\hline 98 & 0.0 & 30 & 11 \\
\hline 95 & 0.0 & 20 & 18 \\
\hline 90 & 0.1 & 10 & 34 \\
\hline 80 & 0.5 & 5 & 60 \\
\hline 70 & 1.2 & 2 & 111 \\
\hline 60 & 2.2 & 1 & 172 \\
\hline 50 & 3.9 & & \\
\hline
\end{tabular}




\section{SALT CREEK NEAR HARRODSBURG, IN}

Location. - Lat $39^{\circ} 00^{\prime} 16^{\prime \prime}$, long 86 $30^{\prime} 31^{\prime \prime}$ referenced to North American Datum of 1927, in NE 1/4 NW 1/4 Sec.34, T.7 N., R.1 W., Monroe County, IN, Hydrologic Unit 05120208, on right bank 0.25 mi downstream from Monroe Lake, 0.9 mi upstream from Clear Creek, 2.2 mi southeast of Harrodsburg, and 25.7 mi upstream from mouth.

Drainage area.- $432 \mathrm{mi}^{2}$.

Period of record.- May 1955 to September 2001.

Average discharge. $-495 \mathrm{ft}^{3} / \mathrm{s}$.

Minimum daily discharge. $-0.0 \mathrm{ft}^{3} / \mathrm{s}$.

Human health (harmonic mean) design flow. - $99 \mathrm{ft}^{3} / \mathrm{s}$.

Remarks. - Flow regulated by U.S. Army Corps of Engineers from Monroe Lake since April 1966. Records of daily discharge provided by U.S. Army Corps of Engineers beginning Oct. 1, 1976. Low-flow statistics are calculated for the regulated period, 1967 to 2001. The 1Q10 (--) was not calculated on account of high skew.

\begin{tabular}{cccc}
\hline \multicolumn{5}{c}{ Magnitude and frequency of annual low flow } \\
\hline \multicolumn{4}{c}{ Lowest average streamflow, in $\mathrm{ft}^{3} / \mathrm{s}$, for indicated period of } \\
consecutive days at an annual nonexceedance probability of 0.1
\end{tabular}




\section{CLEAR CREEK NEAR HARRODSBURG, IN}

Location. - Lat $39^{\circ} 02^{\prime} 03^{\prime \prime}$, long $86^{\circ} 34^{\prime} 03^{\prime \prime}$ referenced to North American Datum of 1927, in NE 1/4 NW 1/4 sec.19, T.7 N., R.1 W., Monroe County, IN, Hydrologic Unit 05120208, on left bank at downstream side of county road bridge, 1.9 mi northwest of Harrodsburg, 3.9 mi upstream from Little Clear Creek, and 5.1 mi upstream from mouth.

Drainage area. $-55.2 \mathrm{mi}^{2}$, of which $6.4 \mathrm{mi}^{2}$ does not contribute directly to surface runoff.

Period of record.- September 1960 to September 1971.

Average discharge. $-72.0 \mathrm{ft}^{3} / \mathrm{s}$.

Minimum daily discharge. $-5.4 \mathrm{ft}^{3} / \mathrm{s}$.

Human health (harmonic mean) design flow. $-24 \mathrm{ft}^{3} / \mathrm{s}$.

Remarks. - Flow regulated by effluent from sewage treatment plant of the city of Bloomington and possibly by pumpage from several rock quarries.

\begin{tabular}{|c|c|c|c|}
\hline \multicolumn{4}{|c|}{ Magnitude and frequency of annual low flow } \\
\hline \multicolumn{4}{|c|}{$\begin{array}{l}\text { Lowest average streamflow, in } \mathrm{ft}^{3} / \mathrm{s} \text {, for indicated period of } \\
\text { consecutive days at an annual nonexceedance probability of } 0.1\end{array}$} \\
\hline 1 & \multicolumn{2}{|c|}{7} & 30 \\
\hline 6.0 & \multicolumn{2}{|c|}{7.7} & 9.3 \\
\hline \multicolumn{4}{|c|}{$\begin{array}{c}\text { Percentage of time streamflow was equaled or exceeded for } \\
\text { the period of record }\end{array}$} \\
\hline $\begin{array}{l}\text { Percentage } \\
\text { of time }\end{array}$ & $\begin{array}{c}\text { Daily mean } \\
\text { streamflow } \\
\left(\mathrm{ft}^{3} / \mathrm{s}\right)\end{array}$ & $\begin{array}{l}\text { Percentage } \\
\text { of time }\end{array}$ & $\begin{array}{l}\text { Daily mean } \\
\text { streamflow } \\
\left(\mathrm{ft}^{3} / \mathrm{s}\right)\end{array}$ \\
\hline 99 & 8.1 & 40 & 39 \\
\hline 98 & 8.5 & 30 & 55 \\
\hline 95 & 9.7 & 20 & 83 \\
\hline 90 & 12 & 10 & 149 \\
\hline 80 & 15 & 5 & 237 \\
\hline 70 & 18 & 2 & 449 \\
\hline 60 & 22 & 1 & 749 \\
\hline 50 & 30 & & \\
\hline
\end{tabular}


Wabash River Basin

\section{INDIAN CREEK NEAR SPRINGVILLE, IN}

Location.- Lat $38^{\circ} 57^{\prime} 01^{\prime \prime}$, long $86^{\circ} 40^{\prime} 30^{\prime \prime}$ referenced to North American Datum of 1927, in SE 1/4 SW 1/4 Sec.18, T.6 N., R.2 W., Lawrence County, IN, Hydrologic Unit 05120208, on left bank at downstream side of bridge on State Highway 54, 0.2 mi downstream from Popcorn Creek, and 4 mi northwest of Springville.

Drainage area. $-60.7 \mathrm{mi}^{2}$.

Period of record.- September 1961 to September 1973.

Average discharge. $-55.3 \mathrm{ft}^{3} / \mathrm{s}$

Minimum daily discharge. $-0.0 \mathrm{ft}^{3} / \mathrm{s}$.

Human health (harmonic mean) design flow. $-2.2 \mathrm{ft}^{3} / \mathrm{s}$.

Remarks.- None.

\begin{tabular}{|c|c|c|c|}
\hline \multicolumn{4}{|c|}{ Magnitude and frequency of annual low flow } \\
\hline \multicolumn{4}{|c|}{$\begin{array}{l}\text { Lowest average streamflow, in } \mathrm{ft}^{3} / \mathrm{s} \text {, for indicated period of } \\
\text { consecutive days at an annual nonexceedance probability of } 0.1\end{array}$} \\
\hline 1 & \multicolumn{2}{|c|}{7} & 30 \\
\hline 0.0 & \multicolumn{2}{|c|}{0.0} & 0.0 \\
\hline \multicolumn{4}{|c|}{$\begin{array}{l}\text { Percentage of time streamflow was equaled or exceeded for } \\
\text { the period of record }\end{array}$} \\
\hline $\begin{array}{l}\text { Percentage } \\
\text { of time }\end{array}$ & $\begin{array}{c}\text { Daily mean } \\
\text { streamflow } \\
\left(\mathrm{ft}^{3} / \mathrm{s}\right)\end{array}$ & $\begin{array}{l}\text { Percentage } \\
\text { of time }\end{array}$ & $\begin{array}{l}\text { Daily mean } \\
\text { streamflow } \\
\left(\mathrm{ft}^{3} / \mathrm{s}\right)\end{array}$ \\
\hline 99 & 0.0 & 40 & 20 \\
\hline 98 & 0.1 & 30 & 34 \\
\hline 95 & 0.3 & 20 & 58 \\
\hline 90 & 0.9 & 10 & 119 \\
\hline 80 & 2.3 & 5 & 213 \\
\hline 70 & 4.0 & 2 & 448 \\
\hline 60 & 7.1 & 1 & 876 \\
\hline 50 & 12 & & \\
\hline
\end{tabular}




\section{EAST FORK WHITE RIVER AT SHOALS, IN}

Location. - Lat $38^{\circ} 40^{\prime} 01^{\prime \prime}$, long 86 $47^{\prime} 31^{\prime \prime}$ referenced to North American Datum of 1927, in SW 1/4 NW 1/4 sec.30, T.3 N., R.3 W., Martin County, IN, Hydrologic Unit 05120208, on upstream left bank, $30 \mathrm{ft}$ upstream of Highway 50 bridge at Shoals, $1.0 \mathrm{mi}$ upstream from Beaver Creek, $6.5 \mathrm{mi}$ downstream from Indian Creek, and at mile 105.4.

Drainage area.- $-4,927 \mathrm{mi}^{2}$.

Period of record.- June 1903 to July 1906, October 1908 to September 1916, June 1923 to current year.

Average discharge. $-5,772 \mathrm{ft}^{3} / \mathrm{s}$.

Minimum daily discharge. $-64 \mathrm{ft}^{3} / \mathrm{s}$.

Human health (harmonic mean) design flow. $-1,450 \mathrm{ft}^{3} / \mathrm{s}$.

Remarks. - Flow partially regulated by upstream reservoirs.

\begin{tabular}{ccc}
\hline \multicolumn{3}{c}{ Magnitude and frequency of annual low flow } \\
\hline $\begin{array}{c}\text { Lowest average streamflow, in } \mathrm{ft}^{3} / \mathrm{s} \text {, for indicated period of } \\
\text { consecutive days at an annual nonexceedance probability of } \mathbf{0 . 1}\end{array}$ \\
\hline $\mathbf{1}$ & $\mathbf{7}$ & $\mathbf{3 0}$ \\
\hline 194 & 275 & 315 \\
\hline
\end{tabular}

Percentage of time streamflow was equaled or exceeded for the period of record

\begin{tabular}{cc|cc}
\hline $\begin{array}{c}\text { Percentage } \\
\text { of time }\end{array}$ & $\begin{array}{c}\text { Daily mean } \\
\text { streamflow } \\
\left(\mathrm{ft}^{3} / \mathbf{s}\right)\end{array}$ & $\begin{array}{c}\text { Percentage } \\
\text { of time }\end{array}$ & $\begin{array}{c}\text { Daily mean } \\
\text { streamflow } \\
\left(\mathrm{ft}^{3} / \mathbf{s}\right)\end{array}$ \\
\hline 99 & 280 & 40 & 4200 \\
\hline 98 & 326 & 30 & 5970 \\
\hline 95 & 411 & 20 & 8810 \\
\hline 90 & 542 & 10 & 15,000 \\
\hline 80 & 827 & 5 & 21,100 \\
\hline 70 & 1270 & 2 & 30,900 \\
\hline 60 & 1890 & 1 & 39,000 \\
\hline 50 & 2870 & & \\
\hline
\end{tabular}


Wabash River Basin

\section{BEAVER CREEK NEAR SHOALS, IN}

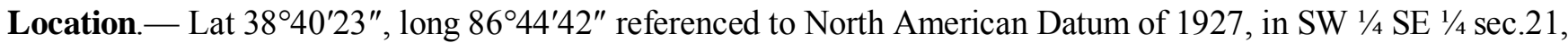
T.3 N., R.3 W., Martin County, IN, Hydrologic Unit 05120208, on right bank inside National Gypsum Property, $70 \mathrm{ft}$ upstream of confluence with small tributary on right, $3.8 \mathrm{mi}$ east of Shoals, and at river mile 7.08.

Drainage area.- $62.1 \mathrm{mi}^{2}$, of which $21.5 \mathrm{mi}^{2}$ probably is noncontributing.

Period of record. - June 2007 to current year.

Average discharge. $-44.7 \mathrm{ft}^{3} / \mathrm{s}$.

Minimum daily discharge. $-0.0 \mathrm{ft}^{3} / \mathrm{s}$.

Human health (harmonic mean) design flow. $-0.3 \mathrm{ft}^{3} / \mathrm{s}$.

Remarks. - This site has less than 10 years of record.

\begin{tabular}{|c|c|c|c|}
\hline \multicolumn{4}{|c|}{ Magnitude and frequency of annual low flow } \\
\hline \multicolumn{4}{|c|}{$\begin{array}{l}\text { Lowest average streamflow, in } \mathrm{ft}^{3} / \mathrm{s} \text {, for indicated period of } \\
\text { consecutive days at an annual nonexceedance probability of } 0.1\end{array}$} \\
\hline 1 & \multicolumn{2}{|c|}{7} & 30 \\
\hline 0 & \multicolumn{2}{|c|}{0} & 0 \\
\hline \multicolumn{4}{|c|}{$\begin{array}{l}\text { Percentage of time streamflow was equaled or exceeded for } \\
\text { the period of record }\end{array}$} \\
\hline $\begin{array}{l}\text { Percentage } \\
\text { of time }\end{array}$ & $\begin{array}{l}\text { Daily mean } \\
\text { streamflow } \\
\left(\mathrm{ft}^{3} / \mathrm{s}\right)\end{array}$ & $\begin{array}{l}\text { Percentage } \\
\text { of time }\end{array}$ & $\begin{array}{l}\text { Daily mean } \\
\text { streamflow } \\
\text { (ft3/s) }\end{array}$ \\
\hline 99 & 0.0 & 40 & 13 \\
\hline 98 & 0.0 & 30 & 20 \\
\hline 95 & 0.0 & 20 & 37 \\
\hline 90 & 0.1 & 10 & 100 \\
\hline 80 & 0.6 & 5 & 188 \\
\hline 70 & 2.1 & 2 & 449 \\
\hline 60 & 4.4 & 1 & 784 \\
\hline 50 & 8.1 & & \\
\hline
\end{tabular}




\section{LOST RIVER NEAR LIEPSIC, IN}

Location.- Lat $38^{\circ} 38^{\prime} 11^{\prime \prime}$, long $86^{\circ} 2^{\prime} 1^{\prime} 55^{\prime \prime}$ referenced to North American Datum of 1927, in NE 1/4 SE 1/4 sec.2, T.2 N., R.1 E., Orange County, IN, Hydrologic Unit 05120208, on left bank at county road bridge on County Road 500 E, about $0.1 \mathrm{mi}$ above Carter Creek tributary, $2.6 \mathrm{mi}$ south of Leipsic, and $6.3 \mathrm{mi}$ southeast of Orleans.

Drainage area. - $34.8 \mathrm{mi}^{2}$ (75.94 river miles), up to a gage height of $12.5 \mathrm{ft}$; $44 \mathrm{mi}^{2}$ above a gage height of $12.5 \mathrm{ft}$.

Period of record.- October 1992 to September 2001, October 20, 2010 to present.

Average discharge. $-45.4 \mathrm{ft}^{3} / \mathrm{s}$.

Minimum daily discharge. $-0.39 \mathrm{ft}^{3} / \mathrm{s}$.

Human health (harmonic mean) design flow. $-5.5 \mathrm{ft}^{3} / \mathrm{s}$.

Remarks. - None.

\begin{tabular}{c}
\hline \multicolumn{4}{c}{ Magnitude and frequency of annual low flow } \\
\hline \multicolumn{4}{c}{ Lowest average streamflow, in $\mathrm{ft}^{3}$ /s, for indicated period of } \\
consecutive days at an annual nonexceedance probability of 0.1
\end{tabular}




\section{LOST RIVER NEAR PROSPECT, IN}

Location. - Lat $38^{\circ} 34^{\prime} 52^{\prime \prime}$, long $86^{\circ} 35^{\prime} 56^{\prime \prime}$ referenced to North American Datum of 1927, in SE 1/4 NW 1/4 sec.26, T.2 N., R.2 W., Orange County, IN, Hydrologic Unit 05120208, on downstream side of bridge on Hwy 50/150 at $0.75 \mathrm{mi}$ east of Prospect, $9 \mathrm{mi}$ west of Paoli, $22 \mathrm{mi}$ southwest of Mitchell, $32 \mathrm{mi}$ southwest of Bedford, and at river mile 38.66.

Drainage area. $-175 \mathrm{mi}^{2}$.

Period of record. - January 2010 to current year.

Average discharge. $-253 \mathrm{ft}^{3} / \mathrm{s}$.

Minimum daily discharge. $-4.3 \mathrm{ft}^{3} / \mathrm{s}$.

Human health (harmonic mean) design flow. $-42 \mathrm{ft}^{3} / \mathrm{s}$.

Remarks. - This site has less than 10 years of record.

\begin{tabular}{ccc}
\hline \multicolumn{3}{c}{ Magnitude and frequency of annual low flow } \\
\hline $\begin{array}{c}\text { Lowest average streamflow, in } \mathrm{ft}^{3} / \mathrm{s} \text {, for indicated period of } \\
\text { consecutive days at an annual nonexceedance probability of } 0.1\end{array}$ \\
\hline $\mathbf{1}$ & $\mathbf{3 0}$ \\
\hline 4.9 & 5.7 & 7.3 \\
\hline
\end{tabular}

Percentage of time streamflow was equaled or exceeded for the period of record

\begin{tabular}{cc|cc}
\hline $\begin{array}{c}\text { Percentage } \\
\text { of time }\end{array}$ & $\begin{array}{c}\text { Daily mean } \\
\text { streamflow } \\
\left(\mathrm{ft}^{3} / \mathbf{s}\right)\end{array}$ & $\begin{array}{c}\text { Percentage } \\
\text { of time }\end{array}$ & $\begin{array}{c}\text { Daily mean } \\
\text { streamflow } \\
\left(\mathrm{ft}^{3} / \mathbf{s}\right)\end{array}$ \\
\hline 99 & 5.9 & 40 & 153 \\
\hline 98 & 6.8 & 30 & 232 \\
\hline 95 & 9.8 & 20 & 336 \\
\hline 90 & 14 & 10 & 549 \\
\hline 80 & 18 & 5 & 894 \\
\hline 70 & 28 & 2 & 1920 \\
\hline 60 & 58 & 1 & 2800 \\
\hline 50 & 106 & & \\
\hline
\end{tabular}




\section{LICK CREEK AT PAOLI, IN}

Location. - Lat 38 $33^{\prime} 21^{\prime \prime}$, long $86^{\circ} 28^{\prime} 33^{\prime \prime}$ referenced to North American Datum of 1983, in NW 1/4 NW 1/4 Sec.1, T.1 N., R.1 W., Orange County, IN, Hydrologic Unit 05120208, on the middle of the bridge over Highway 56, 0.5 mi west of Highway 37, 9.5 mi east of West Baden Springs, and $23.5 \mathrm{mi}$ south of Bedford.

Drainage area. $-21.5 \mathrm{mi}^{2}$.

Period of record. - October 2010 to current year.

Average discharge. $-51.6 \mathrm{ft}^{3} / \mathrm{s}$.

Minimum daily discharge. $-0.28 \mathrm{ft}^{3} / \mathrm{s}$.

Human health (harmonic mean) design flow. $-4.7 \mathrm{ft}^{3} / \mathrm{s}$.

Remarks. - This site has less than 10 years of record.

\begin{tabular}{ccc}
\hline \multicolumn{3}{c}{ Magnitude and frequency of annual low flow } \\
\hline $\begin{array}{c}\text { Lowest average streamflow, in } \mathrm{ft}^{3} / \mathrm{s} \text {, for indicated period of } \\
\text { consecutive days at an annual nonexceedance probability of } \mathbf{0 . 1}\end{array}$ \\
\hline $\mathbf{1}$ & $\mathbf{7}$ & $\mathbf{3 0}$ \\
\hline 0.5 & 0.6 & 0.8 \\
\hline
\end{tabular}

Percentage of time streamflow was equaled or exceeded for the period of record

\begin{tabular}{cc|cc}
\hline $\begin{array}{c}\text { Percentage } \\
\text { of time }\end{array}$ & $\begin{array}{c}\text { Daily mean } \\
\text { streamflow } \\
\left(\mathrm{ft}^{3} / \mathbf{s}\right)\end{array}$ & $\begin{array}{c}\text { Percentage } \\
\text { of time }\end{array}$ & $\begin{array}{c}\text { Daily mean } \\
\text { streamflow } \\
\left(\mathrm{ft}^{3} / \mathbf{s}\right)\end{array}$ \\
\hline 99 & 0.4 & 40 & 25 \\
\hline 98 & 0.6 & 30 & 38 \\
\hline 95 & 1 & 20 & 56 \\
\hline 90 & 1.4 & 10 & 125 \\
\hline 80 & 2 & 5 & 222 \\
\hline 70 & 2.9 & 2 & 407 \\
\hline 60 & 5.7 & 1 & 757 \\
\hline 50 & 12 & & \\
\hline
\end{tabular}




\section{FRENCH LICK CREEK AT FRENCH LICK, IN}

Location. - Lat 38 $32^{\prime} 29^{\prime \prime}$, long 86 $36^{\prime} 45^{\prime \prime}$ referenced to North American Datum of 1983, in NW 1/4 NE 1/4 sec.10, T.1 N., R.2 W., Orange County, IN, Hydrologic Unit 05120208, on upstream side of bridge on 100S, $300 \mathrm{ft}$ south of Highway 145, 1.2 mi east of Highway 56, and 12.5 mi west of Paoli.

Drainage area. $-25.1 \mathrm{mi}^{2}$.

Period of record. - January 2010 to current year.

Average discharge. $-48.3 \mathrm{ft}^{3} / \mathrm{s}$.

Minimum daily discharge. $-0.0 \mathrm{ft}^{3} / \mathrm{s}$.

Human health (harmonic mean) design flow. $-0.8 \mathrm{ft}^{3} / \mathrm{s}$.

Remarks. - This site has less than 10 years of record.

\begin{tabular}{ccc}
\hline \multicolumn{3}{c}{ Magnitude and frequency of annual low flow } \\
\hline $\begin{array}{c}\text { Lowest average streamflow, in } \mathrm{ft}^{3} / \mathrm{s} \text {, for indicated period of } \\
\text { consecutive days at an annual nonexceedance probability of } 0.1\end{array}$ \\
\hline $\mathbf{1}$ & $\mathbf{3 0}$ & 0.0 \\
\hline 0.0 & 0.0 & \\
\hline
\end{tabular}

Percentage of time streamflow was equaled or exceeded for the period of record

\begin{tabular}{cc|cc}
\hline $\begin{array}{c}\text { Percentage } \\
\text { of time }\end{array}$ & $\begin{array}{c}\text { Daily mean } \\
\text { streamflow } \\
\left(\mathrm{ft}^{3} / \mathbf{s}\right)\end{array}$ & $\begin{array}{c}\text { Percentage } \\
\text { of time }\end{array}$ & $\begin{array}{c}\text { Daily mean } \\
\text { streamflow } \\
\left(\mathrm{ft}^{3} / \mathbf{s}\right)\end{array}$ \\
\hline 99 & 0 & 40 & 20 \\
\hline 98 & 0 & 30 & 33 \\
\hline 95 & 0 & 20 & 53 \\
\hline 90 & 0.5 & 10 & 105 \\
\hline 80 & 2.1 & 5 & 167 \\
\hline 70 & 3.8 & 2 & 403 \\
\hline 60 & 6.8 & 1 & 637 \\
\hline 50 & 12 & & \\
\hline
\end{tabular}




\section{FRENCH LICK CREEK AT WEST BADEN SPRINGS, IN}

Location. - Lat $38^{\circ} 33^{\prime} 48^{\prime \prime}$, long $86^{\circ} 37^{\prime} 00^{\prime \prime}$ referenced to North American Datum of 1927, in NW 1/4 SE 1/4 sec.34, T.2 N., R.2 W., Orange County, IN, Hydrologic Unit 05120208, on the upstream side of the bridge over French Lick Creek, 0.2 mi west of Highway 56, at river mile 0.9, $1.3 \mathrm{mi}$ south of Prospect, and $10.1 \mathrm{mi}$ west of Paoli.

Drainage area. $-32.9 \mathrm{mi}^{2}$.

Period of record.- August 26, 2010 to current year.

Average discharge. $-72.1 \mathrm{ft}^{3} / \mathrm{s}$.

Minimum daily discharge. $-0.07 \mathrm{ft}^{3} / \mathrm{s}$.

Human health (harmonic mean) design flow. $-1.6 \mathrm{ft}^{3} / \mathrm{s}$.

Remarks. - This site has less than 10 years of record. -- indicates that there is poor correlation with the index stations, no values determined.

\begin{tabular}{|c|c|c|c|}
\hline \multicolumn{4}{|c|}{ Magnitude and frequency of annual low flow } \\
\hline \multicolumn{4}{|c|}{$\begin{array}{l}\text { Lowest average streamflow, in } \mathrm{ft}^{3} / \mathrm{s} \text {, for indicated period of } \\
\text { consecutive days at an annual nonexceedance probability of } 0 .\end{array}$} \\
\hline 1 & \multicolumn{2}{|c|}{7} & 30 \\
\hline -- & \multicolumn{2}{|c|}{--} & -- \\
\hline \multicolumn{4}{|c|}{$\begin{array}{l}\text { Percentage of time streamflow was equaled or exceeded for } \\
\text { the period of record }\end{array}$} \\
\hline $\begin{array}{l}\text { Percentage } \\
\text { of time }\end{array}$ & $\begin{array}{l}\text { Daily mean } \\
\text { streamflow } \\
\left(\mathrm{ft}^{3} / \mathrm{s}\right)\end{array}$ & $\begin{array}{l}\text { Percentage } \\
\text { of time }\end{array}$ & $\begin{array}{l}\text { Daily mean } \\
\text { streamflow } \\
\left(\mathrm{ft}^{3} / \mathrm{s}\right)\end{array}$ \\
\hline 99 & 0.1 & 40 & 23 \\
\hline 98 & 0.2 & 30 & 38 \\
\hline 95 & 0.3 & 20 & 57 \\
\hline 90 & 0.7 & 10 & 94 \\
\hline 80 & 2 & 5 & 159 \\
\hline 70 & 3.9 & 2 & 684 \\
\hline 60 & 7.4 & 1 & 1180 \\
\hline 50 & 14 & & \\
\hline
\end{tabular}




\section{LOST RIVER NEAR WEST BADEN SPRINGS, IN}

Location.- Lat $38^{\circ} 35^{\prime} 10^{\prime \prime}$, long $86^{\circ} 38^{\prime} 03^{\prime \prime}$ referenced to North American Datum of 1927, in SW 1/4 SE 1/4 Sec.21, T.2 N., R.2 W., Orange County, IN, Hydrologic Unit 05120208, on left bank $20 \mathrm{ft}$ downstream from bridge on U.S. Highway 150, 1.7 mi northwest of West Baden Springs, 3.8 mi downstream from Lick Creek, and at mile 34.8.

Drainage area. $-287 \mathrm{mi}^{2}$.

Period of record.—- December 1964 to September 1993.

Average discharge. $-355 \mathrm{ft}^{3} / \mathrm{s}$.

Minimum daily discharge. $-5.9 \mathrm{ft}^{3} / \mathrm{s}$.

Human health (harmonic mean) design flow. $-60 \mathrm{ft}^{3} / \mathrm{s}$.

Remarks. - Prior to October 1965, published as Lost River near West Baden.

\begin{tabular}{|c|c|c|c|}
\hline \multicolumn{4}{|c|}{ Magnitude and frequency of annual low flow } \\
\hline \multicolumn{4}{|c|}{$\begin{array}{l}\text { Lowest average streamflow, in } \mathrm{ft}^{3} / \mathrm{s} \text {, for indicated period of } \\
\text { consecutive days at an annual nonexceedance probability of } 0.1\end{array}$} \\
\hline 1 & \multicolumn{2}{|c|}{7} & 30 \\
\hline 8.1 & \multicolumn{2}{|c|}{8.9} & 11 \\
\hline \multicolumn{4}{|c|}{$\begin{array}{c}\text { Percentage of time streamflow was equaled or exceeded for } \\
\text { the period of record }\end{array}$} \\
\hline $\begin{array}{l}\text { Percentage } \\
\text { of time }\end{array}$ & $\begin{array}{c}\text { Daily mean } \\
\text { streamflow } \\
\left(\mathrm{ft}^{3} / \mathrm{s}\right)\end{array}$ & $\begin{array}{l}\text { Percentage } \\
\text { of time }\end{array}$ & $\begin{array}{l}\text { Daily mean } \\
\text { streamflow } \\
\left(\mathrm{ft}^{3} / \mathrm{s}\right)\end{array}$ \\
\hline 99 & 10 & 40 & 215 \\
\hline 98 & 12 & 30 & 331 \\
\hline 95 & 16 & 20 & 498 \\
\hline 90 & 22 & 10 & 908 \\
\hline 80 & 34 & 5 & 1470 \\
\hline 70 & 58 & 2 & 2240 \\
\hline 60 & 92 & 1 & 2820 \\
\hline 50 & 141 & & \\
\hline
\end{tabular}




\section{WHITE RIVER AT PETERSBURG, IN}

Location. - Lat $38^{\circ} 30^{\prime} 39^{\prime \prime}$, long $87^{\circ} 17^{\prime} 22^{\prime \prime}$ referenced to North American Datum of 1927, in SE 1/4 SW 1/4 Sec.15, T.1 N., R.8 W., Pike County, IN, Hydrologic Unit 05120202, on the downstream rail on the State Highway 61 bridge, 0.4 mi upstream from Prides Creek, $1.4 \mathrm{mi}$ north of Petersburg, $2.0 \mathrm{mi}$ west of Arda, and at mile 45.7.

Drainage area. $-11,125 \mathrm{mi}^{2}$.

Period of record. - October 1927 to current year.

Average discharge. $-12,660 \mathrm{ft}^{3} / \mathrm{s}$.

Minimum daily discharge. $-573 \mathrm{ft}^{3} / \mathrm{s}$.

Human health (harmonic mean) design flow. - 3,990 $\mathrm{ft}^{3} / \mathrm{s}$.

Remarks. - Flow partially regulated by upstream reservoirs. Published as "at Hazelton" October 1927 to September 1938.

\begin{tabular}{|c|c|c|c|}
\hline \multicolumn{4}{|c|}{ Magnitude and frequency of annual low flow } \\
\hline \multicolumn{4}{|c|}{$\begin{array}{l}\text { Lowest average streamflow, in } \mathrm{ft}^{3} / \mathrm{s} \text {, for indicated period of } \\
\text { consecutive days at an annual nonexceedance probability of } 0 \text {. }\end{array}$} \\
\hline 1 & \multicolumn{2}{|c|}{7} & 30 \\
\hline 791 & \multicolumn{2}{|c|}{837} & 925 \\
\hline \multicolumn{4}{|c|}{$\begin{array}{l}\text { Percentage of time streamflow was equaled or exceeded for } \\
\text { the period of record }\end{array}$} \\
\hline $\begin{array}{l}\text { Percentage } \\
\text { of time }\end{array}$ & $\begin{array}{l}\text { Daily mean } \\
\text { streamflow } \\
\left(\mathrm{ft}^{3} / \mathrm{s}\right)\end{array}$ & $\begin{array}{l}\text { Percentage } \\
\text { of time }\end{array}$ & $\begin{array}{l}\text { Daily mean } \\
\text { streamflow } \\
\left(\mathrm{ft}^{3} / \mathrm{s}\right)\end{array}$ \\
\hline 99 & 851 & 40 & 9790 \\
\hline 98 & 967 & 30 & 13500 \\
\hline 95 & 1210 & 20 & 19300 \\
\hline 90 & 1580 & 10 & 30800 \\
\hline 80 & 2370 & 5 & 43600 \\
\hline 70 & 3400 & 2 & 62800 \\
\hline 60 & 4900 & 1 & 79700 \\
\hline 50 & 7020 & & \\
\hline
\end{tabular}




\section{PATOKA RIVER NEAR HARDINSBURG, IN}

Location. - Lat $38^{\circ} 26^{\prime} 41^{\prime \prime}$, long $86^{\circ} 23^{\prime} 14^{\prime \prime}$ referenced to North American Datum of 1927, in NW 1/4 SE 1/4 sec.10, T.1 S., R.1 E., Orange County, IN, Hydrologic Unit 05120209, on downstream edge of center pier of county road bridge, $0.3 \mathrm{mi}$ downstream from Fudge Creek, $0.7 \mathrm{mi}$ northeast of Valeene, $6.0 \mathrm{mi}$ southwest of Hardinsburg, and at mile 158.0.

Drainage area. $-12.8 \mathrm{mi}^{2}$.

Period of record.- October 1968 to October 2003.

Average discharge. $-25.2 \mathrm{ft}^{3} / \mathrm{s}$.

Minimum daily discharge. $-0.0 \mathrm{ft}^{3} / \mathrm{s}$.

Human health (harmonic mean) design flow. $-0.6 \mathrm{ft}^{3} / \mathrm{s}$.

Remarks. - None.

\begin{tabular}{|c|c|c|c|}
\hline \multicolumn{4}{|c|}{ Magnitude and frequency of annual low flow } \\
\hline \multicolumn{4}{|c|}{$\begin{array}{l}\text { Lowest average streamflow, in } \mathrm{ft}^{3} / \mathrm{s} \text {, for indicated period of } \\
\text { consecutive days at an annual nonexceedance probability of } 0.1\end{array}$} \\
\hline 1 & \multicolumn{2}{|c|}{7} & 30 \\
\hline 0.0 & \multicolumn{2}{|c|}{0.0} & 0.0 \\
\hline \multicolumn{4}{|c|}{$\begin{array}{l}\text { Percentage of time streamflow was equaled or exceeded for } \\
\text { the period of record }\end{array}$} \\
\hline $\begin{array}{l}\text { Percentage } \\
\text { of time }\end{array}$ & $\begin{array}{l}\text { Daily mean } \\
\text { streamflow } \\
\left(\mathrm{ft}^{3} / \mathrm{s}\right)\end{array}$ & $\begin{array}{l}\text { Percentage } \\
\text { of time }\end{array}$ & $\begin{array}{l}\text { Daily mean } \\
\text { streamflow } \\
\left(\mathrm{ft}^{3} / \mathrm{s}\right)\end{array}$ \\
\hline 99 & 0.0 & 40 & 8.9 \\
\hline 98 & 0.0 & 30 & 15 \\
\hline 95 & 0.1 & 20 & 26 \\
\hline 90 & 0.2 & 10 & 54 \\
\hline 80 & 0.7 & 5 & 110 \\
\hline 70 & 1.6 & 2 & 230 \\
\hline 60 & 2.9 & 1 & 369 \\
\hline 50 & 5.2 & & \\
\hline
\end{tabular}




\section{PATOKA RIVER AT JASPER, IN}

Location.- Lat $38^{\circ} 24^{\prime} 49^{\prime \prime}$, long 86 52'36" referenced to North American Datum of 1927, in NW 1/4 SE 1/4 sec.20, T.1 S., R.4 W., Dubois County, IN, Hydrologic Unit 05120209, on left bank 0.3 mi upstream from unnamed outlet of Idlewild Lake, 1.2 mi downstream from county road bridge, $1.2 \mathrm{mi}$ downstream from Beaver Creek, $3.3 \mathrm{mi}$ northeast of Jasper, and at mile 91.5 .

Drainage area. $-262.0 \mathrm{mi}^{2}$.

Period of record. - November 1947 to current year.

Average discharge. $-371 \mathrm{ft}^{3} / \mathrm{s}$.

Minimum daily discharge. $-0.0 \mathrm{ft}^{3} / \mathrm{s}$.

Human health (harmonic mean) design flow. $-100 \mathrm{ft}^{3} / \mathrm{s}$.

Remarks. - Flow regulated by Beaver Creek Reservoir beginning Oct. 11, 1955, and by Patoka Lake beginning Feb. 13, 1978. Low-flow statistics are calculated for the regulated period, 1978 to 2011.

\begin{tabular}{|c|c|c|c|}
\hline \multicolumn{4}{|c|}{ Magnitude and frequency of annual low flow } \\
\hline \multicolumn{4}{|c|}{$\begin{array}{l}\text { Lowest average streamflow, in } \mathrm{ft}^{3} / \mathrm{s} \text {, for indicated period of } \\
\text { consecutive days at an annual nonexceedance probability of } 0.1\end{array}$} \\
\hline 1 & \multicolumn{2}{|c|}{7} & 30 \\
\hline 13 & \multicolumn{2}{|c|}{17} & 21 \\
\hline \multicolumn{4}{|c|}{$\begin{array}{l}\text { Percentage of time streamflow was equaled or exceeded for } \\
\text { the period of record }\end{array}$} \\
\hline $\begin{array}{l}\text { Percentage } \\
\text { of time }\end{array}$ & $\begin{array}{c}\text { Daily mean } \\
\text { streamflow } \\
\left(\mathrm{ft}^{3} / \mathrm{s}\right)\end{array}$ & $\begin{array}{l}\text { Percentage } \\
\text { of time }\end{array}$ & $\begin{array}{l}\text { Daily mean } \\
\text { streamflow } \\
\left(\mathrm{ft}^{3} / \mathrm{s}\right)\end{array}$ \\
\hline 99 & 20 & 40 & 294 \\
\hline 98 & 23 & 30 & 427 \\
\hline 95 & 29 & 20 & 663 \\
\hline 90 & 37 & 10 & 993 \\
\hline 80 & 56 & 5 & 1240 \\
\hline 70 & 97 & 2 & 1630 \\
\hline 60 & 152 & 1 & 2010 \\
\hline 50 & 213 & & \\
\hline
\end{tabular}




\section{HALL CREEK NEAR ST. ANTHONY, IN}

Location. - Lat 38 $21^{\prime} 45^{\prime \prime}$, long 86 $49^{\prime} 43^{\prime \prime}$ referenced to North American Datum of 1927, in NW 1/4 NW 1/4 sec.11, T.2 S., R.4 W., Dubois County, IN, Hydrologic Unit 05120209, on right bank $10 \mathrm{ft}$ downstream of bridge on County Road 125 South, 0.7 mi upstream from Grassy Fork, 3.3 mi north of St. Anthony, and at mile 4.1.

Drainage area. $-21.8 \mathrm{mi}^{2}$.

Period of record. - October 1970 to December 2001.

Average discharge. $-31.6 \mathrm{ft}^{3} / \mathrm{s}$.

Minimum daily discharge. $-0.0 \mathrm{ft}^{3} / \mathrm{s}$.

Human health (harmonic mean) design flow. $-0.5 \mathrm{ft}^{3} / \mathrm{s}$.

Remarks. - None.

\begin{tabular}{ccc}
\hline \multicolumn{3}{c}{ Magnitude and frequency of annual low flow } \\
\hline $\begin{array}{c}\text { Lowest average streamflow, in } \mathrm{ft}^{3} / \mathrm{s} \text {, for indicated period of } \\
\text { consecutive days at an annual nonexceedance probability of } 0.1\end{array}$ \\
\hline $\mathbf{1}$ & $\mathbf{3 0}$ \\
\hline 0.0 & 0.0 & 0.0 \\
\hline
\end{tabular}

Percentage of time streamflow was equaled or exceeded for the period of record

\begin{tabular}{cc|cc}
\hline $\begin{array}{c}\text { Percentage } \\
\text { of time }\end{array}$ & $\begin{array}{c}\text { Daily mean } \\
\text { streamflow } \\
\left(\mathrm{ft}^{3} / \mathbf{s}\right)\end{array}$ & $\begin{array}{c}\text { Percentage } \\
\text { of time }\end{array}$ & $\begin{array}{c}\text { Daily mean } \\
\text { streamflow } \\
\left(\mathrm{ft}^{3} / \mathbf{s}\right)\end{array}$ \\
\hline 99 & 0.0 & 40 & 11 \\
\hline 98 & 0.0 & 30 & 17 \\
\hline 95 & 0.0 & 20 & 29 \\
\hline 90 & 0.3 & 10 & 63 \\
\hline 80 & 0.9 & 5 & 129 \\
\hline 70 & 2.1 & 2 & 307 \\
\hline 60 & 4.1 & 1 & 499 \\
\hline 50 & 7.0 & & \\
\hline
\end{tabular}




\section{FLAT CREEK NEAR OTWELL, IN}

Location.- Lat $38^{\circ} 26^{\prime} 12^{\prime \prime}$, long $87^{\circ} 07^{\prime} 52^{\prime \prime}$ referenced to North American Datum of 1927 , in SE $1 / 4$ SE $1 / 4$ sec.12, T.1 S., R.7 W., Pike County, IN, Hydrologic Unit 05120209, on right bank at upstream side of bridge on State Highway 56; 2.2 mi west of intersection of State Highways 56 and 257; 2.5 mi southeast of Otwell, 6.2 mi east of intersection of State Highways 56 and 61, and at mile 10.9.

Drainage area.- $21.3 \mathrm{mi}^{2}$.

Period of record.- October 1964 to April 1982.

Average discharge. $-22.8 \mathrm{ft}^{3} / \mathrm{s}$.

Minimum daily discharge. $-0.0 \mathrm{ft}^{3} / \mathrm{s}$.

Human health (harmonic mean) design flow. $-0.8 \mathrm{ft}^{3} / \mathrm{s}$.

Remarks. - None.

\begin{tabular}{|c|c|c|c|}
\hline \multicolumn{4}{|c|}{ Magnitude and frequency of annual low flow } \\
\hline \multicolumn{4}{|c|}{$\begin{array}{l}\text { Lowest average streamflow, in } \mathrm{ft}^{3} / \mathrm{s} \text {, for indicated period of } \\
\text { consecutive days at an annual nonexceedance probability of } 0.1\end{array}$} \\
\hline 1 & \multicolumn{2}{|c|}{7} & 30 \\
\hline 0.0 & \multicolumn{2}{|c|}{0.0} & 0.0 \\
\hline \multicolumn{4}{|c|}{$\begin{array}{c}\text { Percentage of time streamflow was equaled or exceeded for } \\
\text { the period of record }\end{array}$} \\
\hline $\begin{array}{l}\text { Percentage } \\
\text { of time }\end{array}$ & $\begin{array}{l}\text { Daily mean } \\
\text { streamflow } \\
\left(\mathrm{ft}^{3} / \mathrm{s}\right)\end{array}$ & $\begin{array}{l}\text { Percentage } \\
\text { of time }\end{array}$ & $\begin{array}{l}\text { Daily mean } \\
\text { streamflow } \\
\left(\mathrm{ft}^{3} / \mathrm{s}\right)\end{array}$ \\
\hline 99 & 0.0 & 40 & 7.0 \\
\hline 98 & 0.0 & 30 & 11 \\
\hline 95 & 0.0 & 20 & 20 \\
\hline 90 & 0.2 & 10 & 46 \\
\hline 80 & 0.9 & 5 & 95 \\
\hline 70 & 2.0 & 2 & 239 \\
\hline 60 & 3.1 & 1 & 398 \\
\hline 50 & 4.7 & & \\
\hline
\end{tabular}




\section{PATOKA RIVER AT WINSLOW, IN}

Location. - Lat $38^{\circ} 22^{\prime} 49^{\prime \prime}$, long $87^{\circ} 13^{\prime} 00^{\prime \prime}$ referenced to North American Datum of 1927, in SW 1/4 SW 1/4 sec.32, T.1 S., R.7 W., Pike County, IN, Hydrologic Unit 05120209, on right bank at abandoned bridge abutment, $65 \mathrm{ft}$ upstream from bridge on State Highway 61, $100 \mathrm{ft}$ downstream from dam of Winslow Water Company, and $41.3 \mathrm{mi}$ above mouth.

Drainage area. - $603 \mathrm{mi}^{2}$.

Period of record. - October 1963 to current year.

Average discharge. $-809 \mathrm{ft}^{3} / \mathrm{s}$.

Minimum daily discharge. $-0.50 \mathrm{ft}^{3} / \mathrm{s}$.

Human health (harmonic mean) design flow. $-162 \mathrm{ft}^{3} / \mathrm{s}$.

Remarks. - Minor diversion by municipal water supply $100 \mathrm{ft}$ above gage. Flow regulated by Patoka Lake. Lowflow statistics are calculated for the regulated period, 1987 to 2011.

\begin{tabular}{ccc}
\hline \multicolumn{3}{c}{ Magnitude and frequency of annual low flow } \\
\hline $\begin{array}{c}\text { Lowest average streamflow, in } \mathrm{ft}^{3} / \mathrm{s} \text {, for indicated period of } \\
\text { consecutive days at an annual nonexceedance probability of } 0.1\end{array}$ \\
\hline $\mathbf{1}$ & $\mathbf{7}$ & $\mathbf{3 0}$ \\
\hline 20 & 22 & 28 \\
\hline
\end{tabular}

Percentage of time streamflow was equaled or exceeded for the period of record

\begin{tabular}{cc|cc}
\hline $\begin{array}{c}\text { Percentage } \\
\text { of time }\end{array}$ & $\begin{array}{c}\text { Daily mean } \\
\text { streamflow } \\
\left(\mathrm{ft}^{3} / \mathbf{s}\right)\end{array}$ & $\begin{array}{c}\text { Percentage } \\
\text { of time }\end{array}$ & $\begin{array}{c}\text { Daily mean } \\
\text { streamflow } \\
\left(\mathbf{f t}^{3} / \mathbf{s}\right)\end{array}$ \\
\hline 99 & 25 & 40 & 694 \\
\hline 98 & 30 & 30 & 1060 \\
\hline 95 & 39 & 20 & 1500 \\
\hline 90 & 57 & 10 & 2210 \\
\hline 80 & 106 & 5 & 2940 \\
\hline 70 & 181 & 2 & 4360 \\
\hline 60 & 276 & 1 & 5520 \\
\hline 50 & 419 & & \\
\hline
\end{tabular}




\section{SOUTH FORK PATOKA RIVER NEAR SPURGEON, IN}

Location.- Lat $38^{\circ} 17^{\prime} 50^{\prime \prime}$, long $87^{\circ} 15^{\prime} 39^{\prime \prime}$ referenced to North American Datum of 1927 in SE 1/4 NE 1/4 Sec.35, T.2 S., R.8 W., Pike County, IN, Hydrologic Unit 05120209, on right bank at downstream side of bridge on State Highway 61, 0.5 mi north of Enos Corner, $3.1 \mathrm{mi}$ north of Spurgeon, and at mile 8.0.

Drainage area.- $42.8 \mathrm{mi}^{2}$.

Period of record.- October 1964 to September 1986.

Average discharge. $-52.0 \mathrm{ft}^{3} / \mathrm{s}$.

Minimum daily discharge. $-0.0 \mathrm{ft}^{3} / \mathrm{s}$.

Human health (harmonic mean) design flow. - $14 \mathrm{ft}^{3} / \mathrm{s}$.

Remarks. - Regulation by coal-washing operation and strip mining above gage.

\begin{tabular}{|c|c|c|c|}
\hline \multicolumn{4}{|c|}{ Magnitude and frequency of annual low flow } \\
\hline \multicolumn{4}{|c|}{$\begin{array}{l}\text { Lowest average streamflow, in } \mathrm{ft}^{3} / \mathrm{s} \text {, for indicated period of } \\
\text { consecutive days at an annual nonexceedance probability of } 0.1\end{array}$} \\
\hline 1 & \multicolumn{2}{|c|}{7} & 30 \\
\hline 2.9 & \multicolumn{2}{|c|}{3.2} & 4.8 \\
\hline \multicolumn{4}{|c|}{$\begin{array}{l}\text { Percentage of time streamflow was equaled or exceeded for } \\
\text { the period of record }\end{array}$} \\
\hline $\begin{array}{l}\text { Percentage } \\
\text { of time }\end{array}$ & $\begin{array}{l}\text { Daily mean } \\
\text { streamflow } \\
\left(\mathrm{ft}^{3} / \mathrm{s}\right)\end{array}$ & $\begin{array}{l}\text { Percentage } \\
\text { of time }\end{array}$ & $\begin{array}{l}\text { Daily mean } \\
\text { streamflow } \\
\left(\mathrm{ft}^{3} / \mathrm{s}\right)\end{array}$ \\
\hline 99 & 3.4 & 40 & 30 \\
\hline 98 & 4.3 & 30 & 42 \\
\hline 95 & 5.4 & 20 & 61 \\
\hline 90 & 6.6 & 10 & 113 \\
\hline 80 & 9.0 & 5 & 190 \\
\hline 70 & 12 & 2 & 352 \\
\hline 60 & 17 & 1 & 510 \\
\hline 50 & 22 & & \\
\hline
\end{tabular}


Wabash River Basin

\section{PATOKA RIVER NEAR PRINCETON, IN}

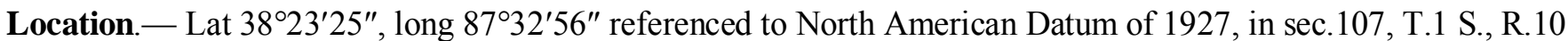
W., Gibson County, IN, Hydrologic Unit 05120209, on right downstream side of bridge on State Highway 65, 0.5 mi downstream from Indian Creek, 2 mi northeast of Princeton, and at mile 21.4.

Drainage area. $-822 \mathrm{mi}^{2}$.

Period of record. - August 1934 to current year.

Average discharge. $-1,066 \mathrm{ft}^{3} / \mathrm{s}$.

Minimum daily discharge. $-0.0 \mathrm{ft}^{3} / \mathrm{s}$.

Human health (harmonic mean) design flow. $-250 \mathrm{ft}^{3} / \mathrm{s}$.

Remarks. - Flow regulated by Patoka Lake. Low-flow statistics are calculated for the regulated period, 1978 to 2011.

\begin{tabular}{c}
\hline \multicolumn{5}{c}{ Magnitude and frequency of annual low flow } \\
\hline \multicolumn{4}{c}{ Lowest average streamflow, in ft 3 /s, for indicated period of } \\
consecutive days at an annual nonexceedance probability of 0.1 \\
\hline $\mathbf{1}$ \\
\hline 33
\end{tabular}




\section{WABASH RIVER AT MOUNT CARMEL, IL}

Location. - Lat $38^{\circ} 23^{\prime} 54^{\prime \prime}$, long $87^{\circ} 45^{\prime} 23^{\prime \prime}$ referenced to North American Datum of 1983, in SE 1/4 NW 1/4 sec.28, T.1 S., R.12 W., Wabash County, IL, Hydrologic Unit 05120113, on upstream right side of SR 64 bridge at Mount Carmel, 0.4 mi downstream from Patoka River, 0.2 mi downstream of Southern Railway bridge, and at mile 94.2.

Drainage area.- $28,635 \mathrm{mi}^{2}$.

Period of record. - October 1927 to current year.

Average discharge. $-29,280 \mathrm{ft}^{3} / \mathrm{s}$.

Minimum daily discharge. $-1,650 \mathrm{ft}^{3} / \mathrm{s}$.

Human health (harmonic mean) design flow. - 11,050 $\mathrm{ft}^{3} / \mathrm{s}$.

Remarks. - Flow partially regulated by upstream reservoirs.

\begin{tabular}{|c|c|c|c|}
\hline \multicolumn{4}{|c|}{ Magnitude and frequency of annual low flow } \\
\hline \multicolumn{4}{|c|}{$\begin{array}{l}\text { Lowest average streamflow, in } \mathrm{ft}^{3} / \mathrm{s} \text {, for indicated period of } \\
\text { consecutive days at an annual nonexceedance probability of } 0.1\end{array}$} \\
\hline 1 & \multicolumn{2}{|c|}{7} & 30 \\
\hline 2480 & \multicolumn{2}{|c|}{2580} & 2880 \\
\hline \multicolumn{4}{|c|}{$\begin{array}{l}\text { Percentage of time streamflow was equaled or exceeded for } \\
\text { the period of record }\end{array}$} \\
\hline $\begin{array}{l}\text { Percentage } \\
\text { of time }\end{array}$ & $\begin{array}{l}\text { Daily mean } \\
\text { streamflow } \\
\left(\mathrm{ft}^{3} / \mathrm{s}\right)\end{array}$ & $\begin{array}{l}\text { Percentage } \\
\text { of time }\end{array}$ & $\begin{array}{l}\text { Daily mean } \\
\text { streamflow } \\
\left(\mathrm{ft}^{3} / \mathrm{s}\right)\end{array}$ \\
\hline 99 & 2560 & 40 & 24100 \\
\hline 98 & 2850 & 30 & 32800 \\
\hline 95 & 3580 & 20 & 46600 \\
\hline 90 & 4570 & 10 & 69400 \\
\hline 80 & 6640 & 5 & 93700 \\
\hline 70 & 9210 & 2 & 130000 \\
\hline 60 & 12900 & 1 & 157000 \\
\hline 50 & 17600 & & \\
\hline
\end{tabular}




\section{BIG CREEK NEAR WADESVILLE, IN}

Location. - Lat $38^{\circ} 04^{\prime} 58^{\prime \prime}$, long $87^{\circ} 46^{\prime} 10^{\prime \prime}$ referenced to North American Datum of 1927, in SW 1/4 SW 1/4 sec.16, T.5 S., R.12 W., Posey County, IN, Hydrologic Unit 05120113, on left bank at downstream side of bridge on State Highway 66, 0.6 mi northwest of Blairsville, 0.8 mi upstream from County Road 250 North, and 1.6 mi southeast of Wadesville.

Drainage area. - $104 \mathrm{mi}^{2}$.

Period of record. - July 1965 to current year.

Average discharge. $-122 \mathrm{ft}^{3} / \mathrm{s}$.

Minimum daily discharge. $-0.0 \mathrm{ft}^{3} / \mathrm{s}$.

Human health (harmonic mean) design flow. $-0.6 \mathrm{ft}^{3} / \mathrm{s}$.

Remarks. - None.

\begin{tabular}{|c|c|c|c|}
\hline \multicolumn{4}{|c|}{ Magnitude and frequency of annual low flow } \\
\hline \multicolumn{4}{|c|}{$\begin{array}{l}\text { Lowest average streamflow, in } \mathrm{ft}^{3} / \mathrm{s} \text {, for indicated period of } \\
\text { consecutive days at an annual nonexceedance probability of } 0.1\end{array}$} \\
\hline 1 & \multicolumn{2}{|c|}{7} & 30 \\
\hline 0.0 & \multicolumn{2}{|c|}{0.0} & 0.0 \\
\hline \multicolumn{4}{|c|}{$\begin{array}{c}\text { Percentage of time streamflow was equaled or exceeded for } \\
\text { the period of record }\end{array}$} \\
\hline $\begin{array}{l}\text { Percentage } \\
\text { of time }\end{array}$ & $\begin{array}{c}\text { Daily mean } \\
\text { streamflow } \\
\left(\mathrm{ft}^{3} / \mathrm{s}\right)\end{array}$ & $\begin{array}{l}\text { Percentage } \\
\text { of time }\end{array}$ & $\begin{array}{l}\text { Daily mean } \\
\text { streamflow } \\
\left(\mathrm{ft}^{3} / \mathrm{s}\right)\end{array}$ \\
\hline 99 & 0.0 & 40 & 32 \\
\hline 98 & 0.0 & 30 & 52 \\
\hline 95 & 0.1 & 20 & 90 \\
\hline 90 & 0.3 & 10 & 217 \\
\hline 80 & 1.5 & 5 & 523 \\
\hline 70 & 3.9 & 2 & 1280 \\
\hline 60 & 9.0 & 1 & 2210 \\
\hline 50 & 18 & & \\
\hline
\end{tabular}




\section{DEEP RIVER AT LAKE GEORGE OUTLET AT HOBART, IN}

Location.- Lat $41^{\circ} 32^{\prime} 10^{\prime \prime}$, long $87^{\circ} 15^{\prime} 25^{\prime \prime}$ referenced to North American Datum of 1927, in NW 1/4 NW 1/4 sec.32, T.36 N., R.7 W., Lake County, IN, Hydrologic Unit 04040001, on left bank at upstream side of bridge on Ridge Road in Hobart, $300 \mathrm{ft}$ upstream from Duck Creek, and $400 \mathrm{ft}$ downstream from Lake George Dam, and $3.3 \mathrm{mi}$ north of Ainsworth, IN.

Drainage area. $-124 \mathrm{mi}^{2}$.

Period of record. - April 1947 to current year.

Average discharge. $-122 \mathrm{ft}^{3} / \mathrm{s}$.

Minimum daily discharge. $-0.0 \mathrm{ft}^{3} / \mathrm{s}$.

Human health (harmonic mean) design flow. $-22 \mathrm{ft}^{3} / \mathrm{s}$.

Remarks. - Flow subject to regulation by operation of Lake George Dam. The 1Q10 and the 7Q10 low-flow values (--) could not be computed due to zero flows caused by extreme regulation and dam construction. These values caused statistical skews to exceed allowable limits.

\begin{tabular}{|c|c|c|c|}
\hline \multicolumn{4}{|c|}{ Magnitude and frequency of annual low flow } \\
\hline \multicolumn{4}{|c|}{$\begin{array}{l}\text { Lowest average streamflow, in } \mathrm{ft}^{3} / \mathrm{s} \text {, for indicated period of } \\
\text { consecutive days at an annual nonexceedance probability of } 0.1\end{array}$} \\
\hline 1 & \multicolumn{2}{|c|}{7} & 30 \\
\hline-- & \multicolumn{2}{|c|}{--} & 9.0 \\
\hline \multicolumn{4}{|c|}{$\begin{array}{l}\text { Percentage of time streamflow was equaled or exceeded for } \\
\text { the period of record }\end{array}$} \\
\hline $\begin{array}{l}\text { Percentage } \\
\text { of time }\end{array}$ & $\begin{array}{l}\text { Daily mean } \\
\text { streamflow } \\
\left(\mathrm{ft}^{3} / \mathrm{s}\right)\end{array}$ & $\begin{array}{l}\text { Percentage } \\
\text { of time }\end{array}$ & $\begin{array}{l}\text { Daily mean } \\
\text { streamflow } \\
\left(\mathrm{ft}^{3} / \mathrm{s}\right)\end{array}$ \\
\hline 99 & 6.6 & 40 & 70 \\
\hline 98 & 8.3 & 30 & 100 \\
\hline 95 & 11 & 20 & 152 \\
\hline 90 & 14 & 10 & 296 \\
\hline 80 & 21 & 5 & 505 \\
\hline 70 & 28 & 2 & 836 \\
\hline 60 & 38 & 1 & 1090 \\
\hline 50 & 51 & & \\
\hline
\end{tabular}




\section{LITTLE CALUMET RIVER AT PORTER, IN}

Location. - Lat $41^{\circ} 37^{\prime} 18^{\prime \prime}$, long $87^{\circ} 05^{\prime} 13^{\prime \prime}$ referenced to North American Datum of 1927, in NE 1/4 NE 1/4 Sec.34, T.37 N., R.6 W., Porter County, IN, Hydrologic Unit 04040001, on right bank at downstream end of county road bridge, $200 \mathrm{ft}$ upstream from bridge on U.S. Highway 20, $0.8 \mathrm{mi}$ northwest of Porter, and $4.5 \mathrm{mi}$ upstream from Salt Creek.

Drainage area. - $66.2 \mathrm{mi}^{2}$.

Period of record.- May 1945 to current year.

Average discharge. $-79.6 \mathrm{ft}^{3} / \mathrm{s}$.

Minimum daily discharge. $-17 \mathrm{ft}^{3} / \mathrm{s}$.

Human health (harmonic mean) design flow. $-48 \mathrm{ft}^{3} / \mathrm{s}$.

Remarks.- None.

\begin{tabular}{c}
\hline \multicolumn{4}{c}{ Magnitude and frequency of annual low flow } \\
\hline \multicolumn{4}{c}{ Lowest average streamflow, in $\mathrm{ft}^{3}$ /s, for indicated period of } \\
consecutive days at an annual nonexceedance probability of 0.1
\end{tabular}




\section{SALT CREEK AT VALPARAISO, IN}

Location. - Lat $41^{\circ} 27^{\prime} 53^{\prime \prime}$, long 8704'14" referenced to North American Datum of 1983, in SE 1/4 SW 1/4 sec.23, T.35 N., R.6 W., Porter County, IN, Hydrologic Unit 04040001, on downstream side of Factory Street Bridge, $0.4 \mathrm{mi}$ upstream from southeast corner of treatment plant property, $0.4 \mathrm{mi}$ from mouth of Sagers Lake Outlet, 14.1 mi east of Merrillville, and at river mile 18.4.

Drainage area. - $16.1 \mathrm{mi}^{2}$.

Period of record. - March 2009 to current year.

Average discharge $-17.5 \mathrm{ft}^{3} / \mathrm{s}$.

Minimum daily discharge. $-4.8 \mathrm{ft}^{3} / \mathrm{s}$.

Human health (harmonic mean) design flow. $-12 \mathrm{ft}^{3} / \mathrm{s}$.

Remarks. - This site has less than 10 years of record.

\begin{tabular}{|c|c|c|c|}
\hline \multicolumn{4}{|c|}{ Magnitude and frequency of annual low flow } \\
\hline \multicolumn{4}{|c|}{$\begin{array}{l}\text { Lowest average streamflow, in } \mathrm{ft}^{3} / \mathrm{s} \text {, for indicated period of } \\
\text { consecutive days at an annual nonexceedance probability of } 0.1\end{array}$} \\
\hline 1 & \multicolumn{2}{|c|}{7} & 30 \\
\hline 4.8 & \multicolumn{2}{|c|}{5.0} & 5.5 \\
\hline \multicolumn{4}{|c|}{$\begin{array}{l}\text { Percentage of time streamflow was equaled or exceeded for } \\
\text { the period of record }\end{array}$} \\
\hline $\begin{array}{l}\text { Percentage } \\
\text { of time }\end{array}$ & $\begin{array}{l}\text { Daily mean } \\
\text { streamflow } \\
\left(\mathrm{ft}^{3} / \mathrm{s}\right)\end{array}$ & $\begin{array}{l}\text { Percentage } \\
\text { of time }\end{array}$ & $\begin{array}{l}\text { Daily mean } \\
\text { streamflow } \\
\left(\mathrm{ft}^{3} / \mathrm{s}\right)\end{array}$ \\
\hline 99 & 5.5 & 40 & 15 \\
\hline 98 & 5.7 & 30 & 17 \\
\hline 95 & 6.3 & 20 & 21 \\
\hline 90 & 7.0 & 10 & 30 \\
\hline 80 & 8.2 & 5 & 44 \\
\hline 70 & 9.6 & 2 & 81 \\
\hline 60 & 11 & 1 & 121 \\
\hline 50 & 13 & & \\
\hline
\end{tabular}




\section{SALT CREEK NEAR MCCOOL, IN}

Location.- Lat $41^{\circ} 35^{\prime} 48^{\prime \prime}$, long $87^{\circ} 08^{\prime} 40^{\prime \prime}$ referenced to North American Datum of 1927, in SE 1/4 SE 1/4 sec.6, T.36 N., R.6 W., Porter County, IN, Hydrologic Unit 04040001, on left bank on downstream side of highway bridge, $50 \mathrm{ft}$ downstream from Conrail Railroad bridge, $1.2 \mathrm{mi}$ north of McCool, and $1.6 \mathrm{mi}$ upstream from Little Calumet River.

Drainage area. $-74.6 \mathrm{mi}^{2}$.

Period of record.- May 1945 to September 1991.

Average discharge. $-76.6 \mathrm{ft}^{3} / \mathrm{s}$.

Minimum daily discharge. $-10 \mathrm{ft}^{3} / \mathrm{s}$.

Human health (harmonic mean) design flow. $-46 \mathrm{ft}^{3} / \mathrm{s}$.

Remarks. - None.

\begin{tabular}{ccc}
\hline \multicolumn{3}{c}{ Magnitude and frequency of annual low flow } \\
\hline $\begin{array}{c}\text { Lowest average streamflow, in } \mathrm{ft}^{3} / \mathrm{s} \text {, for indicated period of } \\
\text { consecutive days at an annual nonexceedance probability of } \mathbf{0 . 1}\end{array}$ \\
\hline $\mathbf{7}$ & $\mathbf{3 0}$ \\
\hline 18 & 19 & 22 \\
\hline
\end{tabular}

Percentage of time streamflow was equaled or exceeded for the period of record

\begin{tabular}{cc|cc}
\hline $\begin{array}{c}\text { Percentage } \\
\text { of time }\end{array}$ & $\begin{array}{c}\text { Daily mean } \\
\text { streamflow } \\
\left(\mathrm{ft}^{3} / \mathbf{s}\right)\end{array}$ & $\begin{array}{c}\text { Percentage } \\
\text { of time }\end{array}$ & $\begin{array}{c}\text { Daily mean } \\
\text { streamflow } \\
\left(\mathrm{ft}^{3} / \mathbf{s}\right)\end{array}$ \\
\hline 99 & 20 & 40 & 57 \\
\hline 98 & 23 & 30 & 69 \\
\hline 95 & 25 & 20 & 89 \\
\hline 90 & 28 & 10 & 140 \\
\hline 80 & 32 & 5 & 226 \\
\hline 70 & 37 & 2 & 382 \\
\hline 60 & 42 & 1 & 520 \\
\hline 50 & 49 & & \\
\hline
\end{tabular}




\section{PORTAGE-BURNS WATERWAY AT PORTAGE, IN}

Location. - Lat $41^{\circ} 37^{\prime} 20^{\prime \prime}$, long $87^{\circ} 10^{\prime} 33^{\prime \prime}$ referenced to North American Datum of 1927, in NE 1/4 NW 1/4 sec.36, T.37 N., R.7 W., Porter County, IN, Hydrologic Unit 04040001, on right bank at an industrial road bridge, 1,300 feet north of U.S. Highway 12, $0.7 \mathrm{mi}$ south of the mouth, $1.2 \mathrm{mi}$ west of the State Road 249 overpass over U.S. Highway 12, $2.4 \mathrm{mi}$ east of County Line Road, and $3.2 \mathrm{mi}$ north of the intersection of Central Avenue and Willow Creek Road in Portage.

Drainage area.- $331 \mathrm{mi}^{2}$.

Period of record.- February 1995 to October 2011.

Average discharge. $-553 \mathrm{ft}^{3} / \mathrm{s}$.

Minimum daily discharge. $-83 \mathrm{ft}^{3} / \mathrm{s}$.

Human health (harmonic mean) design flow. - $384 \mathrm{ft}^{3} / \mathrm{s}$.

Remarks. - Prior to October 2011, published as Burns Ditch at Portage, IN.

\begin{tabular}{|c|c|c|c|}
\hline \multicolumn{4}{|c|}{ Magnitude and frequency of annual low flow } \\
\hline \multicolumn{4}{|c|}{$\begin{array}{l}\text { Lowest average streamflow, in } \mathrm{ft}^{3} / \mathrm{s} \text {, for indicated period of } \\
\text { consecutive days at an annual nonexceedance probability of } 0.1\end{array}$} \\
\hline 1 & \multicolumn{2}{|c|}{7} & 30 \\
\hline 84 & \multicolumn{2}{|c|}{100} & 125 \\
\hline \multicolumn{4}{|c|}{$\begin{array}{l}\text { Percentage of time streamflow was equaled or exceeded for } \\
\text { the period of record }\end{array}$} \\
\hline $\begin{array}{l}\text { Percentage } \\
\text { of time }\end{array}$ & $\begin{array}{c}\text { Daily mean } \\
\text { streamflow } \\
\left(\mathrm{ft}^{3} / \mathrm{s}\right)\end{array}$ & $\begin{array}{l}\text { Percentage } \\
\text { of time }\end{array}$ & $\begin{array}{c}\text { Daily mean } \\
\text { streamflow } \\
\left(\mathrm{ft}^{3} / \mathrm{s}\right)\end{array}$ \\
\hline 99 & 125 & 40 & 474 \\
\hline 98 & 145 & 30 & 547 \\
\hline 95 & 186 & 20 & 675 \\
\hline 90 & 235 & 10 & 949 \\
\hline 80 & 293 & 5 & 1380 \\
\hline 70 & 336 & 2 & 2070 \\
\hline 60 & 379 & 1 & 2720 \\
\hline 50 & 423 & & \\
\hline
\end{tabular}


Lake Michigan Basin

\section{TRAIL CREEK AT MICHIGAN CITY, IN}

Location.- Lat $41^{\circ} 43^{\prime} 00^{\prime \prime}$, long $86^{\circ} 51^{\prime} 35^{\prime \prime}$ referenced to North American Datum of 1927, in NE 1/4 SE 1/4 Sec.27, T.38 N., R.4 W., LaPorte County, IN, Hydrologic Unit 04040001, on right upstream side of bridge on Springland Avenue in Michigan City, 1.0 mi upstream from Otter Creek, and 4.2 mi upstream from mouth.

Drainage area. $-54.1 \mathrm{mi}^{2}$.

Period of record. - June 1969 to October 1994.

Average discharge. $-76.2 \mathrm{ft}^{3} / \mathrm{s}$.

Minimum daily discharge. $-20 \mathrm{ft}^{3} / \mathrm{s}$.

Human health (harmonic mean) design flow. $-55 \mathrm{ft}^{3} / \mathrm{s}$.

Remarks.- None.

Magnitude and frequency of annual low flow

Lowest average streamflow, in $\mathrm{ft}^{3} / \mathrm{s}$, for indicated period of consecutive days at an annual nonexceedance probability of 0.1

\begin{tabular}{ccc}
\hline $\mathbf{1}$ & $\mathbf{7}$ & $\mathbf{3 0}$ \\
\hline 21 & 23 & 26 \\
\hline
\end{tabular}

Percentage of time streamflow was equaled or exceeded for the period of record

\begin{tabular}{cc|cc}
\hline $\begin{array}{c}\text { Percentage } \\
\text { of time }\end{array}$ & $\begin{array}{c}\text { Daily mean } \\
\text { streamflow } \\
\left(\mathrm{ft}^{3} / \mathbf{s}\right)\end{array}$ & $\begin{array}{c}\text { Percentage } \\
\text { of time }\end{array}$ & $\begin{array}{c}\text { Daily mean } \\
\text { streamflow } \\
\left(\mathrm{ft}^{3} / \mathbf{s}\right)\end{array}$ \\
\hline 99 & 27 & 40 & 65 \\
\hline 98 & 29 & 30 & 75 \\
\hline 95 & 31 & 20 & 92 \\
\hline 90 & 34 & 10 & 129 \\
\hline 80 & 40 & 5 & 192 \\
\hline 70 & 45 & 2 & 322 \\
\hline 60 & 51 & 1 & 463 \\
\hline 50 & 58 & & \\
\hline
\end{tabular}


Lake Michigan Basin

\section{TRAIL CREEK AT MICHIGAN CITY HARBOR, IN}

Location. - Lat $41^{\circ} 43^{\prime} 22^{\prime \prime}$, long $86^{\circ} 54^{\prime} 15^{\prime \prime}$ referenced to North American Datum of 1927, in NE 1/4 NW 1/4 sec.29, T.38 N., R.4 W., LaPorte County, IN, Hydrologic Unit 04040001, on right bank in the northeast drawbridge tower, $2000 \mathrm{ft}$ north of Michigan Street, 2,600 ft southeast of lake end of west breakwater, $0.5 \mathrm{mi}$ southwest of Washington Park, and $3000 \mathrm{ft}$ downstream of U.S. Highway 12 bridge in Michigan City.

Drainage area. $-59.1 \mathrm{mi}^{2}$.

Period of record.- October 1994 to current year.

Average discharge. $-116 \mathrm{ft}^{3} / \mathrm{s}$.

Minimum daily discharge. $--95 \mathrm{ft}^{3} / \mathrm{s}$.

Human health (harmonic mean) design flow. $-73 \mathrm{ft}^{3} / \mathrm{s}$.

Remarks. - Positive discharge values indicate flow towards Lake Michigan; negative discharge values indicate flow away from Lake Michigan. The 1-day and 7-day annual low flow values (--) are not valid due to negative flows caused by backwater from Lake Michigan.

\begin{tabular}{cc|cc}
\hline \multicolumn{5}{c}{ Magnitude and frequency of annual low flow } \\
\hline \multicolumn{4}{c}{ Lowest average streamflow, in $\mathrm{ft}^{3} / \mathbf{s}$, for indicated period of } \\
consecutive days at an annual nonexceedance probability of 0.1
\end{tabular}


Lake Michigan Basin

\section{GALENA RIVER NEAR LAPORTE, IN}

Location. - Lat $41^{\circ} 44^{\prime} 54^{\prime \prime}$, long $86^{\circ} 40^{\prime} 30^{\prime \prime}$ referenced to North American Datum of 1927, in SE 1/4 NW 1/4 sec.17, T.38 N., R.2 W., LaPorte County, Hydrologic Unit 04040001, on left bank at downstream side of bridge on County Road 125 East, $1.3 \mathrm{mi}$ upstream from Indiana-Michigan State line, $7.5 \mathrm{mi}$ west of the LaPorte-St. Joseph County line, and 9.8 mi north of the Court House in LaPorte.

Drainage area.- $17.2 \mathrm{mi}^{2}$, of which $2.30 \mathrm{mi}^{2}$ does not contribute directly to surface runoff.

Period of record. - October 1969 to October 2003.

Average discharge. $-25.9 \mathrm{ft}^{3} / \mathrm{s}$.

Minimum daily discharge. $-6.7 \mathrm{ft}^{3} / \mathrm{s}$.

Human health (harmonic mean) design flow. $-20 \mathrm{ft}^{3} / \mathrm{s}$.

Remarks. - None.

\begin{tabular}{|c|c|c|c|}
\hline \multicolumn{4}{|c|}{ Magnitude and frequency of annual low flow } \\
\hline \multicolumn{4}{|c|}{$\begin{array}{l}\text { Lowest average streamflow, in } \mathrm{ft}^{3} / \mathrm{s} \text {, for indicated period of } \\
\text { consecutive days at an annual nonexceedance probability of } 0.1\end{array}$} \\
\hline 1 & \multicolumn{2}{|c|}{7} & 30 \\
\hline 7.5 & \multicolumn{2}{|c|}{8.1} & 9.4 \\
\hline \multicolumn{4}{|c|}{$\begin{array}{c}\text { Percentage of time streamflow was equaled or exceeded for } \\
\text { the period of record }\end{array}$} \\
\hline $\begin{array}{l}\text { Percentage } \\
\text { of time }\end{array}$ & $\begin{array}{l}\text { Daily mean } \\
\text { streamflow } \\
\left(\mathrm{ft}^{3} / \mathrm{s}\right)\end{array}$ & $\begin{array}{l}\text { Percentage } \\
\text { of time }\end{array}$ & $\begin{array}{l}\text { Daily mean } \\
\text { streamflow } \\
\left(\mathrm{ft}^{3} / \mathrm{s}\right)\end{array}$ \\
\hline 99 & 8.8 & 40 & 24 \\
\hline 98 & 9.4 & 30 & 27 \\
\hline 95 & 11 & 20 & 32 \\
\hline 90 & 12 & 10 & 42 \\
\hline 80 & 15 & 5 & 56 \\
\hline 70 & 17 & 2 & 88 \\
\hline 60 & 19 & 1 & 119 \\
\hline 50 & 21 & & \\
\hline
\end{tabular}




\section{LIME LAKE OUTLET AT PANAMA, IN}

Location. - Lat $41^{\circ} 42^{\prime} 46^{\prime \prime}$, long $85^{\circ} 07^{\prime} 10^{\prime \prime}$ referenced to North American Datum of 1927, in NW 1/4 NW 1/4 sec. 35, T.38 N., R.12 E., Steuben County, IN, Hydrologic Unit 04050001, on right bank $10 \mathrm{ft}$ downstream from dam for Lime Lake, $30 \mathrm{ft}$ upstream from bridge on Orland Road, and 0.7 mi northwest of Panama.

Drainage area.- $17.5 \mathrm{mi}^{2}$, of which $3.68 \mathrm{mi}^{2}$ does not contribute directly to surface runoff.

Period of record. - October 1969 to September 1986.

Average discharge. $-8.06 \mathrm{ft}^{3} / \mathrm{s}$.

Minimum daily discharge. $-0.0 \mathrm{ft}^{3} / \mathrm{s}$.

Human health (harmonic mean) design flow. $-0.9 \mathrm{ft}^{3} / \mathrm{s}$.

Remarks. - Occasional regulation by control structure for Lime Lake. Low-flow statistics are calculated for the regulated period, 1969 to 1986.

\begin{tabular}{|c|c|c|c|}
\hline \multicolumn{4}{|c|}{ Magnitude and frequency of annual low flow } \\
\hline \multicolumn{4}{|c|}{$\begin{array}{l}\text { Lowest average streamflow, in } \mathrm{ft}^{3} / \mathrm{s} \text {, for indicated period of } \\
\text { consecutive days at an annual nonexceedance probability of } 0.1\end{array}$} \\
\hline 1 & \multicolumn{2}{|c|}{7} & 30 \\
\hline 0.0 & \multicolumn{2}{|c|}{0.0} & 0.0 \\
\hline \multicolumn{4}{|c|}{$\begin{array}{c}\text { Percentage of time streamflow was equaled or exceeded for } \\
\text { the period of record }\end{array}$} \\
\hline $\begin{array}{l}\text { Percentage } \\
\text { of time }\end{array}$ & $\begin{array}{l}\text { Daily mean } \\
\text { streamflow } \\
\left(\mathrm{ft}^{3} / \mathrm{s}\right)\end{array}$ & $\begin{array}{l}\text { Percentage } \\
\text { of time }\end{array}$ & $\begin{array}{l}\text { Daily mean } \\
\text { streamflow } \\
\left(\mathrm{ft}^{3} / \mathrm{s}\right)\end{array}$ \\
\hline 99 & 0.0 & 40 & 8.6 \\
\hline 98 & 0.0 & 30 & 11 \\
\hline 95 & 0.2 & 20 & 14 \\
\hline 90 & 0.5 & 10 & 20 \\
\hline 80 & 1.6 & 5 & 25 \\
\hline 70 & 2.7 & 2 & 31 \\
\hline 60 & 4.3 & 1 & 39 \\
\hline 50 & 6.2 & & \\
\hline
\end{tabular}


Lake Michigan Basin

\section{PIGEON CREEK NEAR ANGOLA, IN}

Location. - Lat $41^{\circ} 38^{\prime} 04^{\prime \prime}$, long $85^{\circ} 06^{\prime} 35^{\prime \prime}$ referenced to North American Datum of 1927, in NW 1/4 SE 1/4 Sec.26, T.37 N., R.12 E., Steuben County, IN, Hydrologic Unit 04050001, on left bank $5 \mathrm{ft}$ upstream from bridge on U.S. Highway 20, 1.3 mi downstream from outlet of Hogback Lake, $1.3 \mathrm{mi}$ southeast of Flint, and $5.8 \mathrm{mi}$ west of Angola.

Drainage area.- $106 \mathrm{mi}^{2}$, of which $22.5 \mathrm{mi}^{2}$ probably is noncontributing.

Period of record. - October 1945 to current year.

Average discharge. $-87.2 \mathrm{ft}^{3} / \mathrm{s}$.

Minimum daily discharge. $-3.4 \mathrm{ft}^{3} / \mathrm{s}$.

Human health (harmonic mean) design flow. $-37 \mathrm{ft}^{3} / \mathrm{s}$.

Remarks.- Prior to October 1947, published as "near Flint." Published as Pigeon Creek at Hogback Lake Outlet near Angola, October 1947 to September 1971, and Pigeon Creek and Hogback Lake near Angola, October 1971 to September 1974.

\begin{tabular}{|c|c|c|c|}
\hline \multicolumn{4}{|c|}{ Magnitude and frequency of annual low flow } \\
\hline \multicolumn{4}{|c|}{$\begin{array}{l}\text { Lowest average streamflow, in } \mathrm{ft}^{3} / \mathrm{s} \text {, for indicated period of } \\
\text { consecutive days at an annual nonexceedance probability of } 0.1\end{array}$} \\
\hline 1 & \multicolumn{2}{|c|}{7} & 30 \\
\hline 7.7 & \multicolumn{2}{|c|}{8.0} & 9.2 \\
\hline \multicolumn{4}{|c|}{$\begin{array}{l}\text { Percentage of time streamflow was equaled or exceeded for } \\
\text { the period of record }\end{array}$} \\
\hline $\begin{array}{l}\text { Percentage } \\
\text { of time }\end{array}$ & $\begin{array}{l}\text { Daily mean } \\
\text { streamflow } \\
\left(\mathrm{ft}^{3} / \mathrm{s}\right)\end{array}$ & $\begin{array}{l}\text { Percentage } \\
\text { of time }\end{array}$ & $\begin{array}{l}\text { Daily mean } \\
\text { streamflow } \\
\left(\mathrm{ft}^{3} / \mathrm{s}\right)\end{array}$ \\
\hline 99 & 6.8 & 40 & 73 \\
\hline 98 & 8.6 & 30 & 96 \\
\hline 95 & 14 & 20 & 130 \\
\hline 90 & 18 & 10 & 196 \\
\hline 80 & 26 & 5 & 270 \\
\hline 70 & 34 & 2 & 363 \\
\hline 60 & 44 & 1 & 453 \\
\hline 50 & 57 & & \\
\hline
\end{tabular}


Lake Michigan Basin

\section{PRETTY LAKE INLET NEAR STROH, IN}

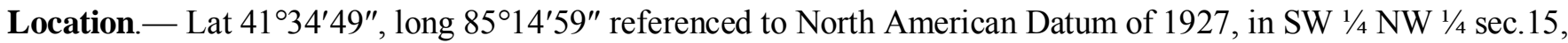
T.36 N., R.11 E., Lagrange County, IN, Hydrologic Unit 04050001, on left bank $400 \mathrm{ft}$ upstream from mouth, 2.6 miles west of Stroh.

Drainage area.- $1.96 \mathrm{mi}^{2}$, of which $1.32 \mathrm{mi}^{2}$ does not contribute directly to surface runoff.

Period of record.- June 1963 to September 1980.

Average discharge. $-0.48 \mathrm{ft}^{3} / \mathrm{s}$.

Minimum daily discharge. $-0.1 \mathrm{ft}^{3} / \mathrm{s}$.

Human health (harmonic mean) design flow. $-0.1 \mathrm{ft}^{3} / \mathrm{s}$.

Remarks.- None.

\begin{tabular}{|c|c|c|c|}
\hline \multicolumn{4}{|c|}{ Magnitude and frequency of annual low flow } \\
\hline \multicolumn{4}{|c|}{$\begin{array}{l}\text { Lowest average streamflow, in } \mathrm{ft}^{3} / \mathrm{s} \text {, for indicated period of } \\
\text { consecutive days at an annual nonexceedance probability of } 0.1\end{array}$} \\
\hline 1 & \multicolumn{2}{|c|}{7} & 30 \\
\hline 0.0 & \multicolumn{2}{|c|}{0.0} & 0.0 \\
\hline \multicolumn{4}{|c|}{$\begin{array}{l}\text { Percentage of time streamflow was equaled or exceeded for } \\
\text { the period of record }\end{array}$} \\
\hline $\begin{array}{l}\text { Percentage } \\
\text { of time }\end{array}$ & $\begin{array}{c}\text { Daily mean } \\
\text { streamflow } \\
\left(\mathrm{ft}^{3} / \mathrm{s}\right)\end{array}$ & $\begin{array}{l}\text { Percentage } \\
\text { of time }\end{array}$ & $\begin{array}{l}\text { Daily mean } \\
\text { streamflow } \\
\left(\mathrm{ft}^{3} / \mathrm{s}\right)\end{array}$ \\
\hline 99 & 0.0 & 40 & 0.4 \\
\hline 98 & 0.0 & 30 & 0.5 \\
\hline 95 & 0.0 & 20 & 0.7 \\
\hline 90 & 0.1 & 10 & 1.1 \\
\hline 80 & 0.1 & 5 & 1.5 \\
\hline 70 & 0.1 & 2 & 2.3 \\
\hline 60 & 0.2 & 1 & 3.3 \\
\hline 50 & 0.3 & & \\
\hline
\end{tabular}


Lake Michigan Basin

\section{PIGEON RIVER NEAR SCOTT, IN}

Location. - Lat 41 $44^{\prime} 56^{\prime \prime}$, long 85³4'35" referenced to North American Datum of 1927, in SE 1/4 NW 1/4 sec.14, T.38 N., R.8 E., LaGrange County, IN, Hydrologic Unit 04050001, on right bank $20 \mathrm{ft}$ downstream from bridge on County Road 750 North, 1,200 ft downstream from Page Ditch, 0.7 mi south of Indiana-Michigan State line, and $1.2 \mathrm{mi}$ northwest of Scott.

Drainage area. $-361 \mathrm{mi}^{2}$, of which $54 \mathrm{mi}^{2}$ probably is noncontributing.

Period of record.- June 1968 to current year.

Average discharge. - $371 \mathrm{ft}^{3} / \mathrm{s}$.

Minimum daily discharge. $-42 \mathrm{ft}^{3} / \mathrm{s}$.

Human health (harmonic mean) design flow. $-258 \mathrm{ft}^{3} / \mathrm{s}$.

Remarks.- None.

\begin{tabular}{|c|c|c|c|}
\hline \multicolumn{4}{|c|}{ Magnitude and frequency of annual low flow } \\
\hline \multicolumn{4}{|c|}{$\begin{array}{l}\text { Lowest average streamflow, in } \mathrm{ft}^{3} / \mathrm{s} \text {, for indicated period of } \\
\text { consecutive days at an annual nonexceedance probability of } 0.1\end{array}$} \\
\hline 1 & \multicolumn{2}{|c|}{7} & 30 \\
\hline 79 & \multicolumn{2}{|c|}{90} & 105 \\
\hline \multicolumn{4}{|c|}{$\begin{array}{c}\text { Percentage of time streamflow was equaled or exceeded for } \\
\text { the period of record }\end{array}$} \\
\hline $\begin{array}{l}\text { Percentage } \\
\text { of time }\end{array}$ & $\begin{array}{l}\text { Daily mean } \\
\text { streamflow } \\
\left(\mathrm{ft}^{3} / \mathrm{s}\right)\end{array}$ & $\begin{array}{l}\text { Percentage } \\
\text { of time }\end{array}$ & $\begin{array}{l}\text { Daily mean } \\
\text { streamflow } \\
\left(\mathrm{ft}^{3} / \mathrm{s}\right)\end{array}$ \\
\hline 99 & 94 & 40 & 350 \\
\hline 98 & 106 & 30 & 419 \\
\hline 95 & 126 & 20 & 514 \\
\hline 90 & 145 & 10 & 696 \\
\hline 80 & 176 & 5 & 875 \\
\hline 70 & 209 & 2 & 1190 \\
\hline 60 & 247 & 1 & 1390 \\
\hline 50 & 295 & & \\
\hline
\end{tabular}


Lake Michigan Basin

\section{LITTLE ELKHART RIVER AT MIDDLEBURY, IN}

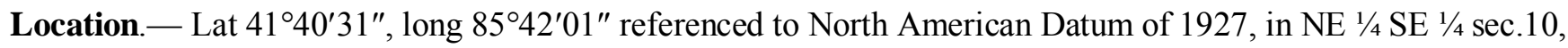
T.37 N., R.7 E., Elkhart County, IN, Hydrologic Unit 04050001, on left bank $15 \mathrm{ft}$ downstream from bridge on County Road 1, $0.1 \mathrm{mi}$ east of Middlebury, $0.4 \mathrm{mi}$ upstream from intersection of State Road 13 bridge and Little Elkhart River, and 1.7 mi downstream from Rowe Eden Ditch.

Drainage area.- $97.6 \mathrm{mi}^{2}$, of which $5.89 \mathrm{mi}^{2}$ does not contribute directly to surface runoff.

Period of record. - October 1979 to October 2003.

Average discharge. $-97.0 \mathrm{ft}^{3} / \mathrm{s}$.

Minimum daily discharge. $-22 \mathrm{ft}^{3} / \mathrm{s}$.

Human health (harmonic mean) design flow. $-69 \mathrm{ft}^{3} / \mathrm{s}$.

Remarks. - None.

\begin{tabular}{|c|c|c|c|}
\hline \multicolumn{4}{|c|}{ Magnitude and frequency of annual low flow } \\
\hline \multicolumn{4}{|c|}{$\begin{array}{l}\text { Lowest average streamflow, in } \mathrm{ft}^{3} / \mathrm{s} \text {, for indicated period of } \\
\text { consecutive days at an annual nonexceedance probability of } 0.1\end{array}$} \\
\hline 1 & \multicolumn{2}{|c|}{7} & 30 \\
\hline 27 & \multicolumn{2}{|c|}{29} & 32 \\
\hline \multicolumn{4}{|c|}{$\begin{array}{c}\text { Percentage of time streamflow was equaled or exceeded for } \\
\text { the period of record }\end{array}$} \\
\hline $\begin{array}{l}\text { Percentage } \\
\text { of time }\end{array}$ & $\begin{array}{l}\text { Daily mean } \\
\text { streamflow } \\
\left(\mathrm{ft}^{3} / \mathrm{s}\right)\end{array}$ & $\begin{array}{l}\text { Percentage } \\
\text { of time }\end{array}$ & $\begin{array}{l}\text { Daily mean } \\
\text { streamflow } \\
\left(\mathrm{ft}^{3} / \mathrm{s}\right)\end{array}$ \\
\hline 99 & 32 & 40 & 87 \\
\hline 98 & 34 & 30 & 101 \\
\hline 95 & 38 & 20 & 122 \\
\hline 90 & 41 & 10 & 164 \\
\hline 80 & 48 & 5 & 220 \\
\hline 70 & 56 & 2 & 327 \\
\hline 60 & 66 & 1 & 454 \\
\hline 50 & 76 & & \\
\hline
\end{tabular}


Lake Michigan Basin

\section{PINE CREEK NEAR ELKHART, IN}

Location.- Lat $41^{\circ} 40^{\prime} 53^{\prime \prime}$, long 85 $52^{\prime} 57^{\prime \prime}$ referenced to North American Datum of 1927, in NE 1/4 NW 1/4 sec.7, T.37 N., R.6 E., Elkhart County, IN, Hydrologic Unit 04050001, on right bank $50 \mathrm{ft}$ upstream from bridge on

County Road 14, 0.3 mi east of the intersection of County Roads 17 and 14, 3.1 mi east of Elkhart, and at mile 2.0.

Drainage area. $-31.0 \mathrm{mi}^{2}$, of which $8.75 \mathrm{mi}^{2}$ does not contribute directly to surface runoff.

Period of record. - October 1979 to October 2003.

Average discharge. $-18.6 \mathrm{ft}^{3} / \mathrm{s}$.

Minimum daily discharge. $-1.8 \mathrm{ft}^{3} / \mathrm{s}$.

Human health (harmonic mean) design flow. $-12 \mathrm{ft}^{3} / \mathrm{s}$.

Remarks.- None.

\begin{tabular}{|c|c|c|c|}
\hline \multicolumn{4}{|c|}{ Magnitude and frequency of annual low flow } \\
\hline \multicolumn{4}{|c|}{$\begin{array}{l}\text { Lowest average streamflow, in } \mathrm{ft}^{3} / \mathrm{s} \text {, for indicated period of } \\
\text { consecutive days at an annual nonexceedance probability of } 0.1\end{array}$} \\
\hline 1 & \multicolumn{2}{|c|}{7} & 30 \\
\hline 2.9 & \multicolumn{2}{|c|}{3.4} & 4.6 \\
\hline \multicolumn{4}{|c|}{$\begin{array}{l}\text { Percentage of time streamflow was equaled or exceeded for } \\
\text { the period of record }\end{array}$} \\
\hline $\begin{array}{l}\text { Percentage } \\
\text { of time }\end{array}$ & $\begin{array}{c}\text { Daily mean } \\
\text { streamflow } \\
\left(\mathrm{ft}^{3} / \mathrm{s}\right)\end{array}$ & $\begin{array}{l}\text { Percentage } \\
\text { of time }\end{array}$ & $\begin{array}{c}\text { Daily mean } \\
\text { streamflow } \\
\left(\mathrm{ft}^{3} / \mathrm{s}\right)\end{array}$ \\
\hline 99 & 3.7 & 40 & 17 \\
\hline 98 & 4.4 & 30 & 20 \\
\hline 95 & 5.8 & 20 & 24 \\
\hline 90 & 7.0 & 10 & 31 \\
\hline 80 & 8.8 & 5 & 42 \\
\hline 70 & 11 & 2 & 63 \\
\hline 60 & 13 & 1 & 92 \\
\hline 50 & 15 & & \\
\hline
\end{tabular}


Lake Michigan Basin

\section{NORTH BRANCH ELKHART RIVER AT COSPERVILLE, IN}

Location. - Lat $41^{\circ} 28^{\prime} 54^{\prime \prime}$, long $85^{\circ} 28^{\prime} 32^{\prime \prime}$ referenced to North American Datum of 1927, in NE 1/4 NW 1/4 sec.22, T.35 N., R.9 E., Noble County, IN, Hydrologic Unit 04050001, on right bank at downstream side of bridge on County Road 900 North at Cosperville, 1,300 ft downstream from Boyd Ditch, 1.7 mi upstream from Hustin Ditch, and $3.1 \mathrm{mi}$ downstream from Waldron Lake.

Drainage area. $-142 \mathrm{mi}^{2}$.

Period of record.- October 1979 to current year.

Average discharge. $-139 \mathrm{ft}^{3} / \mathrm{s}$.

Minimum daily discharge. $-0.76 \mathrm{ft}^{3} / \mathrm{s}$.

Human health (harmonic mean) design flow. $-61 \mathrm{ft}^{3} / \mathrm{s}$.

Remarks. - Flow regulated at times by dam at Waldron Lake.

\begin{tabular}{|c|c|c|c|}
\hline \multicolumn{4}{|c|}{ Magnitude and frequency of annual low flow } \\
\hline \multicolumn{4}{|c|}{$\begin{array}{l}\text { Lowest average streamflow, in } \mathrm{ft}^{3} / \mathrm{s} \text {, for indicated period of } \\
\text { consecutive days at an annual nonexceedance probability of } 0 \text {. }\end{array}$} \\
\hline 1 & \multicolumn{2}{|c|}{7} & 30 \\
\hline 4.8 & \multicolumn{2}{|c|}{6.4} & 12 \\
\hline \multicolumn{4}{|c|}{$\begin{array}{l}\text { Percentage of time streamflow was equaled or exceeded for } \\
\text { the period of record }\end{array}$} \\
\hline $\begin{array}{l}\text { Percentage } \\
\text { of time }\end{array}$ & $\begin{array}{l}\text { Daily mean } \\
\text { streamflow } \\
\left(\mathrm{ft}^{3} / \mathrm{s}\right)\end{array}$ & $\begin{array}{l}\text { Percentage } \\
\text { of time }\end{array}$ & $\begin{array}{c}\text { Daily mean } \\
\text { streamflow } \\
\left(\mathrm{ft}^{3} / \mathrm{s}\right)\end{array}$ \\
\hline 99 & 9.4 & 40 & 134 \\
\hline 98 & 14 & 30 & 166 \\
\hline 95 & 20 & 20 & 211 \\
\hline 90 & 29 & 10 & 297 \\
\hline 80 & 45 & 5 & 369 \\
\hline 70 & 62 & 2 & 475 \\
\hline 60 & 84 & 1 & 589 \\
\hline 50 & 108 & & \\
\hline
\end{tabular}




\section{FORKER CREEK NEAR BURR OAK, IN}

Location. - Lat $41^{\circ} 19^{\prime} 58^{\prime \prime}$, long $85^{\circ} 25^{\prime} 25^{\prime \prime}$ referenced to North American Datum of 1927, in SE 1/4 NE 1/4 Sec.12, T.33 N., R.9 E., Noble County, IN, Hydrologic Unit 04050001, on right bank $300 \mathrm{ft}$ downstream from bridge on State Highway 9, $400 \mathrm{ft}$ downstream from Miller Lake Outlet, $0.8 \mathrm{mi}$ northeast of Burr Oak, and $4.5 \mathrm{mi}$ south of Albion.

Drainage area. $-19.2 \mathrm{mi}^{2}$.

Period of record.- June 1969 to October 2003.

Average discharge. $-17.0 \mathrm{ft}^{3} / \mathrm{s}$.

Minimum daily discharge. $-0.10 \mathrm{ft}^{3} / \mathrm{s}$.

Human health (harmonic mean) design flow. $-2.1 \mathrm{ft}^{3} / \mathrm{s}$.

Remarks. - Occasional regulation at Miller Lake Outlet. Low-flow statistics are calculated for the regulated period, 1969 to 2003.

\begin{tabular}{|c|c|c|c|}
\hline \multicolumn{4}{|c|}{ Magnitude and frequency of annual low flow } \\
\hline \multicolumn{4}{|c|}{$\begin{array}{l}\text { Lowest average streamflow, in } \mathrm{ft}^{3} / \mathrm{s} \text {, for indicated period of } \\
\text { consecutive days at an annual nonexceedance probability of } 0.1\end{array}$} \\
\hline 1 & \multicolumn{2}{|c|}{7} & 30 \\
\hline 0.1 & \multicolumn{2}{|c|}{0.2} & 0.2 \\
\hline \multicolumn{4}{|c|}{$\begin{array}{l}\text { Percentage of time streamflow was equaled or exceeded for } \\
\text { the period of record }\end{array}$} \\
\hline $\begin{array}{l}\text { Percentage } \\
\text { of time }\end{array}$ & $\begin{array}{c}\text { Daily mean } \\
\text { streamflow } \\
\left(\mathrm{ft}^{3} / \mathrm{s}\right)\end{array}$ & $\begin{array}{l}\text { Percentage } \\
\text { of time }\end{array}$ & $\begin{array}{l}\text { Daily mean } \\
\text { streamflow } \\
\left(\mathrm{ft}^{3} / \mathrm{s}\right)\end{array}$ \\
\hline 99 & 0.2 & 40 & 13 \\
\hline 98 & 0.3 & 30 & 18 \\
\hline 95 & 0.5 & 20 & 26 \\
\hline 90 & 0.8 & 10 & 43 \\
\hline 80 & 1.7 & 5 & 66 \\
\hline 70 & 3.3 & 2 & 98 \\
\hline 60 & 5.6 & 1 & 123 \\
\hline 50 & 8.6 & & \\
\hline
\end{tabular}




\section{RIMMEL BRANCH NEAR ALBION, IN}

Location. - Lat $41^{\circ} 23^{\prime} 07^{\prime \prime}$, long $85^{\circ} 22^{\prime} 14^{\prime \prime}$ referenced to North American Datum of 1927, in NE 1/4 SE 1/4 Sec.21, T.34 N., R.10 E., Noble County, IN, Hydrologic Unit 04050001, on right bank $900 \mathrm{ft}$ downstream from culvert on County Road 300 East, $0.75 \mathrm{mi}$ south of State Highway 8, and 3.0 mi east of intersection of State Highway 9 and State Highway 8 in Albion.

Drainage area. $-10.7 \mathrm{mi}^{2}$.

Period of record. - November 1979 to October 2001.

Average discharge. $-9.97 \mathrm{ft}^{3} / \mathrm{s}$.

Minimum daily discharge. $-0.02 \mathrm{ft}^{3} / \mathrm{s}$.

Human health (harmonic mean) design flow. $-1.1 \mathrm{ft}^{3} / \mathrm{s}$.

Remarks. - None.

\begin{tabular}{|c|c|c|c|}
\hline \multicolumn{4}{|c|}{ Magnitude and frequency of annual low flow } \\
\hline \multicolumn{4}{|c|}{$\begin{array}{l}\text { Lowest average streamflow, in } \mathrm{ft}^{3} / \mathrm{s} \text {, for indicated period of } \\
\text { consecutive days at an annual nonexceedance probability of } 0.1\end{array}$} \\
\hline 1 & \multicolumn{2}{|c|}{7} & 30 \\
\hline 0.1 & \multicolumn{2}{|c|}{0.1} & 0.2 \\
\hline \multicolumn{4}{|c|}{$\begin{array}{l}\text { Percentage of time streamflow was equaled or exceeded for } \\
\text { the period of record }\end{array}$} \\
\hline $\begin{array}{l}\text { Percentage } \\
\text { of time }\end{array}$ & $\begin{array}{l}\text { Daily mean } \\
\text { streamflow } \\
\quad\left(\mathrm{ft}^{3} / \mathrm{s}\right)\end{array}$ & $\begin{array}{l}\text { Percentage } \\
\text { of time }\end{array}$ & $\begin{array}{l}\text { Daily mean } \\
\text { streamflow } \\
\left(\mathrm{ft}^{3} / \mathrm{s}\right)\end{array}$ \\
\hline 99 & 0.1 & 40 & 5.8 \\
\hline 98 & 0.2 & 30 & 7.9 \\
\hline 95 & 0.3 & 20 & 12 \\
\hline 90 & 0.4 & 10 & 23 \\
\hline 80 & 0.9 & 5 & 40 \\
\hline 70 & 1.7 & 2 & 72 \\
\hline 60 & 2.8 & 1 & 107 \\
\hline 50 & 4.2 & & \\
\hline
\end{tabular}


Lake Michigan Basin

\section{SOLOMON CREEK NEAR SYRACUSE, IN}

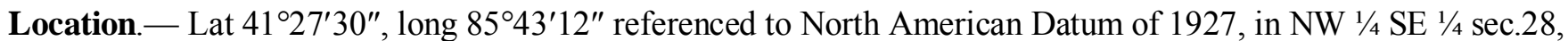
T.35 N., R.7 E., Elkhart County, IN, Hydrologic Unit 04050001, on right bank $40 \mathrm{ft}$ upstream from County Road 52 East bridge over Solomon Creek, and 2.5 mi northeast of Syracuse.

Drainage area.- $36.1 \mathrm{mi}^{2}$.

Period of record.- October 1987 to October 2003.

Average discharge. $-35.6 \mathrm{ft}^{3} / \mathrm{s}$.

Minimum daily discharge. $-6.3 \mathrm{ft}^{3} / \mathrm{s}$.

Human health (harmonic mean) design flow. $-25 \mathrm{ft}^{3} / \mathrm{s}$.

Remarks.- None.

\begin{tabular}{|c|c|c|c|}
\hline \multicolumn{4}{|c|}{ Magnitude and frequency of annual low flow } \\
\hline \multicolumn{4}{|c|}{$\begin{array}{l}\text { Lowest average streamflow, in } \mathrm{ft}^{3} / \mathrm{s} \text {, for indicated period of } \\
\text { consecutive days at an annual nonexceedance probability of } 0.1\end{array}$} \\
\hline 1 & \multicolumn{2}{|c|}{7} & 30 \\
\hline 7.3 & \multicolumn{2}{|c|}{8.3} & 10 \\
\hline \multicolumn{4}{|c|}{$\begin{array}{l}\text { Percentage of time streamflow was equaled or exceeded for } \\
\text { the period of record }\end{array}$} \\
\hline $\begin{array}{l}\text { Percentage } \\
\text { of time }\end{array}$ & $\begin{array}{c}\text { Daily mean } \\
\text { streamflow } \\
\left(\mathrm{ft}^{3} / \mathrm{s}\right)\end{array}$ & $\begin{array}{l}\text { Percentage } \\
\text { of time }\end{array}$ & $\begin{array}{c}\text { Daily mean } \\
\text { streamflow } \\
\left(\mathrm{ft}^{3} / \mathrm{s}\right)\end{array}$ \\
\hline 99 & 10 & 40 & 35 \\
\hline 98 & 11 & 30 & 43 \\
\hline 95 & 12 & 20 & 51 \\
\hline 90 & 14 & 10 & 65 \\
\hline 80 & 17 & 5 & 79 \\
\hline 70 & 21 & 2 & 101 \\
\hline 60 & 25 & 1 & 122 \\
\hline 50 & 30 & & \\
\hline
\end{tabular}


Lake Michigan Basin

\section{TURKEY CREEK NEAR SYRACUSE, IN}

Location. - Lat $41^{\circ} 25^{\prime} 35^{\prime \prime}$, long $85^{\circ} 45^{\prime} 16^{\prime \prime}$ referenced to North American Datum of 1927, in NE 1/4 SE 1/4 Sec.6, T.34 N., R.7 E., Kosciusko County, IN, Hydrologic Unit 04050001, on right bank $75 \mathrm{ft}$ upstream from Main Street bridge in Syracuse and 1,500 ft downstream from dam at outlet of Syracuse Lake.

Drainage area. $-43.8 \mathrm{mi}^{2}$.

Period of record. - October 1969 to September 1987.

Average discharge. $-38.2 \mathrm{ft}^{3} / \mathrm{s}$.

Minimum daily discharge. $-0.82 \mathrm{ft}^{3} / \mathrm{s}$.

Human health (harmonic mean) design flow. - $10 \mathrm{ft}^{3} / \mathrm{s}$.

Remarks. - Flow regulated by dam on Syracuse Lake.

\begin{tabular}{|c|c|c|c|}
\hline \multicolumn{4}{|c|}{ Magnitude and frequency of annual low flow } \\
\hline \multicolumn{4}{|c|}{$\begin{array}{l}\text { Lowest average streamflow, in } \mathrm{ft}^{3} / \mathrm{s} \text {, for indicated period of } \\
\text { consecutive days at an annual nonexceedance probability of } 0.1\end{array}$} \\
\hline 1 & \multicolumn{2}{|c|}{7} & 30 \\
\hline 1.5 & \multicolumn{2}{|c|}{1.8} & 2.4 \\
\hline \multicolumn{4}{|c|}{$\begin{array}{l}\text { Percentage of time streamflow was equaled or exceeded for } \\
\text { the period of record }\end{array}$} \\
\hline $\begin{array}{l}\text { Percentage } \\
\text { of time }\end{array}$ & $\begin{array}{l}\text { Daily mean } \\
\text { streamflow } \\
\left(\mathrm{ft}^{3} / \mathrm{s}\right)\end{array}$ & $\begin{array}{l}\text { Percentage } \\
\text { of time }\end{array}$ & $\begin{array}{l}\text { Daily mean } \\
\text { streamflow } \\
\left(\mathrm{ft}^{3} / \mathrm{s}\right)\end{array}$ \\
\hline 99 & 2.1 & 40 & 42 \\
\hline 98 & 2.4 & 30 & 58 \\
\hline 95 & 3.1 & 20 & 73 \\
\hline 90 & 3.8 & 10 & 91 \\
\hline 80 & 5.2 & 5 & 104 \\
\hline 70 & 7.7 & 2 & 132 \\
\hline 60 & 14 & 1 & 150 \\
\hline 50 & 25 & & \\
\hline
\end{tabular}


Lake Michigan Basin

\section{ELKHART RIVER AT GOSHEN, IN}

Location. - Lat $41^{\circ} 35^{\prime} 36^{\prime \prime}$, long $85^{\circ} 50^{\prime} 55^{\prime \prime}$ referenced to North American Datum of 1927, in NE 1/4 NE 1/4 Sec.8, T.36 N., R.6 E., Elkhart County, IN, Hydrologic Unit 04050001, on right bank $20 \mathrm{ft}$ downstream from River Avenue bridge at Goshen, 0.4 mi upstream from Rock Run, 9.1 mi northwest of Millersburg, and at mile 16.1.

Drainage area. $-594 \mathrm{mi}^{2}$.

Period of record. - April 1931 to current year.

Average discharge. $-547 \mathrm{ft}^{3} / \mathrm{s}$.

Minimum daily discharge. $-7.0 \mathrm{ft}^{3} / \mathrm{s}$.

Human health (harmonic mean) design flow. $-305 \mathrm{ft}^{3} / \mathrm{s}$.

Remarks.- Occasional low-flow regulation at Goshen Dam, 3.4 mi upstream.

\begin{tabular}{ccc}
\hline \multicolumn{3}{c}{ Magnitude and frequency of annual low flow } \\
\hline $\begin{array}{c}\text { Lowest average streamflow, in } \mathrm{ft}^{3} / \mathrm{s} \text {, for indicated period of } \\
\text { consecutive days at an annual nonexceedance probability of } 0.1\end{array}$ \\
\hline $\mathbf{1}$ & $\mathbf{3 0}$ \\
\hline 43 & 85 & 101 \\
\hline
\end{tabular}

Percentage of time streamflow was equaled or exceeded for the period of record

\begin{tabular}{cc|cc}
\hline $\begin{array}{c}\text { Percentage } \\
\text { of time }\end{array}$ & $\begin{array}{c}\text { Daily mean } \\
\text { streamflow } \\
\left(\mathrm{ft}^{3} / \mathbf{s}\right)\end{array}$ & $\begin{array}{c}\text { Percentage } \\
\text { of time }\end{array}$ & $\begin{array}{c}\text { Daily mean } \\
\text { streamflow } \\
\left(\mathrm{ft}^{3} / \mathbf{s}\right)\end{array}$ \\
\hline 99 & 82 & 40 & 495 \\
\hline 98 & 98 & 30 & 623 \\
\hline 95 & 127 & 20 & 817 \\
\hline 90 & 157 & 10 & 1140 \\
\hline 80 & 204 & 5 & 1440 \\
\hline 70 & 253 & 2 & 1980 \\
\hline 60 & 315 & 1 & 2440 \\
\hline 50 & 394 & & \\
\hline
\end{tabular}


Lake Michigan Basin

\section{ST. JOSEPH RIVER AT ELKHART, IN}

Location.- Lat $41^{\circ} 41^{\prime} 30^{\prime \prime}$, long $85^{\circ} 58^{\prime} 30^{\prime \prime}$ referenced to North American Datum of 1927, in SW 1/4 NE 1/4 sec.5, T.37 N., R.5 E., Elkhart County, IN, Hydrologic Unit 04050001, on left bank $30 \mathrm{ft}$ upstream from Main Street bridge located at the intersection of Main Street and Pottawatomi Drive in Elkhart, $370 \mathrm{ft}$ downstream from mouth of Elkhart River, 2,170 ft downstream from mouth of Christiana Creek, and $0.53 \mathrm{mi}$ downstream from Elkhart Hydroelectric Plant.

Drainage area.- $-3,370 \mathrm{mi}^{2}$.

Period of record. - August 1947 to current year.

Average discharge. $-3,309 \mathrm{ft}^{3} / \mathrm{s}$.

Minimum daily discharge. $-336 \mathrm{ft}^{3} / \mathrm{s}$.

Human health (harmonic mean) design flow. $-2,440 \mathrm{ft}^{3} / \mathrm{s}$.

Remarks. - The flow is regulated by Elkhart Hydroelectric Plant.

\begin{tabular}{|c|c|c|c|}
\hline \multicolumn{4}{|c|}{ Magnitude and frequency of annual low flow } \\
\hline \multicolumn{4}{|c|}{$\begin{array}{l}\text { Lowest average streamflow, in } \mathrm{ft}^{3} / \mathrm{s} \text {, for indicated period of } \\
\text { consecutive days at an annual nonexceedance probability of } 0.1\end{array}$} \\
\hline 1 & \multicolumn{2}{|c|}{7} & 30 \\
\hline 673 & \multicolumn{2}{|c|}{875} & 1010 \\
\hline \multicolumn{4}{|c|}{$\begin{array}{l}\text { Percentage of time streamflow was equaled or exceeded for } \\
\text { the period of record }\end{array}$} \\
\hline $\begin{array}{l}\text { Percentage } \\
\text { of time }\end{array}$ & $\begin{array}{l}\text { Daily mean } \\
\text { streamflow } \\
\left(\mathrm{ft}^{3} / \mathrm{s}\right)\end{array}$ & $\begin{array}{l}\text { Percentage } \\
\text { of time }\end{array}$ & $\begin{array}{l}\text { Daily mean } \\
\text { streamflow } \\
\left(\mathrm{ft}^{3} / \mathrm{s}\right)\end{array}$ \\
\hline 99 & 864 & 40 & 3290 \\
\hline 98 & 1000 & 30 & 3860 \\
\hline 95 & 1180 & 20 & 4630 \\
\hline 90 & 1400 & 10 & 5930 \\
\hline 80 & 1720 & 5 & 7090 \\
\hline 70 & 2060 & 2 & 8640 \\
\hline 60 & 2420 & 1 & 9930 \\
\hline 50 & 2830 & & \\
\hline
\end{tabular}


Lake Michigan Basin

\section{JUDY CREEK NEAR SOUTH BEND, IN}

Location. - Lat $41^{\circ} 43^{\prime} 43^{\prime \prime}$, long $86^{\circ} 15^{\prime} 46^{\prime \prime}$ referenced to North American Datum of 1927, in NW 1/4 SE 1/4 sec.23, T.38 N., R.2 E., St. Joseph County, IN, Hydrologic Unit 04050001, on right bank at downstream side of bridge on access road to Izaak Walton League property, $0.1 \mathrm{mi}$ south of Darden Road in Roseland, 0.5 mi northeast of intersection of St. Joseph River and Interstate 80/90, and 0.6 mi from mouth.

Drainage area. $-38 \mathrm{mi}^{2}$.

Period of record.- October 1992 to current year.

Average discharge. $-17.3 \mathrm{ft}^{3} / \mathrm{s}$.

Minimum daily discharge. $-0.59 \mathrm{ft}^{3} / \mathrm{s}$.

Human health (harmonic mean) design flow. $-11 \mathrm{ft}^{3} / \mathrm{s}$.

Remarks. - None.

\section{Magnitude and frequency of annual low flow}

Lowest average streamflow, in $\mathrm{ft}^{3} / \mathrm{s}$, for indicated period of consecutive days at an annual nonexceedance probability of 0.1

\begin{tabular}{ccc}
\hline $\mathbf{1}$ & $\mathbf{7}$ & $\mathbf{3 0}$ \\
\hline 1.6 & 1.8 & 2.2 \\
\hline
\end{tabular}

Percentage of time streamflow was equaled or exceeded for the period of record

\begin{tabular}{cc|cc}
\hline $\begin{array}{c}\text { Percentage } \\
\text { of time }\end{array}$ & $\begin{array}{c}\text { Daily mean } \\
\text { streamflow } \\
\left(\mathrm{ft}^{3} / \mathbf{s}\right)\end{array}$ & $\begin{array}{c}\text { Percentage } \\
\text { of time }\end{array}$ & $\begin{array}{c}\text { Daily mean } \\
\text { streamflow } \\
\left(\mathrm{ft}^{3} / \mathbf{s}\right)\end{array}$ \\
\hline 99 & 2.4 & 40 & 18 \\
\hline 98 & 3.0 & 30 & 22 \\
\hline 95 & 4.0 & 20 & 25 \\
\hline 90 & 5.5 & 10 & 32 \\
\hline 80 & 8.0 & 5 & 39 \\
\hline 70 & 11 & 2 & 48 \\
\hline 60 & 14 & 1 & 57 \\
\hline 50 & 16 & & \\
\hline
\end{tabular}


Lake Erie Basin

\section{FISH CREEK AT HAMILTON, IN}

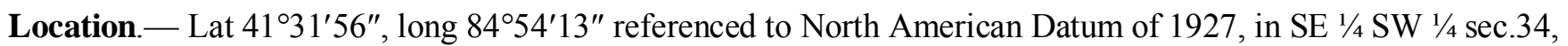
T.36 N., R.14 E., Steuben County, IN, Hydrologic Unit 04100003, on left bank $6 \mathrm{ft}$ upstream from bridge on County Road 775 South, $0.5 \mathrm{mi}$ downstream from Hamilton Lake outlet, $0.5 \mathrm{mi}$ northeast of SR 1 and SR 427 junction, and 0.5 mi southeast of Hamilton.

Drainage area. $-37.5 \mathrm{mi}^{2}$.

Period of record. - October 1969 to current year.

Average discharge. $-34.8 \mathrm{ft}^{3} / \mathrm{s}$.

Minimum daily discharge. $-0.33 \mathrm{ft}^{3} / \mathrm{s}$.

Human health (harmonic mean) design flow. $-7.9 \mathrm{ft}^{3} / \mathrm{s}$.

Remarks. - None.

\section{Magnitude and frequency of annual low flow}

Lowest average streamflow, in $\mathrm{ft}^{3} / \mathrm{s}$, for indicated period of consecutive days at an annual nonexceedance probability of 0.1

\begin{tabular}{ccc}
\hline $\mathbf{1}$ & $\mathbf{7}$ & $\mathbf{3 0}$ \\
\hline 0.9 & 1.1 & 1.6 \\
\hline
\end{tabular}

\begin{tabular}{cc|cc}
\hline Percentage of time streamflow was equaled or exceeded for \\
the period of record
\end{tabular}


Lake Erie Basin

\section{ST. JOSEPH RIVER NEAR NEWVILLE, IN}

Location. - Lat $41^{\circ} 23^{\prime} 07^{\prime \prime}$, long $84^{\circ} 48^{\prime} 06^{\prime \prime}$ referenced to North American Datum of 1927, in SW 1/4 SW 1/4 sec.18, T.5 N., R.1 E., Defiance County, OH, Hydrologic Unit 04100003, on left downstream side at bridge on Ohio State Highway 249, 3.5 mi northeast of Newville, $6.5 \mathrm{mi}$ northwest of Hicksville, $\mathrm{OH}$, and at mile 42.3.

Drainage area. $-610 \mathrm{mi}^{2}$.

Period of record. - October 1946 to current year.

Average discharge. $-557 \mathrm{ft}^{3} / \mathrm{s}$.

Minimum daily discharge. $-14 \mathrm{ft}^{3} / \mathrm{s}$.

Human health (harmonic mean) design flow. $-131 \mathrm{ft}^{3} / \mathrm{s}$.

Remarks.- None.

\begin{tabular}{|c|c|c|c|}
\hline \multicolumn{4}{|c|}{ Magnitude and frequency of annual low flow } \\
\hline \multicolumn{4}{|c|}{$\begin{array}{l}\text { Lowest average streamflow, in } \mathrm{ft}^{3} / \mathrm{s} \text {, for indicated period of } \\
\text { consecutive days at an annual nonexceedance probability of } 0.1\end{array}$} \\
\hline 1 & \multicolumn{2}{|c|}{7} & 30 \\
\hline 21 & \multicolumn{2}{|c|}{22} & 27 \\
\hline \multicolumn{4}{|c|}{$\begin{array}{c}\text { Percentage of time streamflow was equaled or exceeded for } \\
\text { the period of record }\end{array}$} \\
\hline $\begin{array}{l}\text { Percentage } \\
\text { of time }\end{array}$ & $\begin{array}{l}\text { Daily mean } \\
\text { streamflow } \\
\left(\mathrm{ft}^{3} / \mathrm{s}\right)\end{array}$ & $\begin{array}{l}\text { Percentage } \\
\text { of time }\end{array}$ & $\begin{array}{l}\text { Daily mean } \\
\text { streamflow } \\
\left(\mathrm{ft}^{3} / \mathrm{s}\right)\end{array}$ \\
\hline 99 & 24 & 40 & 350 \\
\hline 98 & 28 & 30 & 523 \\
\hline 95 & 39 & 20 & 840 \\
\hline 90 & 52 & 10 & 1530 \\
\hline 80 & 79 & 5 & 2190 \\
\hline 70 & 114 & 2 & 3070 \\
\hline 60 & 170 & 1 & 3850 \\
\hline 50 & 246 & & \\
\hline
\end{tabular}


Lake Erie Basin

\section{ST. JOSEPH RIVER AT CEDARVILLE, IN}

Location. - Lat $41^{\circ} 11^{\prime} 46^{\prime \prime}$, long 8501'27" referenced to North American Datum of 1927, in J. Hackley Reserve, T.32 N., R.13 E., Allen County, IN, Hydrologic Unit 04100003, on left bank $700 \mathrm{ft}$ upstream from highway bridge, $0.4 \mathrm{mi}$ south of Cedarville, $0.5 \mathrm{mi}$ upstream from Cedar Creek, $0.6 \mathrm{mi}$ downstream from Cedarville Dam, and at mile 13.9.

Drainage area. $-763 \mathrm{mi}^{2}$.

Period of record.- January 1931 to May 1932, October 1955 to September 1981.

Average discharge. $-620 \mathrm{ft}^{3} / \mathrm{s}$.

Minimum daily discharge. $-1.6 \mathrm{ft}^{3} / \mathrm{s}$.

Human health (harmonic mean) design flow. $-140 \mathrm{ft}^{3} / \mathrm{s}$.

Remarks. - Flow regulated by Cedarville Reservoir and some flow diverted into storage of Hurshtown Reservoir. Low-flow statistics calculated for the regulated period, 1956 to 1981.

\begin{tabular}{cccc}
\hline \multicolumn{5}{c}{ Magnitude and frequency of annual low flow } \\
\hline \multicolumn{4}{c}{ Lowest average streamflow, in $\mathrm{ft}^{3} / \mathbf{s}$, for indicated period of } \\
consecutive days at an annual nonexceedance probability of 0.1
\end{tabular}


Lake Erie Basin

\section{CEDAR CREEK AT AUBURN, IN}

Location. - Lat $41^{\circ} 21^{\prime} 57^{\prime \prime}$, long $85^{\circ} 03^{\prime} 08^{\prime \prime}$ referenced to North American Datum of 1927, in NE 1/4 NW 1/4 sec.32, T.34 N., R.13 E., DeKalb County, Hydrologic Unit 04100003, on right bank $15 \mathrm{ft}$ downstream from Ninth Street bridge in Auburn, and 2 mi upstream from John Diehl Ditch.

Drainage area. $-87.3 \mathrm{mi}^{2}$.

Period of record. - July 1943 to September 1973.

Average discharge. $-68.5 \mathrm{ft}^{3} / \mathrm{s}$.

Minimum daily discharge. $-0.70 \mathrm{ft}^{3} / \mathrm{s}$.

Human health (harmonic mean) design flow. $-12 \mathrm{ft}^{3} / \mathrm{s}$.

Remarks.- None.

\begin{tabular}{ccc}
\hline \multicolumn{3}{c}{ Magnitude and frequency of annual low flow } \\
\hline $\begin{array}{c}\text { Lowest average streamflow, in } \mathrm{ft}^{3} / \mathrm{s} \text {, for indicated period of } \\
\text { consecutive days at an annual nonexceedance probability of } 0.1\end{array}$ \\
\hline $\mathbf{7}$ & $\mathbf{7}$ & $\mathbf{3 0}$ \\
\hline 1.3 & 1.8 & 2.3 \\
\hline
\end{tabular}

Percentage of time streamflow was equaled or exceeded for the period of record

\begin{tabular}{cc|cc}
\hline $\begin{array}{c}\text { Percentage } \\
\text { of time }\end{array}$ & $\begin{array}{c}\text { Daily mean } \\
\text { streamflow } \\
\left(\mathrm{ft}^{3} / \mathbf{s}\right)\end{array}$ & $\begin{array}{c}\text { Percentage } \\
\text { of time }\end{array}$ & $\begin{array}{c}\text { Daily mean } \\
\text { streamflow } \\
\left(\mathrm{ft}^{3} / \mathbf{s}\right)\end{array}$ \\
\hline 99 & 2.2 & 40 & 39 \\
\hline 98 & 2.8 & 30 & 58 \\
\hline 95 & 3.7 & 20 & 94 \\
\hline 90 & 4.8 & 10 & 180 \\
\hline 80 & 7.5 & 5 & 296 \\
\hline 70 & 12 & 2 & 471 \\
\hline 60 & 18 & 1 & 600 \\
\hline 50 & 27 & & \\
\hline
\end{tabular}


Lake Erie Basin

\section{CEDAR CREEK AT $18^{\text {TH }}$ STREET AT AUBURN, IN}

Location. - Lat $41^{\circ} 21^{\prime} 36^{\prime \prime}$, long $85^{\circ} 02^{\prime} 57^{\prime \prime}$ referenced to North American Datum of 1927, in SW 1/4 NE 1/4 Sec.32, T.34 N., R.13 E., DeKalb County, IN, Hydrologic Unit 04100003, on top of right upstream wingwall of the bridge on 18th Street, 0.3 mi east of downtown Auburn, 1.46 mi above John Diehl Ditch, and at mile 20.94.

Drainage area.- $90.2 \mathrm{mi}^{2}$.

Period of record. - September 2001 to current year.

Average discharge. $-95.2 \mathrm{ft}^{3} / \mathrm{s}$.

Minimum daily discharge. $-2.4 \mathrm{ft}^{3} / \mathrm{s}$.

Human health (harmonic mean) design flow. - $19 \mathrm{ft}^{3} / \mathrm{s}$.

Remarks. - None.

\begin{tabular}{|c|c|c|c|}
\hline \multicolumn{4}{|c|}{ Magnitude and frequency of annual low flow } \\
\hline \multicolumn{4}{|c|}{$\begin{array}{l}\text { Lowest average streamflow, in } \mathrm{ft}^{3} / \mathrm{s} \text {, for indicated period of } \\
\text { consecutive days at an annual nonexceedance probability of } 0.1\end{array}$} \\
\hline 1 & \multicolumn{2}{|c|}{7} & 30 \\
\hline 2.9 & \multicolumn{2}{|c|}{3.3} & 4.1 \\
\hline \multicolumn{4}{|c|}{$\begin{array}{l}\text { Percentage of time streamflow was equaled or exceeded for } \\
\text { the period of record }\end{array}$} \\
\hline $\begin{array}{l}\text { Percentage } \\
\text { of time }\end{array}$ & $\begin{array}{l}\text { Daily mean } \\
\text { streamflow } \\
\left(\mathrm{ft}^{3} / \mathrm{s}\right)\end{array}$ & $\begin{array}{l}\text { Percentage } \\
\text { of time }\end{array}$ & $\begin{array}{l}\text { Daily mean } \\
\text { streamflow } \\
\left(\mathrm{ft}^{3} / \mathrm{s}\right)\end{array}$ \\
\hline 99 & 3.8 & 40 & 58 \\
\hline 98 & 4.3 & 30 & 84 \\
\hline 95 & 5.6 & 20 & 134 \\
\hline 90 & 7.1 & 10 & 251 \\
\hline 80 & 10 & 5 & 395 \\
\hline 70 & 17 & 2 & 592 \\
\hline 60 & 27 & 1 & 777 \\
\hline 50 & 40 & & \\
\hline
\end{tabular}


Lake Erie Basin

\section{CEDAR CREEK NEAR CEDARVILLE, IN}

Location.- Lat $41^{\circ} 13^{\prime} 08^{\prime \prime}$, long $85^{\circ} 04^{\prime} 35^{\prime \prime}$ referenced to North American Datum of 1927, in NW 1/4 NW 1/4 sec.19, T.32 N., R.13 E., Allen County, IN, Hydrologic Unit 04100003, on left bank at downstream side of bridge on Tonkle Road, 3 mi northwest of Cedarville, $5.8 \mathrm{mi}$ upstream from mouth, and $10 \mathrm{mi}$ south of Auburn.

Drainage area. $-270 \mathrm{mi}^{2}$.

Period of record. - October 1946 to current year.

Average discharge. $-263 \mathrm{ft}^{3} / \mathrm{s}$.

Minimum daily discharge. $-13 \mathrm{ft}^{3} / \mathrm{s}$.

Human health (harmonic mean) design flow. $-79 \mathrm{ft}^{3} / \mathrm{s}$.

Remarks.- None.

\begin{tabular}{ccc}
\hline \multicolumn{3}{c}{ Magnitude and frequency of annual low flow } \\
\hline $\begin{array}{c}\text { Lowest average streamflow, in } \mathrm{ft}^{3} / \mathrm{s} \text {, for indicated period of } \\
\text { consecutive days at an annual nonexceedance probability of } \mathbf{0 . 1}\end{array}$ \\
\hline $\mathbf{1}$ & $\mathbf{7}$ & $\mathbf{3 0}$ \\
\hline 19 & 21 & 24 \\
\hline
\end{tabular}

Percentage of time streamflow was equaled or exceeded for the period of record

\begin{tabular}{cc|cc}
\hline $\begin{array}{c}\text { Percentage } \\
\text { of time }\end{array}$ & $\begin{array}{c}\text { Daily mean } \\
\text { streamflow } \\
\left(\mathrm{ft}^{3} / \mathbf{s}\right)\end{array}$ & $\begin{array}{c}\text { Percentage } \\
\text { of time }\end{array}$ & $\begin{array}{c}\text { Daily mean } \\
\text { streamflow } \\
\left(\mathrm{ft}^{3} / \mathbf{s}\right)\end{array}$ \\
\hline 99 & 22 & 40 & 158 \\
\hline 98 & 24 & 30 & 224 \\
\hline 95 & 28 & 20 & 340 \\
\hline 90 & 33 & 10 & 626 \\
\hline 80 & 45 & 5 & 1030 \\
\hline 70 & 62 & 2 & 1700 \\
\hline 60 & 88 & 1 & 2250 \\
\hline 50 & 118 & & \\
\hline
\end{tabular}


Lake Erie Basin

\section{ST. JOSEPH RIVER NEAR FORT WAYNE, IN}

Location. - Lat $41^{\circ} 10^{\prime} 38^{\prime \prime}$, long $85^{\circ} 03^{\prime} 21^{\prime \prime}$ referenced to North American Datum of 1927, in NW 1/4 NE 1/4 Sec.3, T.31 N., R.13 E., Allen County, IN, Hydrologic Unit 04100003, on left bank 0.8 mi downstream from Ely Run, 1.3 mi upstream from Mayhew Road bridge, 8.0 mi northeast of the Fort Wayne Court House, and at mile 10.71.

Drainage area.- $1,060 \mathrm{mi}^{2}$.

Period of record.- October 1983 to current year. August 1941 to September 1955, gage located $1.3 \mathrm{mi}$ downstream at Ely Bridge.

Average discharge. $-1,079 \mathrm{ft}^{3} / \mathrm{s}$.

Minimum daily discharge. $-35 \mathrm{ft}^{3} / \mathrm{s}$.

Human health (harmonic mean) design flow. $-281 \mathrm{ft}^{3} / \mathrm{s}$.

Remarks. - Flow regulated by Cedarville Reservoir and some flow diverted into storage of Hurshtown Reservoir.

\begin{tabular}{|c|c|c|c|}
\hline \multicolumn{4}{|c|}{ Magnitude and frequency of annual low flow } \\
\hline \multicolumn{4}{|c|}{$\begin{array}{l}\text { Lowest average streamflow, in } \mathrm{ft}^{3} / \mathrm{s} \text {, for indicated period of } \\
\text { consecutive days at an annual nonexceedance probability of } 0.1\end{array}$} \\
\hline 1 & \multicolumn{2}{|c|}{7} & 30 \\
\hline 46 & \multicolumn{2}{|c|}{54} & 70 \\
\hline \multicolumn{4}{|c|}{$\begin{array}{c}\text { Percentage of time streamflow was equaled or exceeded for } \\
\text { the period of record }\end{array}$} \\
\hline $\begin{array}{l}\text { Percentage } \\
\text { of time }\end{array}$ & $\begin{array}{l}\text { Daily mean } \\
\text { streamflow } \\
\left(\mathrm{ft}^{3} / \mathrm{s}\right)\end{array}$ & $\begin{array}{l}\text { Percentage } \\
\text { of time }\end{array}$ & $\begin{array}{l}\text { Daily mean } \\
\text { streamflow } \\
\left(\mathrm{ft}^{3} / \mathrm{s}\right)\end{array}$ \\
\hline 99 & 62 & 40 & 671 \\
\hline 98 & 72 & 30 & 990 \\
\hline 95 & 92 & 20 & 1560 \\
\hline 90 & 118 & 10 & 2780 \\
\hline 80 & 169 & 5 & 4080 \\
\hline 70 & 229 & 2 & 5770 \\
\hline 60 & 331 & 1 & 7000 \\
\hline 50 & 469 & & \\
\hline
\end{tabular}


Lake Erie Basin

\section{ST. MARYS RIVER AT DECATUR, IN}

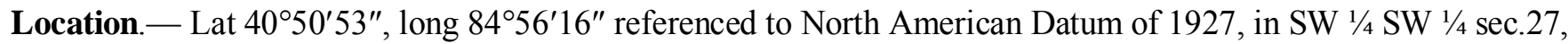
T.28 N., R.14 E., Adams County, IN, Hydrologic Unit 04100004, on left downstream side of bridge on U.S. Highway 27, 0.5 mi upstream from Holthouse Ditch, 1.3 mi north of Decatur, and at mile 29.1.

Drainage area.- $621 \mathrm{mi}^{2}$.

Period of record.- October 1946 to current year.

Average discharge. $-538 \mathrm{ft}^{3} / \mathrm{s}$.

Minimum daily discharge. $-5.4 \mathrm{ft}^{3} / \mathrm{s}$.

Human health (harmonic mean) design flow. $-64 \mathrm{ft}^{3} / \mathrm{s}$.

Remarks. - Flow regulated by Grand Lake. There can be a slight diversion from or into the Wabash River Basin and into the Miami and Erie Canal.

\begin{tabular}{|c|c|c|c|}
\hline \multicolumn{4}{|c|}{ Magnitude and frequency of annual low flow } \\
\hline \multicolumn{4}{|c|}{$\begin{array}{l}\text { Lowest average streamflow, in } \mathrm{ft}^{3} / \mathrm{s} \text {, for indicated period of } \\
\text { consecutive days at an annual nonexceedance probability of } 0.1\end{array}$} \\
\hline 1 & \multicolumn{2}{|c|}{7} & 30 \\
\hline 9.5 & \multicolumn{2}{|c|}{11} & 14 \\
\hline \multicolumn{4}{|c|}{$\begin{array}{l}\text { Percentage of time streamflow was equaled or exceeded for } \\
\text { the period of record }\end{array}$} \\
\hline $\begin{array}{l}\text { Percentage } \\
\text { of time }\end{array}$ & $\begin{array}{l}\text { Daily mean } \\
\text { streamflow } \\
\left(\mathrm{ft}^{3} / \mathrm{s}\right)\end{array}$ & $\begin{array}{l}\text { Percentage } \\
\text { of time }\end{array}$ & $\begin{array}{l}\text { Daily mean } \\
\text { streamflow } \\
\left(\mathrm{ft}^{3} / \mathrm{s}\right)\end{array}$ \\
\hline 99 & 13 & 40 & 223 \\
\hline 98 & 15 & 30 & 395 \\
\hline 95 & 19 & 20 & 745 \\
\hline 90 & 24 & 10 & 1570 \\
\hline 80 & 35 & 5 & 2550 \\
\hline 70 & 54 & 2 & 3880 \\
\hline 60 & 86 & 1 & 4970 \\
\hline 50 & 136 & & \\
\hline
\end{tabular}


Lake Erie Basin

\section{ST. MARYS RIVER NEAR FORT WAYNE, IN}

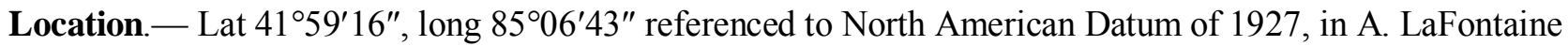
Reserve, T.29 N., R.12 E., Allen County, Hydrologic Unit 04100004, on left bank $130 \mathrm{ft}$ downstream from Anthony Boulevard Extension, 0.8 mi downstream from Houk Ditch, 5 mi south of Fort Wayne, and 10.8 mi upstream from mouth.

Drainage area. $-762 \mathrm{mi}^{2}$.

Period of record. - October 1930 to current year.

Average discharge. $-650 \mathrm{ft}^{3} / \mathrm{s}$.

Minimum daily discharge. $-3.4 \mathrm{ft}^{3} / \mathrm{s}$.

Human health (harmonic mean) design flow. $-70 \mathrm{ft}^{3} / \mathrm{s}$.

Remarks. - The flow is sometimes regulated by Grand Lake. The can be a slight diversion from or into the Wabash River Basin and into Miami and Erie Canal.

\begin{tabular}{|c|c|c|c|}
\hline \multicolumn{4}{|c|}{ Magnitude and frequency of annual low flow } \\
\hline \multicolumn{4}{|c|}{$\begin{array}{l}\text { Lowest average streamflow, in } \mathrm{ft}^{3} / \mathrm{s} \text {, for indicated period of } \\
\text { consecutive days at an annual nonexceedance probability of } 0.1\end{array}$} \\
\hline 1 & \multicolumn{2}{|c|}{7} & 30 \\
\hline 8.9 & \multicolumn{2}{|c|}{10} & 14 \\
\hline \multicolumn{4}{|c|}{$\begin{array}{c}\text { Percentage of time streamflow was equaled or exceeded for } \\
\text { the period of record }\end{array}$} \\
\hline $\begin{array}{l}\text { Percentage } \\
\text { of time }\end{array}$ & $\begin{array}{c}\text { Daily mean } \\
\text { streamflow } \\
\left(\mathrm{ft}^{3} / \mathrm{s}\right)\end{array}$ & $\begin{array}{l}\text { Percentage } \\
\text { of time }\end{array}$ & $\begin{array}{c}\text { Daily mean } \\
\text { streamflow } \\
\left(\mathrm{ft}^{3} / \mathrm{s}\right)\end{array}$ \\
\hline 99 & 13 & 40 & 265 \\
\hline 98 & 15 & 30 & 470 \\
\hline 95 & 20 & 20 & 874 \\
\hline 90 & 26 & 10 & 1900 \\
\hline 80 & 41 & 5 & 3200 \\
\hline 70 & 64 & 2 & 4770 \\
\hline 60 & 101 & 1 & 6050 \\
\hline 50 & 160 & & \\
\hline
\end{tabular}




\section{HARBER DITCH AT FORT WAYNE, IN}

Location.- Lat $41^{\circ} 00^{\prime} 27^{\prime \prime}$, long $85^{\circ} 10^{\prime} 58^{\prime \prime}$ referenced to North American Datum of 1927, in NE 1/4 SW 1/4 sec.33, T.30 N., R.12 E., Allen County, IN, Hydrologic Unit 04100004, on left bank $50 \mathrm{ft}$ upstream from bridge on Baer Road in Fort Wayne, 3.2 mi upstream from mouth. The stream name changes to Fairfield Ditch 0.7 mi downstream at bridge on Lower Huntington Road.

Drainage area. $-21.9 \mathrm{mi}^{2}$.

Period of record.- May 1964 to September 1991.

Average discharge. $-18.6 \mathrm{ft}^{3} / \mathrm{s}$.

Minimum daily discharge. $-0.04 \mathrm{ft}^{3} / \mathrm{s}$.

Human health (harmonic mean) design flow. $-1.1 \mathrm{ft}^{3} / \mathrm{s}$.

Remarks.- None.

\begin{tabular}{|c|c|c|c|}
\hline \multicolumn{4}{|c|}{ Magnitude and frequency of annual low flow } \\
\hline \multicolumn{4}{|c|}{$\begin{array}{l}\text { Lowest average streamflow, in } \mathrm{ft}^{3} / \mathrm{s} \text {, for indicated period of } \\
\text { consecutive days at an annual nonexceedance probability of } 0.1\end{array}$} \\
\hline 1 & \multicolumn{2}{|c|}{7} & 30 \\
\hline 0.1 & \multicolumn{2}{|c|}{0.1} & 0.2 \\
\hline \multicolumn{4}{|c|}{$\begin{array}{c}\text { Percentage of time streamflow was equaled or exceeded for } \\
\text { the period of record }\end{array}$} \\
\hline $\begin{array}{l}\text { Percentage } \\
\text { of time }\end{array}$ & $\begin{array}{l}\text { Daily mean } \\
\text { streamflow } \\
\left(\mathrm{ft}^{3} / \mathrm{s}\right)\end{array}$ & $\begin{array}{l}\text { Percentage } \\
\text { of time }\end{array}$ & $\begin{array}{c}\text { Daily mean } \\
\text { streamflow } \\
\left(\mathrm{ft}^{3} / \mathrm{s}\right)\end{array}$ \\
\hline 99 & 0.1 & 40 & 6.1 \\
\hline 98 & 0.2 & 30 & 11 \\
\hline 95 & 0.3 & 20 & 20 \\
\hline 90 & 0.4 & 10 & 44 \\
\hline 80 & 0.8 & 5 & 87 \\
\hline 70 & 1.5 & 2 & 168 \\
\hline 60 & 2.5 & 1 & 253 \\
\hline 50 & 3.9 & & \\
\hline
\end{tabular}


Lake Erie Basin

\section{ST. MARYS RIVER AT MAIN ST. AT FORT WAYNE, IN}

Location.- Lat $41^{\circ} 04^{\prime} 43^{\prime \prime}$, long $85^{\circ} 09^{\prime} 16^{\prime \prime}$ referenced to North American Datum of 1927 , in SE $1 / 4$ SE $1 / 4 \mathrm{sec} .3$, T.30 N., R.12 E., Allen County, IN, Hydrologic Unit 04100004, on downstream side of Main St. bridge, 2.6 mi west of Wells St, 3.2 mi south of intersection of Coliseum Blvd and Lima Road, 5.4 mi northeast of I-69 and at river mile 1.67 .

Drainage area. $-823 \mathrm{mi}^{2}$.

Period of record. - October 2009 to current year.

Average discharge. $-859 \mathrm{ft}^{3} / \mathrm{s}$.

Minimum daily discharge. $-12 \mathrm{ft}^{3} / \mathrm{s}$.

Human health (harmonic mean) design flow. $-81 \mathrm{ft}^{3} / \mathrm{s}$.

Remarks. - This site has less than 10 years of record.

\begin{tabular}{|c|c|c|c|}
\hline \multicolumn{4}{|c|}{ Magnitude and frequency of annual low flow } \\
\hline \multicolumn{4}{|c|}{$\begin{array}{l}\text { Lowest average streamflow, in } \mathrm{ft}^{3} / \mathrm{s} \text {, for indicated period of } \\
\text { consecutive days at an annual nonexceedance probability of } 0.1\end{array}$} \\
\hline 1 & \multicolumn{2}{|c|}{7} & 30 \\
\hline 10 & \multicolumn{2}{|c|}{12} & 16 \\
\hline \multicolumn{4}{|c|}{$\begin{array}{l}\text { Percentage of time streamflow was equaled or exceeded for } \\
\text { the period of record }\end{array}$} \\
\hline $\begin{array}{l}\text { Percentage } \\
\text { of time }\end{array}$ & $\begin{array}{l}\text { Daily mean } \\
\text { streamflow } \\
\left(\mathrm{ft}^{3} / \mathrm{s}\right)\end{array}$ & $\begin{array}{l}\text { Percentage } \\
\text { of time }\end{array}$ & $\begin{array}{l}\text { Daily mean } \\
\text { streamflow } \\
\left(\mathrm{ft}^{3} / \mathrm{s}\right)\end{array}$ \\
\hline 99 & 22 & 40 & 378 \\
\hline 98 & 24 & 30 & 650 \\
\hline 95 & 28 & 20 & 1230 \\
\hline 90 & 33 & 10 & 2560 \\
\hline 80 & 50 & 5 & 4320 \\
\hline 70 & 74 & 2 & 6470 \\
\hline 60 & 124 & 1 & 7390 \\
\hline 50 & 229 & & \\
\hline
\end{tabular}




\section{SPY RUN CREEK NEAR PARK DRIVE AT FORT WAYNE, IN}

Location.- Lat $41^{\circ} 06^{\prime} 17^{\prime \prime}$, long $85^{\circ} 09^{\prime} 28^{\prime \prime}$ referenced to North American Datum of 1927, in SE 1/4 SE 1/4 SW 1/4 sec.27, T.31 N., R.12 E., Allen County, IN, Hydrologic Unit 04100004, on right bank at top of stairs, $0.5 \mathrm{mi}$ from entrance to Franke Park, 2.5 mi from I-69, and at river mile 2.66.

Drainage area. $-13.5 \mathrm{mi}^{2}$.

Period of record. - May 2008 to current year.

Average discharge. $-17.3 \mathrm{ft}^{3} / \mathrm{s}$.

Minimum daily discharge. $-0.27 \mathrm{ft}^{3} / \mathrm{s}$.

Human health (harmonic mean) design flow. $-2.6 \mathrm{ft}^{3} / \mathrm{s}$.

Remarks. - This site has less than 10 years of record.

\begin{tabular}{|c|c|c|c|}
\hline \multicolumn{4}{|c|}{ Magnitude and frequency of annual low flow } \\
\hline \multicolumn{4}{|c|}{$\begin{array}{l}\text { Lowest average streamflow, in } \mathrm{ft}^{3} / \mathrm{s} \text {, for indicated period of } \\
\text { consecutive days at an annual nonexceedance probability of } 0.1\end{array}$} \\
\hline 1 & \multicolumn{2}{|c|}{7} & 30 \\
\hline 0.6 & \multicolumn{2}{|c|}{0.6} & 0.8 \\
\hline \multicolumn{4}{|c|}{$\begin{array}{l}\text { Percentage of time streamflow was equaled or exceeded for } \\
\text { the period of record }\end{array}$} \\
\hline $\begin{array}{l}\text { Percentage } \\
\text { of time }\end{array}$ & $\begin{array}{l}\text { Daily mean } \\
\text { streamflow } \\
\left(\mathrm{ft}^{3} / \mathrm{s}\right)\end{array}$ & $\begin{array}{l}\text { Percentage } \\
\text { of time }\end{array}$ & $\begin{array}{l}\text { Daily mean } \\
\text { streamflow } \\
\left(\mathrm{ft}^{3} / \mathrm{s}\right)\end{array}$ \\
\hline 99 & 0.4 & 40 & 5.1 \\
\hline 98 & 0.6 & 30 & 7.6 \\
\hline 95 & 0.9 & 20 & 15 \\
\hline 90 & 1.1 & 10 & 38 \\
\hline 80 & 1.7 & 5 & 78 \\
\hline 70 & 2.3 & 2 & 162 \\
\hline 60 & 3 & 1 & 244 \\
\hline 50 & 3.9 & & \\
\hline
\end{tabular}


Lake Erie Basin

\section{SPY RUN CREEK AT FORT WAYNE, IN}

Location.- Lat $41^{\circ} 06^{\prime} 18^{\prime \prime}$, long $85^{\circ} 09^{\prime} 12^{\prime \prime}$ referenced to North American Datum of 1927, in SW1/4SW1/4 sec.26, T.31 N., R.12 E., Allen County, Hydrologic Unit 04100004, on right bank $50 \mathrm{ft}$ upstream from Sherman Boulevard bridge in Fort Wayne, and at mile 2.2.

Drainage area.- $-14.0 \mathrm{mi}^{2}$.

Period of record.- October 1983 to October 2001.

Average discharge. $-18.0 \mathrm{ft}^{3} / \mathrm{s}$.

Minimum daily discharge. $-0.93 \mathrm{ft}^{3} / \mathrm{s}$.

Human health (harmonic mean) design flow. $-5 \mathrm{ft}^{3} / \mathrm{s}$.

Remarks. - None.

\begin{tabular}{|c|c|c|c|}
\hline \multicolumn{4}{|c|}{ Magnitude and frequency of annual low flow } \\
\hline \multicolumn{4}{|c|}{$\begin{array}{l}\text { Lowest average streamflow, in } \mathrm{ft}^{3} / \mathrm{s} \text {, for indicated period of } \\
\text { consecutive days at an annual nonexceedance probability of } 0.1\end{array}$} \\
\hline 1 & \multicolumn{2}{|c|}{7} & 30 \\
\hline 1.1 & \multicolumn{2}{|c|}{1.4} & 2.0 \\
\hline \multicolumn{4}{|c|}{$\begin{array}{l}\text { Percentage of time streamflow was equaled or exceeded for } \\
\text { the period of record }\end{array}$} \\
\hline $\begin{array}{l}\text { Percentage } \\
\text { of time }\end{array}$ & $\begin{array}{l}\text { Daily mean } \\
\text { streamflow } \\
\left(\mathrm{ft}^{3} / \mathrm{s}\right)\end{array}$ & $\begin{array}{l}\text { Percentage } \\
\text { of time }\end{array}$ & $\begin{array}{l}\text { Daily mean } \\
\text { streamflow } \\
\left(\mathrm{ft}^{3} / \mathrm{s}\right)\end{array}$ \\
\hline 99 & 1.6 & 40 & 7.5 \\
\hline 98 & 1.8 & 30 & 11 \\
\hline 95 & 2.1 & 20 & 18 \\
\hline 90 & 2.5 & 10 & 35 \\
\hline 80 & 3.2 & 5 & 70 \\
\hline 70 & 3.7 & 2 & 154 \\
\hline 60 & 4.5 & 1 & 240 \\
\hline 50 & 5.6 & & \\
\hline
\end{tabular}


Lake Erie Basin

\section{MAUMEE RIVER AT COLISEUM BLVD AT FORT WAYNE, IN}

Location.- Lat $41^{\circ} 04^{\prime} 47^{\prime \prime}$, long $85^{\circ} 05^{\prime} 15^{\prime \prime}$ referenced to North American Datum of 1927, in SW 1/4 SE 1/4 Sec.5, T.30 N., R.13 E., Allen County, IN, Hydrologic Unit 04100003, on left bank and downstream side of Coliseum Blvd. bridge, 0.4 mi north of intersection of State Road 14 and Coliseum Blvd., 1.5 mi downstream of Anthony Blvd., 2.7 mi below confluence of St. Joseph and St. Marys Rivers, and at mile 133.4.

Drainage area.- $1,930 \mathrm{mi}^{2}$.

Period of record. - November 2003 to current year.

Average discharge. $-2,124 \mathrm{ft}^{3} / \mathrm{s}$.

Minimum daily discharge. $-41 \mathrm{ft}^{3} / \mathrm{s}$.

Human health (harmonic mean) design flow. $-460 \mathrm{ft}^{3} / \mathrm{s}$.

Remarks. - This site has less than 10 years of record. Flow is regulated by hydropower plant on the St. Joseph River 5.9 mi upstream from the station. Flow is slightly regulated by upstream reservoirs.

\begin{tabular}{|c|c|c|c|}
\hline \multicolumn{4}{|c|}{ Magnitude and frequency of annual low flow } \\
\hline \multicolumn{4}{|c|}{$\begin{array}{l}\text { Lowest average streamflow, in } \mathrm{ft}^{3} / \mathrm{s} \text {, for indicated period of } \\
\text { consecutive days at an annual nonexceedance probability of } 0.1\end{array}$} \\
\hline 1 & \multicolumn{2}{|c|}{7} & 30 \\
\hline 56 & \multicolumn{2}{|c|}{89} & 112 \\
\hline \multicolumn{4}{|c|}{$\begin{array}{l}\text { Percentage of time streamflow was equaled or exceeded for } \\
\text { the period of record }\end{array}$} \\
\hline $\begin{array}{l}\text { Percentage } \\
\text { of time }\end{array}$ & $\begin{array}{l}\text { Daily mean } \\
\text { streamflow } \\
\left(\mathrm{ft}^{3} / \mathrm{s}\right)\end{array}$ & $\begin{array}{l}\text { Percentage } \\
\text { of time }\end{array}$ & $\begin{array}{l}\text { Daily mean } \\
\text { streamflow } \\
\left(\mathrm{ft}^{3} / \mathrm{s}\right)\end{array}$ \\
\hline 99 & 95 & 40 & 1300 \\
\hline 98 & 106 & 30 & 1900 \\
\hline 95 & 124 & 20 & 3230 \\
\hline 90 & 163 & 10 & 6100 \\
\hline 80 & 248 & 5 & 9030 \\
\hline 70 & 392 & 2 & 13010 \\
\hline 60 & 579 & 1 & 15370 \\
\hline 50 & 849 & & \\
\hline
\end{tabular}


Lake Erie Basin

\section{MAUMEE RIVER AT NEW HAVEN, IN}

Location.- Lat $41^{\circ} 05^{\prime} 06^{\prime \prime}$, long $85^{\circ} 01^{\prime} 20^{\prime \prime}$ referenced to North American Datum of 1927 , in SE $1 / 4$ NE $1 / 4 \sec .2$, T.30 N., R.13 E., Allen County, IN, Hydrologic Unit 04100005, on left bank $600 \mathrm{ft}$ upstream from bridge on Landin Road, 1,400 ft upstream from the Norfolk and Western Railroad bridge, $1.1 \mathrm{mi}$ northwest of New Haven, $2.8 \mathrm{mi}$ upstream from Sixmile Creek, and at mile 129.0.

Drainage area.- $1,967 \mathrm{mi}^{2}$.

Period of record.- October 1956 to current year.

Average discharge. $-1,867 \mathrm{ft}^{3} / \mathrm{s}$.

Minimum daily discharge. $-48 \mathrm{ft}^{3} / \mathrm{s}$.

Human health (harmonic mean) design flow. $-432 \mathrm{ft}^{3} / \mathrm{s}$.

Remarks. - The flow is regulated by hydropower plant on the St. Joseph River 10.3 mi upstream from station. Flow is slightly regulated by upstream reservoirs.

\begin{tabular}{cccc}
\hline \multicolumn{5}{c}{ Magnitude and frequency of annual low flow } \\
\hline \multicolumn{4}{c}{ Lowest average streamflow, in $\mathrm{ft}^{3} / \mathrm{s}$, for indicated period of } \\
consecutive days at an annual nonexceedance probability of 0.1
\end{tabular}


Illinois River Basin

\section{KANKAKEE RIVER NEAR NORTH LIBERTY, IN}

Location. - Lat $41^{\circ} 33^{\prime} 50^{\prime \prime}$, long $86^{\circ} 29^{\prime} 50^{\prime \prime}$ referenced to North American Datum of 1927, in NW 1/4 NE $1 / 4 \mathrm{sec} .23$, T.36 N., R.1 W., St. Joseph County, IN, Hydrologic Unit 07120001, on left bank at downstream side of bridge on county highway named "New Road," 2.7 mi upstream from Little Kankakee River, 4 mi northwest of North Liberty, and at mile 126.9 .

Drainage area.- $174 \mathrm{mi}^{2}$, of which $58.2 \mathrm{mi}^{2}$ does not contribute directly to surface runoff.

Period of record.- January 1951 to October 2003.

Average discharge. $-158 \mathrm{ft}^{3} / \mathrm{s}$.

Minimum daily discharge. $-44 \mathrm{ft}^{3} / \mathrm{s}$.

Human health (harmonic mean) design flow. $-129 \mathrm{ft}^{3} / \mathrm{s}$.

Remarks. - None.

\begin{tabular}{ccc}
\hline \multicolumn{3}{c}{ Magnitude and frequency of annual low flow } \\
\hline $\begin{array}{c}\text { Lowest average streamflow, in } \mathrm{ft}^{3} / \mathrm{s} \text {, for indicated period of } \\
\text { consecutive days at an annual nonexceedance probability of } 0.1\end{array}$ \\
\hline $\mathbf{1}$ & $\mathbf{7}$ & $\mathbf{3 0}$ \\
\hline 52 & 57 & 64 \\
\hline
\end{tabular}

Percentage of time streamflow was equaled or exceeded for the period of record

\begin{tabular}{cc|cc}
\hline $\begin{array}{c}\text { Percentage } \\
\text { of time }\end{array}$ & $\begin{array}{c}\text { Daily mean } \\
\text { streamflow } \\
\left(\mathrm{ft}^{3} / \mathbf{s}\right)\end{array}$ & $\begin{array}{c}\text { Percentage } \\
\text { of time }\end{array}$ & $\begin{array}{c}\text { Daily mean } \\
\text { streamflow } \\
\left(\mathrm{ft}^{3} / \mathbf{s}\right)\end{array}$ \\
\hline 99 & 61 & 40 & 155 \\
\hline 98 & 66 & 30 & 174 \\
\hline 95 & 74 & 20 & 203 \\
\hline 90 & 83 & 10 & 259 \\
\hline 80 & 96 & 5 & 322 \\
\hline 70 & 109 & 2 & 416 \\
\hline 60 & 123 & 1 & 488 \\
\hline 50 & 137 & & \\
\hline
\end{tabular}


Illinois River Basin

\section{KINGSBURY CREEK NEAR LAPORTE, IN}

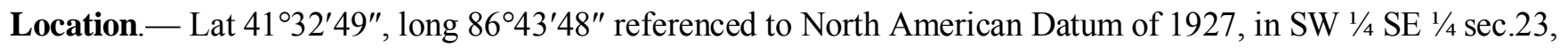
T.36 N., R.3 W., LaPorte County, Hydrologic Unit 07120001, on left bank at upstream side of bridge on County Road 400 South, 0.5 mi east of State Highway 39, 1.5 mi west of U.S. Highway 35, and 3 mi south of LaPorte city limits.

Drainage area.- $7.08 \mathrm{mi}^{2}$, of which $4.07 \mathrm{mi}^{2}$ does not contribute directly to surface runoff.

Period of record. - October 1970 to September 1986.

Average discharge. $-4.26 \mathrm{ft}^{3} / \mathrm{s}$.

Minimum daily discharge. $-0.83 \mathrm{ft}^{3} / \mathrm{s}$.

Human health (harmonic mean) design flow. $-3.2 \mathrm{ft}^{3} / \mathrm{s}$.

Remarks.- None.

\begin{tabular}{ccc}
\hline \multicolumn{3}{c}{ Magnitude and frequency of annual low flow } \\
\hline $\begin{array}{c}\text { Lowest average streamflow, in } \mathrm{ft}^{3} / \mathrm{s} \text {, for indicated period of } \\
\text { consecutive days at an annual nonexceedance probability of } 0.1\end{array}$ \\
\hline $\mathbf{7}$ & $\mathbf{3 0}$ \\
\hline 1.1 & 1.2 & 1.3 \\
\hline
\end{tabular}

Percentage of time streamflow was equaled or exceeded for the period of record

\begin{tabular}{rr|rr}
\hline $\begin{array}{c}\text { Percentage } \\
\text { of time }\end{array}$ & $\begin{array}{c}\text { Daily mean } \\
\text { streamflow } \\
\text { (ft3 } 3 \mathbf{s})\end{array}$ & $\begin{array}{c}\text { Percentage } \\
\text { of time }\end{array}$ & $\begin{array}{c}\text { Daily mean } \\
\text { streamflow } \\
\left(\mathrm{ft}^{3} / \mathbf{s}\right)\end{array}$ \\
\hline 99 & 1.4 & 40 & 4.2 \\
\hline 98 & 1.5 & 30 & 4.8 \\
\hline 95 & 1.6 & 20 & 5.6 \\
\hline 90 & 1.9 & 10 & 7.4 \\
\hline 80 & 2.3 & 5 & 9.0 \\
\hline 70 & 2.7 & 2 & 12 \\
\hline 60 & 3.2 & 1 & 15 \\
\hline 50 & 3.7 & & \\
\hline
\end{tabular}


Illinois River Basin

\section{KANKAKEE RIVER AT DAVIS, IN}

Location. - Lat $41^{\circ} 24^{\prime} 00^{\prime \prime}$, long $86^{\circ} 42^{\prime} 04^{\prime \prime}$ referenced to North American Datum of 1927, in SE 1/4 NE $1 / 4$ Sec.13, T.34 N., R.3 W., Starke County, IN, Hydrologic Unit 07120001, on left bank at downstream side of bridge on U.S. Highway 30 at Davis, 0.5 mi downstream from Mill Creek, 4 mi east of Hanna, and at mile 110.9.

Drainage area. - $537 \mathrm{mi}^{2}$, of which $137 \mathrm{mi}^{2}$ does not contribute directly to surface runoff.

Period of record. - October 1924 to current year.

Average discharge. $-528 \mathrm{ft}^{3} / \mathrm{s}$.

Minimum daily discharge. $-147 \mathrm{ft}^{3} / \mathrm{s}$.

Human health (harmonic mean) design flow. $-433 \mathrm{ft}^{3} / \mathrm{s}$.

Remarks. - None.

\begin{tabular}{ccc}
\hline \multicolumn{3}{c}{ Magnitude and frequency of annual low flow } \\
\hline $\begin{array}{c}\text { Lowest average streamflow, in } \mathrm{ft}^{3} / \mathrm{s} \text {, for indicated period of } \\
\text { consecutive days at an annual nonexceedance probability of } \mathbf{0 . 1}\end{array}$ \\
\hline $\mathbf{1}$ & $\mathbf{7}$ & $\mathbf{3 0}$ \\
\hline 184 & 191 & 211 \\
\hline
\end{tabular}

Percentage of time streamflow was equaled or exceeded for the period of record

\begin{tabular}{cc|cc}
\hline $\begin{array}{c}\text { Percentage } \\
\text { of time }\end{array}$ & $\begin{array}{c}\text { Daily mean } \\
\text { streamflow } \\
\left(\mathrm{ft}^{3} / \mathbf{s}\right)\end{array}$ & $\begin{array}{c}\text { Percentage } \\
\text { of time }\end{array}$ & $\begin{array}{c}\text { Daily mean } \\
\text { streamflow } \\
\left(\mathrm{ft}^{3} / \mathbf{s}\right)\end{array}$ \\
\hline 99 & 206 & 40 & 517 \\
\hline 98 & 220 & 30 & 594 \\
\hline 95 & 248 & 20 & 705 \\
\hline 90 & 277 & 10 & 899 \\
\hline 80 & 322 & 5 & 1070 \\
\hline 70 & 363 & 2 & 1260 \\
\hline 60 & 407 & 1 & 1410 \\
\hline 50 & 455 & & \\
\hline
\end{tabular}


Illinois River Basin

\section{YELLOW RIVER NEAR BREMEN, IN}

Location. - Lat $41^{\circ} 25^{\prime} 11^{\prime \prime}$, long $86^{\circ} 10^{\prime} 14^{\prime \prime}$ referenced to North American Datum of 1927, in NW 1/4 NW 1/4 sec. 10 , T.34 N., R.3 E., Marshall County, Hydrologic Unit 07120001, on left bank at downstream side of bridge on East 4th Road, 0.5 mi downstream from Bunch ditch, 2 mi southwest of Bremen, and 4 mi upstream from Dausman ditch.

Drainage area.- $135 \mathrm{mi}^{2}$.

Period of record. - August 1955 to September 1973.

Average discharge. $-104 \mathrm{ft}^{3} / \mathrm{s}$.

Minimum daily discharge. $-6.2 \mathrm{ft}^{3} / \mathrm{s}$.

Human health (harmonic mean) design flow. $-25 \mathrm{ft}^{3} / \mathrm{s}$.

Remarks. - None.

\begin{tabular}{ccc}
\hline \multicolumn{3}{c}{ Magnitude and frequency of annual low flow } \\
\hline $\begin{array}{c}\text { Lowest average streamflow, in } \mathrm{ft}^{3} / \mathrm{s} \text {, for indicated period of } \\
\text { consecutive days at an annual nonexceedance probability of } 0.1\end{array}$ \\
\hline $\mathbf{1}$ & $\mathbf{7}$ & $\mathbf{3 0}$ \\
\hline 6.0 & 6.3 & 6.9 \\
\hline
\end{tabular}

Percentage of time streamflow was equaled or exceeded for the period of record

\begin{tabular}{cc|cc}
\hline $\begin{array}{c}\text { Percentage } \\
\text { of time }\end{array}$ & $\begin{array}{c}\text { Daily mean } \\
\text { streamflow } \\
\left(\mathrm{ft}^{3} / \mathbf{s}\right)\end{array}$ & $\begin{array}{c}\text { Percentage } \\
\text { of time }\end{array}$ & $\begin{array}{c}\text { Daily mean } \\
\text { streamflow } \\
\left(\mathrm{ft}^{3} / \mathbf{s}\right)\end{array}$ \\
\hline 99 & 7.2 & 40 & 54 \\
\hline 98 & 7.5 & 30 & 78 \\
\hline 95 & 8.4 & 20 & 130 \\
\hline 90 & 10 & 10 & 257 \\
\hline 80 & 14 & 5 & 472 \\
\hline 70 & 21 & 2 & 810 \\
\hline 60 & 29 & 1 & 950 \\
\hline 50 & 39 & & \\
\hline
\end{tabular}


Illinois River Basin

\section{YELLOW RIVER AT PLYMOUTH, IN}

Location. - Lat $41^{\circ} 20^{\prime} 25^{\prime \prime}$, long $86^{\circ} 18^{\prime} 16^{\prime \prime}$ referenced to North American Datum of 1927, in SE 1/4 NW 1/4 sec.13, T.33 N., R.2 E., Marshall County, IN, Hydrologic Unit 07120001, on left bank $50 \mathrm{ft}$ upstream from LaPorte Street footbridge in Plymouth, 1.1 mi downstream from Elmer Seltenright (formerly Baker) Ditch, 8.1 mi upstream from Wolf Creek, and at mile 40.3.

Drainage area.- $294 \mathrm{mi}^{2}$, of which $22 \mathrm{mi}^{2}$ does not contribute directly to surface runoff.

Period of record. - July 1948 to current year.

Average discharge. $-273 \mathrm{ft}^{3} / \mathrm{s}$.

Minimum daily discharge. $-13 \mathrm{ft}^{3} / \mathrm{s}$.

Human health (harmonic mean) design flow. - $94 \mathrm{ft}^{3} / \mathrm{s}$.

Remarks. - None.

\begin{tabular}{|c|c|c|c|}
\hline \multicolumn{4}{|c|}{ Magnitude and frequency of annual low flow } \\
\hline \multicolumn{4}{|c|}{$\begin{array}{l}\text { Lowest average streamflow, in } \mathrm{ft}^{3} / \mathrm{s} \text {, for indicated period of } \\
\text { consecutive days at an annual nonexceedance probability of } 0.1\end{array}$} \\
\hline 1 & \multicolumn{2}{|c|}{7} & 30 \\
\hline 21 & \multicolumn{2}{|c|}{23} & 27 \\
\hline \multicolumn{4}{|c|}{$\begin{array}{l}\text { Percentage of time streamflow was equaled or exceeded for } \\
\text { the period of record }\end{array}$} \\
\hline $\begin{array}{l}\text { Percentage } \\
\text { of time }\end{array}$ & $\begin{array}{l}\text { Daily mean } \\
\text { streamflow } \\
\left(\mathrm{ft}^{3} / \mathrm{s}\right)\end{array}$ & $\begin{array}{l}\text { Percentage } \\
\text { of time }\end{array}$ & $\begin{array}{l}\text { Daily mean } \\
\text { streamflow } \\
\left(\mathrm{ft}^{3} / \mathrm{s}\right)\end{array}$ \\
\hline 99 & 25 & 40 & 179 \\
\hline 98 & 28 & 30 & 244 \\
\hline 95 & 34 & 20 & 365 \\
\hline 90 & 41 & 10 & 677 \\
\hline 80 & 57 & 5 & 1080 \\
\hline 70 & 77 & 2 & 1610 \\
\hline 60 & 103 & 1 & 1990 \\
\hline 50 & 135 & & \\
\hline
\end{tabular}


Illinois River Basin

\section{YELLOW RIVER AT KNOX, IN}

Location. - Lat $41^{\circ} 18^{\prime} 10^{\prime \prime}$, long $86^{\circ} 37^{\prime} 14^{\prime \prime}$ referenced to North American Datum of 1927, in SW 1/4 SW 1/4 sec.14, T.33 N., R.2 W., Starke County, IN, Hydrologic Unit 07120001, on right bank $40 \mathrm{ft}$ upstream from bridge on U.S. Highway 35 in Knox, 0.3 mi north of Knox, 1.4 mi downstream from Eagle Creek, and at mile 11.6.

Drainage area.- $435 \mathrm{mi}^{2}$, of which $51 \mathrm{mi}^{2}$ does not contribute directly to surface runoff.

Period of record.- August 1943 to October 2011.

Average discharge. $-415 \mathrm{ft}^{3} / \mathrm{s}$.

Minimum daily discharge. $-37 \mathrm{ft}^{3} / \mathrm{s}$.

Human health (harmonic mean) design flow. $-226 \mathrm{ft}^{3} / \mathrm{s}$.

Remarks. - None.

\begin{tabular}{|c|c|c|c|}
\hline \multicolumn{4}{|c|}{ Magnitude and frequency of annual low flow } \\
\hline \multicolumn{4}{|c|}{$\begin{array}{l}\text { Lowest average streamflow, in } \mathrm{ft}^{3} / \mathrm{s} \text {, for indicated period of } \\
\text { consecutive days at an annual nonexceedance probability of } 0.1\end{array}$} \\
\hline 1 & \multicolumn{2}{|c|}{7} & 30 \\
\hline 64 & \multicolumn{2}{|c|}{71} & 83 \\
\hline \multicolumn{4}{|c|}{$\begin{array}{l}\text { Percentage of time streamflow was equaled or exceeded for } \\
\text { the period of record }\end{array}$} \\
\hline $\begin{array}{l}\text { Percentage } \\
\text { of time }\end{array}$ & $\begin{array}{l}\text { Daily mean } \\
\text { streamflow } \\
\left(\mathrm{ft}^{3} / \mathrm{s}\right)\end{array}$ & $\begin{array}{l}\text { Percentage } \\
\text { of time }\end{array}$ & $\begin{array}{l}\text { Daily mean } \\
\text { streamflow } \\
\left(\mathrm{ft}^{3} / \mathrm{s}\right)\end{array}$ \\
\hline 99 & 81 & 40 & 338 \\
\hline 98 & 87 & 30 & 432 \\
\hline 95 & 99 & 20 & 585 \\
\hline 90 & 114 & 10 & 891 \\
\hline 80 & 142 & 5 & 1240 \\
\hline 70 & 176 & 2 & 1810 \\
\hline 60 & 220 & 1 & 2200 \\
\hline 50 & 271 & & \\
\hline
\end{tabular}


Illinois River Basin

\section{KANKAKEE RIVER AT DUNNS BRIDGE, IN}

Location.- Lat $41^{\circ} 13^{\prime} 12^{\prime \prime}$, long $86^{\circ} 58^{\prime} 06^{\prime \prime}$ referenced to North American Datum of 1927, in NE 1/4 SE $1 / 4$ Sec.15, T.32 N., R.5 W., Porter County, IN, Hydrologic Unit 07120001, on right bank at downstream side of county road 500E bridge at Dunns Bridge, 1.8 mi north of Tefft, 3.6 mi upstream from Davis Ditch, and at mile 90.8.

Drainage area.- - 1,352 $\mathrm{mi}^{2}$, of which $192 \mathrm{mi}^{2}$ does not contribute directly to surface runoff.

Period of record. - July 1948 to current year.

Average discharge. $-1,393 \mathrm{ft}^{3} / \mathrm{s}$.

Minimum daily discharge. $-280 \mathrm{ft}^{3} / \mathrm{s}$.

Human health (harmonic mean) design flow. $-988 \mathrm{ft}^{3} / \mathrm{s}$.

Remarks.- None.

\begin{tabular}{|c|c|c|c|}
\hline \multicolumn{4}{|c|}{ Magnitude and frequency of annual low flow } \\
\hline \multicolumn{4}{|c|}{$\begin{array}{l}\text { Lowest average streamflow, in } \mathrm{ft}^{3} / \mathrm{s} \text {, for indicated period of } \\
\text { consecutive days at an annual nonexceedance probability of } 0.1\end{array}$} \\
\hline 1 & \multicolumn{2}{|c|}{7} & 30 \\
\hline 337 & \multicolumn{2}{|c|}{352} & 395 \\
\hline \multicolumn{4}{|c|}{$\begin{array}{l}\text { Percentage of time streamflow was equaled or exceeded for } \\
\text { the period of record }\end{array}$} \\
\hline $\begin{array}{l}\text { Percentage } \\
\text { of time }\end{array}$ & $\begin{array}{l}\text { Daily mean } \\
\text { streamflow } \\
\left(\mathrm{ft}^{3} / \mathrm{s}\right)\end{array}$ & $\begin{array}{l}\text { Percentage } \\
\text { of time }\end{array}$ & $\begin{array}{l}\text { Daily mean } \\
\text { streamflow } \\
\left(\mathrm{ft}^{3} / \mathrm{s}\right)\end{array}$ \\
\hline 99 & 372 & 40 & 1360 \\
\hline 98 & 401 & 30 & 1630 \\
\hline 95 & 462 & 20 & 2040 \\
\hline 90 & 541 & 10 & 2680 \\
\hline 80 & 667 & 5 & 3190 \\
\hline 70 & 803 & 2 & 3760 \\
\hline 60 & 968 & 1 & 4270 \\
\hline 50 & 1160 & & \\
\hline
\end{tabular}


Illinois River Basin

\section{KANKAKEE RIVER NEAR KOUTS, IN}

Location.- Lat $41^{\circ} 15^{\prime} 14^{\prime \prime}$, long $87^{\circ} 02^{\prime} 02^{\prime \prime}$ referenced to North American Datum of 1927, in SW 1/4 NE $1 / 4$ sec.6, T.32 N., R.5 W., Jasper County, IN, Hydrologic Unit 07120001, on left bank, $20 \mathrm{ft}$ downstream from bridge on State Highway 49, 0.7 mi upstream from Cook Ditch, 4.5 mi south of Kouts, and at mile 86.7.

Drainage area.- 1,376 $\mathrm{mi}^{2}$, of which $194 \mathrm{mi}^{2}$ does not contribute directly to surface runoff.

Period of record. - October 1974 to current year.

Average discharge. $-1,488 \mathrm{ft}^{3} / \mathrm{s}$.

Minimum daily discharge. $-273 \mathrm{ft}^{3} / \mathrm{s}$.

Human health (harmonic mean) design flow. - 1,060 $\mathrm{ft}^{3} / \mathrm{s}$.

Remarks.- None.

\begin{tabular}{|c|c|c|c|}
\hline \multicolumn{4}{|c|}{ Magnitude and frequency of annual low flow } \\
\hline \multicolumn{4}{|c|}{$\begin{array}{l}\text { Lowest average streamflow, in } \mathrm{ft}^{3} / \mathrm{s} \text {, for indicated period of } \\
\text { consecutive days at an annual nonexceedance probability of } 0.1\end{array}$} \\
\hline 1 & \multicolumn{2}{|c|}{7} & 30 \\
\hline 334 & \multicolumn{2}{|c|}{362} & 431 \\
\hline \multicolumn{4}{|c|}{$\begin{array}{l}\text { Percentage of time streamflow was equaled or exceeded for } \\
\text { the period of record }\end{array}$} \\
\hline $\begin{array}{l}\text { Percentage } \\
\text { of time }\end{array}$ & $\begin{array}{l}\text { Daily mean } \\
\text { streamflow } \\
\left(\mathrm{ft}^{3} / \mathrm{s}\right)\end{array}$ & $\begin{array}{l}\text { Percentage } \\
\text { of time }\end{array}$ & $\begin{array}{l}\text { Daily mean } \\
\text { streamflow } \\
\left(\mathrm{ft}^{3} / \mathrm{s}\right)\end{array}$ \\
\hline 99 & 406 & 40 & 1470 \\
\hline 98 & 441 & 30 & 1750 \\
\hline 95 & 504 & 20 & 2160 \\
\hline 90 & 581 & 10 & 2860 \\
\hline 80 & 712 & 5 & 3450 \\
\hline 70 & 857 & 2 & 4110 \\
\hline 60 & 1050 & 1 & 4540 \\
\hline 50 & 1260 & & \\
\hline
\end{tabular}


Illinois River Basin

\section{COBB DITCH NEAR KOUTS, IN}

Location. - Lat $41^{\circ} 20^{\prime} 19^{\prime \prime}$, long $87^{\circ} 04^{\prime} 30^{\prime \prime}$ referenced to North American Datum of 1927, in NW 1/4 SE 1/4 Sec. 2 , T.33 N., R.6 W., Porter County, IN, Hydrologic Unit 07120001, on left bank $15 \mathrm{ft}$ upstream from bridge on County Road 50 West, 1.6 mi upstream from mouth, 3 mi northwest of Kouts, and 6.5 mi northeast of Hebron.

Drainage area. $-30.3 \mathrm{mi}^{2}$.

Period of record. - July 1968 to October 2003.

Average discharge. $-33.1 \mathrm{ft}^{3} / \mathrm{s}$.

Minimum daily discharge. $-6.0 \mathrm{ft}^{3} / \mathrm{s}$.

Human health (harmonic mean) design flow. $-21 \mathrm{ft}^{3} / \mathrm{s}$.

Remarks. - Prior to October 1971, published as State Ditch near Kouts.

\begin{tabular}{cc|cc}
\hline \multicolumn{5}{c}{ Magnitude and frequency of annual low flow } \\
\hline \multicolumn{4}{c}{ Lowest average streamflow, in $\mathrm{ft}^{3} / \mathbf{s}$, for indicated period of } \\
consecutive days at an annual nonexceedance probability of 0.1
\end{tabular}


Illinois River Basin

\section{KANKAKEE RIVER AT SHELBY, IN}

Location. - Lat $41^{\circ} 10^{\prime} 58^{\prime \prime}$, long $87^{\circ} 20^{\prime} 25^{\prime \prime}$ referenced to North American Datum of 1927, in SW 1/4 NE 1/4 Sec.33, T.32 N., R.8 W., Newton County, IN, Hydrologic Unit 07120001, on right bank at upstream side of Highway 55 bridge, $1.0 \mathrm{mi}$ south of Shelby, $7.8 \mathrm{mi}$ upstream from Beaver Lake Ditch, and at mile 68.0.

Drainage area.- - 1,779 $\mathrm{mi}^{2}$, of which $201 \mathrm{mi}^{2}$ does not contribute directly to surface runoff.

Period of record.- October 1922 to current year.

Average discharge. $-1,720 \mathrm{ft}^{3} / \mathrm{s}$.

Minimum daily discharge. $-260 \mathrm{ft}^{3} / \mathrm{s}$.

Human health (harmonic mean) design flow. - 1,180 $\mathrm{ft}^{3} / \mathrm{s}$.

Remarks. - None.

\begin{tabular}{|c|c|c|c|}
\hline \multicolumn{4}{|c|}{ Magnitude and frequency of annual low flow } \\
\hline \multicolumn{4}{|c|}{$\begin{array}{l}\text { Lowest average streamflow, in } \mathrm{ft}^{3} / \mathrm{s} \text {, for indicated period of } \\
\text { consecutive days at an annual nonexceedance probability of } 0.1\end{array}$} \\
\hline 1 & \multicolumn{2}{|c|}{7} & 30 \\
\hline 393 & \multicolumn{2}{|c|}{416} & 468 \\
\hline \multicolumn{4}{|c|}{$\begin{array}{l}\text { Percentage of time streamflow was equaled or exceeded for } \\
\text { the period of record }\end{array}$} \\
\hline $\begin{array}{l}\text { Percentage } \\
\text { of time }\end{array}$ & $\begin{array}{l}\text { Daily mean } \\
\text { streamflow } \\
\left(\mathrm{ft}^{3} / \mathrm{s}\right)\end{array}$ & $\begin{array}{l}\text { Percentage } \\
\text { of time }\end{array}$ & $\begin{array}{c}\text { Daily mean } \\
\text { streamflow } \\
\left(\mathrm{ft}^{3} / \mathrm{s}\right)\end{array}$ \\
\hline 99 & 450 & 40 & 1670 \\
\hline 98 & 489 & 30 & 2030 \\
\hline 95 & 555 & 20 & 2580 \\
\hline 90 & 640 & 10 & 3420 \\
\hline 80 & 789 & 5 & 4020 \\
\hline 70 & 951 & 2 & 4670 \\
\hline 60 & 1150 & 1 & 5110 \\
\hline 50 & 1390 & & \\
\hline
\end{tabular}


Illinois River Basin

\section{SINGLETON DITCH AT SCHNEIDER, IN}

Location. - Lat $41^{\circ} 12^{\prime} 44^{\prime \prime}$, long $87^{\circ} 26^{\prime} 44^{\prime \prime}$ referenced to North American Datum of 1927, in SW 1/4 NW 1/4 sec.22, T.32 N., R.9 W., Lake County, IN, Hydrologic Unit 07120001, on left bank $15 \mathrm{ft}$ upstream from bridge on Ackerman Avenue, 0.5 mi upstream from Bruce Ditch, 1.5 mi downstream from Cedar Creek, 1.6 mi north of Schneider, and at mile 10.1 .

Drainage area. $-123 \mathrm{mi}^{2}$.

Period of record.- July 1948 to October 2001.

Average discharge. $-115 \mathrm{ft}^{3} / \mathrm{s}$.

Minimum daily discharge. $-3.6 \mathrm{ft}^{3} / \mathrm{s}$.

Human health (harmonic mean) design flow. $-41 \mathrm{ft}^{3} / \mathrm{s}$.

Remarks. - None.

\begin{tabular}{|c|c|c|c|}
\hline \multicolumn{4}{|c|}{ Magnitude and frequency of annual low flow } \\
\hline \multicolumn{4}{|c|}{$\begin{array}{l}\text { Lowest average streamflow, in } \mathrm{ft}^{3} / \mathrm{s} \text {, for indicated period of } \\
\text { consecutive days at an annual nonexceedance probability of } 0.1\end{array}$} \\
\hline 1 & \multicolumn{2}{|c|}{7} & 30 \\
\hline 7.4 & \multicolumn{2}{|c|}{7.9} & 9.4 \\
\hline \multicolumn{4}{|c|}{$\begin{array}{c}\text { Percentage of time streamflow was equaled or exceeded for } \\
\text { the period of record }\end{array}$} \\
\hline $\begin{array}{l}\text { Percentage } \\
\text { of time }\end{array}$ & $\begin{array}{l}\text { Daily mean } \\
\text { streamflow } \\
\left(\mathrm{ft}^{3} / \mathrm{s}\right)\end{array}$ & $\begin{array}{l}\text { Percentage } \\
\text { of time }\end{array}$ & $\begin{array}{c}\text { Daily mean } \\
\text { streamflow } \\
\left(\mathrm{ft}^{3} / \mathrm{s}\right)\end{array}$ \\
\hline 99 & 8.6 & 40 & 80 \\
\hline 98 & 10 & 30 & 107 \\
\hline 95 & 14 & 20 & 152 \\
\hline 90 & 19 & 10 & 254 \\
\hline 80 & 27 & 5 & 411 \\
\hline 70 & 36 & 2 & 696 \\
\hline 60 & 48 & 1 & 922 \\
\hline 50 & 62 & & \\
\hline
\end{tabular}


Illinois River Basin

\section{WEST CREEK NEAR SCHNEIDER, IN}

Location. - Lat $41^{\circ} 12^{\prime} 52^{\prime \prime}$, long $87^{\circ} 29^{\prime} 36^{\prime \prime}$ referenced to North American Datum of 1927, in NW 1/4 NE 1/4 Sec.19, T.32 N., R.9 W., Lake County, IN, Hydrologic Unit 07120001, on left bank at downstream side of county highway bridge, 1.2 mi upstream from Singleton Ditch, and $2.8 \mathrm{mi}$ northwest of Schneider.

Drainage area. $-54.7 \mathrm{mi}^{2}$.

Period of record.— July 1948 to December 1951, January 1954 to September 1972.

Average discharge. $-41.4 \mathrm{ft}^{3} / \mathrm{s}$.

Minimum daily discharge. $-2.6 \mathrm{ft}^{3} / \mathrm{s}$.

Human health (harmonic mean) design flow. $-14 \mathrm{ft}^{3} / \mathrm{s}$.

Remarks. - None.

\begin{tabular}{|c|c|c|c|}
\hline \multicolumn{4}{|c|}{ Magnitude and frequency of annual low flow } \\
\hline \multicolumn{4}{|c|}{$\begin{array}{l}\text { Lowest average streamflow, in } \mathrm{ft}^{3} / \mathrm{s} \text {, for indicated period of } \\
\text { consecutive days at an annual nonexceedance probability of } 0.1\end{array}$} \\
\hline 1 & \multicolumn{2}{|c|}{7} & 30 \\
\hline 3.8 & \multicolumn{2}{|c|}{4.6} & 5.6 \\
\hline \multicolumn{4}{|c|}{$\begin{array}{c}\text { Percentage of time streamflow was equaled or exceeded for } \\
\text { the period of record }\end{array}$} \\
\hline $\begin{array}{l}\text { Percentage } \\
\text { of time }\end{array}$ & $\begin{array}{l}\text { Daily mean } \\
\text { streamflow } \\
\left(\mathrm{ft}^{3} / \mathrm{s}\right)\end{array}$ & $\begin{array}{l}\text { Percentage } \\
\text { of time }\end{array}$ & $\begin{array}{l}\text { Daily mean } \\
\text { streamflow } \\
\left(\mathrm{ft}^{3} / \mathrm{s}\right)\end{array}$ \\
\hline 99 & 4.8 & 40 & 21 \\
\hline 98 & 5.2 & 30 & 29 \\
\hline 95 & 6.2 & 20 & 44 \\
\hline 90 & 7.3 & 10 & 82 \\
\hline 80 & 9.0 & 5 & 159 \\
\hline 70 & 11 & 2 & 306 \\
\hline 60 & 14 & 1 & 442 \\
\hline 50 & 17 & & \\
\hline
\end{tabular}


Illinois River Basin

\section{IROQUOIS RIVER AT ROSEBUD, IN}

Location. - Lat $41^{\circ} 02^{\prime} 00^{\prime \prime}$, long $87^{\circ} 10^{\prime} 49^{\prime \prime}$ referenced to North American Datum of 1927, in NW 1/4 SW 1/4 sec.24, T.30 N., R.7 W., Jasper County, IN, Hydrologic Unit 07120002, on right bank $100 \mathrm{ft}$ downstream from bridge on county road 700W, $0.5 \mathrm{mi}$ north of Rosebud, $0.5 \mathrm{mi}$ downstream from confluence of Swain and Dexter Ditches, 1.5 mi upstream from Davidson Ditch, 2 mi east of Parr, and at mile 93.5.

Drainage area. $-35.6 \mathrm{mi}^{2}$.

Period of record.- July 1948 to October 2003.

Average discharge. $-28.7 \mathrm{ft}^{3} / \mathrm{s}$.

Minimum daily discharge. $-0.50 \mathrm{ft}^{3} / \mathrm{s}$.

Human health (harmonic mean) design flow. - $10 \mathrm{ft}^{3} / \mathrm{s}$.

Remarks. - None.

\begin{tabular}{|c|c|c|c|}
\hline \multicolumn{4}{|c|}{ Magnitude and frequency of annual low flow } \\
\hline \multicolumn{4}{|c|}{$\begin{array}{l}\text { Lowest average streamflow, in } \mathrm{ft}^{3} / \mathrm{s} \text {, for indicated period of } \\
\text { consecutive days at an annual nonexceedance probability of } 0.1\end{array}$} \\
\hline 1 & \multicolumn{2}{|c|}{7} & 30 \\
\hline 2.2 & \multicolumn{2}{|c|}{2.4} & 3.0 \\
\hline \multicolumn{4}{|c|}{$\begin{array}{l}\text { Percentage of time streamflow was equaled or exceeded for } \\
\text { the period of record }\end{array}$} \\
\hline $\begin{array}{l}\text { Percentage } \\
\text { of time }\end{array}$ & $\begin{array}{l}\text { Daily mean } \\
\text { streamflow } \\
\left(\mathrm{ft}^{3} / \mathrm{s}\right)\end{array}$ & $\begin{array}{l}\text { Percentage } \\
\text { of time }\end{array}$ & $\begin{array}{l}\text { Daily mean } \\
\text { streamflow } \\
\left(\mathrm{ft}^{3} / \mathrm{s}\right)\end{array}$ \\
\hline 99 & 2.3 & 40 & 23 \\
\hline 98 & 2.8 & 30 & 31 \\
\hline 95 & 3.5 & 20 & 41 \\
\hline 90 & 4.4 & 10 & 64 \\
\hline 80 & 6.2 & 5 & 93 \\
\hline 70 & 8.8 & 2 & 148 \\
\hline 60 & 13 & 1 & 196 \\
\hline 50 & 18 & & \\
\hline
\end{tabular}


Illinois River Basin

\section{IROQUOIS RIVER NEAR NORTH MARION, IN}

Location. - Lat $41^{\circ} 58^{\prime} 12^{\prime \prime}$, long $87^{\circ} 06^{\prime} 50^{\prime \prime}$ referenced to North American Datum of 1927, in NE 1/4 NW 1/4 sec.16, T.29 N., R.6 W., Jasper County, IN, Hydrologic Unit 07120002, on downstream side of county highway bridge, 1.2 mi upstream from Ryan Ditch, 2 mi east of North Marion, 3.5 mi northeast of Rensselaer, and at mile 87.7.

Drainage area.- $144 \mathrm{mi}^{2}$.

Period of record.- December 1948 to October 1993.

Average discharge. $-139 \mathrm{ft}^{3} / \mathrm{s}$.

Minimum daily discharge. $-1.6 \mathrm{ft}^{3} / \mathrm{s}$.

Human health (harmonic mean) design flow. $-34 \mathrm{ft}^{3} / \mathrm{s}$.

Remarks. - Water from Oliver Ditch, an upstream tributary, can be diverted to Ryan Ditch and enter the Iroquois River below the station. Streamflow affected by irrigation.

\begin{tabular}{ccc}
\hline \multicolumn{3}{c}{ Magnitude and frequency of annual low flow } \\
\hline $\begin{array}{c}\text { Lowest average streamflow, in } \mathrm{ft}^{3} / \mathrm{s} \text {, for indicated period of } \\
\text { consecutive days at an annual nonexceedance probability of } \mathbf{0 . 1}\end{array}$ \\
\hline $\mathbf{1}$ & $\mathbf{7}$ & $\mathbf{3 0}$ \\
\hline 3.6 & 4.4 & 5.9 \\
\hline
\end{tabular}

Percentage of time streamflow was equaled or exceeded for the period of record

\begin{tabular}{cc|cc}
\hline $\begin{array}{c}\text { Percentage } \\
\text { of time }\end{array}$ & $\begin{array}{c}\text { Daily mean } \\
\text { streamflow } \\
\left(\mathrm{ft}^{3} / \mathbf{s}\right)\end{array}$ & $\begin{array}{c}\text { Percentage } \\
\text { of time }\end{array}$ & $\begin{array}{c}\text { Daily mean } \\
\text { streamflow } \\
\left(\mathrm{ft}^{3} / \mathbf{s}\right)\end{array}$ \\
\hline 99 & 4.9 & 40 & 104 \\
\hline 98 & 6.2 & 30 & 141 \\
\hline 95 & 9.5 & 20 & 205 \\
\hline 90 & 14 & 10 & 346 \\
\hline 80 & 23 & 5 & 510 \\
\hline 70 & 35 & 2 & 750 \\
\hline 60 & 54 & 1 & 941 \\
\hline 50 & 76 & & \\
\hline
\end{tabular}


Illinois River Basin

\section{IROQUOIS RIVER AT RENSSELAER, IN}

Location. - Lat $40^{\circ} 56^{\prime} 00^{\prime \prime}$, long $87^{\circ} 07^{\prime} 44^{\prime \prime}$ referenced to North American Datum of 1927, in NW 1/4 SE 1/4 sec.29, T.29 N., R.6 W., Jasper County, IN, Hydrologic Unit 07120002, on right bank $20 \mathrm{ft}$ downstream from bridge on State Highway 114, $0.8 \mathrm{mi}$ east of Rensselaer, $1.5 \mathrm{mi}$ downstream from Ryan Ditch, 5.5 mi upstream from Slough Creek, and at mile 84.9.

Drainage area. $-203 \mathrm{mi}^{2}$.

Period of record. - July 1948 to current year.

Average discharge. $-189 \mathrm{ft}^{3} / \mathrm{s}$.

Minimum daily discharge. $-2.2 \mathrm{ft}^{3} / \mathrm{s}$.

Human health (harmonic mean) design flow. $-45 \mathrm{ft}^{3} / \mathrm{s}$.

Remarks. - Stream flow is affected by irrigation.

\begin{tabular}{ccc}
\hline \multicolumn{3}{c}{ Magnitude and frequency of annual low flow } \\
\hline $\begin{array}{c}\text { Lowest average streamflow, in } \mathrm{ft}^{3} / \mathrm{s} \text {, for indicated period of } \\
\text { consecutive days at an annual nonexceedance probability of } 0.1\end{array}$ \\
\hline $\mathbf{1}$ & $\mathbf{3 0}$ \\
\hline 5.8 & 6.7 & 8.6 \\
\hline
\end{tabular}

Percentage of time streamflow was equaled or exceeded for the period of record

\begin{tabular}{cc|cc}
\hline $\begin{array}{c}\text { Percentage } \\
\text { of time }\end{array}$ & $\begin{array}{c}\text { Daily mean } \\
\text { streamflow } \\
\left(\mathrm{ft}^{3} / \mathbf{s}\right)\end{array}$ & $\begin{array}{c}\text { Percentage } \\
\text { of time }\end{array}$ & $\begin{array}{c}\text { Daily mean } \\
\text { streamflow } \\
\left(\mathrm{ft}^{3} / \mathbf{s}\right)\end{array}$ \\
\hline 99 & 6.7 & 40 & 133 \\
\hline 98 & 8.8 & 30 & 186 \\
\hline 95 & 13 & 20 & 275 \\
\hline 90 & 18 & 10 & 473 \\
\hline 80 & 29 & 5 & 721 \\
\hline 70 & 46 & 2 & 1110 \\
\hline 60 & 69 & 1 & 1370 \\
\hline 50 & 97 & & \\
\hline
\end{tabular}


Illinois River Basin

\section{BICE DITCH NEAR MARION, IN}

Location. - Lat $40^{\circ} 52^{\prime} 00^{\prime \prime}$, long $87^{\circ} 05^{\prime} 32^{\prime \prime}$ referenced to North American Datum of 1927, in NE 1/4 NW 1/4 sec.22, T.28 N., R.6 W., Jasper County, IN, Hydrologic Unit 07120002, on left bank at upstream side of bridge on State Highway 16, 2.3 mi upstream from mouth, 3 mi southeast of South Marion, and 5 mi southeast of Rensselaer.

Drainage area. $-21.8 \mathrm{mi}^{2}$.

Period of record.- December 1948 to September 1993.

Average discharge. $-18.2 \mathrm{ft}^{3} / \mathrm{s}$.

Minimum daily discharge. $-0.0 \mathrm{ft}^{3} / \mathrm{s}$.

Human health (harmonic mean) design flow. $-1.2 \mathrm{ft}^{3} / \mathrm{s}$.

Remarks. - Low flows in summer may be affected by irrigation.

\begin{tabular}{ccc}
\hline \multicolumn{3}{c}{ Magnitude and frequency of annual low flow } \\
\hline $\begin{array}{c}\text { Lowest average streamflow, in } \mathrm{ft}^{3} / \mathrm{s} \text {, for indicated period of } \\
\text { consecutive days at an annual nonexceedance probability of } 0.1\end{array}$ \\
\hline $\mathbf{1}$ & $\mathbf{3 0}$ \\
\hline 0.1 & 0.1 & 0.1 \\
\hline
\end{tabular}

Percentage of time streamflow was equaled or exceeded for the period of record

\begin{tabular}{cc|cc}
\hline $\begin{array}{c}\text { Percentage } \\
\text { of time }\end{array}$ & $\begin{array}{c}\text { Daily mean } \\
\text { streamflow } \\
\left(\mathrm{ft}^{3} / \mathbf{s}\right)\end{array}$ & $\begin{array}{c}\text { Percentage } \\
\text { of time }\end{array}$ & $\begin{array}{c}\text { Daily mean } \\
\text { streamflow } \\
\left(\mathrm{ft}^{3} / \mathbf{s}\right)\end{array}$ \\
\hline 99 & 0.1 & 40 & 8.2 \\
\hline 98 & 0.1 & 30 & 14 \\
\hline 95 & 0.2 & 20 & 22 \\
\hline 90 & 0.4 & 10 & 46 \\
\hline 80 & 1.0 & 5 & 78 \\
\hline 70 & 1.9 & 2 & 137 \\
\hline 60 & 3.2 & 1 & 205 \\
\hline 50 & 5.1 & & \\
\hline
\end{tabular}


Illinois River Basin

\section{SLOUGH CREEK NEAR COLLEGEVILLE, IN}

Location. - Lat $40^{\circ} 53^{\prime} 30^{\prime \prime}$, long $87^{\circ} 09^{\prime} 17^{\prime \prime}$ referenced to North American Datum of 1927, in SE 1/4 NE 1/4 Sec.12, T.28 N., R.7 W., Jasper County, IN, Hydrologic Unit 07120002, on right bank at downstream side of bridge on State Highway 53, 1.5 mi south of Collegeville, 2.2 mi downstream from Bice Ditch, 2.9 mi upstream from Carpenter Creek, and 3.2 mi upstream from mouth.

Drainage area. $-83.7 \mathrm{mi}^{2}$.

Period of record. - July 1948 to December 1951, October 1952 to September 1982.

Average discharge. $-70.1 \mathrm{ft}^{3} / \mathrm{s}$.

Minimum daily discharge. $-0.70 \mathrm{ft}^{3} / \mathrm{s}$.

Human health (harmonic mean) design flow. $-11 \mathrm{ft}^{3} / \mathrm{s}$.

Remarks. - None.

\begin{tabular}{ccc}
\hline \multicolumn{3}{c}{ Magnitude and frequency of annual low flow } \\
\hline $\begin{array}{c}\text { Lowest average streamflow, in } \mathrm{ft}^{3} / \mathrm{s} \text {, for indicated period of } \\
\text { consecutive days at an annual nonexceedance probability of } 0.1\end{array}$ \\
\hline $\mathbf{7}$ & $\mathbf{3 0}$ \\
\hline 1.3 & 1.4 & 1.8 \\
\hline
\end{tabular}

Percentage of time streamflow was equaled or exceeded for the period of record

\begin{tabular}{cc|cc}
\hline $\begin{array}{c}\text { Percentage } \\
\text { of time }\end{array}$ & $\begin{array}{c}\text { Daily mean } \\
\text { streamflow } \\
\left(\mathrm{ft}^{3} / \mathbf{s}\right)\end{array}$ & $\begin{array}{c}\text { Percentage } \\
\text { of time }\end{array}$ & $\begin{array}{c}\text { Daily mean } \\
\text { streamflow } \\
\left(\mathrm{ft}^{3} / \mathbf{s}\right)\end{array}$ \\
\hline 99 & 1.6 & 40 & 34 \\
\hline 98 & 2.0 & 30 & 52 \\
\hline 95 & 3.0 & 20 & 87 \\
\hline 90 & 4.4 & 10 & 176 \\
\hline 80 & 7.1 & 5 & 299 \\
\hline 70 & 11 & 2 & 518 \\
\hline 60 & 16 & 1 & 711 \\
\hline 50 & 23 & & \\
\hline
\end{tabular}


Illinois River Basin

\section{CARPENTER CREEK AT EGYPT, IN}

Location.- Lat $40^{\circ} 51^{\prime} 58^{\prime \prime}$, long $87^{\circ} 12^{\prime} 20^{\prime \prime}$ referenced to North American Datum of 1927, in SE 1/4 SW 1/4 Sec.15, T.28 N., R.7 W., Jasper County, IN, Hydrologic Unit 07120002, on left bank at downstream side of bridge on State Highway 16, 0.5 mi north of Egypt, 4 mi southwest of Collegeville, and at mile 4.0.

Drainage area. $-44.8 \mathrm{mi}^{2}$.

Period of record. - July 1948 to December 1951, October 1952 to September 1982.

Average discharge. $-38.3 \mathrm{ft}^{3} / \mathrm{s}$.

Minimum daily discharge. $-0.0 \mathrm{ft}^{3} / \mathrm{s}$.

Human health (harmonic mean) design flow. - $1.6 \mathrm{ft}^{3} / \mathrm{s}$.

Remarks. - None.

\begin{tabular}{ccc}
\hline \multicolumn{3}{c}{ Magnitude and frequency of annual low flow } \\
\hline $\begin{array}{c}\text { Lowest average streamflow, in } \mathrm{ft}^{3} / \mathrm{s} \text {, for indicated period of } \\
\text { consecutive days at an annual nonexceedance probability of } 0.1\end{array}$ \\
\hline $\mathbf{7}$ & $\mathbf{3 0}$ \\
\hline 0.0 & 0.0 & 0.0 \\
\hline
\end{tabular}

Percentage of time streamflow was equaled or exceeded for the period of record

\begin{tabular}{cc|cc}
\hline $\begin{array}{c}\text { Percentage } \\
\text { of time }\end{array}$ & $\begin{array}{c}\text { Daily mean } \\
\text { streamflow } \\
\left(\mathrm{ft}^{3} / \mathbf{s}\right)\end{array}$ & $\begin{array}{c}\text { Percentage } \\
\text { of time }\end{array}$ & $\begin{array}{c}\text { Daily mean } \\
\text { streamflow } \\
\left(\mathrm{ft}^{3} / \mathbf{s}\right)\end{array}$ \\
\hline 99 & 0.0 & 40 & 18 \\
\hline 98 & 0.1 & 30 & 28 \\
\hline 95 & 0.2 & 20 & 47 \\
\hline 90 & 0.5 & 10 & 97 \\
\hline 80 & 1.3 & 5 & 171 \\
\hline 70 & 3.0 & 2 & 313 \\
\hline 60 & 6.1 & 1 & 435 \\
\hline 50 & 11 & & \\
\hline
\end{tabular}


Illinois River Basin

\section{IROQUOIS RIVER NEAR FORESMAN, IN}

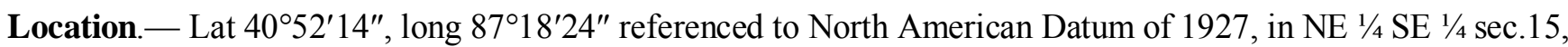
T.28 N., R.8 W., Newton County, IN, Hydrologic Unit 07120002, on right bank at downstream side of bridge on State Highway 55, 0.2 mi north of intersection of State Highways 16 and 55, 0.5 mi downstream from Mosquito Creek, 0.6 mi west of Foresman, 3 mi east of Brook, and at mile 72.7 .

Drainage area.- $449 \mathrm{mi}^{2}$.

Period of record. - July 1948 to current year.

Average discharge. $-420 \mathrm{ft}^{3} / \mathrm{s}$.

Minimum daily discharge. $-6.3 \mathrm{ft}^{3} / \mathrm{s}$.

Human health (harmonic mean) design flow. $-86 \mathrm{ft}^{3} / \mathrm{s}$.

Remarks. - None.

\begin{tabular}{ccc}
\hline \multicolumn{3}{c}{ Magnitude and frequency of annual low flow } \\
\hline $\begin{array}{c}\text { Lowest average streamflow, in } \mathrm{ft}^{3} / \mathrm{s} \text {, for indicated period of } \\
\text { consecutive days at an annual nonexceedance probability of } 0.1\end{array}$ \\
\hline $\mathbf{1}$ & $\mathbf{7}$ & $\mathbf{3 0}$ \\
\hline 11 & 12 & 15 \\
\hline
\end{tabular}

Percentage of time streamflow was equaled or exceeded for the period of record

\begin{tabular}{cc|cc}
\hline $\begin{array}{c}\text { Percentage } \\
\text { of time }\end{array}$ & $\begin{array}{c}\text { Daily mean } \\
\text { streamflow } \\
\left(\mathrm{ft}^{3} / \mathbf{s}\right)\end{array}$ & $\begin{array}{c}\text { Percentage } \\
\text { of time }\end{array}$ & $\begin{array}{c}\text { Daily mean } \\
\text { streamflow } \\
\left(\mathrm{ft}^{3} / \mathbf{s}\right)\end{array}$ \\
\hline 99 & 13 & 40 & 289 \\
\hline 98 & 16 & 30 & 416 \\
\hline 95 & 22 & 20 & 660 \\
\hline 90 & 33 & 10 & 1110 \\
\hline 80 & 56 & 5 & 1580 \\
\hline 70 & 90 & 2 & 2340 \\
\hline 60 & 144 & 1 & 2940 \\
\hline 50 & 205 & & \\
\hline
\end{tabular}


Illinois River Basin

\section{HART DITCH AT DYER, IN}

Location. - Lat $41^{\circ} 30^{\prime} 28^{\prime \prime}$, long 87³0'36" referenced to North American Datum of 1927, in NE 1/4 NE 1/4 Sec.12, T.35 N., R.10 W., Lake County, IN, Hydrologic Unit 07120003, on right bank, $50 \mathrm{ft}$ upstream from 213th Street in Dyer, $0.8 \mathrm{mi}$ upstream from Dyer Ditch, $0.8 \mathrm{mi}$ east of Illinois State line, $3.5 \mathrm{mi}$ east of intersection of U.S. Highway 30 and Interstate 394.

Drainage area. - $37.6 \mathrm{mi}^{2}$.

Period of record. - October 1989 to current year.

Average discharge. $-43.4 \mathrm{ft}^{3} / \mathrm{s}$.

Minimum daily discharge. $-0.61 \mathrm{ft}^{3} / \mathrm{s}$.

Human health (harmonic mean) design flow. $-9.7 \mathrm{ft}^{3} / \mathrm{s}$.

Remarks. - Low flow affected by sewage effluent.

\begin{tabular}{c}
\hline \multicolumn{4}{c}{ Magnitude and frequency of annual low flow } \\
\hline \multicolumn{4}{c}{ Lowest average streamflow, in $\mathrm{ft}^{3}$ /s, for indicated period of } \\
consecutive days at an annual nonexceedance probability of 0.1
\end{tabular}


Illinois River Basin

\section{HART DITCH AT MUNSTER, IN}

Location. - Lat $41^{\circ} 33^{\prime} 40^{\prime \prime}$, long $87^{\circ} 28^{\prime} 50^{\prime \prime}$ referenced to North American Datum of 1927, in SE 1/4 NW 1/4 sec.20, T.36 N., R.9 W., Lake County, IN, Hydrologic Unit 07120003, on left bank, 0.2 mi downstream from Ridge Road, $0.4 \mathrm{mi}$ upstream from mouth, and $0.9 \mathrm{mi}$ south of intersection of Interstate 80/90 and U.S. Highway 41.

Drainage area. $-70.7 \mathrm{mi}^{2}$.

Period of record. - September 1942 to October 2011.

Average discharge. $-75.8 \mathrm{ft}^{3} / \mathrm{s}$.

Minimum daily discharge. $-1.6 \mathrm{ft}^{3} / \mathrm{s}$.

Human health (harmonic mean) design flow. $-16 \mathrm{ft}^{3} / \mathrm{s}$.

Remarks. - Low flow affected by sewage effluent.

\begin{tabular}{|c|c|c|c|}
\hline \multicolumn{4}{|c|}{ Magnitude and frequency of annual low flow } \\
\hline \multicolumn{4}{|c|}{$\begin{array}{l}\text { Lowest average streamflow, in } \mathrm{ft}^{3} / \mathrm{s} \text {, for indicated period of } \\
\text { consecutive days at an annual nonexceedance probability of } 0.1\end{array}$} \\
\hline 1 & \multicolumn{2}{|c|}{7} & 30 \\
\hline 2.5 & \multicolumn{2}{|c|}{3.0} & 4.1 \\
\hline \multicolumn{4}{|c|}{$\begin{array}{l}\text { Percentage of time streamflow was equaled or exceeded for } \\
\text { the period of record }\end{array}$} \\
\hline $\begin{array}{l}\text { Percentage } \\
\text { of time }\end{array}$ & $\begin{array}{l}\text { Daily mean } \\
\text { streamflow } \\
\left(\mathrm{ft}^{3} / \mathrm{s}\right)\end{array}$ & $\begin{array}{l}\text { Percentage } \\
\text { of time }\end{array}$ & $\begin{array}{l}\text { Daily mean } \\
\text { streamflow } \\
\left(\mathrm{ft}^{3} / \mathrm{s}\right)\end{array}$ \\
\hline 99 & 3.5 & 40 & 38 \\
\hline 98 & 4.1 & 30 & 54 \\
\hline 95 & 5.1 & 20 & 82 \\
\hline 90 & 6.4 & 10 & 162 \\
\hline 80 & 10 & 5 & 308 \\
\hline 70 & 15 & 2 & 603 \\
\hline 60 & 20 & 1 & 869 \\
\hline 50 & 28 & & \\
\hline
\end{tabular}


Illinois River Basin

\section{LITTLE CALUMET RIVER AT MUNSTER, IN}

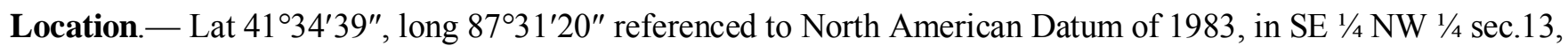
T.36 N., R.10 W., Lake County, IN, Hydrologic Unit 07120003, on Hohman Avenue bridge at north city limits of Munster, 0.4 mi upstream from Indiana-Illinois State line, and 4.6 mi upstream from Thorn Creek

Drainage area. - $90.0 \mathrm{mi}^{2}$.

Period of record.- June 1958 to current year.

Average discharge. $-69.4 \mathrm{ft}^{3} / \mathrm{s}$.

Minimum daily discharge. $-1.9 \mathrm{ft}^{3} / \mathrm{s}$.

Human health (harmonic mean) design flow. $-22 \mathrm{ft}^{3} / \mathrm{s}$.

Remarks. - Flow from eastern part of Little Calumet River Basin is diverted to Lake Michigan by Burns Ditch.

\begin{tabular}{|c|c|c|c|}
\hline \multicolumn{4}{|c|}{ Magnitude and frequency of annual low flow } \\
\hline \multicolumn{4}{|c|}{$\begin{array}{l}\text { Lowest average streamflow, in } \mathrm{ft}^{3} / \mathrm{s} \text {, for indicated period of } \\
\text { consecutive days at an annual nonexceedance probability of } 0.1\end{array}$} \\
\hline 1 & \multicolumn{2}{|c|}{7} & 30 \\
\hline 3.6 & \multicolumn{2}{|c|}{4.1} & 6.3 \\
\hline \multicolumn{4}{|c|}{$\begin{array}{l}\text { Percentage of time streamflow was equaled or exceeded for } \\
\text { the period of record }\end{array}$} \\
\hline $\begin{array}{l}\text { Percentage } \\
\text { of time }\end{array}$ & $\begin{array}{l}\text { Daily mean } \\
\text { streamflow } \\
\left(\mathrm{ft}^{3} / \mathrm{s}\right)\end{array}$ & $\begin{array}{l}\text { Percentage } \\
\text { of time }\end{array}$ & $\begin{array}{l}\text { Daily mean } \\
\text { streamflow } \\
\left(\mathrm{ft}^{3} / \mathrm{s}\right)\end{array}$ \\
\hline 99 & 4.7 & 40 & 44 \\
\hline 98 & 5.8 & 30 & 61 \\
\hline 95 & 7.4 & 20 & 95 \\
\hline 90 & 9.5 & 10 & 175 \\
\hline 80 & 14 & 5 & 275 \\
\hline 70 & 19 & 2 & 438 \\
\hline 60 & 25 & 1 & 556 \\
\hline 50 & 33 & & \\
\hline
\end{tabular}


Illinois River Basin

\section{GRAND CALUMET RIVER AT HOHMAN AVE AT HAMMOND, IN}

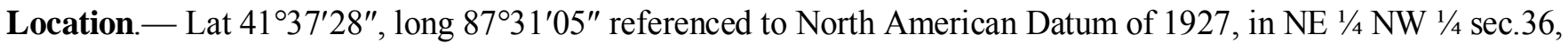
T.37 N., R.10 W., Lake County, IN, Hydrologic Unit 07120003, on left bank, 20 feet upstream of Hohman Avenue, 1,000 feet east of Indiana-Illinois State line, 0.57 mi downstream of U.S. Highway 41, and 0.7 mi north of St. Margaret's Hospital (Hohman Avenue).

Drainage area.- - Indeterminate.

Period of record. - October 1991 to current year.

Average discharge. $-22.4 \mathrm{ft}^{3} / \mathrm{s}$.

Minimum daily discharge. $-0.0 \mathrm{ft}^{3} / \mathrm{s}$.

Human health (harmonic mean) design flow. $-0.5 \mathrm{ft}^{3} / \mathrm{s}$.

Remarks.- None.

\begin{tabular}{|c|c|c|c|}
\hline \multicolumn{4}{|c|}{ Magnitude and frequency of annual low flow } \\
\hline \multicolumn{4}{|c|}{$\begin{array}{l}\text { Lowest average streamflow, in } \mathrm{ft}^{3} / \mathrm{s} \text {, for indicated period of } \\
\text { consecutive days at an annual nonexceedance probability of } 0.1\end{array}$} \\
\hline 1 & \multicolumn{2}{|c|}{7} & 30 \\
\hline 0.0 & \multicolumn{2}{|c|}{0.0} & 0.2 \\
\hline \multicolumn{4}{|c|}{$\begin{array}{l}\text { Percentage of time streamflow was equaled or exceeded for } \\
\text { the period of record }\end{array}$} \\
\hline $\begin{array}{l}\text { Percentage } \\
\text { of time }\end{array}$ & $\begin{array}{l}\text { Daily mean } \\
\text { streamflow } \\
\left(\mathrm{ft}^{3} / \mathrm{s}\right)\end{array}$ & $\begin{array}{l}\text { Percentage } \\
\text { of time }\end{array}$ & $\begin{array}{c}\text { Daily mean } \\
\text { streamflow } \\
\left(\mathrm{ft}^{3} / \mathrm{s}\right)\end{array}$ \\
\hline 99 & 0.0 & 40 & 21 \\
\hline 98 & 0.0 & 30 & 28 \\
\hline 95 & 0.2 & 20 & 42 \\
\hline 90 & 0.7 & 10 & 62 \\
\hline 80 & 2.4 & 5 & 81 \\
\hline 70 & 5.5 & 2 & 104 \\
\hline 60 & 8.9 & 1 & 117 \\
\hline 50 & 15 & & \\
\hline
\end{tabular}




\section{Table 2}

2. Low-flow characteristics for partial-record streamgaging stations in Indiana.

Tables 1 and 2 in Excel file format are available at http://dx.doi.org/10.3133/sir20145242. 
Table 2. Low-flow characteristics for partial-record streamgaging stations in Indiana.

[mi² , square miles; IN, Indiana; Lat, latitude; ', degrees; ', minutes; ", seconds; long, longitude; Sec, Section; T, Township; R, Range; N, North; E, East; S, South; W, West; - , no correlation]

\begin{tabular}{|c|c|c|c|c|c|c|c|}
\hline \multirow{2}{*}{ Station number } & \multirow{2}{*}{ Station name } & \multirow{2}{*}{$\begin{array}{l}\text { Drainage } \\
\text { area }\left(\mathrm{mi}^{2}\right)\end{array}$} & \multirow{2}{*}{ Location } & \multicolumn{4}{|c|}{ Flow characteristic, in cubic feet per second } \\
\hline & & & & 1010 & 7010 & 30010 & $\begin{array}{c}\text { Harmomic } \\
\text { mean }\end{array}$ \\
\hline \multirow[t]{6}{*}{03274800} & Martindale & 58.1 & Lat $39^{\circ} 49^{\prime} 50^{\prime \prime}$, long $85^{\circ} 08^{\prime} 32^{\prime \prime}$, & 1.5 & 1.7 & 2.2 & 14.7 \\
\hline & Creek near & & on a line between Secs 13 and 24, & & & & \\
\hline & Cambridge City, & & T.16N., R.12E., Wayne County, 1.25 & & & & \\
\hline & IN & & miles upstream from U.S. Highway & & & & \\
\hline & & & 40 , and 1.75 miles northeast of & & & & \\
\hline & & & Cambridge City & & & & \\
\hline \multirow[t]{5}{*}{03274900} & Greens Fork at & 66.7 & Lat $39^{\circ} 53^{\prime} 35^{\prime \prime}$, long $85^{\circ} 02^{\prime} 39^{\prime \prime}$, & 2 & 2.3 & 2.7 & 12.4 \\
\hline & Greens Fork, & & in NE1/4 SW1/4 Sec 26, T.17N., & & & & \\
\hline & IN & & R.13E., Wayne County, at State & & & & \\
\hline & & & Highway 38 bridge at west edge of & & & & \\
\hline & & & Greens Fork. & & & & \\
\hline \multirow[t]{5}{*}{03275200} & Salt Creek near & 115 & Lat $39^{\circ} 26^{\prime} 35^{\prime \prime}$, long $85^{\circ} 11^{\prime} 01^{\prime \prime}$, & 0.4 & 0.5 & 0.7 & 5.8 \\
\hline & Metamora, IN & & in SW1/4 Sec 34, T.12N., R.12E., & & & & \\
\hline & & & Franklin County, 0.3 mile south or & & & & \\
\hline & & & U.S. Highway 52, and 2.75 miles & & & & \\
\hline & & & west of Metamora. & & & & \\
\hline \multirow[t]{5}{*}{03275700} & Silver Creek & 9.67 & Lat $39^{\circ} 39^{\prime} 36^{\prime \prime}$, long $84^{\circ} 55^{\prime} 39^{\prime \prime}$, & 0 & 0 & 0 & - \\
\hline & near Liberty, & & on line between $\operatorname{Sec} 31, \mathrm{~T} .12 \mathrm{~N}$., & & & & \\
\hline & IN & & R.1W., and Sec 36, T.12N, R.2W., & & & & \\
\hline & & & Union County, at bridge on U.S. & & & & \\
\hline & & & Highway $27,1.5$ miles north of Liberty. & & & & \\
\hline
\end{tabular}




\section{Low-flow Characteristics for Selected Streams in Indiana}

Table 2. Low-flow characteristics for partial-record streamgaging stations in Indiana.-Continued

[mi², square miles; IN, Indiana; Lat, latitude; ' , degrees; ', minutes; ", seconds; long, longitude; Sec, Section; T, Township; R, Range; N, North; E, East; S, South; W, West; —, no correlation]

\begin{tabular}{|c|c|c|c|c|c|c|c|}
\hline \multirow{2}{*}{ Station number } & \multirow{2}{*}{ Station name } & \multirow{2}{*}{$\begin{array}{l}\text { Drainage } \\
\text { area }\left(\mathrm{mi}^{2}\right)\end{array}$} & \multirow{2}{*}{ Location } & \multicolumn{4}{|c|}{ Flow characteristic, in cubic feet per second } \\
\hline & & & & 1010 & 7010 & 30010 & $\begin{array}{c}\text { Harmomic } \\
\text { mean }\end{array}$ \\
\hline 03275850 & $\begin{array}{l}\text { Hanna Creek } \\
\text { near Roseburg, } \\
\text { IN }\end{array}$ & 22.3 & $\begin{array}{l}\text { Lat } 39^{\circ} 34^{\prime} 58^{\prime \prime} \text {, long } 84^{\circ} 56^{\prime} 48^{\prime \prime} \text {, } \\
\text { in SW1/4 SW1/4 Sec } 25, \mathrm{~T} .11 \mathrm{~N} . \text {, } \\
\text { R.2W., Union County, at bridge on } \\
\text { State Highway } 101,0.5 \text { mile south } \\
\text { of Roseburg. }\end{array}$ & 0.1 & 0.2 & 0.3 & 8.7 \\
\hline 03276630 & $\begin{array}{l}\text { Tanners Creek } \\
\text { near Guilford, } \\
\text { IN }\end{array}$ & 84.1 & $\begin{array}{l}\text { Lat } 39^{\circ} 09^{\prime} 15^{\prime \prime} \text {, long } 84^{\circ} 53^{\prime} 50^{\prime \prime} \text {, } \\
\text { E1/2 Sec } 29, \text { T.6N., R.1W., Dear- } \\
\text { born County, on Pribble Road low- } \\
\text { water bridge crossing } 2.3 \text { miles } \\
\text { southeast of Guilford. }\end{array}$ & 0 & 0 & 0 & 3.6 \\
\hline 03276750 & $\begin{array}{l}\text { Laughery Creek } \\
\text { near Ballstown, } \\
\text { IN }\end{array}$ & 39.8 & $\begin{array}{l}\text { Lat } 39^{\circ} 14^{\prime} 42^{\prime \prime} \text {, long } 85^{\circ} 14^{\prime} 52^{\prime \prime} \text {, } \\
\text { in SW1/4 NE1/4 Sec 12, T.9N., } \\
\text { R.11E., Ripley County, at bridge on } \\
\text { State Highway } 229,0.6 \text { mile south } \\
\text { of Ballstown. }\end{array}$ & 0 & 0 & 0 & 0.2 \\
\hline 03291800 & $\begin{array}{l}\text { Indian-Kentuck } \\
\text { Creek near } \\
\text { Manville, IN }\end{array}$ & 121 & $\begin{array}{l}\text { Lat } 38^{\circ} 47^{\prime} 10^{\prime \prime} \text {, long } 85^{\circ} 16^{\prime} 58^{\prime \prime} \text {, } \\
\text { in SE } 1 / 4 \text { Sec } 15, \text { T. } 4 \text { N., R.11E., Jef- } \\
\text { ferson County, southeast of Man- } \\
\text { ville, } 0.2 \text { mile below mouth of west } \\
\text { fork Indian-Kentuck Creek. }\end{array}$ & 0 & 0 & 0 & - \\
\hline
\end{tabular}


Table 2. Low-flow characteristics for partial-record streamgaging stations in Indiana._Continued

[mi² , square miles; IN, Indiana; Lat, latitude; ', degrees; ', minutes; ", seconds; long, longitude; Sec, Section; T, Township; R, Range; N, North; E, East; S, South; W, West; - , no correlation]

\begin{tabular}{|c|c|c|c|c|c|c|c|}
\hline \multirow{2}{*}{ Station number } & \multirow{2}{*}{ Station name } & \multirow{2}{*}{$\begin{array}{l}\text { Drainage } \\
\text { area }\left(\mathrm{mi}^{2}\right)\end{array}$} & \multirow{2}{*}{ Location } & \multicolumn{4}{|c|}{ Flow characteristic, in cubic feet per second } \\
\hline & & & & 1010 & 7010 & 30010 & $\begin{array}{c}\text { Harmomic } \\
\text { mean }\end{array}$ \\
\hline \multirow[t]{5}{*}{03292400} & Fourteenmile & 97 & Lat $38^{\circ} 27^{\prime} 58^{\prime \prime}$, long $85^{\circ} 37^{\prime} 04^{\prime \prime}$, & 0 & 0 & 0 & - \\
\hline & Creek near & & in SE1/4 SE1/4 of Lot 120, T.2S., & & & & \\
\hline & Charlestown, & & R.9E., Clark County, Clark military & & & & \\
\hline & $\mathrm{IN}$ & & grant at bridge on State Highway 62 & & & & \\
\hline & & & 3 miles northeast of Charlestown. & & & & \\
\hline \multirow[t]{5}{*}{03302600} & Little Indian & 32.5 & Lat $38^{\circ} 11^{\prime} 59^{\prime \prime}$, long $86^{\circ} 05^{\prime} 44^{\prime \prime}$, & 0.2 & 0.2 & 0.2 & 2.6 \\
\hline & Creek near & & NE1/4 Sec 5, T.4S., R.4E., Harrison & & & & \\
\hline & Corydon, IN & & County, at bridge on County High- & & & & \\
\hline & & & way, 0.2 mile south of State High- & & & & \\
\hline & & & way $62,1.75$ miles east of Corydon. & & & & \\
\hline \multirow[t]{6}{*}{03302700} & Middle Fork & 38.4 & Lat $38^{\circ} 32^{\prime} 36^{\prime \prime}$, long $86^{\circ} 05^{\prime} 37^{\prime \prime}$, & 0 & 0 & 0 & - \\
\hline & Blue River near & & in NE1/4 Sec 8, T.1N., R.4E., Wash- & & & & \\
\hline & Salem, IN & & ington County, at bridge on State & & & & \\
\hline & & & Highway $135,1.7$ miles upstream & & & & \\
\hline & & & from confluence with west fork Blue & & & & \\
\hline & & & River and 3.8 miles south of Salem. & & & & \\
\hline
\end{tabular}




\section{Low-flow Characteristics for Selected Streams in Indiana}

Table 2. Low-flow characteristics for partial-record streamgaging stations in Indiana.-Continued

[mi², square miles; IN, Indiana; Lat, latitude; ' , degrees; ', minutes; ", seconds; long, longitude; Sec, Section; T, Township; R, Range; N, North; E, East; S, South; W, West; —, no correlation]

\begin{tabular}{|c|c|c|c|c|c|c|c|}
\hline \multirow{2}{*}{ Station number } & \multirow{2}{*}{ Station name } & \multirow{2}{*}{$\begin{array}{l}\text { Drainage } \\
\text { area }\left(\mathrm{mi}^{2}\right)\end{array}$} & \multirow{2}{*}{ Location } & \multicolumn{4}{|c|}{ Flow characteristic, in cubic feet per second } \\
\hline & & & & 1010 & 7010 & 30010 & $\begin{array}{c}\text { Harmomic } \\
\text { mean }\end{array}$ \\
\hline \multirow[t]{8}{*}{03302730} & South Fork Blue & 64.3 & Lat $38^{\circ} 28^{\prime} 07^{\prime \prime}$, long $86^{\circ} 4^{\prime} 55^{\prime \prime}$, in & 0 & 0 & 0 & 2.8 \\
\hline & River near & & SW1/4 NE1/4 NW1/4 Sec 4, T.1S., & & & & \\
\hline & Palmyra, IN & & R.4E., Washington County at bridge & & & & \\
\hline & & & on Old Palmyra Road, 0.2 mile & & & & \\
\hline & & & north of State Highway 135, 4.7 & & & & \\
\hline & & & miles north of Junction of U.S. & & & & \\
\hline & & & Highway 150 and State Highway & & & & \\
\hline & & & 135 at Palmyra. & & & & \\
\hline \multirow[t]{7}{*}{03302850} & Whiskey Run at & 39.8 & Lat $38^{\circ} 21^{\prime} 08^{\prime \prime}$, long $86^{\circ} 17^{\prime} 01^{\prime \prime}$, & 0.1 & 0.1 & 0.1 & 2.1 \\
\hline & Milltown, IN & & in SE 1/4 SW1/4 Sec 10, T.2S. & & & & \\
\hline & & & R.2E., Crawford County, at bridge & & & & \\
\hline & & & on County Road, 0.25 mile south of & & & & \\
\hline & & & State Highway 64 and 0.8 mile north & & & & \\
\hline & & & of intersection of Main and Station & & & & \\
\hline & & & in Milltown. & & & & \\
\hline \multirow[t]{4}{*}{03302900} & Spring Creek & 36.6 & Lat $38^{\circ} 14^{\prime} 20^{\prime \prime}$, long $86^{\circ} 13^{\prime} 45^{\prime \prime}$, & 5.4 & 6 & 7.5 & 32.4 \\
\hline & near White & & SE1/4 Sec 19, T.35S., R.3E., Harri- & & & & \\
\hline & Cloud, IN & & son County, at county bridge 0.8 & & & & \\
\hline & & & mile north of White Cloud. & & & & \\
\hline
\end{tabular}


Table 2. Low-flow characteristics for partial-record streamgaging stations in Indiana._Continued

[mi² , square miles; IN, Indiana; Lat, latitude; ', degrees; ', minutes; ", seconds; long, longitude; Sec, Section; T, Township; R, Range; N, North; E, East; S, South; W, West; - , no correlation]

\begin{tabular}{|c|c|c|c|c|c|c|c|}
\hline \multirow{2}{*}{ Station number } & \multirow{2}{*}{ Station name } & \multirow{2}{*}{$\begin{array}{l}\text { Drainage } \\
\text { area }\left(\mathrm{mi}^{2}\right)\end{array}$} & \multirow{2}{*}{ Location } & \multicolumn{4}{|c|}{ Flow characteristic, in cubic feet per second } \\
\hline & & & & 1010 & 7010 & 30010 & $\begin{array}{c}\text { Harmomic } \\
\text { mean }\end{array}$ \\
\hline \multirow[t]{5}{*}{03303050} & Bird Hollow & 9.31 & Lat $38^{\circ} 21^{\prime} 02^{\prime \prime}$, long $86^{\circ} 28^{\prime} 01^{\prime \prime}$, & 0 & 0 & 0 & 0.4 \\
\hline & Creek near & & in SE1/4 NW1/4 NW1/4 Sec 13, & & & & \\
\hline & English, IN & & T.2S., R.1W., Crawford County, at & & & & \\
\hline & & & bridge on State Highway 37, 0.7 & & & & \\
\hline & & & mile north of Highway 64. & & & & \\
\hline \multirow[t]{5}{*}{03303160} & Little Blue & 54.4 & Lat $38^{\circ} 16^{\prime} 53^{\prime \prime}$, long $86^{\circ} 28^{\prime} 03^{\prime \prime}$, & 0.1 & 0.1 & 0.2 & 3.8 \\
\hline & River near & & in SW1/4 SE1/4 Sec 1, T.3S., R.1W., & & & & \\
\hline & Grantsburg, IN & & Crawford County, at bridge on State & & & & \\
\hline & & & Highway $37,0.5$ mile south of & & & & \\
\hline & & & Grantsburg. & & & & \\
\hline \multirow[t]{6}{*}{03303190} & Little Blue & 128 & Lat $38^{\circ} 10^{\prime} 01^{\prime \prime}$, long $86^{\circ} 24^{\prime} 57^{\prime \prime}$, & 0.1 & 0.1 & 0.1 & 6.8 \\
\hline & River near & & SW1/4 NE1/4 SE1/4 Sec 16, T.4S., & & & & \\
\hline & Beechwood, & & R.1E., Crawford County, 0.5 mile & & & & \\
\hline & $\mathrm{IN}$ & & above confluence with Turkey Fork, & & & & \\
\hline & & & at county, road 3.0 miles south of & & & & \\
\hline & & & Beechwood. & & & & \\
\hline \multirow[t]{5}{*}{003303240} & Deer Creek near & 8.70 & Lat $37^{\circ} 58^{\prime} 16^{\prime \prime}$, long $86^{\circ} 38^{\prime} 40^{\prime \prime}$, & 0 & 0 & 0 & 0.1 \\
\hline & Cannelton, IN & & in SW1/4 SE1/4 SE1/4 Sec 20 T.6S., & & & & \\
\hline & & & R.2W., Perry County, at county road & & & & \\
\hline & & & bridge, 6.8 miles northeast of Can- & & & & \\
\hline & & & nelton. & & & & \\
\hline
\end{tabular}


Table 2. Low-flow characteristics for partial-record streamgaging stations in Indiana.-Continued

[mi², square miles; IN, Indiana; Lat, latitude; ' , degrees; ', minutes; ", seconds; long, longitude; Sec, Section; T, Township; R, Range; N, North; E, East; S, South; W, West; —, no correlation]

\begin{tabular}{|c|c|c|c|c|c|c|c|}
\hline \multirow{2}{*}{ Station number } & \multirow{2}{*}{ Station name } & \multirow{2}{*}{$\begin{array}{l}\text { Drainage } \\
\text { area }\left(\mathrm{mi}^{2}\right)\end{array}$} & \multirow{2}{*}{ Location } & \multicolumn{4}{|c|}{ Flow characteristic, in cubic feet per second } \\
\hline & & & & 1010 & 7010 & 30010 & $\begin{array}{c}\text { Harmomic } \\
\text { mean }\end{array}$ \\
\hline \multirow[t]{5}{*}{03303950} & Otter Creek near & 30.1 & Lat $38^{\circ} 02^{\prime} 24^{\prime \prime}$, long $87^{\circ} 12^{\prime} 22^{\prime \prime}$, & 0.6 & 0.6 & 0.8 & 2.3 \\
\hline & De Gonia & & in SE1/4 SE1/4 NE1/4 Sec 32 T.5S., & & & & \\
\hline & Springs, IN & & R.7W., Warrick County, at bridge on & & & & \\
\hline & & & State Highway $62,1.5$ miles south- & & & & \\
\hline & & & west of De Gonia Springs. & & & & \\
\hline \multirow[t]{6}{*}{03304000} & Little Pigeon & 150 & Lat $38^{\circ} 02^{\prime} 43^{\prime \prime}$, long $87^{\circ} 07^{\prime} 08^{\prime \prime}$, & 0 & 0 & 0 & - \\
\hline & Creek near & & in NW1/4 Sec 31, T.5S., R.6W., & & & & \\
\hline & Tennyson, IN & & Spencer County, at bridge operated & & & & \\
\hline & & & as continuous-record streamgage, on & & & & \\
\hline & & & State Highway 161 or East 650 & & & & \\
\hline & & & Road, 2.3 miles south of Tennyson. & & & & \\
\hline \multirow[t]{6}{*}{03322050} & Pigeon Creek & 190 & Lat $38^{\circ} 11^{\prime} 38^{\prime \prime}$, long $87^{\circ} 25^{\prime} 28^{\prime \prime}$, & 0 & 0 & 0 & - \\
\hline & near Buckskin, & & on line between Secs 4 and 9, T.4S. & & & & \\
\hline & $\mathrm{IN}$ & & R.9W., Warrick County, at bridge on & & & & \\
\hline & & & State Highway $68,2.3$ miles south & & & & \\
\hline & & & of Buckskin and 6.4 miles west of & & & & \\
\hline & & & Lynnville. & & & & \\
\hline \multirow[t]{4}{*}{03322800} & Bear Creek near & 14.5 & Lat $40^{\circ} 30^{\prime} 53^{\prime \prime}$, long $84^{\circ} 58^{\prime} 23^{\prime \prime}$, & 0 & 0 & 0 & - \\
\hline & Bryant, IN & & SE 1/4 Secs 19, T.24N., R.14E., Jay & & & & \\
\hline & & & County, at bridge on U.S. Highway & & & & \\
\hline & & & $27,1.2$ miles south of Bryant. & & & & \\
\hline
\end{tabular}


Table 2. Low-flow characteristics for partial-record streamgaging stations in Indiana._Continued

[mi² , square miles; IN, Indiana; Lat, latitude; ', degrees; ', minutes; ", seconds; long, longitude; Sec, Section; T, Township; R, Range; N, North; E, East; S, South; W, West; - , no correlation]

\begin{tabular}{|c|c|c|c|c|c|c|c|}
\hline \multirow{2}{*}{ Station number } & \multirow{2}{*}{ Station name } & \multirow{2}{*}{$\begin{array}{l}\text { Drainage } \\
\text { area }\left(\mathrm{mi}^{2}\right)\end{array}$} & \multirow{2}{*}{ Location } & \multicolumn{4}{|c|}{ Flow characteristic, in cubic feet per second } \\
\hline & & & & 1010 & 7010 & 30010 & $\begin{array}{c}\text { Harmomic } \\
\text { mean }\end{array}$ \\
\hline \multirow[t]{5}{*}{03322860} & Loblolly Creek & 67.2 & Lat $40^{\circ} 34^{\prime} 59^{\prime \prime}$, long $84^{\circ} 57^{\prime} 38^{\prime \prime}$, & 0.3 & 0.4 & 0.5 & 3.2 \\
\hline & at Geneva, IN & & NW1/4 NE1/4 Sec 32, T.25N., & & & & \\
\hline & & & R.14E., Adams County, 200 feet & & & & \\
\hline & & & upstream from bridge on U.S. High- & & & & \\
\hline & & & way 27 , at south edge of Geneva. & & & & \\
\hline \multirow[t]{5}{*}{03322880} & Limberlost & 41.7 & Lat $40^{\circ} 34^{\prime} 59^{\prime \prime}$, long $84^{\circ} 57^{\prime} 38^{\prime \prime}$, & 0.1 & 0.2 & 0.2 & 1.6 \\
\hline & Creek at & & NW1/4 Sec 32, T.25N., R.14E. & & & & \\
\hline & Geneva, IN & & Adams County, 200 feet upstream & & & & \\
\hline & & & from bridge on U.S. Highway 27, at & & & & \\
\hline & & & south edge of Geneva. & & & & \\
\hline \multirow[t]{5}{*}{03322980} & Sixmile Creek & 30.0 & Lat $40^{\circ} 42^{\prime} 24^{\prime \prime}$, long $85^{\circ} 05^{\prime} 16^{\prime \prime}$, & 0.1 & 0.2 & 0.2 & 2.4 \\
\hline & near Bluffton, & & in NE1/4 NE1/4 SW1/4 Sec 14, & & & & \\
\hline & IN & & T.26N., R.12E., Wells County, on & & & & \\
\hline & & & County Road 250 south, about 3 & & & & \\
\hline & & & miles southeast of Bluffton. & & & & \\
\hline \multirow[t]{7}{*}{03323700} & Aboite Creek & 52.7 & Lat $40^{\circ} 59^{\prime} 23^{\prime \prime}$, long $85^{\circ} 21^{\prime} 00^{\prime \prime}$, & 0.6 & 0.8 & 1.1 & 6 \\
\hline & near Aboite, & & on line between Secs 1 and 12, & & & & \\
\hline & IN & & T.29N., R.10E., Huntington County, & & & & \\
\hline & & & on County Road 1100 north, & & & & \\
\hline & & & 0.5 mile east of State Highway 37 , & & & & \\
\hline & & & 800 feet above mouth, 2.25 miles & & & & \\
\hline & & & northeast of Roanoke. & & & & \\
\hline
\end{tabular}


Table 2. Low-flow characteristics for partial-record streamgaging stations in Indiana.-Continued

[mi², square miles; IN, Indiana; Lat, latitude; ' , degrees; ', minutes; ", seconds; long, longitude; Sec, Section; T, Township; R, Range; N, North; E, East; S, South; W, West; —, no correlation]

\begin{tabular}{|c|c|c|c|c|c|c|c|}
\hline \multirow{2}{*}{ Station number } & \multirow{2}{*}{ Station name } & \multirow{2}{*}{$\begin{array}{l}\text { Drainage } \\
\text { area }\left(\mathrm{mi}^{2}\right)\end{array}$} & \multirow{2}{*}{ Location } & \multicolumn{4}{|c|}{ Flow characteristic, in cubic feet per second } \\
\hline & & & & 1010 & 7010 & 30010 & $\begin{array}{c}\text { Harmomic } \\
\text { mean }\end{array}$ \\
\hline \multirow[t]{4}{*}{03323800} & Eightmile Creek & 45.8 & Lat $40^{\circ} 54^{\prime} 40^{\prime \prime}$, long $85^{\circ} 17^{\prime} 30^{\prime \prime}$, & 0.2 & 0.2 & 0.3 & 2 \\
\hline & at Zanesville, & & in Sec 4, T.28N., R.11E., Wells & & & & \\
\hline & $\mathrm{IN}$ & & County, at bridge on State Highway & & & & \\
\hline & & & 3, 0.7 mile southwest of Zanesville. & & & & \\
\hline \multirow[t]{5}{*}{03324050} & Clear Creek & 49.0 & Lat $40^{\circ} 54^{\prime} 57^{\prime \prime}$, long $85^{\circ} 32^{\prime} 31^{\prime \prime}$, & 0 & 0 & 0.1 & 2.8 \\
\hline & near & & in NE1/4 NW1/4 NW1/4 Sec 5, & & & & \\
\hline & Huntington, IN & & T.28N., R.9E., Huntington County, & & & & \\
\hline & & & at bridge on State Highway $16,0.75$ & & & & \\
\hline & & & mile west of State Highway 5. & & & & \\
\hline \multirow[t]{5}{*}{03324250} & Salamonie River & 253 & Lat $40^{\circ} 33^{\prime} 33^{\prime \prime}$, long $85^{\circ} 16^{\prime} 43^{\prime \prime}$, & 2.7 & 3.2 & 4.3 & 24.7 \\
\hline & at Montpelier, & & NE 1/4 NE1/4 SE 1/4 Sec 4, T.24N., & & & & \\
\hline & IN & & R.11E., Blackford County, at bridge & & & & \\
\hline & & & on State Highway 303, on north & & & & \\
\hline & & & edge of Montpelier. & & & & \\
\hline \multirow[t]{5}{*}{03324280} & Black Creek & 47.0 & Lat $40^{\circ} 38^{\prime} 55^{\prime \prime}$, long $85^{\circ} 26^{\prime} 02^{\prime \prime}$, & 0 & 0 & 0 & - \\
\hline & near Warren, & & in NW1/4 SE1/4 NE1/4 Sec 6, & & & & \\
\hline & IN & & T.25N., R.10E., Wells County, at & & & & \\
\hline & & & bridge on County Road 1120 West & & & & \\
\hline & & & about 2 miles south of Warren. & & & & \\
\hline
\end{tabular}


Table 2. Low-flow characteristics for partial-record streamgaging stations in Indiana._Continued

[mi², square miles; IN, Indiana; Lat, latitude; ', degrees; ', minutes; ", seconds; long, longitude; Sec, Section; T, Township; R, Range; N, North; E, East; S, South; W, West; --, no correlation]

\begin{tabular}{|c|c|c|c|c|c|c|c|}
\hline \multirow{2}{*}{ Station number } & \multirow{2}{*}{ Station name } & \multirow{2}{*}{$\begin{array}{l}\text { Drainage } \\
\text { area }\left(\mathrm{mi}^{2}\right)\end{array}$} & \multirow{2}{*}{ Location } & \multicolumn{4}{|c|}{ Flow characteristic, in cubic feet per second } \\
\hline & & & & 1010 & 7010 & 30010 & $\begin{array}{c}\text { Harmomic } \\
\text { mean }\end{array}$ \\
\hline 03325700 & $\begin{array}{l}\text { Halfway Creek } \\
\text { near Albany, } \\
\text { IN }\end{array}$ & 19.1 & $\begin{array}{l}\text { Lat } 40^{\circ} 19^{\prime} 37^{\prime \prime} \text {, long } 85^{\circ} 12^{\prime} 56^{\prime \prime} \text {, } \\
\text { in NW1/4 NW1/4 SE1/4 Sec } 29 \text {, } \\
\text { T.22N., R.12E., Delaware County, at } \\
\text { bridge on County Road } 15 \text { East, } 1.0 \\
\text { mile north of State Highway } 67,1.5 \\
\text { miles northeast of Albany. }\end{array}$ & 0 & 0 & 0 & - \\
\hline 03326375 & $\begin{array}{l}\text { Deer Creek at } \\
\text { Marion, IN }\end{array}$ & 45.2 & $\begin{array}{l}\text { Lat } 40^{\circ} 30^{\prime} 30^{\prime \prime} \text {, long } 85^{\circ} 38^{\prime} 15^{\prime \prime} \text {, } \\
\text { in the NE1/4 Sec } 29, \text { T.24N., R.8E., } \\
\text { Grant County, on Lincoln } \\
\text { Boulevard, at the south edge of } \\
\text { Marion. }\end{array}$ & 0 & 0.2 & 0.3 & 2.6 \\
\hline 03326480 & $\begin{array}{l}\text { Lugar Creek at } \\
\text { Marion, IN }\end{array}$ & 30.0 & $\begin{array}{l}\text { Lat } 40^{\circ} 32^{\prime} 20^{\prime \prime} \text {, long } 85^{\circ} 37^{\prime} 40^{\prime \prime} \text {, } \\
\text { in SW1/4 SE1/4 SW1/4 Sec 9, } \\
\text { T.24N., R.8E., Grant County, at } \\
\text { bridge on Stone Road, } 2.0 \text { miles east } \\
\text { of Marion. }\end{array}$ & 0.2 & 0.5 & 0.7 & 2.7 \\
\hline 033275554 & $\begin{array}{l}\text { Johnson Drain } \\
\text { near } \\
\text { Churubusco, } \\
\text { IN }\end{array}$ & 8.67 & $\begin{array}{l}\text { Lat } 41^{\circ} 11^{\prime} 13^{\prime \prime} \text {, long } 85^{\circ} 17^{\prime} 32^{\prime \prime} \text {, } \\
\text { SE1/4 NE1/4 Sec } 31, \text { T.32N., } \\
\text { R.11E., Allen County, at bridge on } \\
\text { State Road } 33,3.3 \text { miles southeast } \\
\text { of Churubusco. }\end{array}$ & 0.2 & 0.3 & 0.4 & - \\
\hline
\end{tabular}


300 Low-flow Characteristics for Selected Streams in Indiana

Table 2. Low-flow characteristics for partial-record streamgaging stations in Indiana.—Continued

[mi², square miles; IN, Indiana; Lat, latitude; ' , degrees; ', minutes; ", seconds; long, longitude; Sec, Section; T, Township; R, Range; N, North; E, East; S, South; W, West; —, no correlation]

\begin{tabular}{|c|c|c|c|c|c|c|c|}
\hline \multirow{2}{*}{ Station number } & \multirow{2}{*}{ Station name } & \multirow{2}{*}{$\begin{array}{l}\text { Drainage } \\
\text { area }\left(\mathrm{mi}^{2}\right)\end{array}$} & \multirow{2}{*}{ Location } & \multicolumn{4}{|c|}{ Flow characteristic, in cubic feet per second } \\
\hline & & & & 1010 & 7010 & 30010 & $\begin{array}{c}\text { Harmomic } \\
\text { mean }\end{array}$ \\
\hline \multirow[t]{5}{*}{03327590} & Eel River near & 77.4 & Lat $41^{\circ} 08^{\prime} 33^{\prime \prime}$, long $85^{\circ} 27^{\prime} 25^{\prime \prime}$, & 3.7 & 5 & 6.5 & 32 \\
\hline & Columbia City, & & NW1/4 SE1/4 NE1/4 Sec 13, & & & & \\
\hline & $\mathrm{IN}$ & & T.13N., R.9E., Whitley County, at & & & & \\
\hline & & & bridge on Old U.S. Highway $30,1.5$ & & & & \\
\hline & & & miles east of junction 9 and 30. & & & & \\
\hline \multirow[t]{6}{*}{03327620} & Blue River near & 35.8 & Lat $41^{\circ} 13^{\prime} 49^{\prime \prime}$, long $85^{\circ} 23^{\prime} 27^{\prime \prime}$, & 1.3 & 1.4 & 1.6 & 6.2 \\
\hline & Churubusco, & & in E1/2 Sec 17, T.32N., R.10E., & & & & \\
\hline & $\mathrm{IN}$ & & Whitley County, at bridge on Ander- & & & & \\
\hline & & & son Road, 4 miles west of Churu- & & & & \\
\hline & & & busco and 0.75 mile east of County & & & & \\
\hline & & & Road 450 East. & & & & \\
\hline \multirow[t]{6}{*}{03327770} & Blue River near & 61.0 & Lat $41^{\circ} 10^{\prime} 52^{\prime \prime}$, long $85^{\circ} 27^{\prime} 24^{\prime \prime}$, & 3.3 & 3.6 & 4 & 12.1 \\
\hline & Columbia City, & & in SW1/4 Sec 35, T.32N., R.9E., & & & & \\
\hline & $\mathrm{IN}$ & & Whitley County, 2.6 miles east of & & & & \\
\hline & & & State Highway 9, 2.2 miles northeast & & & & \\
\hline & & & of Columbia City, 2.5 miles down- & & & & \\
\hline & & & stream from Thorn Creek. & & & & \\
\hline
\end{tabular}


Table 2. Low-flow characteristics for partial-record streamgaging stations in Indiana._Continued

[mi² , square miles; IN, Indiana; Lat, latitude; ', degrees; ', minutes; ", seconds; long, longitude; Sec, Section; T, Township; R, Range; N, North; E, East; S, South; W, West; - , no correlation]

\begin{tabular}{|c|c|c|c|c|c|c|c|}
\hline \multirow{2}{*}{ Station number } & \multirow{2}{*}{ Station name } & \multirow{2}{*}{$\begin{array}{l}\text { Drainage } \\
\text { area }\left(\mathbf{m i}^{2}\right)\end{array}$} & \multirow{2}{*}{ Location } & \multicolumn{4}{|c|}{ Flow characteristic, in cubic feet per second } \\
\hline & & & & 1010 & 7010 & 30010 & $\begin{array}{c}\text { Harmomic } \\
\text { mean }\end{array}$ \\
\hline 03327853 & $\begin{array}{l}\text { Spring Creek } \\
\text { near Columbia } \\
\text { City, IN }\end{array}$ & 8.12 & $\begin{array}{l}\text { Lat } 41^{\circ} 09^{\prime} 55^{\prime \prime} \text {, long } 85^{\circ} 34^{\prime} 05^{\prime \prime} \text {, } \\
\text { in SE1/4 SE1/4 Sec. } 1, \mathrm{~T} .31 \mathrm{~N} . \text {, } \\
\text { R.8E., Whitley County, at bridge on } \\
\text { County Road } 400 \text { West, } 1 \text { mile south } \\
\text { of Highway } 30 \text { and } 4.1 \text { miles west } \\
\text { of intersection of U.S. Highways } 30 \\
\text { and } 109 \text { in Columbia City. }\end{array}$ & 0 & 0 & 0 & 0.7 \\
\hline 03327875 & $\begin{array}{l}\text { Clear Creek } \\
\text { above Spring } \\
\text { Creek at South } \\
\text { Whitley, IN }\end{array}$ & 9.58 & $\begin{array}{l}\text { Lat } 41^{\circ} 05^{\prime} 39^{\prime \prime} \text {, long } 85^{\circ} 37^{\prime} 20^{\prime \prime} \text {, } \\
\text { in SE1/4 NW1/4 SW1/4 Sec } 34 \text {, } \\
\text { T.31N., R.8E., in Whitley County, } \\
\text { upstream of bridge on State Road } \\
\text { 205, approximately } 0.5 \text { mile north of } \\
\text { South Whitley. }\end{array}$ & 0 & 0 & 0 & 0.5 \\
\hline 03327876 & $\begin{array}{l}\text { Spring Creek } \\
\text { below Clear } \\
\text { Creek at South } \\
\text { Whitley, IN }\end{array}$ & 44.0 & $\begin{array}{l}\text { Lat } 41^{\circ} 05^{\prime} 39^{\prime \prime} \text {, long } 85^{\circ} 37^{\prime} 20^{\prime \prime} \text {, } \\
\text { in SW1/4 of Sec } 34, \text { T.31N., R.8E., } \\
\text { in Whitley County, on State High- } \\
\text { way } 205 \text { at the northeast edge of } \\
\text { South Whitley. }\end{array}$ & 2.4 & 2.8 & 3.1 & 7.3 \\
\hline
\end{tabular}


302 Low-flow Characteristics for Selected Streams in Indiana

Table 2. Low-flow characteristics for partial-record streamgaging stations in Indiana.—Continued

[mi², square miles; IN, Indiana; Lat, latitude; ' , degrees; ', minutes; ", seconds; long, longitude; Sec, Section; T, Township; R, Range; N, North; E, East; S, South; W, West; —, no correlation]

\begin{tabular}{|c|c|c|c|c|c|c|c|}
\hline \multirow{2}{*}{ Station number } & \multirow{2}{*}{ Station name } & \multirow{2}{*}{$\begin{array}{l}\text { Drainage } \\
\text { area }\left(\mathrm{mi}^{2}\right)\end{array}$} & \multirow{2}{*}{ Location } & \multicolumn{4}{|c|}{ Flow characteristic, in cubic feet per second } \\
\hline & & & & 1010 & 7010 & 30010 & $\begin{array}{c}\text { Harmomic } \\
\text { mean }\end{array}$ \\
\hline 03327900 & $\begin{array}{l}\text { Sugar Creek at } \\
\text { South Whitley, } \\
\text { IN }\end{array}$ & 30.7 & $\begin{array}{l}\text { Lat } 41^{\circ} 04^{\prime} 31^{\prime \prime} \text {, long } 85^{\circ} 36^{\prime} 54^{\prime \prime} \text {, } \\
\text { on line between Secs } 3 \text { and } 10 \\
\text { T.30N., R.8E., Whitley County, at } \\
\text { bridge on Highway } 14,0.3 \text { mile east } \\
\text { of intersection of State Highways } 5 \\
\text { and } 105 \text { on the southeast edge of } \\
\text { South Whitley. }\end{array}$ & 0.4 & 0.6 & 0.7 & 4 \\
\hline 03328420 & $\begin{array}{l}\text { Paw Paw Creek } \\
\text { near Roann, IN }\end{array}$ & 31.2 & $\begin{array}{l}\text { Lat } 40^{\circ} 53^{\prime} 40^{\prime \prime} \text {, long } 85^{\circ} 53^{\prime} 19^{\prime \prime} \text {, } \\
\text { in SW1/4 NW1/4 NW1/4 Sec } 8 \text {, } \\
\text { T. } 28 \mathrm{~N} ., \text { R.6E., Wabash County, at } \\
\text { bridge on State Highway } 15 \text {, } \\
3.5 \text { miles northwest of intersection } \\
\text { of State Highways } 115 \text { and } 15 \text { near } \\
\text { Roann. }\end{array}$ & 0.2 & 0.2 & 0.3 & 2.8 \\
\hline 03328470 & $\begin{array}{l}\text { Twelve Mile } \\
\text { Creek near } \\
\text { Hoover, IN }\end{array}$ & 53.0 & $\begin{array}{l}\text { Lat } 40^{\circ} 48^{\prime} 31^{\prime \prime} \text {, long } 86^{\circ} 13^{\prime} 22^{\prime \prime} \text {, } \\
\text { in NE1/4 SE1/4, T.27., R.3E., Cass } \\
\text { County, at bridge on County Road } \\
300 \text { North, } 1.2 \text { miles west of } \\
\text { Hoover. }\end{array}$ & 3.7 & 3.9 & 4.5 & 16.4 \\
\hline
\end{tabular}


Table 2. Low-flow characteristics for partial-record streamgaging stations in Indiana._Continued

[mi² , square miles; IN, Indiana; Lat, latitude; ', degrees; ', minutes; ", seconds; long, longitude; Sec, Section; T, Township; R, Range; N, North; E, East; S, South; W, West; - , no correlation]

\begin{tabular}{|c|c|c|c|c|c|c|c|}
\hline \multirow{2}{*}{ Station number } & \multirow{2}{*}{ Station name } & \multirow{2}{*}{$\begin{array}{l}\text { Drainage } \\
\text { area }\left(\mathrm{mi}^{2}\right)\end{array}$} & \multirow{2}{*}{ Location } & \multicolumn{4}{|c|}{ Flow characteristic, in cubic feet per second } \\
\hline & & & & 1010 & 7010 & 30010 & $\begin{array}{c}\text { Harmomic } \\
\text { mean }\end{array}$ \\
\hline 03329100 & $\begin{array}{l}\text { Crooked Creek } \\
\text { near Royal } \\
\text { Center, IN }\end{array}$ & 35.9 & $\begin{array}{l}\text { Lat } 40^{\circ} 48^{\prime} 23^{\prime \prime} \text {, long } 86^{\circ} 29^{\prime} 31^{\prime \prime} \text {, } \\
\text { in NW1/4 Sec } 11, \text { T.27N., R.1W., } \\
\text { Cass County, at triple culverts on } \\
625 \text { West Road, } 4 \text { miles south of } \\
\text { Royal Center. }\end{array}$ & 2.8 & 3 & 3.5 & 15.4 \\
\hline 03329150 & $\begin{array}{l}\text { Crooked Creek } \\
\text { near } \\
\text { Logansport, IN }\end{array}$ & 54.2 & $\begin{array}{l}\text { Lat } 40^{\circ} 45^{\prime} 51^{\prime \prime} \text {, long } 86^{\circ} 29^{\prime} 54^{\prime \prime} \text {, } \\
\text { in NW1/4 Sec } 26, \text { T.27N., R.1W., } \\
\text { Cass County, at bridge on U.S. } \\
\text { Highway } 24,2.5 \text { miles west of } \\
\text { County Road } 600 \text { West, } 6.5 \text { miles } \\
\text { west of Logansport. }\end{array}$ & 11 & 12 & 13 & 33 \\
\hline 03329300 & $\begin{array}{l}\text { Rock Creek } \\
\text { near Rockfield, } \\
\text { IN }\end{array}$ & 80.8 & $\begin{array}{l}\text { Lat } 40^{\circ} 39^{\prime} 10^{\prime \prime} \text {, long } 86^{\circ} 33^{\prime} 30^{\prime \prime} \text {, } \\
\text { in SE1/4 Sec } 22 \text {, T.26N., R.1E., Car- } \\
\text { roll County, at bridge on State High- } \\
\text { way } 25,1.25 \text { miles northeast of } \\
\text { Rockfield. }\end{array}$ & 0.6 & 0.7 & 1 & 11 \\
\hline 03329510 & $\begin{array}{l}\text { Deer Creek near } \\
\text { Lincoln, IN }\end{array}$ & 56.5 & $\begin{array}{l}\text { Lat } 40^{\circ} 36^{\prime} 11^{\prime \prime} \text {, long } 86^{\circ} 12^{\prime} 10^{\prime \prime} \text {, } \\
\text { SW1/4 NW1/4 Sec } 35, \text { T.25N., } \\
\text { R.4E., Cass County, } 1 \text { mile south of } \\
\text { Lincoln. }\end{array}$ & 0.5 & 0.5 & 0.8 & 7.8 \\
\hline
\end{tabular}


304 Low-flow Characteristics for Selected Streams in Indiana

Table 2. Low-flow characteristics for partial-record streamgaging stations in Indiana.—Continued

[mi², square miles; IN, Indiana; Lat, latitude; ' , degrees; ', minutes; ", seconds; long, longitude; Sec, Section; T, Township; R, Range; N, North; E, East; S, South; W, West; —, no correlation]

\begin{tabular}{|c|c|c|c|c|c|c|c|}
\hline \multirow{2}{*}{ Station number } & \multirow{2}{*}{ Station name } & \multirow{2}{*}{$\begin{array}{l}\text { Drainage } \\
\text { area }\left(\mathrm{mi}^{2}\right)\end{array}$} & \multirow{2}{*}{ Location } & \multicolumn{4}{|c|}{ Flow characteristic, in cubic feet per second } \\
\hline & & & & 1010 & 7010 & 30010 & $\begin{array}{c}\text { Harmomic } \\
\text { mean }\end{array}$ \\
\hline \multirow[t]{5}{*}{03329530} & South Fork Deer & 31.6 & Lat $40^{\circ} 34^{\prime} 54^{\prime \prime}$, long $86^{\circ} 11^{\prime} 23^{\prime \prime}$, & 0.4 & 0.5 & 0.6 & 4.5 \\
\hline & Creek at & & SE1/4 NW1/4 SE1/4 Sec 28, T.25N., & & & & \\
\hline & Galveston, IN & & R.3E., Cass County, at bridge on & & & & \\
\hline & & & Highway 35 , at north edge of & & & & \\
\hline & & & Galveston. & & & & \\
\hline \multirow[t]{5}{*}{03329580} & Little Deer & 52.9 & Lat $40^{\circ} 35^{\prime} 26^{\prime \prime}$, long $86^{\circ} 28^{\prime} 02^{\prime \prime}$, & 1 & 1 & 1.3 & 8.9 \\
\hline & Creek near & & in SW1/4 SW1/4 SW1/4 Sec 19, & & & & \\
\hline & Camden, IN & & T.25N., R.1E., Carroll County, at & & & & \\
\hline & & & North Road bridge, approximately 4 & & & & \\
\hline & & & miles southeast of Camden. & & & & \\
\hline \multirow[t]{5}{*}{03329600} & Bachelor Run & 13.5 & Lat $40^{\circ} 32^{\prime} 50^{\prime \prime}$, long $86^{\circ} 29^{\prime} 30^{\prime \prime}$, & 0.2 & 0.2 & 0.3 & 1.5 \\
\hline & near Flora, IN & & on line between Secs 2 and 11, & & & & \\
\hline & & & T.24N., R.1W., Carroll County, at & & & & \\
\hline & & & bridge on State Highway $18,1.2$ & & & & \\
\hline & & & miles east of Flora. & & & & \\
\hline \multirow[t]{6}{*}{03331350} & Big Yellow & 47.8 & Lat $41^{\circ} 10^{\prime} 18^{\prime \prime}$, long $86^{\circ} 07^{\prime} 16^{\prime \prime}$, & 1.8 & 1.9 & 2.2 & 12 \\
\hline & Creek near & & in NE1/4 NW1/4 Sec 6. T.31N., & & & & \\
\hline & Mentone, IN & & R.4E., Fulton County, at bridge on & & & & \\
\hline & & & State Highway 25, 0.1 mile south of & & & & \\
\hline & & & junction of State Highway 110 about & & & & \\
\hline & & & 4.5 miles west of Mentone. & & & & \\
\hline
\end{tabular}


Table 2. Low-flow characteristics for partial-record streamgaging stations in Indiana._Continued

[mi² , square miles; IN, Indiana; Lat, latitude; ', degrees; ', minutes; ", seconds; long, longitude; Sec, Section; T, Township; R, Range; N, North; E, East; S, South; W, West; - , no correlation]

\begin{tabular}{|c|c|c|c|c|c|c|c|}
\hline \multirow{2}{*}{ Station number } & \multirow{2}{*}{ Station name } & \multirow{2}{*}{$\begin{array}{l}\text { Drainage } \\
\text { area }\left(\mathrm{mi}^{2}\right)\end{array}$} & \multirow{2}{*}{ Location } & \multicolumn{4}{|c|}{ Flow characteristic, in cubic feet per second } \\
\hline & & & & 1010 & 7010 & 30010 & $\begin{array}{c}\text { Harmomic } \\
\text { mean }\end{array}$ \\
\hline \multirow[t]{5}{*}{03331375} & Chippewanuck & 43.7 & Lat $41^{\circ} 06^{\prime} 43^{\prime \prime}$, long $86^{\circ} 11^{\prime} 09^{\prime \prime}$, & 5.3 & 5.5 & 6 & 17 \\
\hline & Creek near & & NW1/4 NW1/4 NW1/4 Sec 27, & & & & \\
\hline & Rochester, IN & & T.31N., R.3E., Fulton County, at & & & & \\
\hline & & & bridge on State Highway 25, 3.5 & & & & \\
\hline & & & miles north of Rochester. & & & & \\
\hline \multirow[t]{6}{*}{03331430} & Mud Creek near & 66.9 & Lat $41^{\circ} 03^{\prime} 10^{\prime \prime}$, long $86^{\circ} 19^{\prime} 39^{\prime \prime}$, & 2.9 & 3 & 3.5 & 18 \\
\hline & Bruce Lake, IN & & on line between Secs 8 and 17, & & & & \\
\hline & & & T.30N., R.2E., Fulton County, at & & & & \\
\hline & & & bridge on State Highway 14, 4.5 & & & & \\
\hline & & & miles east of Bruce Lake and 5.5 & & & & \\
\hline & & & miles west of Rochester. & & & & \\
\hline \multirow[t]{7}{*}{03331750} & Quigley Marsh & 14.8 & Lat $41^{\circ} 03^{\prime} 20^{\prime \prime}$, long $86^{\circ} 36^{\prime} 12^{\prime \prime}$, & 6.2 & 6.3 & 6.6 & 12 \\
\hline & Ditch at & & on line between Secs 11 and 12, & & & & \\
\hline & Winamac, IN & & T.30N., R.2W., Pulaski County, at & & & & \\
\hline & & & north edge of Winamac at Indian & & & & \\
\hline & & & Head Motel on U.S. Highway 35 & & & & \\
\hline & & & bridge, 0.3 mile north of State High- & & & & \\
\hline & & & way 14 East of Winamac. & & & & \\
\hline
\end{tabular}


Table 2. Low-flow characteristics for partial-record streamgaging stations in Indiana.-Continued

[mi², square miles; IN, Indiana; Lat, latitude; ' , degrees; ', minutes; ", seconds; long, longitude; Sec, Section; T, Township; R, Range; N, North; E, East; S, South; W, West; -, no correlation]

\begin{tabular}{|c|c|c|c|c|c|c|c|}
\hline \multirow{2}{*}{ Station number } & \multirow{2}{*}{ Station name } & \multirow{2}{*}{$\begin{array}{l}\text { Drainage } \\
\text { area }\left(\mathrm{mi}^{2}\right)\end{array}$} & \multirow{2}{*}{ Location } & \multicolumn{4}{|c|}{ Flow characteristic, in cubic feet per second } \\
\hline & & & & 1010 & 7010 & 30010 & $\begin{array}{c}\text { Harmomic } \\
\text { mean }\end{array}$ \\
\hline \multirow[t]{6}{*}{03331780} & Mill Creek near & 24.8 & Lat $40^{\circ} 57^{\prime} 28^{\prime \prime}$, long $86^{\circ} 20^{\prime} 06^{\prime \prime}$, & 1.6 & 1.9 & 2.2 & 7 \\
\hline & Fulton, IN & & in S1/2 NW1/4 SW1/4 Sec 17, & & & & \\
\hline & & & T.29N., R.2E., at bridge on Fulton & & & & \\
\hline & & & County Road, 1 mile north of State & & & & \\
\hline & & & Road 114, and 5 miles west of Fulton & & & & \\
\hline & & & and 0.75 mile north of Marshtown. & & & & \\
\hline \multirow[t]{5}{*}{03332250} & Indian Creek & 56.6 & Lat $40^{\circ} 55^{\prime} 12^{\prime \prime}$, long $86^{\circ} 31^{\prime} 42^{\prime \prime}$, & 5.6 & 6.6 & 7.8 & 29 \\
\hline & near Thornhope, & & NE1/4 SE1/4 NE1/4 Sec 33. T.29N., & & & & \\
\hline & $\mathrm{IN}$ & & R.1W., Pulaski County, at bridge on & & & & \\
\hline & & & U.S. Highway $35,1 / 3$ mile south of & & & & \\
\hline & & & Thornhope. & & & & \\
\hline \multirow[t]{6}{*}{03332350} & Big Monon & 69.6 & Lat $41^{\circ} 03^{\prime} 21^{\prime \prime}$, long $86^{\circ} 50^{\prime} 02^{\prime \prime}$, & 12 & 13 & 14 & 35 \\
\hline & Creek near & & in NW1/4 NW1/4 Sec 13, T.30N., & & & & \\
\hline & Medaryville, & & R.4W., Pulaski County, at bridge on & & & & \\
\hline & $\mathrm{IN}$ & & State Highway 14, 3 miles east of & & & & \\
\hline & & & Junction of U.S. Highway 421, 3.5 & & & & \\
\hline & & & miles southeast of Medaryville. & & & & \\
\hline
\end{tabular}


Table 2. Low-flow characteristics for partial-record streamgaging stations in Indiana._Continued

[mi² , square miles; IN, Indiana; Lat, latitude; ', degrees; ', minutes; ", seconds; long, longitude; Sec, Section; T, Township; R, Range; N, North; E, East; S, South; W, West; - , no correlation]

\begin{tabular}{|c|c|c|c|c|c|c|c|}
\hline \multirow{2}{*}{ Station number } & \multirow{2}{*}{ Station name } & \multirow{2}{*}{$\begin{array}{l}\text { Drainage } \\
\text { area }\left(\mathrm{mi}^{2}\right)\end{array}$} & \multirow{2}{*}{ Location } & \multicolumn{4}{|c|}{ Flow characteristic, in cubic feet per second } \\
\hline & & & & 1010 & 7010 & 30010 & $\begin{array}{c}\text { Harmomic } \\
\text { mean }\end{array}$ \\
\hline 03332440 & $\begin{array}{l}\text { Hoagland Ditch } \\
\text { near Monon, } \\
\text { IN }\end{array}$ & 72.8 & $\begin{array}{l}\text { Lat } 40^{\circ} 48^{\prime} 52^{\prime \prime} \text {, long } 86^{\circ} 48^{\prime} 51^{\prime \prime} \text {, } \\
\text { in NE1/4 NE1/4 SE1/4 Sec 1, on } \\
\text { line with Sec } 6 \text {, T.27N., R.4W., at } \\
\text { bridge on White County Road, } 3.75 \\
\text { miles south of State Road 16, } 1 \text { mile } \\
\text { west of confluence at Lake Schaefer, } \\
\text { and } 7 \text { miles southeast of Monon. }\end{array}$ & 0.4 & 0.4 & 0.5 & 6.5 \\
\hline 03332480 & $\begin{array}{l}\text { Honey Creek } \\
\text { near Reynolds, } \\
\text { IN }\end{array}$ & 38.4 & $\begin{array}{l}\text { Lat } 40^{\circ} 46^{\prime} 53^{\prime \prime} \text {, long } 86^{\circ} 48^{\prime} 52^{\prime \prime} \text {, } \\
\text { on line between Secs } 19 \text { and } 24 \text {, } \\
\text { T. } 27 \text { N., and on line between R.3W., } \\
\text { and } 4 \mathrm{~W} ., \text { White County, at bridge on } \\
\text { County Road } 300 \text { East, about } 5 \\
\text { miles northwest of Monticello. }\end{array}$ & 0.3 & 0.3 & 0.3 & 2.3 \\
\hline 03332550 & $\begin{array}{l}\text { Pike Creek near } \\
\text { Norway IN }\end{array}$ & 20.6 & $\begin{array}{l}\text { Lat } 40^{\circ} 46^{\prime} 51^{\prime \prime} \text {, long } 86^{\circ} 44^{\prime} 42^{\prime \prime} \text {, } \\
\text { in NE1/4 NE1/4 NW1/4 Sec } 22 \text {, } \\
\text { T.27N., R.3W., White County, at } \\
\text { bridge on State Highway } 39,2.0 \\
\text { miles north of U.S. Highway } 24 .\end{array}$ & 2.9 & 3 & 3.6 & 12 \\
\hline
\end{tabular}


Table 2. Low-flow characteristics for partial-record streamgaging stations in Indiana.-Continued

[mi², square miles; IN, Indiana; Lat, latitude; ' ${ }^{2}$ degrees; ', minutes; ", seconds; long, longitude; Sec, Section; T, Township; R, Range; N, North; E, East; S, South; W, West; -, no correlation]

\begin{tabular}{|c|c|c|c|c|c|c|c|}
\hline \multirow{2}{*}{ Station number } & \multirow{2}{*}{ Station name } & \multirow{2}{*}{$\begin{array}{l}\text { Drainage } \\
\text { area }\left(\mathrm{mi}^{2}\right)\end{array}$} & \multirow{2}{*}{ Location } & \multicolumn{4}{|c|}{ Flow characteristic, in cubic feet per second } \\
\hline & & & & 1010 & 7010 & 30010 & $\begin{array}{c}\text { Harmomic } \\
\text { mean }\end{array}$ \\
\hline \multirow[t]{5}{*}{03332800} & Big Creek near & 55.3 & Lat $40^{\circ} 40^{\prime} 16^{\prime \prime}$, long $86^{\circ} 47^{\prime} 14^{\prime \prime}$, & 0.8 & 0.9 & 1.3 & 14 \\
\hline & Monticello, IN & & in NW1/4 SW1/4 Sec 9, T.26N., & & & & \\
\hline & (previously & & R.3W., White County, at bridge on & & & & \\
\hline & published as & & county road 4.8 miles east of State & & & & \\
\hline & "near Chalmers") & & Highway 43 in Chalmers. & & & & \\
\hline \multirow[t]{6}{*}{03333100} & Moots Creek & 42.7 & Lat $40^{\circ} 34^{\prime} 18^{\prime \prime}$, long $86^{\circ} 48^{\prime} 42^{\prime \prime}$, & 0.4 & 0.4 & 0.6 & 6.2 \\
\hline & near Brookston, & & on line between $\operatorname{Sec} 36$, T.25N., & & & & \\
\hline & IN & & R.4W., and Sec 31, T.25N., R.3W., & & & & \\
\hline & & & White County, at bridge on County & & & & \\
\hline & & & Road 300 East, 3.5 miles southwest & & & & \\
\hline & & & of Brookston. & & & & \\
\hline \multirow[t]{7}{*}{03333400} & Mud Creek near & 74.8 & Lat $40^{\circ} 24^{\prime} 23^{\prime \prime}$, long $85^{\circ} 56^{\prime} 48^{\prime \prime}$, & 0.3 & 0.3 & 0.4 & 3.5 \\
\hline & Windfall, IN & & in NW1/4 NE1/4 Sec 34, T.23N., & & & & \\
\hline & & & R.5E., Tipton County, at bridge on & & & & \\
\hline & & & 500 South Road along Tipton- & & & & \\
\hline & & & Howard County line 0.5 mile east of & & & & \\
\hline & & & State Highway 213 and 3.3 miles & & & & \\
\hline & & & north of Windfall. & & & & \\
\hline \multirow[t]{4}{*}{03334300} & Kilmore Creek & 62.6 & Lat $40^{\circ} 20^{\prime} 40^{\prime \prime}$, long $86^{\circ} 30^{\prime} 25^{\prime \prime}$, & 0 & 0 & - & - \\
\hline & at Kilmore, IN & & in SW1/4 Sec 14, T.22N., R.1W., & & & & \\
\hline & & & Clinton County, at county highway & & & & \\
\hline & & & bridge 0.2 mile south of Kilmore. & & & & \\
\hline
\end{tabular}


Table 2. Low-flow characteristics for partial-record streamgaging stations in Indiana._Continued

[mi² , square miles; IN, Indiana; Lat, latitude; ', degrees; ', minutes; ", seconds; long, longitude; Sec, Section; T, Township; R, Range; N, North; E, East; S, South; W, West; - , no correlation]

\begin{tabular}{|c|c|c|c|c|c|c|c|}
\hline \multirow{2}{*}{ Station number } & \multirow{2}{*}{ Station name } & \multirow{2}{*}{$\begin{array}{l}\text { Drainage } \\
\text { area }\left(\mathrm{mi}^{2}\right)\end{array}$} & \multirow{2}{*}{ Location } & \multicolumn{4}{|c|}{ Flow characteristic, in cubic feet per second } \\
\hline & & & & 1010 & 7010 & 30010 & $\begin{array}{c}\text { Harmomic } \\
\text { mean }\end{array}$ \\
\hline \multirow[t]{4}{*}{03334330} & Kilmore Creek & 77.4 & Lat $40^{\circ} 19^{\prime} 41^{\prime \prime}$, long $86^{\circ} 37^{\prime} 05^{\prime \prime}$, & 0.4 & 0.4 & 0.5 & 8.3 \\
\hline & near Frankfort, & & in the NE1/4 Sec 27, T.22N., R.2W., & & & & \\
\hline & IN & & Clinton County, on Gasline Road & & & & \\
\hline & & & 6.3 miles northwest of Frankfort. & & & & \\
\hline \multirow[t]{7}{*}{03334700} & Middle Fork & 54.0 & Lat $40^{\circ} 25^{\prime} 47^{\prime \prime}$, long $86^{\circ} 36^{\prime} 15^{\prime \prime}$, & 1.7 & 1.8 & 2.3 & 12 \\
\hline & Wildcat Creek & & NE1/4 NE1/4 Sec 23, T.23N., & & & & \\
\hline & near Rossville, & & R.2W., Clinton County, at bridge on & & & & \\
\hline & IN & & U.S. Highway 421, 1.0 mile north- & & & & \\
\hline & & & west of junction of U.S. Highway & & & & \\
\hline & & & 421 and State Highway 26, in Ross- & & & & \\
\hline & & & ville. & & & & \\
\hline \multirow[t]{6}{*}{03334750} & Campbells Run & 18.3 & Lat $40^{\circ} 24^{\prime} 46^{\prime \prime}$, long $86^{\circ} 35^{\prime} 41^{\prime \prime}$, & 0.2 & 0.2 & 0.3 & 2.5 \\
\hline & near Rossville, & & in SE1/4 NW1/4 Sec 25, T.23N., & & & & \\
\hline & IN & & R.2W., Clinton County, at bridge on & & & & \\
\hline & & & U.S. Highway 421, 0.3 mile south of & & & & \\
\hline & & & junction U.S. Highway 326 in Ross- & & & & \\
\hline & & & ville. & & & & \\
\hline \multirow[t]{5}{*}{03335670} & Wea Creek near & 105 & Lat $40^{\circ} 21^{\prime} 46^{\prime \prime}$, long $86^{\circ} 54^{\prime} 17^{\prime \prime}$, & 7.8 & 8.2 & 9.3 & - \\
\hline & Lafayette, IN & & in SE1/4 Sec 7, T.23N., R.4W., & & & & \\
\hline & & & Tippecanoe County, at bridge on & & & & \\
\hline & & & State Highway 43, 4.0 miles south & & & & \\
\hline & & & of Lafayette. & & & & \\
\hline
\end{tabular}




\section{Low-flow Characteristics for Selected Streams in Indiana}

Table 2. Low-flow characteristics for partial-record streamgaging stations in Indiana.-Continued

[mi², square miles; IN, Indiana; Lat, latitude; ' , degrees; ', minutes; ", seconds; long, longitude; Sec, Section; T, Township; R, Range; N, North; E, East; S, South; W, West; —, no correlation]

\begin{tabular}{|c|c|c|c|c|c|c|c|}
\hline \multirow{2}{*}{ Station number } & \multirow{2}{*}{ Station name } & \multirow{2}{*}{$\begin{array}{l}\text { Drainage } \\
\text { area }\left(\mathrm{mi}^{2}\right)\end{array}$} & \multirow{2}{*}{ Location } & \multicolumn{4}{|c|}{ Flow characteristic, in cubic feet per second } \\
\hline & & & & 1010 & 7010 & 30010 & $\begin{array}{c}\text { Harmomic } \\
\text { mean }\end{array}$ \\
\hline \multirow[t]{5}{*}{03335674} & Little Wea & 22.6 & Lat $40^{\circ} 19^{\prime} 36^{\prime \prime}$, long $86^{\circ} 54^{\prime} 17^{\prime \prime}$, in & 1.7 & 1.7 & 2 & 7.9 \\
\hline & Creek near & & the NE1/4 of Sec 30, T.22N., R.4W., & & & & \\
\hline & Lafayette, IN & & Tippecanoe County, on Tippecanoe & & & & \\
\hline & & & County Road 6C, 6.5 miles south of & & & & \\
\hline & & & Lafayette. & & & & \\
\hline \multirow[t]{6}{*}{03335681} & Flint Creek near & 29.9 & Lat $40^{\circ} 20^{\prime} 25^{\prime \prime}$, long $87^{\circ} 04^{\prime} 02^{\prime \prime}$, & 0 & 0.1 & 0.1 & 6.6 \\
\hline & West Point, IN & & in SE1/4 NW1/4 Sec 23, T.22N., & & & & \\
\hline & & & R.6W., Tippecanoe County, at & & & & \\
\hline & & & bridge on County Road 510 South, & & & & \\
\hline & & & 1.2 miles west of State Highway 25 & & & & \\
\hline & & & in Westpoint. & & & & \\
\hline \multirow[t]{6}{*}{03335682} & Indian Creek & 29.0 & Lat $40^{\circ} 25^{\prime} 03^{\prime \prime}$, long $87^{\circ} 02^{\prime} 31^{\prime \prime}$, & 0 & 0 & 0.1 & 1.9 \\
\hline & near Green Hill, & & in SE1/4 SW1/4 SE1/4 Sec 24, & & & & \\
\hline & $\mathrm{IN}$ & & T.23N., R.6W., Tippecanoe County, & & & & \\
\hline & & & at bridge on South River Road, 6.5 & & & & \\
\hline & & & miles west of West Lafayette, 0.2 & & & & \\
\hline & & & mile west of County Road 700 West. & & & & \\
\hline \multirow[t]{5}{*}{03335683} & Kickapoo Creek & 36.4 & Lat $40^{\circ} 19^{\prime} 27^{\prime \prime}$, long $87^{\circ} 14^{\prime} 05^{\prime \prime}$, & 2.5 & 2.6 & 2.8 & 5.9 \\
\hline & near Attica, IN & & in SE1/4 NW1/4 Sec 29, T.22N., & & & & \\
\hline & & & R.7W., Warren County, at bridge on & & & & \\
\hline & & & Kickapoo Road, 0.5 mile north of & & & & \\
\hline & & & Independence Road. & & & & \\
\hline
\end{tabular}


Table 2. Low-flow characteristics for partial-record streamgaging stations in Indiana._Continued

[mi² , square miles; IN, Indiana; Lat, latitude; ', degrees; ', minutes; ", seconds; long, longitude; Sec, Section; T, Township; R, Range; N, North; E, East; S, South; W, West; - , no correlation]

\begin{tabular}{|c|c|c|c|c|c|c|c|}
\hline \multirow{2}{*}{ Station number } & \multirow{2}{*}{ Station name } & \multirow{2}{*}{$\begin{array}{l}\text { Drainage } \\
\text { area }\left(\mathbf{m i}^{2}\right)\end{array}$} & \multirow{2}{*}{ Location } & \multicolumn{4}{|c|}{ Flow characteristic, in cubic feet per second } \\
\hline & & & & 1010 & 7010 & 30010 & $\begin{array}{c}\text { Harmomic } \\
\text { mean }\end{array}$ \\
\hline \multirow[t]{6}{*}{03335800} & Big Shawnee & 42.0 & Lat $40^{\circ} 14^{\prime} 30^{\prime \prime}$, long $87^{\circ} 14^{\prime} 12^{\prime \prime}$, & 6.1 & 6.4 & 7.2 & 19 \\
\hline & Creek near & & in SW1/4 NE1/4 NW1/4 Sec 29, & & & & \\
\hline & Attica, IN & & T.21N., R.7W., Fountain County, at & & & & \\
\hline & & & concrete bridge on County Road 3.7 & & & & \\
\hline & & & miles southeast of Attica and State & & & & \\
\hline & & & Highway 55 at Rob Roy. & & & & \\
\hline \multirow[t]{4}{*}{03335950} & Bear Creek at & 10.4 & Lat $40^{\circ} 13^{\prime} 11^{\prime \prime}$, long $87^{\circ} 20^{\prime} 23^{\prime \prime}$, & 1.2 & 1.3 & 1.4 & 2.5 \\
\hline & Fountain, IN & & in SE1/4 Sec 32, T.21N., R.8W., & & & & \\
\hline & & & Fountain County, on county road at & & & & \\
\hline & & & the southwest edge of Fountain. & & & & \\
\hline \multirow[t]{6}{*}{03339100} & Coal Creek near & 77.6 & Lat $40^{\circ} 08^{\prime} 33^{\prime \prime}$, long $87^{\circ} 15^{\prime} 00^{\prime \prime}$, & 3.9 & 4.1 & 4.8 & 23 \\
\hline & Veedersburg, & & on line between Secs 30 and 31 , & & & & \\
\hline & IN & & T.20N., R.7W., Fountain County, at & & & & \\
\hline & & & bridge on County Road, 0.25 mile & & & & \\
\hline & & & west of U.S. Highway 41, 2.2 miles & & & & \\
\hline & & & northeast of Veedersburg. & & & & \\
\hline
\end{tabular}




\section{Low-flow Characteristics for Selected Streams in Indiana}

Table 2. Low-flow characteristics for partial-record streamgaging stations in Indiana.-Continued

[mi², square miles; IN, Indiana; Lat, latitude; ' , degrees; ', minutes; ", seconds; long, longitude; Sec, Section; T, Township; R, Range; N, North; E, East; S, South; W, West; —, no correlation]

\begin{tabular}{|c|c|c|c|c|c|c|c|}
\hline \multirow{2}{*}{ Station number } & \multirow{2}{*}{ Station name } & \multirow{2}{*}{$\begin{array}{l}\text { Drainage } \\
\text { area }\left(\mathrm{mi}^{2}\right)\end{array}$} & \multirow{2}{*}{ Location } & \multicolumn{4}{|c|}{ Flow characteristic, in cubic feet per second } \\
\hline & & & & 1010 & 7010 & 30010 & $\begin{array}{c}\text { Harmomic } \\
\text { mean }\end{array}$ \\
\hline 03339110 & $\begin{array}{l}\text { East Fork Coal } \\
\text { Creek near } \\
\text { Veedersburg, } \\
\text { IN }\end{array}$ & 60.1 & $\begin{array}{l}\text { Lat } 40^{\circ} 05^{\prime} 48^{\prime \prime} \text {, long } 87^{\circ} 14^{\prime} 34^{\prime \prime} \text {, } \\
\text { in NW1/4 NW1/4 NW1/4 Sec } 17 \text {, } \\
\text { T.19N., R. } 7 W ., \text { Fountain County at } \\
\text { concrete bridge on relocated U.S. } \\
\text { Highway } 41,1.5 \text { miles southeast of } \\
\text { Veedersburg, } 0.5 \text { mile south of inter- } \\
\text { section of U.S. Highways } 41 \text { and } 136 .\end{array}$ & 4.7 & 5.2 & 6.4 & 23 \\
\hline 03339200 & $\begin{array}{l}\text { Sugar Creek } \\
\text { near Kirklin, } \\
\text { IN }\end{array}$ & 41.4 & $\begin{array}{l}\text { Lat } 40^{\circ} 12^{\prime} 29^{\prime \prime} \text {, long } 86^{\circ} 22^{\prime} 03^{\prime \prime} \text {, } \\
\text { SE1/4 NW1/4 Sec } 1, \text { T.20N., R.1E., } \\
\text { Clinton County, at bridge on U.S. } \\
\text { Highway } 421,1 \text { mile north of Kirk- } \\
\text { lin. }\end{array}$ & 0.1 & 0.2 & 0.2 & 4.6 \\
\hline 03339300 & $\begin{array}{l}\text { Prairie Creek at } \\
\text { Thorntown, IN }\end{array}$ & 46.9 & $\begin{array}{l}\text { Lat } 40^{\circ} 07^{\prime} 46^{\prime \prime} \text {, long } 86^{\circ} 36^{\prime} 01^{\prime \prime}, \\
\text { SE1/4 SE1/4 Sec } 35, \text { T. } 20 \text { N., R.2W., } \\
\text { Boone County, at bridge on State } \\
\text { Highway } 47,0.5 \text { mile east of junc- } \\
\text { tion of State Highways } 47 \text { and } 375 \\
\text { at east edge of Thorntown. }\end{array}$ & 1.5 & 1.7 & 2 & 7.9 \\
\hline
\end{tabular}


Table 2. Low-flow characteristics for partial-record streamgaging stations in Indiana._Continued

[mi² , square miles; IN, Indiana; Lat, latitude; ', degrees; ', minutes; ", seconds; long, longitude; Sec, Section; T, Township; R, Range; N, North; E, East; S, South; W, West; - , no correlation]

\begin{tabular}{|c|c|c|c|c|c|c|c|}
\hline \multirow{2}{*}{ Station number } & \multirow{2}{*}{ Station name } & \multirow{2}{*}{$\begin{array}{l}\text { Drainage } \\
\text { area }\left(\mathrm{mi}^{2}\right)\end{array}$} & \multirow{2}{*}{ Location } & \multicolumn{4}{|c|}{ Flow characteristic, in cubic feet per second } \\
\hline & & & & 1010 & 7010 & 30010 & $\begin{array}{c}\text { Harmomic } \\
\text { mean }\end{array}$ \\
\hline \multirow[t]{7}{*}{03339408} & Little Potato & 31.8 & Lat $40^{\circ} 09^{\prime} 48^{\prime \prime}$, long $86^{\circ} 45^{\prime} 08^{\prime \prime}$, & 0 & 0 & 0 & - \\
\hline & Creek near & & on line between Secs 21 and 22, & & & & \\
\hline & Darlington, IN & & T.20N., R.3W., Montgomery & & & & \\
\hline & & & County, on County Road $800 \mathrm{E}$, & & & & \\
\hline & & & 150 feet north of intersection with & & & & \\
\hline & & & County Road 850 N, 5 miles north- & & & & \\
\hline & & & east of Darlington. & & & & \\
\hline \multirow[t]{5}{*}{03339460} & Walnut Fork & 44.8 & Lat $40^{\circ} 02^{\prime} 49^{\prime \prime}$, long $86^{\circ} 51^{\prime} 33^{\prime \prime}$, & 0.2 & 0.2 & 0.3 & 3.4 \\
\hline & Sugar Creek & & in SE1/4 NW1/4 Sec 34, T.19N., & & & & \\
\hline & near & & R.4W., Montgomery County, at & & & & \\
\hline & Crawfordsville, & & bridge on State Highway 32, 2.5 & & & & \\
\hline & IN & & miles east of Crawfordsville. & & & & \\
\hline \multirow[t]{5}{*}{03340200} & Sugar Mill & 39.5 & Lat $39^{\circ} 58^{\prime} 03^{\prime \prime}$, long $87^{\circ} 10^{\prime} 28^{\prime \prime}$, & 0.3 & 0.4 & 0.7 & 11 \\
\hline & Creek near & & in SW1/4 SW1/4 SE1/4 Sec 26, & & & & \\
\hline & Wallace, IN & & T.18N., R.7W., Fountain County at & & & & \\
\hline & & & bridge on State Road 234, 1.4 miles & & & & \\
\hline & & & west of State Road 341. & & & & \\
\hline
\end{tabular}




\section{Low-flow Characteristics for Selected Streams in Indiana}

Table 2. Low-flow characteristics for partial-record streamgaging stations in Indiana.-Continued

[mi², square miles; IN, Indiana; Lat, latitude; ' , degrees; ', minutes; ", seconds; long, longitude; Sec, Section; T, Township; R, Range; N, North; E, East; S, South; W, West; —, no correlation]

\begin{tabular}{|c|c|c|c|c|c|c|c|}
\hline \multirow{2}{*}{ Station number } & \multirow{2}{*}{ Station name } & \multirow{2}{*}{$\begin{array}{l}\text { Drainage } \\
\text { area }\left(\mathrm{mi}^{2}\right)\end{array}$} & \multirow{2}{*}{ Location } & \multicolumn{4}{|c|}{ Flow characteristic, in cubic feet per second } \\
\hline & & & & 1010 & 7010 & 30010 & $\begin{array}{c}\text { Harmomic } \\
\text { mean }\end{array}$ \\
\hline \multirow[t]{6}{*}{03340651} & Big Raccoon & 46.4 & Lat $39^{\circ} 54^{\prime} 32^{\prime \prime}$, long $86^{\circ} 47^{\prime} 58^{\prime \prime}$, & 0 & 0 & 0 & 7 \\
\hline & Creek near & & in SE1/4 SE1/4 SW1/4 Sec 18, & & & & \\
\hline & Ladoga, IN & & T.17N., R.3W., Montgomery & & & & \\
\hline & & & County, at bridge on county road, & & & & \\
\hline & & & 0.25 mile south of Ladoga, and & & & & \\
\hline & & & 200 feet downstream from Monon RR. & & & & \\
\hline \multirow[t]{5}{*}{03341420} & Brouillettes & 331 & Lat $39^{\circ} 37^{\prime} 09^{\prime \prime}$, long $87^{\circ} 26^{\prime} 08^{\prime \prime}$, & 1.1 & 1.3 & 1.9 & 21 \\
\hline & Creek near & & in SW1/4 NE1/4 Sec 32, T.14N., & & & & \\
\hline & Universal, IN & & R.9W., Vermillion County, on down- & & & & \\
\hline & & & stream side of bridge on State High- & & & & \\
\hline & & & way $63,0.7$ mile east of Universal. & & & & \\
\hline \multirow[t]{6}{*}{03341580} & Honey Creek & 64.0 & Lat $39^{\circ} 23^{\prime} 40^{\prime \prime}$, long $87^{\circ} 23^{\prime} 54^{\prime \prime}$, & 0.2 & 0.3 & 0.5 & 3.6 \\
\hline & near Terre & & SW1/4 Sec 15, T.11N., R.9W., Vigo & & & & \\
\hline & Haute, IN & & County, at bridge on U.S. Highway & & & & \\
\hline & & & 41 , at north edge of Allendale and & & & & \\
\hline & & & about 3 miles south of the south city & & & & \\
\hline & & & limits of Terre Haute. & & & & \\
\hline 03341800 & Prairie Creek & 22.4 & Lat $39^{\circ} 16^{\prime} 50^{\prime \prime}$, long $87^{\circ} 29^{\prime} 53^{\prime \prime}$, & 0 & 0 & 0 & - \\
\hline$(391650087295$ & near Prairie & & NW1/4 SW1/4 Sec 26, T.10N., & & & & \\
\hline \multirow[t]{3}{*}{ 300) } & Creek, IN & & R.10W., Vigo County, at bridge on & & & & \\
\hline & & & State Highway $63,0.3$ mile north of & & & & \\
\hline & & & Prairie Creek. & & & & \\
\hline
\end{tabular}


Table 2. Low-flow characteristics for partial-record streamgaging stations in Indiana._Continued

[mi² , square miles; IN, Indiana; Lat, latitude; ', degrees; ', minutes; ", seconds; long, longitude; Sec, Section; T, Township; R, Range; N, North; E, East; S, South; W, West; - , no correlation]

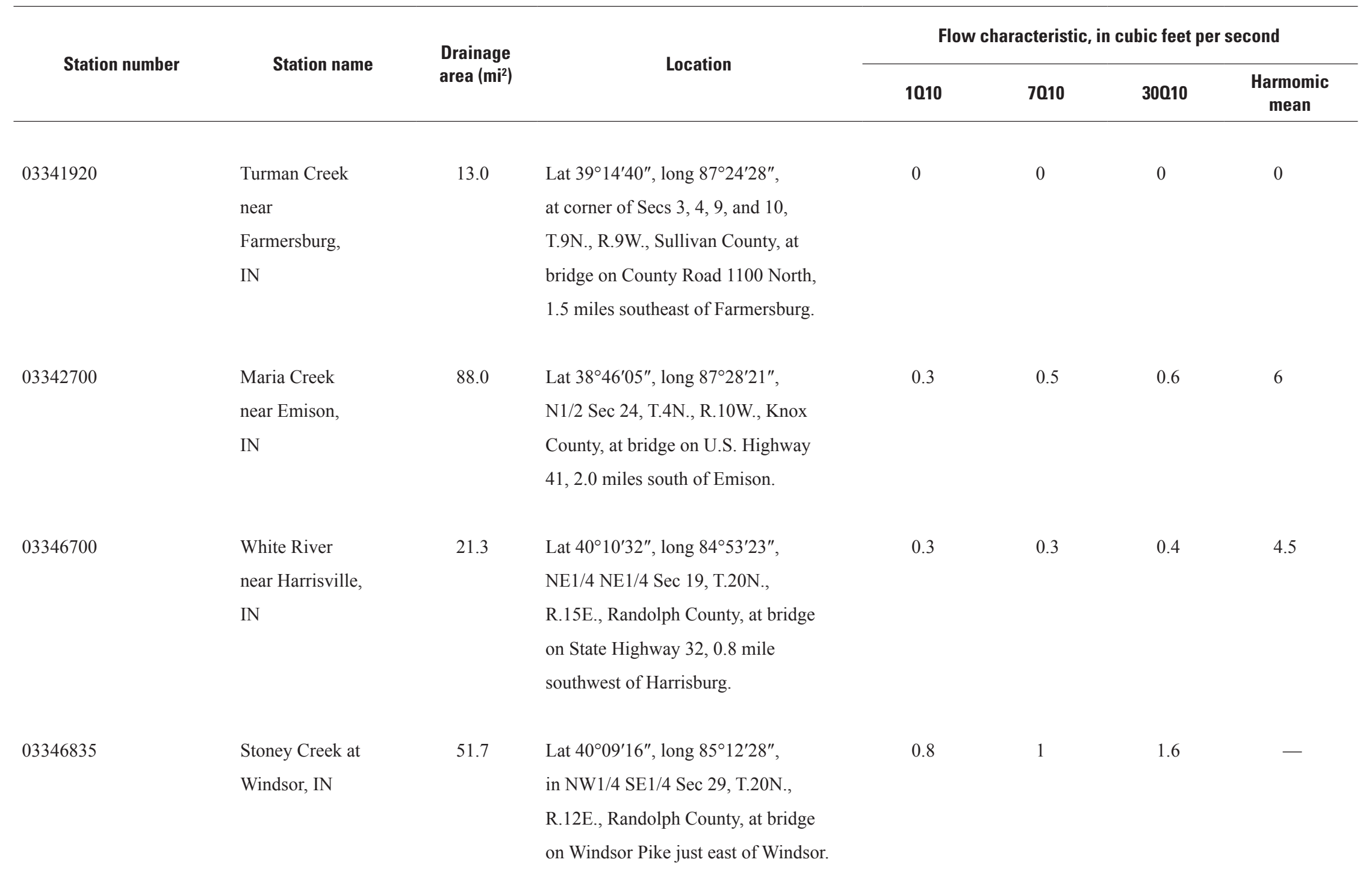




\section{Low-flow Characteristics for Selected Streams in Indiana}

Table 2. Low-flow characteristics for partial-record streamgaging stations in Indiana.-Continued

[mi², square miles; IN, Indiana; Lat, latitude; ' , degrees; ', minutes; ", seconds; long, longitude; Sec, Section; T, Township; R, Range; N, North; E, East; S, South; W, West; —, no correlation]

\begin{tabular}{|c|c|c|c|c|c|c|c|}
\hline \multirow{2}{*}{ Station number } & \multirow{2}{*}{ Station name } & \multirow{2}{*}{$\begin{array}{l}\text { Drainage } \\
\text { area }\left(\mathrm{mi}^{2}\right)\end{array}$} & \multirow{2}{*}{ Location } & \multicolumn{4}{|c|}{ Flow characteristic, in cubic feet per second } \\
\hline & & & & 1010 & 7010 & 30010 & $\begin{array}{c}\text { Harmomic } \\
\text { mean }\end{array}$ \\
\hline \multirow[t]{5}{*}{03347595} & Bell Creek near & 44.7 & Lat $40^{\circ} 08^{\prime} 43^{\prime \prime}$, long $85^{\circ} 27^{\prime} 03^{\prime \prime}$, & 1 & 1.1 & 1.4 & 11 \\
\hline & Yorktown, IN & & in SW1/4 NE1/4 Sec 36, T.20N., & & & & \\
\hline & & & R.9E., Delaware County at bridge on & & & & \\
\hline & & & Bell Creek Road, 0.2 mile west of & & & & \\
\hline & & & County Road 325 West. & & & & \\
\hline \multirow[t]{5}{*}{03348100} & Killbuck Creek & 97.8 & Lat $40^{\circ} 08^{\prime} 18^{\prime \prime}$, long $85^{\circ} 39^{\prime} 44^{\prime \prime}$, & 7.2 & 7.8 & 9 & 28 \\
\hline & near Anderson, & & in SW1/4 Sec 31, T.20N., R.8E., & & & & \\
\hline & $\mathrm{IN}$ & & Madison County, at bridge on State & & & & \\
\hline & & & Road 109, 2.3 miles northeast of & & & & \\
\hline & & & Anderson. & & & & \\
\hline \multirow[t]{5}{*}{03348300} & Pipe Creek near & 44.8 & Lat $40^{\circ} 16^{\prime} 40^{\prime \prime}$, long $85^{\circ} 38^{\prime} 34^{\prime \prime}$, & 1.4 & 1.6 & 2 & 9.2 \\
\hline & Alexandria, IN & & on line between Secs 7 and 8 , & & & & \\
\hline & & & T.21N., R.8E., Madison County, at & & & & \\
\hline & & & bridge on State Highway 28, 2 miles & & & & \\
\hline & & & northeast of Alexandria. & & & & \\
\hline \multirow[t]{6}{*}{03348400} & Duck Creek at & 98.9 & Lat $40^{\circ} 08^{\prime} 17^{\prime \prime}$, long $85^{\circ} 56^{\prime} 22^{\prime \prime}$, & 0.9 & 1.1 & 1.5 & 19 \\
\hline & Strawtown, IN & & on line between Secs 34 and 35 , & & & & \\
\hline & & & T.20N., R.5E., Hamilton County, at & & & & \\
\hline & & & bridge on State Highway 213, 600 & & & & \\
\hline & & & feet upstream from White River, 0.6 & & & & \\
\hline & & & mile south of East 246th Street. & & & & \\
\hline
\end{tabular}


Table 2. Low-flow characteristics for partial-record streamgaging stations in Indiana.-Continued

[mi², square miles; IN, Indiana; Lat, latitude; ' , degrees; ', minutes; ", seconds; long, longitude; Sec, Section; T, Township; R, Range; N, North; E, East; S, South; W, West; —, no correlation]

\begin{tabular}{|c|c|c|c|c|c|c|c|}
\hline \multirow{2}{*}{ Station number } & \multirow{2}{*}{ Station name } & \multirow{2}{*}{$\begin{array}{l}\text { Drainage } \\
\text { area }\left(\mathrm{mi}^{2}\right)\end{array}$} & \multirow{2}{*}{ Location } & \multicolumn{4}{|c|}{ Flow characteristic, in cubic feet per second } \\
\hline & & & & 1010 & 7010 & 30010 & $\begin{array}{c}\text { Harmomic } \\
\text { mean }\end{array}$ \\
\hline 03349200 & $\begin{array}{l}\text { Cicero Creek } \\
\text { near Tipton, IN }\end{array}$ & 80.2 & $\begin{array}{l}\text { Lat } 40^{\circ} 16^{\prime} 16^{\prime \prime} \text {, long } 86^{\circ} 03^{\prime} 02^{\prime \prime} \text {, } \\
\text { SE1/4 NE1/4 Sec } 15, \text { T.21N., R.4E., } \\
\text { Tipton County, } 45 \text { feet west of } \\
\text { bridge on County Road } 200 \text { West, } \\
0.5 \text { mile west of State Highway 19, } \\
\text { and County Home. }\end{array}$ & 0.4 & 0.4 & 0.6 & 4.8 \\
\hline 03351300 & $\begin{array}{l}\text { Crooked Creek } \\
\text { at Augusta, IN }\end{array}$ & 7.01 & $\begin{array}{l}\text { Lat } 39^{\circ} 53^{\prime} 43^{\prime \prime} \text {, long } 86^{\circ} 12^{\prime} 53^{\prime \prime} \text {, } \\
\text { in NE1/4 NW1/4 Sec } 29 \text { T.17N., } \\
\text { T.3E., Marion County, at bridge on } \\
\text { U.S. Highway } 421,0.4 \text { mile north of } \\
\text { Augusta. }\end{array}$ & 0 & 0 & 0 & - \\
\hline 03351490 & $\begin{array}{l}\text { Lick Creek near } \\
\text { Fortville, IN }\end{array}$ & 36.6 & $\begin{array}{l}\text { Lat } 39^{\circ} 57^{\prime} 22^{\prime \prime}, \text { long } 85^{\circ} 50^{\prime} 36^{\prime \prime} \text {, } \\
\text { on line between Secs } 3 \text { and } 4 \text {, } \\
\text { T.17N., R.6E., Madison County, at } \\
\text { bridge on State Highway 13, } 1.5 \\
\text { miles north of U.S. Highway } 36 \text { in } \\
\text { Fortville. }\end{array}$ & 1.1 & 1.3 & 1.8 & 20 \\
\hline 03351900 & $\begin{array}{l}\text { Indian Creek } \\
\text { near Oaklandon, } \\
\text { IN }\end{array}$ & 18.3 & $\begin{array}{l}\text { Lat } 39^{\circ} 51^{\prime} 51^{\prime \prime} \text {, long } 85^{\circ} 58^{\prime} 07^{\prime \prime} \text {, } \\
\text { in SW1/4 NW1/4 Sec } 3, \mathrm{~T} .16 \mathrm{~N} \text {., } \\
\text { R.5E., Marion County, at bridge on } \\
\text { Old Highway } 67,1 \text { mile southwest of } \\
\text { Oaklandon. }\end{array}$ & 0 & 0 & 0 & - \\
\hline
\end{tabular}


318 Low-flow Characteristics for Selected Streams in Indiana

Table 2. Low-flow characteristics for partial-record streamgaging stations in Indiana.—Continued

[mi², square miles; IN, Indiana; Lat, latitude; ' , degrees; ', minutes; ", seconds; long, longitude; Sec, Section; T, Township; R, Range; N, North; E, East; S, South; W, West; —, no correlation]

\begin{tabular}{|c|c|c|c|c|c|c|c|}
\hline \multirow{2}{*}{ Station number } & \multirow{2}{*}{ Station name } & \multirow{2}{*}{$\begin{array}{l}\text { Drainage } \\
\text { area }\left(\mathrm{mi}^{2}\right)\end{array}$} & \multirow{2}{*}{ Location } & \multicolumn{4}{|c|}{ Flow characteristic, in cubic feet per second } \\
\hline & & & & 1010 & 7010 & 30010 & $\begin{array}{c}\text { Harmomic } \\
\text { mean }\end{array}$ \\
\hline \multirow[t]{5}{*}{03353631} & Little Buck & 8.28 & Lat $39^{\circ} 39^{\prime} 55^{\prime \prime}$, long $86^{\circ} 06^{\prime} 06^{\prime \prime}$, & 0 & 0 & 0 & - \\
\hline & Creek near & & on line between Secs 8 and 17 , & & & & \\
\hline & Southport, IN & & T.14N., R.4E., Marion County, at & & & & \\
\hline & & & bridge on Southport Road, 0.5 mile & & & & \\
\hline & & & east of Southport. & & & & \\
\hline \multirow[t]{5}{*}{03353650} & Pleasant Run at & 4.93 & Lat $39^{\circ} 37^{\prime} 53^{\prime \prime}$, long $86^{\circ} 06^{\prime} 58^{\prime \prime}$, & 0 & 0 & 0 & - \\
\hline & Greenwood, & & in NW1/4 Sec 29, T.14N., R.4E., & & & & \\
\hline & $\mathrm{IN}$ & & Johnson County, at bridge on State & & & & \\
\hline & & & Highway $431,0.5$ mile north of & & & & \\
\hline & & & Greenwood. & & & & \\
\hline \multirow[t]{7}{*}{03353665} & Stotts Creek & 60.1 & Lat $39^{\circ} 30^{\prime} 02^{\prime \prime}$, long $86^{\circ} 19^{\prime} 57^{\prime \prime}$, & 0.2 & 0.2 & 0.4 & 4.6 \\
\hline & near & & NW1/4 NE1/4 Sec 8, T.12N., R.8E., & & & & \\
\hline & Martinsville, & & Morgan County, at 220-foot concrete & & & & \\
\hline & IN & & bridge on State Highway 37, 7.2 & & & & \\
\hline & & & miles northeast of Martinsville and & & & & \\
\hline & & & 250 feet from confluence with White & & & & \\
\hline & & & River. & & & & \\
\hline
\end{tabular}


Table 2. Low-flow characteristics for partial-record streamgaging stations in Indiana.-Continued

[mi² , square miles; IN, Indiana; Lat, latitude; ', degrees; ', minutes; ", seconds; long, longitude; Sec, Section; T, Township; R, Range; N, North; E, East; S, South; W, West; - , no correlation]

\begin{tabular}{|c|c|c|c|c|c|c|c|}
\hline \multirow{2}{*}{ Station number } & \multirow{2}{*}{ Station name } & \multirow{2}{*}{$\begin{array}{l}\text { Drainage } \\
\text { area }\left(\mathrm{mi}^{2}\right)\end{array}$} & \multirow{2}{*}{ Location } & \multicolumn{4}{|c|}{ Flow characteristic, in cubic feet per second } \\
\hline & & & & 1010 & 7010 & 30010 & $\begin{array}{c}\text { Harmomic } \\
\text { mean }\end{array}$ \\
\hline \multirow[t]{5}{*}{03353670} & White Lick & 28.7 & Lat $39^{\circ} 51^{\prime} 56^{\prime \prime}$, long $86^{\circ} 23^{\prime} 42^{\prime \prime}$ & 0 & 0 & 0 & - \\
\hline & Creek near & & on line between Secs 2 and 34, & & & & \\
\hline & Brownsburg, & & T.16N., R.1E., Hendricks County, at & & & & \\
\hline & $\mathrm{IN}$ & & bridge on county highway, 0.5 mile & & & & \\
\hline & & & north of Brownsburg. & & & & \\
\hline \multirow[t]{6}{*}{03353900} & East Fork White & 37.4 & Lat $39^{\circ} 38^{\prime} 47^{\prime \prime}$, long $86^{\circ} 20^{\prime} 47^{\prime \prime}$, & 0.7 & 0.9 & 1.2 & 5.9 \\
\hline & Lick Creek near & & in SE1/4 Sec 18, T.134N., R.2E., & & & & \\
\hline & Mooresville, & & Hendricks County, at bridge on & & & & \\
\hline & $\mathrm{IN}$ & & Mooresville Road, 0.8 mile west of & & & & \\
\hline & & & Friendswood, 3.0 miles northeast of & & & & \\
\hline & & & Mooresville. & & & & \\
\hline \multirow[t]{7}{*}{03354140} & Lambs Creek & 31.0 & Lat $39^{\circ} 25^{\prime} 26^{\prime \prime}$, long $86^{\circ} 28^{\prime} 01^{\prime \prime}$, & 0 & 0 & 0 & 2 \\
\hline & near & & in NE1/4 NW1/4 Sec 1, T.11N., & & & & \\
\hline & Martinsville, & & R.1W., Morgan County, on county & & & & \\
\hline & IN & & road parallel to and upstream from & & & & \\
\hline & & & State Highway $67,2.5$ miles west of & & & & \\
\hline & & & Martinsville and 0.5 mile northeast & & & & \\
\hline & & & of intersection with Mosier Road. & & & & \\
\hline
\end{tabular}




\section{Low-flow Characteristics for Selected Streams in Indiana}

Table 2. Low-flow characteristics for partial-record streamgaging stations in Indiana.-Continued

[mi², square miles; IN, Indiana; Lat, latitude; ' , degrees; ', minutes; ", seconds; long, longitude; Sec, Section; T, Township; R, Range; N, North; E, East; S, South; W, West; —, no correlation]

\begin{tabular}{|c|c|c|c|c|c|c|c|}
\hline \multirow{2}{*}{ Station number } & \multirow{2}{*}{ Station name } & \multirow{2}{*}{$\begin{array}{l}\text { Drainage } \\
\text { area }\left(\mathrm{mi}^{2}\right)\end{array}$} & \multirow{2}{*}{ Location } & \multicolumn{4}{|c|}{ Flow characteristic, in cubic feet per second } \\
\hline & & & & 1010 & 7010 & 30010 & $\begin{array}{c}\text { Harmomic } \\
\text { mean }\end{array}$ \\
\hline \multirow[t]{6}{*}{03354200} & Indian Creek & 19.4 & Lat $39^{\circ} 22^{\prime} 05^{\prime \prime}$, long $86^{\circ} 13^{\prime} 50^{\prime \prime}$, & 0 & 0 & 0 & - \\
\hline & near & & in NW1/4 Sec 29, T.11N., R.3E., & & & & \\
\hline & Morgantown, & & Johnson County, at bridge on High- & & & & \\
\hline & $\mathrm{IN}$ & & way 100 upstream from Barnes & & & & \\
\hline & & & Creek, 1.6 miles east of Morgan- & & & & \\
\hline & & & town. & & & & \\
\hline \multirow[t]{5}{*}{03357180} & Fish Creek near & 49.4 & Lat $39^{\circ} 12^{\prime} 45^{\prime \prime}$, long $86^{\circ} 53^{\prime} 48^{\prime \prime}$, & 0 & 0 & 0 & - \\
\hline & Freedom, & & in SW1/4 SE1/4 Sec 18, T.9N., & & & & \\
\hline & IN & & R.4W., Owen County, at bridge on & & & & \\
\hline & & & county road 2 miles west of Free- & & & & \\
\hline & & & dom, Ind. & & & & \\
\hline \multirow[t]{5}{*}{03357300} & Big Walnut & 119 & Lat $39^{\circ} 49^{\prime} 51^{\prime \prime}$, long $86^{\circ} 41^{\prime} 15^{\prime \prime}$, & 1.3 & 1.5 & 1.9 & 14 \\
\hline & Creek near & & in NW1/4 Sec 18, T.16N., R.2W., & & & & \\
\hline & Barnard, IN & & Hendricks County, at bridge on Put- & & & & \\
\hline & & & nam-Hendricks County line, 1.25 & & & & \\
\hline & & & miles southeast of Barnard. & & & & \\
\hline
\end{tabular}


Table 2. Low-flow characteristics for partial-record streamgaging stations in Indiana._Continued

[mi² , square miles; IN, Indiana; Lat, latitude; ', degrees; ', minutes; ", seconds; long, longitude; Sec, Section; T, Township; R, Range; N, North; E, East; S, South; W, West; - , no correlation]

\begin{tabular}{|c|c|c|c|c|c|c|c|}
\hline \multirow{2}{*}{ Station number } & \multirow{2}{*}{ Station name } & \multirow{2}{*}{$\begin{array}{l}\text { Drainage } \\
\text { area }\left(\mathrm{mi}^{2}\right)\end{array}$} & \multirow{2}{*}{ Location } & \multicolumn{4}{|c|}{ Flow characteristic, in cubic feet per second } \\
\hline & & & & 1010 & 7010 & 30010 & $\begin{array}{c}\text { Harmomic } \\
\text { mean }\end{array}$ \\
\hline 03357385 & $\begin{array}{l}\text { Miller Creek } \\
\text { near Fillmore, } \\
\text { IN }\end{array}$ & 11.0 & $\begin{array}{l}\text { Lat } 39^{\circ} 42^{\prime} 52^{\prime \prime} \text {, long } 86^{\circ} 45^{\prime} 04^{\prime \prime} \text {, } \\
\text { in NW1/4 NE1/4 Sec 33, T.15N., } \\
\text { R.3W., Putnam County, on county } \\
\text { road } 2 \text { miles north of Fillmore, } 0.1 \\
\text { mile east of Fillmore Road and } 0.5 \\
\text { mile upstream from confluence with } \\
\text { Clear Creek }\end{array}$ & 0.1 & 0.1 & 0.1 & 0.7 \\
\hline 03357400 & $\begin{array}{l}\text { Big Walnut } \\
\text { Creek near } \\
\text { Greencastle, } \\
\text { IN }\end{array}$ & 199 & $\begin{array}{l}\text { Lat } 39^{\circ} 40^{\prime} 43^{\prime \prime} \text {, long } 86^{\circ} 48^{\prime} 39^{\prime \prime} \text {, } \\
\text { in SW1/4 Sec 1, T.14N., R.4W., at } \\
\text { Pinhook Bridge, } 0.5 \text { mile upstream } \\
\text { from Monon railroad bridge and } 2.5 \\
\text { miles northeast of Greencastle. }\end{array}$ & 0.9 & 1.2 & 1.5 & 18 \\
\hline 03357700 & $\begin{array}{l}\text { Mud Creek near } \\
\text { Little Point, IN }\end{array}$ & 34.8 & $\begin{array}{l}\text { Lat } 39^{\circ} 34^{\prime} 34^{\prime \prime} \text {, long } 86^{\circ} 37^{\prime} 54^{\prime \prime} \text {, } \\
\text { on line between Secs } 9 \text { and } 10 \text {, } \\
\text { T.13N., R.2W., Morgan County, at } \\
\text { bridge on County Road } 1100 \text { West, } \\
0.8 \text { mile north of Little Point and } 1.7 \\
\text { miles south of the Hendricks-Mor- } \\
\text { gan County Line. }\end{array}$ & 0.8 & 0.9 & 1.2 & 4.6 \\
\hline
\end{tabular}


322 Low-flow Characteristics for Selected Streams in Indiana

Table 2. Low-flow characteristics for partial-record streamgaging stations in Indiana.—Continued

[mi², square miles; IN, Indiana; Lat, latitude; ' , degrees; ', minutes; ", seconds; long, longitude; Sec, Section; T, Township; R, Range; N, North; E, East; S, South; W, West; —, no correlation]

\begin{tabular}{|c|c|c|c|c|c|c|c|}
\hline \multirow{2}{*}{ Station number } & \multirow{2}{*}{ Station name } & \multirow{2}{*}{$\begin{array}{l}\text { Drainage } \\
\text { area }\left(\mathrm{mi}^{2}\right)\end{array}$} & \multirow{2}{*}{ Location } & \multicolumn{4}{|c|}{ Flow characteristic, in cubic feet per second } \\
\hline & & & & 1010 & 7010 & 30010 & $\begin{array}{c}\text { Harmomic } \\
\text { mean }\end{array}$ \\
\hline 03359980 & $\begin{array}{l}\text { Jordan Creek } \\
\text { near Jordan, } \\
\text { IN }\end{array}$ & 25.9 & $\begin{array}{l}\text { Lat } 39^{\circ} 24^{\prime} 09^{\prime \prime} \text {, long } 86^{\circ} 55^{\prime} 33^{\prime \prime} \text {, } \\
\text { in NW1/4 SE1/4 Sec 11., T.11N., } \\
\text { R.4W., Owen County, at bridge on } \\
\text { county road } 0.9 \text { mile east of Owen- } \\
\text { Clay County line, and } 0.5 \text { mile } \\
\text { northwest of Jordan. }\end{array}$ & 0.4 & 0.5 & 0.8 & 4.4 \\
\hline 03360050 & $\begin{array}{l}\text { Birch Creek } \\
\text { near Ashboro, } \\
\text { IN }\end{array}$ & 40.0 & $\begin{array}{l}\text { Lat } 39^{\circ} 24^{\prime} 14^{\prime \prime} \text {, long } 87^{\circ} 06^{\prime} 41^{\prime \prime} \text {, } \\
\text { in NE1/4 SE1/4 SE1/4 Sec 7, } \\
\text { T.11N., R.6W., Clay County, at } \\
\text { bridge on State Highway 59, } 0.5 \\
\text { mile northwest of Ashboro. }\end{array}$ & 0.5 & 0.6 & 0.9 & 4.5 \\
\hline 03360076 & $\begin{array}{l}\text { Birch Creek } \\
\text { near Old Hill, } \\
\text { IN }\end{array}$ & 71.3 & $\begin{array}{l}\text { Lat } 39^{\circ} 19^{\prime} 36^{\prime \prime} \text {, long } 87^{\circ} 10^{\prime} 47^{\prime \prime} \text {, } \\
\text { in SE1/4 NE1/4 NW1/4 Sec 10, } \\
\text { T.10N., R.7W., Clay County, at } \\
\text { county road bridge and } 0.7 \text { mile } \\
\text { from the mouth. }\end{array}$ & 0.7 & 0.8 & 1.1 & 4.9 \\
\hline 03360115 & $\begin{array}{l}\text { Lick Creek near } \\
\text { Coal City, IN }\end{array}$ & 47.0 & $\begin{array}{l}\text { Lat } 39^{\circ} 10^{\prime} 48^{\prime \prime} \text {, long } 86^{\circ} 59^{\prime} 47^{\prime \prime} \text {, } \\
\text { in SW1/4 SW1/4 Sec } 29, \text { T.9N., } \\
\text { R.5W., Owen County, on Owen } \\
\text { County Road, } 1.5 \text { miles upstream } \\
\text { from confluence with Beech Creek } \\
\text { and } 4.1 \text { miles southeast of Coal City. }\end{array}$ & 0 & 0 & 0 & - \\
\hline
\end{tabular}


Table 2. Low-flow characteristics for partial-record streamgaging stations in Indiana._Continued

[mi² , square miles; IN, Indiana; Lat, latitude; ', degrees; ', minutes; ", seconds; long, longitude; Sec, Section; T, Township; R, Range; N, North; E, East; S, South; W, West; - , no correlation]

\begin{tabular}{|c|c|c|c|c|c|c|c|}
\hline \multirow{2}{*}{ Station number } & \multirow{2}{*}{ Station name } & \multirow{2}{*}{$\begin{array}{l}\text { Drainage } \\
\text { area }\left(\mathrm{mi}^{2}\right)\end{array}$} & \multirow{2}{*}{ Location } & \multicolumn{4}{|c|}{ Flow characteristic, in cubic feet per second } \\
\hline & & & & 1010 & 7010 & 30010 & $\begin{array}{c}\text { Harmomic } \\
\text { mean }\end{array}$ \\
\hline 03360120 & $\begin{array}{l}\text { Connelly Ditch } \\
\text { near Jasonville, } \\
\text { IN }\end{array}$ & 31.0 & $\begin{array}{l}\text { Lat } 39^{\circ} 12^{\prime} 45^{\prime \prime} \text {, long } 87^{\circ} 07^{\prime} 40^{\prime \prime} \text {, } \\
\text { in NE1/4 NE1/4 NE1/4, Sec } 24 \text {, } \\
\text { T.9N., R.7W., Clay County, on Clay } \\
\text { County Road } 1.75 \text { miles above } \\
\text { mouth and } 7.5 \text { miles northeast of } \\
\text { Jasonville. }\end{array}$ & 0.2 & 0.2 & 0.3 & - \\
\hline 03360200 & $\begin{array}{l}\text { Lattas Creek } \\
\text { near Switz City, } \\
\text { IN }\end{array}$ & 32.7 & $\begin{array}{l}\text { Lat } 39^{\circ} 02^{\prime} 40^{\prime \prime} \text {, long } 87^{\circ} 02^{\prime} 38^{\prime \prime} \text {, } \\
\text { in SW1/4 Sec } 14 \text {, T.7N., R.6W., } \\
\text { Greene County, at bridge on State } \\
\text { Highway } 67,0.9 \text { mile northeast of } \\
\text { Switz City. }\end{array}$ & 0 & 0 & 0 & - \\
\hline 03360800 & $\begin{array}{l}\text { Prairie Creek } \\
\text { near } \\
\text { Washington, } \\
\text { IN }\end{array}$ & 120 & $\begin{array}{l}\text { Lat } 38^{\circ} 43^{\prime} 01^{\prime \prime} \text {, long } 87^{\circ} 10^{\prime} 00^{\prime \prime} \text {, } \\
\text { in SW1/4 SW1/4 Sec 2, T.3N., } \\
\text { R. } 7 \text { W., Daviess County, at bridge } \\
\text { on State Highway } 57,4.0 \text { miles } \\
\text { north of Washington. }\end{array}$ & 0 & 0 & - & - \\
\hline 03360920 & $\begin{array}{l}\text { Big Blue River } \\
\text { near New } \\
\text { Castle, IN }\end{array}$ & 15.8 & $\begin{array}{l}\text { Lat } 40^{\circ} 00^{\prime} 15^{\prime \prime} \text {, long } 85^{\circ} 20^{\prime} 48^{\prime \prime} \text {, } \\
\text { on line between Secs } 23 \text { and } 24 \text {, } \\
\text { T.18N., R.10E., Henry County, } 100 \\
\text { feet downstream from U.S. Highway } \\
36 \text { bridge, } 5.5 \text { miles north of New } \\
\text { Castle. }\end{array}$ & 2.2 & 2.3 & 2.9 & 8 \\
\hline
\end{tabular}




\section{Low-flow Characteristics for Selected Streams in Indiana}

Table 2. Low-flow characteristics for partial-record streamgaging stations in Indiana.-Continued

[mi², square miles; IN, Indiana; Lat, latitude; ' , degrees; ', minutes; ", seconds; long, longitude; Sec, Section; T, Township; R, Range; N, North; E, East; S, South; W, West; —, no correlation]

\begin{tabular}{|c|c|c|c|c|c|c|c|}
\hline \multirow{2}{*}{ Station number } & \multirow{2}{*}{ Station name } & \multirow{2}{*}{$\begin{array}{l}\text { Drainage } \\
\text { area }\left(\mathrm{mi}^{2}\right)\end{array}$} & \multirow{2}{*}{ Location } & \multicolumn{4}{|c|}{ Flow characteristic, in cubic feet per second } \\
\hline & & & & 1010 & 7010 & 30010 & $\begin{array}{c}\text { Harmomic } \\
\text { mean }\end{array}$ \\
\hline 03360965 & Duck Creek at & 24.9 & Lat $39^{\circ} 52^{\prime} 46^{\prime \prime}$, long $85^{\circ} 28^{\prime} 03^{\prime \prime}$, & 1.8 & 2 & 2.7 & 9 \\
\hline$(395246085280$ & Greensboro, & & in SW1/4 NE1/4 Sec 35, T.17N., & & & & \\
\hline \multirow[t]{3}{*}{ 400) } & $\mathrm{IN}$ & & R.9E., Henry County, at bridge on & & & & \\
\hline & & & County Road 350 South at west side & & & & \\
\hline & & & of Greensboro. & & & & \\
\hline \multirow[t]{5}{*}{03361400} & Little Blue & 97.0 & Lat $39^{\circ} 33^{\prime} 16^{\prime \prime}$, long $85^{\circ} 43^{\prime} 08^{\prime \prime}$, & 0.6 & 0.8 & 1 & - \\
\hline & River near Rays & & on line between Secs 23 and 26, & & & & \\
\hline & Crossing, IN & & T.13N., R.7E., Shelby County, at & & & & \\
\hline & & & bridge on County Highway, 2.8 & & & & \\
\hline & & & miles west of Rays Crossing. & & & & \\
\hline \multirow[t]{5}{*}{03361600} & Brandywine & 24.3 & Lat $39^{\circ} 51^{\prime} 30^{\prime \prime}$, long $85^{\circ} 44^{\prime} 17^{\prime \prime}$, & 0 & 0 & 0.1 & 2 \\
\hline & Creek near & & on line between Secs 3 and 10, & & & & \\
\hline & Maxwell, IN & & T.16N., R.7E., Hancock County, at & & & & \\
\hline & & & county highway bridge 1.6 miles & & & & \\
\hline & & & east of Maxwell. & & & & \\
\hline \multirow[t]{6}{*}{03361700} & Sugar Creek & 130 & Lat $39^{\circ} 38^{\prime} 49^{\prime \prime}$, long $85^{\circ} 55^{\prime} 09^{\prime \prime}$, & 5.4 & 5.9 & 6.9 & 30 \\
\hline & near Pleasant & & in E1/2 Sec 24, T.14N., R.5E., & & & & \\
\hline & View, IN & & Shelby County, tributary to Drift- & & & & \\
\hline & & & wood River south of I-74 bridge, & & & & \\
\hline & & & 1.75 miles southeast of Pleasant & & & & \\
\hline & & & View. & & & & \\
\hline
\end{tabular}


Table 2. Low-flow characteristics for partial-record streamgaging stations in Indiana.-Continued

[mi² , square miles; IN, Indiana; Lat, latitude; ', degrees; ', minutes; ", seconds; long, longitude; Sec, Section; T, Township; R, Range; N, North; E, East; S, South; W, West; - , no correlation]

\begin{tabular}{|c|c|c|c|c|c|c|c|}
\hline \multirow{2}{*}{ Station number } & \multirow{2}{*}{ Station name } & \multirow{2}{*}{$\begin{array}{l}\text { Drainage } \\
\text { area }\left(\mathrm{mi}^{2}\right)\end{array}$} & \multirow{2}{*}{ Location } & \multicolumn{4}{|c|}{ Flow characteristic, in cubic feet per second } \\
\hline & & & & 1010 & 7010 & 30010 & $\begin{array}{c}\text { Harmomic } \\
\text { mean }\end{array}$ \\
\hline 03361800 & $\begin{array}{l}\text { Buck Creek } \\
\text { near New } \\
\text { Bethel, IN }\end{array}$ & 51.0 & $\begin{array}{l}\text { Lat } 39^{\circ} 43^{\prime} 34^{\prime \prime} \text {, long } 85^{\circ} 58^{\prime} 21^{\prime \prime} \text {, } \\
\text { on line between Secs } 21 \text { and } 28 \text {, } \\
\text { T.15N., R.5E., Marion County, at } \\
\text { bridge on East Troy Avenue } 2.4 \\
\text { miles northeast of New Bethel. }\end{array}$ & 0.1 & 0.1 & 0.2 & 10 \\
\hline 03361900 & $\begin{array}{l}\text { Hurricane Creek } \\
\text { near Franklin, } \\
\text { IN }\end{array}$ & 14.0 & $\begin{array}{l}\text { Lat } 39^{\circ} 29^{\prime} 53^{\prime \prime} \text {, long } 86^{\circ} 01^{\prime} 34^{\prime \prime} \text {, } \\
\text { on line between Secs } 7 \text { and } 12 \text {, } \\
\text { T.12N., R.5E., Johnson County, } 400 \\
\text { East Road at bridge } 1.0 \text { mile north- } \\
\text { east of Franklin. }\end{array}$ & 0 & 0 & 0 & - \\
\hline 03363200 & $\begin{array}{l}\text { Flatrock River } \\
\text { near Lewisville, } \\
\text { IN }\end{array}$ & 47.8 & $\begin{array}{l}\text { Lat } 39^{\circ} 48^{\prime} 24^{\prime \prime} \text {, long } 85^{\circ} 21^{\prime} 29^{\prime \prime} \text {, } \\
\text { NW1/4 SE1/4 Sec } 25, \mathrm{~T} .16 \mathrm{~N} . \text {, } \\
\text { R.10E., Henry County, at bridge on } \\
\text { U.S. Highway } 40 \text { at Lewisville. }\end{array}$ & 2.3 & 2.6 & 3.1 & 14 \\
\hline 03363400 & $\begin{array}{l}\text { Flatrock River } \\
\text { near Rushville, } \\
\text { IN }\end{array}$ & 167 & $\begin{array}{l}\text { Lat } 39^{\circ} 36^{\prime} 15^{\prime \prime} \text {, long } 85^{\circ} 26^{\prime} 39^{\prime \prime} \text {, } \\
\text { NW1/4 SW1/4 Sec } 5, \text { T.13N., } \\
\text { R.10E., Rush County, at bridge on } \\
\text { U.S. Highway 52, } 0.3 \text { mile south of } \\
\text { court house in Rushville. }\end{array}$ & 4.2 & 4.8 & 5.8 & 24 \\
\hline
\end{tabular}


Table 2. Low-flow characteristics for partial-record streamgaging stations in Indiana.-Continued

[mi², square miles; IN, Indiana; Lat, latitude; ' , degrees; ', minutes; ", seconds; long, longitude; Sec, Section; T, Township; R, Range; N, North; E, East; S, South; W, West; —, no correlation]

\begin{tabular}{|c|c|c|c|c|c|c|c|}
\hline \multirow{2}{*}{ Station number } & \multirow{2}{*}{ Station name } & \multirow{2}{*}{$\begin{array}{l}\text { Drainage } \\
\text { area }\left(\mathrm{mi}^{2}\right)\end{array}$} & \multirow{2}{*}{ Location } & \multicolumn{4}{|c|}{ Flow characteristic, in cubic feet per second } \\
\hline & & & & 1010 & 7010 & 30010 & $\begin{array}{c}\text { Harmomic } \\
\text { mean }\end{array}$ \\
\hline \multirow[t]{6}{*}{03363450} & Little Flatrock & 34.8 & Lat $39^{\circ} 29^{\prime} 49^{\prime \prime}$, long $85^{\circ} 28^{\prime} 24^{\prime \prime}$, & 0 & 0 & 0 & 2.3 \\
\hline & River at Milroy, & & in NW1/4 NE1/4 NW1/4 Sec 13, & & & & \\
\hline & $\mathrm{IN}$ & & T.21N., R.9E., Rush County, at & & & & \\
\hline & & & bridge on State Highway 244, 800 & & & & \\
\hline & & & feet east of State Highway 3 at the & & & & \\
\hline & & & west edge of Milroy. & & & & \\
\hline \multirow[t]{6}{*}{03364711} & Little Sand & 43.1 & Lat $39^{\circ} 07^{\prime} 44^{\prime \prime}$, long $85^{\circ} 51^{\prime} 21^{\prime \prime}$, in & 0 & 0 & 0.1 & 1 \\
\hline & Creek near & & NE1/4 NE1/4 Sec.21, T.8N., R.6E., & & & & \\
\hline & Elizabethtown, & & Bartholomew County, at bridge on & & & & \\
\hline & $\mathrm{IN}$ & & county road, 1.1 miles west of U.S. & & & & \\
\hline & & & Highway 31 , and 2.5 miles west of & & & & \\
\hline & & & Elizabethtown. & & & & \\
\hline \multirow[t]{5}{*}{03364800} & Sand Creek near & 91.6 & Lat $39^{\circ} 20^{\prime} 55^{\prime \prime}$, long $85^{\circ} 26^{\prime} 51^{\prime \prime}$, & 0 & 0 & 0 & - \\
\hline & Greensburg, & & NE1/4 Sec 6, T.10N., R.10E., Deca- & & & & \\
\hline & $\mathrm{IN}$ & & tur County, at county highway & & & & \\
\hline & & & bridge 2.5 miles northeast of & & & & \\
\hline & & & Greensburg. & & & & \\
\hline \multirow[t]{5}{*}{03365600} & White Creek & 96.2 & Lat $38^{\circ} 58^{\prime} 46^{\prime \prime}$, long $86^{\circ} 00^{\prime} 58^{\prime \prime}$, & 0 & 0 & 0 & 0.9 \\
\hline & near Cortland, & & on line between Secs 6 and 7, T.6N., & & & & \\
\hline & IN & & R.5E., Jackson County, at bridge on & & & & \\
\hline & & & State Highway 258, 3 miles west of & & & & \\
\hline & & & Cortland. & & & & \\
\hline
\end{tabular}


Table 2. Low-flow characteristics for partial-record streamgaging stations in Indiana._Continued

[mi² , square miles; IN, Indiana; Lat, latitude; ', degrees; ', minutes; ", seconds; long, longitude; Sec, Section; T, Township; R, Range; N, North; E, East; S, South; W, West; - , no correlation]

\begin{tabular}{|c|c|c|c|c|c|c|c|}
\hline \multirow{2}{*}{ Station number } & \multirow{2}{*}{ Station name } & \multirow{2}{*}{$\begin{array}{l}\text { Drainage } \\
\text { area }\left(\mathrm{mi}^{2}\right)\end{array}$} & \multirow{2}{*}{ Location } & \multicolumn{4}{|c|}{ Flow characteristic, in cubic feet per second } \\
\hline & & & & 1010 & 7010 & 30010 & $\begin{array}{c}\text { Harmomic } \\
\text { mean }\end{array}$ \\
\hline \multirow[t]{4}{*}{03366300} & Big Creek near & 96.9 & Lat $38^{\circ} 46^{\prime} 47^{\prime \prime}$, long $85^{\circ} 32^{\prime} 57^{\prime \prime}$, & 0 & 0 & 0.1 & 1.4 \\
\hline & Volga, IN & & NE1/4 NW1/4 Sec 20, T.4N., R.9E., & & & & \\
\hline & & & Jefferson County, at county highway & & & & \\
\hline & & & bridge, 1.7 miles west of Volga. & & & & \\
\hline \multirow[t]{6}{*}{033673005} & Stucker Fork at & 76.2 & Lat $38^{\circ} 41^{\prime} 41^{\prime \prime}$, long $85^{\circ} 45^{\prime} 24^{\prime \prime}$, & 0 & 0 & - & - \\
\hline & Scottsburg, IN & & on line between Secs 16 and 17, & & & & \\
\hline & & & T.3N., R.7E., Scott County, at & & & & \\
\hline & & & county highway bridge, 0.6 mile & & & & \\
\hline & & & north of State Highway 56, 1 mile & & & & \\
\hline & & & east of Scottsburg. & & & & \\
\hline \multirow[t]{6}{*}{0336940050} & Otter Creek near & 70.7 & Lat $38^{\circ} 59^{\prime} 20^{\prime \prime}$, long $85^{\circ} 34^{\prime} 32^{\prime \prime}$, & 0 & 0 & 0 & 1.3 \\
\hline & Vernon, IN & & in SE1/4 Sec 1, T.6N., R.8E., Jen- & & & & \\
\hline & (formerly & & nings County, 500 feet below bridge & & & & \\
\hline & called South & & on county road, 2 miles east of & & & & \\
\hline & Fork Vernon & & Vernon Bridge known as & & & & \\
\hline & Fork) & & Hinchman's Ford Bridge. & & & & \\
\hline \multirow[t]{6}{*}{03371525} & Guthrie Creek & 68.9 & Lat $38^{\circ} 47^{\prime} 22^{\prime \prime}$, long $86^{\circ} 21^{\prime} 33^{\prime \prime}$, & 0 & 0 & 0 & 1.2 \\
\hline & near Tunnelton, & & SW1/4 SE1/4 SW1/4, Sec 12, T.4N., & & & & \\
\hline & $\mathrm{IN}$ & & R.11E., 100 feet below county road & & & & \\
\hline & & & bridge, 0.8 mile north of intersection & & & & \\
\hline & & & with road from Buddha to Tunnelton, & & & & \\
\hline & & & 1.1 miles northwest of Tunnelton. & & & & \\
\hline
\end{tabular}


Table 2. Low-flow characteristics for partial-record streamgaging stations in Indiana.-Continued

[mi², square miles; IN, Indiana; Lat, latitude; ' , degrees; ', minutes; ", seconds; long, longitude; Sec, Section; T, Township; R, Range; N, North; E, East; S, South; W, West; —, no correlation]

\begin{tabular}{|c|c|c|c|c|c|c|c|}
\hline \multirow{2}{*}{ Station number } & \multirow{2}{*}{ Station name } & \multirow{2}{*}{$\begin{array}{l}\text { Drainage } \\
\text { area }\left(\mathrm{mi}^{2}\right)\end{array}$} & \multirow{2}{*}{ Location } & \multicolumn{4}{|c|}{ Flow characteristic, in cubic feet per second } \\
\hline & & & & 1010 & 7010 & 30010 & $\begin{array}{c}\text { Harmomic } \\
\text { mean }\end{array}$ \\
\hline \multirow[t]{5}{*}{03371530} & Leatherwood & 37.6 & $\begin{array}{l}\text { Lat } 38^{\circ} 50^{\prime} 23^{\prime \prime} \text {, long } 86^{\circ} 28^{\prime} 38^{\prime \prime}, \text { SE } 1 / 4 \\
\text { SW1/4 NW1/4 Sec. } 25 \text {, }\end{array}$ & 0.1 & 0.1 & 0.1 & 1.4 \\
\hline & Creek at & & T.5N., R.1W., Lawrence County at & & & & \\
\hline & Bedford, IN & & bridge on county road, 1.6 miles & & & & \\
\hline & & & southeast from court house in & & & & \\
\hline & & & Bedford. & & & & \\
\hline \multirow[t]{4}{*}{03371550} & Middle Fork & 38.3 & $\begin{array}{l}\text { Lat } 39^{\circ} 05^{\prime} 37^{\prime \prime} \text {, long } 86^{\circ} 12^{\prime} 30^{\prime \prime} \text {, in SE1/4 } \\
\text { SE1/4 Sec 9, T.8N., R.3E., }\end{array}$ & 0 & 0 & 0 & - \\
\hline & Salt Creek at & & Brown County, Ohio River Basin at & & & & \\
\hline & Story, IN & & Bridge on State Highway $135,0.5$ & & & & \\
\hline & & & mile southeast of Story. & & & & \\
\hline \multirow[t]{4}{*}{03373600} & Lick Creek near & 18.9 & Lat $38^{\circ} 32^{\prime} 42^{\prime \prime}$, long $86^{\circ} 26^{\prime} 56^{\prime \prime}$, & 0.3 & 0.3 & 0.4 & - \\
\hline & Paoli, IN & & SW1/4 Sec 6, T.1N., R.1E., Orange & & & & \\
\hline & & & County, at bridge on county road, & & & & \\
\hline & & & 1.3 miles southeast of Paoli. & & & & \\
\hline \multirow[t]{7}{*}{04093900} & Coffee Creek at & 15.0 & Lat $41^{\circ} 36^{\prime} 24^{\prime \prime}$, long $87^{\circ} 03^{\prime} 03^{\prime \prime}$, & 4.7 & 5 & 5.4 & 12 \\
\hline & Chesterton, IN & & on line between Secs 1 and 36, & & & & \\
\hline & & & T.37N., R.6W., Porter County, at & & & & \\
\hline & & & bridge on Porter Avenue, 0.5 mile & & & & \\
\hline & & & east of intersection with Calumet & & & & \\
\hline & & & Road (Old State Highway 49), in & & & & \\
\hline & & & Chesterton. & & & & \\
\hline
\end{tabular}


Table 2. Low-flow characteristics for partial-record streamgaging stations in Indiana._Continued

[mi², square miles; IN, Indiana; Lat, latitude; ', degrees; ', minutes; ", seconds; long, longitude; Sec, Section; T, Township; R, Range; N, North; E, East; S, South; W, West; --, no correlation]

\begin{tabular}{|c|c|c|c|c|c|c|c|}
\hline \multirow{2}{*}{ Station number } & \multirow{2}{*}{ Station name } & \multirow{2}{*}{$\begin{array}{l}\text { Drainage } \\
\text { area }\left(\mathrm{mi}^{2}\right)\end{array}$} & \multirow{2}{*}{ Location } & \multicolumn{4}{|c|}{ Flow characteristic, in cubic feet per second } \\
\hline & & & & 1010 & 7010 & 30010 & $\begin{array}{c}\text { Harmomic } \\
\text { mean }\end{array}$ \\
\hline 04099070 & $\begin{array}{l}\text { Pigeon Creek } \\
\text { near Hamilton, } \\
\text { IN }\end{array}$ & 43.3 & $\begin{array}{l}\text { Lat } 41^{\circ} 36^{\prime} 16^{\prime \prime} \text {, long } 84^{\circ} 56^{\prime} 32^{\prime} \text {, } \\
\text { in NW1/4 SW1/4 SE1/4 Sec } 5 \text {, } \\
\text { T.36N., R.14E., } 3.3 \text { miles southeast } \\
\text { of Angola, then south } 1.5 \text { miles to a } \\
\text { "y" road, then northeast } 0.3 \text { mile to } \\
\text { bridge. }\end{array}$ & 1.9 & 2 & 2.3 & 12 \\
\hline 04099692 & $\begin{array}{l}\text { Rowe Ditch } \\
\text { near Howe, IN }\end{array}$ & 7.52 & $\begin{array}{l}\text { Lat } 41^{\circ} 42^{\prime} 32^{\prime \prime} \text {, long } 85^{\circ} 26^{\prime} 18^{\prime \prime} \text {, in } \\
\text { NE1/4 SE1/4 Sec } 5 \text {, T.38N., R.9E., } \\
\text { Lagrange County, at pipe culvert on } \\
\text { County Road } 450 \text { North, } 9 \text { miles } \\
\text { south of intersection with Highway } \\
\text { 120, } 1.9 \text { miles southwest of Howe. }\end{array}$ & 1.9 & 2 & 2.4 & 5.2 \\
\hline 04099805 & $\begin{array}{l}\text { Little Elkhart } \\
\text { River near } \\
\text { Middlebury, } \\
\text { IN }\end{array}$ & 60.6 & $\begin{array}{l}\text { Lat } 41^{\circ} 39^{\prime} 15^{\prime \prime} \text {, long } 85^{\circ} 40^{\prime} 14^{\prime \prime} \text {, } \\
\text { on line between Secs } 13 \text { and } 24 \text {, } \\
\text { T.37N., R.7E., Elkhart County, at } \\
\text { bridge on U.S. Highway } 20,2.2 \\
\text { miles southeast of Middlebury. }\end{array}$ & 14 & 15 & 16 & 26 \\
\hline 04100490 & $\begin{array}{l}\text { Turkey Creek } \\
\text { near New Paris, } \\
\text { IN }\end{array}$ & 169 & $\begin{array}{l}\text { Lat } 41^{\circ} 30^{\prime} 01^{\prime \prime} \text {, long } 85^{\circ} 50^{\prime} 32^{\prime \prime} \text {, } \\
\text { in SW1/4 Sec } 9 \text {, T.35N., R.6E., } \\
\text { Elkhart County, at county highway } \\
\text { bridge, } 0.4 \text { mile west of New Paris. }\end{array}$ & 22 & 24 & 27 & 76 \\
\hline
\end{tabular}


330 Low-flow Characteristics for Selected Streams in Indiana

Table 2. Low-flow characteristics for partial-record streamgaging stations in Indiana.—Continued

[mi², square miles; IN, Indiana; Lat, latitude; ' , degrees; ', minutes; ", seconds; long, longitude; Sec, Section; T, Township; R, Range; N, North; E, East; S, South; W, West; —, no correlation]

\begin{tabular}{|c|c|c|c|c|c|c|c|}
\hline \multirow{2}{*}{ Station number } & \multirow{2}{*}{ Station name } & \multirow{2}{*}{$\begin{array}{l}\text { Drainage } \\
\text { area }\left(\mathrm{mi}^{2}\right)\end{array}$} & \multirow{2}{*}{ Location } & \multicolumn{4}{|c|}{ Flow characteristic, in cubic feet per second } \\
\hline & & & & 1010 & 7010 & 30010 & $\begin{array}{c}\text { Harmomic } \\
\text { mean }\end{array}$ \\
\hline \multirow[t]{5}{*}{04100800} & Yellow Creek at & 32.4 & Lat $41^{\circ} 38^{\prime} 44^{\prime \prime}$, long $85^{\circ} 56^{\prime} 00^{\prime \prime}$, & 2.2 & 2.4 & 2.8 & 7.4 \\
\hline & Dunlap, IN & & in NE1/4 NE1/4 NE1/4 Sec 22, & & & & \\
\hline & & & T.37N., R.5E., Elkhart County, at & & & & \\
\hline & & & bridge on U.S. Highway 33 at north- & & & & \\
\hline & & & west edge of Dunlap. & & & & \\
\hline \multirow[t]{6}{*}{04177800} & Fish Creek near & 95.8 & Lat $41^{\circ} 29^{\prime} 13^{\prime \prime}$, long $84^{\circ} 50^{\prime} 13^{\prime \prime}$, & 1.9 & 2.4 & 3.3 & 19 \\
\hline & Artic, IN & & SE1/4 SE1/4 Sec 18, T.35N., & & & & \\
\hline & & & R.15E., De Kalb County Road No. 12 & & & & \\
\hline & & & bridge, 1.7 miles northwest of Artic, & & & & \\
\hline & & & and 1.9 miles east of Jerusalem & & & & \\
\hline & & & School. & & & & \\
\hline \multirow[t]{7}{*}{04177900} & Big Run at & 16.7 & Lat $41^{\circ} 26^{\prime} 09^{\prime \prime}$, long $84^{\circ} 52^{\prime} 08^{\prime \prime}$, & 0.8 & 0.8 & 1 & 3 \\
\hline & Butler, IN & & SW1/4 NW1/4 Sec 1, T.34N., & & & & \\
\hline & & & R.14E., De Kalb County at bridge on & & & & \\
\hline & & & State Highway 1, 0.6 mile due north & & & & \\
\hline & & & of intersection of U.S. Highway 6 , & & & & \\
\hline & & & and State Highway 1, north of But- & & & & \\
\hline & & & ler. & & & & \\
\hline
\end{tabular}


Table 2. Low-flow characteristics for partial-record streamgaging stations in Indiana._Continued

[mi² , square miles; IN, Indiana; Lat, latitude; ', degrees; ', minutes; ", seconds; long, longitude; Sec, Section; T, Township; R, Range; N, North; E, East; S, South; W, West; - , no correlation]

\begin{tabular}{|c|c|c|c|c|c|c|c|}
\hline \multirow{2}{*}{ Station number } & \multirow{2}{*}{ Station name } & \multirow{2}{*}{$\begin{array}{l}\text { Drainage } \\
\text { area }\left(\mathrm{mi}^{2}\right)\end{array}$} & \multirow{2}{*}{ Location } & \multicolumn{4}{|c|}{ Flow characteristic, in cubic feet per second } \\
\hline & & & & 1010 & 7010 & 30010 & $\begin{array}{c}\text { Harmomic } \\
\text { mean }\end{array}$ \\
\hline 04178400 & $\begin{array}{l}\text { Bear Creek near } \\
\text { Saint Joe, IN }\end{array}$ & 23.9 & $\begin{array}{l}\text { Lat } 41^{\circ} 18^{\prime} 58^{\prime \prime} \text {, long } 84^{\circ} 53^{\prime} 32^{\prime \prime} \text {, } \\
\text { in SE1/4 SE1/4 Sec } 15, \text { T.33N., } \\
\text { R.14E., De Kalb County, at bridge on } \\
\text { State Highway 1, } 0.1 \text { mile north of } \\
\text { B \& O Railroad and } 0.4 \text { mile east of } \\
\text { Saint Joe. }\end{array}$ & 0.9 & 1.1 & 1.4 & 4.5 \\
\hline 04178500 & $\begin{array}{l}\text { St. Joseph River } \\
\text { at Hursh, IN }\end{array}$ & 734 & $\begin{array}{l}\text { Lat } 41^{\circ} 14^{\prime} 47^{\prime \prime} \text {, long } 84^{\circ} 58^{\prime} 23^{\prime \prime} \text {, } \\
\text { in Sec } 12, T .32 N ., \text { R.13E., Allen } \\
\text { County, near center of span on } \\
\text { downstream side of Hubstown } \\
\text { bridge at Hursh, and } 5.5 \text { miles } \\
\text { upstream from Cedar Creek. }\end{array}$ & 33 & 35 & 42 & 168 \\
\hline 04179308 & $\begin{array}{l}\text { Dibbling Ditch } \\
\text { near Waterloo, } \\
\text { IN }\end{array}$ & 12.9 & $\begin{array}{l}\text { Lat } 41^{\circ} 27^{\prime} 03^{\prime \prime} \text {, long } 85^{\circ} 02^{\prime} 25^{\prime \prime} \text {, } \\
\text { in NW1/4 NW1/4 Sec 33, T.35N., } \\
\text { R.13E., De Kalb County, at bridge on } \\
\text { County Road 22, } 0.8 \text { mile west of } \\
\text { County Road 35, } 1.6 \text { miles north- } \\
\text { west of Waterloo. }\end{array}$ & 0.5 & 0.6 & 0.6 & 2.9 \\
\hline 04179310 & $\begin{array}{l}\text { Cedar Creek at } \\
\text { Waterloo, IN }\end{array}$ & 48.8 & $\begin{array}{l}\text { Lat } 41^{\circ} 26^{\prime} 14^{\prime \prime} \text {, long } 85^{\circ} 01^{\prime} 03^{\prime \prime} \text {, } \\
\text { NW1/4 Sec 3, T.34N., R.13E., } \\
\text { De Kalb County, at bridge on U.S. } \\
\text { Highway 427, } 2.3 \text { miles northeast of } \\
\text { Waterloo. }\end{array}$ & 1.6 & 1.8 & 2.2 & 11 \\
\hline
\end{tabular}




\section{Low-flow Characteristics for Selected Streams in Indiana}

Table 2. Low-flow characteristics for partial-record streamgaging stations in Indiana.-Continued

[mi², square miles; IN, Indiana; Lat, latitude; ' , degrees; ', minutes; ", seconds; long, longitude; Sec, Section; T, Township; R, Range; N, North; E, East; S, South; W, West; —, no correlation]

\begin{tabular}{|c|c|c|c|c|c|c|c|}
\hline \multirow{2}{*}{ Station number } & \multirow{2}{*}{ Station name } & \multirow{2}{*}{$\begin{array}{l}\text { Drainage } \\
\text { area }\left(\mathrm{mi}^{2}\right)\end{array}$} & \multirow{2}{*}{ Location } & \multicolumn{4}{|c|}{ Flow characteristic, in cubic feet per second } \\
\hline & & & & 1010 & 7010 & 30010 & $\begin{array}{c}\text { Harmomic } \\
\text { mean }\end{array}$ \\
\hline \multirow[t]{5}{*}{04179800} & Little Cedar & 72.3 & Lat $41^{\circ} 16^{\prime} 08^{\prime \prime}$, long $85^{\circ} 08^{\prime} 07^{\prime \prime}$, & 3.4 & 3.7 & 4.2 & 15 \\
\hline & Creek near & & on line between Secs 33 and 34, & & & & \\
\hline & Garrett, IN & & T.33N., R.12E., De Kalb County, at & & & & \\
\hline & & & bridge on U.S. Highway 27, 0.25 mile & & & & \\
\hline & & & north of Allen-De Kalb County line. & & & & \\
\hline \multirow[t]{6}{*}{04179900} & Willow Creek & 19.0 & Lat $41^{\circ} 14^{\prime} 37^{\prime \prime}$, long $85^{\circ} 10^{\prime} 03^{\prime \prime}$, & 0.2 & 0.2 & 0.3 & 4.2 \\
\hline & near & & in SE1/4 NW1/4 Sec 8, T.32N., & & & & \\
\hline & Huntertown, & & R.12E., Allen County, at bridge on & & & & \\
\hline & IN & & State Highway 3, about 300 feet & & & & \\
\hline & & & north of Shoaf Road, 2.1 miles north & & & & \\
\hline & & & of Huntertown. & & & & \\
\hline \multirow[t]{6}{*}{04181100} & Blue Creek near & 78.5 & Lat $40^{\circ} 44^{\prime} 49^{\prime \prime}$, long $84^{\circ} 49^{\prime} 20^{\prime \prime}$, & 0.4 & 0.6 & 0.9 & 4.7 \\
\hline & Pleasant Mills, & & in NE1/4 NE1/4 Sec 4, T.26N., & & & & \\
\hline & IN & & R.5E., Adams County, at bridge on & & & & \\
\hline & & & State Highway 124, 1.5 miles west & & & & \\
\hline & & & of Willshire, Ohio, and 2.2 miles & & & & \\
\hline & & & southeast of Pleasant Mills. & & & & \\
\hline \multirow[t]{6}{*}{04181600} & Holthouse Ditch & 34.0 & Lat $40^{\circ} 50^{\prime} 48^{\prime \prime}$, long $84^{\circ} 56^{\prime} 44^{\prime \prime}$, & 0 & 0 & 0 & - \\
\hline & near Decatur, & & in the NE1/4 NW1/4 NE1/4 Sec 32, & & & & \\
\hline & IN & & T.28N., R.14E., Adams County, at & & & & \\
\hline & & & Winchester Road bridge, 0.4 mile & & & & \\
\hline & & & upstream from mouth, and 0.5 mile & & & & \\
\hline & & & northwest of Decatur. & & & & \\
\hline
\end{tabular}


Table 2. Low-flow characteristics for partial-record streamgaging stations in Indiana._Continued

[mi² , square miles; IN, Indiana; Lat, latitude; ', degrees; ', minutes; ", seconds; long, longitude; Sec, Section; T, Township; R, Range; N, North; E, East; S, South; W, West; - , no correlation]

\begin{tabular}{|c|c|c|c|c|c|c|c|}
\hline \multirow{2}{*}{ Station number } & \multirow{2}{*}{ Station name } & \multirow{2}{*}{$\begin{array}{l}\text { Drainage } \\
\text { area }\left(\mathrm{mi}^{2}\right)\end{array}$} & \multirow{2}{*}{ Location } & \multicolumn{4}{|c|}{ Flow characteristic, in cubic feet per second } \\
\hline & & & & 1010 & 7010 & 30010 & $\begin{array}{c}\text { Harmomic } \\
\text { mean }\end{array}$ \\
\hline 04181800 & $\begin{array}{l}\text { Nickelsen Creek } \\
\text { near Poe, } \\
\text { IN }\end{array}$ & 25.6 & $\begin{array}{l}\text { Lat } 40^{\circ} 55^{\prime} 36^{\prime \prime} \text {, long } 85^{\circ} 03^{\prime} 57^{\prime \prime} \text {, } \\
\text { in SE1/4 NW1/4 Sec 33, T.29N., } \\
\text { R.13E., Allen County, at bridge on } \\
\text { Winchester Road, } 1.0 \text { mile southeast } \\
\text { of Poe. }\end{array}$ & 0 & 0 & 0 & - \\
\hline 04181900 & $\begin{array}{l}\text { Houk Ditch near } \\
\text { Hessen Cassel, } \\
\text { IN }\end{array}$ & 16.3 & $\begin{array}{l}\text { Lat } 40^{\circ} 59^{\prime} 27^{\prime \prime} \text {, long } 85^{\circ} 05^{\prime} 32^{\prime \prime} \text {, } \\
\text { SW1/4 SW1/4 Sec 5, T.29N., } \\
\text { R.13E., Allen County, at U.S. High- } \\
\text { way 27-33 bridge, } 0.4 \text { mile upstream } \\
\text { from mouth, } 1.2 \text { miles northwest of } \\
\text { Hessen Cassel, and } 7 \text { miles south- } \\
\text { east of Fort Wayne. }\end{array}$ & 0 & 0 & 0 & - \\
\hline 04191340 & $\begin{array}{l}\text { Flatrock Creek } \\
\text { near Townley, } \\
\text { IN }\end{array}$ & 47.1 & $\begin{array}{l}\text { Lat } 41^{\circ} 00^{\prime} 51^{\prime \prime} \text {, long } 84^{\circ} 51^{\prime} 06^{\prime \prime} \text {, } \\
\text { in NW1/4 SE1/4 Sec 32, T.30N., } \\
\text { R.15E., Allen County, at U.S. High- } \\
\text { way } 30 \text { bridge crossing, } 0.6 \text { mile } \\
\text { east of State Highway, } 1.2 \text { miles } \\
\text { southeast of Townley. }\end{array}$ & 0 & 0 & 0.1 & 1 \\
\hline 04191360 & $\begin{array}{l}\text { Hoffman Creek } \\
\text { at Townley, IN }\end{array}$ & 41.7 & $\begin{array}{l}\text { Lat } 41^{\circ} 01^{\prime} 16^{\prime \prime} \text {, long } 84^{\circ} 52^{\prime} 16^{\prime \prime} \text {, } \\
\text { in NE1/4 Sec } 31 \text {, T.30N., R.15E., } \\
\text { Allen County, at bridge on U.S. } \\
\text { Highway } 30 \text {, at Townley. }\end{array}$ & 0 & 0 & 0 & - \\
\hline
\end{tabular}




\section{Low-flow Characteristics for Selected Streams in Indiana}

Table 2. Low-flow characteristics for partial-record streamgaging stations in Indiana.-Continued

[mi², square miles; IN, Indiana; Lat, latitude; ' , degrees; ', minutes; ", seconds; long, longitude; Sec, Section; T, Township; R, Range; N, North; E, East; S, South; W, West; —, no correlation]

\begin{tabular}{|c|c|c|c|c|c|c|c|}
\hline \multirow{2}{*}{ Station number } & \multirow{2}{*}{ Station name } & \multirow{2}{*}{$\begin{array}{l}\text { Drainage } \\
\text { area }\left(\mathrm{mi}^{2}\right)\end{array}$} & \multirow{2}{*}{ Location } & \multicolumn{4}{|c|}{ Flow characteristic, in cubic feet per second } \\
\hline & & & & 1010 & 7010 & 30010 & $\begin{array}{c}\text { Harmomic } \\
\text { mean }\end{array}$ \\
\hline \multirow[t]{4}{*}{05515100} & Little Kankakee & 33.8 & Lat $41^{\circ} 34^{\prime} 15^{\prime \prime}$, long $86^{\circ} 34^{\prime} 27^{\prime \prime} \mathrm{m}$ & 22 & 24 & 26 & 45 \\
\hline & River near Mill & & Sec 18, T.36N., R.2W., LaPorte & & & & \\
\hline & Creek, IN & & County, at bridge on State Highway & & & & \\
\hline & & & 24, 2.5 miles west of Mill Creek. & & & & \\
\hline \multirow[t]{6}{*}{05515499} & Whitham Ditch & 43.0 & Lat $41^{\circ} 25^{\prime} 08^{\prime \prime}$, long $86^{\circ} 42^{\prime} 27^{\prime \prime}$, & 21 & 22 & 24 & 46 \\
\hline & near Hanna, IN & & LaPorte County, at bridge on & & & & \\
\hline & & & County Road 1300, 1.25 miles south & & & & \\
\hline & & & of State Road 39, 4 miles east of & & & & \\
\hline & & & Hanna, and 0.5 mile east of 100 & & & & \\
\hline & & & West and 1300 South intersection. & & & & \\
\hline \multirow[t]{6}{*}{05516300} & Dausman Ditch & 53.4 & Lat $41^{\circ} 22^{\prime} 58^{\prime \prime}$, long $86^{\circ} 07^{\prime} 02^{\prime \prime}$, & 1.9 & 2.1 & 2.6 & 13 \\
\hline & near Bremen, & & on line between Sec 19 T.34N., & & & & \\
\hline & $\mathrm{IN}$ & & R.4E., and Sec 24 T.34N., R.3E., & & & & \\
\hline & & & Marshall County, at bridge on State & & & & \\
\hline & & & Highway $331,4.5$ miles south of & & & & \\
\hline & & & Bremen. & & & & \\
\hline \multirow[t]{6}{*}{05516650} & Wolf Creek near & 31.1 & Lat $41^{\circ} 15^{\prime} 40^{\prime \prime}$, long $86^{\circ} 18^{\prime} 32^{\prime \prime}$, & 4.5 & 4.8 & 5.5 & 13 \\
\hline & Argos, IN & & on line between Sec 32 and 33, & & & & \\
\hline & & & T.33N., R.2E., Marshall County, at & & & & \\
\hline & & & bridge on South Muckshaw Road, & & & & \\
\hline & & & 1.6 miles north of Highway 31 in & & & & \\
\hline & & & Argos. & & & & \\
\hline
\end{tabular}


Table 2. Low-flow characteristics for partial-record streamgaging stations in Indiana._Continued

[mi² , square miles; IN, Indiana; Lat, latitude; ', degrees; ', minutes; ", seconds; long, longitude; Sec, Section; T, Township; R, Range; N, North; E, East; S, South; W, West; - , no correlation]

\begin{tabular}{|c|c|c|c|c|c|c|c|}
\hline \multirow{2}{*}{ Station number } & \multirow{2}{*}{ Station name } & \multirow{2}{*}{$\begin{array}{l}\text { Drainage } \\
\text { area }\left(\mathrm{mi}^{2}\right)\end{array}$} & \multirow{2}{*}{ Location } & \multicolumn{4}{|c|}{ Flow characteristic, in cubic feet per second } \\
\hline & & & & 1010 & 7010 & 30010 & $\begin{array}{c}\text { Harmomic } \\
\text { mean }\end{array}$ \\
\hline 05516950 & $\begin{array}{l}\text { Eagle Creek } \\
\text { near } \\
\text { Grovertown, } \\
\text { IN }\end{array}$ & 31.9 & $\begin{array}{l}\text { Lat } 41^{\circ} 18^{\prime} 44^{\prime \prime} \text {, long } 86^{\circ} 31^{\prime} 27^{\prime \prime} \text {, } \\
\text { in NE1/4 SE1/4 NE1/4 Sec 16, } \\
\text { T.33N., R.1W., Starke County, at } \\
\text { bridge on State Highway } 23,5.2 \\
\text { miles south of U.S. Highway } 30 \text { out } \\
\text { of Grovertown. }\end{array}$ & 5.8 & 6.4 & 7.6 & 22 \\
\hline 05517550 & $\begin{array}{l}\text { Reeves Ditch } \\
\text { near La Crosse, } \\
\text { IN }\end{array}$ & 44.1 & $\begin{array}{l}\text { Lat } 41^{\circ} 19^{\prime} 03^{\prime \prime}, \text { long } 86^{\circ} 55^{\prime} 49^{\prime \prime} \text {, } \\
\text { on line between Secs } 12 \text { and } 13 \text {, } \\
\text { T.33N., R.5W., Porter-LaPorte } \\
\text { County line, at bridge on State High- } \\
\text { way } 8,2.0 \text { miles west of La Crosse. }\end{array}$ & 5.9 & 6.4 & 7.9 & 41 \\
\hline $\begin{array}{l}05517750 \\
(411720087000 \\
500)\end{array}$ & $\begin{array}{l}\text { Crooked Creek } \\
\text { near Kouts, IN }\end{array}$ & 69.9 & $\begin{array}{l}\text { Lat } 41^{\circ} 17^{\prime} 20^{\prime \prime}, \text { long } 87^{\circ} 00^{\prime} 05^{\prime \prime} \text {, } \\
\text { on line between Secs } 21 \text { and } 28 \text {, } \\
\text { T.33N., R.5W., Porter County, at } \\
\text { bridge on County Road } 1000 \text { South, } \\
0.55 \text { mile east of intersection with } \\
\text { County Road } 75 \text { East, } 2.3 \text { miles } \\
\text { southeast of Kouts. }\end{array}$ & 24 & 26 & 27 & 53 \\
\hline
\end{tabular}




\section{Low-flow Characteristics for Selected Streams in Indiana}

Table 2. Low-flow characteristics for partial-record streamgaging stations in Indiana.-Continued

[mi², square miles; IN, Indiana; Lat, latitude; ' , degrees; ', minutes; ", seconds; long, longitude; Sec, Section; T, Township; R, Range; N, North; E, East; S, South; W, West; —, no correlation]

\begin{tabular}{|c|c|c|c|c|c|c|c|}
\hline \multirow{2}{*}{ Station number } & \multirow{2}{*}{ Station name } & \multirow{2}{*}{$\begin{array}{l}\text { Drainage } \\
\text { area }\left(\mathrm{mi}^{2}\right)\end{array}$} & \multirow{2}{*}{ Location } & \multicolumn{4}{|c|}{ Flow characteristic, in cubic feet per second } \\
\hline & & & & 1010 & 7010 & 30010 & $\begin{array}{c}\text { Harmomic } \\
\text { mean }\end{array}$ \\
\hline 05517880 & $\begin{array}{l}\text { Wolf Creek near } \\
\text { Kouts, IN }\end{array}$ & 13.8 & $\begin{array}{l}\text { Lat } 41^{\circ} 21^{\prime} 44^{\prime \prime} \text {, long } 87^{\circ} 04^{\prime} 59^{\prime \prime} \text {, } \\
\text { in SW1/4 SW1/4 Sec } 26, \text { T.34N., } \\
\text { R.6W., Porter County, at corrugated } \\
\text { metal-pipe culvert on County Road } \\
100 \text { West, } 1.1 \text { miles north of inter- } \\
\text { section with } 600 \text { South, } 4.1 \text { miles } \\
\text { northwest of Kouts. }\end{array}$ & 2.9 & 3.1 & 3.4 & 6.9 \\
\hline 05518500 & $\begin{array}{l}\text { Singleton Ditch } \\
\text { near Hebron, } \\
\text { IN }\end{array}$ & 34.2 & $\begin{array}{l}\text { Lat } 41^{\circ} 17^{\prime} 00^{\prime \prime} \text {, long } 87^{\circ} 15^{\prime} 00^{\prime \prime} \text {, } \\
\text { on line between Secs } 20 \text { and } 29 \text {, } \\
\text { T.33N., R.7W., Lake County, at } \\
\text { State Highway } 2 \text { bridge, } 3.2 \text { miles } \\
\text { southwest of Hebron. }\end{array}$ & 2.3 & 2.5 & 2.8 & 8.7 \\
\hline 05525650 & $\begin{array}{l}\text { Beaver Creek } \\
\text { near Morocco, } \\
\text { IN }\end{array}$ & 41.0 & $\begin{array}{l}\text { Lat } 40^{\circ} 57^{\prime} 57^{\prime \prime} \text {, long } 87^{\circ} 27^{\prime} 00^{\prime \prime} \text {, } \\
\text { in NW1/4 SW1/4 NW1/4 Sec } 15 \text {, } \\
\text { T.29N., R.9W., at bridge over Bea- } \\
\text { ver Creek on U.S. } 41 \text {, Newton } \\
\text { County, about } 1.4 \text { miles north of } \\
\text { Morocco. }\end{array}$ & 0.9 & 1 & 1.4 & 7 \\
\hline
\end{tabular}




\section{Index by station name}

\begin{tabular}{|c|c|}
\hline 03371520 & BACK CREEK AT LEESVILLE, IN .. \\
\hline 03353180 & BEAN CREEK AT INDIANAPOLIS, IN .. \\
\hline 03354500 & BEANBLOSSOM CREEK AT BEANBLOSSOM, IN . \\
\hline 03356000 & BEANBLOSSOM CREEK AT DOLAN, IN.. \\
\hline 03355000 & BEAR CREEK NEAR TREVLAC, IN.. \\
\hline 03373508 & BEAVER CREEK NEAR SHOALS, IN .... \\
\hline 05523000 & BICE DITCH NEAR SOUTH MARION, IN.. \\
\hline 03361000 & BIG BLUE RIVER AT CARTHAGE, IN..... \\
\hline 03361500 & BIG BLUE RIVER AT SHELBYVILLE, IN.. \\
\hline 03378550 & BIG CREEK NEAR WADESVILLE, IN...... \\
\hline 03326070 & BIG LICK CREEK NEAR HARTFORD CITY, IN... \\
\hline 03332400 & BIG MONON CREEK NEAR FRANCESVILLE, IN ... \\
\hline 03335700 & BIG PINE CR NR WILLIAMSPORT IN........... \\
\hline 03341300 & BIG RACCOON CREEK AT COXVILLE, IN ....... \\
\hline 03341000 & BIG RACCOON CREEK AT MANSFIELD, IN... \\
\hline 03340900 & BIG RACCOON CREEK NEAR FERNDALE, IN.... \\
\hline 03340800 & BIG RACCOON CREEK NEAR FINCASTLE IN.. \\
\hline 03357500 & BIG WALNUT CREEK NEAR REELSVILLE, IN ..... \\
\hline 03357330 & BIG WALNUT CREEK NEAR ROACHDALE, IN . \\
\hline 03302800 & BLUE RIVER AT FREDERICKSBURG, IN ................. \\
\hline 03303000 & BLUE RIVER NEAR WHITE CLOUD, IN....... \\
\hline 03368000 & BRUSH CREEK NEAR NEBRASKA, IN ..................... \\
\hline 03361850 & BUCK CREEK AT ACTON, IN .................. \\
\hline 03347500 & BUCK CREEK NEAR MUNCIE, IN \\
\hline 03302220 & BUCK CREEK NEAR NEW MIDDLETOWN, IN ......... \\
\hline 03342300 & 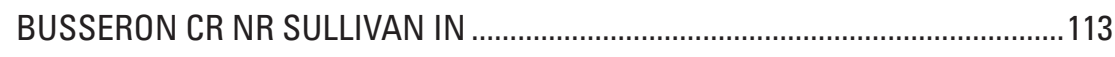 \\
\hline 03342500 & BUSSERON CREEK NEAR CARLISLE, IN \\
\hline 03342100 & BUSSERON CREEK NEAR HYMERA, IN..................... \\
\hline 05524000 & CARPENTER CREEK AT EGYPT, IN............................ \\
\hline 04179520 & CEDAR CREEK AT 18TH STREET AT AUBURN, IN \\
\hline 04179500 & CEDAR CREEK AT AUBURN, IN ................................. \\
\hline 04180000 & CEDAR CREEK NEAR CEDARVILLE, IN ............ \\
\hline 03349510 & CICERO CREEK AT ARCADIA, IN.................. \\
\hline 03350500 & CICERO CREEK AT NOBLESVILLE, IN........... \\
\hline 03349210 & CICERO CREEK AT TIPTON, IN ...................... \\
\hline 03349500 & CICERO CREEK NEAR ARCADIA, IN \\
\hline 03372700 & 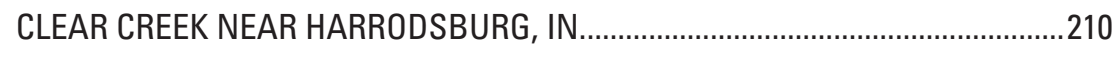 \\
\hline 03364500 & CLIFTY CREEK AT HARTSVILLE, IN \\
\hline 03364650 & CLIFTY CREEK NEAR COLUMBUS, IN \\
\hline 03339120 & COAL CREEK AT COAL CREEK, IN \\
\hline 05517890 & COBB DITCH NEAR KOUTS, IN \\
\hline 03351310 & CROOKED CREEK AT INDIANAPOLIS, IN \\
\hline
\end{tabular}




\begin{tabular}{|c|c|}
\hline 303400 & ROOKED CREEK NEAR SANTA CLAUS, IN ........ \\
\hline 04093000 & DEEP RIVER AT LAKE GEORGE OUTLET AT HOBART, IN ..... \\
\hline 03329700 & EER CREEK NEAR DELPHI, IN..... \\
\hline 03359500 & EER CREEK NEAR PUTNAMVILLE, IN .. \\
\hline 03353635 & DERBYSHIRE CREEK AT SOUTHPORT, IN ..... \\
\hline 03363000 & DRIFTWOOD RIVER NEAR EDINBURGH IN.. \\
\hline 03353460 & EAGLE CREEK AT CLERMONT, IN ...... \\
\hline 03353500 & EAGLE CREEK AT INDIANAPOLIS, IN.. \\
\hline 03353200 & EAGLE CREEK AT ZIONSVILLE, IN..... \\
\hline 03339108 & EAST FORK COAL CREEK NEAR HILLSBORO, IN ... \\
\hline 03353885 & EAST FORK WHITE LICK CREEK AT BRIDGEPORT, IN.................. \\
\hline 03353890 & EAST FORK WHITE LICK CREEK NEAR CAMBY, IN ... \\
\hline 03353910 & EAST FORK WHITE LICK CREEK NEAR MOORESVILLE, IN ........... \\
\hline 03364000 & EAST FORK WHITE RIVER AT COLUMBUS, IN..... \\
\hline 03365500 & EAST FORK WHITE RIVER AT SEYMOUR IN ......... \\
\hline 03373500 & EAST FORK WHITE RIVER AT SHOALS, IN ....... \\
\hline 03371500 & EAST FORK WHITE RIVER NEAR BEDFORD, IN ............ \\
\hline 03275600 & EAST FORK WHITEWATER RIVER AT ABINGTON, IN... \\
\hline 03276000 & 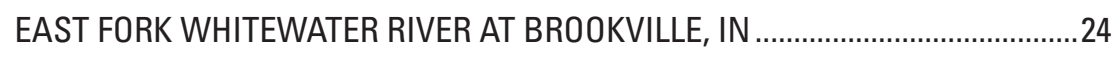 \\
\hline 03275500 & EAST FORK WHITEWATER RIVER AT RICHMOND, IN .. \\
\hline 03360000 & EEL RIVER AT BOWLING GREEN, IN .................... \\
\hline 03328000 & EEL RIVER AT NORTH MANCHESTER, IN \\
\hline 03328500 & EEL RIVER NEAR LOGANSPORT, IN .............. \\
\hline 04100500 & ELKHART RIVER AT GOSHEN, IN.... \\
\hline 033356725 & ELLIOTT DITCH NEAR ELSTON, IN....... \\
\hline 03335671 & ELLIOTT DITCH NEAR LAFAYETTE, IN .. \\
\hline 03353583 & FALCON CREEK 30TH ST. AT INDIANAPOLIS, IN........ \\
\hline 03352875 & FALL CREEK AT 16TH STREET AT INDIANAPOLIS, IN.... \\
\hline 03352500 & FALL CREEK AT MILLERSVILLE, IN ................. \\
\hline 03351500 & FALL CREEK NEAR FORTVILLE, IN... \\
\hline 04177720 & FISH CREEK AT HAMILTON, IN ........... \\
\hline 03376260 & FLAT CREEK NEAR OTWELL, IN.. \\
\hline 03363900 & FLATROCK RIVER AT COLUMBUS, IN..... \\
\hline 03363500 & FLATROCK RIVER AT ST. PAUL, IN........ \\
\hline 04100252 & FORKER CREEK NEAR BURR OAK, IN....... \\
\hline 03373686 & FRENCH LICK CREEK AT FRENCH LICK, IN .......... \\
\hline 03373695 & FRENCH LICK CREEK AT WEST BADEN SPRINGS, IN....... \\
\hline 04096100 & GALENA RIVER NEAR LAPORTE, IN ......... \\
\hline 03366000 & 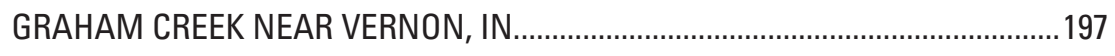 \\
\hline 05536357 & GRAND CALUMET RIVER AT HOHMAN AVE AT HAMMOND, IN .......................289 \\
\hline 03353560 & 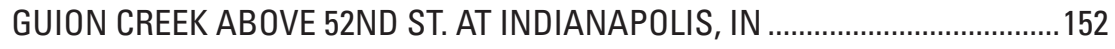 \\
\hline 03375800 & HALL CREEK NEAR ST. ANTHONY, IN......... \\
\hline 04182590 & HARBER DITCH AT FORT WAYNE, IN \\
\hline 03366200 & HARBERTS CREEK NEAR MADISON, IN...... \\
\hline 05536179 & IART DITCH AT DYER, IN ............................. \\
\hline
\end{tabular}




\begin{tabular}{|c|c|}
\hline 05536190 & HART DITCH AT MUNSTER, IN.. \\
\hline 03364042 & HAW CREEK AT HOPE, IN . \\
\hline 03364200 & HAW CREEK NEAR CLIFFORD, IN.. \\
\hline 03350100 & HINKLE CREEK NEAR CICERO, IN . \\
\hline 03302500 & INDIAN CREEK NEAR CORYDON, IN .. \\
\hline 03335678 & INDIAN CREEK NEAR MONTMORENCI, IN . \\
\hline 03373200 & INDIAN CREEK NEAR SPRINGVILLE, IN ....... \\
\hline 03291780 & INDIAN-KENTUCK CREEK NR CANAAN, IN.. \\
\hline 05522500 & IROQUOIS RIVER AT RENSSELAER, IN....... \\
\hline 05521000 & IROQUOIS RIVER AT ROSEBUD, IN.. \\
\hline 05524500 & IROQUOIS RIVER NEAR FORESMAN, IN.... \\
\hline 05522000 & IROQUOIS RIVER NEAR NORTH MARION, IN.. \\
\hline 04101370 & JUDY CREEK NEAR SOUTH BEND, IN ............... \\
\hline 05515500 & KANKAKEE RIVER AT DAVIS, IN........ \\
\hline 05517500 & KANKAKEE RIVER AT DUNNS BRIDGE, IN.... \\
\hline 05518000 & KANKAKEE RIVER AT SHELBY, IN .. \\
\hline 05515000 & KANKAKEE RIVER NEAR NORTH LIBERTY, IN .... \\
\hline 05517530 & 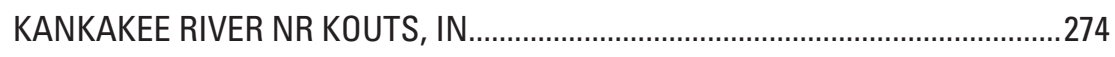 \\
\hline 03348020 & KILLBUCK CREEK NEAR GASTON, IN........ \\
\hline 05515400 & KINGSBURY CREEK NR LAPORTE, IN..... \\
\hline 03333600 & KOKOMO CREEK NEAR KOKOMO, IN...... \\
\hline 03277000 & LAUGHERY CREEK NEAR FARMERS RETREAT, IN..... \\
\hline 03361638 & LEARY-WEBER DITCH AT MOHAWK, IN .......................... \\
\hline 03353620 & LICK CREEK AT INDIANAPOLIS, IN.... \\
\hline 03373610 & LICK CREEK AT PAOLI, IN .......................... \\
\hline 04097970 & LIME LAKE OUTLET AT PANAMA, IN ....................... \\
\hline 03353636 & LITTLE BUCK CREEK AT SOUTHPORT, IN............................... \\
\hline 03353637 & LITTLE BUCK CREEK NEAR INDIANAPOLIS, IN \\
\hline 03353630 & LITTLE BUCK CREEK NEAR SOUTHPORT, IN \\
\hline 05536195 & LITTLE CALUMET RIVER AT MUNSTER, IN \\
\hline 04094000 & LITTLE CALUMET RIVER AT PORTER, IN \\
\hline 03349700 & LITTLE CICERO CREEK NEAR ARCADIA, IN \\
\hline 03353551 & 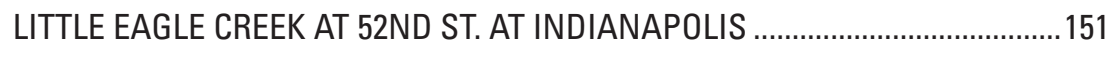 \\
\hline 03353600 & LITTLE EAGLE CREEK AT SPEEDWAY, IN \\
\hline 04099808 & LITTLE ELKHART RIVER AT MIDDLEBURY, IN \\
\hline 03302300 & LITTLE INDIAN CREEK NEAR GALENA, IN \\
\hline 03332300 & LITTLE INDIAN CREEK NEAR ROYAL CENTER, IN .......... \\
\hline 03325311 & LITTLE MISSISSINEWA RIVER AT UNION CITY, IN ................. \\
\hline 03335679 & LITTLE PINE CREEK AT GREEN HILL, IN .......................... \\
\hline 033356786 & 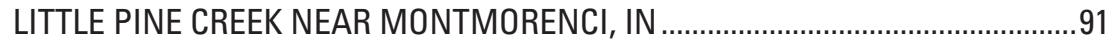 \\
\hline 03341200 & LITTLE RACCOON CREEK NEAR CATLIN, IN \\
\hline 03324000 & LITTLE RIVER NEAR HUNTINGTON, IN \\
\hline 03339150 & LITTLE VERMILLION RIVER NEAR NEWPORT, IN \\
\hline 03335673 & LITTLE WEA CREEK AT SOUTH RAUB, IN \\
\hline 03274950 & LITTLE WILLIAMS CREEK AT CONNERSVILLE, IN \\
\hline
\end{tabular}




\begin{tabular}{|c|c|}
\hline 03373530 & LOST RIVER NEAR LEIPSIC, IN ........ \\
\hline 03373560 & LOST RIVER NEAR PROSPECT, IN.. \\
\hline 03373700 & LOST RIVER NR. WEST BADEN SPRINGS, IN........ \\
\hline 03335677 & MARSHALL DITCH NEAR MONTMORENCI, IN... \\
\hline 04182950 & 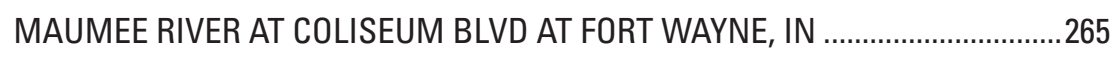 \\
\hline 04183000 & MAUMEE RIVER AT NEW HAVEN, IN... \\
\hline 03303300 & MIDDLE FORK ANDERSON RIVER AT BRISTOW, IN..... \\
\hline 03358000 & MILL CREEK NEAR CATARACT, IN... \\
\hline 03359000 & MILL CREEK NEAR MANHATTAN, IN ....... \\
\hline 03326500 & MISSISSINEWA RIVER AT MARION, IN .. \\
\hline 03327000 & MISSISSINEWA RIVER AT PEORIA, IN ..... \\
\hline 03326000 & MISSISSINEWA RIVER NEAR EATON, IN... \\
\hline 03325500 & MISSISSINEWA RIVER NEAR RIDGEVILLE, IN .... \\
\hline 03342250 & MUD CR NR DUGGER IN... \\
\hline 03352200 & MUD CREEK AT INDIANAPOLIS, IN.... \\
\hline 03335690 & MUD PINE CREEK NEAR OXFORD, IN ... \\
\hline 03366500 & MUSCATATUCK RIVER NEAR DEPUTY, IN ...... \\
\hline 04100222 & NB ELKHART RIVER AT COSPERVILLE, IN....... \\
\hline 03371650 & NORTH FORK SALT CREEK AT NASHVILLE, IN .... \\
\hline 03372000 & NORTH FORK SALT CREEK NEAR BELMONT, IN . \\
\hline 03375500 & PATOKA RIVER AT JASPER, IN ..................... \\
\hline 03376300 & PATOKA RIVER AT WINSLOW, IN . \\
\hline 03374455 & PATOKA RIVER NEAR HARDINSBURG, IN... \\
\hline 03376500 & PATOKA RIVER NEAR PRINCETON, IN. \\
\hline 03322011 & PIGEON CREEK NEAR FORT BRANCH... \\
\hline 04099510 & PIGEON CREEK NR ANGOLA, IN . \\
\hline 04099750 & PIGEON RIVER NEAR SCOTT, IN . \\
\hline 04099850 & PINE CREEK NEAR ELKHART, IN . \\
\hline 03348350 & PIPE CREEK AT FRANKTON, IN .......... \\
\hline 03327520 & PIPE CREEK NEAR BUNKER HILL, IN.......... \\
\hline 03353120 & PLEASANT RN AT ARLINGTON AVE AT INPLS, IN........ \\
\hline 03353160 & PLEASANT RN AT BROOKVILLE RD AT INPLS, IN..... \\
\hline 03357350 & PLUM CREEK NEAR BAINBRIDGE, IN \\
\hline 03352988 & POGUES RUN AT VERMONT STREET AT INDIANAPOLIS, IN ............................ 143 \\
\hline 04095090 & PORTAGE-BURNS WATERWAY AT PORTAGE, IN ......................... \\
\hline 03339280 & PRAIRIE CREEK NEAR LEBANON, IN ........... \\
\hline 03327503 & PRAIRIE DITCH AT PERU, IN .......................... \\
\hline 03327507 & PRAIRIE DITCH NEAR PERU, IN ................. \\
\hline 04099610 & PRETTY LAKE INLET NEAR STROH, IN.......... \\
\hline 03329400 & RATTLESNAKE CREEK NEAR PATTON, IN ....... \\
\hline 04100295 & RIMMELL BRANCH NEAR ALBION, IN.............. \\
\hline 03324500 & SALAMONIE RIVER AT DORA, IN .................. \\
\hline 03324200 & SALAMONIE RIVER AT PORTLAND, IN \\
\hline 03324300 & SALAMONIE RIVER NEAR WARREN, IN ................. \\
\hline 04094400 & SALT CREEK AT VALPARAISO, IN................... \\
\hline
\end{tabular}




\begin{tabular}{|c|c|}
\hline 03372500 & SALT CREEK NEAR HARRODSBURG IN ... \\
\hline 04094500 & ALT CREEK NEAR MCCOOL, IN .. \\
\hline 03365000 & SAND CREEK NEAR BREWERSVILLE, IN ...... \\
\hline 03294000 & SILVER CREEK NEAR SELLERSBURG, IN ... \\
\hline 05519000 & SINGLETON DITCH AT SCHNEIDER, IN......... \\
\hline 05523500 & SLOUGH CREEK NEAR COLLEGEVILLE, IN.. \\
\hline 04100377 & 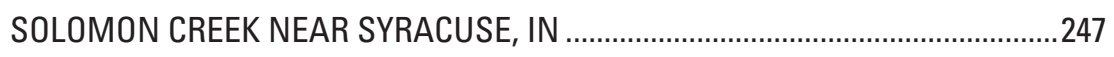 \\
\hline 03376350 & SOUTH FORK PATOKA RIVER NEAR SPURGEON, IN ..... \\
\hline 03371600 & SOUTH FORK SALT CREEK AT KURTZ, IN ............................ \\
\hline 03334500 & SOUTH FORK WILDCAT CREEK NEAR LAFAYETTE, IN............. \\
\hline 03276700 & SOUTH HOGAN CREEK NEAR DILLSBORO, IN.......................... \\
\hline 04182810 & SPY RUN CREEK AT FORT WAYNE, IN...... \\
\hline 04182808 & SPY RUN CREEK NEAR PARK DRIVE AT FORT WAYNE, IN ……............................ \\
\hline 04178000 & ST JOSEPH R NR NEWVILLE IN .......... \\
\hline 04179000 & ST. JOSEPH RIVER AT CEDARVILLE, IN....... \\
\hline 04101000 & ST. JOSEPH RIVER AT ELKHART, IN...... \\
\hline 04180500 & ST. JOSEPH RIVER NEAR FORT WAYNE, IN ..... \\
\hline 04181500 & ST. MARYS RIVER AT DECATUR, IN ... \\
\hline 04182769 & ST. MARYS RIVER AT MAIN ST. AT FORT WAYNE, IN ..... \\
\hline 04182000 & ST. MARYS RIVER NEAR FORT WAYNE, IN ....... \\
\hline 03372300 & STEPHENS CREEK NEAR BLOOMINGTON, IN................. \\
\hline 03350655 & STONY CREEK AT FISHERSBURG, IN ...... \\
\hline 03350700 & STONY CREEK NEAR NOBLESVILLE, IN..... \\
\hline 03339500 & SUGAR CREEK AT CRAWFORDSVILLE, IN ... \\
\hline 03361650 & SUGAR CREEK AT NEW PALESTINE, IN....... \\
\hline 03340000 & SUGAR CREEK NEAR BYRON, IN ................ \\
\hline 03362500 & SUGAR CREEK NEAR EDINBURGH, IN....... \\
\hline 03351400 & SUGAR CREEK NEAR MIDDLETOWN, IN .......... \\
\hline 03330241 & TIPPECANOE RIVER AT NORTH WEBSTER, IN..... \\
\hline 03332555 & TIPPECANOE RIVER AT NORWAY, IN ......... \\
\hline 03330500 & TIPPECANOE RIVER AT OSWEGO, IN.......... \\
\hline 03331753 & TIPPECANOE RIVER AT WINAMAC, IN ........................ \\
\hline 03332605 & TIPPECANOE RIVER BELOW OAKDALE DAM, IN.... \\
\hline 03333050 & TIPPECANOE RIVER NEAR DELPHI, IN ................... \\
\hline 03332500 & TIPPECANOE RIVER NEAR MONTICELLO, IN ........ \\
\hline 03331500 & TIPPECANOE RIVER NEAR ORA, IN ............................. \\
\hline 04095380 & TRAIL CREEK AT MICHIGAN CITY HARBOR, IN........ \\
\hline 04095300 & TRAIL CREEK AT MICHIGAN CITY, IN ............... \\
\hline 04100465 & 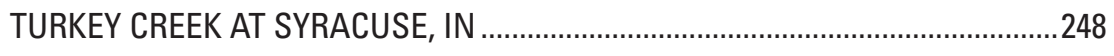 \\
\hline 03369500 & VERNON FORK MUSCATATUCK RIVER AT VERNON, IN \\
\hline 03369000 & VERNON FORK MUSCATATUCK RIVER NR BUTLERVILLE, IN ............................... 201 \\
\hline 03323000 & WABASH RIVER AT BLUFFTON, IN ................... \\
\hline 03336000 & WABASH RIVER AT COVINGTON, IN........ \\
\hline 03329500 & WABASH RIVER AT DELPHI, IN................. \\
\hline 03323500 & WABASH RIVER AT HUNTINGTON IN.... \\
\hline
\end{tabular}




\begin{tabular}{|c|c|}
\hline 5500 & ER AT LAFAYETTE IN.. \\
\hline 03322900 & WABASH RIVER AT LINN GROVE, IN . \\
\hline 03329000 & WABASH RIVER AT LOGANSPORT, IN.. \\
\hline 03340500 & WABASH RIVER AT MONTEZUMA, IN.. \\
\hline 03377500 & WABASH RIVER AT MT. CARMEL, IL.. \\
\hline 03327500 & WABASH RIVER AT PERU, IN .. \\
\hline 03342000 & WABASH RIVER AT RIVERTON IN ....... \\
\hline 03341500 & WABASH RIVER AT TERRE HAUTE, IN... \\
\hline 03343000 & WABASH RIVER AT VINCENNES, IN ..... \\
\hline 03325000 & WABASH RIVER AT WABASH, IN .... \\
\hline 03322985 & WABASH RIVER NEAR BLUFFTON, IN ... \\
\hline 03322500 & WABASH RIVER NEAR NEW CORYDON, IN.. \\
\hline 03331110 & WALNUT CREEK NEAR WARSAW, IN ............ \\
\hline 03328430 & WEESAU CREEK NEAR DEEDSVILLE, IN.. \\
\hline 05519500 & WEST CREEK NEAR SCHNEIDER, IN ........ \\
\hline 03302680 & WEST FORK BLUE RIVER AT SALEM, IN.... \\
\hline 03342150 & 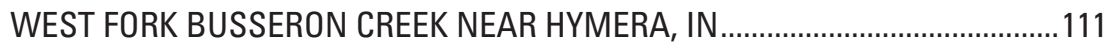 \\
\hline 03353700 & WEST FORK WHITE LICK CREEK AT DANVILLE, IN......... \\
\hline 03302849 & 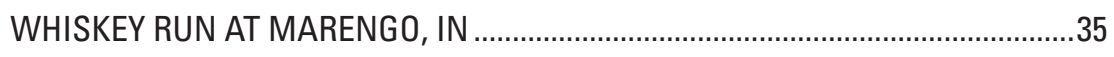 \\
\hline 03353800 & WHITE LICK CREEK AT MOORESVILLE, IN .......................... \\
\hline 03353611 & WHITE R. AT STOUT GEN. STN. AT INDIANAPOLIS, IN.......... \\
\hline 03350800 & WHITE RIVER AT 146TH ST NEAR NOBLESVILLE, IN..... \\
\hline 03348000 & WHITE RIVER AT ANDERSON, IN......................... \\
\hline 03353000 & WHITE RIVER AT INDIANAPOLIS, IN ......... \\
\hline 03352953 & WHITE RIVER AT MICHIGAN STREET AT INDIANAPOLIS, IN ...........................142 \\
\hline 03347000 & WHITE RIVER AT MUNCIE, IN \\
\hline 03360500 & WHITE RIVER AT NEWBERRY, IN .................... \\
\hline 03349000 & WHITE RIVER AT NOBLESVILLE, IN ................. \\
\hline 03374000 & 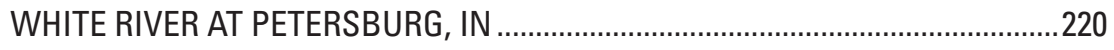 \\
\hline 03348130 & 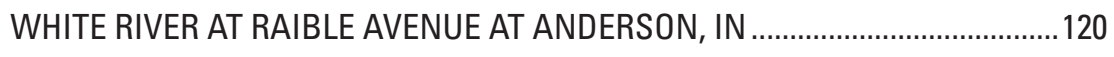 \\
\hline 03357000 & 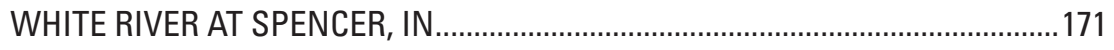 \\
\hline 03353660 & WHITE RIVER AT WAVERLY, IN ................................. \\
\hline 03354000 & WHITE RIVER NEAR CENTERTON, IN..................... \\
\hline 03348500 & WHITE RIVER NEAR NOBLESVILLE IN ............ \\
\hline 03351000 & WHITE RIVER NEAR NORA, IN ................................ \\
\hline 03276500 & WHITEWATER RIVER AT BROOKVILLE, IN............. \\
\hline 03275000 & WHITEWATER RIVER NEAR ALPINE, IN ..................... \\
\hline 03274650 & WHITEWATER RIVER NEAR ECONOMY, IN \\
\hline 03274750 & 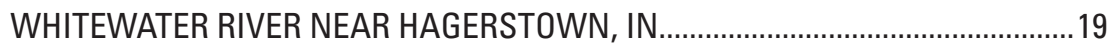 \\
\hline 03333500 & WILDCAT CREEK AT GREENTOWN, IN \\
\hline 03333700 & WILDCAT CREEK AT KOKOMO, IN \\
\hline 03334000 & WILDCAT CREEK AT OWASCO, IN \\
\hline 03333450 & WILDCAT CREEK NEAR JEROME, IN \\
\hline 03335000 & WILDCAT CREEK NEAR LAFAYETTE, IN \\
\hline 03350660 & 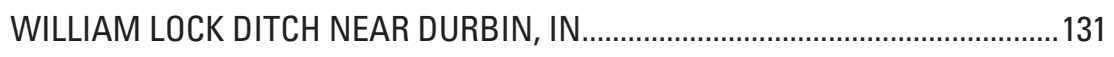 \\
\hline
\end{tabular}


03351072 WILLIAMS CREEK AT 96TH STREET, INDIANAPOLIS, IN .................................135

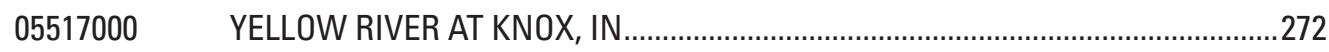

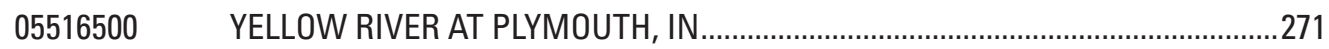

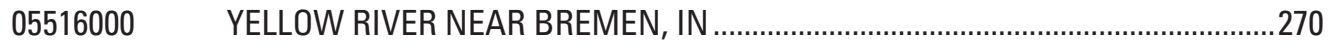

$03362000 \quad$ YOUNGS CREEK NEAR EDINBURGH IN ……......................................................... 185 


\section{Index by station number}

\begin{tabular}{|c|c|}
\hline 03274650 & R RIVER NEAR ECONOMY, IN . \\
\hline 03274750 & NHITEWATER RIVER NEAR HAGERSTOWN, IN. \\
\hline 03274950 & ITTLE WILLIAMS CREEK AT CONNERSVILLE, IN. \\
\hline 03275000 & VHITEWATER RIVER NEAR ALPINE, IN .. \\
\hline 03275500 & EAST FORK WHITEWATER RIVER AT RICHMOND, IN . \\
\hline 03275600 & EAST FORK WHITEWATER RIVER AT ABINGTON, IN. \\
\hline 03276000 & EAST FORK WHITEWATER RIVER AT BROOKVILLE, IN . \\
\hline 03276500 & WHITEWATER RIVER AT BROOKVILLE, IN... \\
\hline 03276700 & SOUTH HOGAN CREEK NEAR DILLSBORO, IN.. \\
\hline 03277000 & LAUGHERY CREEK NEAR FARMERS RETREAT, IN. \\
\hline 03291780 & INDIAN-KENTUCK CREEK NR CANAAN, IN. \\
\hline 03294000 & SILVER CREEK NEAR SELLERSBURG, IN .... \\
\hline 03302220 & BUCK CREEK NEAR NEW MIDDLETOWN, IN \\
\hline 03302300 & LITTLE INDIAN CREEK NEAR GALENA, IN .. \\
\hline 03302500 & INDIAN CREEK NEAR CORYDON, IN . \\
\hline 03302680 & WEST FORK BLUE RIVER AT SALEM, IN ... \\
\hline 03302800 & BLUE RIVER AT FREDERICKSBURG, IN . \\
\hline 03302849 & WHISKEY RUN AT MARENGO, IN .......... \\
\hline 03303000 & BLUE RIVER NEAR WHITE CLOUD, IN. \\
\hline 03303300 & MIDDLE FORK ANDERSON RIVER AT BRISTOW, IN. \\
\hline 03303400 & CROOKED CREEK NEAR SANTA CLAUS, IN.. \\
\hline 03322011 & PIGEON CREEK NEAR FORT BRANCH ........ \\
\hline 03322500 & WABASH RIVER NEAR NEW CORYDON, IN.. \\
\hline 03322900 & WABASH RIVER AT LINN GROVE, IN .............. \\
\hline 03322985 & WABASH RIVER NEAR BLUFFTON, IN ....... \\
\hline 03323000 & WABASH RIVER AT BLUFFTON, IN .................. \\
\hline 03323500 & WABASH RIVER AT HUNTINGTON IN... \\
\hline 03324000 & LITTLE RIVER NEAR HUNTINGTON, IN ....... \\
\hline 03324200 & SALAMONIE RIVER AT PORTLAND, IN ...... \\
\hline 03324300 & SALAMONIE RIVER NEAR WARREN, IN ....... \\
\hline 03324500 & SALAMONIE RIVER AT DORA, IN ................. \\
\hline 03325000 & WABASH RIVER AT WABASH, IN ................... \\
\hline 03325311 & LITTLE MISSISSINEWA RIVER AT UNION CITY, IN .......... \\
\hline 03325500 & MISSISSINEWA RIVER NEAR RIDGEVILLE, IN ............... \\
\hline 03326000 & MISSISSINEWA RIVER NEAR EATON, IN ........... \\
\hline 03326070 & BIG LICK CREEK NEAR HARTFORD CITY, IN ........ \\
\hline 03326500 & MISSISSINEWA RIVER AT MARION, IN ............... \\
\hline 03327000 & 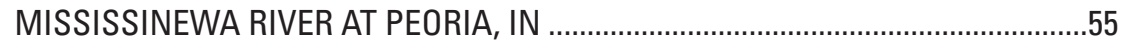 \\
\hline 03327500 & WABASH RIVER AT PERU, IN \\
\hline 327503 & DITCH AT \\
\hline 3327507 & PRAIRIE DITCH NEAR PERU, I \\
\hline
\end{tabular}




\begin{tabular}{|c|c|}
\hline 03327520 & NEAR BUNKER HILL, IN.. \\
\hline 03328000 & EEL RIVER AT NORTH MANCHESTER, IN. \\
\hline 03328430 & WEESAU CREEK NEAR DEEDSVILLE, IN . \\
\hline 03328500 & EEL RIVER NEAR LOGANSPORT, IN . \\
\hline 03329000 & WABASH RIVER AT LOGANSPORT, IN .... \\
\hline 03329400 & RATTLESNAKE CREEK NEAR PATTON, IN . \\
\hline 03329500 & WABASH RIVER AT DELPHI, IN ......... \\
\hline 03329700 & DEER CREEK NEAR DELPHI, IN... \\
\hline 03330241 & TIPPECANOE RIVER AT NORTH WEBSTER, IN .... \\
\hline 03330500 & TIPPECANOE RIVER AT OSWEGO, IN.. \\
\hline 03331110 & WALNUT CREEK NEAR WARSAW, IN .. \\
\hline 03331500 & TIPPECANOE RIVER NEAR ORA, IN... \\
\hline 03331753 & TIPPECANOE RIVER AT WINAMAC, IN.... \\
\hline 03332300 & LITTLE INDIAN CREEK NEAR ROYAL CENTER, IN .. \\
\hline 03332400 & BIG MONON CREEK NEAR FRANCESVILLE, IN ..... \\
\hline 03332500 & TIPPECANOE RIVER NEAR MONTICELLO, IN ........ \\
\hline 03332555 & TIPPECANOE RIVER AT NORWAY, IN ................... \\
\hline 03332605 & TIPPECANOE RIVER BELOW OAKDALE DAM, IN.... \\
\hline 03333050 & TIPPECANOE RIVER NEAR DELPHI, IN ... \\
\hline 03333450 & WILDCAT CREEK NEAR JEROME, IN ....... \\
\hline 03333500 & WILDCAT CREEK AT GREENTOWN, IN .... \\
\hline 03333600 & KOKOMO CREEK NEAR KOKOMO, IN ... \\
\hline 03333700 & WILDCAT CREEK AT KOKOMO, IN.......... \\
\hline 03334000 & WILDCAT CREEK AT OWASCO, IN.... \\
\hline 03334500 & SOUTH FORK WILDCAT CREEK NEAR LAFAYETTE, IN... \\
\hline 03335000 & WILDCAT CREEK NEAR LAFAYETTE, IN...... \\
\hline 03335500 & WABASH RIVER AT LAFAYETTE IN ............. \\
\hline 03335671 & ELLIOTT DITCH NEAR LAFAYETTE, IN ....... \\
\hline 033356725 & ELLIOTT DITCH NEAR ELSTON, IN ............................ \\
\hline 03335673 & LITTLE WEA CREEK AT SOUTH RAUB, IN \\
\hline 03335677 & MARSHALL DITCH NEAR MONTMORENCI, IN.............. \\
\hline 03335678 & INDIAN CREEK NEAR MONTMORENCI, IN ........................ \\
\hline 033356786 & LITTLE PINE CREEK NEAR MONTMORENCI, IN ........... \\
\hline 03335679 & LITTLE PINE CREEK AT GREEN HILL, IN ................. \\
\hline 03335690 & MUD PINE CREEK NEAR OXFORD, IN ....................... \\
\hline 03335700 & BIG PINE CR NR WILLIAMSPORT IN ...................... \\
\hline 03336000 & WABASH RIVER AT COVINGTON, IN ............................... \\
\hline 03339108 & EAST FORK COAL CREEK NEAR HILLSBORO, IN ........... \\
\hline 03339120 & COAL CREEK AT COAL CREEK, IN ........................................... \\
\hline 03339150 & LITTLE VERMILLION RIVER NEAR NEWPORT, IN .............. \\
\hline 03339280 & PRAIRIE CREEK NEAR LEBANON, IN ..................... \\
\hline 03339500 & SUGAR CREEK AT CRAWFORDSVILLE, IN ......... \\
\hline 03340000 & SUGAR CREEK NEAR BYRON, IN \\
\hline 03340500 & WABASH RIVER AT MONTEZUMA, IN \\
\hline 03340800 & IG RACCOON CREEK NEAR FINCASTLE IN \\
\hline
\end{tabular}




\begin{tabular}{|c|c|}
\hline 340900 & RACCOON CREEK NEAR FERNDALE, IN .. \\
\hline 03341000 & SFIELD, IN . \\
\hline 03341200 & TTLE RACCOON CREEK NEAR CATLIN, IN.... \\
\hline 3341300 & G RACCOON CREEK AT COXVILLE, IN... \\
\hline 03341500 & IABASH RIVER AT TERRE HAUTE, IN ........ \\
\hline 03342000 & IABASH RIVER AT RIVERTON IN \\
\hline 03342100 & USSERON CREEK NEAR HYMERA, IN......... \\
\hline 03342150 & VEST FORK BUSSERON CREEK NEAR HYMERA, IN .. \\
\hline 03342250 & UD CR NR DUGGER IN....... \\
\hline 03342300 & USSERON CR NR SULLIVAN IN . \\
\hline 03342500 & USSERON CREEK NEAR CARLISLE, IN ..... \\
\hline 03343000 & VABASH RIVER AT VINCENNES, IN \\
\hline 03347000 & VHITE RIVER AT MUNCIE, IN..... \\
\hline 03347500 & NEAR MUNCIE, IN. \\
\hline 03348000 & WHITE RIVER AT ANDERSON, IN...... \\
\hline 03348020 & KILLBUCK CREEK NEAR GASTON, IN... \\
\hline 03348130 & WHITE RIVER AT RAIBLE AVENUE AT ANDERSON, IN ... \\
\hline 03348350 & PIPE CREEK AT FRANKTON, IN. \\
\hline 03348500 & WHITE RIVER NEAR NOBLESVILLE IN ... \\
\hline 03349000 & WHITE RIVER AT NOBLESVILLE, IN .. \\
\hline 03349210 & CICERO CREEK AT TIPTON, IN ........... \\
\hline 03349500 & CICERO CREEK NEAR ARCADIA, IN. \\
\hline 03349510 & CICERO CREEK AT ARCADIA, IN........ \\
\hline 03349700 & LITTLE CICERO CREEK NEAR ARCADIA, IN. \\
\hline 03350100 & HINKLE CREEK NEAR CICERO, IN .................. \\
\hline 03350500 & CICERO CREEK AT NOBLESVILLE, IN. \\
\hline 03350655 & STONY CREEK AT FISHERSBURG, IN.......... \\
\hline 03350660 & WILLIAM LOCK DITCH NEAR DURBIN, IN... \\
\hline 03350700 & STONY CREEK NEAR NOBLESVILLE, IN ........ \\
\hline 03350800 & WHITE RIVER AT 146TH ST NEAR NOBLESVILLE, IN \\
\hline 03351000 & 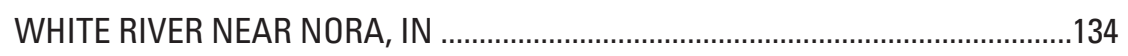 \\
\hline 03351072 & WILLIAMS CREEK AT 96TH STREET, INDIANAPOLIS, IN .......... \\
\hline 03351310 & 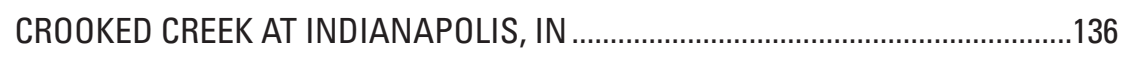 \\
\hline 03351400 & SUGAR CREEK NEAR MIDDLETOWN, IN ...... \\
\hline 03351500 & FALL CREEK NEAR FORTVILLE, IN .................... \\
\hline 03352200 & MUD CREEK AT INDIANAPOLIS, IN ................ \\
\hline 03352500 & FALL CREEK AT MILLERSVILLE, IN \\
\hline 03352875 & FALL CREEK AT 16TH STREET AT INDIANAPOLIS, IN \\
\hline 03352953 & WHITE RIVER AT MICHIGAN STREET AT INDIANAPOLIS, IN ............................142 \\
\hline 03352988 & 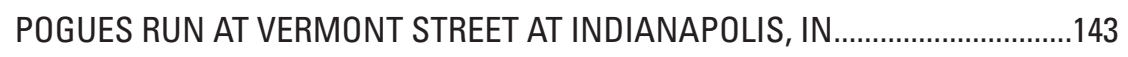 \\
\hline 03353000 & 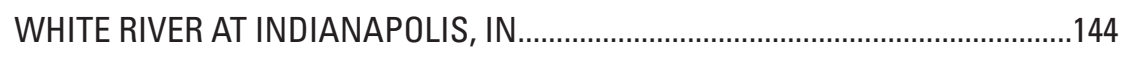 \\
\hline 03353120 & PLEASANT RN AT ARLINGTON AVE AT INPLS, IN \\
\hline 03353160 & PLEASANT RN AT BROOKVILLE RD AT INPLS, IN \\
\hline 03353180 & BEAN CREEK AT INDIANAPOLIS, IN \\
\hline 03353200 & EAGLE CREEK AT ZIONSVILLE, IN .......... \\
\hline
\end{tabular}




\begin{tabular}{|c|c|}
\hline 3460 & EEK AT CLERMONT, IN . \\
\hline 03353500 & AGLE CREEK AT INDIANAPOLIS, IN .. \\
\hline 03353551 & ITTLE EAGLE CREEK AT 52ND ST. AT INDIANAPOLIS.. \\
\hline 03353560 & IUION CREEK ABOVE 52ND ST. AT INDIANAPOLIS, IN \\
\hline 03353583 & FALCON CREEK 30TH ST. AT INDIANAPOLIS, IN... \\
\hline 03353600 & LITTLE EAGLE CREEK AT SPEEDWAY, IN . \\
\hline 03353611 & WHITE R. AT STOUT GEN. STN. AT INDIANAPOLIS, IN .... \\
\hline 03353620 & LICK CREEK AT INDIANAPOLIS, IN.. \\
\hline 03353630 & LITTLE BUCK CREEK NEAR SOUTHPORT, IN .... \\
\hline 03353635 & DERBYSHIRE CREEK AT SOUTHPORT, IN... \\
\hline 03353636 & LITTLE BUCK CREEK AT SOUTHPORT, IN... \\
\hline 03353637 & LITTLE BUCK CREEK NEAR INDIANAPOLIS, IN.... \\
\hline 03353660 & WHITE RIVER AT WAVERLY, IN ............ \\
\hline 03353700 & WEST FORK WHITE LICK CREEK AT DANVILLE, IN... \\
\hline 03353800 & WHITE LICK CREEK AT MOORESVILLE, IN........ \\
\hline 03353885 & EAST FORK WHITE LICK CREEK AT BRIDGEPORT, IN... \\
\hline 03353890 & EAST FORK WHITE LICK CREEK NEAR CAMBY, IN ......... \\
\hline 03353910 & EAST FORK WHITE LICK CREEK NEAR MOORESVILLE, IN .. \\
\hline 03354000 & WHITE RIVER NEAR CENTERTON, IN...... \\
\hline 03354500 & BEANBLOSSOM CREEK AT BEANBLOSSOM, IN ... \\
\hline 03355000 & BEAR CREEK NEAR TREVLAC, IN ..... \\
\hline 03356000 & BEANBLOSSOM CREEK AT DOLAN, IN.... \\
\hline 03357000 & WHITE RIVER AT SPENCER, IN ................... \\
\hline 03357330 & BIG WALNUT CREEK NEAR ROACHDALE, IN.... \\
\hline 03357350 & 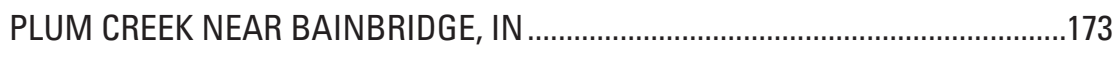 \\
\hline 03357500 & BIG WALNUT CREEK NEAR REELSVILLE, IN .... \\
\hline 03358000 & MILL CREEK NEAR CATARACT, IN ............................ \\
\hline 03359000 & MILL CREEK NEAR MANHATTAN, IN ......... \\
\hline 03359500 & DEER CREEK NEAR PUTNAMVILLE, IN ................ \\
\hline 03360000 & 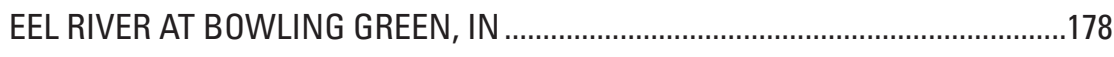 \\
\hline 03360500 & WHITE RIVER AT NEWBERRY, IN .......................... \\
\hline 03361000 & BIG BLUE RIVER AT CARTHAGE, IN ............................ \\
\hline 03361500 & BIG BLUE RIVER AT SHELBYVILLE, IN ................ \\
\hline 03361638 & LEARY-WEBER DITCH AT MOHAWK, IN ......... \\
\hline 03361650 & SUGAR CREEK AT NEW PALESTINE, IN............ \\
\hline 03361850 & 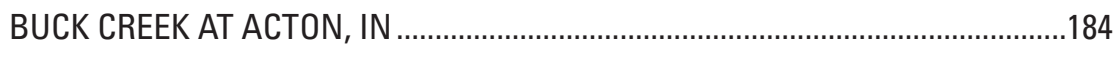 \\
\hline 03362000 & YOUNGS CREEK NEAR EDINBURGH IN ........... \\
\hline 03362500 & SUGAR CREEK NEAR EDINBURGH, IN .............. \\
\hline 03363000 & DRIFTWOOD RIVER NEAR EDINBURGH IN .......... \\
\hline 03363500 & 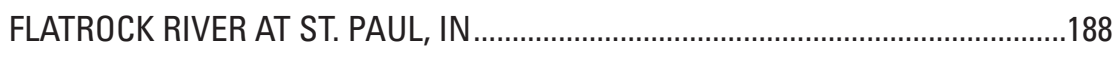 \\
\hline 03363900 & 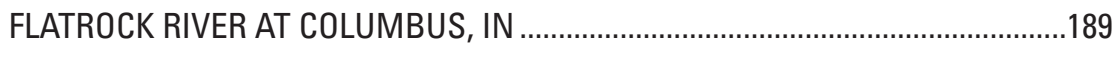 \\
\hline 03364000 & EAST FORK WHITE RIVER AT COLUMBUS, IN ........... \\
\hline 03364042 & 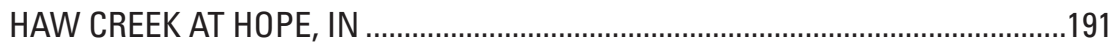 \\
\hline 03364200 & HAW CREEK NEAR CLIFFORD, IN .................. \\
\hline 03364500 & LIFTY CREEK AT HARTSVILLE, IN ..... \\
\hline
\end{tabular}




\begin{tabular}{|c|c|}
\hline 4650 & FTY CREEK NEAR COLUMBUS, IN ........ \\
\hline 365000 & AND CREEK NEAR BREWERSVILLE, IN .. \\
\hline 365500 & AST FORK WHITE RIVER AT SEYMOUR IN.... \\
\hline 366000 & IAHAM CREEK NEAR VERNON, IN... \\
\hline 03366200 & IARBERTS CREEK NEAR MADISON, IN... \\
\hline 3366500 & USCATATUCK RIVER NEAR DEPUTY, IN . \\
\hline 03368000 & BRUSH CREEK NEAR NEBRASKA, IN...... \\
\hline 03369000 & VERNON FORK MUSCATATUCK RIVER NR BUTLERVILLE, IN ... \\
\hline 03369500 & VERNON FORK MUSCATATUCK RIVER AT VERNON, IN.... \\
\hline 03371500 & EAST FORK WHITE RIVER NEAR BEDFORD, IN .. \\
\hline 03371520 & BACK CREEK AT LEESVILLE, IN .............. \\
\hline 03371600 & SOUTH FORK SALT CREEK AT KURTZ, IN.. \\
\hline 03371650 & NORTH FORK SALT CREEK AT NASHVILLE, IN.... \\
\hline 03372000 & NORTH FORK SALT CREEK NEAR BELMONT, IN . \\
\hline 03372300 & STEPHENS CREEK NEAR BLOOMINGTON, IN ...... \\
\hline 03372500 & SALT CREEK NEAR HARRODSBURG IN .. \\
\hline 03372700 & CLEAR CREEK NEAR HARRODSBURG, IN..... \\
\hline 03373200 & INDIAN CREEK NEAR SPRINGVILLE, IN . \\
\hline 03373500 & EAST FORK WHITE RIVER AT SHOALS, IN... \\
\hline 03373508 & BEAVER CREEK NEAR SHOALS, IN.. \\
\hline 03373530 & LOST RIVER NEAR LEIPSIC, IN ........... \\
\hline 03373560 & LOST RIVER NEAR PROSPECT, IN . \\
\hline 03373610 & LICK CREEK AT PAOLI, IN................... \\
\hline 03373686 & FRENCH LICK CREEK AT FRENCH LICK, IN . \\
\hline 03373695 & FRENCH LICK CREEK AT WEST BADEN SPRINGS, IN .... \\
\hline 03373700 & LOST RIVER NR. WEST BADEN SPRINGS, IN . \\
\hline 03374000 & 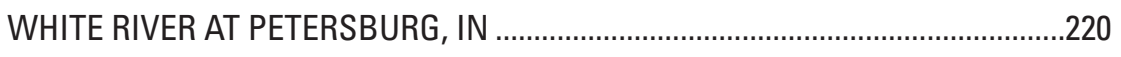 \\
\hline 03374455 & PATOKA RIVER NEAR HARDINSBURG, IN. \\
\hline 03375500 & PATOKA RIVER AT JASPER, IN ............. \\
\hline 03375800 & HALL CREEK NEAR ST. ANTHONY, IN... \\
\hline 03376260 & FLAT CREEK NEAR OTWELL, IN .............. \\
\hline 03376300 & PATOKA RIVER AT WINSLOW, IN... \\
\hline 03376350 & SOUTH FORK PATOKA RIVER NEAR SPURGEON, IN ..... \\
\hline 03376500 & PATOKA RIVER NEAR PRINCETON, IN ... \\
\hline 03377500 & WABASH RIVER AT MT. CARMEL, IL ..... \\
\hline 03378550 & BIG CREEK NEAR WADESVILLE, IN .... \\
\hline 04093000 & DEEP RIVER AT LAKE GEORGE OUTLET AT HOBART, IN .. \\
\hline 04094000 & LITTLE CALUMET RIVER AT PORTER, IN... \\
\hline 04094400 & SALT CREEK AT VALPARAISO, IN............... \\
\hline 04094500 & SALT CREEK NEAR MCCOOL, IN..... \\
\hline 04095090 & PORTAGE-BURNS WATERWAY AT PORTAGE, IN ... \\
\hline 04095300 & TRAIL CREEK AT MICHIGAN CITY, IN ..... \\
\hline 04095380 & TRAIL CREEK AT MICHIGAN CITY HARBOR, IN \\
\hline 04096100 & GALENA RIVER NEAR LAPORTE, IN ..................... \\
\hline 04097970 & ME LAKE OUTLET AT PANAMA, IN....... \\
\hline
\end{tabular}




\begin{tabular}{|c|c|}
\hline 04099510 & PIGEON CREEK NR ANGOLA, IN ... \\
\hline 04099610 & INLET NEAR STROH, IN.. \\
\hline 04099750 & IGEON RIVER NEAR SCOTT, IN..... \\
\hline 4099808 & ITLE ELKHART RIVER AT MIDDLEBURY, IN ..... \\
\hline 04099850 & PINE CREEK NEAR ELKHART, IN ................................ \\
\hline 04100222 & JB ELKHART RIVER AT COSPERVILLE, IN. \\
\hline 04100252 & 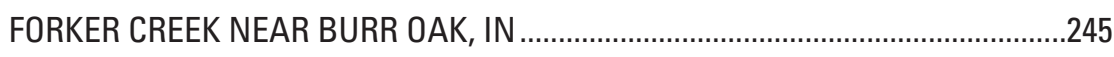 \\
\hline 04100295 & RIMMELL BRANCH NEAR ALBION, IN. \\
\hline 04100377 & SOLOMON CREEK NEAR SYRACUSE, IN ....... \\
\hline 04100465 & TURKEY CREEK AT SYRACUSE, IN .. \\
\hline 04100500 & ELKHART RIVER AT GOSHEN, IN.......... \\
\hline 04101000 & ST. JOSEPH RIVER AT ELKHART, IN ... \\
\hline 04101370 & JUDY CREEK NEAR SOUTH BEND, IN ...... \\
\hline 04177720 & FISH CREEK AT HAMILTON, IN ....... \\
\hline 04178000 & ST JOSEPH R NR NEWVILLE IN .............. \\
\hline 04179000 & ST. JOSEPH RIVER AT CEDARVILLE, IN. \\
\hline 04179500 & CEDAR CREEK AT AUBURN, IN.................. \\
\hline 04179520 & CEDAR CREEK AT 18TH STREET AT AUBURN, IN...... \\
\hline 04180000 & CEDAR CREEK NEAR CEDARVILLE, IN ............................. \\
\hline 04180500 & ST. JOSEPH RIVER NEAR FORT WAYNE, IN .. \\
\hline 04181500 & 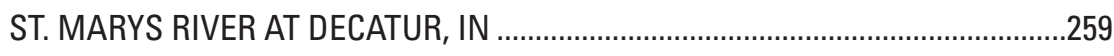 \\
\hline 04182000 & ST. MARYS RIVER NEAR FORT WAYNE, IN... \\
\hline 04182590 & HARBER DITCH AT FORT WAYNE, IN................ \\
\hline 04182769 & ST. MARYS RIVER AT MAIN ST. AT FORT WAYNE, IN................ \\
\hline 04182808 & SPY RUN CREEK NEAR PARK DRIVE AT FORT WAYNE, IN ....... \\
\hline 04182810 & 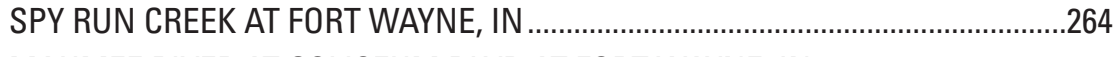 \\
\hline 04182950 & MAUMEE RIVER AT COLISEUM BLVD AT FORT WAYNE, IN .... \\
\hline 04183000 & MAUMEE RIVER AT NEW HAVEN, IN ... \\
\hline 05515000 & KANKAKEE RIVER NEAR NORTH LIBERTY, IN ..... \\
\hline 05515400 & KINGSBURY CREEK NR LAPORTE, IN... \\
\hline 05515500 & KANKAKEE RIVER AT DAVIS, IN............. \\
\hline 05516000 & YELLOW RIVER NEAR BREMEN, IN .. \\
\hline 05516500 & YELLOW RIVER AT PLYMOUTH, IN..... \\
\hline 05517000 & YELLOW RIVER AT KNOX, IN... \\
\hline 05517500 & KANKAKEE RIVER AT DUNNS BRIDGE, IN .. \\
\hline 05517530 & KANKAKEE RIVER NR KOUTS, IN... \\
\hline 05517890 & COBB DITCH NEAR KOUTS, IN ........ \\
\hline 05518000 & KANKAKEE RIVER AT SHELBY, IN .. \\
\hline 05519000 & SINGLETON DITCH AT SCHNEIDER, IN........ \\
\hline 05519500 & WEST CREEK NEAR SCHNEIDER, IN ... \\
\hline 05521000 & 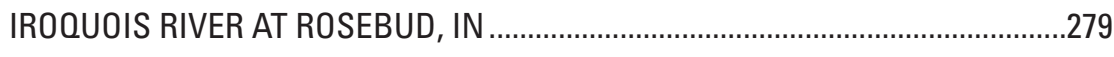 \\
\hline 05522000 & IROQUOIS RIVER NEAR NORTH MARION, IN ..... \\
\hline 05522500 & 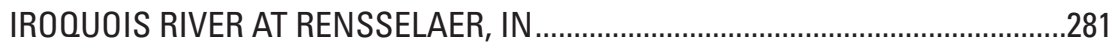 \\
\hline 05523000 & BICE DITCH NEAR SOUTH MARION, IN............ \\
\hline 05523500 & LOUGH CREEK NEAR COLLEGEVILLE, IN ... \\
\hline
\end{tabular}




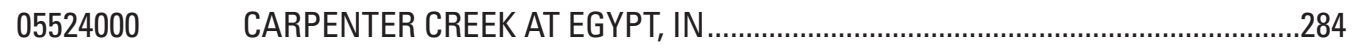

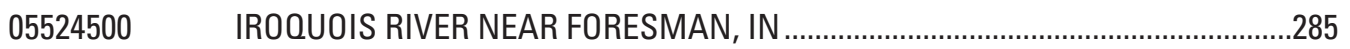

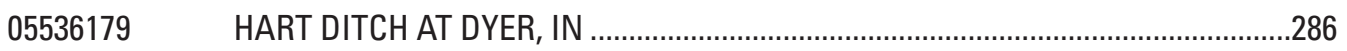

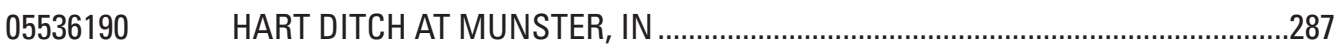

$05536195 \quad$ LITTLE CALUMET RIVER AT MUNSTER, IN.........................................................28

05536357 GRAND CALUMET RIVER AT HOHMAN AVE AT HAMMOND, IN.........................289 


\section{Index of partial-record streamgaging stations by name and number}

\begin{tabular}{|c|c|}
\hline 03323700 & boite Creek nr Aboite, IN.. \\
\hline 03329600 & Bachelor Run nr Flora, IN . \\
\hline 03335950 & Bear Creek at Fountain, IN . \\
\hline 03322800 & Bear Creek nr Bryant, IN.. \\
\hline 04178400 & Bear Creek nr Saint Joe, IN.. \\
\hline 05525650 & Beaver Creek nr Morocco, IN.. \\
\hline 03347595 & Bell Creek nr Yorktown, IN.... \\
\hline 03360920 & Big Blue River nr New Castle, IN.. \\
\hline 03332800 & Big Creek nr Monticello, IN.. \\
\hline 03366300 & Big Creek nr Volga, IN ............ \\
\hline 03332350 & Big Monon Creek nr Medaryville, IN . \\
\hline 03340651 & Big Raccoon Creek nr Ladoga, IN ..... \\
\hline 04177900 & 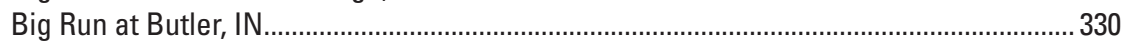 \\
\hline 03335800 & Big Shawnee Creek nr Attica, IN... \\
\hline 03357300 & Big Walnut Creek nr Barnard, IN... \\
\hline 03357400 & Big Walnut Creek nr Greencastle, IN. \\
\hline 03331350 & Big Yellow Creek nr Mentone, IN....... \\
\hline 03360050 & Birch Creek nr Ashboro, IN .. \\
\hline 03360076 & Birch Creek nr Old Hill, IN .... \\
\hline 03303050 & Bird Hollow Creek nr English, IN . \\
\hline 03324280 & Black Creek nr Warren, IN............. \\
\hline 04181100 & Blue Creek nr Pleasant Mills, IN..... \\
\hline 03327620 & Blue River nr Churubusco, IN... \\
\hline 03327770 & Blue River nr Columbia City, IN ........ \\
\hline 03361600 & Brandywine Creek nr Maxwell, IN . \\
\hline 03341420 & Brouillettes Creek nr Universal, IN. \\
\hline 03361800 & Buck Creek nr New Bethel, IN ............. \\
\hline 03334750 & Campbells Run nr Rossville, IN .. \\
\hline 04179310 & Cedar Creek at Waterloo, IN ....... \\
\hline 03331375 & Chippewanuck Creek nr Rochester, IN..... \\
\hline 03349200 & 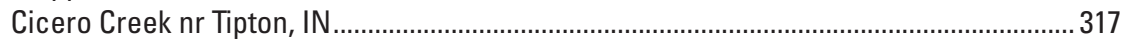 \\
\hline 03327875 & Clear Creek above Spring Creek at South Whitley, IN..... \\
\hline 03324050 & Clear Creek nr Huntington, IN. \\
\hline 03339100 & Coal Creek nr Veedersburg, IN .. \\
\hline 04093900 & Coffee Creek at Chesterton, IN .. \\
\hline 03360120 & Connelly Ditch nr Jasonville, IN..... \\
\hline 03351300 & Crooked Creek at Augusta, IN........ \\
\hline 05517750 & Crooked Creek nr Kouts, IN ............ \\
\hline 03329150 & Crooked Creek nr Logansport, IN . \\
\hline 03329100 & Crooked Creek nr Royal Center, IN.. \\
\hline 05516300 & Dausman Ditch nr Bremen, IN ............ \\
\hline 03326375 & Deer Creek at Marion, IN ................ \\
\hline 03303240 & Deer Creek nr Cannelton, IN ........ \\
\hline 03329510 & Deer Creek nr Lincoln, IN..... \\
\hline 04179308 & Dibbling Ditch nr Waterloo, IN ... \\
\hline 03360965 & Duck Creek at Greensboro, IN ..... \\
\hline 03348400 & Duck Creek at Strawtown, IN........ \\
\hline 05516950 & Eagle Creek nr Grovertown, IN ....... \\
\hline 03339110 & East Fork Coal Creek nr Veedersburg, IN......... \\
\hline 03353900 & East Fork White Lick Creek nr Mooresville, IN... \\
\hline 03327590 & Eel River nr Columbia City, IN .................................. \\
\hline 03323800 & Eightmile Creek at Zanesville, IN ........................... \\
\hline 04177800 & Fish Creek nr Artic, IN ............... \\
\hline 03357180 & Fish Creek nr Freedom, IN ........... \\
\hline 04191340 & Flatrock Creek nr Townley, IN .... \\
\hline 03363200 & Flatrock River nr Lewisville, IN ......... \\
\hline 03363400 & Flatrock River nr Rushville, IN \\
\hline 03335681 & Flint Creek nr West Point, IN .......... \\
\hline
\end{tabular}


03292400

03274900

03371525

03325700

03275850

03332440

04191360

04181600

03332480

03341580

04181900

03361900

03335682

03354200

03351900

03332250

03291800

033275554

03359980

03335683

03348100

03334300

03334330

03354140

03360200

03276750

03371530

03360115

03351490

03373600

03322880

03303190

03303160

03361400

03353631

04179800

03329580

04099805

03363450

03302600

05515100

03304000

03339408

03364711

03335674

03322860

03326480

03342700

03274800

03302700

03371550

03334700

03331780

03357385

03333100

03331430

03357700

03333400

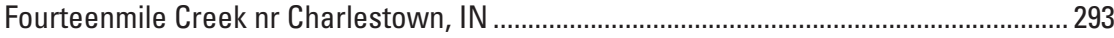

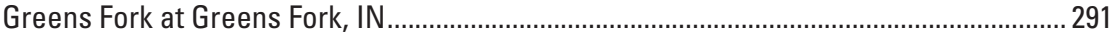

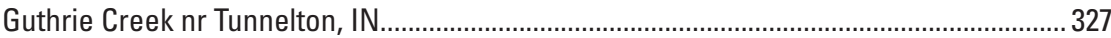

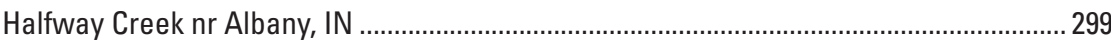

Hanna Creek nr Roseburg, IN ............................................................................................ 292

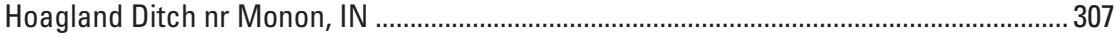

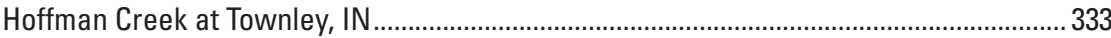

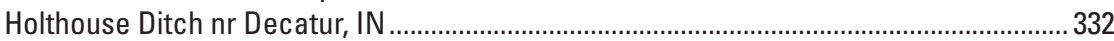

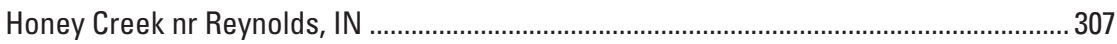

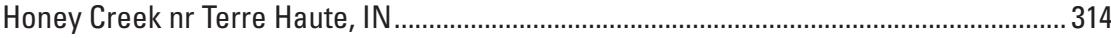

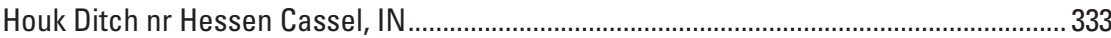

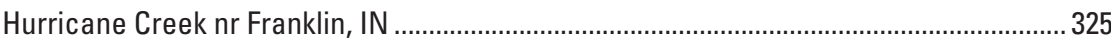

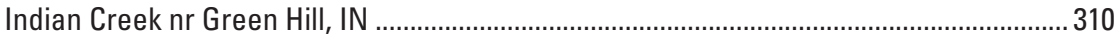

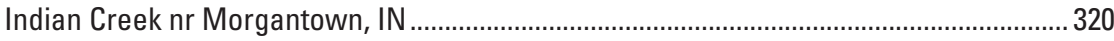

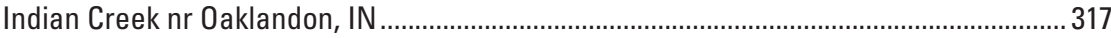

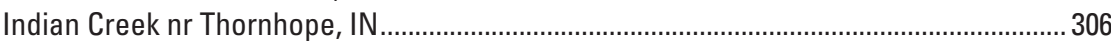

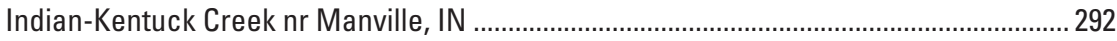

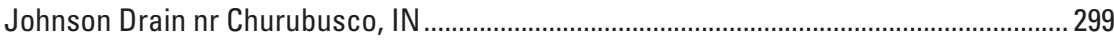

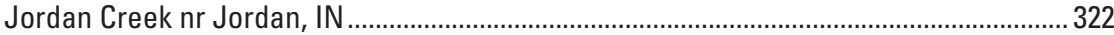

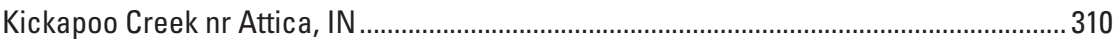

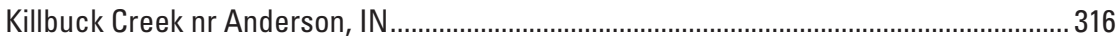

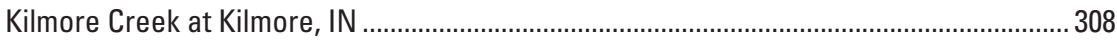

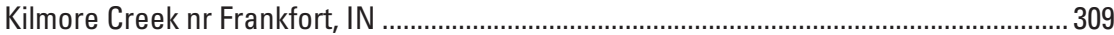

Lambs Creek nr Martinsville, IN ............................................................................................. 319

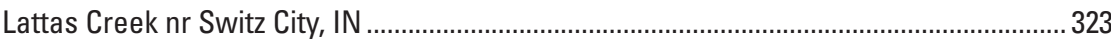

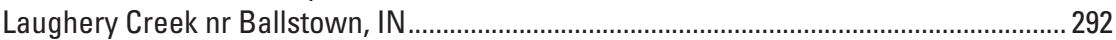

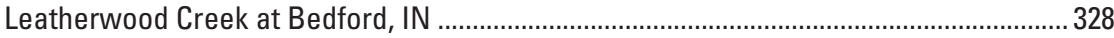

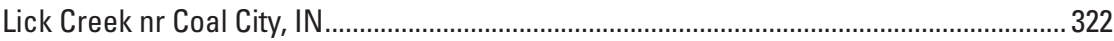

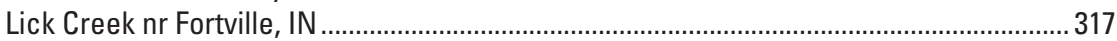

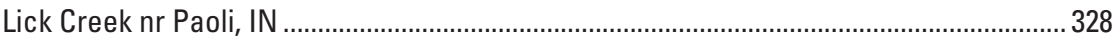

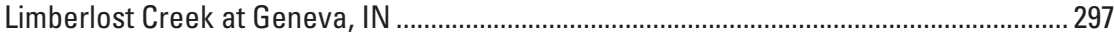

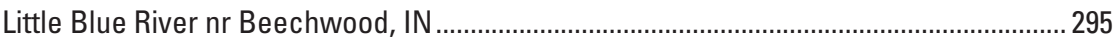

Little Blue River nr Grantsburg, IN .................................................................................... 295

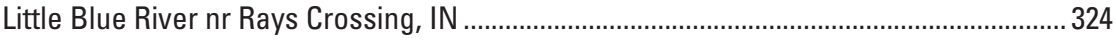

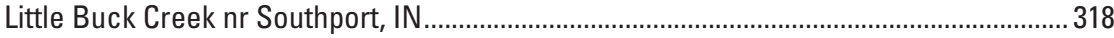

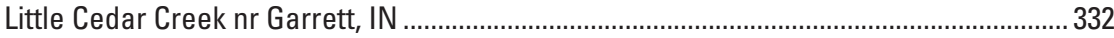

Little Deer Creek nr Camden, IN ............................................................................................... 304

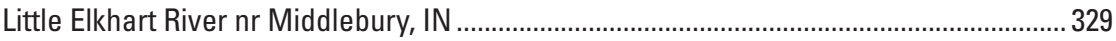

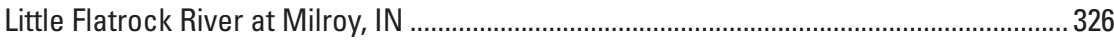

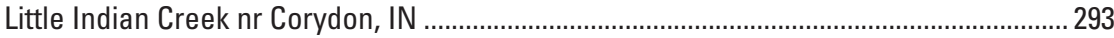

Little Kankakee River nr Mill Creek, IN ................................................................................... 334

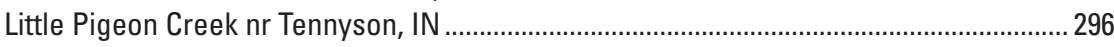

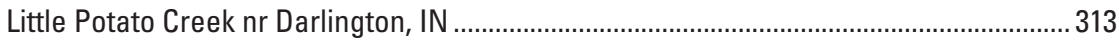

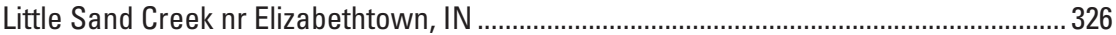

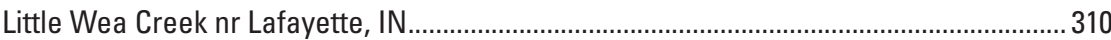

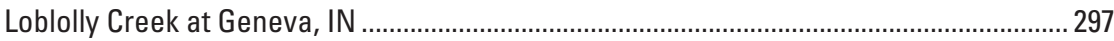

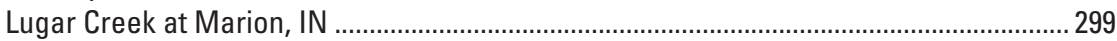

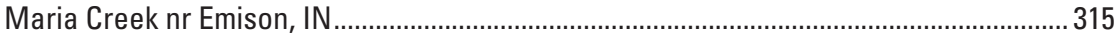

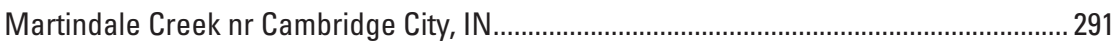

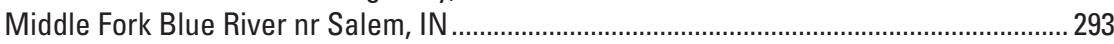

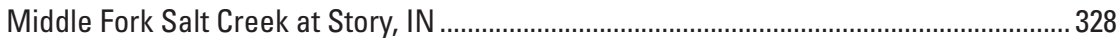

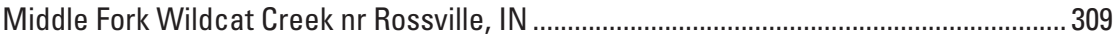

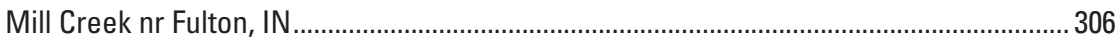

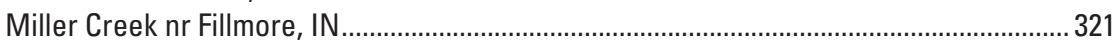

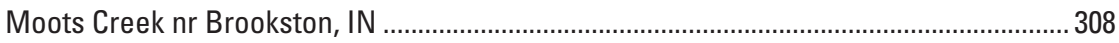

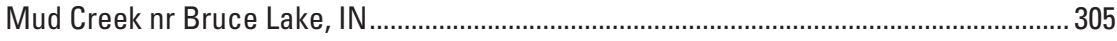

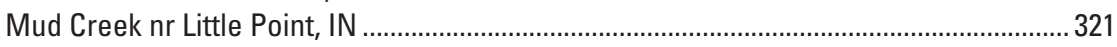

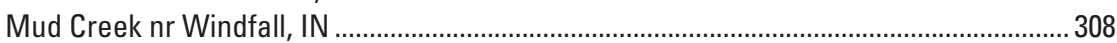




\begin{tabular}{|c|c|}
\hline 04181800 & Jickelsen Creek nr Poe, IN \\
\hline 03303950 & Otter Creek nr DeGonia Springs, IN . \\
\hline 0336940050 & 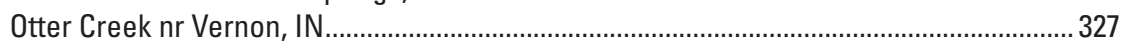 \\
\hline 3328420 & aw Paw Creek nr Roann, IN. \\
\hline 03322050 & Pigeon Creek nr Buckskin, IN . \\
\hline 4099070 & Pigeon Creek nr Hamilton, IN. \\
\hline 3332550 & Pike Creek nr Norway, IN... \\
\hline 3348300 & Pipe Creek nr Alexandria, IN .... \\
\hline 03353650 & Pleasant Run at Greenwood, IN . \\
\hline 3339300 & Prairie Creek at Thorntown, IN ... \\
\hline 3341800 & Prairie Creek nr Prairie Creek, IN.. \\
\hline 03360800 & Prairie Creek nr Washington, IN.. \\
\hline 3331750 & Quigley Marsh Ditch at Winamac, IN. \\
\hline 05517550 & Reeves Ditch nr LaCrosse, IN . \\
\hline 3329300 & ek nr Rockfield, IN..... \\
\hline 4099692 & owe Ditch nr Howe, IN.......... \\
\hline 03324250 & ie River at Montpelier, IN. \\
\hline 03275200 & alt Creek nr Metamora, IN............ \\
\hline 03364800 & and Creek nr Greensburg, IN .. \\
\hline 03275700 & ver Creek nr Liberty, IN ............ \\
\hline 05518500 & ngleton ditch nr Hebron, IN. \\
\hline 03322980 & Sixmile Creek nr Bluffton, IN .... \\
\hline 03302730 & South Fork Blue River nr Palmyra, IN \\
\hline 03329530 & South Fork Deer Creek at Galveston, IN \\
\hline 03327876 & Spring Creek below Clear Creek at South Whitley, IN................. \\
\hline 03327853 & Spring Creek nr Columbia City, IN ... \\
\hline 03302900 & ring Creek nr White Cloud, IN.... \\
\hline 04178500 & St. Joseph River at Hursh, IN .. \\
\hline 03346835 & Stoney Creek at Windsor, IN ....... \\
\hline 03353665 & Stotts Creek nr Martinsville, IN.... \\
\hline 033673005 & Stucker Fork at Scottsburg, IN..... \\
\hline 03327900 & Sugar Creek at South Whitley, IN.. \\
\hline 03339200 & Sugar Creek nr Kirklin, IN .. \\
\hline 03361700 & Sugar Creek nr Pleasant View, IN ... \\
\hline 03340200 & Sugar Mill Creek nr Wallace, IN ....... \\
\hline 03276630 & inners Creek nr Guilford, IN... \\
\hline 04100490 & Turkey Creek nr New Paris, IN........ \\
\hline 03341920 & Turman Creek nr Farmersburg, IN. \\
\hline 03328470 & Twelve Mile Creek nr Hoover, IN .... \\
\hline 03339460 & Walnut Fork Sugar Creek nr Crawfordsville, IN \\
\hline 03335670 & Wea Creek nr Lafayette, IN .......... \\
\hline 03302850 & Whiskey Run at Milltown, IN ........... \\
\hline 03365600 & White Creek nr Cortland, IN ..... \\
\hline 03353670 & reek nr Brownsburg, IN .. \\
\hline 03346700 & White River nr Harrisville, IN................ \\
\hline 05515499 & Whitham Ditch nr Hanna, IN ........... \\
\hline 04179900 & $\ldots$ \\
\hline 05516650 & Wolf Creek nr Argos, IN ... \\
\hline 05517880 & Wolf Creek nr Kouts, IN....... \\
\hline 04100800 & Yellow Creek at Dunlap, IN .. \\
\hline
\end{tabular}




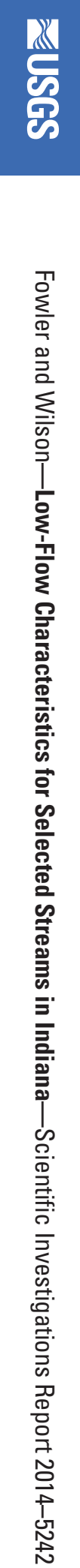